\title{
Linguistic functions of head and body movements in Austrian Sign Language (ÖGS). A corpus-based analysis.
}

\section{Dissertation}

zur Erlangung des Doktorgrades der Philosophie an der Karl-Franzens-Universität Graz

eingereicht von Mag. ${ }^{\text {a Andrea Lackner }}$

am

Institut für Sprachwissenschaft

Erstbegutachter: O.Univ.-Prof. Dr. Bernhard Hurch Zweitbegutachterin: Prof. Dr. Ronnie B. Wilbur 


\section{Acknowledgements}

In the following, I would like to thank all the 'helping hands' for their guidance and support in identifying, describing, and documenting all these insights into the Austrian Sign Language.

First of all, I want to say thanks to the members of the 'Pongauer Gehörlosenverein' ('Pongau Deaf Club') who engaged in both signing various utterances as well as annotating these signed texts with the greatest enthusiasm. Their work resulted in various natural and authentic recordings and very detailed annotation entries in ELAN. In particular, I would like to thank the Deaf club’s chairman Hans Scharfetter and his wife Elisabeth. They took the initiative and organized a group of Deaf informants who were willing to perfom in front of a camera. They also arranged all the recording and annotating sessions, and they most intensively discussed each language-relevant head and body movement with me. They have always been very welcoming to me on my home visits to the Salzburg area, where I grew up. We have shared many hours of discussions with respect to sign language or Deaf culture. I am very much looking forward to continuing this close cooperation!

Also sincere thanks go to my supervisors Prof. Ronnie Wilbur, Purdue University, and Prof. Bernhard Hurch, University of Graz, who both supported me in various ways. Ronnie Wilbur, thank you very much for commenting and discussing ALL the new findings on head and body movements in the Austrian Sign Language in MORE than one run-through. Also, I want to thank you for providing me with a large variety of sign language research and for motivating and supporting me in writing this thesis in English. Bernhard Hurch, thank you very much for welcoming me to do sign language research at the Department of Linguistics in Graz, for providing comments and open discussions on my thesis, and for showing confidence in my research work. At the University of Graz, I also want to say thanks to the other professors and assistant professors doing their work in different 
linguistic fields. I thank you all for always being willing to give me linguistic advice. A sincere thank-you also goes to Prof. Utz Maas in particular.

As for the other universities involved, I want to thank in particular those who have cordially participated in a vivid exchange about my sign language research. This positive feedback has motivated me in going on with my investigations on the nonmanuals in ÖGS for both my $\mathrm{PhD}$ thesis and the project on segmentation and structuring of ÖGS-texts which I am responsible for, and which is primarily conducted by me. In this course, I want to thank Prof. em. Dr. Bernd Heine, University of Cologne, Dr. Penny Boyes Braem, Centre of Sign Language Research in Basel, and Dr. O.A. Onno Crasborn, Radboud University Nijmegen, for their inspiring conversations on doing sign language research. Also a thank-you goes to all those researchers whom I met at various sign language conferences and meetings (i.e. the members of the COST action IS1006 meetings arranged in order to design a blue print for a sign language grammar) and who provided me with various information regarding sign language and motivated me in going on with my research work. I am looking forward to collaborating with some of you in the near future in order to do further fascinating cross-linguistic sign language research.

A special thank goes to Wolfgang Puschnig who recorded all the sessions, cut and converted a part of the data, and who supported me whenever technical advice was needed.

I would like to thank all the members of the ongoing project on segmentation and structuring ÖGS-texts who supported me by doing annotations, revising parts of my thesis, and discussing contents with me. To begin with, I want to thank Christian Stalzer who has always been present for discussing sign language-relevant matters. Thanks to Anna Wiener, Lena Mallinger, and Isabel Graf for revising my thesis and assisting with the data annotation and translation. A sincere thank-you goes to Nikolaus Riemer who supported me by annotating the nonmanuals of parts of the data. Also, I want to thank Prof. Franz Dotter, University of Klagenfurt, who facilitated the implementation of my thesis paper 
into the context of the project on segmentation and structuring of ÖGS-text, which he is the head of. The results surely will support the findings of this project.

Several colleagues and friends also supported me in various matters. A great thank you goes to Kristina Henschke and Andrea Schmidt who revised various chapters of my thesis. Also, I want to thank Petra Hödl for revising the reference list and constantly offering support on various linguistic matters. A special thank-you goes to Ingeborg Fink, University of Cologne, who discussed various linguistic matters with me - and, first of all, I want to thank her for being there with me on my roller coaster ride through the 'writing a thesis' project. I do hope we will continue our 'working sessions' - even if we have to do this via Skype now and not comfortably at one of our homes. Also, I want to thank Claudia Kreš-Edlinger for discussing linguistic matters and offering support when writing the thesis, as well as Anke Jackschinak for joining in on the mutual motivation for writing a (MA / PhD) thesis.

Furthermore, I want to thank the Federal State Government of Salzburg for a financial support of 1.000 Euro which was a nice compensation for the Deaf informants for doing their annotation tasks.

Finally, I really thank my family who supported me in doing this work. In particular, I thank both grannies and grandpa who took care of my kids while I took the time to continue my research work.

Concluding, I want to dedicate my thesis first to my family - Franz and my boys Florian and Jakob. Florian, you entered our lives in 2006, and in the same year I finished my MA thesis and started my linguistic work on sign language research. Jakob, you joined our family in 2011. In this year our proposal for the project on segmentation and structuring ÖGS-text was accepted by the Austrian Science Fund (FWF) and consequently, a new stage in sign language research started for me. In addition, in that year I did most of the annotation tasks with the Deaf annotators and started to realize the enormous scope of 
contents which can be coded by head and body movements in a sign language. These results are now all put on paper and show brand-new insights of coding various functions in Austrian Sign Language research, but new findings in sign language research are also present in general.

This thesis is also dedicated to the Austrian Deaf Community - especially to the Deaf Community of Pongau. You should be proud on your abilities to code an enormous scope of contents in YOUR language by non-manual means. 


\title{
Dedication
}

\author{
Gewidmet der \\ Österreichischen Gehörlosen- und Gebärdensprachgemeinschaft \\ und meiner Familie „Franz, Florian und Jakob“
}

(Dedicated to

the Austrian Deaf and Signing Community and to my family 'Franz, Florian, and Jakob’) 


\section{Abstract}

This thesis presents various functions of head and body movements in Austrian Sign Language (ÖGS) identified within a corpus-based analysis.

With regard to methodology, an empirical and inductive approach was followed. First, head and body movements recorded in narratives and lectures from 13 fluent ÖGS signers were identified by various Deaf annotators. They were described with regard to their form, meaning, context of occurrence and co-occurrence with other markers. Based on the common characteristics three groups of markers were identified: the first comprises headshakes coding negation, implying negative contrast, expressing lack of knowledge in wh-questions, incredibility and a few more, head nods coding assertion and implying positive contrast, chin down indicating direct polar questions, chin up and/or head forward indicating direct and embedded content questions, head forward coding embedded polar interrogatives and also conditionals, and head backward coding a special interrogative. The second group includes head and body markers which have in common that they are directed by the signing space. They are used to indicate hypothetical thoughts and alternatives. The third classified group is a set of head and body markers used to code modality. It includes an assertive head marker, a non-assertive head marker, a speculative body marker, a timitive head marker (all coding epistemic modality) and a possibility marker (coding deontic modality).

The thesis concludes with summarizing that the 'head' is one of the most structured nonmanual markers in ÖGS. It is of special interest that different constructions are indicated by the same marker. For instance, positioning the head forward can indicate interrogativity, irreality, conditionality, and exclamation - all together functions which show semantic contiguity/relation. 


\section{Abstract in German}

Die vorliegende Dissertation ist eine korpusbasierte Beschreibung und Analyse verschiedener Kopf- und Körperbewegungen in der Österreichischen Gebärdensprache (ÖGS).

Einem empirisch-induktiven Ansatz folgend, werden zuerst sprachrelevante Kopf- und Körperbewegungen von muttersprachlichen Informanten identifiziert und beschrieben. Im Anschluss wird jede Kopf- und Körperbewegung hinsichtlich ihrer Form, ihrer Funktion, ihrem Auftreten in verschiedenen Kontexten und ihrer Kookkurrenz mit anderen nichtmanuellen Komponenten analysiert. In der Folge werden die Kopf- und Körperbewegungen anhand ihrer Charakteristika klassifiziert. Generalisierungen zeigen das Ausmaß, in dem die non-manuellen Indikatoren in die Struktur der ÖGS integriert sind. Die identifizierten Kopf- und Körperbewegungen lassen sich in drei Gruppen kategorisieren. Parameter der Klassifizierung sind neben der Funktion auch die Regelmäßigkeit der Bewegungsausführung und die Beziehung der non-manuellen Marker zum Gebärdenraum. Die erste Gruppe umfasst jene Kopf- und Körperbewegungen, welche Negation, Assertion, Interrogativität, Irrealität und Konditionalität ausdrücken und regelmäßig in ihrer Ausführung sind. Der zweiten Gruppe gehören jene Sprachelemente an, die auf den Gebärdenraum kalibriert sind und folglich den Artikulator wechseln können. Sie werden eingesetzt, um hypothetische Gedanken oder Alternativen anzuzeigen. Die dritte Gruppe enthält jene Kopf- und Körperbewegungen, welche vorrangig epistemische Modalität kodieren und unregelmäßig in der Art der Ausführung sind.

Die wichtigsten Ergebnissen der Arbeit sind: Die bisher in der Forschung wenig beachteten Kopfmarker zählen zu den meist strukturierten nicht-manuellen Komponenten in der ÖGS. Bestimmte Sprachfunktionen, welche eine semantische Kontiguität aufweisen, werden mit demselben Kopfmarker kodiert. 


\section{Table of contents}

Acknowledgments $\quad 2$

Dedication $\quad 6$

$\begin{array}{ll}\text { Abstracts } & 7\end{array}$

Table of contents $\quad 9$

List of figures $\quad 15$

List of tables $\quad 17$

$\begin{array}{lr}\text { List of abbreviations } & 18\end{array}$

$\begin{array}{ll}\text { Annotation conventions } & 20\end{array}$

1 Introduction 24

1.1 Subject matter, research question, hypotheses, and objectives 24

1.2 Research on Austrian Sign Language 28

$\begin{array}{ll}1.3 \text { Organization of the present thesis } & 29\end{array}$

2 Research objective and methodology 34

2.1 The research objective $\quad 34$

2.1.1 Possible head and body movements $\quad 34$

2.1.1.1 Head movements $\quad 34$

2.1.1.2 Body movements 37

2.1.1.3 Shoulder movements 38

2.1.2 Sign Language research on head and body movements 39

2.1.3 Language-relevant head and body movements in ÖGS 42

2.1.3.1 Head movements in ÖGS 42

2.1.3.2 Body movements in ÖGS 46

2.1.3.3 Shoulder movements in ÖGS 49

2.2 Methodology 51

2.2.1 The approach $\quad 51$

2.2.2 The Deaf informants/annotators 58

2.2.3 Excursion: The valley of 'Großarl' 60

2.2.4 The data 64

2.2.4.1 Video recording process and data preparation 64

2.2.4.2 Resulting data 66

2.2.4.3 Procedure of data coding $\quad 68$

$\begin{array}{ll}\text { 2.2.4.4 Results of data coding } & 71\end{array}$ 


\section{Negation and assertion coded by headshakes and head nods}

$\begin{array}{ll}3.1 \text { Negation } & 75\end{array}$

$\begin{array}{ll}3.1 .1 \text { Introduction } & 75\end{array}$

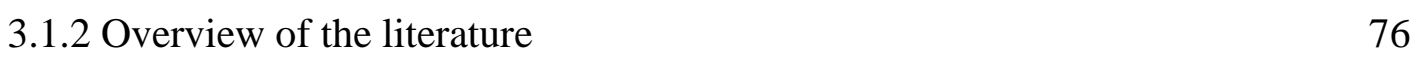

$\begin{array}{ll}\text { 3.1.3 Clause negation coded by headshakes } & 78\end{array}$

3.1.3.1 Different types of negative headshakes $\quad 78$

$\begin{array}{ll}\text { 3.1.3.2 Clause negation as main function of headshakes } & 79\end{array}$

3.1.3.3 Clause negation marked by co-occurring negative 81 headshakes

3.1.3.4 Negative headshakes with frequently co-occurring 85 negative signs

3.1.3.5 Negative headshakes spreading along the entire clause 91

3.1.3.6 Addition: Tricky negation 99

$\begin{array}{lr}\text { 3.1.4 Speech act negation coded by headshakes } & 100\end{array}$

$\begin{array}{ll}\text { 3.1.5 Negative contrast coded by headshakes } & 101\end{array}$

$\begin{array}{ll}\text { 3.1.6 Other functions coded by headshakes } & 108\end{array}$

$\begin{array}{lr}3.1 .7 \text { Conclusions } & 110\end{array}$

3.2 Assertion 113

3.2.1 Introduction 113

$\begin{array}{ll}3.2 .2 \text { Overview of the literature } & 114\end{array}$

$\begin{array}{ll}\text { 3.2.3 Assertion coded by head nods } & 115\end{array}$

3.2.3.1 Different types of assertive head nods and places of 115 occurrence

3.2.3.2 Co-occurrence of assertive head nods $\quad 118$

3.2.3.3 Assertive head nods and other head movements coding 119 other functions

$\begin{array}{ll}\text { 3.2.3.4 Other assertive markers } & 120\end{array}$

$\begin{array}{ll}\text { 3.1.4 Speech act assertion coded by head nods } & 121\end{array}$

$\begin{array}{ll}\text { 3.2.5 Positive contrast coded by head nods } & 122\end{array}$

$\begin{array}{ll}\text { 3.2.6 Distinguishing between assertion and confirmation } & 123\end{array}$

$\begin{array}{ll}3.2 .7 \text { Conclusions } & 125\end{array}$

$\begin{array}{lr}3.3 \text { Assertion and negation compared } & 127\end{array}$ 


\section{Interrogativity coded by head markers}

4.1 Overview

$\begin{array}{lr}\text { 4.1.1 Introduction } & 130\end{array}$

$\begin{array}{ll}\text { 4.1.2 Literature overview } & 131\end{array}$

4.1.3 Investigated interrogatives in ÖGS 134

4.2 Direct questions in ÖGS 136

4.2.1 Polar questions 136

4.2.1.1 Marking polar question with ‘chin down’ 136

4.2.1.2 Marking polar question and other co-occurring head 138 markers

4.2.1.3 Discourse control with polar questions $\quad 141$

$\begin{array}{ll}\text { 4.2.2 Content questions } & 143\end{array}$

4.2.2.1 Marking content questions with ‘chin up’ and/or 'head 143 forward'

4.2.2.2 Marking content question and other co-occurring head $\quad 145$ markers

$\begin{array}{lc}\text { 4.2.3 Conclusions on direct questions } & 148\end{array}$

4.3 Embedded interrogatives in ÖGS 150

$\begin{array}{lr}\text { 4.3.1 Embedded polar interrogatives } & 150\end{array}$

$\begin{array}{ll}\text { 4.3.1.1 Introduction } & 150\end{array}$

4.3.1.2 The interrogative markers 153

4.3.1.2.1 The marker 'head forward' 154

4.3.1.2.2 The additional marker 'brow raise' 167

4.3.1.2.3 The marker 'ob' ('whether') 171

4.3.1.3 The characteristics of the embedded clause 173

4.3.1.3.1 Semantics of alternativity 173

4.3.1.3.2 Filled syntactic positions $\quad 177$

4.3.1.3.3 Special use of the hypothetical space $\quad 180$

$\begin{array}{ll}\text { 4.3.2 Embedded content interrogatives } & 181\end{array}$

$\begin{array}{ll}\text { 4.3.2.1 Introduction } & 181\end{array}$

4.3.2.2 Marking of embedded content interrogatives with 'chin 181

up' and/or 'head forward'

4.3.2.3 The characteristics of the embedded clause 185

4.3.2.4 Distinguishing embedded content interrogatives from 186 other constructions

$\begin{array}{ll}\text { 4.3.3 Conclusions on embedded interrogatives } & 191\end{array}$

4.4 A special interrogative marked with 'head backward' 195

4.4.1 Marking with 'head backward' 195

4.4.2 The characteristics when marking an interrogative with 'head 197

backward'

4.4.3 Conclusions on interrogatives marker with 'head backward' 198 
5 Conditionality coded by a head marker

5.1 Introduction 201

5.2 Literature overview 202

5.3 The types of conditionals 204

5.4 The conditional markers 209

5.4.1 The marker 'head forward' 209

5.4.2 The alternative/additional marker 'brow raise' 212

$\begin{array}{ll}\text { 5.4.3 The additional marker 'shoulder forward' } & 214\end{array}$

5.4.4 The maker 'wenn' ('if') 215

$\begin{array}{ll}5.5 \text { Consequent clauses } & 216\end{array}$

$\begin{array}{ll}\text { 5.5.1 Statement as consequent } & 217\end{array}$

$\begin{array}{ll}\text { 5.5.2 Interrogative or other clauses as consequent } 218 & 217\end{array}$

5.6 Other functions marked within conditionals 222

$\begin{array}{ll}\text { 5.6.1 Marking negation and assertion in conditionals } & 222\end{array}$

$\begin{array}{ll}\text { 5.6.2 Marking alternativity in conditionals } & 230\end{array}$

5.6.3 Marking modality in conditionals 232

5.7 Conclusions on conditionals 235

\section{Constructions based on space and coded by head and body markers}

6.1 Introduction 238

$\begin{array}{ll}\text { 6.2 Multifunctional use of space } & 238\end{array}$

$\begin{array}{ll}\text { 6.2.1 Spatial and linguistic use of space } & 238\end{array}$

6.2.2 Research on the spatial and linguistic use of space in ÖGS 239

6.2.3 Interferences of spatial and linguistic use of space 241

$\begin{array}{ll}\text { 6.2.4 Multifunctional use of spatial indicators } & 243\end{array}$

6.3 The syntactic/textual/discourse use of space $\quad 244$

6.3.1 The various linguistic uses of the signing space on 244 syntactic/textual/discourse level

$\begin{array}{ll}\text { 6.3.2 Common characteristics } & 245\end{array}$

$\begin{array}{ll}\text { 6.3.3 Resulting indicators } & 248\end{array}$

6.3.3.1 Orientation-toward-indicators 249

6.3.3.2 Moving-toward-indicators 253

6.3.3.3 Pointing elements to location 254

6.3.4 Conclusions on the syntactic/textual/discourse use of space 256

6.4 Space as discrete medium 259

6.4.1 The hypothetical space and the alternative space 259

6.4.2 Characteristics of the indicators for the hypothetical and 259 alternative space 
6.4.2.1 Beginning or domain marking

6.4.2.2 Orientation toward location marking / moving toward location marking / pointing

6.4.3 The hypothetical space

6.4.3.1 Location of the hypothetical space 260

6.4.3.2 Indicators and characteristics $\quad 262$

6.4.3.3 Functional use 268

6.4.3.4 Conclusions on the hypothetical space 270

$\begin{array}{ll}\text { 6.4.4 The alternative space } & 270\end{array}$

6.4.4.1 Locations of the alternative space 270

6.4.4.2 Indicating one or more alternative locations 275

$\begin{array}{ll}\text { 6.4.4.3 Indicators and characteristics } & 279\end{array}$

6.4.4.4 Functional use $\quad 290$

6.4.4.5 The underlying subject matters of the alternative space 300

6.4.4.6 Differentiation of listing 301

6.4.4.7 Conclusions on the alternative space 304

6.5 Conclusions on spatial cues primarily coded by head and body markers 304

\section{Modality coded by head and body markers 309}

$\begin{array}{ll}7.1 \text { Introduction } & 309\end{array}$

$\begin{array}{ll}\text { 7.2 Literature overview } & 310\end{array}$

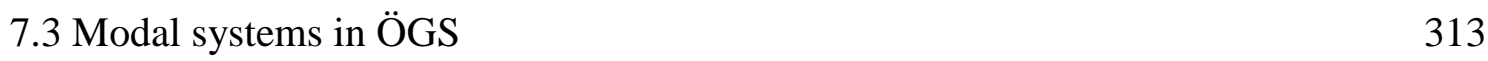

$\begin{array}{ll}\text { 7.3.1 Modality signs } & 314\end{array}$

$\begin{array}{ll}\text { 7.3.2 Modality coded by nonmanuls } & 317\end{array}$

7.4 Types of modality coded by the head and body movements in ÖGS 324

$\begin{array}{ll}\text { 7.4.1 Introduction } & 324\end{array}$

$\begin{array}{ll}\text { 7.4.2 Convinced-assertive head marker } & 326\end{array}$

$\begin{array}{ll}\text { 7.4.3 Non-assertive head marker } & 333\end{array}$

$\begin{array}{ll}\text { 7.4.4 Speculative body marker } & 338\end{array}$

$\begin{array}{ll}\text { 7.4.5 Timitive head marker } & 345\end{array}$

$\begin{array}{ll}\text { 7.4.6 Possibility head/body marker } & 347\end{array}$

7.5 Conclusions on modality markers in ÖGS 351 


\section{New insights, summary and generalizations on head and body markers}

8.1 New insights 355

8.2 Summary 358

$\begin{array}{ll}\text { 8.3 Generalizations on head and body markers } & 369\end{array}$

8.3.1 The 'head' as one of the most structured non-manual markers in $\quad 370$

ÖGS

8.3.2 Classification of head and body movements/positions 370

8.3.3 Semantic contiguity 373

8.3.4 Co-occurrence and sequential occurrence of head/body marker $\quad 379$

$\begin{array}{ll}8.4 \text { Future research } & 381\end{array}$

Appendix A: Corpora 386

Appendix B: Metadata of the participants from Großarl 388

$\begin{array}{ll}\text { Appendix C: Questionnaire } & 390\end{array}$

Appendix D: Preliminary study on the identification of head and body 396

movements in ÖGS

References 


\section{List of figures}

Figure 2.1 Possible head movements 35

$\begin{array}{lll}\text { Figure 2.2 Movements of the trunk } 38 & 38\end{array}$

Figure 2.3 Lateral extension achieved by sideward movements 38

Figure 2.4 Possible shoulder movements 39

Figure 2.5 Up/down movement/s of the head 43

Figure 2.6 Sideward movement/s of the head 44

Figure 2.7 Tilting movements of the head 44

Figure 2.8 Head forward/backward positions/movements 45

Figure 2.9 Special forms of head movements 46

Figure 2.10 Forward movement/s of the body 47

Figure 2.11 Turn movements of the body 47

Figure 2.12 Sideward movement/s of the body 48-49

Figure 2.13 Shoulder positions and movement/s; body straightening 50

Figure 2.14 Illustration of non-manual markers in sign language literature 52

Figure 2.15 Photographs of the Deaf inhabitants of the valley Großarl 61

Figure 2.16 Annotations of the different Deaf annotators with regard to the $\begin{array}{ll}\text { various head and body tiers } & 70\end{array}$

Figure 3.1 Co-occurrence of negative headshakes and a negation sign 82

Figure 3.2 Possible spreading of the non-manual negator headshakes in 83

Figure 3.3 The manual negator NEIN (NO) 85

Figure 3.4 Sign of cognition with manual negator NO 86

$\begin{array}{lll}\text { Figure } 3.5 & \text { Sign of knowledge and negation } & 87\end{array}$

$\begin{array}{lll}\text { Figure } 3.6 & \text { The manual negator NICHT/KEIN (NOT/NO) } & 88\end{array}$

$\begin{array}{lll}\text { Figure 3.7 Morphological negation } & 90\end{array}$

Figure 3.8 Scalar of assertive/positive versus non-assertive/negative presupposition compared to assertive/positive contexts versus non-assertive/negative contexts $\quad 94$

$\begin{array}{lll}\text { Figure 3.9 } & \text { Marking of contrast in adversative constructions } & 104\end{array}$

$\begin{array}{lll}\text { Figure 3.10 The signs RATHER and ANYWAY } & 107\end{array}$

$\begin{array}{lll}\text { Figure } 3.11 & \text { A fast single headshake } & 109\end{array}$

Figure 3.12 Affirmative 'body lean forward' with negation 127

Figure 3.13 Head nods and headshakes compared 129

$\begin{array}{lll}\text { Figure 4.1 } & \begin{array}{l}\text { Marking of an embedded polar interrogative versus a direct } \\ \text { polar question }\end{array} & 138\end{array}$

$\begin{array}{lll}\text { Figure 4.2 Head markers in polar questions } & 139\end{array}$

$\begin{array}{lll}\text { Figure 4.3 The marker for content questions } & 144\end{array}$

Figure 4.4 The marker 'chin up' (\& slightly 'head forward') 145

$\begin{array}{lll}\text { Figure 4.5 Intra-individual perception of marking embedded polar } & 151 \\ & \text { interrogatives by 'head forward' }\end{array}$ 
Figure 4.6 Illustration of positioning forward of the head during the requested part

Figure 4.7 Prototypical arrangement of the lexical elements covered by the hf-marker

Figure 4.8 Br-marker covers one offered option

Figure 4.9 Br-marker covers two offered options

Figure 4.12 Lexical elements of the embedded part (or complement clause)

Figure 4.13 Prototypical illustration of polar interrogatives in direct communication

Figure 4.14 The embedded interrogative markers 'head forward' and 'chin up'

Figure 4.15 Head forward covering the entire embedded content interrogative construction

Figure 4.17 Embedded content interrogative characterized by chin up / head forward, gaze up or to the front, and being linked to an expression of perception

Figure 4.18 The interrogative markers 'head backward'

Figure 5.1 Marking of a hypothetical conditional

Figure 6.1 Spatial and linguistic use of the signing space

Figure 6.5 Head marker 'head turn sideward' used for indicating the referential space 
Figure 6.14 Indicating two alternative spaces

$282-283$

Figure 6.15 Pointing sign for alternative space 285

Figure 6.16 Dominance reversal and buoys used for alternative spaces 286

$\begin{array}{lll}\text { Figure 6.17 Moving of the listing signs towards the three alternative spaces } & 288\end{array}$

$\begin{array}{lll}\text { Figure 6.18 Listing signs constitute the the three lateral alternative spaces } & 289\end{array}$

Figure 6.19 Opposing activities by using the alternative space 293

Figure 6.20 A and B constitute C 295

Figure 6.21 Use of space for displaying two temporal sequential activities 296

Figure 6.22 Use of space for displaying two complex temporal sequential $\begin{array}{ll}\text { activities } & 297\end{array}$

Figure 6.23 Listing question signs 303

Figure 6.24 Indicating to the 'hypothetical space' 305

$\begin{array}{lll}\text { Figure 7.1 } & \text { Fast, small head nodding movements } & 327\end{array}$

$\begin{array}{lll}\text { Figure 7.2 Slow, large head nodding movements } & 327\end{array}$

$\begin{array}{lll}\text { Figure 7.3 Co-occurring nonmanuals with the convinced-assertive marker } & 329\end{array}$

Figure 7.4 The speculative marker with deliberative meaning 339

Figure 7.5 The speculative marker with indecisive/doubtful meaning 339

Figure 7.6 The timitive marker 346

Figure 7.7 POSSIBLE and MAYBE in ÖGS 349

Figure 7.8 POSSIBLE and MAYBE in ASL 349

Figure 7.9 Nonmanuals coding modality meaning 352

Figure 8.1 Semantic mapping of head markers in ÖGS 375

$\begin{array}{lll}\text { Figure 8.2 Antecedent-consequent-sequence } 378 & 375\end{array}$

$\begin{array}{lll}\text { Figure 8.3 Question-answer-sequence or antecedent-consequent-sequence } & 378\end{array}$

Figure 8.4 Ongoing head nods/rotations downward to upward 383

Figure 8.5 Correlation of movements of different articulators 384

\section{List of tables}

Table 5.1 Negation and implying negative constrast in conditionals

Table 6.1 Differences/similarities between the alternative/hypothetical space and the referential space

Table 7.1 A set of head and body markers coding modality 


\section{List of abbreviations}

\section{Abbreviations of sign languages:}

$\begin{array}{ll}\text { ASL } & \text { American Sign Language } \\ \text { Auslan } & \text { Australian Sign Language } \\ \text { BSL } & \text { British Sign Language } \\ \text { [DSL } & \text { Danish Sign Language] } \\ \text { DGS } & \text { German Sign Language (Deutsche Gebärdensprache) } \\ \text { FinSL } & \text { Finnish Sign Language } \\ \text { GSL } & \text { Greek Sign Language } \\ \text { HKSL } & \text { Hong Kong Sign Language } \\ \text { HZJ } & \text { Croatian Sign Language (Hrvatski znakovni jezik) } \\ \text { IPSL } & \text { Indo-Pakistani Sign Language } \\ \text { ISL } & \text { Irish Sign Language } \\ \text { ISL } & \text { Israeli Sign Language } \\ \text { JSL } & \text { Japanese Sign Language } \\ \text { Kata Kolok } & \text { Kata Kolok (village-based sign language in Bali) } \\ \text { LIS } & \text { Italian Sign Language (Lingua dei Segni Italiana) } \\ \text { LIU } & \text { Jordanian Sign Language (Lughat al-Ishāra al-Urdunia) } \\ \text { LSB } & \text { Brazilian Sign Language (Lingua Brasileira de Sinais, or LIBRAS) } \\ \text { LSC } & \text { Catalan Sign Language (Llengua de signes catalana) } \\ \text { LSE } & \text { Spanish Sign Language (Lengua de signos española) } \\ \text { LSM } & \text { Mexican Sign Language (Lenuga de señas mexicana) } \\ \text { LSQ } & \text { Quebec Sign Language (Langue des Signes Québécoise) } \\ \text { NGT } & \text { Sign Language of the Netherlands (Nederlandse Gebarentaal) } \\ \text { NZSL } & \text { New Zealand Sign Language } \\ \text { ÖGS } & \text { Austrian Sign Language (Österreichische Gebärdensprache) } \\ \text { DSGS } & \text { Swiss German Sign Language (Deutschschweizer Gebärdensprache) } \\ \text { SSL } & \text { Swedish Sign Language } \\ \text { TSL } & \text { Taiwan Sign Language } \\ \text { TİD } & \text { Turkish Sign Language (Türk İşaret Dili) } \\ \text { VGT } & \text { Flemish Sign Language (Vlaams Gebarentaal) } \\ & \end{array}$

\section{Linguistic abbreviations:}

$\begin{array}{ll}\text { IP } & \text { intonational phrase } \\ \text { LFT } & \text { lower face tension } \\ \text { LOC } & \text { locus } \\ \text { NEG } & \text { negation } \\ \text { NP } & \text { noun phrase } \\ \text { NPI } & \text { negative polarity item } \\ \text { PP } & \text { prosodic phrase }\end{array}$


PPI positive polarity item

PRED predicate

PredP predicate phrase

QE question element

$\mathrm{REF}_{\mathrm{HYP}} \quad$ hypothetical reference

$\mathrm{REF}_{\mathrm{LOC}} \quad$ locative reference

\section{Other abbreviations:}

ECHO European Cultural Heritage Online (Project)

ELAN EUDICO (European Distributed Corpora Project) Linguistic Annotator

FWF Austrian Science Fund

SL(s) sign language(s)

SpL(s) spoken language(s)

TISLR Theoretical Issues on Sign Language Research (Conference)

ZGH Center for Sign Language and Deaf Communication (Zentrum für

Gebärdensprache und Hörbehindertenkommunikation, Klagenfurt) 


\section{Annotation conventions}

\section{Glossing}

SIGN

SIGN-SIGN

SIGN1, SIGN 2

SIGN+SIGN

SIGN+, SIGN++

s-i-g-n

CL-description-of-action

IX (e.g. IX-up)

$\mathrm{PU} / \mathrm{HO}$

SIGN-h

h-SIGN

SIGN-a

SIGN-r

XXX

$<$ hands/arms are ...> identification term for a sign

identification term for a sign which requires more than one word for describing the sign in German/English there is only one identification term for a sign in German/English composition of two signs sign has one or several repetitions which have a meaning/function

fingerspelling

classifier index(ing) palm-up / Handflächen-nach-oben sign (mostly hand form and place of articulation) is held starting position of the sign is held sign is started and suddenly discontinued but is identifiable sign is produced in a reduced form but is identifiable the sign is not identifiable hands are held in a resting position

\section{Annotations of the nonmanuals}

\section{head parameters:}

cu / cd / cu-large / cd-large

hn-up / hn(-d)

hn-fw

hns

hns-slow

hns-fast

hns-fast,small

hns-slow, large

hns-trembling

hf / hb / hf-large

hfs chin up / chin down / large upward movement of the chin / large downward movement of the chin head nod upward / head nod downward (if only ' $h n$ ' is annotated the movement is downward) head nod with forward movement head nods performed at regular speed head nods performed at low speed (regular size) head nods performed at high speed (regular size) head nods performed at high speed, small in size head nods performed at low speed, large in size head nods performed in a trembling way

head forward / head backward / intensified forward movement of the head head forward movements 
hfs-alt-r/l

ht-l / ht-r /
ht-l-large / ht-r-large

hs

hs-fast

hs-slow

hs-fast,large

hs-fast,small

hs- slow,small,(tentative)

hs-alpha-shaped

hti-l / hti-r (hti-l/r)

htis

hrots (hrots-forward,upward) head forward movements alternating to the right and left

head turn to the left / head turn to the right

head turn intensified to the left / head turn intensified to the right

head shake/s performed at regular speed, regular in size head shake/s performed at high speed (tend/s to be smaller in size)

head shake/s performed at low speed (tend/s to be larger in size)

single headshake performed at high speed, large in size headshakes performed at high speed, small in size

head shakes performed in a tentative way at low speed, small in size

side-to-side-shakes with alpha-movement

head tilt to the left / head tilt to the right (head tilt to the left or to the right)

ongoing head tilting movements to the side

head rotation movements forward and upward

body lean forward / body lean backward ongoing forward movements of the body

step forward / step backward

body turn to the left / body turn to the right

ongoing body turn movements

body lean to the left / body lean to the right ongoing body sways to the side body sways to the side performed at high speed weight shift to the left / weight shift to the right ongoing shifting of weight to the side

step to the left / step to the right

both shoulders upward / right shoulder upward / left shoulder upward both shoulders forward / right shoulder forward / left shoulder forward 
shb / shb-r / shb-l

shu+; shu++

shu-alt+; shu-alt++

bstr-u

\section{eye gaze parameters:}

gaze-l / gaze-r

gaze-up / gaze-d(own)

gaze-f

gaze-up/f

gaze-r/f

gaze-f-d

gaze-up-r / gaze-up-l

gaze-h

gaze-c

gaze-a

in dialogues:

+gaze / -gaze

\section{eye aperture parameters:}

b

(almost)b

eye-w

eye-s

eye-s-strong

eye-s(or b)

\section{eye brows parameters:}

br

bf

bf-raised inward (bf-r.in.)

br-bf

\section{other face parameter:}

nose-w

cheeks-p

cheeks-i both shoulders backward / right shoulder backward / left shoulder backward

one or several upward movements of the shoulders

alternating upward movements of the shoulders

body straitening up

gaze to the left / gaze to the right

gaze upward / gaze downward

gaze somewhere to the front (to gaze into space)

gaze varies between upward and somewhere to the front

gaze varies between to the right and somewhere to the

front

gaze directed somewhere to the front and downward gaze directed upward to the right / to the left gaze directed toward the hands

gaze toward the camera

gaze toward the audience (including panoramic view)

eye contact /no eye contact with the dialogue partner

blinking movements and all occurrences of closed eyes eyes are almost closed

eye(s) are wide open

squinted eye(s)

eyes are squinted in an intensified way

eyes are squinted and it is not identifiable if the eyelids are already closed

raised brows

furrowed brows

furrowed brows which are raised inward

brows are raised and furrowed

wrinkling one's nose

puffed cheeks

cheeks inward 


\section{additions to parameters:}

(slightly)

(with tension), (tensed)

marker/marker (e.g. hti-r/bl-r) the particular marker is performed in a slight way the particular marker is performed with tension of the articulator (e.g. head, body)

two markers co-occur

\author{
annotated mouth actions ${ }^{1}$ : \\ cl / open \\ str-down / str-down-large \\ str-down-r / str-down-l \\ str-up /str-up-r /str-up-l \\ round \\ forward (fw) \\ round,forward \\ cl.fw / open,forward \\ cl.str-down \\ open,str-down \\ open,str (op.str) \\ op.str-up-l \\ (cl.)compr. \\ ao \\ cl.ao \\ cl.fw.ao
}

mouth closed / mouth open

lips are stretched, corners of the mouth are lowered / lips are stretched, corners are lowered in an intensified way lips are stretched, right corner down / left corner down lips are stretched, corners up / right corner up / left corner up

lips are round

lips are pushed forward

lips are round and pushed forward

mouth is closed, lips are pushed forward / mouth is open, lips are pushed forward mouth is closed, lips are stretched, corners are lowered mouth is open, lips are stretched, corners are lowered mouth is open, lips are stretched mouth is open, lips are stretched, left corner of the mouth is pulled up

(closed mouth) compressed lips air comes out of the mouth mouth is (nearly) closed, air comes out of the mouth mouth is (nearly) closed, lips are pushed forward, air comes out of the mouth

\section{annotation of mouthing:}

e.g. Haus (house)

e.g. o(ffen) (o(pen)) mouthing taken from German, even though not all sounds are visible; sounds which are obviously not produced, but belong to the German term, are annotated in brackets

\footnotetext{
${ }^{1}$ The used terminology follows Nonhebel et al. (2004) who introduced within the ECHO Project annotation conventions for mouth actions in the British Sign Language (BSL) and the Sign Language of the Netherlands (NGT).
} 


\section{Introduction}

\subsection{Subject matter, research question, hypotheses, and objectives}

In the last two decades sign language researchers have shifted their attention to include nonmanuals ${ }^{2}$. In doing so, they started to pay attention to the form and meaning/function of non-manual means of coding as well as to their place of occurrence or co-occurrence (see, among other activities, the 'Workshop on Nonmanuals in Sign Languages', 2009 in Frankfurt). Much research on nonmanuals is done from a typological cross-linguistic perspective (cf., among others, Zeshan 2006a). In the course of investigating nonmanuals and functions which are primarily coded by non-manual means like interrogativity or negation, some sign language researchers have found that possible means of coding linguistic information are articulated by the head or body (cf. 2.1.2).

My observation and awareness that Deaf Austrian Sign Language signers frequently code a lot of linguistic information by head movements/positions ${ }^{3}$ inspired me to do the present investigation. To be on the safe side my first intention was to ask some Deaf informants to produce a certain kind of text which seemed to evoke many different head and body movements. So, in a pilot study I asked them to think about a situation in which they sign that they are performing a long activity. During this activity they were supposed to think of a situation and sign the trains of thoughts with different attitudes towards these lines of thoughts. Afterwards they could keep on narrating whatever they wanted. And indeed, the Deaf informants produced a wide variety of head and body movements.

Thus, I implemented a (large-scale) investigation on head and body movements including recording various signed texts, annotating a selection of the signed texts by different Deaf individuals with focus on the head and body movements, and analyzing the form and function of head and body movements as well as the contexts in which the head and body

\footnotetext{
${ }^{2}$ One of the articles which is frequently referred to, which clearly points out the non-manual elements in sign languages, is Wilbur's article on 'Phonological and prosodic layering of nonmanuals in American Sign Language', published in 2000.

3 The term 'movement' is used in general to refer to all motions carried out by the articulators of head and body. The distinction between 'movement' and 'positions' is used to clarify whether a movement is performed in one action or whether a longer hold lies in between the movement. This is done in order to reflect the feedback from the Deaf annotators.
} 
movements (co-)occur. I was assisted in this process by several Deaf annotators who contributed their perceptions and suggestions with respect to meaning and function.

A couple of head movements/positions have already been reported and analyzed for Austrian Sign Language. Those which have been described more comprehensively are 'chin down' ${ }^{4}$ for polar questions, 'chin up' and/or 'head forward' for content questions (cf. Schalber 2002, 2006) and 'headshakes' for negation (Hofstätter \& Stalzer 2001; Skant et al. 2002, 110-102, 183-235). As a matter of fact, the video recordings and the annotations of the Deaf participants in this study show that first of all, many more head and body movements exist in ÖGS and secondly, that a movement along a body plane like 'headshakes' can have many different forms and different meanings/functions. The overall research questions of the present thesis have thus derived from the above mentioned subject matter and are as follows:

- Which head and body movements and positions exist in Austrian Sign Language?

- Are there different phonetic variations of each form, which all express the same meaning/function? The other way around, is there one form which possesses different meanings/functions?

Consequently, the main goal of the present thesis is to describe the observed head and body movements/positions and to illustrate in which contexts they occur. In doing so, the perspective on head/body movement along a plane becomes more differentiated. So, the movement directions around an axis and along a plane are the main features of a head/body form. However, there are further features included, such as the size or the speed of a production (cf. 2.2.4.4). The various features of a head or body movement/position are all responsible for identifying distinctive language-relevant head and body elements. So, the assumption is, for instance, that 'headshake' is not 'headshake' (i.e. all headshakes are not the same), or 'head forward' is not 'head forward' (i.e. all the instances in which the head is positioned forward are not the same).

\footnotetext{
${ }^{4}$ When a marker is first described, it is put in quotation marks. For reasons of simplifying the reading flow the quotation marks are omitted in the following text.
} 
After having answered these main research questions and consequently described the various observed head and body movements/positions together with their context of (co-) occurrence (chapter 3 to 7), in the final conclusions (chapter 8) I will address the following hypotheses. These refer to the common characteristics as well as to differences of the identified language elements and support the intended classification of the various head and body movements/positions.

Concluding, I hypothesize that

- one of the most systematically structured non-manual articulators in Austrian Sign Language (ÖGS) is the 'head'. Some head movements or head positions display particular linguistic structures. In addition, there are body movements or positions in ÖGS which code linguistic information.

- ÖGS includes head and body movements which are both more and less clear in their form and meaning/function. The first classified group constitutes several head and body movements/positions which possess a clear form-function-pair and cooccur with syntactic constituents. It is possible that two distinct forms exist which are used in different contexts, but which possess the same function - or one form expresses different functions. The second classified group is composed of head and body movements/positions which are identified through their form which, however, can vary in their phonetic implementation; they can possess a broader or narrower meaning/function and they co-occur with whole utterances to which they are associated. The head and body movements/positions of both groups are languagerelevant distinctive head and body markers, that is, they are identified by the Deaf signers due to their form and meaning/function. A third group of head and body movements/positions which are not the immediate subject matter of the present thesis are those head and body movements which don't possess these characteristics.

- some of the linguistic structures marked with the same non-manual marker have a functional common ground, that is, their functions have a semantic/pragmatic contiguity. For example, this can be applied to the ÖGS-marker 'head forward' 
which occurs in direct and embedded content interrogatives, embedded polar interrogatives, conditionals, and exclamatory utterances.

As a consequence the following goals are aimed at with regard to the present thesis:

- First, by describing all the functional contexts in which the respective head and body movements occur, the systematically structured use of head and body movements/positions can be illustrated.

- Second, those head and body movements/positions are discussed first which convey well-known functions like expressing negation, assertion, interrogativity, irreality, or conditionality. Then, those head and body movements/positions which have a relation to the signing space as they indicate the referential, the alternative, or the hypothetical space and which show common characteristics are dealt with. Finally, those head and body movements for which the form and function have not been easily recognizable at first sight are analyzed. From these, a set of head and body markers is identified as a means to code modality. Other identified head and body movements/positions whose form and function are not investigated and analyzed in detail or whose form, meaning, or both have been unclear up to now are briefly discussed in the section on future research (cf. 8.4).

- Third, in the concluding chapter common characteristics of the various observed head and body markers are analyzed and subsequently, the head and body movements/positions are classified. Furthermore, I will suggest an explanation on the matter 'why some constructions are indicated by the same head marker'.

Before introducing the organization of the present thesis, the object of study, Austrian Sign Language (ÖGS), is described by giving an overview of the research that has already been conducted on it. 


\subsection{Research on Austrian Sign Language}

In Austria, the University of $\mathrm{Graz}^{5}$ and the Centre for Sign Language and Deaf Communication (ZGH) of Klagenfurt University ${ }^{6}$ have carried out SL research since the 1990s. In Graz the focus has been put on interpreting, lexicography, sign language didactics and sociolinguistics. Klagenfurt has conducted various projects on life and education of Deaf people. It runs an on-line dictionary of ÖGS ${ }^{7}$. Further, a first volume on ÖGS grammar (Skant et al. 2002) has been published. At the present, the ZGH is implementing a project on 'Segmentation and Structuring of ÖGS-texts' ${ }^{8}$, financed by the Austrian Science Fund (FWF), led by Prof. Franz Dotter, and conducted by this author. Furthermore, a project on the Common European Framework of Reference for ÖGS (SignLef) $^{9}$ and a project on creating a learning tool for media professionals (Sign Media) ${ }^{10}$, conducted with European partners, are being implemented. Krausneker (Vienna) has done research on sociolinguistics including language policies and bilingualism (cf., for instance, Krausneker 2006, 2008). Schalber (Graz, and also Purdue) has analyzed linguistic subjects, like modals (Hunger, Schalber \& Wilbur 2000), interrogatives (2006), adverbial nonmanuals (Schalber \& Grose 2008) or possession (Schalber \& Hunger 2008). Lackner (Graz, Klagenfurt) has been working on text organization (turn-taking, dialogue structure, definitional structure), word/sign formation and functions of head and body movements (see Lackner 2007, 2008, 2009a, 2009b, 2009c, 2009d; Lackner \& Stalzer 2010; Lackner et al. in prep.). Moreover, Wilbur, Purdue University, USA, initiated investigations on typological comparison in which she included Austrian Sign Language. This resulted in a special volume in 'Sign Language and Linguistics' (Volume 9, Number 1-2, 2006) including Austrian and Croatian Sign Language, and some articles like a comparison of marking interrogatives (cf. Šarac et al. 2007) or comparing the phrase structure of American and Austrian Sign Language (Wilbur 2002).

\footnotetext{
${ }^{5}$ cf. http://www.uni-graz.at/uedoawww/

${ }^{6} \mathrm{cf}$. http://www.uni-klu.ac.at/zgh/inhalt/1.htm

${ }^{7}$ cf. http://www.uni-klu.ac.at/zgh/inhalt/251.htm; cf. http://ledasila.uni-klu.ac.at/

${ }^{8} \mathrm{cf}$. http://www.fwf.ac.at/de/abstracts/abstract.asp?L=D\&PROJ=P23867

${ }^{9} \mathrm{cf}$. http://signlef.aau.at/

${ }^{10} \mathrm{cf}$. http://signmedia.eu/node/65
} 


\section{INTRODUCTION}

A more detailed description of investigations on head and body movements together with their functions in ÖGS conducted up to now, is found at the beginning of each subchapter (cf. 3.1.2, 4.1.3, 6.2.2, 7.3).

\subsection{Organization of the present thesis}

The present thesis focuses on describing distinctive language-relevant head and body movements/positions in Austrian Sign Language. Oriented towards the respective functions which these elements fulfill, the present thesis first of all focuses on those head and body movements/positions which are clear in form and meaning (chapter 3 to 5). Subsequently, those head and body elements which depend on the signing space (chapter 6) as well as those elements whose form is less regularly produced, which can possess different phonetic variations, and which function to code modality meaning (chapter 7) are analyzed. In the conclusions (chapter 8) these head and body movements/positions are classified, the contiguity of some of these elements is discussed, and the characteristics of these elements are described.

With regard to the description of all head and body movements/positions in all chapters, the particular phenomenon is illustrated with various exemplified instances, in which the respective head or body movement/position occurs. Hence, the form, the function, its cooccurrence with lexical elements as well as additional ${ }^{11}$ (non-manual) elements, and its place of occurrence is illustrated and described. In doing so, the present thesis also aims to

\footnotetext{
${ }^{11}$ In this thesis the term 'additional marker' is used when another non-manual marker which codes the same or another function co-occurs. If an 'additional marker' codes the same function, the co-occurrence may be caused by language-internal or -external reasons (like the setting). So, the co-occurrence may cause a change of the intensity of perception of the function, but it may have other effects/impacts too. If an 'additional marker' codes another function, the terms 'other' or 'further' are used. The term 'alternative marker' is used when different markers which code the same function occur in turns. If markers which code the same function co-occur, the terms 'primary' and 'secondary' are avoided. On the one hand, this could imply that one of the markers is ascribed to be the more dominant marker due to its frequency of occurrence or the intuitive feeling of the annotators. As various reasons can be responsible for the additional co-occurrence of several markers coding the same function, just the term 'additional' is used. On the other hand, the use of the terms 'primary' and 'secondary' could imply that the ascribed functions are analyzed as first and second function. For instance, the marker 'head forward' in embedded polar interrogatives (cf. 4.3.1) is analyzed as interrogative marker as well as irrealis marker. This observation is described, but it is avoided to ascribe one of the functions to be 'primary' or 'secondary'. This depends on the way of interpretation. To sum up, the term 'additional markers' is used and meant in its purely descriptive way, i.e. as 'another additionally co-occurring marker' possessing the same or another function.
} 
show the respective functional contexts and to give, for instance, reasons for alternation or co-occurrence of additional elements. These can be other head/body markers or other nonmanual markers. The illustrations of the signed examples are either figures displaying photos of the informants when articulating the particular markers, or examples in which the co-occurrence of the markers is illustrated. If both possibilities are used for showing one example, frequently some signs are left out in the figure, but included in the example.

A literature overview on the particular function and its means of coding in various sign languages in which these aspects have already been investigated precedes chapters 3 to 7 . In addition, the findings on ÖGS, if existing, are mentioned as well.

After introducing my subject matter, research question, hypotheses, and objectives and after giving a brief introduction to the existing research on Austrian Sign Language in Chapter 1, the remaining chapters are organized as follows:

In Chapter 2 the research object, i.e. possible head and body movements, is described. This includes first, an anatomic view on possible head, body and shoulder movements, second, a description of head, body and shoulder movements which have already been described in other sign languages, and third, a characterization of head, body and shoulder movements which have been identified and analyzed as language-relevant elements for Austrian Sign Language, investigated in this thesis. The second part of chapter 2 addresses the methodology. It comprises a brief discussion of the theoretical and methodological approach, provides information on the Deaf informants/annotators as well as the uniqueness of their particular dialect, and demonstrates the process of data coding.

Chapter 3 will focus on the functions 'negation' and 'assertion'. In doing so, the respective head movements (headshakes, head nods) are presented. Thus, the two identified forms of headshakes functioning as negators are described together with their interaction with the manual negation signs. I will also demonstrate that these negative headshakes function as clause negators. They are distinguished from headshakes functioning as 'speech act negation' or 'implying negative contrast'. In addition, negative 
headshakes are demarcated from other headshakes conveying different forms and/or functions. The same approach is done with head nods functioning as means of assertion. Their form and function, co-occurrence with other means of assertion, co-occurrence with other head markers, a description of covered parts (always a constituent), and contexts in which they frequently occur are given. Finally, assertive head nods are delimited from confirmative head nods.

In Chapter 4 the interrogative and the irrealis function, coded by head positions, are detailed. First, the existing findings on head markers indicating direct polar and content questions are elaborated. In addition, co-occurring head markers (which are described as second interrogative markers by Schalber $(2006,136)$ ) which convey different functions but tend to occur in the context of polar or content questions, are described. Consequently, the reasons for the use of additional nonmanuals (especially head markers) are given.

The second part of chapter 4 comprises embedded interrogatives (polar and content interrogatives) and the co-occurrence of head markers. In the case of embedded polar interrogatives, these are marked by a different head marker than the one which is used in direct polar questions. This head marker is interpreted as an 'interrogative marker' as well as 'irrealis marker'. Its form and function, co-occurrence with other head markers, its spreading across other sign in the context, the different intensified productions, and the repeated occurrence are described in detail. Also, the regularly co-occurring brow marker and the question element 'ob' ('whether') are shown. In addition, a characterization of the embedded clause (implying alternatives or linking with the 'hypothetical space') is given. With embedded content questions, first, the two possible head markers which are similar to the direct content question are described. Then, the characteristics of embedded content interrogatives are discussed and various instances of occurrence are given.

In the final part of chapter 4, a special interrogative construction is described, which is marked by a head marker different from all other polar and content interrogative markers.

Chapter 5 deals with the conditional function, which is also primarily coded by a head marker covering the antecedent of the conditional construction. To this, the consistently 
co-occurring elements in conditional constructions (primarily additional nonmanuals) are described. What is more, the different clauses which function as consequents are described as well, as it is interesting to see what happens if a polar or content question, which are also indicated by head markers, is attached and constitutes the consequent. Also, further functions like negation, modality, or phenomenon such as the uses of spatial indicators, which can co-occur with conditionals, are analyzed.

In chapter 6 I will dwell on the syntactic/textual/discourse use of space, for which the primary means of coding are head and/or body markers. Independent of the articulators (whether head or body) and independent of the exact functions of these indicators, the common characteristics of these spatial markers are analyzed - resulting in orientationtoward, moving-toward, and pointing indicators. Furthermore, two functional uses of the space, indicated primarily by head and/or body markers, are described. These are the 'hypothetical space' (space of thoughts) and the 'alternative space' - indicated by phrasal beginning or domain markers. In describing both uses of space I will conclude that the use of the respective indicators and the direction of implementation are directed by the signing space itself. This fact is demarcated from head and/or body movements which are used for listing items and whose forward movement is not directed by the signing space.

In chapter 7 I will distinguish two modal systems which are used in ÖGS. The first comprises modality signs (modal verbs and signs which display expressions of cognition, emotion, or perception that imply such a modality meaning), the second includes nonmanuals which code modality meaning. With regard to the latter system, I will describe for the first time a set of non-manual markers which is primarily used to code 'epistemic modality'. These nonmanual markers are produced with the 'head' or 'body' (in addition to other nonmanuals) in my data. They primarily serve to mark propositional modality, that is, the signer's knowledge and/or degree of confidence of the truth value of a proposition can be shown by these possibilities: convinced-assertive head marker, non-assertive head marker, speculative body marker, and timitive head marker. In addition, a possibility head marker is also presented, coding event modality. 
After having described in detail various head and body movements/positions coding different functions, in the final chapter 8, I will summarize the new insights of my findings in sign language research, followed by findings which confirm existing findings in ÖGS and other sign languages. Then, I intend to draw conclusions on the findings. In doing so, I will first determine that the articulator head is, according to the present stage of research, one of the most systematically structured non-manual markers in ÖGS; second, I will classify the various identified head and body movements/positions; third, the contiguity of different functions (and constructions) coded by the same head marker is described; forth, common characteristics of the various head and body markers are treated; finally, in the section on future research, head and body movements are discussed which have not yet been investigated in detail, and which will definitely be a part in my upcoming research on head and body movements/positions. 


\section{Research objective and methodology}

This chapter deals with first the research objective and second the methodological approach for identifying language-relevant elements of various head and body movements in Austrian Sign Language.

\subsection{The research objective}

The trajectory of three-dimensional head and body movements is very complex. Consequently, in the following part, the anatomic possibilities of movement are briefly described followed by a description of head and body movements as applied in the present thesis. Subsequently, those movements/positions which are language-relevant in Austrian Sign Language are presented in detail.

\subsubsection{Possible head and body movements}

The following head and body rotational movements appear within the context of possibilities.

\subsubsection{Head movements}

The basic movement possibilities of the head are rotation movements, thrusting (pulling / pushing / sliding) movements, or a combination of both.

These possibilities are illustrated in Figure 2.1. 


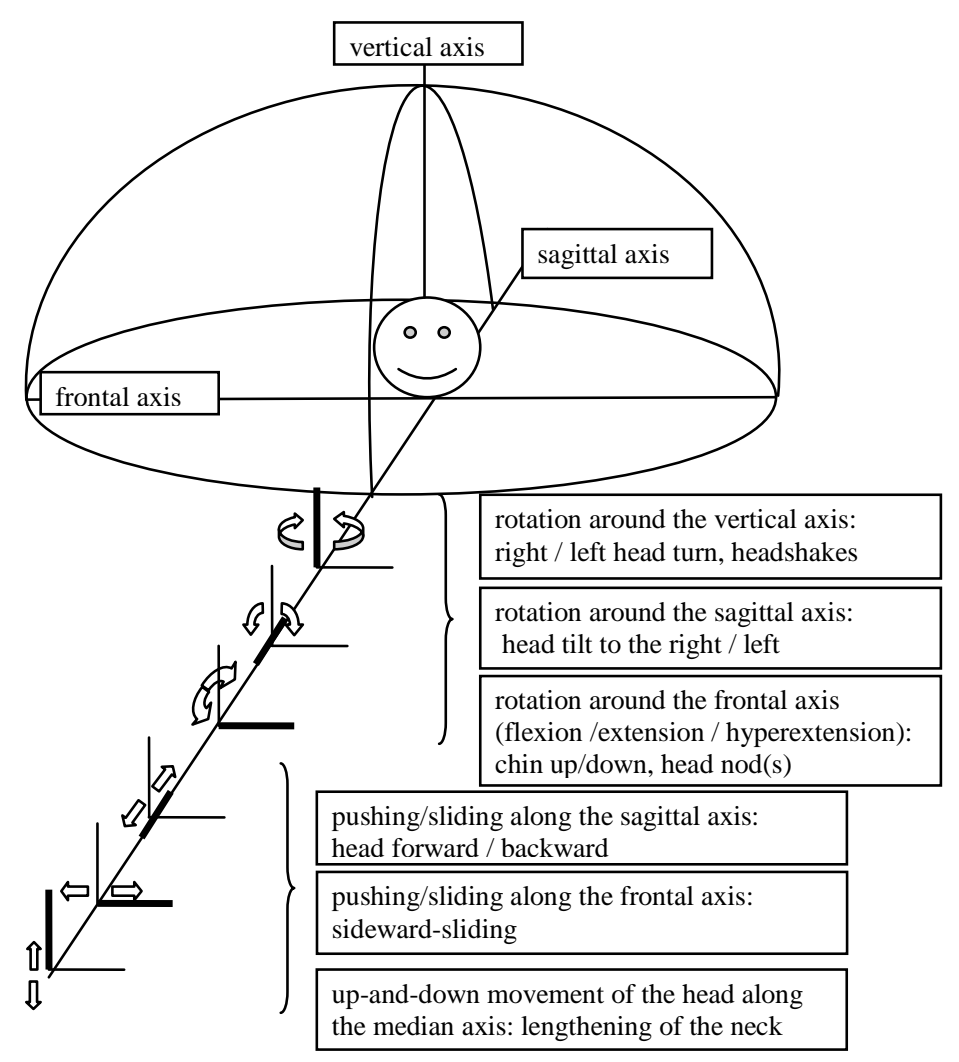

Figure 2.1 Possible head movements

The first movement possibility of the head is a rotation along one of the three axes. Movements of the head (or the neck) are at the joint between the cervical vertebrae and in the atlanto-occipital joint. Just like movements of the body (trunk) head movements are carried out along the three axes (Richmond \& Vidal 1988, 3-5; Schünke et al. 2006, 98).

Around the frontal axis (and in the sagittal plane) the head can be bent to the chest, brought back to neutral position, or the occiput is bent toward the back resulting in the three movements 'flexion', 'extension' and 'hyperextension' (McGinnis 2005, 164-165). The resulting movements are labeled 'head nod (downward or upward)'. If the movement is repeated it is named 'head nods'. If the up or down movement is held for a while the position of the head is defined as 'chin down' and 'chin up'. This does not mean that the jaw alone moves downward, but that the most significant point of the head - the chin - is positioned upward or downward. 
Around the sagittal axis (in the frontal plane) the head can be bent toward the shoulders, resulting in a lateral flexion to the right or to the left (McGinnis 2005, 166). In this thesis these movements are denoted by 'tilting the head sideward'. If the side-to-side movements are repeated, the description used is 'head tilts (sideward)'.

Finally, the head can be rotated around the vertical axis in the transverse plane (Saladin 2007, 237), resulting in a right or left head turn. If the right and left turning movement is performed as one contiguous movement and if this entire movement is performed one after the other, the term used is 'headshakes'.

The second movement possibility of the head is to push it into one direction on one of the three axes. A forward movement of the head along the sagittal axis, parallel to the frontal plane, is possible due to the hyperextension of the cervical spine. To be exact, the suboccipital, posterior cervical, the upper trapezius and the splenius capitis muscles contract (and shorten), so that the head undergoes a slight extension movement which allows the eyes to gaze forward (Simons et al. 1999, 262). Colloquially holding the head constantly in this forward position is known as 'forward head posture' or 'Reading Neck' or 'Scholar's Neck'. This movement performance is labeled 'positioning the head forward' in the present thesis. If the forward positioning is performed several times, this is labeled 'head forward movements'.

In contrast, positioning the cervical spine backwards results in a superior head movement along the sagittal axis, parallel to the frontal plane (Saladin 2007, 236) ${ }^{12}$. This is defined as 'positioning the head backward'.

Finally, the head can slide to the side along the frontal axis, parallel to the sagittal plane (lateral excursion) (Saladin 2007, 235-236) or can even be moved upward along the median axis, parallel to the transverse plane resulting in lengthening of the neck (cf. Campbell 2005, 350). As both head positions do not occur in my ÖGS data these two positions are not analyzed any further.

The third movement possibility of the head is a combination of two rotation movements or a combination of a rotation movement with a pushing movement.

12 Saladin $(2007,236)$ describes the forward and backward movement of the head as protraction and retraction of the mandible. 
For instance, rotation movements to the right and left around the vertical axis combined with rotation movements (flexion and extension) around the frontal axis result in an alphashaped movement of the head (like a Greek alpha letter), or a combination of a flexion and extension movement around the frontal axis (nodding movement) together with a forward and back movement of the head result in a circling rotation movement of the chin along the saggital axis. Both movements are identified as language-relevant elements in Austrian Sign Language.

\subsubsection{Body movements}

The spinal column can move in all three planes and is often accompanied by hip or pelvic movements when the whole trunk is in movement. Hence, the mobility of the upper part of the body is caused by the mobility of the spine and the rotation movement at the hip joints. Bending the upper part of the body forward is labeled 'flexion' (around the frontal axis, in the sagittal plane), moving the body back from flexion is denoted 'extension' (Dimon 2008, 85). These movements are labeled here as 'body lean forward' and 'body lean backward'. If the forward movement is performed several times the description used is 'body leans forward'.

Second, the upper part of the body can be bending towards one side (along the frontal plane, around the sagittal axis). This is labeled 'lateral flexion' (Dimon 2008, 85). In this thesis bending the body to one side and holding this position is labeled as 'body lean sideward'. If the side-to-side bending movements is repeated, it is labeled as 'body sways'. Finally, the upper part of the body can be rotated (around a vertical axis in the transverse plane) due to the flexibility of the spine. The results are rotations to the left or to the right (Dimon 2008, 85). This is labeled in the present research as 'body turn'.

These movements of the trunk are illustrated in Figure 2.2. 

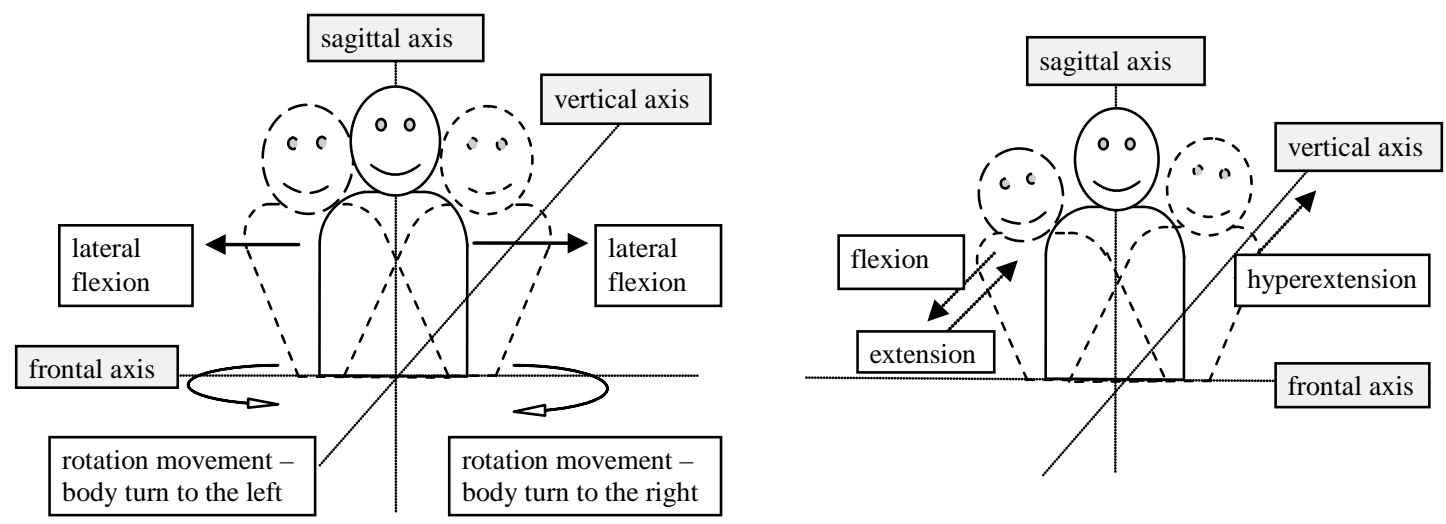

Figure 2.2 Movements of the trunk

The same movement can be achieved by moving the entire body. This is implemented by steps, weight shift, and even hip shift. In the following those movements of the entire body which also occur in Austrian Sign Language are illustrated. So, lateral extension can be achieved by stepping sideward, weight shift, or even hip shift (see Figure 2.3). Forward or backward extension can be achieved by stepping forward/backward.
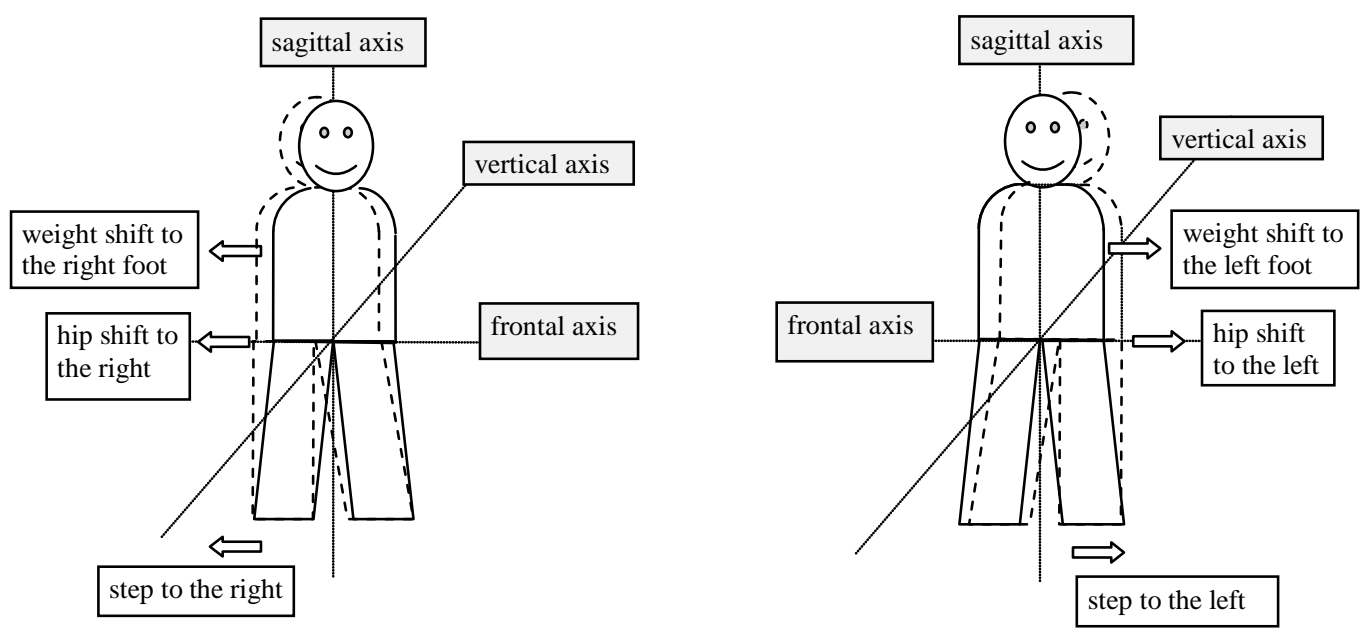

Figure 2.3 Lateral extension achieved by sideward movements

\subsubsection{Shoulder movements}

The human shoulders can be moved in various directions and are the most moveable joints due to the mobility of the joint and the contributive muscles. Basically, the shoulders can 
be moved into different directions and they also can be rotated. The variety of possibilities is anatomically defined as follows:

(Scapular) retraction is the backward movement of one or both shoulder(s) along the sagittal axis by moving the shoulder joint(s) posteriorly. (Scapular) protraction is the forward movement of the shoulder joints (anterior) along the sagittal axis. (Scapular) elevation is the raising of the shoulders (shoulder shrugging) while (scapular) depression is the lowering of the raised shoulders. One or both shoulders can be rotated outward medial, resulting in an outward medial rotation, or inward medial, resulting in an inward medial rotation, or one or both shoulders can be rotated forward or backward with arms, resulting in a circumduction movement (Palastanga et al. 2006, 146-151).

These movement varieties are illustrated in Figure 2.4.

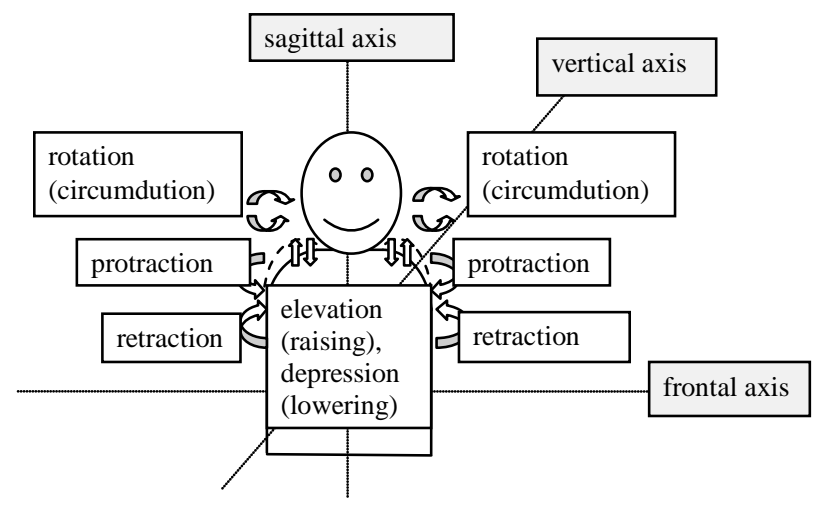

Figure 2.4 Possible shoulder movements

In this thesis the language-relevant movements of the shoulders are raising the shoulders one or several times. This is labeled as 'shoulders up' or 'ongoing shoulder up movements'. Another language-relevant shoulder movement is moving the shoulders forward. This is labeled as 'shoulders forward'.

\subsubsection{Sign language research on head and body movements}

In various sign languages the articulator 'head' has been investigated primarily in the course of describing linguistic functions in which the articulator head is one of the means of encoding. The most frequently mentioned head movements are 'headshakes' conveying 
a negation function (cf. 3.1.2), different head positions coding interrogativity (cf. 4.1.2), or head nods that are used as means of coding assertion, confirmation, aspect, phrasal boundary marking and so forth (see literature overview in chapter 3).

With regard to the articulator 'body' a few studies focus on this articulator and describe its relative function (see 'the various linguistic uses of the signing space on syntactic/textual/discourse level' in chapter 6).

With regard to head and body movements/positions as one of the 'layering of nonmanuals' Liddell $(1977,1978)$ as well as Baker \& Padden (1978) are some of the first researchers to clearly describe several language-relevant distinctive head and body movements/positions in ASL. Wilbur (2000, 226-227) offers an overview on head and body movements (among other nonmanuals), allocating an edge or domain marking function. These non-manual markers are head tilt, head nod(s), headshake(s), head thrust, body leans (forward, backward, sideward in various variations and possibly implemented as step forward or backward in standing position too (see also Wilbur \& Patschke 1998)), or body shifting. Further studies on one of these head or body markers show that there are more formatives of the particular head or body movement possible. So, for instance, in ASL there are various headshakes which differ in form and meaning (see, for instance, Watson 2010).

Possible head and body markers have been listed in the course of describing the language structure of various sign languages. For instance, Johnston \& Schembri (2007, 97 and xiv) list language-distinctive head markers in their description of Australian Sign Language (Auslan). These are headshakes, head nod(s), head turn to the left or right, head tilt rightward, leftward, forward or backward, and moving the head forward, backward or sideward. The language-relevant body markers are leans along the different axes, namely, leaning forward, leaning backward, and leaning sideward. With regard to languagerelevant shoulder movements the following are listed: moving the shoulders forward or backward, turning them to the left or right, and shoulder shrugging. 
Some research has been done on individual sign languages with specific focus on the head or body movements/positions:

Ichida (2004) describes the elements head nods (up and down), headshakes, head thrust, and change in head position as language-relevant movements for Japanese Sign Language (JSL); Positioning the chin up, down, forward, or back are described as relevant position elements.

Wilbur \& Patschke (1998 for ASL) and Kooij et al. (2006 for the Sign Language of the Netherlands (NGT)) describe the various functions of body leans. In both languages body leans are used for prosodic, lexical, semantic, and pragmatic purposes. Thus, in both languages they can be used for marking stress, expressing involvement/non-involvement, inclusion/exclusion, and affirmation/denial. However, the direction of the leaning movement concerning some special functions differs. The latter authors even take into consideration the influence of the interactive communication and describe some of the body leans from a pragmatic point of view.

Boyes Braem (1999) observes ‘body sways’ occurring in narratives in Swiss German Sign Language (DSGS). She reports that in discourse texts a single body sway to one direction constitutes a prosodic unit. Continuous side-to-side movements of the body indicate a discourse unit.

Body shifts have been treated in the course of 'constructed actions' or 'embodiment' too (Metzger 1995 for ASL; Quinto-Pozos et al. 2009 on constructed actions in ASL, BSL and Mexican Sign Language (LSM); Cormier \& Smith 2011 on defining and annotating these phenomena).

In this thesis the possible segmentation or rhythmic function of head and body movements is not treated, but mentioned in the section on future research (cf. 8.4) ${ }^{13}$. For the sake of completeness sign language research on these functions is briefly mentioned here. So,

\footnotetext{
${ }^{13}$ Since October 2011 a project on 'Segmentation and Structuring of Austrian Sign Language texts' has been implemented. This project is financed by the Austrian Science Fund (FWF), led by Prof. Franz Dotter, head of the Centre for Sign Language and Deaf Communication (ZGH) of Klagenfurt University, and conducted by the author of this thesis. The focus in this project is on head and body movements/positions (and their change) functioning as segmentation and/or structuring cues.
} 
among others, Sze (2004) investigated the segmentation function of head movements/positions in Hong Kong Sign Language (HKSL); Nespor \& Sandler (1999) and Dachkovsky \& Sandler (2009) report that the change of head, body and further nonmanual markers indicate a prosodic boundary in Israeli Sign Language (ISL) and mention the possible rhythmic function of them; Fenlon (2010) investigated segmentation cues in British Sign Language (BSL).

\subsubsection{Language-relevant head and body movements in ÖGS}

As discussed in each of the following subchapters different linguistic functions that are indicated by head and body movements have been observed for ÖGS too (cf. each literature overview of the chapters 3 to 7 ).

In this overview those head and body movements and positions which have been identified by the Deaf annotators are listed. Each of these head and body movements/positions has been identified as a language-relevant and distinctive element by them.

\subsubsection{Head movements in ÖGS}

Language-relevant positions of the head around the frontal axis (and in the sagittal plane) are the markers 'chin up' and 'chin down'. The data clearly show that the direction of the 'head position', i.e. 'up’ or ‘down', is used for linguistic purposes. In some instances the upward movement is perceived as more intensely performed and therefore, perceived as distinctive compared to the regular marker 'chin up'. This instance is annotated as 'chin up-large'. A combination of up-and-down movement or down-and-up movement is perceived as 'head nod'. Ongoing up-and-down movements of the head around the frontal axis are identified as 'head nods'. One further head marker has been identified by the Deaf annotators. This is ongoing head movements which tend to be very small nodding or head forward movements which are performed in a trembling way. They provide modality meaning (cf. 7.4.5). 


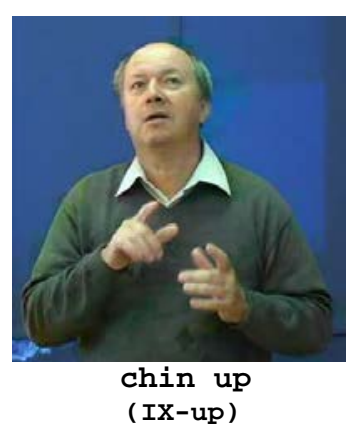

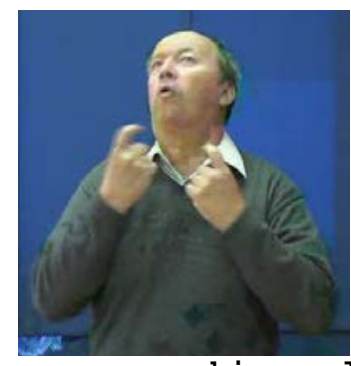

(HOPE)

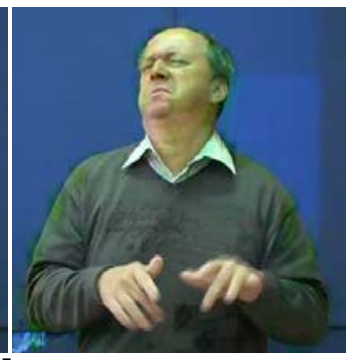

$(\text { EXIST })^{14}$

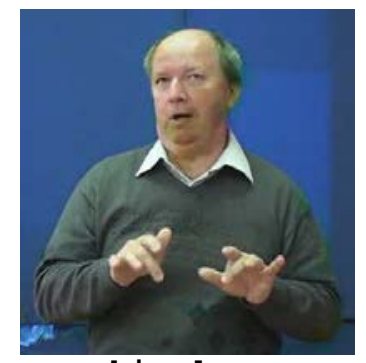

chin down

(EXIST)

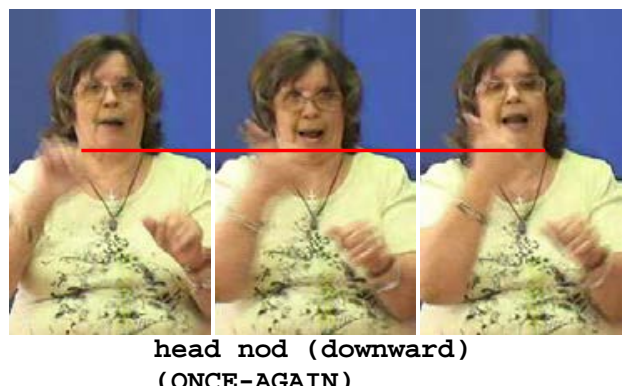

(ONCE-AGAIN)

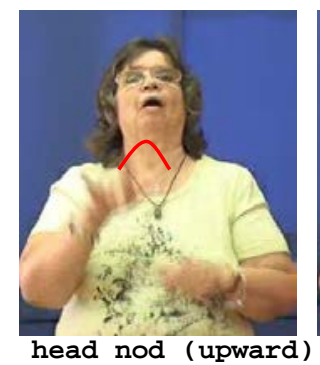

(EXIST)

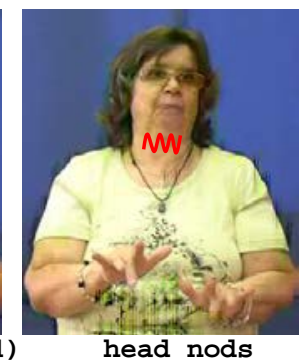

(EXIST)

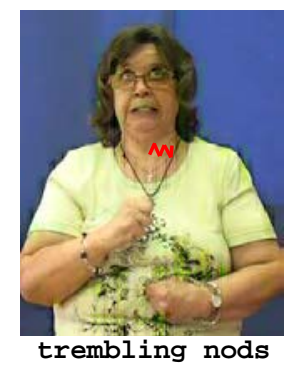

(CERTAIN)

Figure 2.5 Up/down movement/s of the head

The second possible head rotation around an axis - the vertical axis (in the transverse plane) - results in the marker 'head turn' which can be opposed to the unmarked form (no head turn) or to the opposite implementation of the turning movement, that is, 'head turn to the other side'. If the sideward movement is implemented in an intensified way and if this is perceived as meaningful by the annotators, the larger implementation is annotated. Ongoing head turn movements are identified as distinctive language elements. The annotators identified different forms of shaking movements to which they allocated different meanings/functions. The headshakes described in this thesis are fast and slow headshakes functioning as negators (cf. 3.1.3.1), a fast single headshake to underline the realized speed of an action (cf. 3.1.6), small, fast headshakes co-occurring with signs like RATHER (cf. 3.1.5), fast, non-tensed headshakes occurring in content questions (cf. 4.2.2.2), and small, slow and tentative headshakes displaying a non-assertive modality marker (cf. 7.4.3).

\footnotetext{
${ }^{14}$ Both THERE-IS and EXIST are translations of the German ID-gloss DA which are used in this thesis.
} 


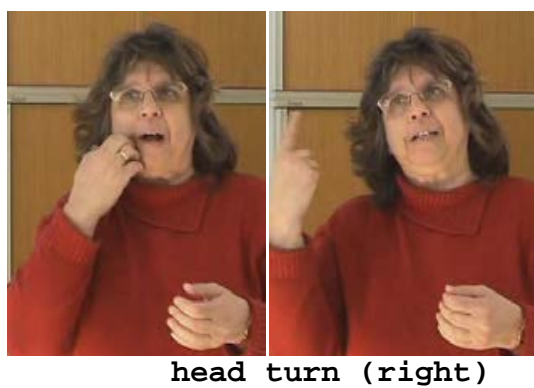

(HUSBAND)
(HIMSELF)

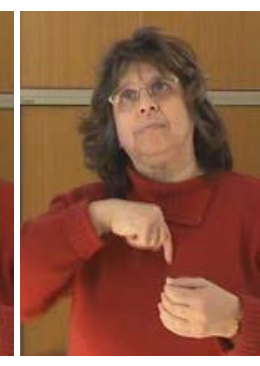

(новBY)

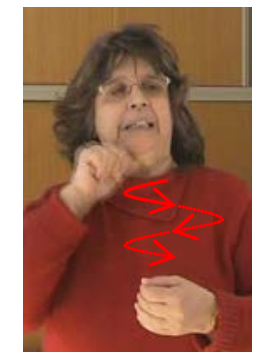

(NOT)

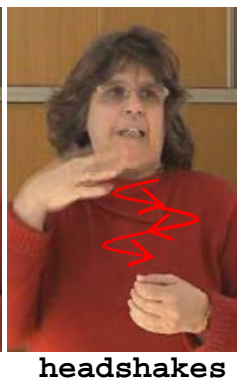

(NO)

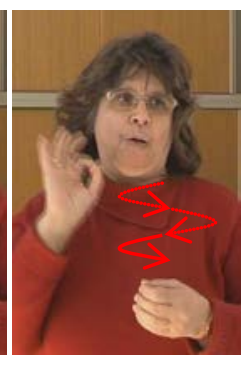

(GOOD)

Figure 2.6 Sideward movement/s of the head

The third language-relevant movement is a head rotation movement or position around an axis - the sagittal axis (in the frontal plane) - resulting in tilting the head sideward. Head tilt sideward can be opposed to an unmarked form or to the opposite tilting movement. A more intensified head tilt sideward which is perceived as distinctive from the regular side tilt has not been found in the present data. Ongoing side-to-side tilting movements also have been detected as a language-distinctive marker. The annotators identified fast and slow tilting movements. Comparable to headshakes functioning as negator, those tilting movements which cover several signs are mostly performed more slowly (and more unequally) while tilting movements which only accompany one sign or stand on their own (that is, they occur together with 'palm up', a rest position, or the holding hand configuration of the preceding sign) are performed in a faster, more regular way.
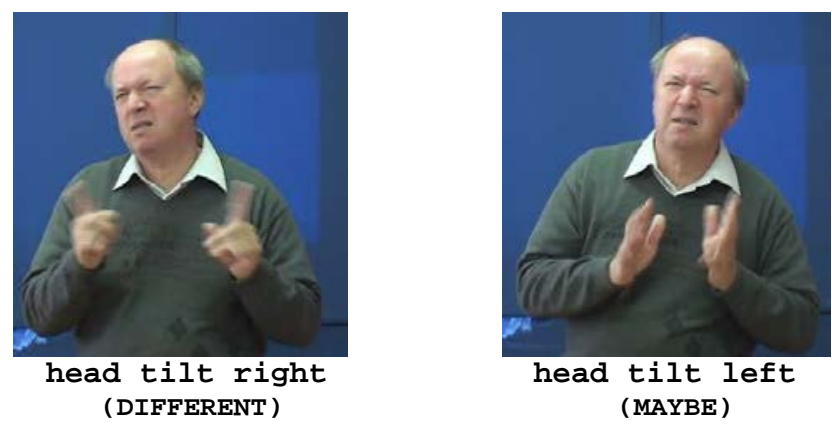

Figure 2.7 Tilting movements of the head

A further language-relevant distinctive head marker is positioning or moving the head forward or backward along the sagittal axis, parallel to the frontal plane. The annotators 
identified a stronger forward positioning of the head which has a language-relevant function. Moreover, in the ÖGS data different ongoing forward movements of the head were identified. First, the annotators identified intensively performed head forward movements of which each forward movement co-occurs with a lexical item. These can function for listing (cf. 6.4.4.6) or display the first of the two existing forms of the convinced-assertive marker (cf. 7.4.2). Second, fast and small forward movements were identified. These display one of the phonetic implementations of the second of the two existing forms of the convinced-assertive modality marker (cf. 7.4.2).

Positions:

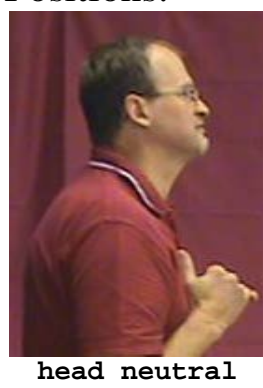

(HIKE)

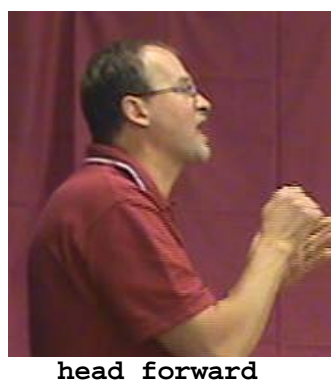

(ALPS)

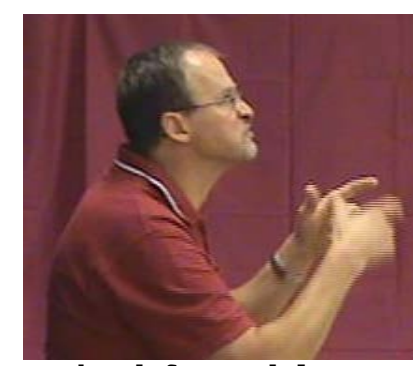

head forward large (MILK)

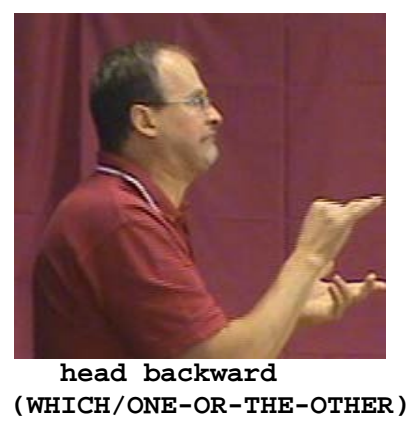

Movements:

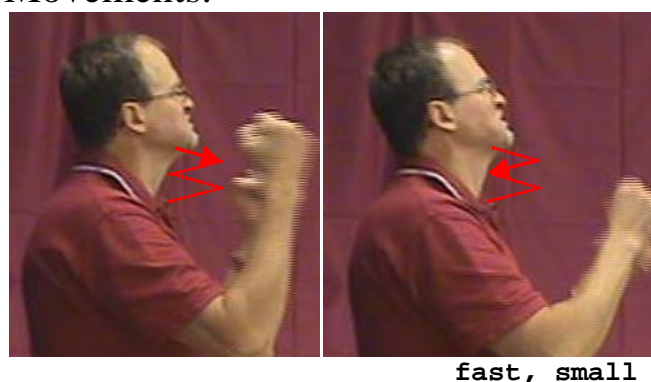

(CERTAIN-beginning) (CERTAIN-end)

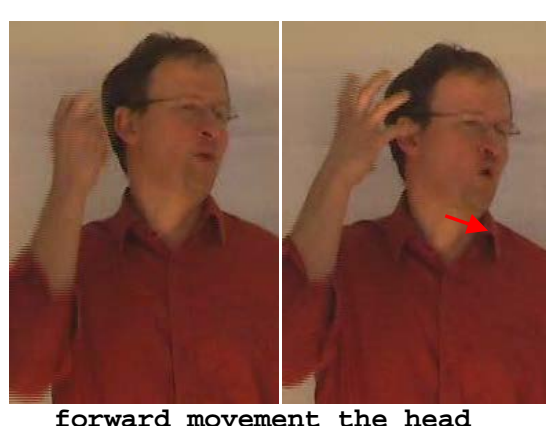

(HARD-OF-HEARING while itemizing)

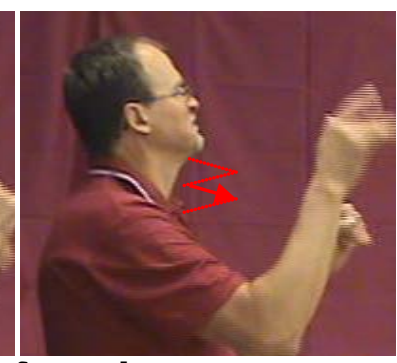

(EXIST-beginning)

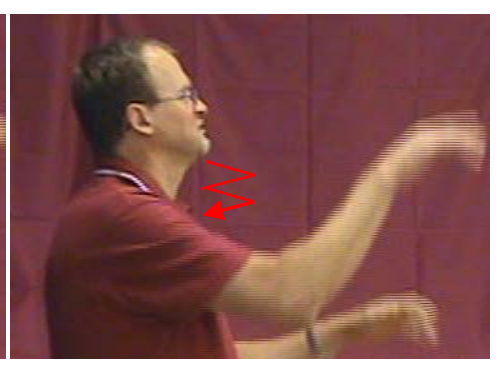

(EXIST - end)

Figure 2.8 Head forward/backward positions/movements 
Finally, some special head movements are present in the ÖGS data which are identified as distinctive, language-relevant elements. These are, among others, ongoing head rotations forward and upward, side-to-side movements performed in an alpha-shaped movement of the head, or head forward movements which alternate between the right and left side forward. The first serves as a phonetic variation of the convinced-assertive modality marker (cf. 7.4.2), the second and third show the regular and consistent aspect of walking movements, implemented by different Deaf individuals.

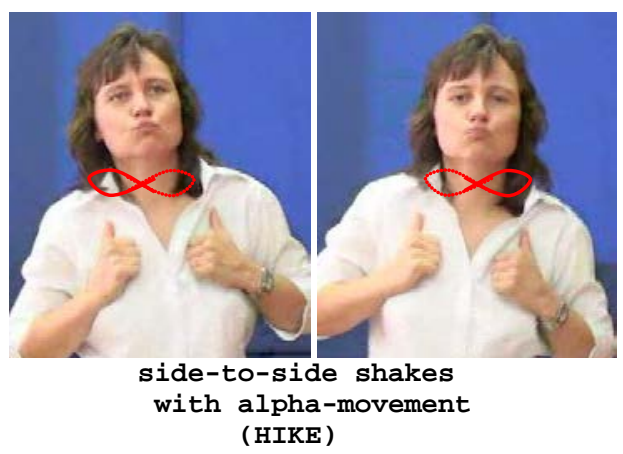

Figure 2.9 Special forms of head movements

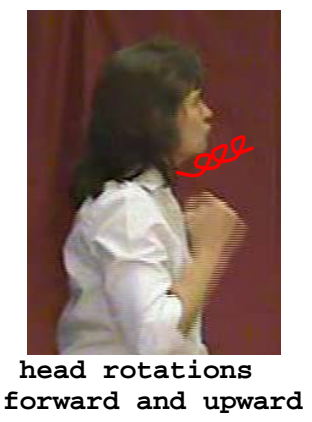

(CERTAIN)

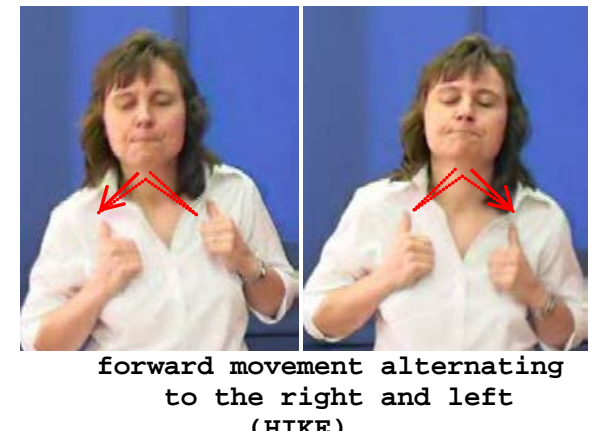

(HIKE)

\subsubsection{Body movements in ÖGS}

In the following all elements are listed which are implemented by a part of the body or the body as a whole and which are perceived as language-relevant elements. They are grouped together by one of the three axes.

Forward or backward movements or positions of the upper part of the body are implemented by the markers 'body lean forward or backward' (cf. 6.4.4.3). Ongoing body leans forward are also identified as language-relevant elements. These occur in listings and can alternate with head forward movements (cf. 6.4.4.6). 


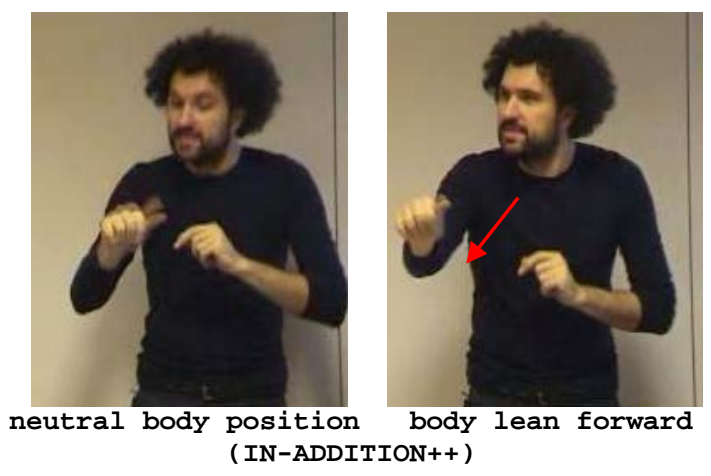

Figure 2.10

Forward movement/s of the body

Rotating the upper part of the body around a vertical axis in the transverse plane results in the language-relevant element 'body turn'. As this marker as well as the marker 'head turn' and 'gaze directed sideward' is primarily used for reference purposes (cf. 6.3.3.1), the sideward direction (rightward or leftward) is added in the annotations as this provides information about the established reference space.
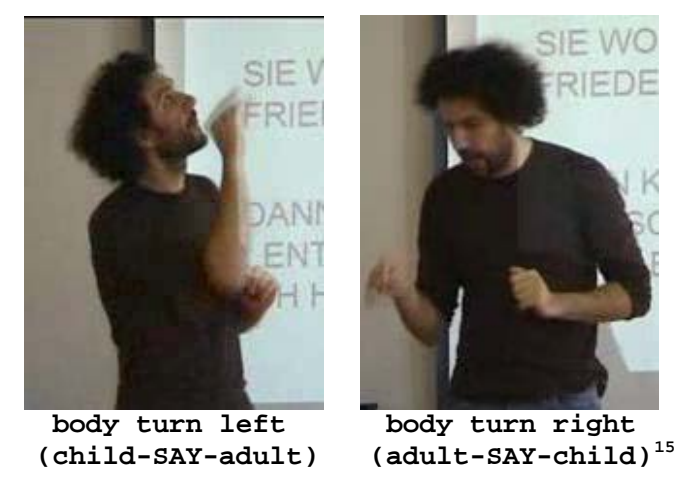

Figure 2.11 Turn movements of the body

Moving or positioning the body - i.e. the upper part of the body or the body as a whole - in the frontal plane results in some sign language-relevant elements. These elements include

\footnotetext{
${ }^{15}$ Figure 2.11 includes an additional upward and downward positioning of the head together with looking upward and downward. Schalber (2006, 140-141) observed that height can be indicated by both gaze direction and chin position in ÖGS. If the head position is used for indicating a polar or content interrogative, the addressee height is only indicated by gaze or the head marker is performed in an intensified way.
} 
'body leans sideward', 'shifting of weight', and 'step sideward' ${ }^{16}$ (cf. 6.4.4.3 and 7.4.4). In one instance a signer even moves the hip sideward which is perceived as a languagerelevant element. In many instances, the particular side movement is opposed to an unmarked body position, or it is opposed to the opposite side movement of the body. Language-relevant ongoing body movements from side to side are 'body sways', that is, the upper part of the body is swaying from side to side, or 'weight shifts' from side to side (cf. 7.4.4).
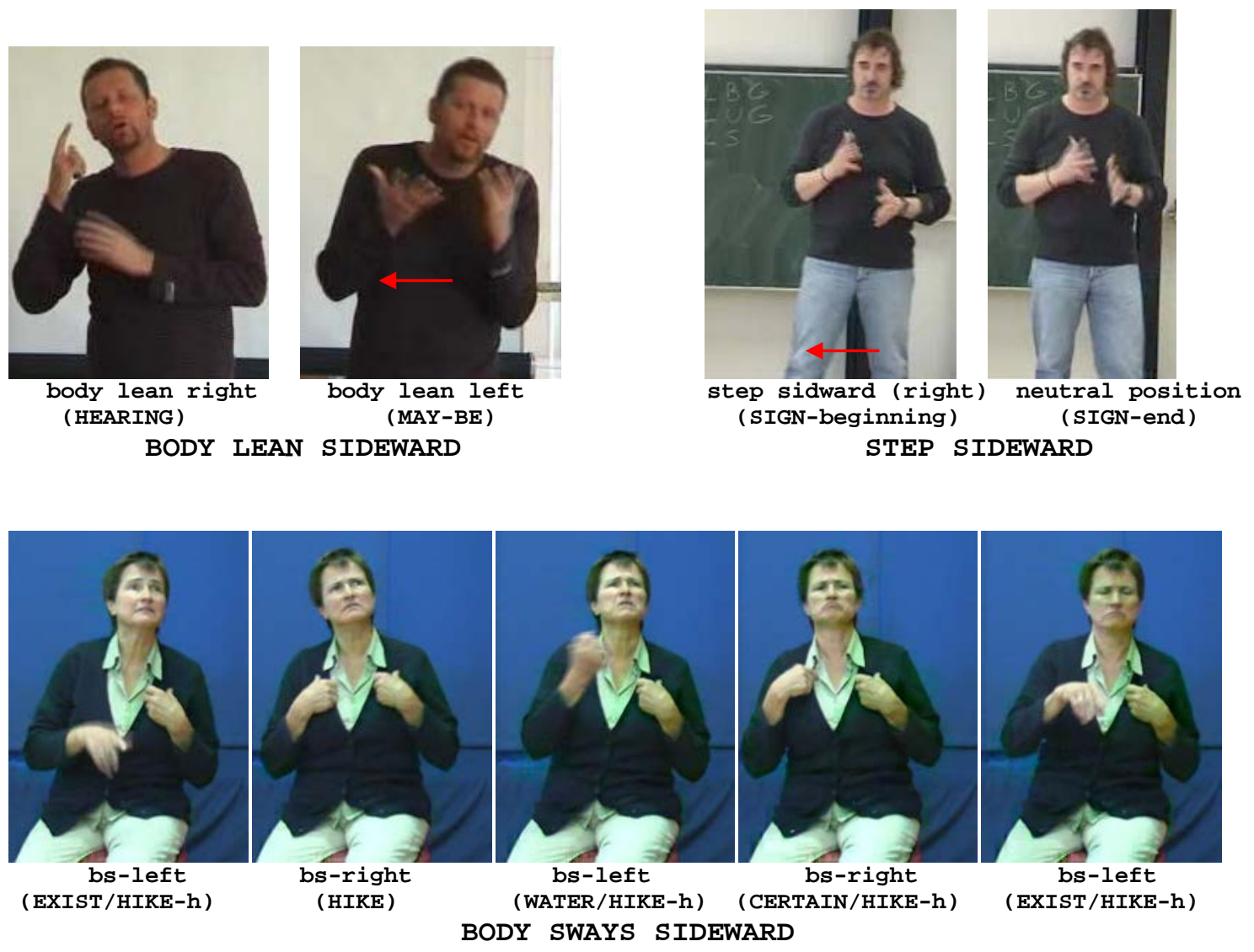

\footnotetext{
${ }^{16}$ Body leans, shifting of weight, and steps sideward were annotated in one tier in ELAN as these positions or movements exclude each other or are produced together (e.g. body lean to the right goes together with weight shift to the right). Whether the upper part of the body or the body as a whole is moved depends on various facts. Some of the already noticed ones are the position of the signer (sitting or standing position), the distance of the audience (more steps in the educational corpus), and the language style of the relevant signer. In chapter 6 spatial means of coding (head and body movements) are listed which can vary in their use. However, the variation of these articulators needs further investigations but would extend beyond the aim of the present thesis.
} 


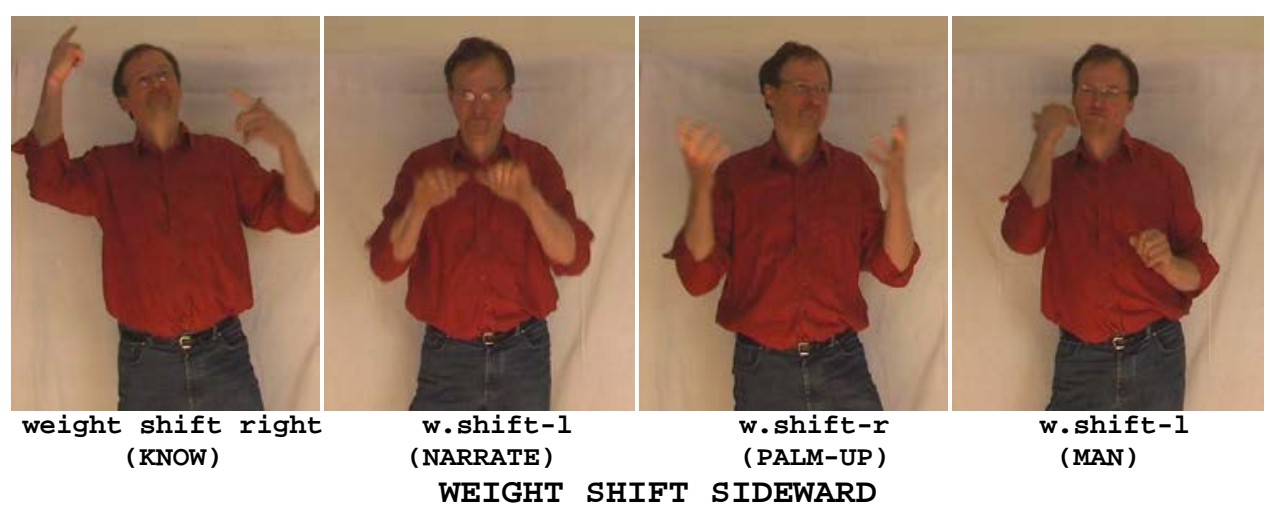

Figure 2.12 Sideward movement/s of the body

\subsubsection{Shoulder movements in ÖGS}

Moving one or both shoulders upward (one time, or several times in an equal or alternating way), moving both shoulders forward, and straightening the body up ${ }^{17}$ are all identified as language-relevant elements in ÖGS. The meaning/function of these elements is only partly solved. What is clear is that raising the shoulders can convey the meaning of 'lack of knowledge'; shoulder shrugging can possess the same meaning or the meaning of doing an activity in a continuous way (cf. 4.2.2.2 and 4.3.1.2.2). Moving the shoulders forward is a marker which can co-occur in the if-clause of conditionals (cf. 5.4.3). Straightening the body up and down while performing some signs is one of the segmentation cues which is mentioned by annotators conducting a segmentation task ${ }^{18}$. These language distinctive elements are illustrated in the following.

\footnotetext{
${ }^{17}$ This marker occurs in the existing ÖGS corpus when the signers are sitting.

${ }^{18}$ This segmentation task is part of the project 'Segmentation and Structuring of ÖGS-texts'.
} 


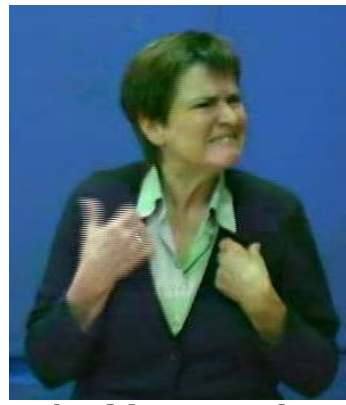

shoulder upward

(HIKE-h)

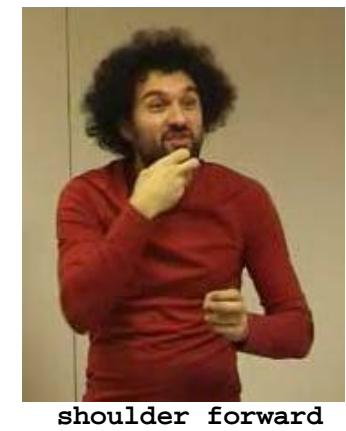

(WHO)
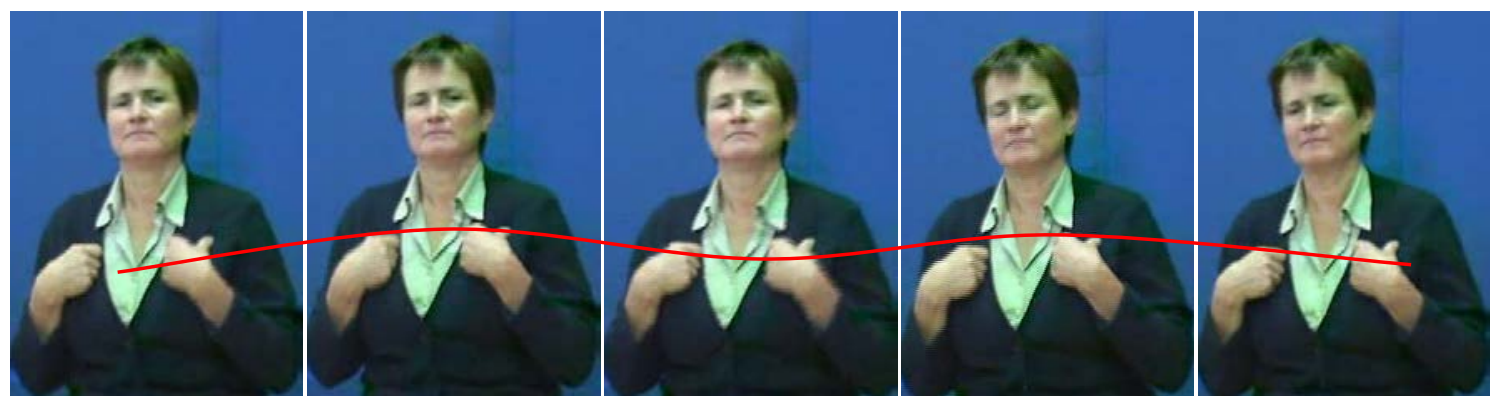

ongoing shoulder shrugging (HIKE)

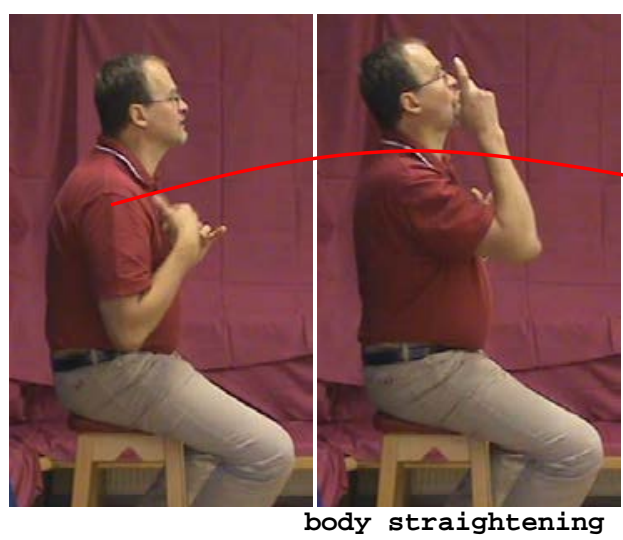

(I)

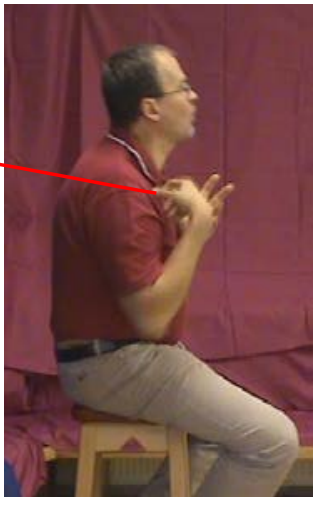

(I)

Figure 2.13 Shoulder positions and movement/s; body straightening 


\subsection{Methodology}

In the following, the theoretical approach and methodological approach of the present research on head and body movements is discussed and subsequently described in detail. In doing so, first, the information about the Deaf informants/annotators is indicated including a description of the former and actual living situation of the Deaf individuals in the valley 'Großarl' - as this is unique in Europe. Second, the data analysis is described step-by-step involving the process of data recording and coding, the presentation of the resulting corpora, and finally the implementation for identifying language-relevant head and body movements/positions.

\subsubsection{The approach}

To begin with, I would like to illustrate four different observations which I made in the course of the data coding with Deaf annotators and analyzing and comparing the identified head and body movements. These observations have led me to the primary goal of my thesis and consequently to the intended methodological approach.

The first crucial observation is that in the course of the data coding, the Deaf annotators identified various nonmanuals which 'constantly co-occurred'. In other words, they always identified several 'co-occurring non-manual elements'.

When comparing cross-linguistically the co-occurrence of non-manual elements with regard to the particular identified function in ÖGS (e.g. the functions interrogativity, negation, modality, and so forth) it turns out that some of these functions have already been described in several sign languages. But in doing so, almost exclusively the 'only' relevant non-manual marker is focused on and its co-occurrence with the lexical elements is described. This results in language descriptions like the following, which exemplarily illustrates the description of negation. 


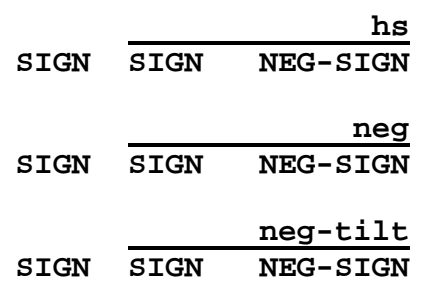

Figure 2.14 Illustration of non-manual markers in sign language literature

In Figure 2.14, the co-occurring non-manual element which (primarily) codes the function 'negation' is described with regard to its form, its function, or both. In addition, the mode of description provides information on the possible spreading of the non-manual marker. However, in the course of ÖGS data coding with Deaf annotators it has turned out that a description of an utterance most frequently looks like this:

$(1)^{19}$

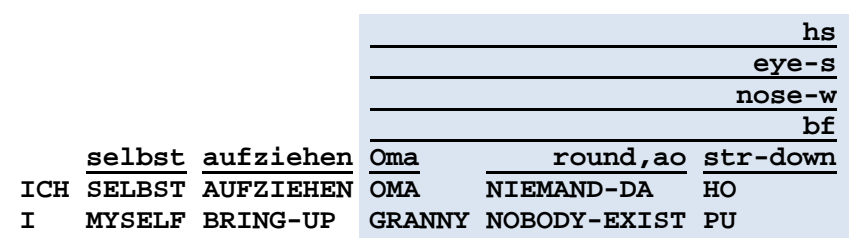

Ich habe sie selbst groß gezogen. Es gab keine Oma.

I have brought them up alone. There was no granny.

(F001_Film1_Szene7_d_informal_story_01.04-01.07)

Example (1) illustrates that frequently various non-manual elements co-occur. In this example several non-manual elements ${ }^{20}$ are present which seem to be connected with negation, that is, more elements refer to 'negativity' than just the marker 'headshakes'. This fact has brought me to the conclusion that all co-occurring elements have to be taken into consideration in order to do justice to the various language phenomena - like 'negation' in this example.

The second observation is the following: In several sign languages linguists have noticed that other nonmanuals can co-occur in constructions (like negative constructions or

\footnotetext{
${ }^{19}$ Example (1) is described in detail in chapter 3 (and quoted again as example (8)).

${ }^{20}$ My current data compilation includes only the clearly identified co-occurring non-manual elements, which implies that it is very likely that further non-manual elements exist, which have not yet been identified.
} 
interrogative constructions) which are indicated by one primary non-manual marker. These nonmanuals are mentioned in the construction description, but frequently described as additional or even secondary markers (cf. 1.2 and 4.2.3). When annotating all the cooccurring nonmanuals and the different contexts of occurrence during the process of data coding, it has turned out that some of these co-occurring nonmanuals function as alternative markers, others as additional markers, and again others possess a different function which, however, tends to occur in the particular described construction. Consequently, a detailed annotation of each non-manual element and a description of each context of occurrence lead to the exact function of the particular co-occurring non-manual element.

A further observation resulted from data coding by the Deaf annotators. This observation refers to the prerequisite that languages contain lexical and functional/grammatical categories (cf. Heine \& Narrog 2010b, 18). The following two observations will illustrate that this lexicon-grammar interface is not always that clear. First, some of the non-manual elements are characterized by the fact that they can occur on their own and function as a lexical element, occur on their own but function as a marker that refers to a preceding or following clause, or co-occur with lexical elements / syntactic constituent on which they associate. For example, in ÖGS 'headshakes' occurring without a lexical sign can provide the semantic information 'NO'; a clause can be followed by 'headshakes' (occurring on its own) which function as negator; finally, 'headshakes' can co-occur with a clause or part of it and negate it. Second, some bundles of non-manuals constantly co-occur with a common starting and ending point and without any manual signs ${ }^{21}$. These instances are mostly interpreted by the Deaf annotators as a lexical element. For example, co-occurring 'wrinkled nose', 'squinted eyes' and the mouth action 'stretched-down' is interpreted as KNOW-NO/INSECURE. When annotating these elements in ELAN (description of data coding follows) the Deaf annotators could not understand why that should not be put into the glossing tier (which refers in the present annotation conventions only to the manuals)

\footnotetext{
${ }^{21}$ The hands are held in a rest position, a palm-up position, or the preceding sign is held.
} 
and get its own gloss. Heine \& Narrog (2010b, 18) observe that these two categories, i.e. the lexical and grammatical categories, are postulated as two discrete categories in all theoretical frameworks which are included in their volume ${ }^{22}$. Only some linguists treat this differentiation more accurately. For instance, Langacker $(2010,102)$ does not focus on that dichotomy. In his studies, lexicon and grammar are part of 'a continuum of meaningful structures' (cf. also Heine \& Narrog 2010b, 18). For now it is noted that for the Deaf annotators the clear ascription of non-manual elements to lexical or grammatical categories is not as clear as it often seems to be in sign language literature.

The final striking observation in the present data is the following: As the various multisigner data are texts (i.e. longer signed utterances) and one of the main goals has been to achieve a very 'natural signing flow' (see below all the conditions for recording the data) the data shows that 'a lot of information is coded by nonmanuals'. This becomes especially obvious after doing the annotations of the non-manual tiers in ELAN ${ }^{23}$. The following two examples show this phenomenon (i.e. layering of several non-manual elements).

\section{(3)}

\begin{tabular}{|c|c|c|}
\hline shf & & shf \\
\hline$\frac{\text { cd-large }}{\text { hti-1/bl-1 }}$ & shu & hti-1/b]-1 \\
\hline$\overline{\mathrm{hf}}$ & & $\frac{\mathrm{hf}}{\mathrm{hf}}$ \\
\hline eye-s-strong & & eye-s \\
\hline nose-W & & \\
\hline Mann & cl>open & open \\
\hline JETZT $\overline{\text { KOMM }}$ & WAS/HO & WAS/HO-h \\
\hline HUSBAND NOW & WHAT/PU & WHAT/HO-h \\
\hline
\end{tabular}

Wenn dein Mann kommt, was würdest du tun? If your husband comes, what would you do? (M007_04.05.08_edu.course_part01_12.59-13.02)

Wenn dein Mann jetzt kommt, was würdest du tun?

If your husband comes now, what would you do?

(M007_04.05.08_edu.course_part01_13.05-13.07)

\footnotetext{
${ }^{22}$ Heine \& Narrog's (2010a) volume on 'Linguistic Analysis' includes the entire spectrum of theoretical frameworks, ranging from Cognitive Grammar to Function Discourse Grammar to Formal Generative Typology.

${ }^{23}$ In addition, the Deaf annotators' personal feedback is that the data looks like very natural signing. They argue that this judgment is based on the fact that the various Deaf informants code a lot of information by nonmanuals and less information is given by 'the hands'.
} 
Both sign language examples date from a lecture in which the signer gives examples which include modality. First, the signer copies an example of a Deaf student and confirms that this is a way in which modality is expressed (see example 2). He adds that when signing in this way more information is offered by successively produced signs. The non-manual elements are produced with reduced intensity or they are omitted. Subsequently, the Deaf lecturer repeats the content (see example 3 in which additionally the lexical information NOW is included) and states that this is the same information, but the way of signing looks more natural - a statement which is totally confirmed by the entire Deaf audience. The most significant aspect of these two conditional constructions is that in example (2) more information is coded successively by the hands while in the example (3) a lot of information is coded simultaneously by nonmanuals. The observation that a lot of information is coded simultaneously is well-known for sign languages (cf., among others, Vermeerbergen et al. 2007 on simultaneity in signed languages). Heine \& Narrog (2010b, 19), in their handbook on linguistic analysis, point out that many linguistic schools focus on the linear arrangement of elements in clauses and put strong attention on the basic word order. Sign language researchers also apply theoretical frameworks which are based on spoken language that more likely tend to provide linearly arranged information ${ }^{24}$. The intention of my thesis is to show the high degree of simultaneously provided semantic information. In doing so, the thesis is a contribution in sign language research which shifts the focus from 'linearly arranged information' to the 'comprehensive layering of nonmanuals'. My wish is that in the near future the simultaneity of co-occurring elements and thus, the simultaneity of providing semantic information is more manifested in sign

\footnotetext{
${ }^{24}$ Some linguists tried to create their own model on sign language or adopted a model to sign language which originally was created for spoken languages. For an overview on linguistic analysis on visual-gestural languages see Wilcox \& Wilcox (2010, 740-745) who, for example, list some phonological modals like the move-hold model by Liddell (1984), the autosegmental model by Sandler (1986), the moraic model by Perlmutter (1989), or the prosodic model by Brentari (1998). The latter model, for instance, focuses equally on sequential and simultaneous components of a sign, is based on real sign language examples, and probably can expand the non-manual part. In addition, the authors mention that Stokoe (1974) favored a 'gestural model' in the sense of semantic phonology.
} 
language examples, and consequently, in theoretical frameworks and their models which are used in sign language research ${ }^{25}$.

All these observations and conclusions have made it clear to me that first a detailed language description is required which includes a careful description of the various language-relevant non-manual elements before an interpretation on any phenomenon with regard to the language structure can be made. Thus, following Boas' approach ${ }^{26}$ in the sense of describing carefully all the phenomena within a language without being influenced by structures of other languages (cf. Maas 2009), the intention of my thesis is to present language phenomena descriptively. To be more specific, particular head and body movements are described in detail with regard to their exact meaning/function, their form, their co-occurrence with further elements and their occurrence in a special functional construction.

It is not my intention to enter a discussion whether a framework-free grammatical description and analysis, postulated by Haspelmath (2010) or a framework-bound analysis (whether more or less restrictive) (for an overview see Heine \& Narrog 2010a) is to be preferred; the first one is even under discussion whether it is possible at all. Comments of researchers who work on cross-linguistic comparison have supported my intention to start off with a clear language description. So, for instance, Bowerman (2011) who was researching the field of cross-linguistic comparison of language acquisition, demonstrated that if a careful description of language phenomena is used as a basis, wrong hypotheses and prerequisites can be avoided in advance.

Naturally, this thesis is also based on a presupposition which is the following: special functional contexts require special indicators. As the focus of the present thesis is on head and body movements the question is 'which functional contexts require which head and/or body movement(s) / position(s)'? Thus, the assumption is that an element (in my case a

\footnotetext{
${ }^{25}$ Of course, I have to add that I have the highest respect for what linguists have contributed to sign language research up to now. I just want to indicate my intention and contribution to sign language research which is focusing on the high degree of co-occurring elements in sign languages.

${ }^{26}$ As noted in his graduation examination but also in his work on 'Kosmographie', Boas was inspired by Wilhelm von Humbold's works like 'Über die Verschiedenheit des menschlichen Sprachbaus' (cf. Maas 2009, 259).
} 
specified language-relevant head or body movement/position) fulfills a certain function, if it occurs in a special functional context.

Concluding, following an empirical and inductive approach, the aim is to achieve welldescribed, cross-linguistic comparable data. In doing so, first language phenomena are identified and described, second, they are classified, and third, generalizations on the results are made. Finally, to some extent, explanations of some generalizations are included too, such as the fact that a special marker is used for different constructions which possess contiguity. Following these steps my implemented approach concurs with Croft's approach which he calls ‘empirical scientific approach’ (Croft 2003, 2). This approach is recommended as a basis for typological comparison of languages.

Different ways of operationalization are applied in order to determine the languagerelevant distinctive head and body elements. First, Deaf informants are asked to produce more or less the same content, assuming that they make use of the same or similar nonmanuals concerning head and/or body movement(s) / position(s). Second, these videos are shown to Deaf fluent signers in order to ask them to identify language-relevant distinctive head and body markers. Deaf individuals doing this identification task are labeled 'Deaf annotators' in the present thesis while a person being in the role of producing a signed text is termed 'Deaf informant' or just 'signer'. Following these two steps, a paradigmatic definition of the head and/or body movement(s) / position(s) in question has become possible.

At this point it has to be mentioned that a preliminary study on the identification of language-relevant head and body movements in ÖGS, conducted by Lackner \& Stalzer (2010), preceded the current one. In this study, the same sequence of signs was presented, but each time the signs were used with a different head/body movement/position. After each presentation of an example, an answer pair was offered from which the Deaf viewers had to choose the correct one. The correct answer included the same information as provided in the offered example, however, this information was coded by hands instead of being coded by head or body. The results were presented at TISLR 2010 and are included in Appendix D. 


\subsubsection{The Deaf informants/annotators}

The Deaf signers who participated in the present investigation (except the educational training corpus) have in common that they all live in the same mountain valley or are related to it. This means that they were either born in the valley, or they live there today, or they stay in close contact to a Deaf person of the valley (by marriage, close friendship). Moreover, the participants meet regularly and also take part in the official meetings in the Pongauer Gehörlosenverein ('Pongauer Deaf club’) ${ }^{27}$. Consequently, the main focus of the present investigation is on this Salzburg variety of the Austrian Sign Language (ÖGS ${ }^{28}$.

The present thesis is based on three sessions in which video recordings were produced and two two-week sessions during which the annotations for one corpus were conducted. In the first recording session, conducted in March 2005, six male and three female fluent Deaf signers who stay in close contact with each other and who have a close relation to the Großarl valley performed dialogues on various topics. In the second recording session, carried out in September 2010, five male and three female signers participated. This group was composed of the same individuals, except for one female Deaf signer who was not able to participate that day. As a substitute, one female joined the group. One male Deaf informant died in 2008. The third recording session was implemented for reasons of clarification of the observed head and body markers. This follow-up session, recorded in April 2011, included two male and two female Deaf signers who took part in the sessions 2005 and 2010. For annotating one part of the session, recorded in September 2010, two female Deaf signers and three male Deaf signers - who all had participated in the recording sessions - collaborated and conducted the annotation task in March 2011.

In 2005, the participants were aged between 43 and 70 years and the average age was 55 years. In 2010, the participants from Salzburg were aged between 38 and 75 years and the

\footnotetext{
${ }^{27}$ Pongau is a region situated in the North Alps and constitutes one of the five counties of the federal state Salzburg which is one of the nine federal states of Austria.

${ }^{28}$ The first investigation on that variety of ÖGS started with my MA thesis on turn-taking, published in 2007. The first video data on that variety of ÖGS dates from 2005, which is used for the present thesis too.
} 
average was 58 years. In the follow-up session as well as in the annotation $\operatorname{task}^{29}$ the participants were aged between 57 and 68 years with an average age of 62 years. The generalized metadata on the participants as well as the questionnaire used (including demographic data and information on the use of ÖGS) are attached in Appendix B and Appendix C. For the sake of completeness it has to be added that the questionnaire was requested in ÖGS by one of the Deaf participants who also filled in the particular form. In addition, all the participants consented to the use of the recordings for research purposes. As the way of signing would have been influenced if the participants had known that the data would be used for teaching or other purposes, consent for using the data for further purposes was not requested. If of interest, permission for using selected data for further purposes will be asked for.

Apart from the different signed texts of the Salzburg variety of ÖGS, the present investigation on head and body movements includes a corpus from an educational training course $^{30}$. This corpus is composed of three Deaf signers of Austria who presented selected topics in the linguistic and pedagogical field in a university course for sign language teachers at the Alpen-Adria-University in Klagenfurt. Out of that corpus, three male Deaf individuals who come from Styria and Vienna are included in the present analysis of body and head movements/positions. They have all grown up with sign language (one of them having Deaf parents), and use it as their first means of communication. They also teach sign language in courses ranging from children to university students. They have an average age of 38 years.

Of course, one of the investigations in the near future will be to analyze whether head and body markers described in this thesis, as well as insufficiently described ones (i.e. their form, function, co-occurrence and occurrence are not yet clear) (cf. 8.4), are the same and are used in each dialectal variety of ÖGS. As the present head and body markers are (only)

\footnotetext{
${ }^{29}$ The same participants took part in the follow-up and annotation sessions except for one male Deaf signer who only collaborated in doing part of the annotations.

${ }^{30}$ Around 30 minutes of the educational training course corpus were annotated and used for a study on 'definitional structures in ÖGS' (cf. Lackner 2009c, 2009d).
} 
those which are clearly identified in form and meaning, at the present stage of research, it can be concluded (based on the data from the Deaf informants from Salzburg, Styria and Vienna) that the head and body markers of this $\mathrm{PhD}$ thesis are present at least in all these ÖGS varieties. With regard to the Carinthian variety of ÖGS it can be determined that at least those head and body markers which were mentioned by Skant et al. (2002) coincide in form and meaning with the present head and body movements/positions.

Prior to describing the data coding process in detail, a descriptive excursion to the valley of 'Großarl' is inserted. This is of particular interest because, apart from the participants in the educational training course, all the participants come from that valley or are connected to it in some way. The interesting fact is that in this valley there used to be a high percentage of Deaf people about 30 to 50 years ago. As this situation of having a higher percentage of Deaf individuals all located in one valley seems to be unique (to my knowledge) in Europe, it seems important to introduce this area, in the following excursion.

\subsubsection{Excursion: The valley of 'Großarl'}

About 50 years ago, 30 Deaf individuals used to live in 'Großarltal' ('Großarl valley'). Most of them settled in Hüttschlag, a smaller village at the end of the valley. The others lived in the village called Großarl, the main village in the valley from which it derived its name. Nowadays 13 Deaf individuals still live in the 'Großarl valley'. Some Deaf individuals who used to live in the valley have settled in villages outside the valley or even in Vienna, and some of them have already died.

This higher percentage of Deaf individuals is probably due to hereditary deafness and consanguineous marriages which, among other factors, correlated with the situation that until around 1960 the valley 'Großarl' was difficult to access for motorized vehicles. 

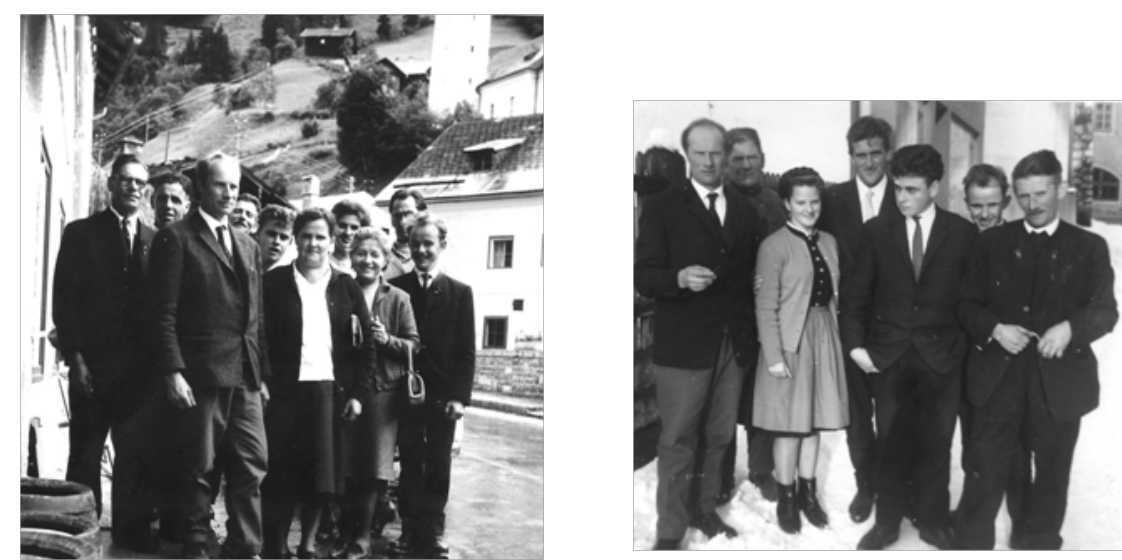

Figure 2.15 Photographs of the Deaf inhabitants of the valley Großarl ${ }^{31}$

The number of Deaf inhabitants (50 years ago) in the village Hüttschlag (the population in 1961 in Hüttschlag was 781 inhabitants)32 can be compared with a small village in North Bali, Bengkala. Following Kortschak (2010, 76), in 200846 Deaf people were counted, out of 2,450 inhabitants in Bali. The language used in Bengkala - besides the official spoken language - is 'Kata Kolok' ('Deaf Talk’), a village-based sign language used by the Deaf and hearing population, but of course with different levels of competence. The extent to which the hearing population was able to use the locally present sign language in Hüttschlag cannot be assessed nowadays. Deaf signers in the present investigation indicate that on the different farms the locals knew the basic signs and also used non-manual means of communication; Even the mayor of Hüttschlag at that time could sign (to some extent) and employed two Deaf individuals at the village hall of Hüttschlag. However, it can only be assumed that the level of knowledge of the hearing population was lower than that of the hearing population in Bali, as the Deaf children of the Großarl valley attended the school for the Deaf in the city of Salzburg from the age of 6 to 15 (nine years in total), stayed at the school's boarding house and just came back to their homes during holidays

\footnotetext{
${ }^{31}$ The two photos are taken in the 50s and 60s. The first picture shows Deaf inhabitants of the valley Großarl (one Deaf person comes from Salzburg) in the centre of the village Hüttschlag. The second picture also shows Deaf inhabitants of the valley Großarl in the centre of the village Großarl.

${ }^{32}$ Hüttschlag: 1961 - 781 inhabitants, 2011 - 915 inhabitants; Großarl: 1961 - 2,574 inhabitants, 2011 3,693 inhabitants (cf. Statistics Austria)
} 
while the Deaf children of Bali are educated in the local school. Thus, the hearing kids of the Großarl valley were not exposed to sign language at school, which is the case today in the village school of Bengkala ${ }^{33}$. First and foremost it has to be stated, due to this educational situation, the sign language used in the Großarl valley today is not a purely village-based one. Because of the school situation the sign language variety in the corpora has been strongly influenced by the Salzburg (city) variety of ÖGS.

The difference between the present sign language and the one 50 years ago is not documented and is based on the informal narratives of the Deaf participants. On the lexical level clear statements on the use of different signs can be made. For instance, example (4) shows a sentence, given in my MA thesis (Lackner 2007, 8), in which the 'older generation' would use different signs for FATHER and FARM than the younger generation. Both generations use the same signs for WORK and FARM-SERVANT.

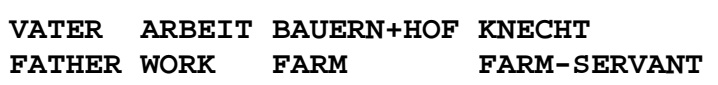

(cf. Lackner 2007, 8)

Structural differences cannot be deduced. Intuitively, the participants of the investigation expressed that less mouthing was used. Only one interesting aspect is still present in the data: One of the Deaf signers (born in 1936) signs differently from the other Deaf signers of the investigated group, who attribute his way of signing to 'the older signing generation'. In addition to this very intuitive evaluation, the annotations of dialogues show that with this person the dialogue partners sign in a different way. For instance, the sign FERTIG (FINISH), conveying an aspectual meaning, occurs more frequently in these dialogues. However, finding differences between these dialogues and those of 'the next generation' (which has already an average age of 55 to 60 years) requires investigation in detail.

\footnotetext{
${ }^{33}$ Since 2007 the village school in Bengkala has been an inclusive one. Since that, both Deaf and hearing kids have attended the same school (De Vos 2011).
} 
The current communication situation of the Deaf individuals who still live in the valley Großarl shows a different picture as compared to 50 years ago. Due to extensive tourism (especially skiing tourism), improvements of transport connections, commuting to work outside the valley, to list only some of the most important factors, the general conditions for the people of the valley have changed and, as a consequence, changes have also affected the Deaf inhabitants.

Nowadays, hardly any hearing inhabitant knows any signs; only the companies that still employ Deaf people use some elementary signs. Consequently, the Deaf community itself gets along well with one another. This is shown in the questionnaire in which the participants indicate that their narrow circle of friends is primarily constituted by Deaf individuals.

At this point the present situation of ÖGS and of the Deaf people living in Austria needs to be referred to. First, the Austrian Sign Language (ÖGS) has no standard variety; in most cases the Deaf individuals allocate themselves to a signing variety according to the standard of one of the nine federal states of Austria, although these varieties are also composed of several regional varieties. Following Jarmer (2004/5, 7), there are 8,000 Deaf people in Austria. These individuals, if they use ÖGS, together with about 10,000 hearing individuals who use ÖGS (at least as a second language), may communicate in ÖGS. In 2005 the Austrian Sign Language was recognized as one of the official languages in Austria. However, ÖGS has not yet been established in the Austrian curriculum for Deaf children (Lehrplan der Sonderschule für Gehörlose) in the way that Deaf children have the option to choose a bilingual (ÖGS and German) education (cf. Krausneker \& Schalber 2007). 


\subsubsection{The data}

After having introduced the Deaf informants/annotators and their special living situation that has influenced their dialectal variety of ÖGS, in the present subchapter the process of recording, data preparing and implementation of data coding is described.

\subsubsection{Video recording process and data preparation}

All recordings were conducted in places which are familiar to the Deaf participants. So, in 2005 the recordings took place in the home of the author of this thesis, which the Deaf participants were already familiar with. Subsequently recordings took place in the Deaf club in 'St. Johann im Pongau', situated in the county of Salzburg.

Apart from the third follow-up session, all the recordings were done with three cameras. When the Deaf informants performed a dialogue, the main camera filmed both participants while the other two cameras were positioned in an angular way (forming an angle of $90^{\circ}$ ) and so, always faced one of the dialogue partners while the second one was in the peripheral field of view. When the Deaf informants produced a monologue, one camera was positioned in the front, one laterally (with an angle of $180^{\circ}$ ), and one at an angle of $90^{\circ}$. In doing so, the various head and body movements in the different directions as well as gaze movements were recorded in an optimal way. The recordings always took place in one part of the room while all the other Deaf participants were able to watch the filming procedure. This circumstance led to the fact that the Deaf people very often started to sign with the filmed person(s), or the filmed person himself/herself commented on an ongoing discussion of the other Deaf individuals, or the watching individuals were inspired and wanted to immediately sign this input which constantly led to a 'change on the fly'. This familiar atmosphere (being in a well-known place, being watched when narrating - even from a farther distance, commenting on an ongoing discussion of signers who stand somewhere apart) is one of the very reasons which reduced the shyness in front of the camera and led to a variety of different signed discourse texts, which were evaluated afterwards by various Deaf individuals as very natural and authentic recordings. 
The recordings were always conducted by a hard of hearing person who grew up with sign language and who is familiar with the Deaf culture. He also offers sign language classes for adults. The instructions were given by him or by two of the Deaf informants who grew up in Hüttschlag and who have Deaf parents ${ }^{34}$. The instructions comprised the following: First, the signers were informed to always produce texts - an instruction which automatically happened in that setting and moreover, no single sentences in German were offered. Second, the Deaf informants were asked to sign dialogues on various topics, monologues on informal and formal stories, and trains of thoughts while going in for a long activity.

Moreover, the informal narrations, both in a dialogue and monologue setting, implied contents of the informants' childhood, their lives in the valley Großarl, about Deaf community in former times, and so on. Formal narrations possessed a special narrating structure. These were Deaf jokes or curricula vitae.

With regard to the short stories the Deaf informants were asked to sign a longer action, e.g. hiking, driving by car, going by train etc. During this longer action they were asked to think about a situation. In doing so, they were instructed to think about a thing or a situation, wonder whether a situation will/would occur, or formulate conditions under which a situation would occur. These trains of thought were then expressed with different attitudes or knowledge about the thought thing/situation like being unaware of a situation, being uncertain about the occurrence of a situation, being full of hope that the thoughts will be fulfilled and so on. The instruction was given twice, once by a video in which a Deaf lecturer described the task and once by a Deaf participant who coordinated the video production process and who constantly guided the Deaf informants through the recordings. As the individual recordings took place in the Deaf club and the particular filmed informant was visible to the other informants, the instruction was clear to everyone and there was not much difference between the signed contents. So, all the individuals mostly took 'hiking' as a long action and produced lines of thoughts which go together with this

\footnotetext{
${ }^{34}$ I was also constantly present as the official conductor of the filming sessions. To be one-hundred percent sure that there was no influence by hearing culture, I took a back seat, observed the ongoing process, and only interjected occasionally, to inform the camera man / instructor when something particular was required to be carried out additionally.
} 
activity. In doing so, contexts were produced which are easily comparable. One reason for doing the follow-up study was to ask the participants of the clarification study to produce some additional long activities during which trains of thoughts were formulated and to think about totally different situations. Thus, the informants were reflecting on other activities and other lines of thoughts.

The recordings were implemented in sitting and standing position, that is, the Deaf informants were instructed to sign in standing position and afterwards to repeat the signed content in the sitting position. The shorter narrations (like a train of thought) had to be signed twice in the particular positions.

The first step of data preparation generally included cutting the videos from the three camera views to make them time aligned and, if required, converting them. Subsequently, the recordings were put into ELAN (EUDICO Linguistic Annotator), a multimedia annotator, which makes it possible to view more videos simultaneously and to fill in all the required annotations into separate tiers which are time aligned with the videos. Thereby, both the sequential and the simultaneous occurrence of different manuals and nonmanuals can be analyzed.

\subsubsection{Resulting data}

The present $\mathrm{PhD}$ thesis is based on video material which is derived from three sessions of recordings in Salzburg and from four weekend blocks of educational training courses. With regard to the Salzburg variety the following video material has been used:

First, 13 annotated dialogues, lasting for three to ten minutes, in total 75 minutes have been used; this data has been annotated for the MA thesis on 'turn-taking signals' by the author of this thesis, together with Deaf individuals of the Pongauer Deaf club (see Corpus 1 in Appendix A). Furthermore, for this thesis six annotated monologues (curriculum vitae, jokes, and informal stories) of fifteen minutes have been used (see Corpus 4 in Appendix A). This material has been annotated in the course of a project on segmentation and structuring of ÖGS-texts by sign language interpreter students and in addition by two Deaf 
individuals via video. The main corpus (see Corpus 3 in Appendix A), which has been primarily used for identifying head and body markers in ÖGS, is composed of seven recordings of three to nine minutes on the average and around 40 minutes in total ${ }^{35}$. These recordings include short stories which are composed of a narrated part and a part in which trains of thoughts are expressed. This data has been annotated by four (some of them by five) Deaf annotators per recording. When doing this task, the Deaf participants annotated the videos produced by other members of the Pongauer Deaf club and their own signed data. As a result, the annotation was done from the Deaf informant's perspective and from an addressee's perspective. Finally, out of the clarification data the utterances of particular interest were cut out and annotated (see Corpus 5 in Appendix A). These amount to five minutes annotated material in total, done by sign language interpreter students and in addition by two Deaf individuals via video.

Second, with regard to the educational corpus (see Corpus 2 in Appendix A), around 30 minutes already annotated material has been used for the present research. This material dates from a study on 'linguistic terminology and its definitional structure in an educational training course', conducted by the author of this thesis (cf. Lackner 2009c, 2009d).

In conclusion, it has to be stated that the analyses of head and body markers are all based on signed texts. No elicited sentences are taken into consideration. As the following description of data coding shows, also the identification of head and body movements is done by Deaf individuals.

\footnotetext{
${ }^{35}$ It has to be added here that the Deaf informants always paused for a while until they repeated a line of thoughts or they started a new line of thoughts. Thus, the actual time of active signing is much shorter.
} 


\subsubsection{Procedure of data coding}

The procedure of the data coding is implemented as follows: The various parameters are described in ELAN first on an interpretative level (a more 'phonological level') and second, on a linguistic functional level. If perceptual details are relevant for the interpretative level, an additional tier is added to include the descriptive level (i.e. a more 'phonetic level') ${ }^{36}$. On the 'descriptive' level, the annotation of the parameters is based on rather detailed visual perception. On the 'interpretative' level, the annotation is a more holistic description in terms of a perceivable 'action' (cf. Figure 2.16) ${ }^{37}$. For example, 'head nod' is annotated on the 'phonological level', 'affirmation' on the 'functional level' and, if required, 'head down>up' on the 'phonetic level'. Beside the parameters ${ }^{38}$ glossleft-hand, gloss-right-hand, mouth movement(s) / positions (including a separate tier for 'mouthing' and 'mouth gesture') ${ }^{39}$, eye gaze movement(s), eye aperture and eye brow position / movement(s) ${ }^{40}$ the following head and body position(s) / movement(s) ${ }^{41}$ are annotated and analyzed in detail by the Deaf annotators (cf. Figure 2.16): head tilt-

\footnotetext{
${ }^{36}$ As a phonetic description for each head and body movement, indicated as language-relevant element by the Deaf annotators, would go beyond the possibilities of the present thesis and is not the subject of this thesis, this aspect will not be taken into any further consideration.

${ }^{37}$ On the interpretative level, e.g., a 'head turn rightward' is annotated while on the descriptive level sideward movements and holding position are annotated (head neutral $>$ head rightward $>$ head turn right moving $>$ head moving leftward $>$ head neutral). Using symbols like arrows for describing the movement contour would be another possibility (cf. Braffort 2008, 17-19). An exact phonetic measurement with, for instance, motion capture equipment would transcend the possibilities of this thesis. As expressed above, in my thesis the phonetic implementation of the identified head and body movements is not dealt with any further.

${ }^{38}$ Transcription conventions primarily follow those of the ECHO Project (cf. Nonhebel et al. 2004) and the Annotation Guidelines of the Auslan corpus (cf. Johnston 2010) as well as further annotation conventions discussed at the $3^{\text {rd }}$ workshop of the Sign Linguistics Corpus Network, 14-16 June 2010 in Stockholm.

39 In this thesis the two categories 'mouthing' and 'mouth gesture' are used. Crasborn et al. (2008) distinguish five categories of mouth actions namely 'mouthings', 'semantic empty mouth gestures', 'adverbial mouth gestures', 'whole face mouth actions' and 'enacting mouth gestures'. For reasons of efficiency and as mouth action is not the main goal of this thesis, the last four mouth actions are summarized in the category 'mouth gesture' in this thesis.

${ }^{40}$ The identified and annotated brow movements/positions are 'raised brows', 'furrowed brows', 'raised and furrowed brows', and 'furrowed brows - raised inward'. Lowering the brows was not distinguished from furrowing the brows. But, this could be of relevance as in ASL (cf. Weast 2008). I still keep using the more frequently used terminology 'furrowed brows' as the general term in this thesis. When having more findings on language-relevant distinctive eyebrow movements in ÖGS, the terminology will certainly become more precise. In addition, I keep the terms 'lowered' or 'furrowed' when quoting the respective researchers' description of the eyebrow movements.

${ }^{41}$ Both position(s) and movement(s) of one parameter have been annotated within one tier.
} 
forward/backward, chin up/down, head tilt-right/left, head turn-right/left, head rotation/etc.; body turn-left/right, body lean forward/backward, body leansideward/sways/shifting of weight/step, shoulder(s)/body straitening-up. Thus, each language-relevant instance of head position or movement(s) and body position or movement(s) along a body axis is annotated in separate tiers. In order to assure interrater reliability, several head and body tiers have been annotated on the phonological level by multiple viewers. This means that the annotators ${ }^{42}$ (four - for some videos five - Deaf annotators per video) were instructed to identify the different relevant head and body movement(s) and positions(s) - together with the exact beginning and ending points - and the current possible meaning/function of the particular parameters. The other tiers were annotated by the particular first Deaf annotator who started the annotation work and were then corrected by the other participants, if required. Each Deaf annotator got a separate list of body and head tiers which they had to annotate. The annotation of head and body movements and positions of the other annotators was concealed. This process resulted in at least four different annotations of the various head and body tiers which were compared afterwards as illustrated in the following:

\footnotetext{
${ }^{42}$ As the Deaf native signers were not familiar with ELAN before, I did the administrative work for them by typing into ELAN what they told me to enter. This means that the Deaf annotators described which movements and positions of the articulators head and body were relevant for them, the beginning and ending point of the movements and positions as well as further relevant characteristics, like a culmination on the last sign etc. In addition, the Deaf annotators described the meaning of the identified element. In some instances the clear function could be described like the function 'negation'. Frequently further contexts were offered in which the respective element could occur.
} 


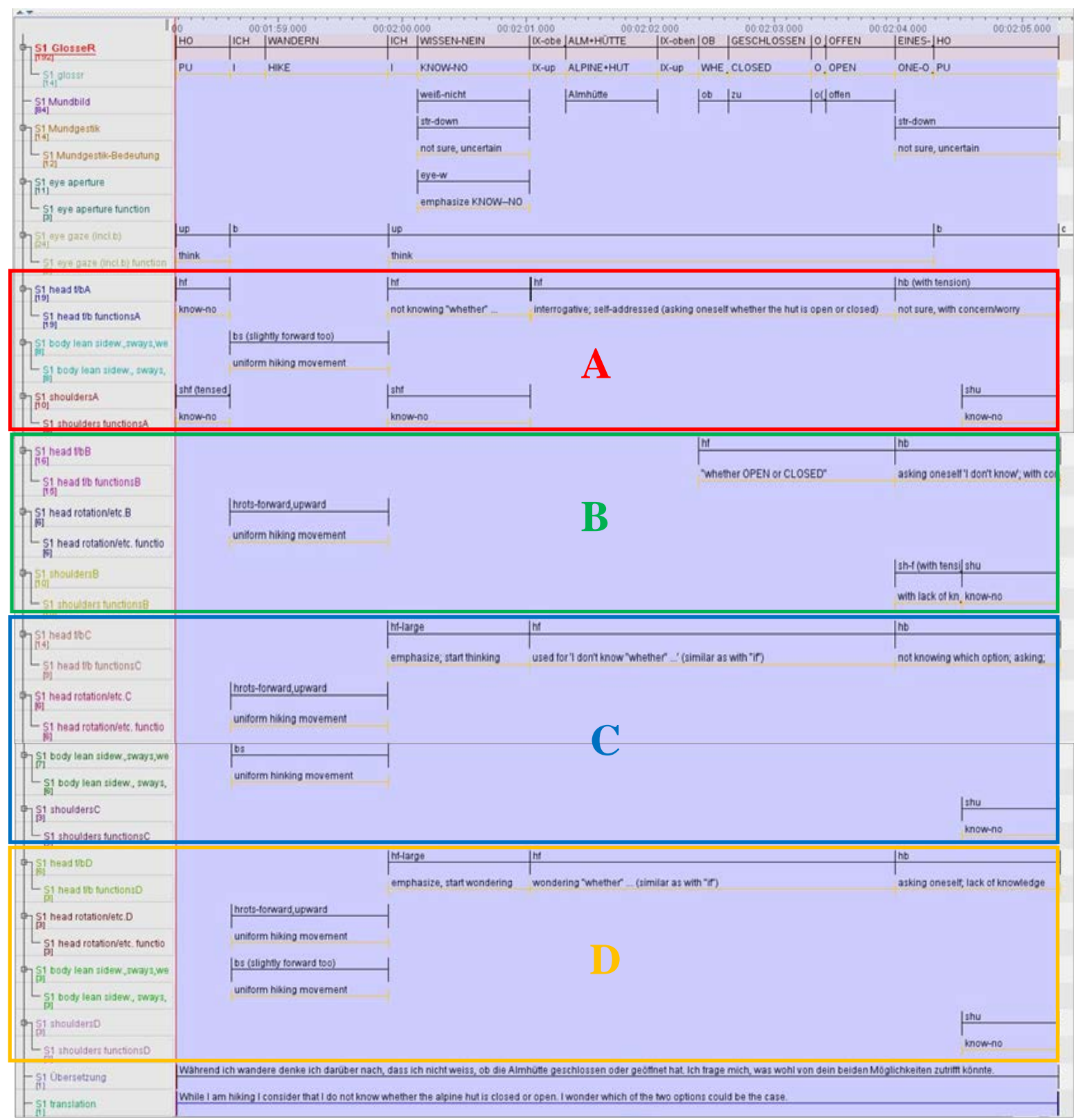

Figure 2.16 Annotations of the different Deaf annotators with regard to the various head and body tiers $^{43}$

Figure 2.16 illustrates the annotated tiers of four Deaf annotators in ELAN (all annotation tiers of annotator A are edged red, of annotator B green, of annotator C blue, and of

43 The annotators' descriptions for the particular meaning/function of an element are annotated in German in the data, but have been translated into English for this illustration. 
annotator D orange). The particular identified head and body movements are underlined black, the annotators' descriptions of the particular meaning/function are underlined yellow. To do the annotation task more quickly and more efficiently, the Deaf annotators used a set of abbreviations for the description of the forms of head and body movements (cf. annotation abbreviations). To sum up, all annotations show that the annotators frequently identified the same head and body movements such as the marker 'head forward', present in Figure 2.16, functioning for coding embedded polar interrogatives (cf. 4.3.1.2.1).

\subsubsection{Results of data coding}

The first and most striking outcome was that in almost all cases the different Deaf annotators identified the same head and body movements/positions along the same body axis - i.e. the same language-relevant, distinctive markers. Moreover, in most cases the Deaf annotators determined the same starting and ending points of these head and body movements/positions.

The identified language-relevant distinctive head and body movements/positions of the Deaf annotators show the following characteristics:

- First, one of the most significant facts is that with 'all' the Deaf annotators, independent of each other, (in nearly all cases) the beginning and ending point of the particular head or body movements were determined by the lexical entries as illustrated in example (3). Consequently, the high percentage of time-aligned entries in ELAN of the head and body markers with the lexical entries on which they refer to, shows that there must be a high tendency in sign languages of alignment between non-manual components with lexical entries, which they associate to - in the production but, most notably in the perception of the signing flow.

- Second, the movement direction of the respective language-relevant head and body markers has been clearly allocated to one direction on the three body axes. Thus, the identified relevant movements along these axes are the two contrastive options, namely 
upward versus downward, rightward versus leftward, forward versus backward. The data shows that nearly all the head and body movements/positions which display a language-relevant element can be performed in the opposite direction and display a distinctive language-relevant element. For instance, positioning the head forward is distinctive from positioning the head backward. Both are perceived as languagerelevant distinctive elements. Other examples are the markers ‘chin down' versus 'chin up', or a head nod performed with an upward-down movement versus a head nod performed with a down-upward movement, and so forth. Some of the identified head and body movements/positions can only be performed into one direction. So, the Deaf annotators identified the marker 'head rotations forward-upward' as language-relevant whereas rotating the head forward-downward can be articulated (only with great difficulty), but it is not a language-relevant element in ÖGS conveying a special meaning/function.

- Third, some movements or positions, performed in one direction, have a regular form and an intensified form. If a movement or a position is perceived as a very intense and distinctive language element by the Deaf annotators, the abbreviation of the annotation entry is modified with the additional information 'large', attached with a hyphen.

- Fourth, the relevant distinctive components of the identified head and body elements in ÖGS are the movement or positioning component, the direction of the movement/positioning (i.e. forward versus backward, upward versus downward, right versus left), the intensified performance or the size of performance (e.g. positioning the head forward versus positioning the head forward in an intensified way, or producing headshakes with a small radius versus headshakes with a large radius), the speed of performance (e.g. producing fast headshakes versus producing slow headshakes), the degree of body tension (e.g. producing non-tensed headshakes or performing head nods in an trembling way with a tensed body), and an additional movement component (e.g. head nods with trembling movement or headshakes with alpha-movement).

- Fifth, some language-relevant elements possess the same function and vary depending on special factors. Thus, head tilt sideward, body lean sideward and step sideward can 
all be used as means of marking the 'alternative space' (cf. 6.4.4). The use of the respective marker depends on facts like being in sitting or standing position, distance of the audience, individual style and probably other reasons too. Other markers cannot vary. So, body sways functioning as modality marker (cf. 7.4.4) cannot be replaced by weight shift sideward while body sways used in narratives for discourse-structuring and/or rhythmical purposes can be implemented by weight shifting too.

- Sixth, the annotations of the same tiers by the different Deaf annotators show that to a high degree the Deaf annotators identified the same movements/positions of the head and/or body along the same body axis - i.e. the same language-relevant, distinctive markers.

- Seventh, in a considerable number of cases different markers of the same articulator, primarily the head, were identified by the Deaf annotators as conveying different functions. This phenomenon is displayed in the following example (5) in which three head markers simultaneously occur.

(5)

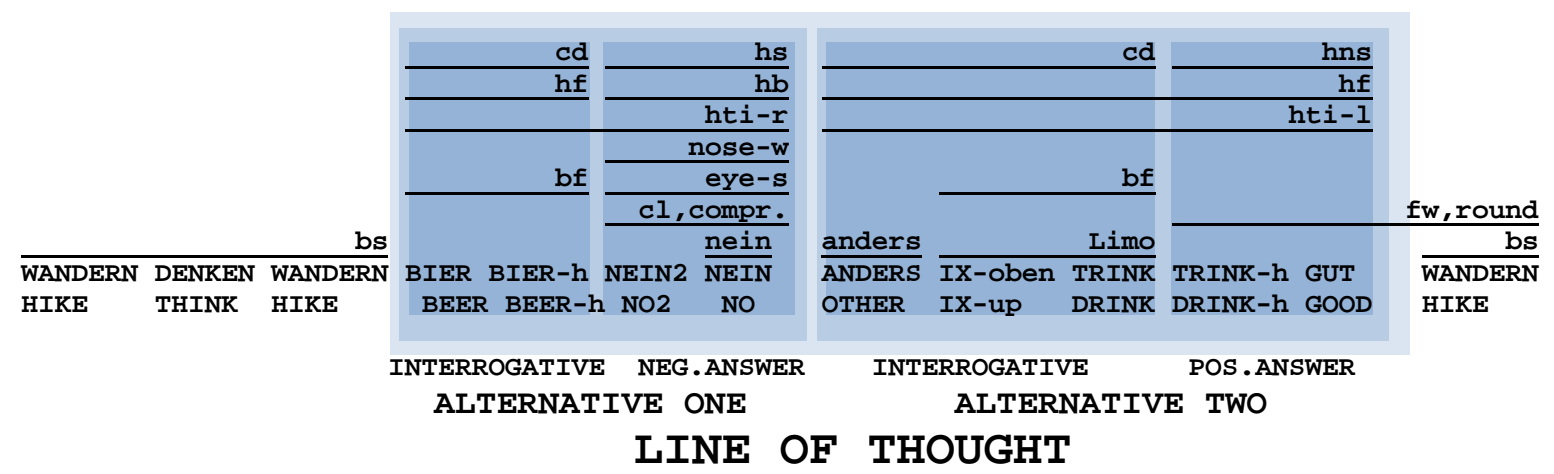

Während ich wandere überlege ich, ob ich ein Bier drinken soll. Ich denke nein, lieber etwas anderes - ja eher eine Limonade.

While I am hiking I think whether I should drink a beer. I think no, better something else - yes, better a lemonade.

(F004_117,1209_m_thoughts_03.26-03.37)

In example (5), three different head markers simultaneously cover the sign BEER and its holding position (color-coded dark blue, first box). These are 'chin down', 'head forward' and 'tilting the head rightwards'. One of these head markers ('head tilt sideward') is maintained during the following two negative signs, a further head marker is added ('head backward'). The subsequently produced signs of the line of thoughts (color-coded light 
blue) are also covered by three head markers. Two of them - i.e. 'head tilt leftward' and 'head forward' (color-coded mid-blue, second box) - co-occur with the remaining signs of the line of thought; The signs OTHER IX-up DRINK have 'chin down' (color-coded dark blue, third box) as the third head marker, while the head is additionally nodding (also color-coded dark blue, last box) while holding the sign for DRINK and signing GOOD. At the present stage, the particular functions of the various head markers are not completely clarified. Tilting the head first rightward, then leftward (the two boxes color-coded midblue) is unmistakably used for expressing two alternatives. The 'headshakes' are used for negation, the head nods are used for assertion. Following the Deaf annotators feedback, the marker 'chin down', which occurs twice, is used to express the interrogative. The interpretation for positioning the head forward, backward, and again forward has not yet been clarified. One interpretation of the use of the head markers forward and backward is to express affirmation versus rejection. A further interpretation is that the first contrastive head marker pair (head forward and subsequently head backward) are used to demonstrate the question-answer-pair. Furthermore, possibly positioning the head forward in the interrogatives is done to formulate twice an 'embedded two-option interrogative' and moving the head backward (while signing 'no' twice) or staying forward (while holding the sign DRINK and adding GOOD) expresses the negative or positive outcome of the thought situation.

Recognizing all these characteristics of head and body movements/positions which convey a language-relevant function was first of all determined by the Deaf annotators' identification and their description of 'what each of the identified elements does in their sense'. Second, all the contexts in which each head and/or body marker occurred were brought together and compared. In doing so, it was possible to clarify the particular function of several of the head and body markers. These identified functions of the various head and body movements/positions are the main research object of the present thesis. In the following, example contexts in which the head and body markers occur are described in detail within each subchapter. Thereby, the form and meaning/function of each identified head and body marker is shown. 


\section{Negation and assertion coded by headshakes and head nods}

In ÖGS texts, head movements to the sides as well as up and down, or down and up are frequent. These are on the one hand 'headshakes', which in most cases a negation function is attributed to, and on the other hand 'head nods' which an asserting function. Taking a closer look at both non-manual elements, it becomes obvious that neither forms nor functions of these headshakes/nods are the same. In this chapter, I will describe those headshakes which have a clear clause negating function and those head nods which convey an assertive function. For reasons of clear illustration, the negated constituents are colorcoded blue, the asserted constituents green.

\subsection{Negation}

\subsubsection{Introduction}

Negation has been investigated in a lot of sign languages, and this in detail. Zeshan (2006b, 30-32) lists 37 sign languages which have published data on that topic. She states it as a significant fact that non-manual marking for negative constructions is primarily done by head movements: In all the investigated sign languages, moving the head sideways resulting in headshakes is at least one possibility for clause negation. It is this non-manual marker that I want to emphasize in the present chapter on 'negative constructions'.

First, I briefly present the literature on negation in sign languages (primarily referring to Zeshan's typological conclusions). Subsequently, the non-manual marker headshakes is described in detail, including its types, its clause-negating function, its co-occurrence, its frequently co-occurring negative signs, and some possible reasons for its spreading along the entire clause. Also, speech act negation, negative contrast and other functions - all coded by headshakes - are discussed within this section. 


\subsubsection{Overview of the literature}

Negation in sign languages is coded by manual signs as well as by non-manual elements. Liddell (1977) observed that negative headshakes in American Sign Language (ASL) can co-occur with a negated clause and reinforce the negativity of that clause. Veinberg \& Wilbur (1990) broaden and deepen the investigations on negative headshakes in ASL. They observed that manual negative elements are 'redundant' to non-manual headshakes. Further, many other non-manual behaviors co-occur with negative clauses, most frequently mouth actions such as 'putting the mouth corners downward', 'slightly closing the mouth', or 'stretching the lips'. Also other non-manual behaviors are occasionally present like 'furrowed brows', ‘closed eyes', 'head back', ‘contracted chin', ‘squinted eyes', 'wrinkled nose', or 'head forward'. Most relevant to this investigation, Veinberg \& Wilbur observed that headshakes implemented by Deaf signers differ from those by hearing speakers. The gestural headshakes performed by the hearing individuals occurred less frequently, less uniformly, and without relation to the syntactic constituents in the speech stream.

Based on a typological comparison, Zeshan (2006b, 42-43) summarizes that the head movement most frequently used for coding negation in sign language is the side-to-side movement which changes a statement from positive to negative. But these headshakes have different grammatical status within the respective sign languages. In few sign languages, an alternative head marker is used for negation. Eastern Mediterranean sign languages including Greek Sign Language (GSL), Turkish Sign Language (TID), Jordanian Sign Language (LIU), and probably further Eastern Mediterranean sign languages use backward tilting of the head as an alternative negative marker. This is likely an areal phenomenon (Antzakas 2006, 261-268 on GSL; Zeshan 2006c, 160-163 and Gökgöz $2011^{44}$ on TID; and Hendriks 2007a, 115-117 on LIU). Other negative nonmanual markers like 'wrinkled nose', 'eye brows furrowed', or 'corners of the mouth

\footnotetext{
${ }^{44}$ Gökgöz $(2011,68)$ shows that in TiD the two head negation markers 'headshakes' and 'backward head tilt' differ. The first can occur as grammatical or lexical marker; the latter is only a lexical marker due to its non-spreading character and its non-consistent occurrence in the data (only in half of the investigated negative sentences). Further, he lists other nonmanuals like a 'single head turn', interpreted as a lexical component of one of the negation signs, and 'non-neutral brow positions', interpreted as showing the syntactic domain of negation.
} 
stretched down' occur in several sign languages (Zeshan 2006b, 42). The interplay of negation and these non-manual phenomena (apart from the head markers) have hardly been investigated, making cross-linguistic comparison not yet possible ${ }^{45}$.

For ÖGS the findings on negation in Croatian Sign Language (HZJ) are of interest, as both sign languages have a historical relation (cf. Šarac Kuhn \& Wilbur 2006, $164^{46}$ ). Hrastinski (2010) observed several negative signs and co-occurring negative nonmanuals in HZJ, the latter defined as 'secondary negation markers'. These are head movements such as 'headshakes' and a 'single head turn sideward' as well as mouth actions like 'mouth corners stretched down', 'pursed lips', and 'open mouth with showing the upper teeth'.

While some research on negation in ÖGS has been done, the focus has been on the manual elements and their contexts of occurrence. Hofstätter \& Stalzer (2001) describe semantic aspects of negation in ÖGS. They include a statistic evaluation of the frequency of manual negative signs compared to negative headshakes with or without a manual negative sign. The results show that headshakes are present in $86 \%$ of these negative constructions ${ }^{47}$. Skant et al. (2002, 110-102, 183-235) describe negation as a sentence type and various negation elements including a detailed and categorized list of manual negation elements along with their context of occurrence and the non-manual element 'headshake/s'. They list two functions of headshakes: either, it can negate a statement, or, in an interactive context, one or more headshake/s may function as an answer or a kind of interactive reaction. In order to negate a statement, the headshakes may cover or follow the statement as shown in the examples in Skant et al. (2002, 184-185):

\footnotetext{
${ }^{45}$ In chapter 3, 4 and 5 the occurrence and possible functions in ÖGS of these listed non-manual markers is briefly described, but requires more in-depth research.

${ }^{46}$ In the $19^{\text {th }}$ century Deaf students and even teachers of Croatia attended the Deaf institute in Vienna. There, they were exposed to ÖGS during the lectures. This very likely caused mutual influence resulting in commonalities. Common non-manual interrogative markers were identified (Šarac Kuhn et al. 2007).

${ }^{47}$ In absolute numbers, 24 instances without headshakes are compared to 147 instances in which headshakes occur alone or with a sign (Hofstätter \& Stalzer 2001, 11).
} 
$(6)^{48}$

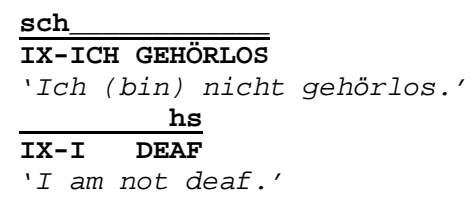

VARIANT 1

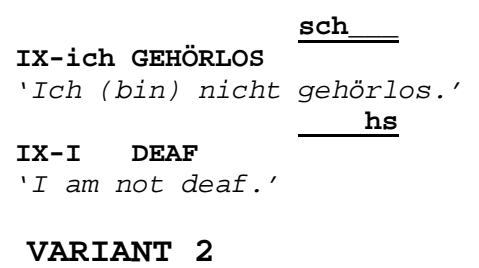

(Skant et al. 2002, 184-185)

The element headshakes may occur by itself, it may occur with the statement which it negates, or it may occur along with manual negation elements. As Skant et al. (2002) add, the 'shaking movement' can be interpreted as a movement which shifts between the articulators. Thus, the 'shaking movement' of the head can move to the hands. Also, head shaking can be an accompanying element of several negative signs.

In the following, I focus on headshakes used for clause negation. Also further functions coded by headshakes are described.

\subsubsection{Clause negation coded by headshakes}

\subsubsection{Different types of negative headshakes}

In the ÖGS data headshakes are frequently used for negation. The annotators note that the shaking movements differ in size and speed ${ }^{49}$. Thus, when a single or several headshakes are perceived to be performed smaller or larger than the regular size, or when they are perceived to be performed slower or faster than the regular speed, this information is added to the annotations. This difference is illustrated in (7):

\footnotetext{
${ }^{48}$ The German term for headshakes is ‘(Kopf)schütteln', abbreviated as 'sch' in Skant et al. (2002).

${ }^{49}$ One or more headshakes functioning for clause negation are annotated as headshakes. However, if a signer performs a head turn sideward which tends to be performed in a larger size and with slower speed, this instance is annotated as 'head turn sideward' - even if it is used for negation purpose. In these cases the annotators described an emphasized negative function. The data shows that the 'single head turn sideward' tends to occur with negative signs like DARF-NICHT (MUST-NOT) more often. Moreover, this movement co-occurs with negative signs which imply only one movement excursion, that is, they do not include several movement iterations.
} 
(7)

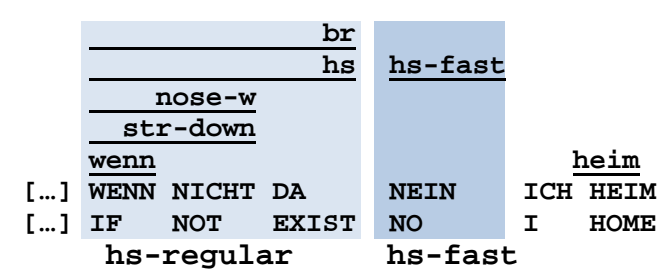

[...] Wenn es nichts gibt, nein (dann bleibe ich nicht), dann gehe ich heim.

[...] If there is nothing, no (then $I$ won't stay), then $I^{\prime} I I$ go home

(F001_092,1184_m_thoughts_ex12b_02.54-02.58)

In example (7), the signer produces a conditional clause in which the entire if-clause is covered by regular headshakes. Afterwards fast headshakes, constituting a part of the consequent, follow. These headshakes are perceived to have been performed faster, demonstrating one of the conditions under which a difference in speed is perceived. First, when several signs are covered by headshakes, the tendency is for the headshakes to be performed at regular speed, while if only one sign is covered by headshakes, they tend to be performed faster. Also, if a clause or a predicate (mostly constituting a verb and a negation element like GEBEN NEIN (GIVE NO)) are negated, headshake movements at a regular speed are perceived, whereas if only a negation element is covered by headshakes functioning as in example (7) or headshakes occur by themselves and follow a clause which they negate (cf. second variant of example (6)), then the headshakes are produced in a fast way.

\subsubsection{Clause negation as main function of headshakes}

The marker headshakes may negate a predicate or an entire clause even without a manual negative sign. In most cases however, headshakes and a manual negator co-occur. Thus, headshakes may negate a clause, but they may also 'reinforce negation' (cf. Fischer 2006, 186). In a few cases, only a manual negator occurs without headshakes. This may be due to socio-linguistic reasons, the filming situation, etc. The annotators clearly understood it as negative despite the absence of negative headshakes. This finding supports the analysis by Hofstätter \& Stalzer (2001, 10-12). 
In the following, I first provide an example of the most frequent case from the data which has both headshakes and negation manual sign. Then I provide an example with headshakes only.

$(8)^{50}$

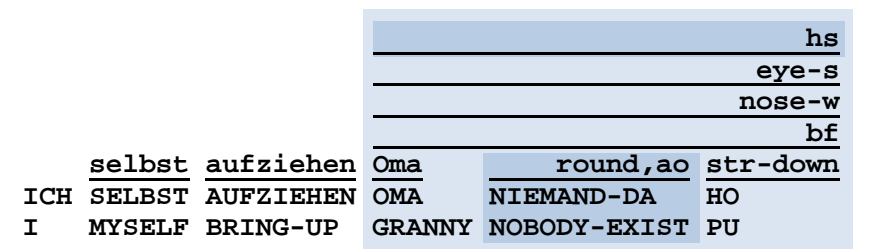

Ich habe sie selbst groß gezogen. Es gab keine Oma.

I brought them up alone. There was no granny.

(F001_Film1_Szene7_d_informal_story_01.04-01.07)

In example (8), the signer narrates that she had to bring up her children alone as both grandmothers had died before. The headshakes co-occur with the preceding noun (about whom the statement is made), the negation element and the element PALM-UP ${ }^{51}$. Both the negative headshakes (color-coded dark blue) and the negative sign (also color-coded dark blue) mark clause negation (the negated clause is color-coded light blue). Three other nonmanual markers accompany the negated clause. These are 'squinted eyes', 'wrinkled nose', and 'furrowed brows'. All three markers frequently co-occur in a negated clause in ÖGS. Their grammatical status, their possible negative or other function such as expressing modality, and the influence of the co-occurring nonmanuals on the negative sign and the negative headshakes require further investigations (cf.7.3.2).

With regard to the negative signs in example (7) the following observation has to be mentioned: Skant et al. (2002, 199-200) observe that some manual negators may convey the meaning of 'non-existing' or 'non-possessing'. The present example (8) shows that the sign NOBODY-EXIST has that meaning and is used in a predicative way. In addition, the negative sign is accompanied by the mouth action 'round lips, air coming out'. This mouth

\footnotetext{
50 The sign NOBODY-EXIST is produced with a two-handed B-hand form. The palm of the dominant hand faces downward, the palm of the non-dominant upward. The movement is that both hands rub in a contrary circle movement in front of the chest. In addition, the mouth is open, the lips are moved forward, and air flows out of the mouth.

${ }^{51}$ The phenomenon that signs following the negated predicate are included in that part, which is covered by headshakes, is described for other sign languages too. For instance, Zeshan (2006c, 162) describes that in Turkish Sign Language pronominal index pointing or PALM-UP which follow the negated predicate may be covered by negative headshakes.
} 
action frequently occurs with negative signs which express non-existence or lack of something.

The second example on clause negation is one in which only the non-manual marker is used to negate the clause.

(9)

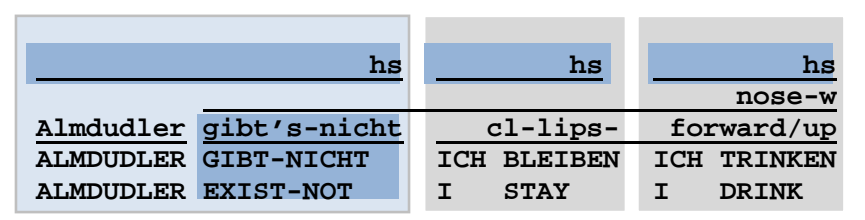

Es gibt keinen Almdudler, ich bleibe nicht, ich trinke nichts.

There is no almdudler, I won't stay, I won't drink anything.

(M007_1120_m_thoughts_ex.03a_00.00-00.06)

In (9), the informant produces three successive statements. All these are covered by headshakes. The breaks between the particular headshakes demonstrate that the signer always restarts with the shaking movements. In the first statement, both are present, the marker headshakes and a manual negation element which constitutes a verb that is conducted with an alpha-movement and consequently conveys the additional meaning of negativity. The second and third statements are composed of the subject (about which a statement is made), a verb and co-occurring headshakes causing each clause to be negated. In other words, the polarity of the second and third clause is reversed, i.e. from positive to negative, by the marker headshakes.

\subsubsection{Clause negation marked by co-occurring negative headshakes}

It bears repeating that while headshakes may occur without any manual negation sign, more frequently they co-occur with a manual negator. However, headshakes can also be completely lacking when a manual negation sign is produced. So, most frequently, headshakes co-occur with manual negation signs and further lexical elements. Both, the non-manual and the manual negators occur in order to negate the clause. This is exemplified in Figure 3.1. 

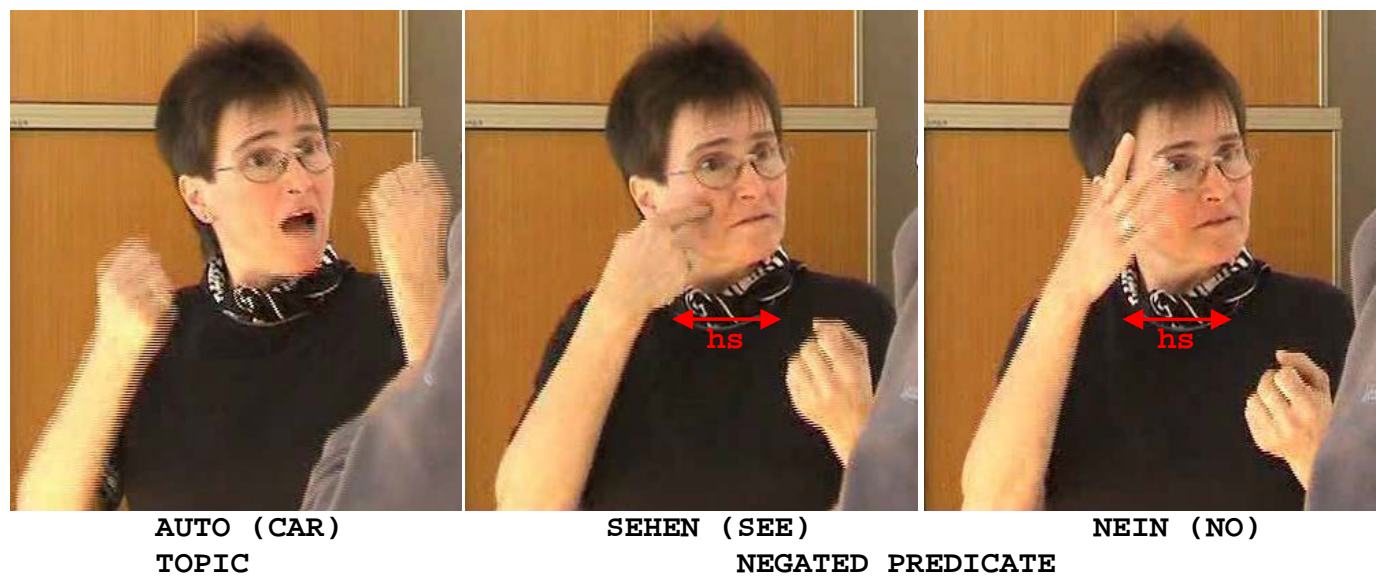

(F001_Film1_Szene1_d_story_informel_00.43)

Figure 3.1 Co-occurrence of negative headshakes and a negation sign

As illustrated, the informant asks the dialogue partner if he could see anything when driving (because it was snowing heavily). The entire clause is covered by 'chin-up' (head slightly tilted ${ }^{52}$, too), indicating a direct polar question. The sign CAR is additionally covered by 'raised eyebrows', indicating this element as the topic ${ }^{53}$. The subsequent sign SEE is followed by the negative sign NO. Both signs receive the negative marker headshakes.

In this subchapter, I focus on the marker headshakes used for clause negation. To begin with, the possible spreading ${ }^{54}$ of the non-manual negation marker is illustrated (cf. this sub-chapter). Subsequently, the negative signs which frequently co-occur with negative

\footnotetext{
${ }^{52}$ cf. 4.2.1.2 and 7.4.6

${ }^{53}$ In the present data the topic is frequently marked by 'raised brows'. But more investigations are required to make clear statements on how topicalization functions in ÖGS.

${ }^{54}$ In this subchapter I use the terms 'co-occurrence' and 'covering' for describing the phenomenon that lexical elements are accompanied by non-manual markers like headshakes or head nods. The term 'spreading' is used to describe the phenomenon that a non-manual marker like headshakes co-occurs with more lexical elements than just the negative manual sign. Consequently, the term 'spreading' focuses on the phonological aspect of the non-manual marker. It is used in accordance with Pfau \& Quer's (2004) study on the syntax of negation. The term '(semantic) scope' is used in accordance with Lehmann (2012) and refers to the semantic relation, specifically, to the syntagmatic relation of an 'operator' (e.g. headshakes and head nods) and the 'operand' (especially the 'extent' of the operand, thus, the unit covered by the nonmanual marker).
} 


\section{NEGATION AND ASSERTION CODED BY HEADSHAKES AND HEAD NODS}

headshakes are described (cf. 3.1.3.4). Then, some reasons for spreading of negative headshakes along the entire clause are discussed (cf. 3.1.3.5).

The following prototypical illustration shows the possible spreading of 'headshakes'. The syntactic element covered by headshakes is the entire predicate (color-coded blue).

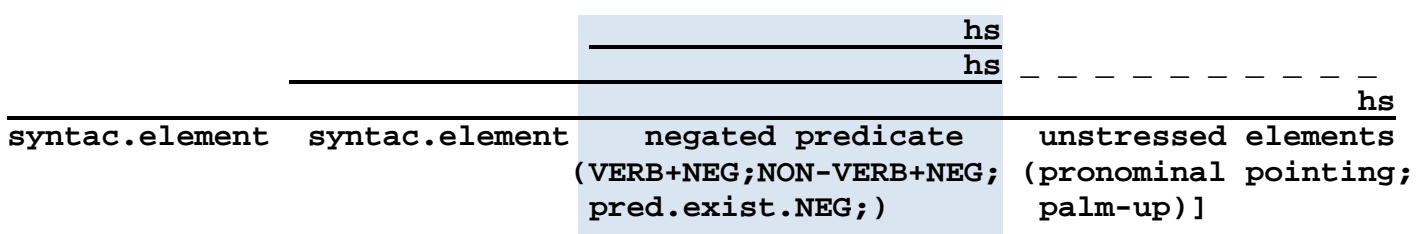

Figure 3.2 Possible spreading of the non-manual negator headshakes in ÖGS

The following three examples demonstrate the possible spreading of the non-manual marker headshakes occurring in the context of NEIN (NO), evident in the present data.

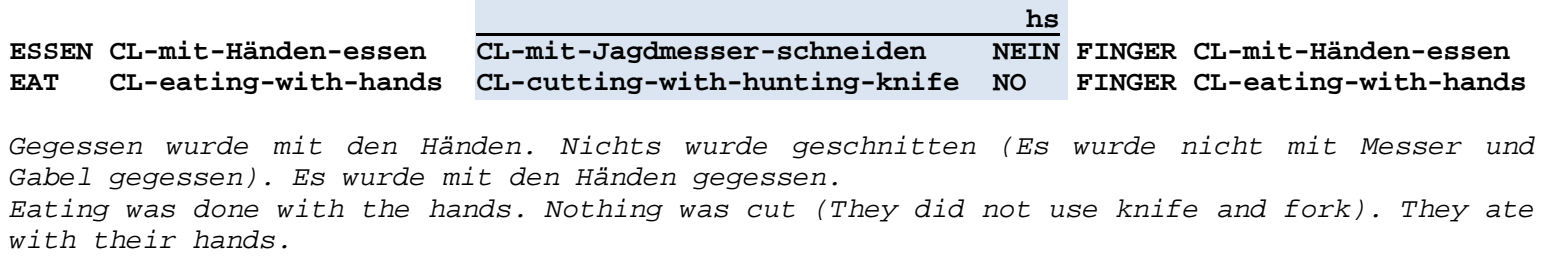

(M005_Film1_Szene2_d_story_informal_04.54-04.59)

In example (10), the headshakes cover the classifier predicate and the manual negator. Thus, the syntactic constituent (displaying the negated predicate), composed of these two signs, is negated. Spreading is required with the use of NO.

As illustrated in Figure 3.2, negative headshakes can spread to the end of the sentence. This is exemplified in (11).

\begin{tabular}{|c|c|c|c|c|c|}
\hline \multirow[t]{3}{*}{ +gaze } & & gaze-up & \multicolumn{3}{|r|}{ +gaze } \\
\hline & & cu & & & \\
\hline & & $\overline{b r}$ & hs & hs & hns \\
\hline $\begin{array}{l}\text { KLEIN-SEIN } \\
\text { BEING-SMALL }\end{array}$ & $\begin{array}{l}\text { WIR-BEIDE } \\
\text { WE-BOTH }\end{array}$ & $\begin{array}{l}\text { Zwillinge } \\
\text { ZWILLINGE } \\
\text { TWINS }\end{array}$ & $\begin{array}{ll}\text { verSch } & \\
\text { VERSTEHEN+NEIN } & \text { PERSON } \\
\text { UNDERSTAND+NO } & \text { PERSON }\end{array}$ & $\begin{array}{l}\text { versch } \\
\text { VERSTEHEN+NEIN WIR-BEIDE } \\
\text { UNDERSTAND+NO WE-BOTH }\end{array}$ & $\begin{array}{l}\text { HO } \\
\text { PU }\end{array}$ \\
\hline
\end{tabular}

[...] Als wir noch klein waren, wurden wir (von Erwachsenen) gefragt, ob wir Zwillinge wären. Wir beide verstanden sie nicht (was sie damit meinten).

[...] Being small, we were asked (by adults) if we were twins. We both could not understand them (what they meant).

(M001_Film1_Szene5_d_story_informel_01.38-01.41) 
The context of (11) a sister and a brother narrate about their time at the Deaf boarding school. When they first arrived, the educators thought that they were twins, but at their young age, they didn't know what 'twins' were. In this context, the first sign is a time reference indicating 'when we were little'. The next two signs display the repetition of the content which they did not understand. This is formulated from the perspective of children, indicated by the non-manual markers 'gaze up' and 'chin up'. The comment that 'they could not understand (what the adults meant with twins)' is as a whole accompanied by headshakes ${ }^{55}$. This comment is made up of two subsequently following syntactic constituents (each color-coded blue), constituting two successively occurring predicates, each of which is followed by a pronominal element (PERSON and WE-BOTH) (encircled red). As illustrated, the negative headshakes spread along these elements, conveying a referential meaning. This phenomenon is also described for other sign languages (compare hereunto Zeshan 2006c, 162).

Headshakes can spread to the beginning of the clause. In the following example (12), two successively produced clauses (each color-coded blue) are present. Both have the negated predicate in clause-final position (encircled red). In the second one, the negation sign even fulfills a predicative negative existential function. In both clauses, the sign of location (i.e. where people come from; encircled green) is covered by the headshakes too. Moreover, the example demonstrates that if two clauses successively occur and both require the nonmanual negative marker, the shaking movement is maintained during the second constituent, in example (12) within one prosodic movement contour.

\footnotetext{
${ }^{55}$ If two subsequently produced phrases or clause are negated, two possible prosodic patterns are present in the data. First, the shaking movements maintain and constitute 'one prosodic contour' which covers both phrases (see example (12)). The second option is that the intensity of shaking restarts when the second phrase or clause starts, resulting in two 'prosodic contours' (see example (11)). At the present stage of research it cannot be determined in which cases a single and in which two movement contours are present. The reason for the presence of one or two prosodic contours is very likely the signing rate (cf. Wilbur 2009).
} 
$(12)^{56}$

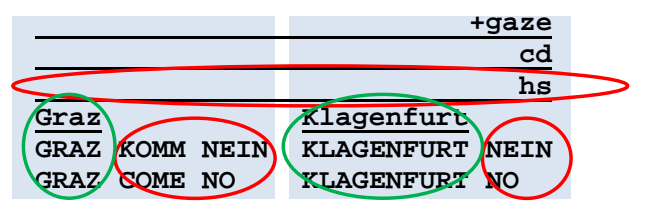

Grazer kamen keine? Klagenfurter (kamen) auch nicht?

There were no people from Graz? None from Klagenfurt either?

(M001_Film1_Szene3_d_story_informel_01.05-01.09)

\subsubsection{Negative headshakes with frequently co-occurring negative signs}

In the present subchapter, the negation elements which most frequently occur in the context of negative headshakes are briefly described. First, the signs NICHT/KEIN (NOT/NO) and NEIN (NO) are analyzed as they are primarily used to negate a clause. In addition, negative modals and suppletive negative signs are described with regard to the co-occurrence of negative headshakes. A more detailed description of various possible negation elements is found in Skant et al. (2002, 185-237) and Stalzer (in prep.).

\section{a) The sign NEIN (NO)}

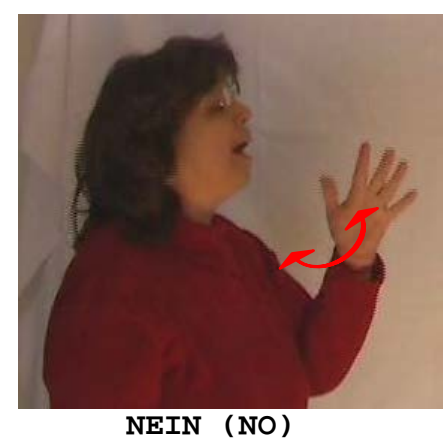

Figure 3.3 The manual negator NEIN (NO)

The variant of NEIN (NO) illustrated above is one of the three NO-signs listed by Skant et al. (2002, 185-186 and 192-194). They note that the sign is accompanied by headshakes and can be combined with various mouth gestures, depending on the intensity of 'negativity'. The manual negator may have the status of a sentence. In this case, the negator precedes the following utterance which displays a correction. The sign may also

\footnotetext{
${ }^{56}$ The marker 'chin down' (abbreviated as 'cd') indicates a polar question (cf. 4.2.1.1).
} 
negate elements of a sentence (but not verbs). In these instances the negator follows the non-verbal elements of a sentence.

The findings of Skant et al. (2002) are confirmed by the present data insofar as the sign NO occurs as a sentence of its own or negates a syntactic element. However, the data also shows that NO is primarily used for clause negation, that is, NO very frequently follows a verb which it negates.

This sign has some interesting aspects: First, it has the tendency to follow various verbs, especially expressions of cognition like knowing, believing or understanding. Second, the manual negator tends to be cliticized to the element. This means that the sign immediately follows the preceding verb and is performed at the place of articulation of the preceding sign or has its starting point there (see Figure 3.4). In some cases, like the sign WISSENNEIN (KNOW-NO), it is already merged with the sign of knowledge in that the sign has the hand form of NO - except for the middle finger, which is pointed down and which displays the characteristic of the sign WISSEN (KNOW); further, the movement starts at the place of articulation of the sign KNOW (at the temple) and goes downward (as is normally done when signing KNOW) while the shaking movement of the hand (being a component of the sign NO) starts (see Figure 3.5).

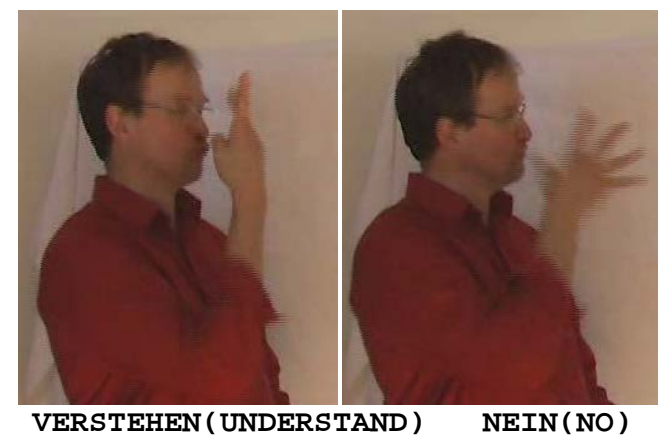

Figure 3.4 Sign of cognition with manual negator NO 


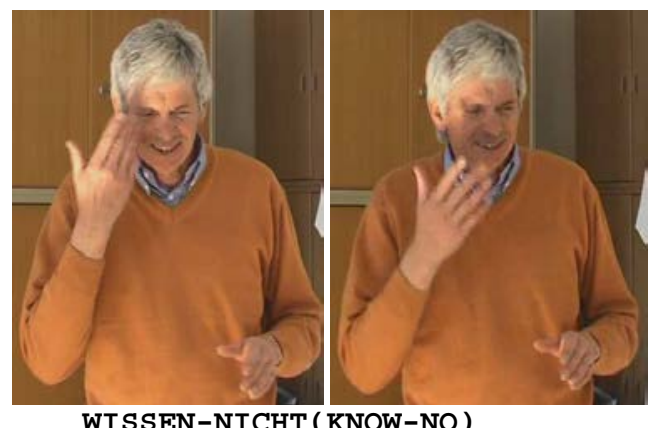

WISSEN - NICHT ( KNOW-NO)

Figure 3.5 Sign of knowledge and negation

\section{b) The sign NICHT/KEIN (NOT/NO)}

The second negation sign quite frequently functioning as clause negator is the sign NICHT/KEIN (NOT/NO) ${ }^{57}$ as depicted in the following (Figure 3.6). Skant et al. (2002, 195-201) identify two signs which they allocate to the category NICHT (NOT). One of these signs is the one illustrated here, NICHT (NOT). Regularly a single headshake cooccurs with the sign. It may be accompanied by mouthing 'nicht' ('not'), 'kein' ('no'), or it has no mouthing. According to Skant et al. the sign can negate a verb by preceding it, following it, or preceding and following the verb. Further, the manual negator may also negate non-verbal elements by preceding this element. Furthermore, a correlation with existence and possession is apparent in the sense that it can be used without a verb of existence or possession in the sense of 'non-existing' or 'non-possessing'.

The described findings coincide with the kind and place of occurrence in the data. As illustrated in Figure 3.6, the manual negator both precedes and follows a sign and negates the predicate or clause. Further, the manual negator also occurs without other elements to refer to or negate and has a predicative use in the sense of 'non-existing' or 'nonpossessing ${ }^{58}$.

\footnotetext{
${ }^{57}$ In the following, only the term NICHT (NOT) is used when describing NICHT/KEIN (NOT/NO).

${ }^{58} \mathrm{NOT} / \mathrm{NO}$ in predicative use, conveying non-existing or non-possessing, has a noticeably longer duration in production in the examples, present in the data. This may also be due to the fact that this negator is always found in clause-final positional when it is used predicatively in the corpus. Thus, there may be processes like 'phrase final lengthening' or more prosodic weight on the final position of a clause which cause the lengthening of the manual negator.

Nespor \& Sandler (1999, 153-172) describe final lengthening of manuals for ISL in the form of reduplication or holding of the last sign of a prosodic phrase (PP) or a longer hold or more iterations at the end of intonational phrase (IP). According to this aspect, Kooij \& Crasborn $(2008,1308)$ determine for
} 


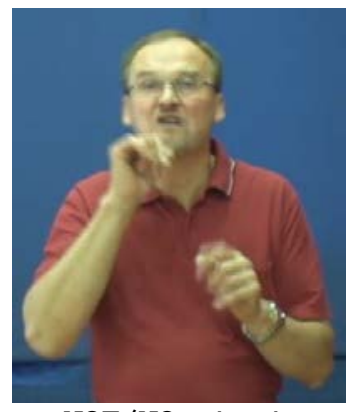

NOT/NO-start

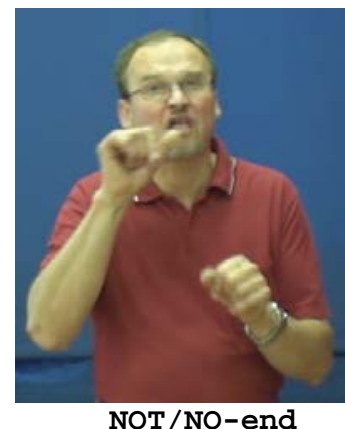

NOT/NO-end

Figure 3.6 The manual negator NICHT/KEIN (NOT/NO)

In the following, in example (13) and (14) the manual negator precedes the negated element, and in (15) it stands on its own. In examples (13) and (15), the sign NOT - even with the mouthing 'kein' ('no') in (13) - has the meaning of 'non-existing'. Only in example (14), the sign for existence is expressed too and the manual negator modifies it. In all examples the manual negator and headshakes are encircled red, the negated syntactic constituent is marked grey.

$(13)^{59}$

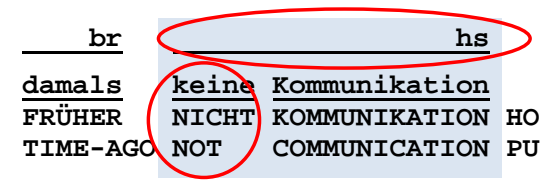

Damals gab es keine Kommunikation(smittel).

In the old days, there used to be no (means of) communication.

$(14)^{60}$

(M002_Film2_Szene14_d_story_informal_04.37-04.40)

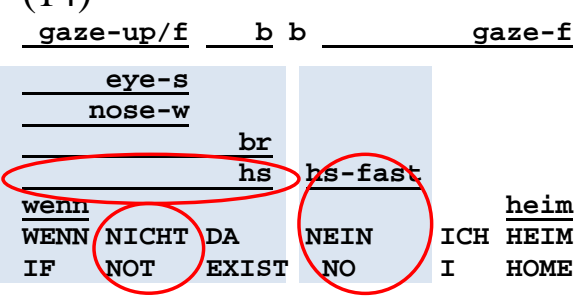

Wenn es nichts gibt, nein (dann bleibe ich nicht), dann gehe ich heim.

If there isn't anything available, no (then I won't stay), then I'll go home.

(F001_092,1184_m_thoughts_ex11a2_02.55-02.58)

NGT that the final position of a clause or sentence has more 'prosodic weight' - they refer to the statements of Wilbur (1999) and Petronio (1993) about ASL and Miller (2004) about LSQ. Originally 'Phrase Final Lengthening' was described for sign languages by Liddell (1978 and 1980).

${ }^{59}$ Whether the negated element in (13) is a verb or a noun requires further investigations and lies beyond the scope of this thesis. A first study on noun-verb pairs in ÖGS has been conducted by Hunger (2003).

${ }^{60}$ The example (14) is also given in chapter 5 on conditionals (see example (96)). Two shaking movements are present; the second one, covering NO, is produced even faster than the preceding one. Examples (14) and (15) are produced successively (only an assertive conditional intervenes). 
(15)

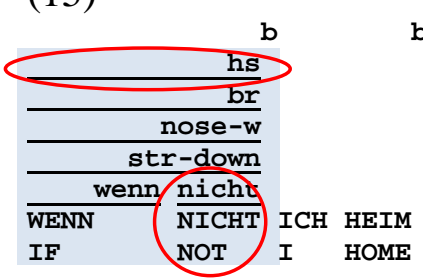

Wenn es nichts gibt, gehe ich heim.

If there isn't anything available, I'll go home.

(F001_092,1184_m_thoughts_ex12a2_03.06-03.08)

Summing up, both manual negators, i.e. NO and NOT, function as predicate or clause negators. However, NO is much more frequently used, especially in postposition of a verb.

As shown below, NO can also co-occur with the mouthing 'nicht' ('not').

$(16)^{61}$

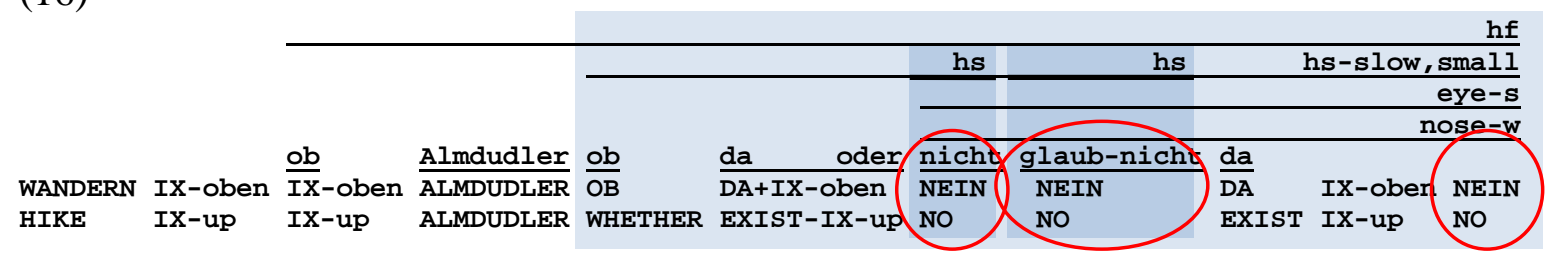

Während ich wandere überlege ich, ob es Almdudler gibt. Ich glaube aber nicht, dass es Almdudler gibt.

While I am hiking I wonder whether there will be almdudler (a kind of soft drink) available or not. I think there won't be.

(M002_106,1198_m_thoughts_ex02_00.29-00.40)

The signer narrates that he is hiking and expressing his thoughts. First, he wonders whether a specific kind of drink will be available, but he quickly convinces himself that this is more likely not the case. The first manual negator NO has the mouthing 'nicht' ('not'), the second the mouthing 'glaub-nicht' ('believe-not'), and the third is produced without mouthing.

Summing up, this example illustrates that both manual negators are used for clause negation, and that in syntactic positions like in (16), both can be used. All in all, the tendency is much higher to use the manual negator NO in non-elicited discourse texts.

\footnotetext{
61 The annotators clearly perceive that the last sign (NO) of the interrogative as well as the first lexical elements (NO++ together with the mouthing 'believe-not') of the following statement are covered by more regular and more definitely performed headshakes (color-coded blue) (cf. 7.4.3, example (137)).
} 


\section{c) Irregular negatives}

Zeshan (2006b, 49-52) describes that in most investigated sign languages, there are irregular negatives which constitute both negative suppletion and morphological negation. This is also the case for Austrian Sign Language.

ÖGS has both simultaneous and sequential morphological negation. The first group is a restricted category containing some verbs - mostly modals - which have changed their sublexical movement component. Thus, the negative form includes an alpha-movement as illustrated in Figure 3.7. This group contains the signs KANN-NEIN (CAN-NO), STIMMT-NEIN (CORRECT-NO), GIBT-NEIN (EXIST-NO), MÖGLICH-NEIN (POSSIBLE-NO), and DARF-NEIN (MAY-NO).

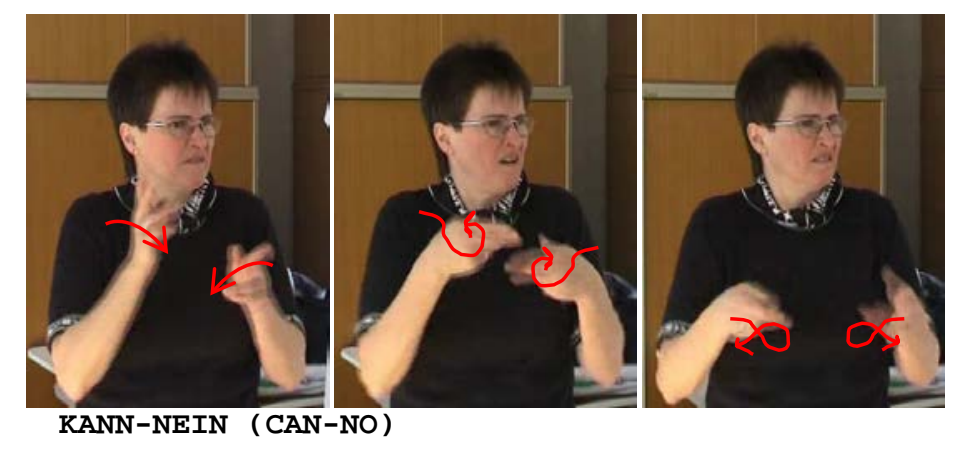

Figure 3.7 Morphological negation

As a rule these negatives are covered by headshakes. Negated modals can be doubled ${ }^{62}$, so they occur in clause-initial and clause-final position:

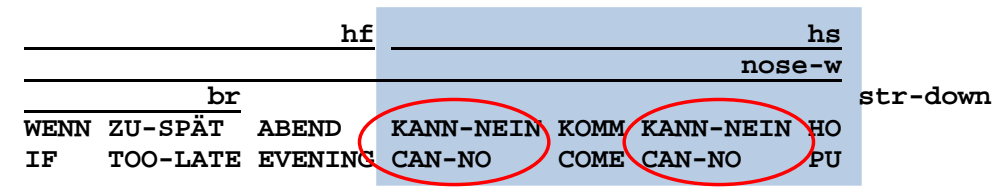

Wenn es zu spät am Abend wird, kann er nicht kommen.

If it's too late in the evening, he can't come.

(F001_Film1_Szene7_d_informel_story_02.45-02.48)

In (17), the signer explains that under a certain condition her husband cannot come to the recording session. The consequent of the conditional has the sign suppletive negative

\footnotetext{
${ }^{62}$ Doubling of modals was described for ÖGS by Schalber \& Hunger (2000).
} 
KANN-NEIN (CAN-NO) in clause-initial position. This sign is repeated in clause-finial position, only followed by an unstressed PALM-UP. The entire consequent is covered by headshakes.

The second group (sequential morphological negation) consists of verbs which go together with a negative suffix, that is, a NO-affix is attached to the predicate stem, always in postposition. This phenomenon has already been mentioned above (cf. Figure 3.4).

\subsubsection{Negative headshakes spreading along the entire clause}

What is striking in the data is that in constructions such as polar questions or the antecedent of conditionals negative headshakes has a high tendency to cover the entire clause. In this section, I draw my attention to this observation and discuss possible reasons for this phenomenon.

As the data show, the non-manual negator headshakes can spread to the clause-initial position as illustrated in Figure 3.2 and in example (12). The question arises whether the spreading is favored by factors such as coding of negative epistemic presupposition, or occurrence in special linguistic constructions (like interrogatives) which show similarities with negative polarity contexts, or displaying semantic-pragmatic reasons, or supporting turn-construction and turn-taking. In other words, in which cases does the negative marker headshakes cover the entire clause and in which only the negated predicate? Based on the present data some conclusions can be drawn.

To begin with, in the context of special language constructions like direct polar questions (cf. 4.2.1), the antecedent of conditionals (cf. 5.6.1), and the apodosis of adversative constructions (cf. 3.1.5) there is a high tendency that the entire clause is covered by headshakes. On a first view, these constructions have common that they tend to involve a negative and positive aspect. Thus, polar questions request an assertive or non-assertive (or negated) answer; Conditional clauses imply 'if X' or 'if not X'; Adversative constructions evoke a contrast and consequently can imply values like negative or positive. For the present sub-chapter, the latter (adversative constructions) are excluded as in these constructions the headshakes clearly function for implying negative contrast. This is 
described in section 3.1.5. In the following, first, two examples are described which illustrate the spreading of negative headshakes along a polar question and a protasis of a conditional construction. Then, possible interpretations for the reasons of the spreading of negative headshakes along the entire clause are discussed.

In polar questions which are covered by negative headshakes there is a high tendency that the entire clause is covered by this marker. This is demonstrated in the previous example (12) as well as in the following example (18).

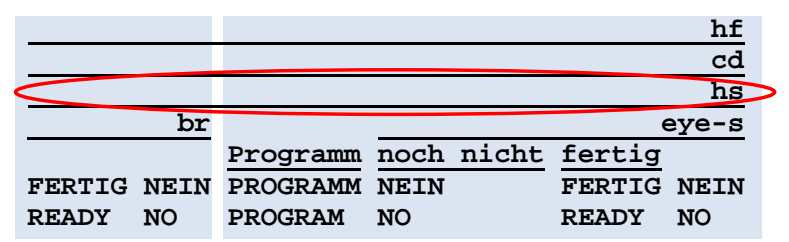

Ist das Programm noch nicht fertig? (Ist das Programm schon fertig?)

Isn't the program finished yet? (Is the programm finished yet?)

(M001_Film1_Szene3_d_story_informel_01.56-01.58)

In (18), the entire polar question, indicated by 'chin down' and 'head forward' (emphasizing the interrogative character) $^{63}$, is covered by negative headshakes. Moreover the question READY NO, which is additionally marked by 'raised eyebrows', is repeated. The headshake movements are maintained during the entire question and display one prosodic movement contour. This has the same duration as the other head markers.

Also, if the protasis of a conditional includes a negated predicate which is covered by negative headshakes, there is a high tendency that the entire protasis is covered by negative headshakes. This is illustrated in example (19).

${ }^{63}$ cf. 4.2.1.1 
$(19)^{64}$

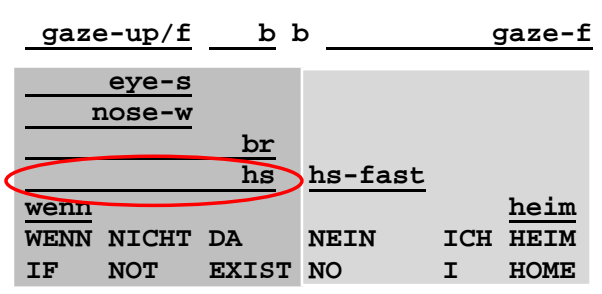

Wenn es nichts gibt, nein (dann bleibe ich nicht), dann gehe ich heim. If there is anything available, no (then I won't stay), then I'll go home.

(F001_092,1184_m_thoughts_ex11a2_02.55-02.58)

In (19), the entire antecedent (color-coded dark grey), constituting the negative condition, is covered by negative headshakes (encircled red).

First, a possible reason for the spreading of the headshakes along the entire clause may be due to negative epistemic presupposition.

In example (19) clearly negative headshakes are present as the clause is negated. But the negative headshakes seem to imply a non-assertive/negative epistemic presupposition in the sense that the signer seems to be convinced that nothing will be available. This negative presupposition may be alone or additionally responsible for the spreading of the headshakes along the entire clause as already at the beginning of the conditional it is obvious that the negative outcome is presupposed by the signer.

Also in example (18) the signer, asking the dialogue partner whether the program is not finished yet, may imply a negative epistemic presupposition in the sense of 'I assume the program is not finished yet'.

Israel (2011, 61 and 126-127) who, among other matters, describes the interplay of (negative/positive) 'polarity' and 'modality', notes that when talking about the factual status of a proposition the speaker (here signer) has to make a judgment on the proposition. Both polarity and modality are 'scalar phenomena' in his interpretation. Following Israel's deliberations, a semantic contiguity between '(polarity) contexts' and 'presuppositions' and consequently also between 'negative contexts' (including negated contexts) and ‘negative epistemic presupposition’ may be supposed. This possible semantic contiguity is

\footnotetext{
${ }^{64}$ The example (19) is described in detail in chapter 5 by example (94).
} 
illustrated in Figure 3.8. Then, the possible interplay of positive/negative presuppositions and positive/negative contexts is described and applied to the ÖGS data.

\begin{tabular}{lll}
$\begin{array}{l}\text { assertive/positive } \\
\text { assertive/negative }\end{array}$ & PRESUPPOSITION & non- \\
$\begin{array}{l}\text { assertive/positive } \\
\text { assertive/negative }\end{array}$ & CONTEXTS & non- \\
\hline
\end{tabular}

Figure 3.8 Scalar of assertive/positive versus non-assertive/negative presupposition compared to assertive/positive contexts versus non-assertive/negative contexts

As illustrated in Figure 3.8, it may be supposed that assertive/positive and nonassertive/negative presuppositions, both being associated with a proposition, are not only two poles on a scale. Rather these epistemic presuppositions display a semantic gradual scale lasting from assertive/positive to non-assertive/negative presupposition on a proposition. To this, it may be supposed that the particular scalar end points also display assertive/positive and non-assertive/negative contexts which are good environments for assertive markers and non-assertive markers, if these markers exist in a language. The nonassertive/negative context is also a good environment for negative markers as negated elements imply that a positive, assertive content is reversed to a negative, non-assertive content.

This possible semantic contiguity is supported by the following observation: In conditional clauses the co-occurrence of the negative marker headshakes and (epistemic) modality markers is obvious. In negated antecedents of conditionals frequently also modality markers such as 'wrinkled nose' (cf. 7.3.2) which seems to code negative epistemic modality, or 'squinted eyes' (cf. 7.3.2) which seems to imply knowledge or lack of knowledge on a proposition, co-occur. The co-occurrence of these markers with negative headshakes is illustrated in example (19). In chapter 5 on conditionals (cf. 5.6.1) all described examples in which negative headshakes occur along or within the antecedent of a conditional (see from example (92) to (98)) also have the nose wrinkled and/or the eyes squinted. This high degree of co-occurrence shows that there very likely is a semantic contiguity between negation and negative epistemic presupposition on a proposition. Thus, 


\section{NEGATION AND ASSERTION CODED BY HEADSHAKES AND HEAD NODS}

it might be concluded that negative epistemic presupposition might influence the negative marker and consequently supports the spreading of negative headshakes along the entire clause with which the negative epistemic presupposition is associated.

What is more, the data show a close relationship between negative headshakes and nonassertive headshakes, both differing in form and meaning. The first codes negation with slow or fast headshakes which are performed with a uniform shaking movement; the latter codes non-assertive epistemic attitude on a proposition with slow headshakes which tend to be small in size and which are performed in a rather tentative way. If within a nonassertive utterance which is covered by non-assertive headshakes a part is negated and has to be marked by the negative headshakes, the negative marker clearly overlays the modality marker.

When listing the contexts in which the negative headshakes spreads along the entire clause, it becomes apparent that these contexts look very similar to those contexts which Buyssens (1959) first labels 'negative contexts' ${ }^{65}$. These are good environments in which 'negative polarity items' (NPIs) ${ }^{66}$ tend to occur. As the above described phenomenon deals with a negative marker which can spread along a clause and not with NPIs which occur in special contexts that need not to be negated, a relation between the spreading of the negation

\footnotetext{
${ }^{65}$ Horn $(2001,176)$ finds it more appropriate to use the term 'non-assertive' for the semantic features of the several contexts which license the occurrence of the respective NPIs.

One of the first, Edmondson (1981), identified contexts which hierarchically increase in their degree of negativity, constituting 'comparatives', 'conditionals', 'interrogatives', and - with the highest degree of negativity - 'negatives'. For an overview on identified negative polarity contexts see Wouden (1994) and Hoeksema (2012); the latter focus on English, German and Dutch.

${ }^{66}$ The term 'negative polarity item' (NPI) was introduced by Baker (1970), but was already circumscribed by Klima (1964). Since then, a lot of research on NPIs has taken place (cf., among others, Fauconnier 1975, Ladusaw 1980, Krifka 1994 and 1995, Israel 2011). Some linguists have tried to renew the term like Haspelmath (1997), labeling NPIs as 'scale reversal items' appearing in 'scale reversal contexts'. As these terms could be misleading, the term NPI is still kept as the primarily used term for describing this phenomenon.

Concluding, negative polarity items (NPIs) and positive polarity items (PPIs) are classes of expressions that tend to occur in such negative/positive polarity contexts. For instance, in ÖGS the sign NOBODY-EXIST (see example (3)) only can occur in negative polarity context. The field of NPIs in sign languages has hardly been explored. One of the few articles is on the negative polarity item N'IMPORTANT-Q in Sign Language of Quebec (LSQ) (cf. Nassira 2008).
} 
marker and negative polarity contexts is not really indicated. Also, negative contexts are always brought into relation with negative polarity items. To be exact, negative contexts are frequently defined by NPIs which occur in these contexts (for a discussion on what are negative contexts and how they are constituted see Wouden (1994, 21-27). If, however, the semantic contiguity between non-assertive/negative presupposition and nonassertive/negative contexts (as illustrated in Figure 3.8) is taken into consideration, the mutual influence of both phenomena and of the particular markers (those coding nonassertive/negative presupposition and those coding negation) is apparent.

Second, another possible reason for the spreading of negative headshakes along an entire clause, especially along an entire polar question, might be a pragmatic-communicative one. Krifka (2011) observes that negation in polar question can be used for clause negation, but also for pragmatic-communicative reasons. If the latter function is fulfilled, he labels them 'negative polar questions ${ }^{\text {, } 7}$. This observation goes back to Ladd (1981) who found that, for instance in English, a negative polarity question can get an ambiguous interpretation by using different means of prosody (i.e. different intonation) or additional particles. On the one hand the interpretation is that the proposition is negated; on the other hand the interpretation is that the negation goes beyond the proposition. Krifka (2011, to appear) describes the latter interpretation in terms of 'speech-act denegation'. Following both linguists, the latter interpretation is that in negative polarity questions the speaker (here signer) expresses his/her expectation in receiving an affirmation or a disaffirmation by using a negation marker. With regard to English, this means that negated polar question with a different intonational pattern or a special additional particle function for both clause negation and speech act denial.

\footnotetext{
${ }^{67}$ In accordance with Krifka (2011), the second question in the following example (20) displays a 'negative polarity question' of which the scope goes beyond the proposition, while the first question has its scope within the clause. Following Ladd (1981) and Krifka (2011), in English, prosody and particles are responsible for the interpretation of 'negative polarity questions'. Thus, adding 'either' in example (20) implies that the speaker wants the negated content to be confirmed, while adding 'too' implies that the speaker wants the affirmation that there were people from Graz.

(20) a) clause negation: Kamen keine Leute aus Graz?

(Were there any people from Graz?)

b) speech-act denegation: Kamen nicht Leute aus Graz?

(Were there any people from Graz (either/too)?)
} 
Following this pragmatic-communicative function, the negative headshakes in polar question can express the signer's expectation in perceiving a positive or a negative answer, in the way of confirming or disconfirming the content of the question. In example (20), two ways of interpretation are possible. On the one side, the proposition is negated and the pragmatic-communicative interpretation is that the signer wants to receive the confirmation that 'Yes /No, the program is/isn't already finished'; on the other side, the proposition is negated and the pragmatic-communicative interpretation is that the signer wants to receive the confirmation that 'Yes, of course (German 'doch'), the program is already finished'. Which pragmatic-communicative interpretation is the correct one, requires further investigations. But the important fact is that possibly headshakes can possess both the clause negation function as well as the pragmatic-communicative function.

Following Krifka (2011), the spreading of the negative headshakes along the entire clause can also be evoked for pragmatic-communicative reasons.

Third, the interactive character of questions allows me to add a possible further reason that probably causes the co-occurrence of negative headshakes along the entire question, too.

In dialogues the dialogue partners wants to be understood correctly and probably therefore the entire question is covered by headshakes in order to express the negation more clearly; another reason for this may be that interposed questions in a dialogue setting prompt the counterpart in a brief and direct fashion to provide a particular detail.

Fourth, a possible additional factor why the negation marker headshakes spreads over the entire clause is that it acts to clarify who has the turn or to whom the turn is addressed. This phenomenon is very obvious in constructed dialogues. Thus, in addition to signing in the different perspectives of the imagined dialogue partners or to making clear that a statement is self-addressed or to the dialogue partner (within a constructed action), the entire turn or a turn unit ${ }^{68}$ is covered by headshakes, in cases where negation is required.

\footnotetext{
${ }^{68}$ This is a part of a turn which has a transition relevance place at the end, but it is not required that the dialogue partner takes the turn. Often, at these places the dialogue partner, being in listening/viewing position, adds a feedback, short comment, etc.
} 


\section{NEGATION AND ASSERTION CODED BY HEADSHAKES AND HEAD NODS}

This is the same with assertion, as described in the following subchapter. The next example (21) shows that the particular turn unit from the perspective of one imagined dialogue partner (the grandfather) within a constructed dialogue is covered by different head movements or positions.

$(21)^{69}$

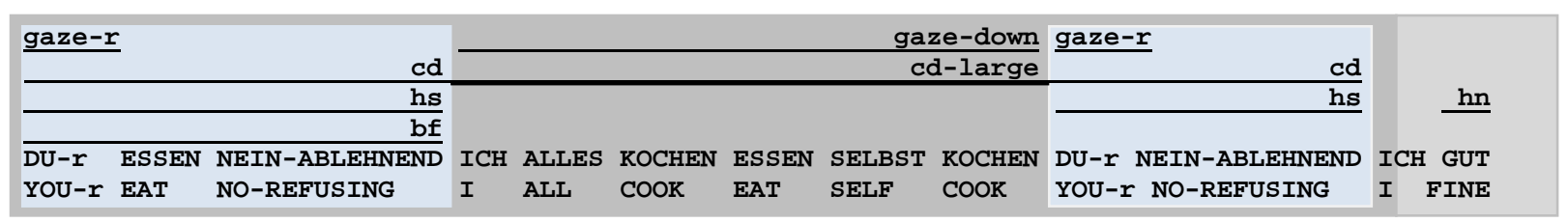

(Mein Großvater sagte zu mir:) "Du brauchst nicht das Essen vorzubereiten, ich werde alles selbst vorbereiten (kochen), du brauchst nichts zu tun." Ich antwortete: "Gut so." (My grandfather said me:) "You needn't prepare the meal, I will prepare (cook) everything myself, you needn't do anything." I answered: "That's fine."

(M005_Film1_Szene2_d_story_informel_00.18-00.22)

In example (21), the signer narrates about the times when he helped his grandfather saving hay. With help of a constructed action he signs what his grandfather told him (color-coded dark grey). This entire unit is indicated by tilting the head downward, resulting in the marker "chin down, ${ }^{70}$. The turns of the grandfather constitute both turn units which are addressed to the grandchild (color-coded blue) and also those which are self-addressed. The turn units addressed to the grandchild are indicated by indexing to the right space and looking to the right, while the self-addressed turn unit is indicated by looking downward and intensifying the downward position of the chin. The following answer from the grandchild to his grandfather is started with a neutral head position and followed by a head nod (color-coded light grey).

\footnotetext{
${ }^{69}$ Gaze direction in constructed dialogues appears to be a promising field for further investigation. The present example is taken from a dialogue setting in which the turns change very frequently. Thus, the actual signer is regularly briefly looking to the dialogue partner. In example (21), the negated clauses which are addressed to the grandchild (from the perspective of the grandfather) are accompanied by looking to the right (when signing YOU) and looking at the actual dialogue partner. The thoughts which are selfaddressed are accompanied by looking downward.

The pointing sign YOU is not addressed to the actual dialogue partner, but to the grandchild from the perspective of the grandfather.

${ }^{70}$ Which indicators are all possible for displaying the particular role in a constructed dialogue requires further investigations in ÖGS. In example (21) the gaze direction, the pointing sign YOU, and the chin position are those cues which make it clear to whom the turn is addressed.
} 


\section{NEGATION AND ASSERTION CODED BY HEADSHAKES AND HEAD NODS}

This example illustrates that probably for reasons of distinctiveness and clarity the particular turns or turn units are covered as a whole by the head movements, whether headshakes or head nods are required for negation or assertion respectively.

In sum, the most striking point is that in the various constructions such as polar questions or conditional clauses there is a high tendency that negative headshakes spread along the entire clause. Various reasons for this are discussed in the present section. With conditional clauses the influence of negative epistemic presupposition seems to be an obvious reason. With negated polar question the influence of speech-act denegation also may be a deciding reason. However, in conclusion, it can be noted that at present none of the listed reasons can be said to be the only right interpretation for the spreading. Further studies are required in order to support one or more of the listed interpretations.

\subsubsection{Addition: Tricky negation}

As in the following, negation can sometimes be a very tricky thing.

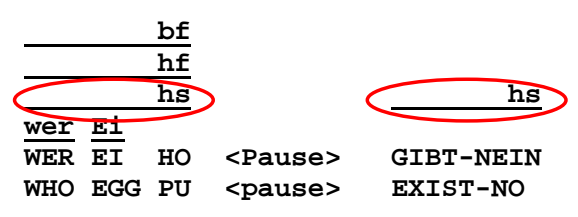

Wer hat das Ei gelegt? - Das gibt es gar nicht.

Who has laid the egg? - That does not exist at all.

(M001_086_m_story_formal_joke_ex12a2_07.37-07.42)

Telling a joke, a signer narrates that two roosters (each of them) laid an egg on a mountain. These rolled down to the valley. There, they only found again one egg and discussed who has laid that egg. After telling this episode, the narrator asks the audience (the camera man) whether they (he) know(s) who had laid the egg. (The answer being neither, as roosters can’t lay eggs.)

The interpretation of the headshakes co-occurring with the question could either be 'You do not know who has laid the egg?' OR it is interpreted as 'anticipated negation'. For this, the following negation, expressed through 'EXIST-NO', is already produced when asking for the answer. Following the second interpretation, the narrator already pre-empts the 
answer. If that is the case, headshakes may be a very tricky thing, if they show your thoughts in advance.

After having focused on clause negation, the following sections describe further functions of headshakes. First, speech act negation is described, second, implying negative contrast by headshakes is dealt with, and third, other functions of headshakes are discussed.

\subsubsection{Speech act negation coded by headshakes}

In a handful of cases headshakes occur on their $\mathrm{own}^{71}$. Some of them function as clause negators. In these instances a clause is negated by subsequent headshakes (cf. example (6) variant 2). In some of the latter cases the following headshakes go together with the sign NO, both elements functioning as negator of the preceding clause.

Example (23) illustrates following headshakes which function as clause negators. Example (24) shows what happens when headshakes precede a clause which is to be negated. Example (25) demonstrates the interactive character of following headshakes.

\begin{tabular}{|c|c|c|}
\hline \multirow[t]{2}{*}{ eye-w } & b & \multirow[b]{2}{*}{ cu/hb } \\
\hline & hf & \\
\hline br & & $\overline{\mathrm{hs}}$ \\
\hline open & Zwi(lling) & \\
\hline$\overline{\mathrm{AHA}}$ & $\overline{\text { ZWILLING }}$ & NEIN \\
\hline I -SEE & TWIN & NO \\
\hline
\end{tabular}

Aha, (nein, ) Zwillinge sind wir nicht. I see, (no,) we are not twins.

In example (23), the utterance is negated by following headshakes (encircled red) and the sign NO which starts with a short delay compared to the headshakes. In addition, the informant tilts the head backward resulting in positioning the chin upward and moving the head slightly backward. To this, the annotator ascribed a denial meaning ${ }^{72}$.

\footnotetext{
${ }^{71}$ Single headshakes most frequently occur within a dialogue or within a constructed dialogue, in which the counterpart answers with shaking the head, just saying 'no'.

72 The exact function of the element 'cu/hb' requires further investigations. An exclamative function has been allocated to the marker 'head forward' ('hf').
} 


\section{NEGATION AND ASSERTION CODED BY HEADSHAKES AND HEAD NODS}

The second example demonstrates that if headshakes without any manual negative sign precede a clause which is to be negated, the headshakes must spread on the clause.

$\begin{array}{ll}\text { hs } & \text { hs } \\ \text { CL-Brotstück-essen } \\ \text { CL-to-eat - a-piece-of-bread }\end{array}$

Nein, du hast kein Brotstück gegegessen. No, you have not eaten a piece of bread.

(M006_Film1_Szene2_d_informal_story_00.44-00.45)

Prior to example (24), the narrator signs that he has not eaten a piece of bread. In (24), the dialogue partner copies this content. In doing so, he starts shaking his head on its own (color-coded dark blue) and afterwards adds the sign and maintains the shaking movement (color-coded light blue).

The third example shows that headshakes can follow an utterance which ought to be negated.

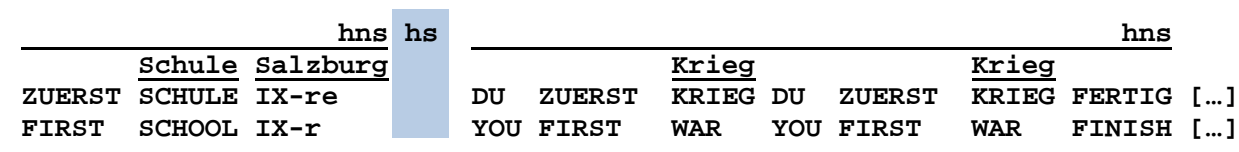

Zuerst erzählst du von der Schule in Salzburg, (ach) nein, zuerst vom Krieg.

First, you talk about the school in Salzburg, (oh) no, first about the war.

(M005_Film1_Szene6_d_informal_story_00.08-00.13)

In (25), the signer formulates something, realizes that he is wrong, shakes the head (marked blue), and adds the right content. In this case head shaking first functions as means of correction, thus repairing the utterance.

To sum up, headshakes following a clause show more speech act function than clause negation.

\subsubsection{Negative contrast coded by headshakes}

The logically-semantic relation of two clauses can be, among others, adversative or causal. Adversative coordinating constructions imply a contrast or at least a constraint. Causal 


\section{NEGATION AND ASSERTION CODED BY HEADSHAKES AND HEAD NODS}

coordinating or subordinating constructions ${ }^{73}$ imply causality (see, among others, Lang 1991 on adversative constructions ${ }^{74}$ or Haspelmath 2007 on coordination ${ }^{75}$ ). In the present subchapter, I focus on negative headshakes resulting from negative contrast which occurs in adversative constructions but also in conditional constructions.

In Austrian Sign Language the following adversative constructions which imply contrastive negative coordination are present in the data in which it can be seen that the negative contrasted clause is covered as a whole by negative headshakes. This phenomenon is illustrated by three examples:

In (26), in the first clause the repeated content is accompanied by assertive head nods, the entire following clause by negative headshakes.

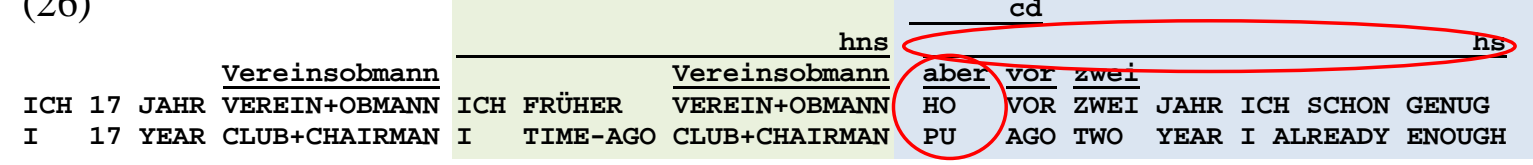

Ich war 17 Jahre lang Vereinsobmann, aber vor zwei Jahren hatte ich genug davon.

I was for 17 years chairman of the club, but two years ago I was sick of it.

(M001_Film1_Szene3_d_story_informel_01.25-01.29)

In example (26), a signer narrates in a dialogue setting that he had been sick of holding the leadership. The entire clause is covered by headshakes which, however, do not negate the clause. The reason for the negative headshakes (encircled red) in example (26) is based on a 'contrastive negative coordination', that is, an 'adversative construction' is on hand. Adversative constructions can imply a reversal of semantics under special conditions or with regard to a restricted aspect. So, new semantic-pragmatic information can be added

\footnotetext{
${ }^{73}$ In German, for instance, both structure-forming adversative constructions are evident. Conditionals display subordinating constructions; 'Denn'-constructions ('for/because'-constructions) display a coordinating construction (Haspelmath 2004, 6).

${ }^{74}$ Lang's (1991) description of adversative constructions and his classification of adversative types is based on the adversative adverb 'but' and refers and implies further/different aspects to the study of Lakoff (1971) on the conjunction 'but'. Lang's classification in different types was, for instance, applied in the study on adversative constructions in Russian and German by Petkova-Schick (1998).

Based on the logically-semantic relation, in German different adverbs are used for adversative construction. Along with the element 'aber' ('but'), the element 'doch' can be used. The adverb 'sondern' is used when the first clause is negated or has a constraint. 'Dagegen' or 'hingegen' are used when the clauses semantically contrast. The adverb 'jedoch' is used when a constraint is implied.

${ }^{75}$ Haspelmath (2007, 17-19 and 46-48) includes a description on 'contrastive negative coordination' and a discussion on the differentiation of coordination versus subordination/dependency from a semantic point of view.
} 


\section{NEGATION AND ASSERTION CODED BY HEADSHAKES AND HEAD NODS}

conveying values like something is positive or negative, something is advantageous or disadvantageous for a contextual evaluation (Petkova-Schick 1998, 45). When formulating that 'he was holding the leadership, but two years ago he was sick of it', the signer adds some information, conveying negative connotation. The result is a contrastive coordination of which the first statement gets the value positive and the second statement the value negative. This contrast is clearly implemented by assertive head nods co-occurring with the repeated positive statement (color-coded green) and negative headshakes co-occurring with the negative statement (color-coded blue). Further, the mouthing 'aber' ('but'; encircled red) is an additional clear indicator for an adversative construction.

The second example implies a semantic contrast too. Adversative constructions can express semantic contrast by contradiction (Lang 1991, 617-618). For example, when formulating that 'I stay but you go home' I imply the semantic contrastive action of what each of us is doing. In this sense, the following example (27) implies that the first person did something that the second person denies having done.

$(27)^{76}$

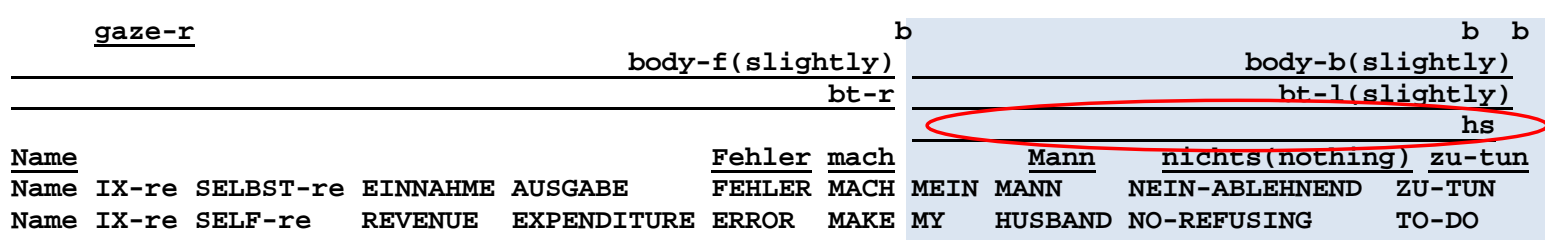

'Name' hat selbst einen Fehler in der Einnahmen-Ausgaben-Rechnung gemacht. Mein Mann hat nichts damit zutun.

'Name' made a mistake himself when doing the cash accounting. My husband has nothing to do with that.

(F002_Film1_Szene4_d_story_informel_02.48-02.54)

The signer wants to clarify that her husband has nothing to do with the mistake. To make this contradiction explicit, the entire clause is covered by headshakes. Thus, beside the obligatorily marked lexical elements (here negative quantifier and verb), the subject ('my husband') is covered by headshakes too. Additionally, this clause is indicated by a body turn to the left, referring to the opposite location of the person who has made the mistake.

\footnotetext{
${ }^{76}$ The sign's place of articulation in the first statement is displaced into a signing space to the right, while the sign's place of articulation in the second statement is displaced into a signing space to the left.
} 
Also, during the first body turn to the right the body slightly leans forward, while during the body turn to the left the body slightly leans backward. This contrastive lean provides the first part of the utterance with an affirmative character, the second part with denial character. This is illustrated in Figure 3.9.

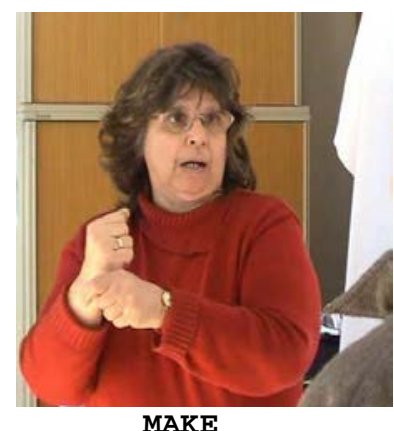

MAKE

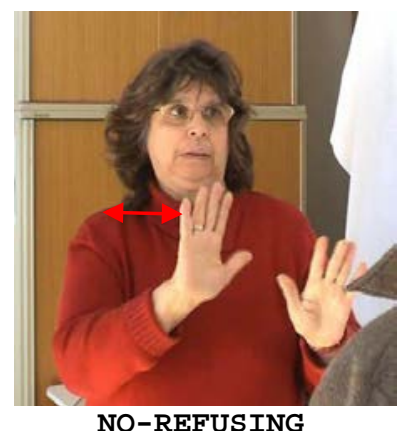

NO-REFUSING

Figure 3.9 Marking of contrast in adversative constructions ${ }^{77}$

In conclusion, it is that for reasons of semantic contrast (of the doing or not doing the action), that the non-manual negation marker tends to cover all involved syntactic elements to make as clear as possible the refusal or denial of having done something wrong.

Furthermore, in adversative constructions the contrast can be implied by canceling a conclusion which ordinarily would be the case (Lang 1991, 619). This is illustrated in example (28). In addition, the example includes several elements that are associated with an adversative construction. These are the elements 'aber' ('but'), 'doch' ('but still'), and the negative headshakes displaying the contrast.

\footnotetext{
${ }^{77}$ Figure 3.9 illustrates the contrast with two pictures (the sign MAKE and NO-REFUSING) taken from example (27). These illustrate the different body leans (forward versus backward), body turns (right versus left) and the negative headshakes covering the second part of the utterance.
} 
$(28)^{78}$

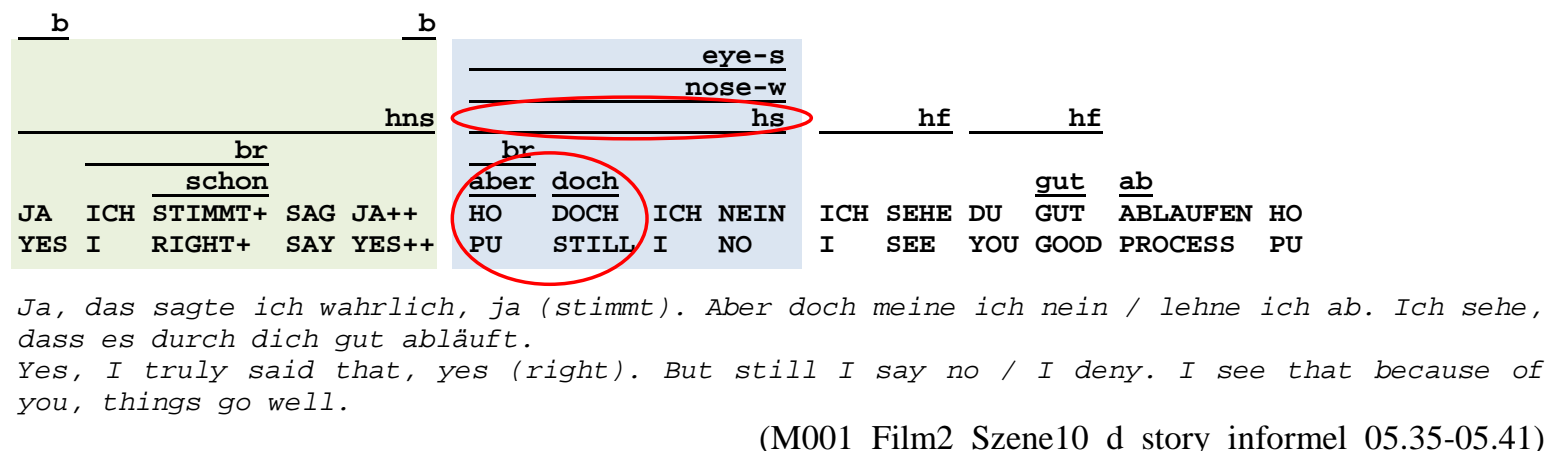

In example (28), the two dialogue partners discuss the leadership of the Deaf community, which both of them have previously held. As the present leader offers the dialogue partner the position, the counterpart affirmed that he had promised to take the leadership (colorcoded green). However, he adds that now he does not want it anymore (color-coded blue). The contrast of the two statements is induced by adding an unexpected conclusion. Thus, as a rule somebody keeps a promise. This usual action is rejected and causes in the present example (28) the negative headshakes (encircled red) which go along the entire denial. Apart from the negative headshakes, the signer uses the elements 'aber doch' ('but still'; encircled red). Both elements are used in order to making the contrast explicit; the sign DOCH additionally can imply causality in the form of giving a causal statement to the preceding utterance.

The second aspect I want to focus on in the present subchapter is the implication of a semantic contrast in a subordinated construction, i.e. in conditionals. In the following, I illustrate the inclusion of semantic contrast induced by negative headshakes along the entire consequent. Thus, including headshakes changes 'if $\mathrm{X}$, then $\mathrm{Y}$ ' into 'if $\mathrm{X}$, then $\mathrm{Y}$ but not Z’’.

\footnotetext{
${ }^{78}$ The manual negator NEIN (NO) is used predicatively, just expressing negation (denial) of the offer. The first part of example (28) is covered by assertive head nods, discussed in example (40) in detail.
} 


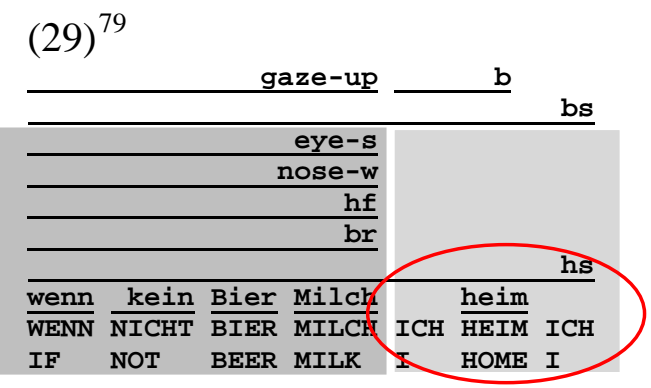

Wenn es in der Almhütte weder Bier noch Milch gibt, dann gehe ich heim, (aber bleibe nicht).

If in the hut there is neither beer nor milk, then $I^{\prime} l 1$ go home, (but won't stay).

In (29), both, the antecedent and the consequent are covered by the marker headshakes. The headshakes co-occurring with the protasis function for clause negation and display the 'negative polarity context'. The question arises why the headshakes also co-occur with the apodosis, where the clause itself is not negated. In (29), the headshakes covering the antecedent do not express that 'the person does not go home'. Rather, this can be interpreted as implying negative semantic contrast. Formulating the consequence of a condition can be done in a positive or negative way. If this is done in an assertive way, the headshakes associate with the non-assertive consequence. Consequently, in example (29), the negative evaluation of the opposed meaning of 'going home' - i.e. '(but) not staying' is shown by negative headshakes.

Concluding, it is obvious that implying a negative semantic contrast is the very reason for spreading of headshakes along the entire coordinating clause of adversative constructions and the optional co-occurrence of negative headshakes along the entire consequent of a conditional construction.

A further construction, which falls within the scope of negative contrast, is present in the data. In the various present ÖGS recording, some signs always co-occur with 'small, fast headshakes' as non-manual lexical component. In this category, we find the signs LIEBER

\footnotetext{
${ }^{79}$ The example (29) is also quoted in chapter 7 on conditionals.
} 
(RATHER) and SOWIESO (ANYWAY), which are always covered by small, fast headshakes in the present ÖGS corpora ${ }^{80}$.

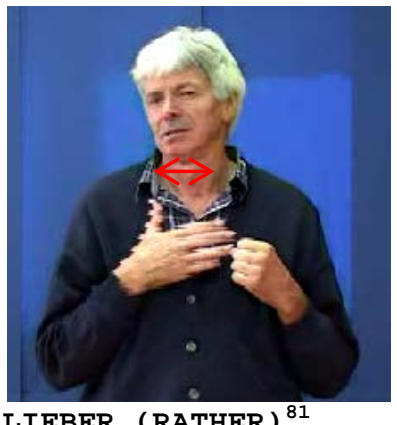

LIEBER (RATHER) ${ }^{81}$

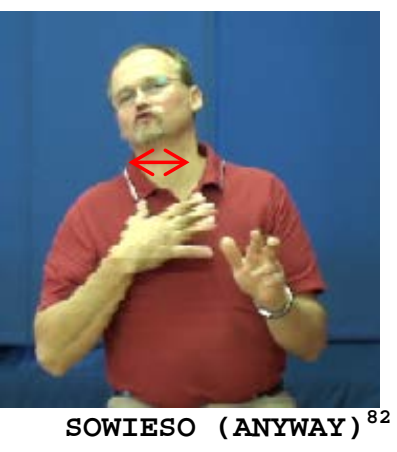

SOWIESO (ANYWAY) $)^{82}$

Figure 3.10 The signs RATHER and ANYWAY

Both signs appear in the following illustrated constructions:

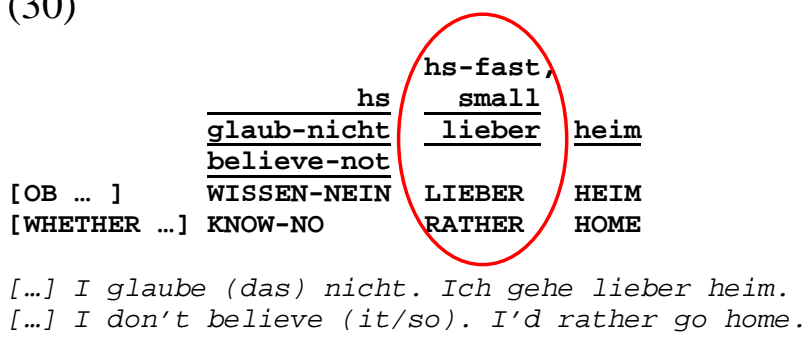

(M002_114,1206_m_thoughts_ex05_05.34-05.38)

Example (30) shows the context of the sign RATHER. The sign is used to connect two clauses which ought to be compared. Thus, in contexts like the quoted one, RATHER connects a negative statement with a positive one. Consequently, a negative contrast is implied in the way of 'if $\mathrm{X}$ condition does not hold, then I will do $\mathrm{Y}$ '. In this sense and following Haspelmath $(2007,28)$, the present construction displays a semantic subtype of adversative constructions in which two coordinators (here clauses) are contrasted.

\footnotetext{
${ }^{80}$ Skant et al. (2002, 194 and 198) cites two examples in which the sign LIEBER (RATHER) and the following statement are covered by nodding movements. In both instances, the preceding statements is negated and covered by negative headshakes.

${ }^{81}$ The sign RATHER can be performed with B-hand form or L-hand form. It is accompanied by the mouthing 'lieber' ('rather') and fast small headshakes.

${ }^{82}$ The sign ANYWAY is performed with B-hand form. It is accompanied by the mouthing 'sowieso' ('anyway') and fast small headshakes.
} 
(31)

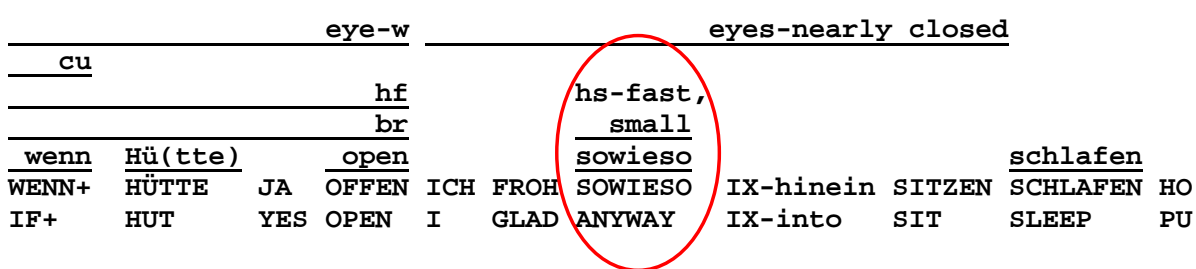

Wenn die Hütte geöffnet ist, bin ich Troh. Auf jeden Fall würde ich mich hineinsetzen und dort übernachten.

If the hut is open, I'll be glad. Anyway, I would sit down inside for sure and also stay for the night.

(M001_091,1183_m_thoughts_ex10_03.13-03.17)

In (31), the signer expresses that independent of the circumstances he would sit down in the hut and stay overnight. The interpretation 'It does not matter whether X or Y, I will do Z' is implied. Consequently, the contrast refers to the preceding statement (independent whether asserted or negated) and the contrasted statement ('I will do Z').

Concluding, both RATHER and ANYWAY connect two coordinating clauses and induce a negative semantic contrast between them. Only the connecting element is covered by negative headshakes. It can be assumed that this lexical marker covers the lexeme due to its above described function.

\subsubsection{Other functions coded by headshakes}

A single or several headshakes may have functions other than clause negation, too. I want to exemplify three functions, the first of which has a close contiguity to negation, while the second and third are performed for their own purposes.

To begin with, the data shows that there are occurrences of a clause covered by headshakes where no manual negator is present, and the meaning of the clause is not negated. This is displayed in the following:

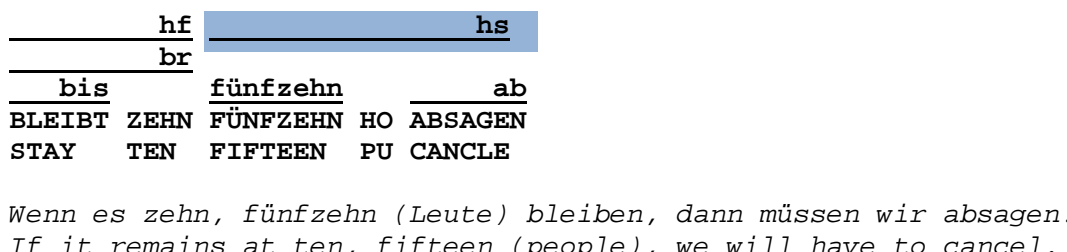

(M007_1120_m_thoughts_ex.03a_00.00-00.06) 


\section{NEGATION AND ASSERTION CODED BY HEADSHAKES AND HEAD NODS}

As example (32) demonstrates, the headshakes do not necessarily negate the clause. Comparable with the phenomenon of 'psychological or affective negation', described by Fischer (2006, 191-192) ${ }^{83}$ for ASL, the present example shows that in ÖGS a lexeme conveying a negative meaning may evoke additional headshakes. These, however, do not negate the clause, but reinforce the existing negative aspect of the sign.

In contrast to this, one or more headshakes may also occur without having a connection to negativity. As follows, a single headshake ${ }^{84}$, huge in size and performed with a very fast backward movement from the side, can be used to underline the 'realized speed of an action'.

Illustrated in the following Figure 3.11, a signer narrates a joke in which a bull escapes/flees in a hurry. The hurried aspect of the action is implemented by performing a rapid movement and in addition by the described fast headshake.
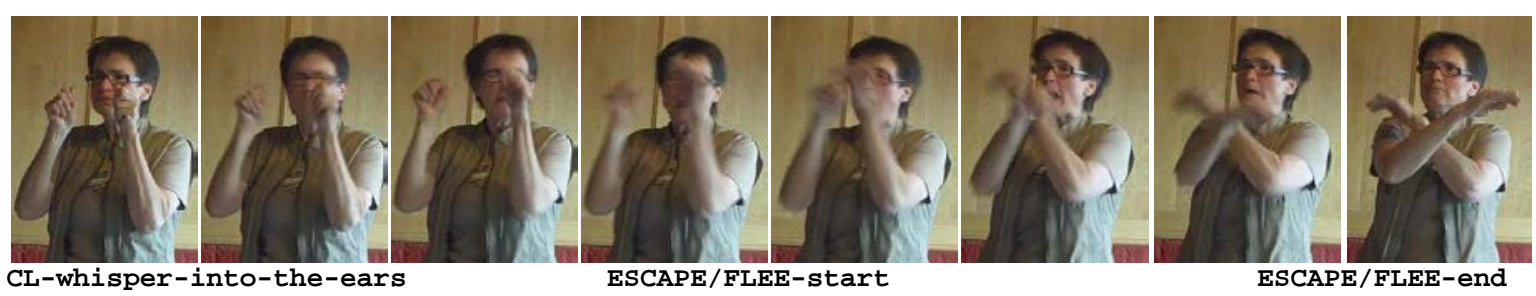

Figure 3.11 A fast single headshake ${ }^{85}$

I have to add that this sign has a negative aspect. As the data shows, signs with implicit negativity can induce negative headshakes in ÖGS, as demonstrated in example (32). Thus, negative signs which possess opposites are good candidates for inducing negative headshakes. In the present example, the annotators do not ascribe a negative aspect to the single headshake. Rather, they focus on the hurried aspect which is expressed by both the fast implementation of the sign and the single headshake.

\footnotetext{
${ }^{83}$ Indeed, it was Klima (1964) who described this phenomenon the first time referring to English.

${ }^{84}$ The present head movement is described as a 'single headshake'. Another description to this would be a 'single head turn sideward'.

${ }^{85}$ The photo series shows that the turn back of the headshake goes together with the movement of the sign FLÜCHTEN (ESCAPE/FLEE).
} 
Other headshakes are present in the data which provide the additional information 'incredible' according to the annotators. In these instances, a single or two lexemes are covered by one or more fast headshakes which are small in size. This is illustrated in example (33).

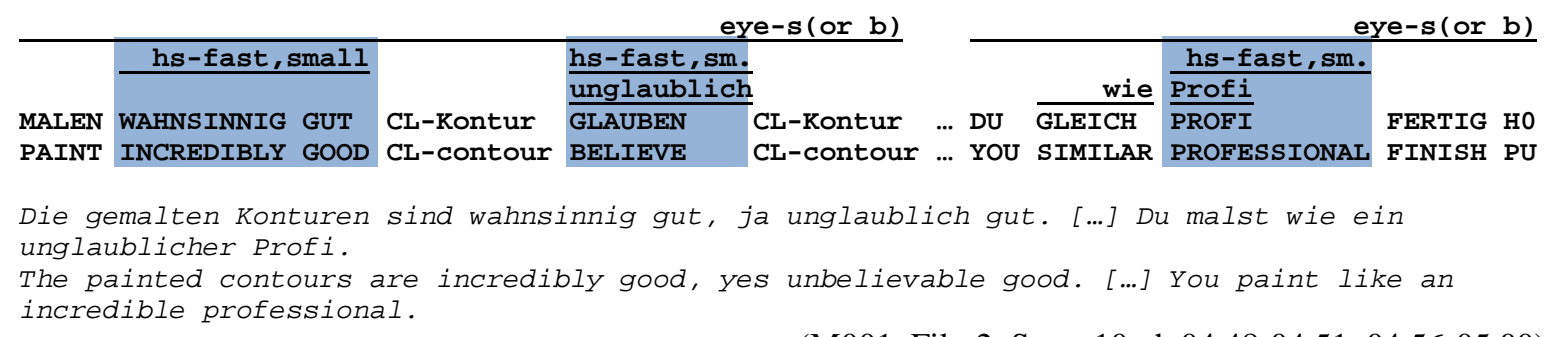

(M001_Film2_Szene10_d_04.48-04.51_04.56-05.00)

In (33), the three headshakes covering INCREDIBLY GOOD, BELIEVE together with the mouthing 'unglaublich' ('unbelievable') and PROFESSIONAL are performed in a very fast speed. To these, the annotators describe the meaning 'incredible'. While performing the headshakes the face is tensed as a whole. In addition, the particular clauses are accompanied by squinted eyes and/or closed eyes. To be exact, 'incredible' means 'not believable / not credible' and in that sense, a negative aspect is implied.

Further functions coded by headshakes and described within this thesis are headshakes displaying a non-assertive modality marker (cf. 7.4.3), and headshakes which frequently co-occurring in wh-questions (cf. 4.2.2.2) and seem to be associated with the degree of knowledge.

\subsubsection{Conclusions}

To sum up, in the data negative headshakes are used for

- clause negation

- speech act negation

- displaying negative contrast

The data shows that one or more headshakes can also have other functions than the listed ones such as showing affective negation or underline the realized speed of an action. 
What is confirmed (with regard to the findings of Skant et al. 2002 and Hofstätter \& Stalzer 2001) by the present analysis is that clause negation in ÖGS can be done only by negative headshakes covering a syntactic constituent or by both headshakes and negative signs, reinforcing negativity. The negative headshakes have to cover the entire negated predicate and can spread along the entire clause.

Zeshan (2006b, 43) distinguishes between two prototypical types of negative constructions showing their particular characteristics of which the main aspect is whether negation is coded by manual or non-manual means. The first type is labeled 'non-manual dominant system of negation', the second 'manual dominant system of negation'. As in the present data headshakes alone, but also together with negative signs indicate negation, ÖGS can be allocated to the first non-manual based type.

What is new for ÖGS is the observation that in signed texts co-occurring headshakes primarily function for clause negation while subsequently occurring headshakes tend to function as speech act negation. Furthermore, the data shows that the most frequently manual negator co-occurring with negative headshakes is the sign NO which follows the verb. Less frequently the sign NOT which precedes the verb is used for clause negation.

Also, the observations that there is a high tendency that negative headshakes spread along special constructions, if negated, such as polar questions, conditional clauses or apodosis of adversative constructions is a new observation for ÖGS, and surely for sign language research in general too. It is shown that first, these headshakes possess different functions (clause negation or implying negative contrast) and second, interpretations for the spreading are offered which are supported by various further findings.

1. To be exact, what is special is that in some constructions such as polar questions or the antecedent of conditionals there is a high tendency that negative headshakes cover the entire clause. The interpretation for conditional clauses is that this phenomenon is influenced by negative epistemic presupposition. This is supported by the high degree of co-occurrence of other epistemic modality markers in these negated if-clauses. In 
addition, the semantic contiguity of coding negation and negative epistemic presupposition is supported by the observation that in the environment of non-assertive epistemic headshakes (occurring in trains of thoughts which imply negative attitude towards /evaluation of a proposition) negative headshakes also occur frequently which precede, follow or intervene - to be exact, overlay - the non-assertive headshakes. Consequently, if an entire clause is to be negated, there is no possibility for the nonassertive headshakes to occur as this epistemic modality marker is overlaid by the negative marker.

With polar questions, it is shown that the spreading of negative headshakes along the entire interrogative can also be caused by pragmatic-communicative reasons.

2. It is also clearly demonstrated that headshakes covering the entire apodosis of adversative or conditional constructions can function for implying negative contrast. An exception displays a semantic subtype of adversative construction which includes the sign RATHER or ANYWAY as a connector between the coordinated clauses. The induced semantic contrast is implied by negative headshakes (or assertive head nods) which only cover the connecting sign.

Finally, one or more headshakes are used for other purposes too. For instance, a fast single headshake is used to illustrate the speed of an action, or small, fast headshakes covering a lexeme are used to add the meaning 'incredible'. 


\subsection{Assertion ${ }^{86}$}

\subsubsection{Introduction}

In language, it is possible to express content or statements in a negative way. That is, first, the meaning of a proposition is negated. In other words, the truth value of a clause can be reversed. Second, it is possible to reject what another person says/signs. In this case, it is not the meaning that is negated; it is the statement in general that is rejected. For instance, this may be due to reasons of ethical values. Thus, it is a negative reaction to a request, a proposition, and so forth (Glück 2000, 467).

In contrast, something may be formulated in a positive way. First, this can be an ‘assertion'. Following Glück (2000, 67), an 'assertion’ expresses that the propositional content of a statement is asserted. Technically, an assertion indicates that the speaker believes that the content of the proposition is true. This is possible in a monologue situation when a person asserts his/her own statement, or it is possible to approve the statement of a dialogue partners. Second, for any reasons a statement can be confirmed in that a positive reaction to a request, a proposition, and so on is expressed. Thus, a person's opinion, his/her action, etc. is affirmed, approved, or agreed.

Concluding, in languages it should be possible (even if not always coded separately) to express negation, denial, assertion, and confirmation (or affirmation) ${ }^{87}$.

These different functions may be coded by the same means of coding, e.g. a negation marker for negation and denial. It is also possible that no means of coding is existent in a language. This is very likely due to 'assertion' as the prototypical statement very often is seen as 'being assertive'.

In the present subchapter, I want to focus on 'assertion', that is, utterances which are marked by one or more head nods for reasons of asserting a proposition.

First, a short literature overview on research on assertion within sign languages is given. Second, the types of assertive head nods, the part which they cover, and the co-occurrence

\footnotetext{
${ }^{86}$ In this thesis the term 'assertion' is used to describe that the truth value of a proposition is asserted. The term 'confirmation' and 'affirmation' are used to describe somebody's reaction to a statement. Thus, somebody can confirm, approve or agree to a statement.

${ }^{87}$ For reasons of clarification of the different functions, I use the four listed terms, following the particular definition of Glück (2000, 67 and 467) in this thesis.
} 
with other head movements are described. Also, other assertive markers in ÖGS are briefly mentioned. Third, head nods which more likely possess a speech act function and head nods which imply positive contrast are analyzed. Finally, assertive head nods are distinguished from confirmative ones.

\subsubsection{Overview of the literature}

As mentioned above, headshakes functioning as negation marker in sign languages have been investigated much more thoroughly than head movements functioning for assertion. The present ÖGS data shows that nods are much more frequent than headshakes, especially in dialogues. But what do they express? - Only confirmation or agreement ${ }^{88}$ within a dialogue setting?

Sutton-Spence \& Woll (1999, 92-93) determine that in British Sign Language (BSL) one, two, or more nods of the head may have different functions too. A head nod may be identified as a lexical element, meaning 'yes', it may have affirmative/agreeing function in discourse, and it may have grammatical functions. With regard to the latter, a single head nod may function as a segmentation cue (in fact, they describe that the head nod fulfils a completing function), fast head nods may be used to insist on the truth value of a proposition $^{89}$, and a single head nod may mark a topic, while a head dip may signal first person.

Johnston \& Schembri $(2007,212)$ observe that a negative statement can be responded to with an 'affirmation' in Australian Sign Language (Auslan). This means that the signer asserts the truth value of the statement. This phenomenon, defined as 'affirmation', is illustrated by them. In doing so, the authors add that the head nods can spread over the entire sentence - similar to headshakes.

\footnotetext{
${ }^{88}$ But not only head nods may be used to express agreement and confirmation. Wilbur \& Patschke (1998, 297-299) for ASL as well as Kooij et al. for NGT $(2006,1609-1610)$ report that leaning the body forward or back is used for expressing agreement/confirmation or denial. This is based on the dialogue partner's opinion concerning the truth value of the proposition of the counterpart.

${ }^{89}$ A comparable marker in form and meaning is found in the ÖGS data too. This is identified as a convincedassertive marker, coding epistemic modality (cf. 7.4.2).
} 


\section{NEGATION AND ASSERTION CODED BY HEADSHAKES AND HEAD NODS}

Due to the long research history (compared with other sign languages), diverse functions have been identified for one or more head nods in ASL. Liddell $(1977,1980)$ observed that nodding may mark emphasis, assertion, and existence. Furthermore, he noticed that when a verb is missing, a head nod may identify this syntactic position. Wilbur (1991) points out that head nods may signal a segmentation boundary and in this position a focus too. With regard to counterfactual conditionals, Liddell (1986) first identifies fast nodding movements in counterfactual conditionals, possessing an assertive character. Wilbur (2000, 229-230) describes small, high frequency nodding movement, used for "hedging”. She concludes that these nodding movements mark a "scope domain" constituting a lexical element, a phrase, or a clause. Wilbur $(2000,230)$ summarizes three main functions of head nods. First, they may signal a boundary; second, they may mark focus and in this function they are produced slower with and without lexical element; third, they express the truth value of the signer's proposition in that large nodding movements express 'strong assertion' while fast and small performed nodding movements are used for counterfactual conditionals and hedging. The third function will be the one on which the present subchapter focuses.

For ÖGS, Skant et al. (2002, 98) notes that a one or more nodding movements may accompany assertive/affirmative signs like JA (YES) or STIMMT (RIGHT). For reasons of intensifying the positive character of a statement or for affirming a self-made statement, the entire clause is covered by head nods or a briefly produced single head nod follows the statement (cf. Skant et al. 2002, 108-109).

\subsubsection{Assertion coded by head nods}

\subsubsection{Different types of assertive head nods and places of occurrence}

In the data two clearly distinguishable forms of assertive head nodding movements are obvious. The characteristics of the first form are slow, intensively performed nodding movements going together with one sign or a sign and an unstressed element. This is illustrated in the following. 
(34)

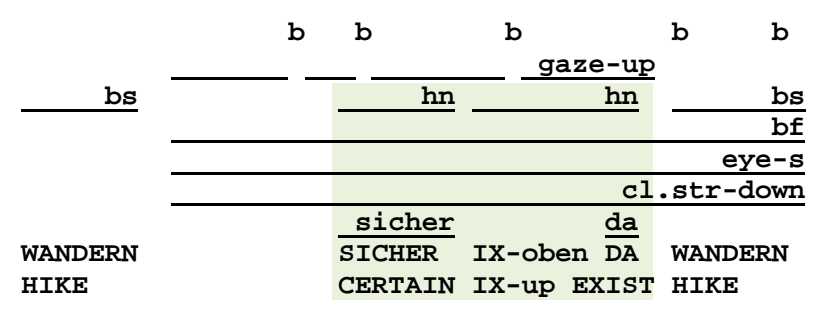

Während ich wandere bin ich überzeugt, dass es oben sicherlich etwas gibt.

While I am hiking I am convinced that (up) there certainly will be something.

(F001_92,1184_m_thoughts _ex.01_00.21-00.25)

The first head nod goes together with the sign CERTAIN, the second head nod with the signs IX-up EXIST. This sign of existence follows a pointing element, which is also covered by the second nodding movement. The nodding movements provide the information that the proposition ('there will certainly be something') is asserted.

The much more frequent form of assertive nodding is fast, small nodding movements which co-occur with a syntactic constituent, forming a single lexical element, a phrase, a clause, or a sentence. This phenomenon is demonstrated in most of the following examples in which head nods function as assertive markers.

In the following example (35) both form of head nodding movements are present. First, the signer performs fast, small movements when signing that he is 'really, in an assertive way recommending something'. Afterwards, the addition, that the dialogue partner is now retired, is not covered by head movements. But, the first sign of the option 'what the counterpart is now able to do' is covered by a single large assertive nodding movement. The example also shows that first, there is a high tendency that modal verbs like KANN (CAN) are covered by one or more head nods. Second, the example demonstrates that in co-/subordinating causal constructions a head nod is very often found in clause-initial position of the second clause. This is the case in conditionals as well as in constructions like the following. 
$(35)^{90}$

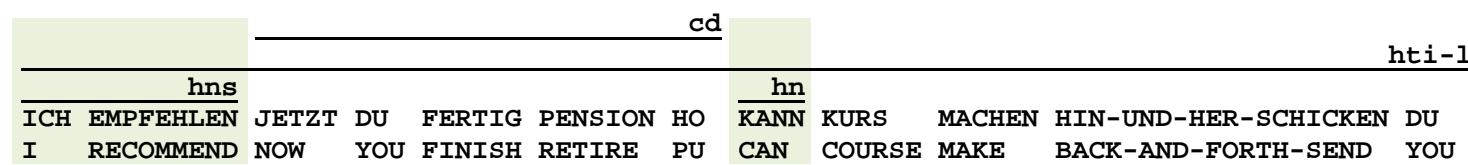

Ich empfehle dir, da du ja jetzt mit dem Arbeiten abgeschlossen hast und in Pension bist, dass du einen (Fern-)Kurs machen kannst, indem du sie (die Zeichnungen) hinschickst. I recommend you, as you have finished your work process and as you are retired now, that you go in for the (correspondence) course by sending it (your paintings) to them.

(M001_Film2_Szene10_d_informel_story_04.24-04.30)

Summing up, in the ÖGS data, the signers frequently produce a single head nod together with modal verbs like KANN (CAN), MUSS (MUST), or SOLL (SHOULD) while negated modals are covered by headshakes.

With conditionals it is obvious that in a number of these constructions in the data the apodosis is either covered by assertive nodding movements ${ }^{91}$ or negative headshakes. Consequently, there seems to be a high tendency that in ÖGS the positive or negative consequence of conditionals is marked (cf. 5.6.1). Also, if the consequence in conditionals is positive, the apodosis frequently is introduced by a head nod which implies an assertive character as it only occurs with positive consequences (cf. 5.6.1).

A comparable phenomenon is present in wh-clefts ${ }^{92}$. Following Wilbur (1996) wh-clefts in ASL display focused structures and are characterized by a focused phrase which stands outside the wh-clause. An ÖGS example of such a structure is offered in the following: $(36)^{93}$

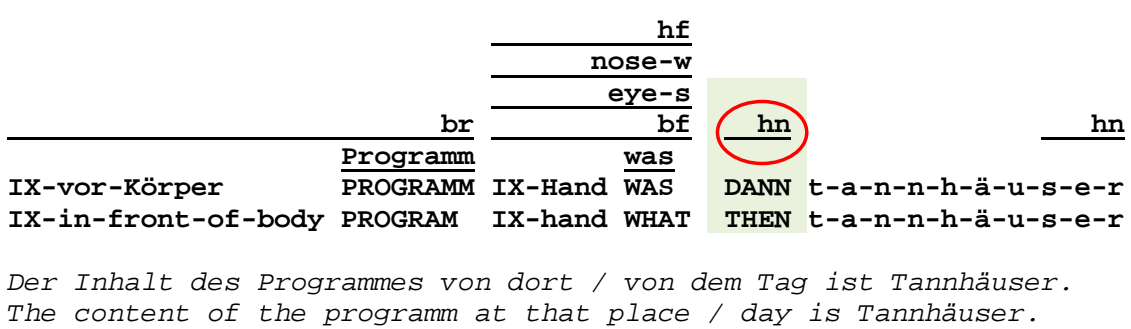

(M001_Film1_Szene3_d_02.35-02.42)

\footnotetext{
${ }^{90}$ Tilting the head to the left may be due to the possibility of the utterance (cf. 7.4.6). Positioning the chin downward might have a conditional-like function (cf. 5.4.1).

${ }^{91}$ There are no instances in the present ÖGS data in which the protasis is covered by assertive head nods.

${ }^{92}$ In 4.3.2.4 wh-clefts are distinguished from content interrogatives. This impression might be evoked by the wh-question sign.

${ }^{93}$ The pointing to the hand refers to the content of the program.
} 
In (36), the focused information is the source of the content of the program. The focused phrase follows the wh-clause. The marker 'head forward', covering IX-hand and WHAT, is described as being more prominent than the preceding signs. The annotators observe that the marker is used to put emphasis on these elements. They do not ascribe an interrogative function to the marker and note that it does not cover the entire clause including IX-infront-of -body PROGRAM IX-hand WHAT. A single head nod, performed in a minimal way, together with the element DANN (THEN), which looks like a short 'backswing' movement of the hand, precede the new information. This nod and/or manual element introducing the focused phrase of a wh-cleft sometimes occur in the data. The annotators ascribe a positive/assertive character to the nodding movement. A single headshake is never found in this position. In (36) also, a single head nod co-occurs at the end of the finger-spelled element, very likely functioning as boundary marker. Wh-cleft-clauses which frequently occur in the data (especially in the educational corpus) are INHALT WAS (CONTENT WHAT) or BEDEUTET WAS (MEAN WHAT) ${ }^{94}$.

\subsubsection{Co-occurrence of assertive head nods}

With regard to co-occurring assertive head nods only the part that has the head nods on it is asserted. The data show that in monologues as well as in dialogues assertive nodding cooccurs in all instances with an entire syntactic constituent, constituting one lexical element up to a whole sentence. The semantic scope of assertion always associates with that part which is covered by the assertive head nods. Following Wilbur (2000, 229), this is also the case with assertive head nods in ASL. Following her interpretation, assertive head nods “mark a scope domain”. Example (37) illustrates that assertive head nods cover exactly that part which is asserted while negative headshakes only cover the negated predicate but negate the entire clause.

\footnotetext{
${ }^{94}$ Only the glosses are given as the nonmanuals covering these glosses vary in several instances of wh-clefts in the data.
} 
$(37)^{95}$

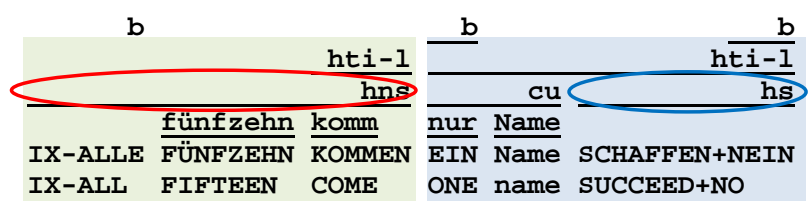

Alle fünfzehn (Kartenspieler) kamen. Lediglich 'Name' schaffte es nicht (dabei zu sein). All fifteen (card players) came. Only 'name' could not make it.

(F002_Film1_Szene4_d_story_informal_01.25-01.29)

In (37), the signer asserts the content that fifteen participants came to play cards; only one Deaf person could not make it. The entire asserted clause (color-coded green) is covered by small constantly performed nodding movements (exactly ten head nods are counted; encircled red). In contrast, the negative headshakes (encircled blue) of the subsequently following clause only co-occur with the elements SUCCEED+NO, but they negate the entire clause (color-coded blue).

\subsubsection{Assertive head nods and other head movements coding other functions}

The most frequent co-occurrence of two different head movements, conveying different functions, is that assertive head nods co-occur with the marker 'chin-down' or 'head forward'. This is exemplified in the following:

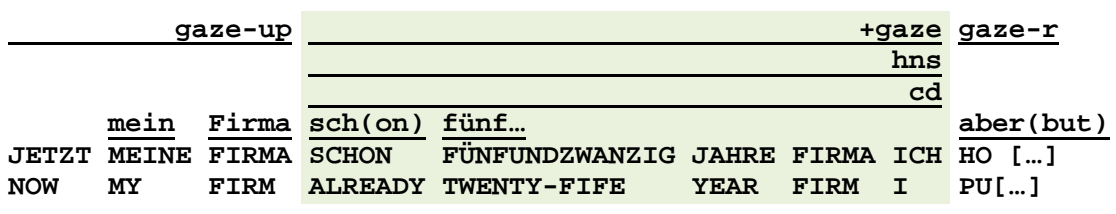

Jetzt hat meine Firma, bei der ich schon seit 25 Jahren bin, (sentence not completed) aber... Now, my company where $I^{\prime}$ ve been employed since 25 years, (sentence not completed) but ...

(M001_Film1_Szene3_d_story_informal_04.18-04.22)

When narrating about how things go on in the company, the signer adds that he has already worked for 25 years for the firm. This additional comment is covered by the non-manual markers 'head nods' and 'chin-down'. The first indicates the assertion of the statement; the latter is probably a means of temporal coding which provides the information that from the

\footnotetext{
${ }^{95}$ During the signs IX-ALL FIFTEEN the signer looks rightward. Only before the blink, which intervenes, the signer looks briefly at her dialogue partner. During signing the name and SUCCEED, the signer looks upward and to the right. The function of the both head tilting movements to the left is not clear up to now.
} 
present view (from 'now') he has already worked 25 years for this particular firm. Interestingly, exactly when formulating this inserted clause, the signer looks at his dialogue partner.

In example (39) different head markers occur in sequence.

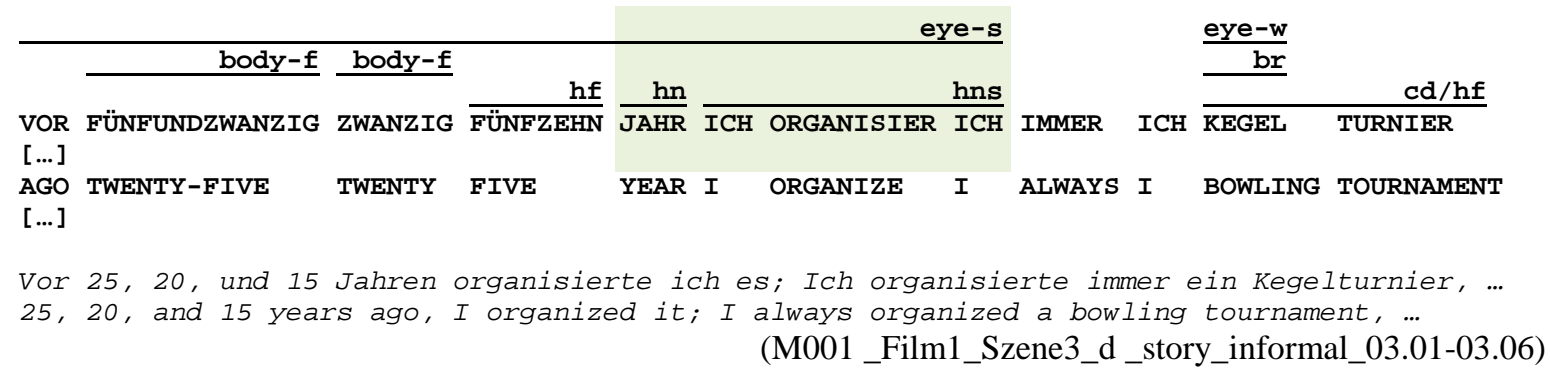

In example (39), the signer narrates that he used to organize festivals for the Deaf club. When signing this, he first lists all years in which he organized the festivals. All itemized numbers are covered by forward movements of the body or head. The subsequently following signs are covered by head nods. First, the sign YEAR is covered by an intensified head nod. As this first intensified head nod is found in clause-final position, a clear segmentation function can be allocated to it. Whether the intensified head nod has further functions can't be determined at the present stage of research. The following small and fast nodding movements possess an assertive function.

\subsubsection{Other assertive markers}

Assertion is expressed by further indicators too. First, the lexical element JA (YES), performed by single or more nodding movements, can co-occur in the context of assertive head nods. The second indicator in the data is a special blinking pattern which occasionally occurs in the context of assertion. This is demonstrated in the following: 
(40)

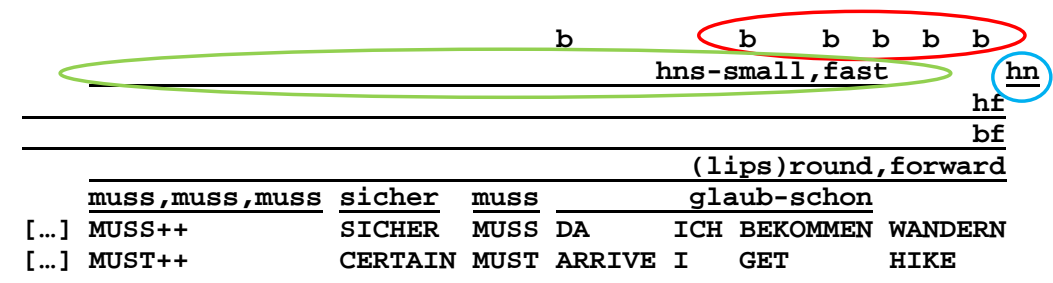

Während ich wandere denke ich, dass [...]. Ich bin überzeugt, es muss so sein und ist sicher so, dass ich etwas bekommen werde.

While I am hiking I think that [...]. I am convinced and it must be and certainly is that $I$ will get something there.

(F001_092,1184_m_thoughts_ex07a_01.51-01.56)

The signer wonders whether she will get something in the inn (expressed in a preceding 'embedded interrogative' which is not illustrated in the example). She is convinced that she will get something. This is indicated by the epistemic marker 'fast, small head nods' (encircled green), functioning as convinced-assertive marker (cf. 7.4.2). The final head nod (encircled blue) functions as terminal signal of the entire utterance. The short, successively blinking movements at the end of the utterance (encircled red) are described as a means of assertion by the annotators, expressing 'yes, yes, yes'. This blinking pattern is presented in this thesis, but further investigations on blinking movements in ÖGS are still an open field of investigations.

\subsubsection{Speech act assertion coded by head nods}

In various cases - especially in dialogues, one or more head nods occur on their own or together with the signs JA (YES) and precede or follow a clause whose content is asserted by the nods. This is demonstrated in the example (41).

$(41)^{96}$

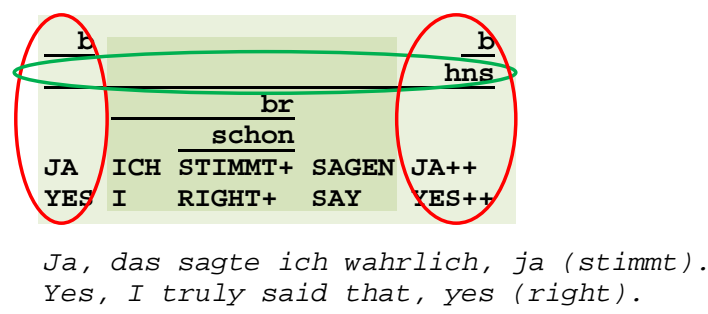

(M001_Film2_Szene10_d_story_informel_05.35-05.38)

\footnotetext{
${ }^{96}$ Example (41) displays the first part of example (28).
} 


\section{NEGATION AND ASSERTION CODED BY HEADSHAKES AND HEAD NODS}

In a discussion on the leadership of the Deaf community, one of the dialogue partners mentions that two years ago his counterpart promised to take the leadship of the Deaf community. As illustrated in (41), this promise is affirmed and the truth value of the content is asserted by the dialogue partner. This is first expressed by the sign YES which precedes and follows the clause, meaning 'yes, I truly said that, yes'. Also, assertive head nods and closed eyes cover the signs YES (both times encircled red). Also the nodding movements are maintained (encircled green) and cover the entire clause (I RIGHT SAY) where they clearly possess an assertive function, in contrast to the preceding and following assertive markers which more likely show a speech act assertion. Thus, similar to the interpretation of negative headshakes which precede or follow a clause (cf. 3.1.4) the present head nods which precede or follow a clause - independent of whether they occur on their own or together with the sign YES - more likely show a speech act function than clause assertion.

\subsubsection{Positive contrast coded by head nods}

The data shows that in a number of cases the apodosis of adversative constructions is covered by assertive head nods. This is caused by semantic contrast. This phenomenon is comparable to the already described negative headshakes induced by negative contrast (cf. 3.1.5).

The occurrence of assertive head nods in these constructions is illustrated in the following: $(42)^{97}$

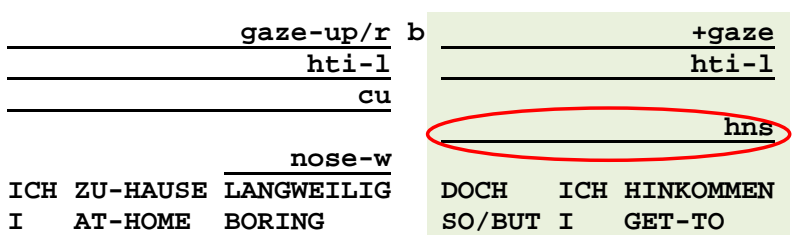

Mir war langweilig, so bin ich doch hingegangen. I felt boring at home, so $I$ went to the place.

(F002_Film1_Szene4_d_story_informal_00.12-00.15)

\footnotetext{
${ }^{97}$ The markers 'chin up' ('cu') and 'gaze up' (the signer looks upward to the right) refer to the space of thoughts (cf. 6.4.3). The function of the head tilt, covering each of the two clauses, may be due to the alternative space (cf. 6.4.4) and may display the same phenomenon as tilting the head sideward in conditionals (cf. 5.6.2). Thus, tilting the head sideward during each of the adversative clauses makes the contrasted coordinating clauses clear.
} 


\section{NEGATION AND ASSERTION CODED BY HEADSHAKES AND HEAD NODS}

In example (42), the entire second clause is covered by head nods (encircled red) of which each nodding movement accompanies one sign. The last head nod is more intensively performed $^{98}$. As already described in the sub-chapter on negative headshakes (cf. 3.1.5), adversative construction can imply a reversal of semantics under special conditions or with regard to a restricted aspect. When giving new information, positive or negative values can be implied resulting in a contrastive coordination (Petkova-Schick 1998, 45). In (42), the first statement gets the negative value, the second statement the positive value. This results in assertive head nods in the second clause (color-coded green). In this example the sign DOCH (SO/BUT) implies that an unexpected action follows.

But not all nodding movements express assertion of a proposition. In the following, assertive head nods are distinguished from confirmative nodding.

\subsubsection{Distinguishing between assertion and confirmation}

In the ÖGS data, especially in dialogues, a lot of head nods can be found ${ }^{99}$. Frequently, these nodding movements are caused by the confirmative style of a dialogue setting. But not all head nods signal confirmation of what the dialogue partner signs. As described above, head nods fulfill further functions too. With regard to assertion and confirmation, the following statement can be made: There is a semantic similarity between both functions. With assertion, the truth value of a proposition is asserted, while with confirmation, a request, statement, etc. - mostly formulated by a dialogue partner (it may be something formulated to somebody himself/herself too) - is approved/agreed. Consequently, this formulated proposition is asserted too. Both functions are expressed by head nods in ÖGS.

\footnotetext{
98 The intensified implementation of the last assertive head nod may be cause by different reasons. First, the last head nod is in clause-final position as well as in prosodic final position, both might cause the intensification. Further, based on the present data it can be affirmed that as a rule a single head nod cooccurs with verbs of existence and arrival (here HINKOMMEN (GET-TO)). The phenomenon that a head nod expresses existence was described for ASL (cf. Chen Pichler et al. 2008).

99 The data shows that due to the strong affirmative character of dialogues in general, there is much stronger tendency for nodding (occurring on its own) than for the negating feedback that is given by headshakes.
} 
In the following example (43), the co-occurring head nods function as an 'indirect confirmation' of the dialogue partner's request. At the same time the signer intends to express the assertion of the proposition.

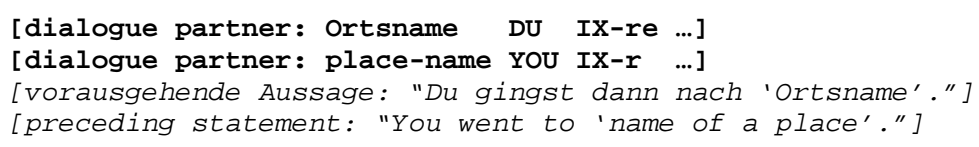

In (43), on the one side the nodding movements express assertion in the sense of 'yes, I wanted to go in for the carpenter apprenticeship'; on the other side, the nodding movements imply a confirmative character. Thus, the signer approves the request by nodding, expressing 'yes, yes in 'place name', I wanted to be apprenticed to a carpenter'. The clause-final head nod indicates the termination of the side comment as well as the end of the utterance.

In many instances in the data head nods clearly function as means of confirmation. When head nods occur in this function, the data shows that the nodding movements do not exclusively cover syntactic constituents, as head nods functioning as means of assertion do. This is exemplified in (44). After having requested confirmation whether a new player participates in playing cards, the following answer and continuation is signed, partly covered by head nods. 
$(44)^{100}$

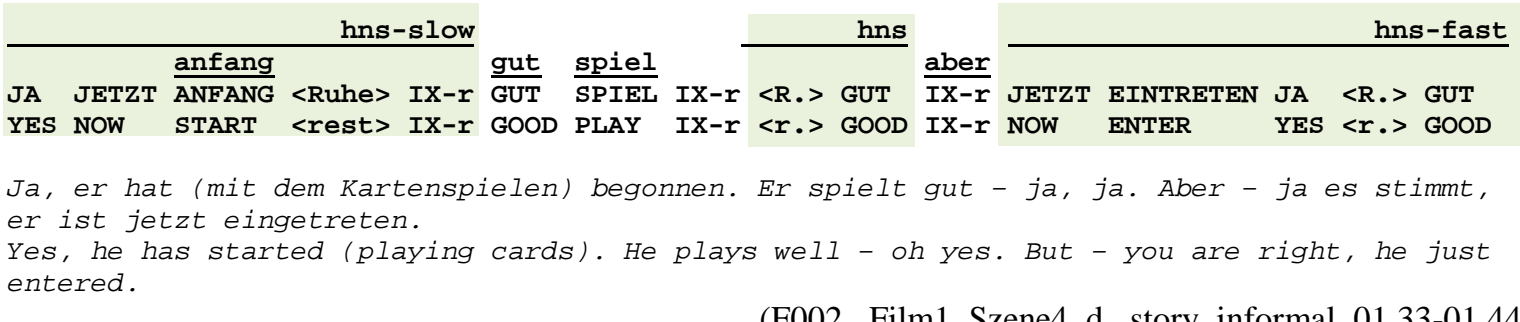

In (44), the various head nods fulfill a confirmative function, even though the signed content is asserted at the same time. The signer produces different nodding movements. The first ones are slow, large movements while the latter nodding movements are small and fast. In addition, the first head nods co-occur with the signs, but they remain too, when the signer rests. In the present example the first and last nodding movements are a confirmation on the counterpart's request. The head nods in between are both assertion of the content and self-confirmation. That is, the signer confirms that he (her husband) is a good card player.

Summing up, the confirmative head nods are not restricted to syntactic constituents, but assertive head nods are. Confirmative nodding movements can also be produced without any lexical signs. Further, they may vary in their size and speed, depending on the person's intention of confirmation. Thus, the annotators identify large and slow nodding movements, which possess an intensified confirmative character. Regular and small head nods are described as a general confirmative attitude toward the dialogue partner or concerning that what s/he signs. Confirmation is mostly addressed to the dialogue partner, but may be addressed to the signer himself/herself too.

\subsubsection{Conclusions}

To sum up, assertive head nods are used for

- assertion (i.e. asserting the truth value of a content)

- speech act assertion

- displaying positive contrast

\footnotetext{
${ }^{100}$ The annotation $<$ Ruhe $>(<$ rest $>)$ abbreviated as $<$ R. $>(<$ r. $>)$ means that the signer holds the hands in a rest position.
} 
The findings in the present data confirm Skant et al.'s (2002, 108-109) observation that cooccurring head nods or a single subsequently occurring head nod are used for asserting the content and affirming a self-made statement in ÖGS. The analysis in this thesis points out that with one or more head nods preceding or following a clause which is to be asserted, these head nods more likely show speech act function. This interpretation is based on the observation that these head nods occur in communicative settings and in these contexts more likely refer to the speech act than to the assertion of a proposition (although the clause is asserted by the preceding/following head nod(s) too).

Similar to ASL (cf. Wilbur 2000, 229) only the part that has the head nods on it is asserted.

What is new for ÖGS and for various aspects for sign language research too, is that the present thesis describes some special 'contexts' which tend to be covered by assertive head nods. First, there is a high tendency for marking the positive (or negative) consequence of conditionals by assertive head nods (or negative headshakes) (cf. 5.6.1). Also, if the consequence in conditionals is positive, the apodosis frequently is introduced by a nod which implies an assertive character as it only occurs with positive consequences (cf. 5.6.1). The same is true for wh-clefts, in which the focused information is frequently introduced by a nod. Also, positive modal verbs like CAN, MUST or SHOULD are frequently covered by a nod to which the annotators ascribe an assertive character. Finally, in a number of instances the apodosis of adversative constructions is covered by head nods induced by positive semantic contrast (similar to headshakes induced by negative semantic contrast).

Furthermore, the present thesis clearly describes the difference between 'assertive' and 'confirmative' head nods in ÖGS, primarily based on the observation that the latter is not restricted to syntactic constituents. Also, in this thesis coding 'assertion' is distinguished from coding 'positive epistemic presupposition'. In this sub-chapter, assertive nods which are used for asserting the truth value of a proposition are shown. In chapter 7 , the 'convinced-assertive marker' will be described, which is used for expressing the signer's 


\section{NEGATION AND ASSERTION CODED BY HEADSHAKES AND HEAD NODS}

judgment/evaluation on a proposition and consequently indicates positive epistemic presupposition. Both markers are distinguished by the annotators due to their different forms. However, in some instances the annotators described that the assertive nods also imply the signer's positive presupposition on the outcome of the situation/event/etc.

With regard to affirmation, the following Figure 3.12 illustrates that a clause can be negated and at the same time the statement is affirmed.

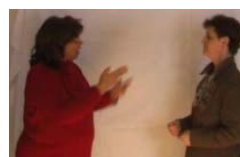

S1: T00-BAD

S2:

S2:

S1:

S2:

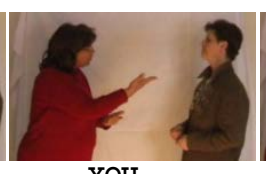

YOU

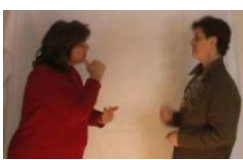

YESTERDAY

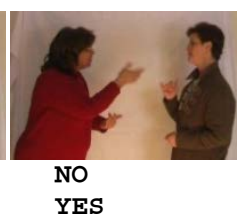

$\mathrm{Ja}$, ja, weil ich

there yesterday.

Yes, yes because I...

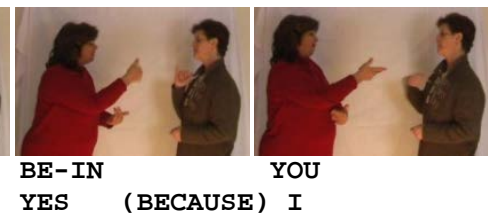

Figure 3.12 Affirmative 'body lean forward' with negation ${ }^{101}$

In Figure 3.12, Signer 1 comments to her dialogue partner that it was too bad that she was not at the Deaf club meeting. This is agreed by Signer 2 and an excuse is offered. While negating the statement, the body leans forward in course of affirming the self-made statement. It also possesses communicative-pragmatic function in the sense of perceiving an affirmative answer. Affirmative body lean forward in the context of negation is also described for ASL (cf. Wilbur \& Patschke 1998, 297-299).

\subsection{Assertion and negation compared}

To sum up, a comparison between head nods functioning as means of assertion and headshakes functioning as negator is briefly discussed.

To begin with, both non-manual markers may cover a construction and provide it with negation or assertion without the presence of a manual negator or a manual assertion sign (i.e. the sign YES). If the sign YES is used in assertive contexts, its syntactic position is frequently the same as that of the sign NO in negated contexts. For instance, if a question

101 Figure 3.12 was quoted in the present thesis author's MA thesis (Lackner 2007, 125) in which the example was quoted to show that body lean forward (the utterance as a whole too) functions as turn-taking cue. 


\section{NEGATION AND ASSERTION CODED BY HEADSHAKES AND HEAD NODS}

is additionally negated/asserted by a manual sign, this assertion/negation sign (YES or NO) follows the question.

Second, the ÖGS data shows that both markers have a syntactic constituent as semantic scope. While the semantic scope of assertion coincides with the constituent, which is covered by assertive head nods, the semantic scope of negation can differ with the part that is covered by negative headshakes.

Third, it is significant that both markers tend to occur in the same constructions. In these, they tend to cover the entire clause. This is the case in polar questions, the consequent of conditionals, and the apodosis of adversative constructions. However, in these environments the negative/assertive marker can possess different functions such as clause negation/assertion, displaying negative/positive contrast or being used for speech-act denegation/affirmation ${ }^{102}$.

Finally, the question arises whether assertive head nods have to be present to assert the meaning of content while it is certain that negative headshakes nearly always have to be present to negate a clause. The following example (45) and Figure 3.13 give some hints to that question:

(45) ${ }^{103}$

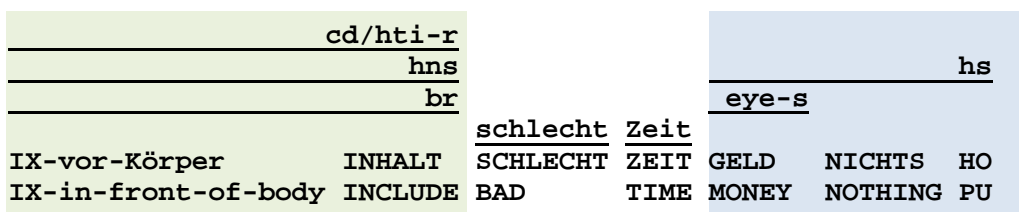

[...] Wahrlich, damals waren schlechte Zeiten. Es gab kein Geld (einfach nichts).

[...] Truly, the times were bad. There was no money left (just nothing).

(M002_Film2_Szene10_d_story_informal_04.01-04.05)

\footnotetext{
102 Krifka (2011) reports that negation in polar question can also possess a pragmatic-communicative function. Consequently, it may be assumed that assertion in polar questions can also be used in order to receive an affirmative/refusing answer.

103 Actually, the left eye is squinted while the other one is hardly squinted.
} 

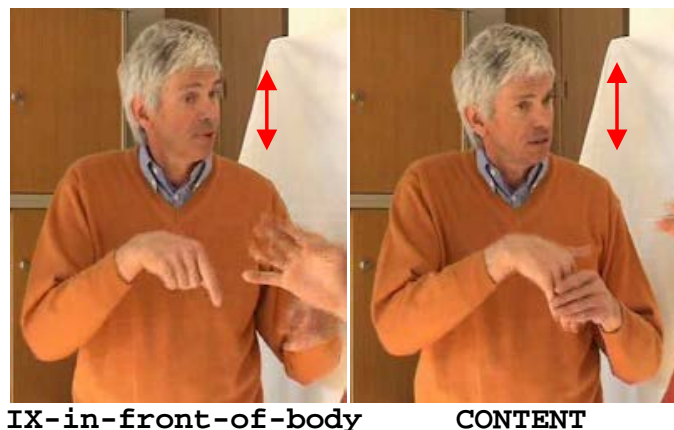

CONTENT

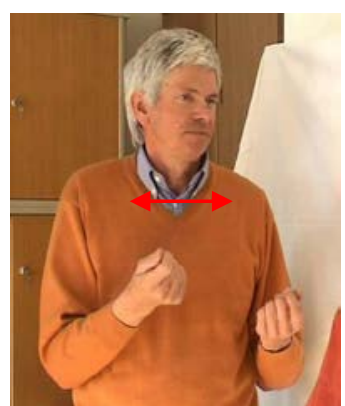

MONEY

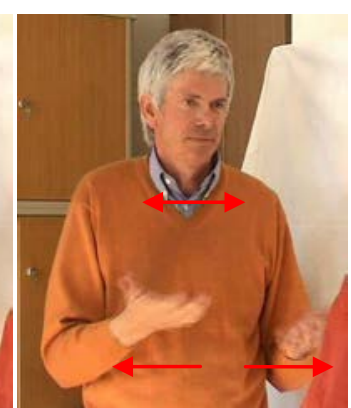

NOTHING

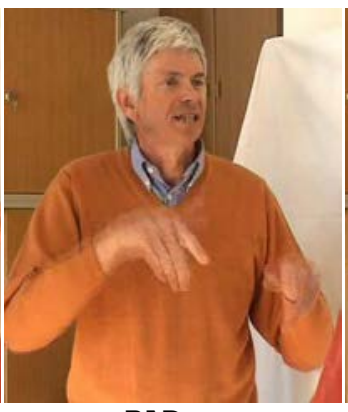

BAD

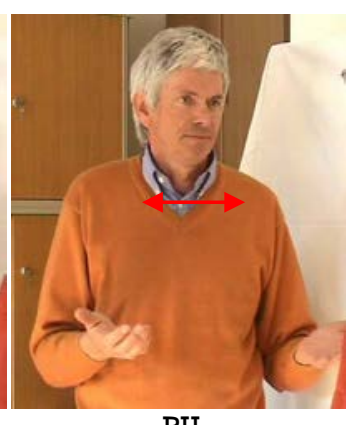

Figure 3.13 Head nods and headshakes compared

In example (45), the second statement ('about how things went on at that time') is covered by headshakes. These clearly negate content (color-coded blue in the example; second line of sequence of pictures). But, the first statement, conveying a positive way of reading, is only partly covered by assertive head nods. Only the pointing sign for establishing a temporal reference and the sign CONTENT are covered by head nods (color-coded green in the example; first two pictures of the illustration), while the statement about the time is not covered by nodding movements. Hence, both syntactic constituents are interpreted in a positive way. This shows that for a positive interpretation head nods are not obligatory. However, for a clear assertive interpretation, they have to be produced. In (45), this means that the part which the signer absolutely wants to be understood in an assertive way is covered by assertive head nods. In doing so, he expresses: 'Truly, at that time ...'. 


\section{Interrogativity coded by head markers}

\subsection{Overview}

\subsubsection{Introduction}

In Austrian Sign Language, not only negation and assertion are coded by movements of the head; a further function that is clearly fulfilled by the head as an articulator is the ‘interrogative function’.

Doubtlessly all languages have some means for expressing that somebody is asking for an answer. Means of coding interrogativity have been investigated in various sign languages. Following Zeshan's (2006a, 40-42) typological comparison on interrogatives ${ }^{104}$, in more or less all sign languages interrogatives are coded by non-manuals, apart from manual question signs. These means of coding are most frequently raised eye brows, wide open eyes, eye contact with the addressee, and positioning the head or body forward.

Research on interrogatives in Austrian Sign Language has already been conducted by Skant et.al. (2002, 109-110 and 157-182), Schalber (2002, 2006), and Lackner (2007, 120 122 - within a larger study investigating turn-taking structures). Following Schalber (2006) and verified and complemented by additional head positions by Lackner (2007 and in the present thesis) the main means of coding interrogatives are different head positions in Austrian Sign Language.

Therefore, the intention of the present subchapter on interrogatives is to show the current state of research on interrogatives and to add the latest findings. These include additional head positions like head forward for embedded polar interrogatives and head backward for a special kind of embedded content interrogatives. Further, the recently identified functions, the reasons for co-occurring head markers in interrogatives, and the additional or alternative use of these head markers (most of these head positions have been mentioned

\footnotetext{
${ }^{104}$ In this thesis the term 'interrogative' is used as a general term for all sentence types that convey the superordinate concept of interrogativity. The term 'question' is primarily used for those interrogatives which are directed to an addressee, that is, the term is primarily used for 'direct questions' being part of a question-answer-sequence. In addition, only polar and content questions are taken into considerations in the literature overview. Further question types like rhetorical questions or the phenomenon of wh-cleft are not focused on. Only when describing the co-occurrence of other nonmanuals in ÖGS-questions does the thesis writer refer to these other question types.
} 
by Schalber 2006 as secondary markers) are described. The present conclusions show that these additional head markers also possess an interrogative function and occur under special conditions, or that these additional head markers convey other functions which occur in the context of interrogatives and whose functions are related to the interrogative function. Moreover, the forms and possible functions of other non-manual markers apart from the head markers occurring in interrogatives are described. This is important because these can be hints as to why an interrogative construction does not represent a 'real interrogative' which prototypically conveys the intention of receiving a response to a question.

Of course, for reasons of providing an overall impression of marking interrogatives in sign languages and especially in ÖGS, the present sub-chapter starts with the latest findings on coding interrogatives in sign languages, focusing on those that code the interrogative information by the head. Also the previously identified markers in Austrian Sign Language (based on the findings of Schalber 2006) are briefly mentioned. For a comprehensive overview on coding interrogativity in sign languages see Zeshan (2006a) and on Austrian Sign Language see Schalber (2006).

\subsubsection{Literature overview}

Following Zeshan's (2004, 2006a) typological comparison of interrogatives, it becomes obvious that there is a high tendency to code this function by nonmanuals (apart from manual question signs). When comparing the non-manual means of coding interrogatives in those sign languages which are described in detail in Zeshan (2006a) ${ }^{105}$ it becomes obvious that most researchers start their description of the non-manual interrogative markers with the eye area in describing the gaze direction (which is directed to the addressee), the gaze aperture and especially the brow movements. The second area which is focused on is the head (and sometimes body too). It is particularly striking that in these sign languages, the head is always involved in marking interrogatives and displays an inherent part of the non-manual configuration of interrogatives - except for ASL (in which

\footnotetext{
${ }^{105}$ The volume comprises descriptions on interrogatives of the following sign languages (SLs): New Zealand SL (NZSL), Japanese SL (JSL), Turkish SL (TID), American SL (ASL), Hong Kong SL (HKSL), Flemish SL (VGT), Brazilian SL (LSB), Finnish SL (FinSL), and Indo-Pakistani SL (IPSL).
} 
the head or body only displays an optional interrogative marker). Furthermore, in most of the sign languages described by Zeshan (2006a) different head positions are used for distinguishing polar questions and content questions. Sign languages that use the same head marker for both types of direct questions are less common ${ }^{106}$.

In the following, some possible head positions marking different types of interrogatives are described, through examples from different sign languages which use distinctive head positions as interrogative markers:

In Croatian Sign Language (HZJ), polar questions are indicated by 'chin down' while content questions are marked by 'chin up'. In addition, less prominent markers for the first labeled question type are 'head forward' and 'wide eyes'. Content questions can be accompanied in addition by 'head forward', 'headshakes', 'shoulders up' and 'closed eyes' (Šarac Kuhn \& Wilbur 2006). With regard to HZJ it should be noted that in the $19^{\text {th }}$ century Deaf Croatian students attended the Deaf institute in Vienna. This circumstance has very likely led to the result that today some features of HZJ are similar to ÖGS (Schalber 2006; Šarac Kuhn \& Wilbur 2006; Šarac et.al. 2007). This is the case with the interrogative markers ‘chin down’ and 'chin up’.

In Quebec Sign language (LSQ), 'head forward' marks polar questions, while 'head backward' marks content questions. A further possible interrogative marker in polar question is 'raised brows', but other brow positions also occur. Content questions tend to have 'furrowed brows'. Brow positions are not as consistent in distinguishing question types as the head positions are. Also, the signer must look at the addressee at least at the beginning and end position of an interrogative (Dubuisson et al. 1991; Dubuisson \& Miller 1992).

\footnotetext{
${ }^{106}$ One example for this is Finnish Sign Language (FinSL) in which 'head tilt forward' is used for both question types. Also, the alternative marker 'head forward' occurs in both question types in special contexts (Savolainen 2006, 284-294).
} 
In Turkish Sign Language (TID), both types of questions are characterized by positioning the head forward, but content questions are additionally indicated by a side-to-side headshake that is different in form and meaning from negative headshakes in TID. Other interrogative markers for both types are gaze contact with the addressee and widened eyes. Raising or furrowing the brows also occurs in interrogatives, especially in polar questions, but they are not an obligatory marker (Zeshan 2006c, 132-134 and 136-137).

Following Morgan (2006, 100-103), who refers to Kimura \& Ichida (1995), in Japanese Sign Language (JSL) the head marker for polar questions is a 'head nod' or 'chin tuck'; it only accompanies the last $\operatorname{sign}^{107}$ of the interrogative while the marker 'raised brows' accompanies the entire polar question. Possible head markers for content questions are a 'chin thrust' which is frequently implemented together with 'tilting the head sideward' and a 'side-to-side tremolo chin wag'. The brows can be raised or furrowed. These content question markers can occur in different configurations.

In Brazilian Sign Language (LSB), polar questions are characterized by 'raised brows' and slight 'tilt downwards of the head', while content questions are characterized also by 'raised brows' and a slight 'head forward' and 'chin up'. The most interesting aspect of LSB for the present thesis lies in its embedded (content ${ }^{108}$ ) interrogatives: These possess a special non-manual configuration, namely a backward tilt the head (resulting in 'chin up'), lowered eyebrows and pursed lips, and a unique question sign (Müller De Quadros 2006, 271-272 and 278-280).

\footnotetext{
${ }^{107}$ Referring to Kimura \& Ichida (1995) Morgan $(2006,100)$ mentions that if a pointing sign follows, both the preceding sign and the pointing sign are covered by the head marker. Consequently, the present thesis writer supposes that the last stressed element (sign), including the following unstressed element, is covered by the head marker.

108 Müller De Quadros (2006, 278-280) does not mention whether the embedded interrogatives are polar/alternative interrogatives or content interrogatives. From the examples given and as the occurrence of a special question sign is mentioned, it can be concluded that these constructions are embedded content interrogatives.
} 
Summing up, the ways of marking interrogatives by head markers vary among the languages. There is a strong tendency to use positioning forward of the head for indicating at least one of the question types, as observed by Zeshan (2006b, 40). Further, the descriptions of the various head indicators show that polar and content questions tend to be indicated by the opposite head positions, as is the case in HZJ ('chin down' versus 'chin up') or in LSQ ('head forward' versus 'head backward'). Finally, the sign languages described so far show that different head positions or movements can be involved even for one question type. So, in TİD for example, content questions are characterized by both 'head forward' and 'side-to-side shake of the head', or in JSL, content questions can involve several head positions/movements within their interrogative configuration (i.e. 'chin forward', 'head tilt', and a special 'chin tremolo movement').

Going through the possible head positions and movements utilized in interrogative constructions that were mentioned above and described in Zeshan (2006a), it becomes obvious that various researchers focus on different aspects of the marker:

- First, the head marker can be described as a whole, which means that the movement of the entire head is described. This results in descriptions like 'the head tilts downward'.

- Second, the focus can also be put on that part of the head which most determines the change of position or movement. This results in descriptions like 'chin down', which implies that the entire head has to move in order to move down the chin as the most striking part, rather than implying that only the jaw goes down while the upper part of the head stays still.

- Third, either the holding position or the movement of the head marker can be emphasized, resulting in descriptions like head 'forward' versus head 'thrust' (compare to this 2.2.4.3).

\subsubsection{Investigated interrogatives in ÖGS}

To begin with, Schalber $(2002,2006)$ made a similar observation in her MA thesis that the 'head' is the most striking marker for indicating polar and content sentences. Skant et al. 
(2002, 109-110 and 157-182) reported that the change in eye brow movement is one of the important markers of coding interrogative information. However, Schalber (2002, 2006) figured out that the primary and dominant interrogative marker(s) is 'chin down' for polar questions and 'chin up' and/or 'head forward' for content questions. These results are based on published educational material containing different variant of ÖGS and elicited data (short stories and paragraphs) of the Styrian variety of ÖGS. Further, Schalber (2006, 139 and 144-146) describes additional non-manual markers occurring in interrogatives. The head markers which additionally can occur in interrogative constructions are the following:

- 'head forward' in polar questions

- 'head tilt sideward' in both polar and content questions

- 'headshakes' in content questions

Whether these headshakes are due to an assimilation process of the shaking movement of the hands (however, not all question signs which are covered by headshakes have an inherent shaking movement) or whether other reasons are responsible for that shaking movement is an open research question for Schalber. In subchapter 4.2.2.2, I will try to find some interpretations for these additional headshakes.

Further non-manual markers in both interrogative types are 'squinted eyes' and different eye brow positions, i.e. raised, furrowed or neutral brows. Schalber (2006, 145-146) speculates that a possible function of eyebrow position is 'expressing the signer's attitude'.

As mentioned in the introduction, the present chapter on interrogatives in Austrian Sign Language should enhance existing findings insofar as other head markers for interrogatives are described and some of the functions of the 'additional non-manual head markers' are identified. This is done by showing example contexts in which they do occur. For reasons of clarification direct polar questions are color-coded green, direct content questions bright orange, embedded polar interrogatives rose, embedded content interrogatives violet, and interrogatives marked with head backward blue. 


\subsection{Direct questions in ÖGS}

\subsubsection{Polar questions}

Beginning with special attention to head markers occurring in interrogatives, the kind of occurrence of the dominant interrogative marker 'chin down' as well as the text types of its occurrence are briefly described. Subsequently, the form, meaning and context of the cooccurring head markers are illustrated. Finally, two question types are listed which are coded by the interrogative marker chin down, but whose goal is not primarily receiving an answer from the addressee.

\subsubsection{Marking polar questions with 'chin down'}

As mentioned above the dominant head marker in polar questions in ÖGS is the marker 'chin down'. This is briefly illustrated in the following:

In a dialogue setting an informant consecutively produces twice nearly the same utterances. The first has no head marker (color-coded grey); the second is covered by chin down (color-coded green) (and includes the modal sign MAY). The covered utterance clearly displays a 'polar question'.

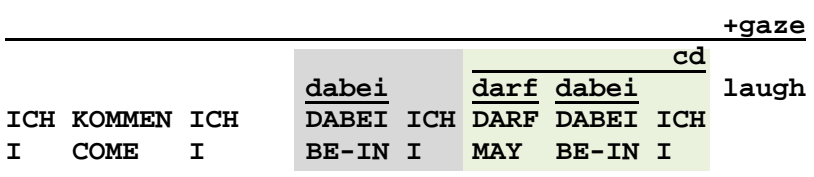

Ich komme hin. Ich bin dabei. Darf ich überhaupt dabei sein?

I come. I am in. May I even be in?

(M001_Film2_Szene10_d_00.48-00.51)

In example (46), the signer states that he will take part in an event. Just then he starts to joke with his dialogue partner by repeating the statement in an interrogative way expressing 'whether he is even allowed to participate'. In doing so, he repeats the sentence adding the modal sign MAY and tilting his head downward towards the chest resulting in the marker chin down. This covers the entire interrogative clause. Thus, he expressed first a declarative and subsequently a polar interrogative. 
This prominent interrogative marker occurs in dialogues as well as in monologues in the present data.

In dialogues the interrogative marker can be influenced by the turn-taking process (cf. Lackner 2007, 120-123). For instance, the additional marker 'head forward' co-occurs with chin down (cf. example (48)) or the dominant marker is held. The latter case is exemplified in example (47):

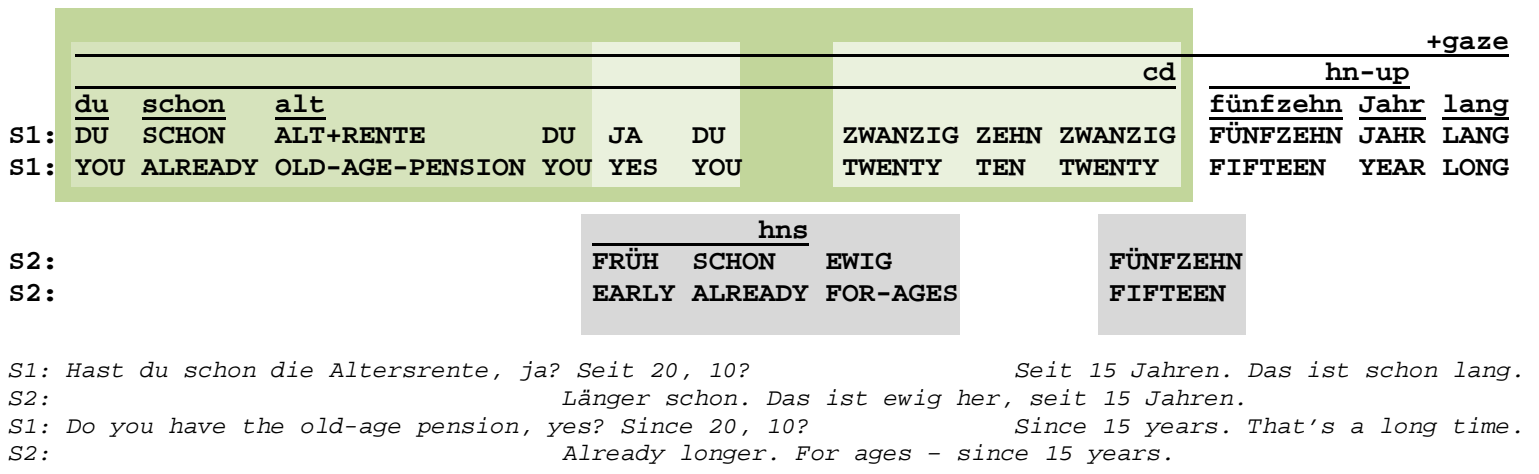

(F001_Film1_Szene1_d_01.06-01.12)

In (47), the annotators identify one question marker, namely chin down indicating a polar question (color-coded dark green, the entire green block). Following the feedback constituting the short turns of the dialogue partner (S2) the first answer (color-coded grey, first block) starts after the main part of the question (color-coded mid green) and maintains during the first extension (color-coded light green, first block). The second answer (colorcoded grey, second block) follows the second extension of the question (color-coded light green, second block). This example illustrates that the interrogative marker can be maintained while a question is extended and it can be maintained while waiting for the response of the dialogue partner (see also example (48) in which the marker chin down is maintained together with the pointing sign YOU while the counterpart answers).

In monologues the marker only occurs if the question is addressed to an (imagined) addressee. This is the case in constructed dialogues. If a question is addressed to the signer himself/herself and would evoke a yes/no-answer (that is, include polarity or alternativity), a distinctive head marker covers the self-addressed interrogative construction (described in 
4.3.1). This different use of two head markers frequently occurs in the corpus containing trains of thoughts. In this data the various signers produce short stories. In several of these narratives the informants sign a long activity during which they ask themselves something. In doing so, they use a different interrogative marker, namely head forward. Afterwards, they want to check whether their self-addressed question is fulfilled or not. They continue the story and in most cases they slip into a role and ask an imagined counterpart whether the requested thing is present/the case/etc. or not. This results in a constructed action in which the dominant interrogative marker of polar questions (chin down) is used. Both implementations within one short story are illustrated in Figure 4.1 in which first, the signer wonders whether a special beverage is available and second, he asks in the form of a constructed dialogue whether this special beverage is available. The first is marked by head forward; the latter is marked by chin down.
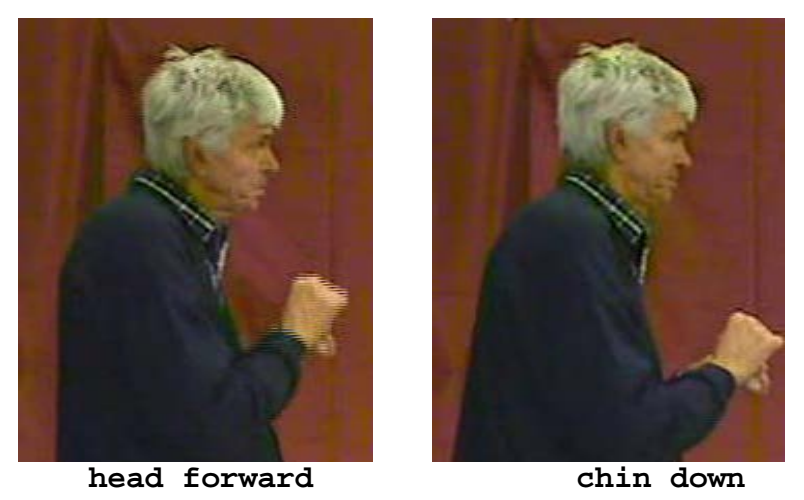

Figure 4.1 Marking of an embedded polar interrogative versus a direct polar question

\subsubsection{Marking polar questions and other co-occurring head markers}

As Schalber (2002, 2006) describes, other head markers are frequently present in polar questions. Two frequently co-occurring head markers are the dominant marker chin down together with head forward (cf. Figure 4.2 and example (48)) and chin down together with head tilt sideward (cf. Figure 4.2 and the examples (49) and (50)).

The single occurrence and the co-occurrence of head markers in polar questions are illustrated in Figure 4.2. A neutral head position precedes as reference. 


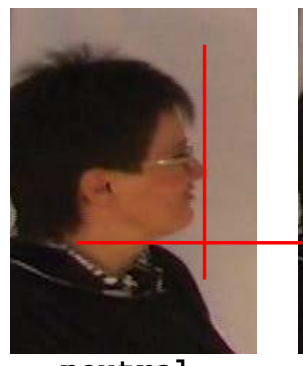

neutral

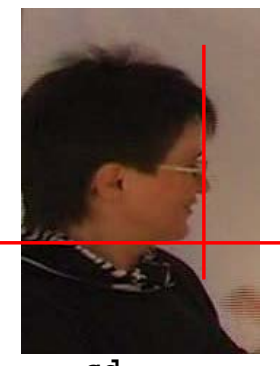

cd

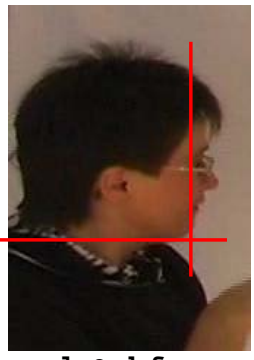

cd \& hf

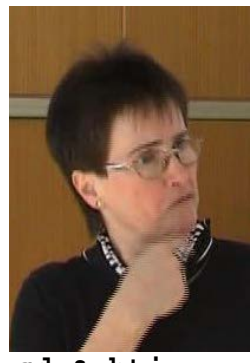

cd \& hti

Figure 4.2 Head markers in polar questions

The ÖGS data shows that if a person asks in sequence the same type of question, the head marker chin down is just maintained. In those instances in which the signer asks the dialogue partner a polar question in a more emotional way, s/he additionally positions the head forward and cranes his/her neck. This gives the interrogative construction a more 'exclamative' character. Both phenomena are illustrated in example (48):

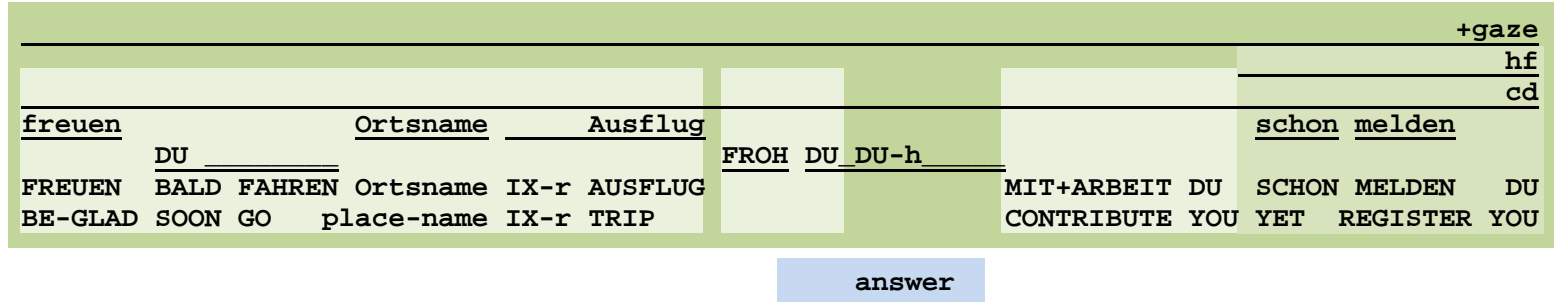

Freust du dich schon auf das Ausflugfahren nach 'Orstname'? Freust dich? Arbeitest du mit? Hast du dich schon angemeldet?!

Are you looking forward to going to 'place name'? Are you looking forward? Do you contribute? Have you already registered?!

(F001_Film1_Szene7_d_03.46-03.52)

Example (48) illustrates four consecutively produced polar questions. They are all accompanied by the marker chin down which is maintained during all four questions. But the last question additionally receives the marker head forward. This additional marker provides the last question with a stronger emotion. The signer asks forcefully whether her dialogue partner has already registered. So, one function of positioning the head forward in interrogatives can be to give the construction a stronger affective and reinforced character. But, the additional marker head forward could also be interpreted as a marker that conveys a meaning beyond expressing interrogativity, that is, the additional head marker could be interpreted as an 'exclamative marker'. 
With regard to the additional head marker 'head tilt sideward' two contexts could be clarified in which the additional marker gives an additional meaning to the interrogative construction. The first function is to provide an interrogative with the notion of foresight or probability (cf. example (49)). The second function is to express in addition politeness (cf. example (50)).

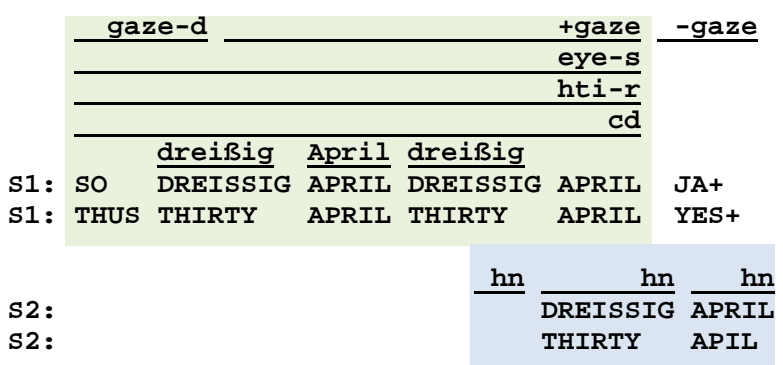

S1: So, voraussichtlich/wahrscheinlich (findet es) am 30. April (statt)?

S2: $\mathrm{Ja}$ am 30. April.

S1: Thus, (it) approximately/probably (takes place) on the $30^{\text {th }}$ of April? S2:

(M001/M002_Film1_Szene3_d_00.11-00.14)

During a dialogue one of the partners requests the counterpart on the date of an event. This is clearly indicated by the head marker chin down. It seems that he knows the date but he is not completely sure about it. As far as I know, this extra information (apart from the interrogative meaning) seems to be provided by the additional non-manual markers. First, possessing some knowledge can be coded by squinted eyes (cf. 7.3.2). Further, the modality meaning 'probable/estimated' of the question can be coded by tilting the head sideward. To this, the probability refers to the event or it refers to the signer's insecure attitude towards the proposition (more on that in chapter 7).

The second function of tilting the head sideward during an interrogative is demonstrated in the following: 
(50)

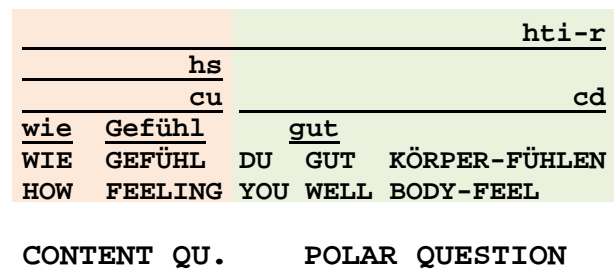

(Ich weiß nicht) Wie ist das Gefühl? Hast du ein gutes Gefühl?

(I don't know) How is the feeling? Have you got a good feeling?

(M001_Film1_Szene3_d_03.38-03.42)

In (50), a signer asks his dialogue partner about a mental state. In doing so, the first question is covered by the marker chin up, the second by the marker chin down. Both questions are additionally accompanied by the marker head tilt sideward. In contexts like the present one, the annotators suggest that tilting of the head sideward implies/expresses 'politeness'.

Other head markers which occur several times in polar questions are negative headshakes (for a discussion on the high tendency of spreading of negative headshakes along the entire polar question see 3.1.3.5). Also, in some instances assertive head nods co-occur in polar questions (cf. 3.3).

Summing up, additional non-manual markers in polar questions can function as an additional 'interrogative marker' which conveys an interrogative meaning and possibly an additional meaning (like the marker head forward occurring in polar questions), or they possess a different function, but tend to occur in polar questions (like the marker head tilt sideward). The listed functions are those which have been determined up to now. Further functions of these additional head markers are possible.

\subsubsection{Discourse control with polar questions}

Questions marked by chin down are in many cases clearly polar questions in the ÖGS data. So, their intention is to receive an answer from the dialogue partner. The data also show that interrogatives indicated by chin down can have other intentions. In the following, two 
interrogatives are briefly presented which are marked by chin down, but whose aim is not to receive an answer from the dialogue partner.

The first question type is labeled 'confirmation question' by the thesis writer as the signer only aims to receive a confirmation from the counterpart on the signed content. No answer in the form of new information is requested.

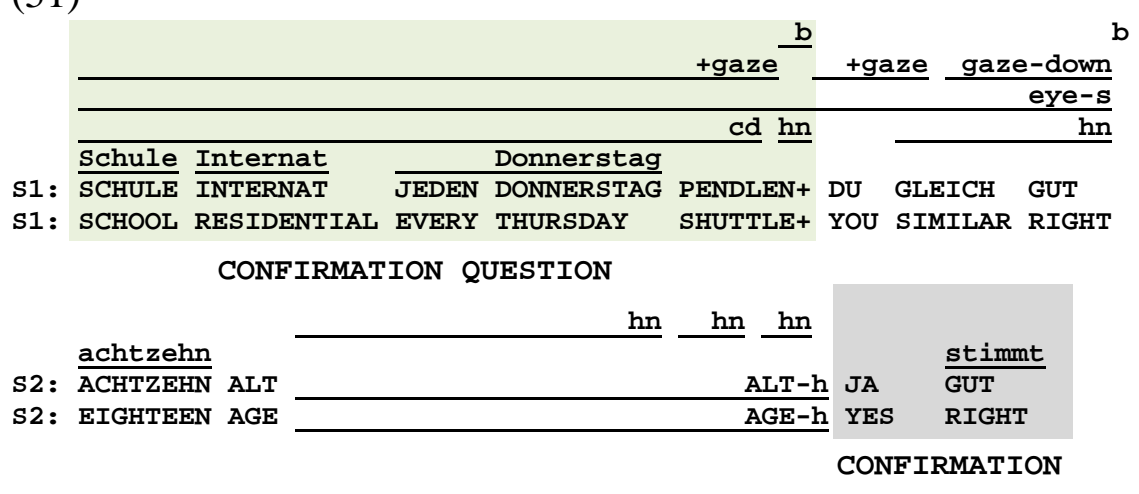

S1: Zum (Berufs-)Schulinternat pendeltet ich jeden Donnerstag./? Du ebenfalls, stimmt.

S2: $\quad$ Ja, stimmt.

S1: To the (professional) residential school I shuttled every Thursday./? You too, right.

S2: $\quad$ Yes, right.

(M001/F001_Film2_Szene5_d_02.07-02.11)

Example (51) shows that the 'confirmation question' (color-coded green) is covered by the marker chin down and terminated by a head nod. The pattern that a question ends with an assertive nod or a negative headshake occurs frequently in the ÖGS data. With this, the content of the question is asserted or negated. Significant in example (51) is that no change of the eye brows is present, however the eyes are squinted. The non-occurrence of furrowed or raised eyebrows as well as the presence of the non-manual marker squinted eyes reveals that a 'confirmation question' is present. That is, the signer does not really ask the dialogue partner for new information about the described context. The signer aims to receive a confirmation (color-coded grey) about what is known by both dialogue partners. So, the use of the marker squinted refers to the 'common knowledge' and the non-presence of brow marker shows that there is no intentions to get a 'real answer', but only to receive a confirmation.

So, the present 'confirmation question' is from the semantic perspective both a question and a declarative statement. With regard to the marker chin down the utterance is clearly a 
question, however with consideration of the other present and non-present non-manual markers, the pure question character, i.e. asking the counterpart for an answer, is not the intention of the present confirmation question.

The second question type which is covered by chin down but which intention is not to receive an answer from the addressee is in the present defined 'attention-getter question' in narratives. The sign of knowledge together with the pointing sign for you, covered by the interrogative marker chin down, are put in front of a narrative as exemplified:

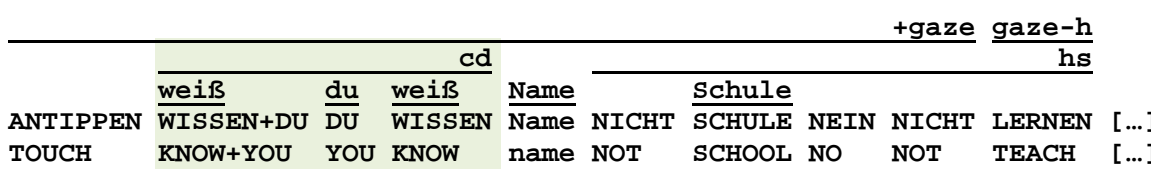

Hey, weißt du (Folgendes):? 'Name' hat keine Schule besucht, nicht gelernt. ...

Hey, do you know (the following):? 'Name' has never attended a school, never learned ...

(M006_Film1_Szene2_d_05.35-05.41)

In example (52) one dialogue partner narrates about a mutual friend who died. In doing so, the turn holder asks the counterpart whether he knows - but he does not continue the question, but keeps on narrating about the late friend. This pattern frequently occurs in the ÖGS corpora. The intention of the 'started question' is to attract attention to the narrated content.

Both described question types show that the dominant polar question marker can be present but no 'polar question' in the proper sense is at hand.

Now I turn my attention to the other type of direct question.

\subsubsection{Content questions}

\subsubsection{Marking content questions with 'chin up' and/or 'head forward'}

The various content questions in the present ÖGS corpora confirm Schalber's $(2006,142)$ findings, that is, content questions are marked by 'chin up', or 'head forward', or both markers together. Figures 4.3 and 4.4 show clearly that an exact differentiation between the single occurrence of chin up and the co-occurrence of chin up together with head forward is not always distinguishable by the annotators. 


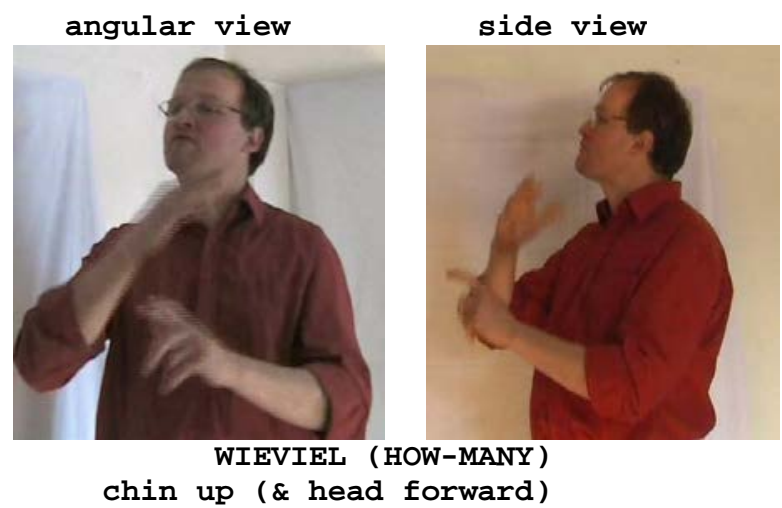

Figure 4.3 The marker for content questions

Further, Schalber's (2006, 142) findings that the dominant content marker can only accompany the question sign or else cover the entire interrogative clause can be confirmed by the present data.

In example (53) and Figure 4.4 three content questions (color-coded bright orange) are illustrated. They all occur within a dialogue and are consecutively produced. The first is produced without a question sign, the second and third with different question signs. The co-occurring non-manual markers in these three questions are always the dominant head marker chin up, gaze directed to the addressee, and partly 'furrowed brows ${ }^{109}$.

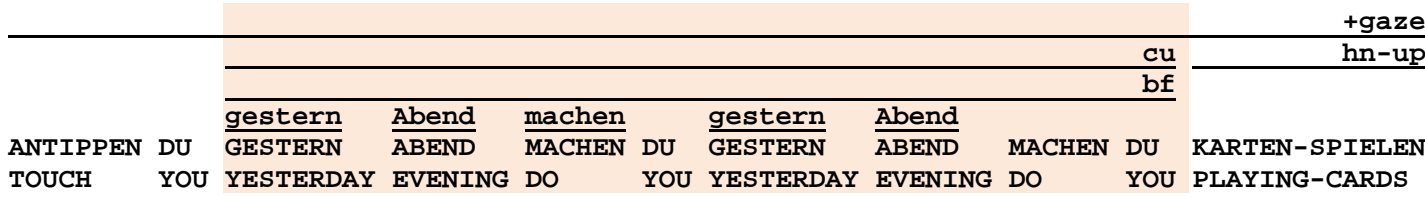

\begin{tabular}{|c|c|c|c|c|c|c|c|c|}
\hline \multirow{3}{*}{$\frac{c u}{\text { bf(slightly }}$} & b & & & & & \multicolumn{2}{|l|}{ b } & tgaze \\
\hline & hn-up & & & & cu & & hn-up & hn-up \\
\hline & & & & & & & $\overrightarrow{b r}$ & $\overrightarrow{b r}$ \\
\hline $\begin{array}{l}\text { WO } \\
\text { WO } \\
\text { WHERE }\end{array}$ & $\begin{array}{l}\text { aso } \\
\text { GUT } \\
\text { WELL }\end{array}$ & $\begin{array}{l}\frac{\text { wieviel }}{\text { WIEVIEL }} \\
\text { HOW-MANY }\end{array}$ & $\begin{array}{l}\text { Leute } \\
\text { LEUTE } \\
\text { PEOPLE }\end{array}$ & $\begin{array}{l}\text { KARTEN - SPIELEN } \\
\text { PLAYING - CARDS }\end{array}$ & $\begin{array}{l}\text { WIEVIEL } \\
\text { HOW-MANY }\end{array}$ & $\begin{array}{l}\text { fünfzehn } \\
\text { FÜNFZEHN } \\
\text { FIFTEEN }\end{array}$ & $\begin{array}{l}\text { GUT } \\
\text { WELL }\end{array}$ & $\begin{array}{l}\overline{\text { Viel }} \\
\text { VIEL } \\
\text { A-LOT }\end{array}$ \\
\hline
\end{tabular}

Hey, was hast du gestern gemacht? Ach, Kartenspielen. Wo? Ach so, gut. Wieviel Leute haben mitgespielt? Fünfzehn, das sind viele.

Hey, what did you do yesterday? Oh, playing cards. Where? oh, well. How many people were playing cards? Fifteen, that's a lot.

(F001_Film1_Szene1_d_00.01-00.10)

109 The function of the brow markers is still an unresolved matter in ÖGS. Skant et al. (2002, 109-110 and 157-182) stated that 'hochgezogene Augenbrauen' ('raised brows') indicate polar questions, 'zusammengezogene Augenbrauen' ('furrowed brows') content questions. Schalber (2006, 145-146) determines that furrowed or raised brows are not the dominant question markers and very likely give information about the signer's attitude. 
In example (53) in all three content questions the marker chin up indicates the interrogative construction. It can be clearly distinguished from the interposed utterances which in most cases display a copy of the dialogue partner's answer. Each of these interposed utterances is accompanied by a single head nod movement upward. During all three content questions the signer looks at the dialogue partner. Blinks occur at the end of the second and third content question.

Furthermore, example (53) should demonstrate that a wh-sign is not always required for a content question (see first question in the example). That is the very reason why in the present thesis the term 'content question' instead of 'wh-question' is used. But the present data show that in most content questions a wh-sign occurs.

In Figure 4.4 the second to fourth picture illustrate the dominant head marker chin up occurring in example (53). Always the first sign in the content interrogative is pictured. The first and last picture of the series show the first and last sign of example (53) in which the annotators did not perceive a separate head marker.
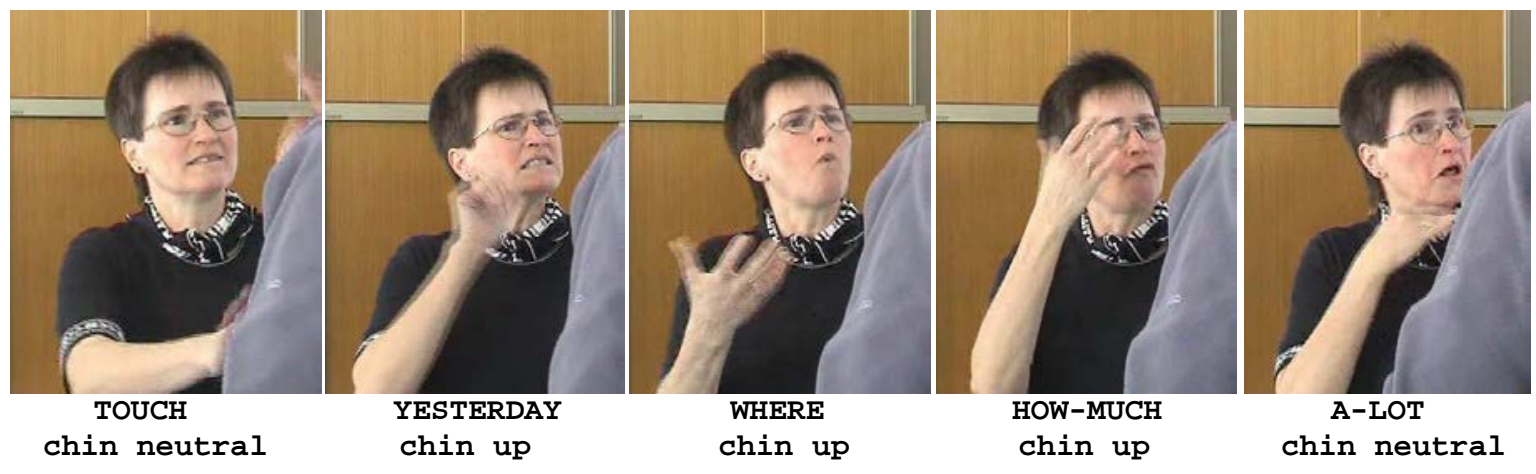

Figure 4.4 The marker 'chin up' ( $\&$ slightly 'head forward')

\subsubsection{Marking content questions and other co-occurring head markers}

Again and again co-occurring head markers in content interrogatives are the markers 'headshakes' and 'head tilt sideward' which also occurs in polar questions.

The marker head tilt sideward has already been illustrated in (50) in which it covers both the content question and the following polar question. Example (54) shows that the marker head tilt sideward can also follow the content question. Similar to (50) the additional head 
marker head tilt sideward is an expression of politeness conveying a notion of modality (cf. 8.2.3 on semantic contiguity).

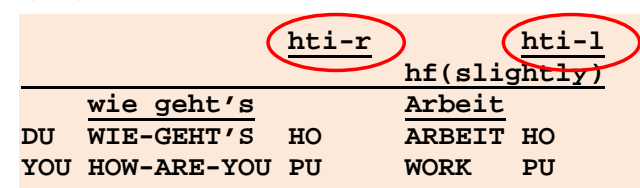

Wie geht's dir bei der Arbeit?

How are you [how things go on] at work?

(M002_Film1_Szene3_d_04.12-04.15)

The second additional head marker in content questions that repeatedly occurs is headshakes which does not function as negator ${ }^{110}$. This phenomenon is illustrated in example (50) in which the headshakes accompany the signs HOW FEELING and co-occur with the dominant interrogative marker chin up and the additional head marker head tilt sideward. As mentioned above, Schalber $(2006,145)$ has already described 'slight turns of the neck to right and left' that sometimes cover the ÖGS content signs expressing what, why, or how. Headshakes co-occurring in content questions in the present data have the form of fast, non-tensed shaking movements. They cover only the question sign or the entire interrogative like in example (50) and (57). In order to find an interpretation of the head shaking movement in content questions, the examples (55), (56) and (57) are described. They all include the sign WAS (WHAT) which is differently performed and which is covered by different non-manual markers. However, all these examples have one thing in common, that is, the requesting person expresses 'his/her unawareness of a fact/situation/circumstance’.

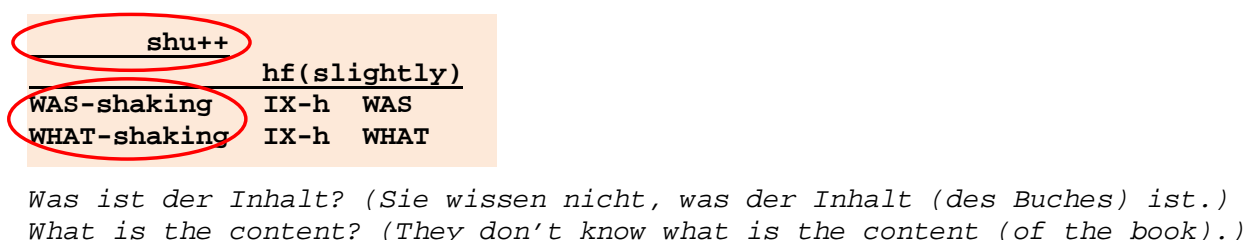

(M003_Film1_Szene3_d_02.43-02.45)

\footnotetext{
${ }^{110}$ Of course, content questions can also be covered by negative headshakes which clearly function as a clause negator. These rarely occur in the present ÖGS data.
} 


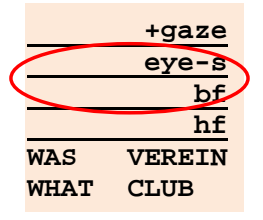

Welchen Verein? (Ich weiß jetzt nicht, welchen (Gehörlosen-)Verein du meinst.) Which club? ( $I$ actually don't know which (Deaf) club do you mean.)

(M001_Film1_Szene3_d_00.05-00.08)

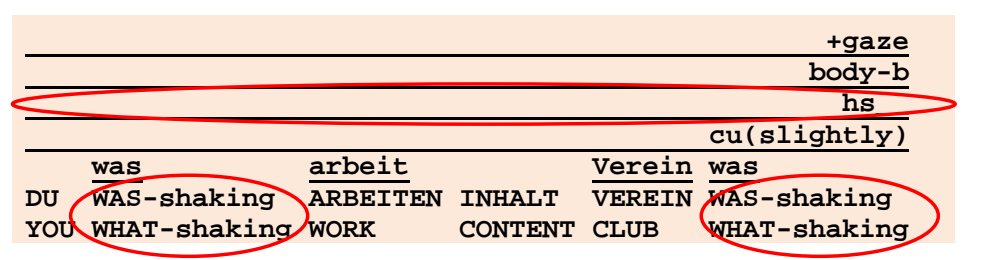

Ich weiß gar nicht, (als) was du arbeitest im verein.

I even don't know, (as) what are you working in the club.

(M001_Film1_Szene3_d_01.11-01.14)

In example (55), the signer shakes the hands sideward when signing WHAT. In addition, he is shrugging his shoulders. In doing so, he expresses the audience's unawareness of the content of a book. To express this, the signer slips into the role of the audience. In example (56), the signer performs the sign WHAT without shaking movements, however the interrogative is covered by furrowed brows and squinted eyes. In doing so, the signer expresses his unawareness of the Deaf club about which the dialogue partner is talking about. In example (57) the signer produces twice the sign WHAT with fast shaking movements sideward. In addition the content question is accompanied by fast, non-tensed headshakes. In doing so, the signer expresses his unawareness about the counterpart's function in the Deaf club.

Based on the annotators' feedback as well as on the fact that the described components (shaking the hands when signing WHAT, shrugging the shoulders, furrowing the brows together with squinting the eyes, and shaking the head) occur by turns in content questions and yet express the same meaning (somebody's unawareness) as illustrated in (55) to (57), the interpretation that the 'head and hands both convey the same function and therefore they do not both have to be present' is easily conceivable. Whether the shaking movement of the head originally is due to an assimilation process in which the shaking movement of 
the hands are adopted by the head, or whether both shaking movements (of the hands and of the head) have their own origin cannot be answered at the present stage. It is striking that also the shoulder movements comprise an on-going shaking movement, but along the vertical axis.

In general, further investigations on the co-occurrence of different non-manual markers are required for clarifying whether the above-listed co-occurring nonmanuals all possess the same, similar, comparable, related or different functions and whether their co-occurrence depends on certain functional contexts or whether other reasons are responsible for the cooccurrence.

\subsubsection{Conclusions on direct questions}

The present subchapter on direct questions confirms Schalber's (2002, 2006) findings that the dominant interrogative markers in polar and content questions are head markers. Polar questions are indicated by 'chin down', content questions by 'chin up' and/or 'head forward'.

What is similar to other sign languages, but not clearly described for ÖGS, is that with direct questions, the gaze is always directed at the counterpart (cf. Zeshan 2006b, 40). Only for reasons of turn-taking (cf. Lackner 2007, 110-119) and probably other causes too, the gaze direction may change.

Also, the present findings show that other non-manual head markers such as head forward in polar questions, head tilt sideward in both polar and content questions, or non-negative headshakes in content questions frequently co-occur with the head markers of direct questions. This was observed for ÖGS by Schalber (2006), but also for other sign languages frequently co-occurring head markers are described (see, for instance, Watson 2010 for ASL). 
What is new in this thesis concerning other head markers ${ }^{111}$ which frequently occur in the context of direct questions is that it is shown that most of these markers have their own non-interrogative function, but they tend to occur in interrogatives.

More precisely, marking a polar question with chin down and additionally positioning the head forward increases the affective character of the interrogative. This results in asking in a more emotional way that even might be interpreted as being an interrogative as well as an exclamative. Tilting the head sideward in polar or content questions can bring an additional function into the interrogative construction. Two of the previously identified functions are providing the construction with potentiality, and expressing a notion of possibility in order to achieve greater politeness. Shaking the head in non-negated content questions along the wh-question sign or along the entire clause is interpreted at the present research stage as additionally expressing the signer's lack of knowledge about the requested matter. As a basic principle the character of an interrogative is expressing lack of knowledge of a matter by asking about it. However, additional headshakes as well as the other possible cooccurring components underline this lack of knowledge.

In conclusion, the section on direct polar questions intends to go beyond a generalized description of co-occurring nonmanuals as ‘additional or secondary interrogative markers'. Rather, contexts are illustrated in which non-interrogative markers co-occur with the interrogative marker(s). However, these non-interrogative markers frequently occur in the context of interrogativity.

\footnotetext{
${ }^{111}$ Other frequently co-occurring nonmanuals which are not articulated by the 'head' (like different brow positions) are not focused on in the present subchapter. A description of their occurrence in the different contexts together with their meanings and functions requires a deeper look at them.
} 


\subsection{Embedded interrogatives in ÖGS}

The interrogatives described so far have one thing in common. They are all directed to a counterpart. This is a real dialogue partner or in monologues an imagined dialogue partner to whom the question is addressed. The latter case is normally implemented by a constructed dialogue, even if this is implemented by a single question.

Now, the present data show an interesting finding. When a signer is asking something to himself/herself or an imagined addressee who is located in the space of thoughts (cf. 6.4.3), a different head marker occurs. Both polar and content interrogatives which are selfaddressed or take place in the space of thoughts are labeled 'embedded interrogatives' as both are embedded from a syntactic perspective and linked to a sign of cognition, emotion, or perception.

\subsubsection{Embedded polar interrogatives}

\subsubsection{Introduction}

One part of the ÖGS corpus contains monologues that comprise reflections on different situations. Many of these trains of thoughts reflect on a situation that might be possible or not while having different estimations on the options of being possible or not.

While analyzing the signed reflections of the videos, the annotators identified that in many constructions the head of the particular signer in the videos is positioned forward and covers the thoughts, part of the thought or the thoughts together with a modality expression. As a result, a construction became obvious that definitely requires the marker 'head forward', abbreviated as 'hf'. This identified construction is labeled 'embedded polar interrogative ${ }^{, 12}$ in the present thesis.

After initiating the chapter with a prototypical example of the present interrogative construction, the interrogative markers head forward, raised brows and 'ob’ ('whether') are

\footnotetext{
112 The term 'polar' interrogative is used as it is a widely used and well-known terminology. Indeed, the present construction shows the semantic characteristic 'alternativity' rather than 'polarity' (cf. 4.3.1.3.1).
} 
introduced and described in detail. Then, the characteristics of the embedded interrogative clause are discussed.

In the following Figure 4.5 a signer is hiking and expresses his insecurity about the situation whether a hut is open or closed. Based on the video that was presented to the different annotators and based on the glosses ${ }^{113}$, the particular annotators added their perceived head/body movements or position together with their interpretation of the possible function of these elements. In this way, a clear head marker became apparent as shown in the following screenshot of ELAN.

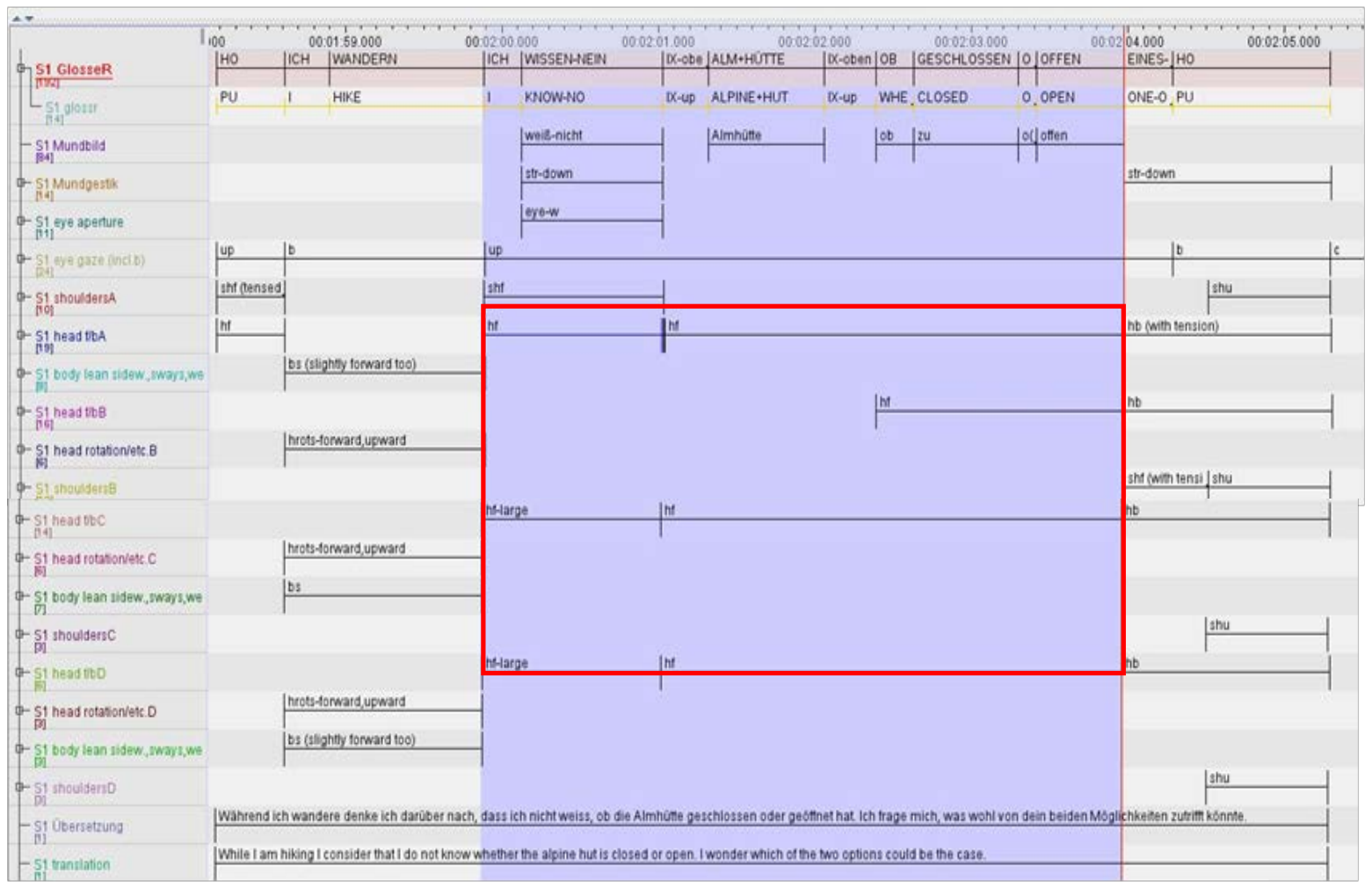

Figure 4.5 Intra-individual perception of marking embedded polar interrogatives by 'head forward'

In Figure 4.5, positioning the head forward is perceived by all annotators as a distinctive, language-relevant element. For three annotators this head marker covers the signs I

\footnotetext{
${ }^{113}$ The annotation of the glosses is realized by the first annotator and corrected by the following annotators, if required (cf. 2.2.4.3).
} 
KNOW-NO (first moving forward of the head) IX-up ALPINE+HUT IX-up WHETHER CLOSED OR OPEN (second positioning forward of the head) - all together constituting an embedded polar interrogative ('I don't know whether the alpine hut is closed or open'). One of the four annotators perceived the starting point from WHETHER onwards. As the starting and ending point of the marker head forward was put into relation with the lexical items, the hf-marker's start/end has the same starting/ending point as the particular sign. For all annotators this head forward marker is the main criterion why the present construction is interpreted as an interrogative.

During the whole short story the signer expresses his uncertainty signing KNOW+NO or INDECISIVE ${ }^{114}$ or articulating mouth actions like 'lips that are closed and stretcheddown', conveying insecurity about the situation.

In addition, the whole short story is accompanied by body sways and shoulder shrugs occur in the beginning and the end of the utterance. The ongoing movements of the body (labeled 'body sways') are described as a further distinctive language-relevant marker (abbreviated as 'bs') that expresses something like 'maybe' or 'possibly'; the shoulder shrugs (abbreviated as 'shu') are perceived as language-relevant elements too, conveying 'being unaware of something'.

The annotators observe that the short story contains a general description about the uncertainty of the situation, implemented by diverse signs, mouth actions, shoulder shrugs and body sways. But they notice also that this general description of the situation and attitude of the person is interrupted twice by two clear trains of thoughts. The first time, the signer asks himself whether the hut is open or closed; the second time, he thinks in a hopeful way that the hut is open. The first thought is accompanied by positioning the head forward as described above. In addition, the eye brows are raised. The collective occurrence of all elements together, placed in the context of uncertainty and primarily identified by positioning the head forward, is described in the present thesis as a

\footnotetext{
${ }^{114}$ The sign UNENTSCHLOSSEN (INDECISIVE) conveys the meaning that a person is of two minds about something and that the person's feels insecure about the evaluation on this.
} 
construction that is embedded in a context of lack of knowledge (mostly paired with uncertainty, doubt or hope), self-addressed (to the signer himself) by referring to a special 'space of thoughts', conveying interrogativity as well as irreality and implying alternatives. In the following the form and meaning of the present construction are described in detail:

\subsubsection{The interrogative markers}

The main concept the present construction conveys is the concept of interrogativity. Hence, the main function of the construction is expressing lack of knowledge about special information which is asked for in a reflective way but which remains unanswered. In doing so, the particular individuals show their lack of knowledge. In addition, the construction expresses the questionability of a proposition which conveys a hypothetical thought and consequently, implies the irreality of an event. Furthermore, it has to be mentioned that when a person is unaware of information for which s/he asks himself/herself or another person, additional aspects are packed into the construction. This additional information (beyond the concepts of interrogativity and irreality) may be expectations towards the (truth value of the) possible answer or the different feelings like uncertainty, doubt, or hope which go together with the lack of knowledge of the situation, but which may depend on the degree of lack of knowledge. If this extra information is coded by other elements like modality signs or nonmanuals expressing modality, it is easier to separate all this extra information from the concepts of interrogativity and irreality. In the case that no further modality element is available that conveys this extra information, the analyst has to be aware that the concepts of interrogativity and irreality may also signify this extra information. Even so, if there are means for coding modality, the concept of interrogativity in an 'embedded polar interrogative construction' may convey a modality aspect which is closely interrelated with the concepts of interrogativity and irreality. One aspect hereunto is 'potentiality' that - at least to a certain extent - goes together with interrogativity and irreality being expressed in such a context. Probably this aspect is not perceived as being relevant for the concept of interrogativity in direct communication, when a person directly asks somebody about something unknown and in the regular way the question is answered. In interrogatives which are addressed to the person him/herself the absence of an answer 
and consequently the maintenance of the lack of knowledge may provide the interrogative construction with a feeling of potentiality, uncertainty, for instance.

In the following subchapters the three markers that provide the construction with interrogativity/irreality are described - first the 'head forward marker' (abbreviated as hfmarker) which is obligatory, subsequently the marker 'brow raise' which optionally cooccurs with the hf-marker, but which has a special interplay with the hf-marker, and finally the question element 'ob' ('whether') which occurs in half of the present interrogative constructions in the data and which has an obligatory status when a modality expression is not preceding, following or co-occurring.

\subsection{The marker 'head forward'}

\section{The hf-marker}

In the corpus - composed of short stories in which the informants think about a situation an 'embedded polar interrogative' was identified 37 times. In 35 cases $^{115}$ the annotators observed that the head was positioned forward covering some lexical elements. This positioning forward of the head was perceived and identified as a language-relevant, distinctive element, labeled in the present thesis as 'head forward marker' ('hf-marker'). The annotators explained that due to that marker they are certain that the signer is asking himself in a reflective and interrogative way whether a situation will occur or not. Thus, both the self-addressed interrogativity as well as the questionability of the proposition is implied in the present head marker. In this sense, the head marker can be interpreted as an 'interrogative marker' as well as 'irrealis marker'. This is due to the fact that on the one hand, the marker is characterized as a means of expressing a self-addressed thought in an interrogative way; on the other hand, the marker refers to the questionability of a proposition, expressed within a train of thought. Thus, the marker does not possess the same interrogative function as those markers occurring in direct questions, i.e. 'asking

\footnotetext{
${ }^{115}$ Just in two instances the annotators were indecisive whether an hf-marker is present or not. In one of the two cases the signer tilts the head sideward instead of forward. The function of the head marker was interpreted similar to that of the hf-marker. Furthermore, the annotators supposed that the lexical sign HOFFEN (HOPE), having head tilting as component, influences the implementation of the head marker.
} 
somebody for something'. In addition, the marker is also used in non-interrogative constructions like in 'hope-that-constructions'.

In Figure $4.6^{116}$, the positioning forward of the head is depicted:
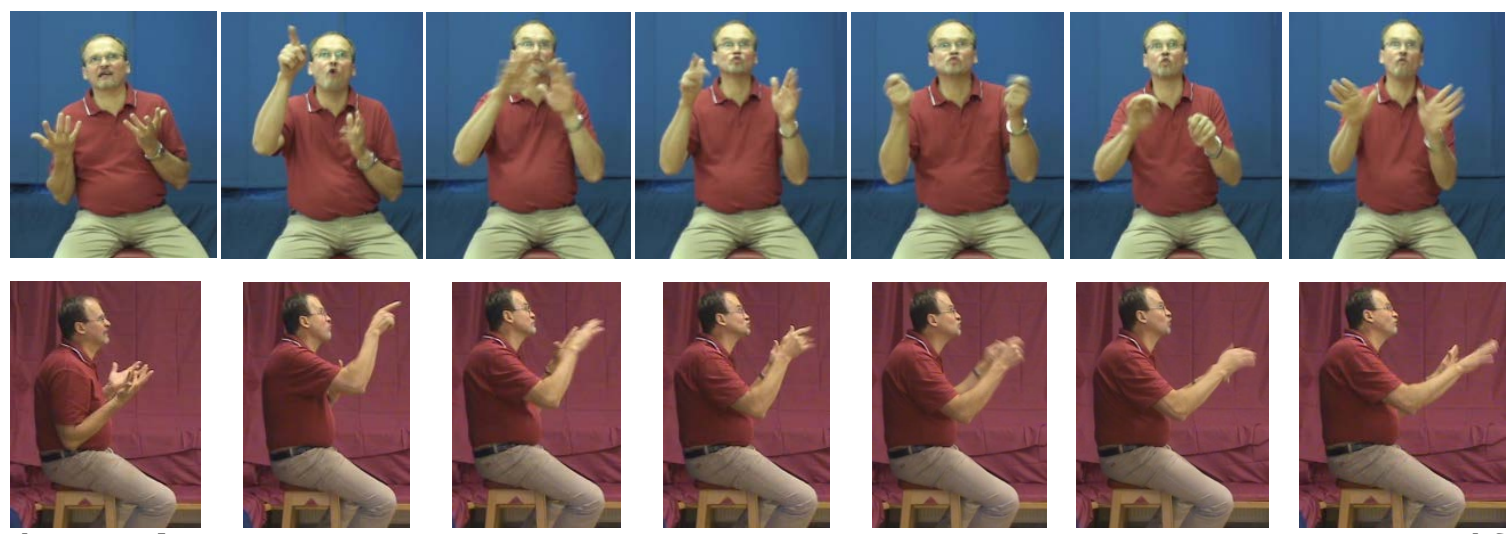

h-neutral

PU

IX-oben/ob

IX-up/whether
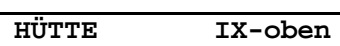

IX-up

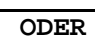

OR

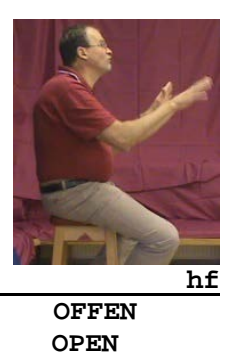

Ich weiß nicht / Ich bin mir unsicher, ob die Hütte offen oder geschlossen ist.

I don't know / I am uncertain whether the hut is open or closed.

(M001_091,1183_m_thoughts_ex05a_01.47-01.57)

Figure 4.6 Illustration of positioning forward of the head during the requested part

\section{Covering/spreading of the hf-marker}

In all 35 cases the head marker covers the part of the construction being questionned. In most cases the entire embedded clause, constituting the element/s of reference, question and request, is covered by the embedded polar interrogative marker head forward. In some instances the head marker spreads over the preceding or following expressions of uncertainty, doubt or hope, respectively. In three cases the hf-marker co-occurs with the entire train of thought, i.e. the following non-interrogative part which is expressed with the interrogative one within one train of thought.

As illustrated in Figure 4.7, the following possible covering/spreading of the hf-marker with regard to the lexemes is present in the corpus ${ }^{117}$ :

\footnotetext{
116 The first picture sequence displays the frontal view, the second picture sequence the side view.

117 This is a prototypical arrangement of lexical elements which are covered by the hf-marker. Other different arrangements of the lexical elements are present in the corpus too.
} 


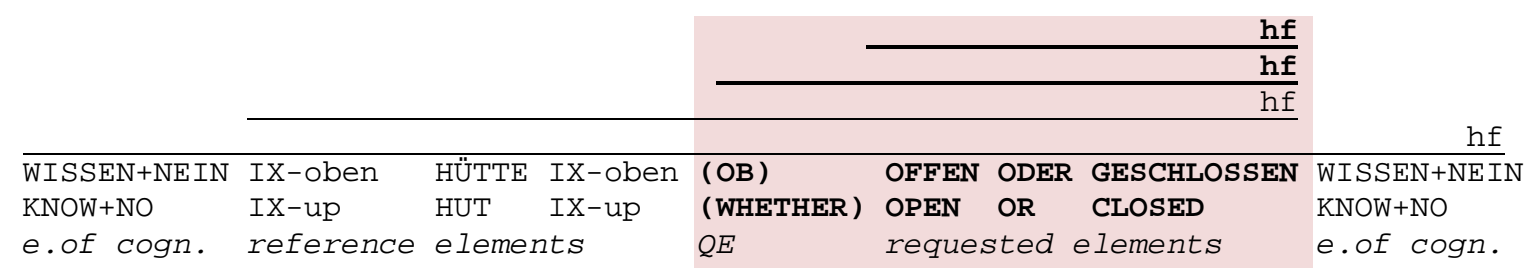

Figure 4.7 Prototypical arrangement of the lexical elements covered by the hf-marker ${ }^{118}$

As depicted, the hf-marker may cover the expression of cognition which precedes and/or follows the requested part, the reference elements (the reference object and/or the pointing sign referring to the reference object or to the 'hypothetical space'), the question element (the sign OB (WHETHER) or the mouthing 'ob' ('whether')) and the requested elements. But, in all constructions the question sign, if present and the requested elements are covered by the hf-marker (color-coded rose).

\section{a) The hf-marker only covers the embedded clause or part of it}

In several cases of the embedded polar interrogatives just the requested part and the question sign 'ob' ('whether') - if present - are covered by the hf-marker while the main clause including the expressions of cognition, sometimes also the signs of reference do not receive the hf-marker. If the reference indicator of the hypothetical space (IX-up) precedes the embedded clause, it is sometimes covered by the hf-marker, sometimes not. Head forward covering the embedded clause is shown in (58).

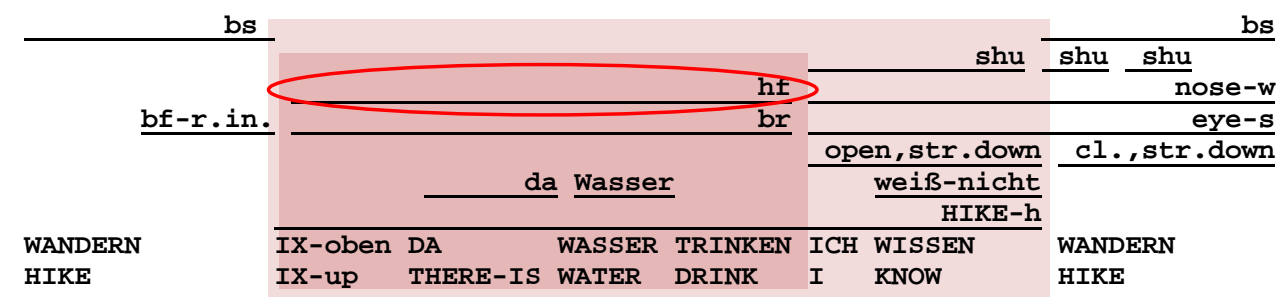

Ich bin am Wandern und weiß nicht, ob ich wasser zum Trinken bekomme.

While I am hiking I don't know whether I'll get water to drink.

(F001_92,1184_m_thoughts_ex06_01.34-01.43)

$\overline{118}$ Abbreviations: e. of cogn. - element of cognition; QE - question element (sign or mouthing); 
While hiking the signer wonders whether she will get some water (to drink in the hut). In addition, she expresses her unawareness of the situation. The signs IX-up THERE-IS WATER DRINK which constitute the embedded clause (color-coded dark rose) are covered by head forward (encircled red). I KNOW accompanied by the mouthing 'weißnicht' ('don't know') and a shoulder shrug do not receive the hf-marker (main and embedded clause are color-coded bright rose).

\section{b) The hf-marker covers more than only the requested elements and the question element}

In several cases other parts beside the requested elements and the question element are covered by the hf-marker too. The following example (59) illustrates these possibility and shows that further components may co-occur with the construction like the nonmanual mouth action 'closed, stretched-down' and holding the non-dominant hand, respectively.

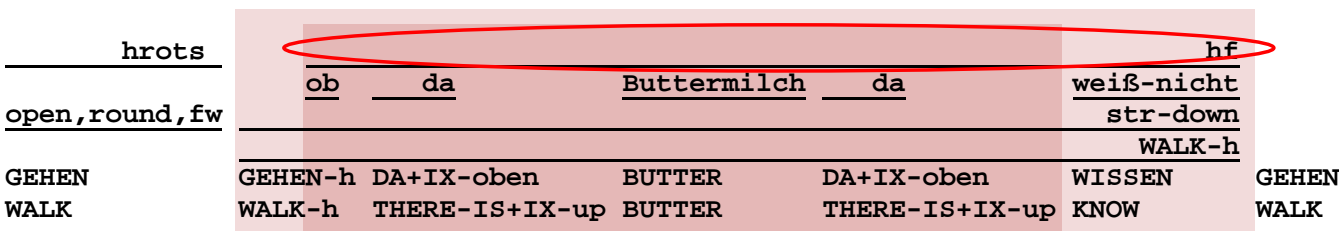

Während ich gehe frage ich mich, ob ich wasser zum Trinken bekommen werde.

While I am walking I wonder whether $I^{\prime} I l$ get water to drink.

(F003_109_m_thoughts_ex02_02.16-02.27)

In (59), the mouth action 'stretched down' (annotated as 'str-down'), meaning that somebody is not certain about a situation about which s/he is thinking, precedes the train of thought, but is maintained during the thoughts (color-coded bright rose). These thoughts start with the mouthing 'ob' ('whether') ${ }^{119}$ and are followed by the lexical signs THEREis+IX-up BUTTER THERE-IS together with the mouthing 'da Buttermilch da' ('there buttermilk there'). These signs constitute the embedded clause (color-coded dark rose). Finally, the sign KNOW together with the mouthing 'weiß-nicht' ('don’t know'), wrinkled nose and squinted eyes - all elements which are perceived as language-relevant, distinctive ones - are produced. The interrogative construction concludes with a blink. The hf-marker

${ }^{119}$ During the mouth action 'closed, stretched-down' and the mouthing 'ob' the hands are maintained in the walking position. 
starts with the mouthing 'ob' ('whether') and co-occurs with all the afterwards listed elements (encircled red). Positioning the head forward is described by the different annotators as 'if the signer is asking herself whether she will get buttermilk in the hut or whether not'. Interestingly, the head is perceived as one constant marker, even though the head is slightly moved forward and downward three times, having its perceived culmination on the signs THERE-IS+IX-there, THERE-IS and KNOW. The annotators added that the slightly forward movement produced three times just indicated to them that the particular lexical elements of the different articulators belong together, resulting in the chunks 'whether THERE-IS+IX-there', 'butter+MILK THERE-IS', 'KNOW-not'. 120

Concerning the non-dominant hand, it has to be noticed that the sign HIKE starts twohanded, afterwards the hand form and hand position of the sign is held during the entire utterance and co-occurs with the mouth action 'closed, stretched-down'. On this matter, the annotators explained that it becomes obvious for them that the informant is expressing her thoughts while keeping on walking.

An interpretation why the hf-marker may cover the entire construction - including the predicate of cognition, emotional state or attention - is the presence of the phonological process of 'assimilation', both progressive assimilation with regard to the following elements and regressive assimilation with regard to the preceding elements. It may be interpreted that the preceding/following head element, which is a neutral one in this example, is changed to head forward. Anyway, the illustrative examples show that the marker head forward can spread on the preceding/following sign(s) of cognition.

From a syntactic perspective the present construction can be identified as an 'embedded' one according to the following formative findings:

- First, the embedded clause is characterized by a beginning marker or domain marker indicating the hypothetical dimension of the proposition, the interrogative marker(s)

\footnotetext{
${ }^{120}$ Actually, the thesis writer is conducting a project on segmentation of ÖGS-texts. In a pilot study the annotators segmented some texts referring to these forward movements of the head and listed, among others, these movements as for them relevant segmentation cues.
} 
and a predicate (phrase). Moreover, all possible syntactic positions which a 'wellformed’ ÖGS interrogative clause requires are filled.

- Second, the syntactic cohesion of the part of the construction that is put into question is marked by the obligatory marker head forward and frequently by the other interrogative markers too. In addition, the markers (IX-up, gaze up) and the displacement of the signs (located in a higher signing space during formulating the hypothetical thoughts) show cohesion of the construction.

- Third, the modality expression which precedes or follows the interrogative clause, can be covered by the interrogative marker head forward too. In some instances also the hypothetical space marker 'gaze up' co-occurs with the modality expression. The possible co-occurrence of both markers with the modality expression is a further hint which shows that the embedded interrogative clause is linked with that modality expression, because neither an interrogative interpretation is read into the modality predicate, nor a hypothetical statement is implied in the modality predicate. Consequently, the expression of cognition/emotion/attention comprises a core argument in the way of an interrogative complement clause.

Also, non-manual modality expression may co-occur with the entire interrogative construction. This possible covering of the diverse formatives on the entire construction shows that even from a syntactic point of view the proposition is 'embedded' in the predicate phrase of the modality expression.

- Fourth, frequently the form and performance location of the non-dominant hand, displaying an ongoing activity, is held while producing an embedded interrogative clause. Thus, the entire interrogative clause is produced with the dominant hand, again showing a kind of cohesion.

- Fourth, the question element 'ob' ('whether') only can occur in these self-addressed interrogatives. Otherwise the direct polar question marker chin down would be present. Consequently, positioning the head forward implies that the signer is evaluating or judging about his/her degree of knowledge or his/her degree of confidence about a situation. Thus, the interrogative element 'ob' ('whether') has a complementizer 
function in the sense that the interrogative clause becomes a complement to the predicate (phrase) and consequently to the modality predicate that is complemented by what is not known or what is insecure.

\section{Intensity ${ }^{121}$ in production of the hf-marker}

The data shows that the hf-marker is perceived to be performed with different force. Consequently, the annotators distinguished between the markers 'head forward' and 'head forward large' (abbreviated as 'hf-large') In all these cases the intensified production is determined by comparing it with a more regular production of the hf-marker. Two different reasons for producing the forward movement of the head in an intensified way become obvious in the present data:

- First, a part of the embedded interrogative is covered by an intensified hf-marker. This part is that one which conveys the requested lexical items (see example (60)).

- Second, a modality element which precedes, follows, or comes in between the embedded interrogative clause, is accentuated by an intensified forward movement of the head, functioning to emphasize this element (see example (61)).

In addition, it should be mentioned that the annotators consistently note that the hf-marker used as interrogative/irrealis marker is perceived as a 'position marker' which has its culmination at the end of the production. This fact may be due to the process of final lengthening (cf. 3.1.3.4) and/or the fact that the predicate is in most cases in the end position in the interrogative constructions and/or further reasons are responsible for this

\footnotetext{
${ }^{121}$ In this thesis the term 'intensification' is used in the case that the annotators perceive an intensified way of production of the particular manual or nonmanul component. Thus, in the present thesis the term refers to the form of an element.

The term 'prominence' (or 'prominent') and the term 'salience' (or 'salient') are used when one or more elements in reference to other elements are produced in a special way (e.g. in an intensified way) or get extra features (e.g. a tone in SpLs or an additional non-manual component in SLs). Thus, on the formative side something is going on which draws the attention to one or more elements.

The term 'stress' is applied in the same way as 'prominence' or 'salience', that is, 'stress marking' refers to the phonological form, but it is primarily applied on the prosodic level (cf., among others, Wilbur 1999). The term 'emphasis' is used when giving one or more elements a special importance concerning the entire content of an utterance. So, it displays the function of the prominent/salient/stressed formative(s), e.g. doubling a wh-sign is used to express 'emphasis' in SLs (cf. Wilbur 2006, Petronio 1993).
} 
perceived culmination. As this aspect has not been the main interest of the present thesis, further investigations are required.

In the following, the two possible occurrences of the hf-large-marker are illustrated:

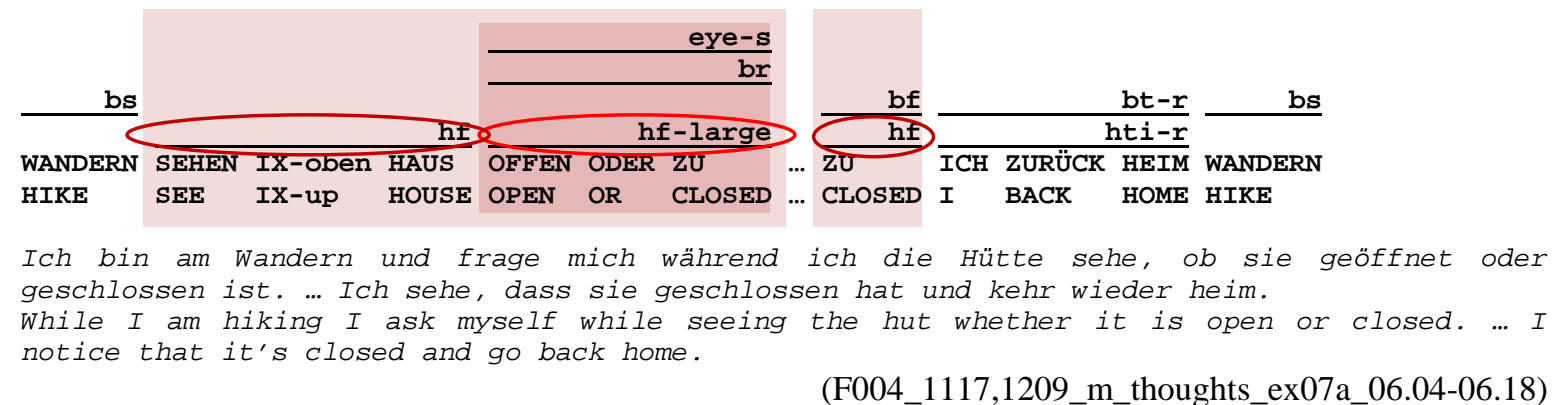

In (60), the signs SEE IX-up HOUSE (color-coded bright rose) are marked by positioning the head forward (encircled dark red). The part of the train of thought that is in focus and contains the elements which are put into question (i.e. OPEN OR CLOSED; color-coded dark rose) are covered by an intensified positioning forward of the head (encircled red). This part is accompanied by other markers, namely squinted eyes and raised brows.

The difference in positioning the head forward is perceived as hf and hf-large markers ${ }^{122}$. The difference in production or perception for all instances in the corpus, exemplified by (60), is analyzed as follows: The part of the interrogative construction conveying the actual requested elements obligatorily requires the hf-marker. The remaining part of the interrogative construction optionally receives the hf-marker. If the non-obligatory part is already covered by the hf-marker, there is a strong tendency that the hf-marker of the obligatorily marked part is intensified. The explanation is based on the annotators' feedback indicating that the intensified production makes obvious to them which part of the interrogative construction is in focus. It has to be added that the actual requested

\footnotetext{
${ }^{122}$ From a prosodic point of view, it can be stated that highly probably two prosodic units are obvious. The annotators indicate that due to the different intensity in production the particular lexical items are grouped together and consequently, the segmentation boundaries are located before and after the hf-marker produced in a regular way and before/after the hf-marker produced in an intensified way. This recognition is based on a pilot investigation about segmentation of ÖGS-texts (cf. 2.1.2).
} 
elements of the embedded interrogative can additionally be marked by raised brows (cf. 4.3.1.2.2) instead of positioning the head forward in an intensified way.

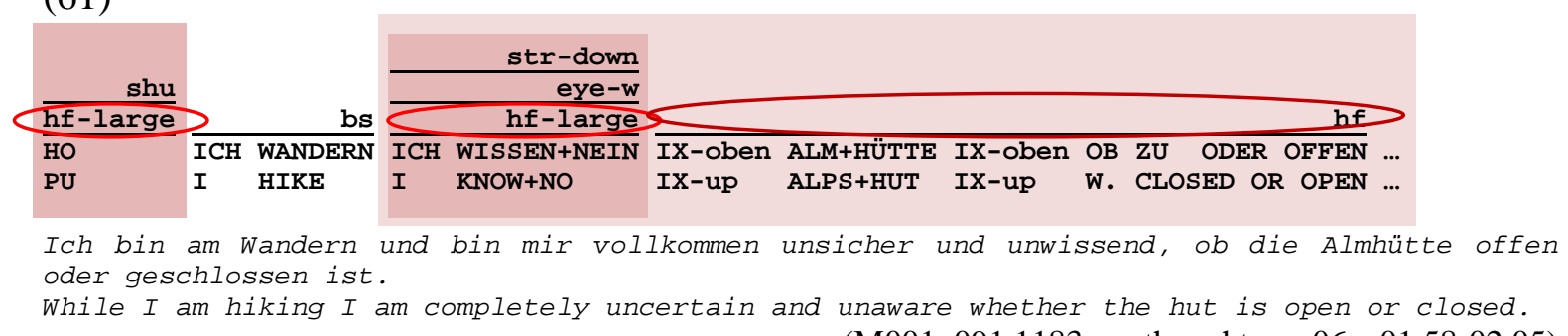

(M001_091,1183_m_thoughts_ex06a_01.58-02.05)

In (61), different movements or positions of the head along the sagittal axis, parallel to the frontal plane are obvious. The annotators identify three distinctive, language-relevant markers: two stronger head forward movements (abbreviated as hf-large; encircled red) of which one is produced in the beginning of the short story and co-occurs with PALM-UP (PU) and a shoulder shrug and one that co-occurs with the sign KNOW+NO, one head forward positioning (abbreviated as hf; encircled dark red) that covers a part of the line of thought and one head backward positioning (abbreviated as hb), which is not illustrated in (61), that covers the remaining line of thought (cf. 4.4).

According to the feedback of the annotators, the two intensified head forward movements function to accentuate the modality expression. Positioning the head forward in a regular way is suggested to express a kind of question which has two options and which the signer asks himself/herself.

\section{Repeated occurrence of the hf-marker}

In seven instances of interrogative constructions the hf-marker is performed twice in the short story. The particular units that are accompanied by the hf-marker occur successively without a pause, in series with a pause in between, or with further lexical elements that occur in between. 
The following example (62) demonstrates the presence of two successively occurring hfmarkers. An intervening pause is seen during the reference sign, which indicates the starting point for the following question sign ${ }^{123}$.

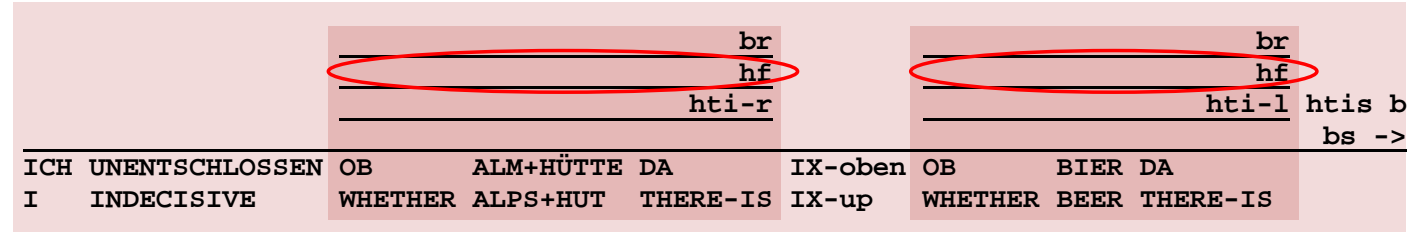

Ich bin unentschlossen, ob es etwas auf der Almhütte gibt, vor allem ob es ein Bier dort gibt.

I am indecisive whether they have something in the hut, especially whether they'll have some beer.

(M002_114,126_m_thoughts_ex04a_05.05-05.18)

In (62), the annotators identify two parts of the interrogative constructions that are covered by the hf-marker (each encircled red). The hf-marker is produced in series with a short pause in between during which the signer points upward. In addition, the head is tilted rightward during the first unit and leftward during the second unit (cf. 6.4.4 and 7.4.6). These head tilts are followed by fast, alternating head tilts sideward (abbreviated as htis) which are interpreted as the lexical output 'maybe/possible'. Twice the particular requested elements are additionally covered by the marker brows raised ${ }^{124}$. The entire embedded interrogative construction is concluded by a blink.

In the following example (63) the arrangement of the lexical items ${ }^{125}$ is more or less reversed to (62). Once again the annotators indicate two successively produced embedded interrogative clauses (color-coded dark rose), covered by the markers head forward (each

\footnotetext{
${ }^{123}$ Concerning movements along the frontal axis, the preceding sign WANDERN (HIKE) (before the interrogative construction) is accompanied by 'weight shifts sideward'. The marker 'body sways' starts with the interrogative construction and also accompanies the following signs GLAUBEN-NEIN (BELIEVE-NO) which are covered by 'headshakes'.

${ }^{124}$ The perceived culmination of the br-marker is on the particular first signs of the two units, i.e. the sign WHETHER. The perceived culmination of the hf-marker is in the first unit on the last sign THERE-IS, in the second unit on the first sign WHETHER and slightly on the last sign THERE-IS.

125 The signs which are covered by the hf-marker are performed in a higher position in the signing space. Thus, this is a further factor which gives the construction (structural) cohesion.
} 
encircled red) and raised brows. This time no lexical element intervenes between the two clauses $^{126}$.

(63) ${ }^{127}$

\begin{tabular}{|c|c|c|c|c|c|c|c|}
\hline & & & br & & br & & \\
\hline & & & hf & & hf & & $\frac{\text { htis }}{\text { bs }}$ \\
\hline überlegen & $\mathbf{X X X}$ & trinken & Bier ob & & Almhütte da & & cl.str-down \\
\hline ÜBERLEGEN & $\overline{\mathrm{ICH}}$ & TRINKEN & BIER & IX-oben & ALM+HÜTTE & IX-oben & UNSICHER \\
\hline THINK & I & DRINK & BEER & IX-up & ALPS+HUT & IX-up & INSECURE \\
\hline
\end{tabular}

Ich überlege, ob ich Bier trinken werde bzw. ob es überhaupt etwas auf der Almhütte gibt. Jedenfalls bin ich mir unsicher.

I wonder whether I'll drink a beer or whether something will be available in the hut at all. Anyway, I am uncertain.

(M002_114,126_m_thoughts_ex05a_05.25-05.38)

Both examples (62) and (63) clearly show the interplay of syntax and prosody in SLs, at least in ÖGS. The annotators clearly segment the positioning forward of the head in two prosodic units, however the syntactic interpretation of the sentence meaning by the annotators is that the signer is in doubt whether he will get something to drink in the hut, especially whether he will get beer. Consequently, in both interrogative constructions both trains of thoughts formulated in an interrogative/irreal way refer to the preceding and following modality elements (i.e. THINK, INSECURE, head tilts conveying the meaning of possibility/eventuality).

\section{Hf-marker and other head markers}

In the corpus in which the informants express their thoughts, in various constructions the articulator head possesses more language-relevant, distinctive movements which are produced equally. Thus, the articulator head may convey more functions simultaneously.

In ten instances the head was not only positioned forward, but tilted sideward (in seven cases), upward (twice, resulting in positioning the chin upward) and downward (once, resulting in positioning the chin down). In these ten cases, the annotators identified the hfmarker and the additional marker (hti-l/r, cu, cd), both conveying different functions. Only

\footnotetext{
${ }^{126}$ The body sways (bs) are performed with slow movements which have short stops at the end points and start with the sign THINK and end with the sign INSECURE (cf. 7.4.4). These body sways precede other body sways which are performed in a uniform alpha-movement and which co-occur with the sign HIKE.

${ }^{127}$ IX-up refers to the hypothetical space - i.e. the space of thoughts - and/or to a location somewhere up. A clear identification for a pointing sign used to refer to the hypothetical space can only be made if the requested matter does not have its absolute location in the upper space.
} 
in two cases, the annotators could not decide whether an hf-marker or a cu-marker or both together occurred as well as whether the head marker expresses an interrogative construction or just a reflection construction or both together. A possible co-occurrence of the embedded polar interrogative markers and other head markers is shown in (64).

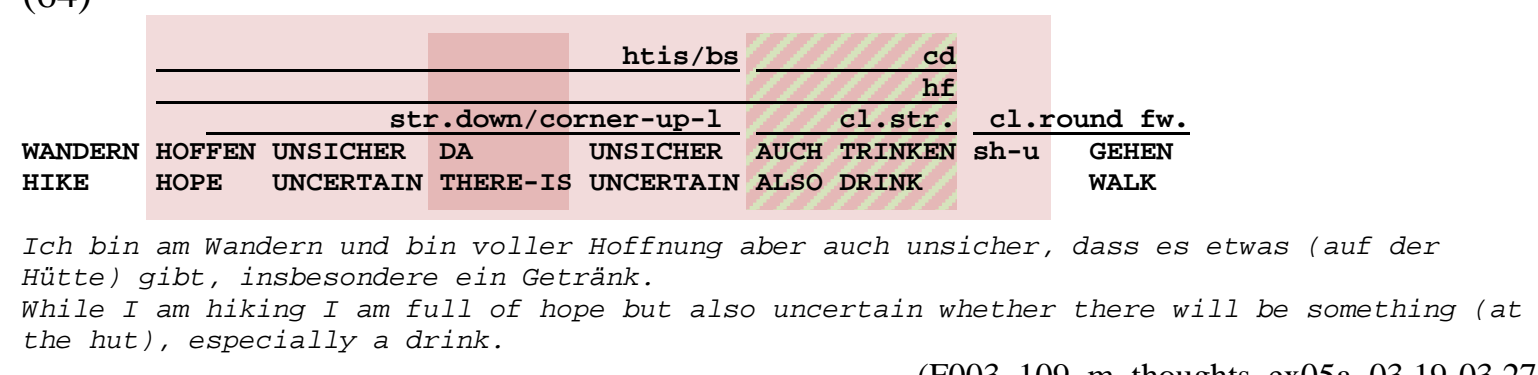

(F003_109_m_thoughts_ex05a_03.19-03.27)

In (64), a signer expresses her hope that she will get something in the hut, especially a drink. During the whole train of thought the signer's head is perceived as being positioned forward except form the following shoulder shrug (color-coded bright rose). In addition, head tilts (htis) together with body sways (bs) co-occur with the first part of the interrogative construction. These express possibility in the sense of maybe yes or maybe not. The second part of the interrogative construction is covered by the remaining hfmarker as well as by the marker chin down (cd) (color-coded dark rose and green). The annotators explain that the marker chin down function as question marker. In their perception, the signer adds that she is asking herself whether she will get something to drink, too. Example (64) makes clear that if already a construction is covered by the interrogative/irrealis marker and if the signer wants to express an additional interrogative thought during the self-addressed train of thoughts, the interrogative marker which occurs in direct polar questions is additionally used.

Also, the data shows that the interrogative marker head forward can change its form if the preceding modality sign has head tilt sideward as non-manual component. In such a case, 
the data show that the annotators identify the marker head tilt sideward ${ }^{128}$ to which they allocate an interrogative function as shown in (65).

$(65)^{129}$

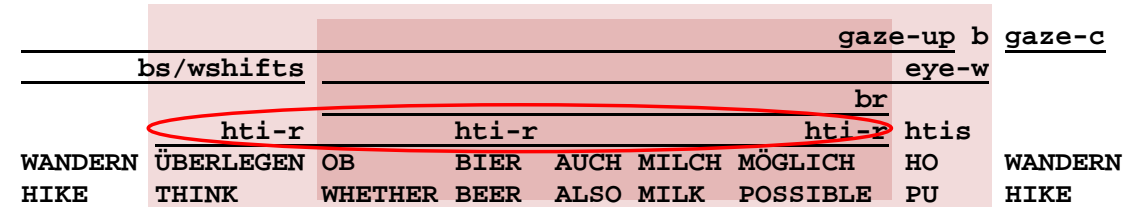

Ich bin am Wandern und überlege, ob es Bier bzw. auch Milch gibt. Es ist möglich.

While I am hiking I wonder whether there will be beer or even milk. It is possible.

(M002_114,126_m_thoughts_ex03a_03.47-03.57)

In (65), an embedded polar interrogative (color-coded bright rose) is present. The annotator perceives a language-relevant, distinctive head tilting sideward (rightward) positioning which is interpreted as an interrogative marker (encircled red). This covers the preceding expression of cognition (THINK) and the embedded interrogative clause (color-coded dark rose). The following expression of possibility (head tilts) is not covered by the interrogative marker. Tilting the head sideward is interpreted as an interrogative/irrealis marker. All annotators add that in their view tilting the head results from the sign THINK and the individual style of the signer who prefers head tilting due to his body height. As the annotators clearly identify an interrogative/irrealis function in the construction, it may be supposed that the head tilting dominates and thus, the forward positioning of the head is not perceived anymore.

In addition, the trains of thoughts - without the preceding modality expression - are covered by raised brows. The annotators describe two clear upward movements (culminations) of the br-marker, one in the beginning on the sign WHETHER and one on the sign ALSO. This additional marker is described in detail in the following sub-chapter. Moreover, the annotators identify that widely opened eyes (a language-relevant, distinctive element) co-occur with the second part of the construction. The entire interrogative construction is terminated by a blink.

\footnotetext{
128 Additionally, the annotators indicate that a slightly forward movement goes together with the tilting movement. In the side view of the video a forward positioning of the head is clearly identifiable.

129 The marker head tilt sideward is perceived as one marker. However, the sideward positioning has three movement excursions. The end points are indicated by each abbreviation (hti-r) in the example (65).
} 


\subsection{The additional marker 'brow raise'}

\section{The br-marker}

In 22 instances out of the 37 embedded polar interrogatives, in addition to the always present interrogative marker head forward, the eye brows are raised. Raising the eye brows (as well as furrowing them or raising the inside of the eye brows) is perceived as a language-relevant, distinctive element by the annotators. The various particular functions of the markers 'brow raise', 'brow furrow' or 'brow furrow and raised inward' have not yet been investigated in ÖGS. But, for raised eye brows covering the present interrogative construction and for which no further function becomes obvious, it can be concluded that they are an additional marker for the embedded polar interrogative. In most examples from the corpus the entire or a larger part of the interrogative construction is covered by the hfmarker while a smaller part - all in all the part of the construction that is put into question - is covered by the br-marker.

\section{Covering of the br-marker}

As illustrated and exemplified in the Figures 4.8, 4.9, and 4.10 ${ }^{130}$, the following possible coverings of the br-marker in embedded polar interrogative constructions are obvious.

\section{a) The br-marker covers one offered option}

As Figure 4.8 shows the requested elements together with the pointing sign for the hypothetical space as well as the question sign can be covered by the br-marker. The requested elements display a polar statement in form that something exists ${ }^{131}$.

\begin{tabular}{lllll} 
& & & \multicolumn{1}{c}{ br } & \\
\cline { 2 - 4 } WISSEN+NEIN & IX-oben OB & TRINKEN DA & WISSEN+NEIN \\
KNOW+NO & IX-up & W. & DRINK THERE-IS KNOW+NO \\
e.of cogn. & ref.e. & QE & requested e. & e.of cogn.
\end{tabular}

Figure 4.8 Br-marker covers one offered option

\footnotetext{
${ }^{130}$ The present illustrations are prototypical arrangements of the lexical elements which can be covered by the br-marker. The obligatory co-occurrence of the hf-marker is omitted in the illustrations.

Abbreviations of Figures 4.8 to 4.10: e.of. cogn. - element of cognition; ref.e. - reference element/s, QE question element (sign or mouthing), requested e. - requested elements, ev. e. - eventuality expression, conj. - conjunction, altern. - alternative;

${ }^{131}$ In one instance a signer adds the second option (which is otherwise not signed but interpreted as an implied option) - i.e. 'non-existing'. Both options receive the br-marker.
} 
This pattern is shown in example (66), in which the two non-manual interrogative markers (raised brows and head forward) are produced for different durations. This instance is used to demonstrate that the hf-marker can cover the entire interrogative construction, including the preceding and/or following modality expression, while the br-marker just covers the embedded clause.

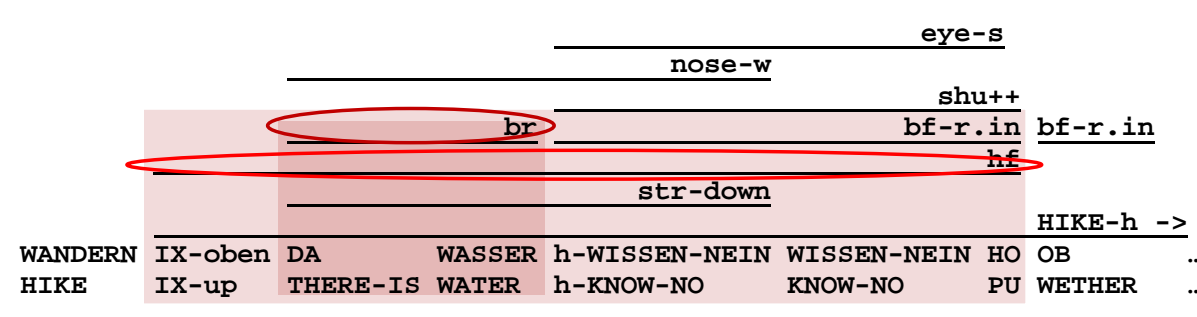

Während ich wandere frage ich mich unsicher, ob es wasser dort gibt. ...

While I am hiking I wonder with uncertainty whether there will be water. ...

(F001_92,1184_m_thoughts_ex10_02.27-02.37)

In (66), the entire interrogative construction (color-coded bright rose) is covered by positioning the head forward (encircled red), including the pointing $\operatorname{sign}^{132}$, the requested matter of subject, and the expressions of uncertainty. The part of the construction that contains the signs that are 'put into question' and that forms the nucleus of the interrogative construction (color-coded dark rose) gets the marker brow raise (encircled dark red), while the part of the construction expressing the uncertainty about this proposition receive the marker 'furrowed brows which are raised inward'. The postpositioned interrogative sign WHETHER is not covered by the hf-marker, however the preceding marker 'brow furrow and raised inward' is maintained and performed in a less intensive way.

\section{b) The br-marker covers two options offered in form of two sequential predicates}

Figure 4.9 and example (67) demonstrate that the br-marker also can cover the two options offered in form of two sequential predicates together with the question element. In the corpus the pointing sign is in some cases covered by the br-marker, in others not.

\footnotetext{
${ }^{132}$ In this example the pointing sign refers to the topical space, i.e. the hut in the Alps, and/or at the hypothetical space, i.e. the space of thoughts (cf. 6.4.3).
} 
\begin{tabular}{llllll} 
& & \multicolumn{2}{c}{ br } \\
WISSEN+NEIN IX-oben HÜTTE & $\bar{I} X-\mathrm{ob} e \bar{n}$ & & (OB) & OFFEN ODER GESCHLOSSEN & WISSEN+NEIN \\
KNOW+NO & IX-up HUT IX-up & (WHETHER) OPEN OR CLOSED & KNOW+NO \\
e.of cogn. & reference elements & QE & requested elements & e.of cogn.
\end{tabular}

Figure 4.9 Br-marker covers two offered options

In (67), it is possible to see the options of occurrence of the br-marker as well as the context of occurrence of the br-marker compared with the hf-large-marker. Moreover, in this example all three possible interrogative indicators occur - i.e. the markers head forward, raised brows and the question element 'ob' ('whether').

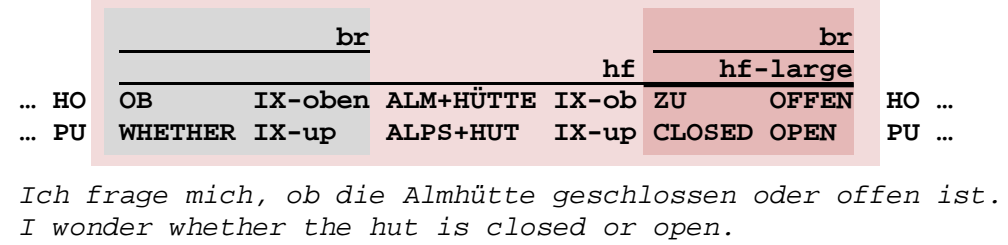

(M001_091,1183_m_thoughts_ex07a_02.11-02.24)

In (67), the entire interrogative construction (color-coded bright rose) is covered by the marker head forward. No expression of cognition precedes, follows or co-occurs with the requested part. If a person is 'asking him/herself something', in none of the examples the sign FRAGEN (ASK) is used, only the interrogative indicators are present. In these cases always the question element 'ob' ('whether') is present.

In (67), the requested information (color-coded dark rose) is covered by an intensified forward positioning of the head. Exactly the same part of the construction is covered by raised brows too. The data shows that in those embedded polar interrogatives in which the br-marker occurs, exactly that part of the construction which conveys the requested elements is covered by raised brows. Consequently, both the br-marker and the hf-largemarker can be used for covering that part of construction that is put into question while the hf-marker can cover the entire construction including the expressions of perception, cognition or emotional state.

In (67) WHETHER and IX-up (indicating the hypothetical space as well as the topographical space) are covered by raised brows too (color-coded grey). It is not clear if 
this first raising of the brows functions as emphasizing the beginning of the interrogative, or if both parts of the interrogative which are additionally covered by the brow marker display the real nucleus of the interrogative (which is WHETHER IX-up OPEN CLOSED), or if another reason causes this.

\section{c) The br-marker covers two alternative options}

As Figure 4.10 and example (68) demonstrate, the br-marker may cover the two options in the form of two sequential predicates together with their argument(s). In addition, the question element and the modality elements VIELLEICHT (MAYBE) ${ }^{133}$ or MÖGLICH (POSSIBLE) are covered by the br-marker, respectively. However, elements of cognition, emotional state or perception which may express lack of knowledge like WISSEN+NEIN (KNOW+NO), insecurity like UNSICHER (INSECURE), or indecisiveness like UNENTSCHLOSSEN (INDECISIVE) are not covered by the br-marker.

\begin{tabular}{llllll} 
& & & \multicolumn{2}{c}{ br } \\
WISSEN+NEIN & \multicolumn{1}{c}{ CB/VIELLEICHT DA } & COLA ODER VIELLEICHT DA & MILCH WISSEN+NEIN \\
KNOW+NO & WHETHER/MAYBE THERE-IS COCA OR MAYBE & THERE-IS MILCH KNOW+NO \\
e. of cogn. & QE/ev.e. & 1.altern. & conj. ev.e. & 2.altern. & e. of cogn.
\end{tabular}

Figure 4.10 Br-marker covers two alternative options

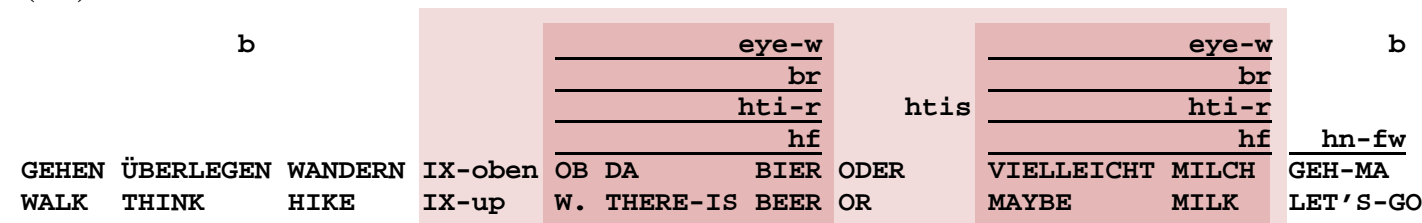

Während ich wandere überlege ich, ob es dort Bier oder vielleicht Milch gibt. Ich schau es mir mal an.

While I am hiking I wonder whether there will be beer or even milk. Let's go and see.

(M002_114,126_m_thoughts_ex02a_03.24-03.37)

In (68), both alternatives (color-coded dark rose) are covered by the marker head forward and raised brows. Interestingly, the particular peaks of the raise of the brows are found in clause-initial position (i.e. with the signs WHETHER and MAYBE). The additional

\footnotetext{
${ }^{133}$ If the sign VIELLEICHT (MAYBE) is used, the question element 'ob' ('whether') is not present. Also, the sign VIELLEICHT (MAYBE) can be found in clause-final position of the embedded clause.
} 
marker widely opened eyes (eye-w) co-occurs with the two non-manual interrogative markers.

Referring to the various eye brow movements an in-depth analysis on their possible occurrences together with their possible function(s) requires a detailed investigation and goes beyond the scope of the present thesis.

In the following subchapter the third interrogative element is described.

\subsection{The marker 'ob' ('whether')}

In half of the identified embedded polar interrogatives a lexical item that is only used in this construction is present, namely the sign OB (WHETHER) and/or the mouthing 'ob' ('whether'). Almost exclusively the lexical item 'ob' follows the expressions of thinking, uncertainty, and doubt and consequently, is found in clause-initial position of the embedded clause. In some exceptional cases the item occurs intermediately or in final position of the construction. It has to be concluded that the element 'ob' is a question sign which signals (in addition to the obligatory hf-marker) that an embedded polar interrogative is present.

The item can be compared to the conjunction 'ob' used in German and probably has its origin (at least the mouthing) from that language. In German the conjunction 'ob' is used as a linking word that connects two clauses. Often an 'ob-sentence' in German corresponds with an 'embedded interrogative clause' such as 'Ich frage mich, ob das der Fall ist.' ('I ask myself whether that's the case.'). An 'ob-sentence' may also follow expressions of question, uncertainty or doubt like 'Ich bin mir unsicher, ob das der Fall ist.' ('I am uncertain whether that's the case.'). These constructions have an independent clause which is augmented by a dependent clause. Both clauses are linked by the conjunction 'ob'.

In most examples of the corpus in which the lexical item 'ob' occurs, the item introduces the embedded clause. In a number of cases the item follows expressions of thinking, uncertainty or doubt and might be interpreted in the same way as the 'ob-conjunction' in German in the sense that it links the first clause, e.g. I KNOW+NO with the second clause WHETHER .... But, in some cases, no utterance with an expression of cognition precedes. 
The various informants sign that s/he is hiking and thinking about a situation in an interrogative way. These trains of thoughts start with the sign and/or mouthing 'ob' and in addition, the head is positioned forward. Consequently, no linking between two constituents is obvious. But in the cases in which no expression of cognition is present, the question element 'ob' is always present. Consequently, it has to be concluded that in these cases the question element is obligatory while in interrogative constructions with a preceding or following expression of cognition it is optional.

Remarkable is a relation between the lexical item 'ob' and the pointing sign IX-up which indicates the hypothetical space (cf. 6.4.3). First, both elements occur in initial position of the part of the construction that is put into question. In 24 cases out of the 37 cases the sign WHETHER, or the pointing sign together with the mouthing 'ob', or just the pointing sign occurs in initial position of the part of the construction that is put into question. In those cases in which these elements are not present the sign(s) (I) HOPE (three times), the sign MAYBE (three times) or the issue about which the signer makes a statement - i.e. the drink which might be there or not (four times) or the state of being open or closed (once) is placed in the initial position.
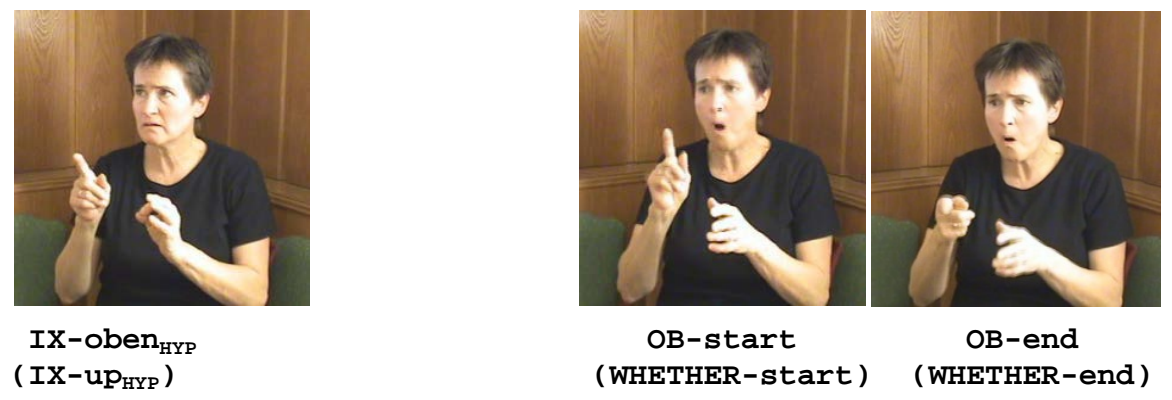

Figure 4.11 Illustration of IX-oben (IX-up) and OB (WHETHER)

Second, the pointing sign and the sign WHETHER have the same IX-hand form (except in one case in which the signer uses a 'B-hand form'), the same place of occurrence in the signing space, and even the same starting position. The pointing signs are always directed towards the upper mostly rightward signing space. Exactly this position is used as starting position for the question sign WHETHER. However, the movement of the wrist goes 
downward, so that the index finger is downward in the end position of the sign. In four instances the annotators could not decide whether the pointing sign or the question sign WHETHER is present.

Third, in the 37 embedded polar interrogatives the lexical item 'ob' occurs in 17 constructions of which 13 have the sign WHETHER together with the mouthing 'ob'. Actually, the sign together with the mouthing occurs 24 times given repetition. In two constructions the sign WHETHER is produced solely with the manual articulator, twice each. In five constructions the mouthing 'ob' is produced alone or together with the pointing sign IX-up. Specifically, the mouthing is produced alone three times and together with the reference sign four times.

Summing up, it has to be concluded that a relationship exists between the question item 'ob' ('whether') and the pointing sign IX-up with respect to the production, the place of occurrence within the construction and the partly simultaneous production of the elements. Also a semantic relationship is implied as both elements can be associated with an irreal/hypothetical proposition.

\subsubsection{The characteristics of the embedded clause}

\subsection{Semantics of alternativity}

The present interrogative constructions have in common that the signers ask themselves about information which they do not have. In contrast to interrogatives in interactive settings, the asker does not get an informative answer from a counterpart. They only have the opportunity to express their thoughts about the potential occurrence of options.

Based on the information and consequently based on the inquiry propositions, the present interrogative constructions, characterized by positioning the head forward, include alternatives. The signers list the alternatives or imply the alternatives. Thus, the main characteristic of the embedded clause is 'alternativity' while direct polar questions can focus on expressing polarity or alternatives. For example, Bolinger (1978) lists several semantic-pragmatic contexts in which polar questions do not imply alternatives. For 
instance, when requesting 'May I see the passport?', when drawing conclusions like 'Is it sunny outside?' (when somebody comes in with an umbrella), or when extending an invitation like 'Do you want a drink?' the (negative) alternative is not really implied. Concluding, in these contexts it would not be appropriate to say 'May I see the passport or not?', 'Is it sunny outside or not?', and 'Do you want a drink or not?'. In several cases the informants formulate a polar question which actually only referred to one alternative. In example (46), for instance, the dialogue partner is joking and asking his counterpart whether he may come. Of course, due to the semantic-pragmatic context 'not coming' is not really intended to be implied. By contrast, in the 'embedded polar interrogatives' alternatives are always implied, regardless of whether all alternatives are mentioned or not. The following possibilities are present:

First, in various examples the signers query themselves about whether something exists. In doing so, an alternative is offered while its negation is implied. Consequently, the signers ask themselves for the truth of the proposition by floating the two options, the second implicit - i.e. (something) 'being-there' or 'not-being-there'. Asking for the truth value may be answered by 'yes, it's there' or 'no, it's not there'. But, in the present examples the signers do not receive an answer, and they can only remain with their considerations. However, in many instances the signers carry on their story and inform the imagined audience whether their expectations are fulfilled or not.

In $(66)^{134}$, for instance, the signer asks herself whether water is available in the hut, signing IX-there THERE-IS WATER. These lexical items are covered by the hf-marker. In another example $^{135}$ a signer asks herself whether she will get something in the hut, signing WHETHER THERE-IS+IX-up, covered by the hf-marker.

\footnotetext{
134 (F001_92,1184_m_thoughts_ex06_01.34-01.43)

135 (F003_109_m_thoughts_ex06_03.39-03.44); Out of the context it is clear that the informant asks herself 'whether she will get something in the hut' in spite of 'whether the hut is there or not'. But, if only the present utterance is present and the remaining part of the story is missing, the second interpretation is possible too.
} 
Second, in several interrogatives the informants offer different options that might occur and consider which one will be true. In all these cases, just two options are opposed, not more. As a result, the number of questioned possibilities is limited. The signed alternatives in the data are either the availability of two opposed items or the occurrence of two opposed states. When the signer queries which of the two items are available the following productions are present in the data: The informant signs the particular items together with the sign of existence which precedes or follows the particular item, or just the items that are possibly available. In addition, the question sign WHETHER, the coordinating conjunction ODER, or further signs expressing the attitude or possibility may precede/intervene/follow both alternatives. This is illustrated in (69) and (70):

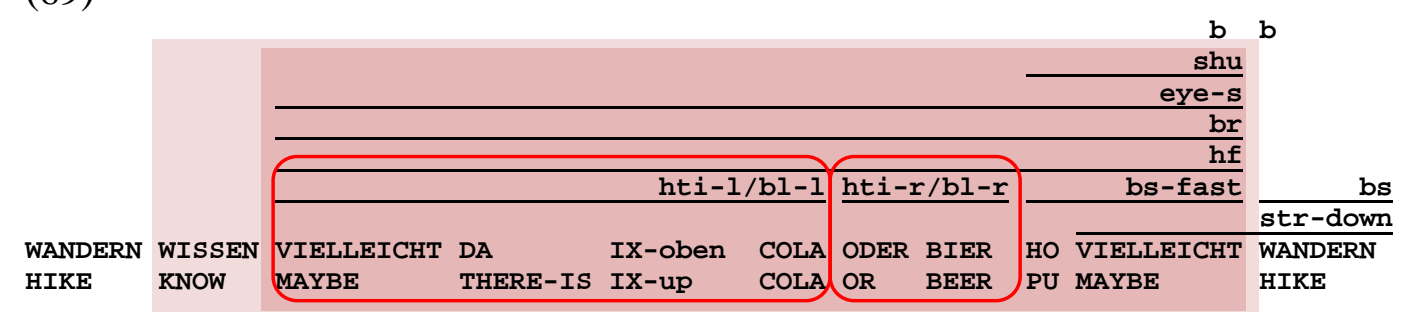

Während ich wandere denke ich, ob/dass es dort in der Hütte vielleicht Cola oder Bier gibt. Ich bin mir unsicher.

While I am hiking I wonder/think whether/that at the hut there might be cola or beer available. I am uncertain.

(F004_1117,1209_m_thoughts_ex06a_05.24-05.37)

In (69), the offered options, indicated by tilting/leaning the head/body to the left/right (encircled red), are two beverages, however two other options are implied in the construction. Following, among others, Biezma \& Rawlins (2012) the choice of possible answers of alternative questions is more than the offered alternatives. Also the choice 'both options' or 'neither of the options' are two further alternatives which are implied in an alternative interrogative. With regard to (69) these two additional alternatives could also be the case. In other words, the options that 'both cola and beer' or 'neither cola nor beer' can be implied in the train of thought.

The second example (70) contains both options of opposed states, i.e. of 'being open' versus 'being closed' (second block color-coded dark rose). The signer queries herself which of the two different states is true. In addition, in the beginning of the train of 
thoughts a further embedded polar interrogative (first block color-coded dark rose) precedes in which one alternative is labeled and the negated alternative is implied.

\begin{tabular}{|c|c|c|c|c|c|c|}
\hline & & & & & & gaze-up \\
\hline \multirow[t]{2}{*}{ str-down } & & str-down & & & hf-strong & $\frac{\text { str-down }}{\text { hf }}$ \\
\hline & $\begin{array}{ll}\text { OB } & \text { DA } \\
\text { WHETHER } & \text { THERE - IS }\end{array}$ & $\begin{array}{l}\text { UNSICHER } \\
\text { INSECURE }\end{array}$ & $\begin{array}{l}\text { ZU } \\
\text { CLOSED }\end{array}$ & $\begin{array}{l}{[\text { Mb. 'oder'] }} \\
\text { [mouth. 'or'] }\end{array}$ & $\begin{array}{l}\text { OFFEN } \\
\text { OPEN }\end{array}$ & $\begin{array}{l}\text { UNSICHER } \\
\text { INSECURE }\end{array}$ \\
\hline
\end{tabular}

Ich bin mir unsicher, ob es (eine Hütte oder ein Getränk in der Hütte) da ist, und ob es (die Hütte) geschlossen oder geöffnet ist.

I am unsure whether it (a hut or a drink in the hut) is there and whether it (the hut) is closed or open.

(F003_109_m_thoughts_ex12_05.15-05.22)

In (70), the second embedded interrogative clause contains two offered options. Compared with (69), only these two alternatives can be taken under consideration as neither 'both' nor 'none' of the options is possible.

In the corpus both possible options are more frequently signed when two lexical items convey a semantically similar but clear contrast. For instance, the options 'being open' opposed to 'being closed' constitute a contrast pairing that occurs quite often in ÖGS and presents a clear contrast. As further data ${ }^{136}$ shows, the contrast of the opposed items is in most cases implemented by a forward vs. backward movement of the head and/or body (see Figure 6.12 in 6.4.4.1). When the two options occur subsequently in an embedded polar interrogative, both options are covered by positioning the head forward while the forward vs. backward movement is not present.

Lexical items like signs for 'existence' or signs for 'performing an activity' are - in the majority of cases - the only option in the interrogative construction (present in the corpus on which these results are based on), although 'non-existence' or 'inactivity' can be expressed by lexical items too. The very reason for only mentioning one option in these cases may be due to the fact that if one pole is expressed the other option is clearly the counterpart. If alternatives are presented, there might be several options, even though in all cases in the corpus of self-addressed thoughts merely two alternatives are opposed.

\footnotetext{
136 This observation is based on a basilectal corpus containing dialogues with the same informants.
} 
Finally, in half of the cases of the embedded polar interrogatives in which both alternatives are offered, the sign ODER (OR) and/or the mouthing 'oder' ('or') occurs between the two options.

Summing up, the embedded polar interrogatives present in the ÖGS data always offer more alternatives. Apart from the possibility that the options 'both' or 'neither of them' can also can be implied (occurs in some cases), most of the interrogatives of this class have in common that two options are offered or implied.

\subsection{Filled syntactic positions}

The next obvious comparison is between the embedded self-addressed polar interrogatives and non-embedded polar interrogatives addressed to a dialogue partner. From this comparison, one aspect immediately leaps to the eye: in embedded self-addressed polar interrogatives the tendency is very high for all possible syntactic positions to be filled. This observation is demonstrated by the following illustration ${ }^{137}$ :

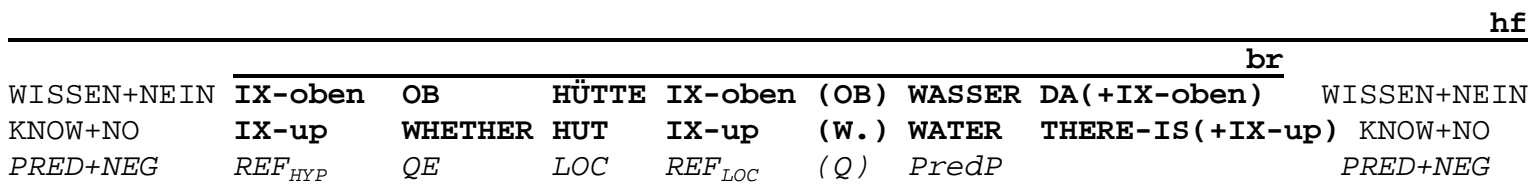

Ich weiß nicht, ob es auf der Hütte Wasser gibt.

I don't know whether there is water in the hut.

Figure 4.12 Lexical elements of the embedded part (or complement clause)

Figure 4.12 illustrates prototypically which lexemes may occur in the complement clause i.e. the embedded interrogative clause - and their syntactic arrangement, as present in the data. These lexemes are first the question element (QE) which directly precedes the requested elements and consequently is in initial position of all trains of thoughts and the complement clause (that follows the expression of cognition and negator). If the pointing

\footnotetext{
${ }^{137}$ Abbreviations in Figure 4.12: PRED - predicate, NEG - negator, QE - question element, LOC - locus (place in the signing space to where the event takes place or where the referent is allocated to), $\mathrm{REF}_{\mathrm{LOC}}-$ locative reference (pointing element which points to the locus where the event takes place or where the referent is located; LOC and $\mathrm{REF}_{\mathrm{LOC}}$ syntactically fall within one phrase), $\mathrm{REF}_{\mathrm{HYP}}$ - hypothetical reference (pointing element which points to the hypothetical space, i.e. the space of thoughts), PredP (predicate phrase);
} 
sign indicating the hypothetical space of thoughts is present, in most cases the question element is in second position, in some cases however it precedes. It may also be found directly preceding the requested elements.

Second, in the illustration the pointing sign(s) may refer to the hypothetical space or to a topographical upper space in the signing space. When it refers to the space of thoughts it is labeled 'hypothetical index' ( $\mathrm{IX}_{\mathrm{HYP}}$ ) which is found in initial position of the train of thought, that is, $\mathrm{IX}_{\mathrm{HYP}}$ occurs in clause-initial position of the complement clause. $\mathrm{IX}_{\mathrm{HYP}}$ may also start in the beginning of the thoughts and cover the entire train of thoughts. When the pointing sign refers to a topographical space (here: the hut up there in the Alps) or to a location where the referent is located, the element is labeled 'locative index' (IX $\mathrm{LOC}_{\mathrm{LO}}$ ). $\mathrm{IX}_{\mathrm{LOC}}$ can precede or/and follow the expression of locus (LOC). In example (61) ${ }^{138}$ in which a statement about the hut is made ('whether the hut is open or closed'), from a syntactic point of view the 'hut' displays the locus as well as functioning as the subject about which a statement is made. In both instances, the locative index precedes or follows the locus (LOC) or the subject including a locus ( $\left.\mathrm{SUBJ}_{\mathrm{LOC}}\right)$.

Finally, the requested elements - in Figure 4.12 consisting of the existential expression and the object which does or does not exist - constitute a predicate phrase (PredP). The sign THERE-IS can be produced in two different ways, first with a B-hand form with a bend at the joint between the back of the hand and the fingers, and second, with an 'open-8, 139 hand configuration. This second existential is glossed by many annotators as THEREIS+IX-there. This shows that in the present corpus the second existential sign can include a locative reference and consequently displays a 'locative predicate'.

This train of thoughts may precede or follow the elements of cognition, emotional state or perception. It is covered by the hf-marker and may additionally be covered by the brmarker (cf. 4.3.1.2.2).

As mentioned in the beginning, the 'embedded self-addressed polar interrogative' can be compared to a 'non-embedded dialogue partner-addressed polar interrogative'. This direct

\footnotetext{
138 (M001_091,1183_m_thoughts_ex06a_01.58-02.05)

139 Schalber et al. $(2006,457)$ described that sign for ÖGS. The fingers are held in an open stage, the middle finger is directed down ward, and the index finger is directed upward. A possible implied additional locative interpretation of the predication has not been described.
} 
comparison is available in the corpus in which the informants express their train of thoughts during performing a long activity. In many cases after having formulated the trains of thoughts (with or without interrogativity) the signers keep on telling the imagined audience the outcome or true value of their assumptions. In doing so, they construct a dialogue. For instance, in the case that a signer is wondering whether s/he will get something to drink in the hut or whether the hut is open or closed, s/he continues telling the audience that s/he goes on hiking, arrives at the hut and asks the dairymaid if she can get something to drink or if the hut is open at all by performing a constructed dialogue. This question directly addressed to a person (in the data in form of a constructed dialogue) is prototypically illustrated in the following Figure $4.13^{140}$ :
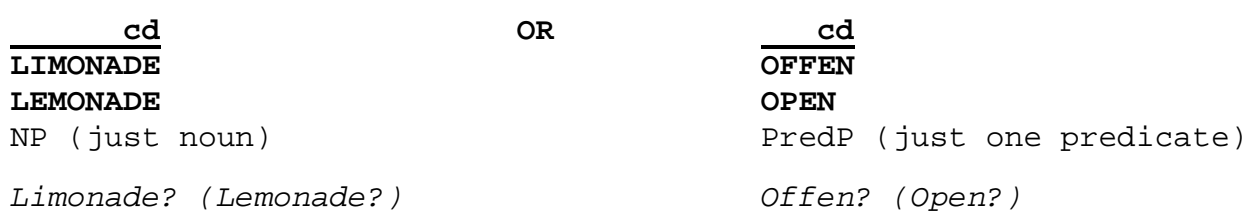

OR

$\overline{\text { OFFEN }}$

OPEN

PredP (just one predicate)

offen? (Open?)

Figure 4.13 Prototypical illustration of polar interrogatives in direct communication

In conclusion, the differences between 'embedded polar interrogatives' and 'direct polar questions', based on the present ÖGS data, are listed:

- Both constructions use different interrogative markers: polar questions have the marker chin down while embedded polar interrogatives have the marker head forward.

- Only in embedded polar interrogatives can modality elements precede, co-occur or follow the requested element(s).

- The (nominal) predicate phrase of polar questions is frequently composed of only the predicate or only the 'nominal element'. For example, in Figure 4.13 no predicate of existence follows the nominal element LEMONADE and no reference subject precedes the predicate OPEN. In embedded polar constructions in most cases both the nominal element and the predicate are signed.

\footnotetext{
${ }^{140}$ Abbreviations: NP (noun phrase), PredP (predicate phrase);
} 
- In embedded polar interrogatives frequently the locus and the reference sign pointing to that locus are present, while in direct polar questions these elements are lacking.

- In polar questions the co-occurring non-manual markers conveying grammatical functions are less or not so systematically organized ${ }^{141}$ as in embedded interrogatives.

- When listing alternatives, in embedded polar interrogatives frequently two options are signed or implied while in the setting of dialogues partner-addressed polar questions mostly only offer one option, as illustrated in Figure 4.13.

- Finally, there are no characteristics (i.e. occurrence of $\mathrm{REF}_{\mathrm{HYP}}$ or marker gaze-up; signing in higher signing space) linking the interrogative with a hypothetical space in direct polar questions, but instead there is direct eye-contact with the dialogue partner or, in constructed dialogues, with the imagined dialogue-partner.

\subsection{Special use of the hypothetical space}

The present embedded polar interrogatives are all not addressed to a dialogue partner instead they display a line of thoughts which a person considers about a situation, about its fulfillment, or about its truth value. All these self-requested chains of thoughts are allocated to a mental space. This hypothetical space or space of thoughts is indicated by different markers which occur in the beginning of the train of thoughts or which co-occur during them. With regard to thoughts asked in an interrogative way the following markers occur in the data: first, the pointing sign IX-up, defined as REF $F_{\text {HYP }}$ (mentioned above), which mostly precedes the chain of thoughts, and second, the marker gaze up which is directed towards that mental space and in most instances co-occurs with the chain of thoughts. The marker chin up which occurs in non-interrogative train of thoughts is not present in the embedded polar interrogatives. In these the marker head forward co-occurs with the construction. Additionally, the signs allocated to the line of thoughts are produced in a higher position in the signing space (a detailed description on the hypothetical space is in 6.4.4).

\footnotetext{
${ }^{141}$ For example, the interplay of the hf-marker and the br-marker conveys a more specified syntactic notion. Regarding the part which they can cover they provide information about the syntactic constituents and their relation (cf. 4.3.1.2.2).
} 


\subsubsection{Embedded content interrogatives}

\subsubsection{Introduction}

In both dialogues and monologues, embedded content interrogatives which are associated with the preceding main clause are obvious and will be described in this section.

First, the head marker(s) which obligatory mark an embedded content interrogative are described. This includes a description of the marker chin up and/or head forward which is similar with direct content questions. Also, the possible spreading of this head marker along the main clause is included in the discussion. Then, the characteristics of the embedded content clause are presented.

\subsubsection{Marking of embedded content interrogatives with 'chin up' and/or 'head forward'}

Similar to direct content questions, embedded content interrogatives which are associated with another clause are covered by 'chin up' and/or 'head forward'. This is illustrated in Figure 4.14.

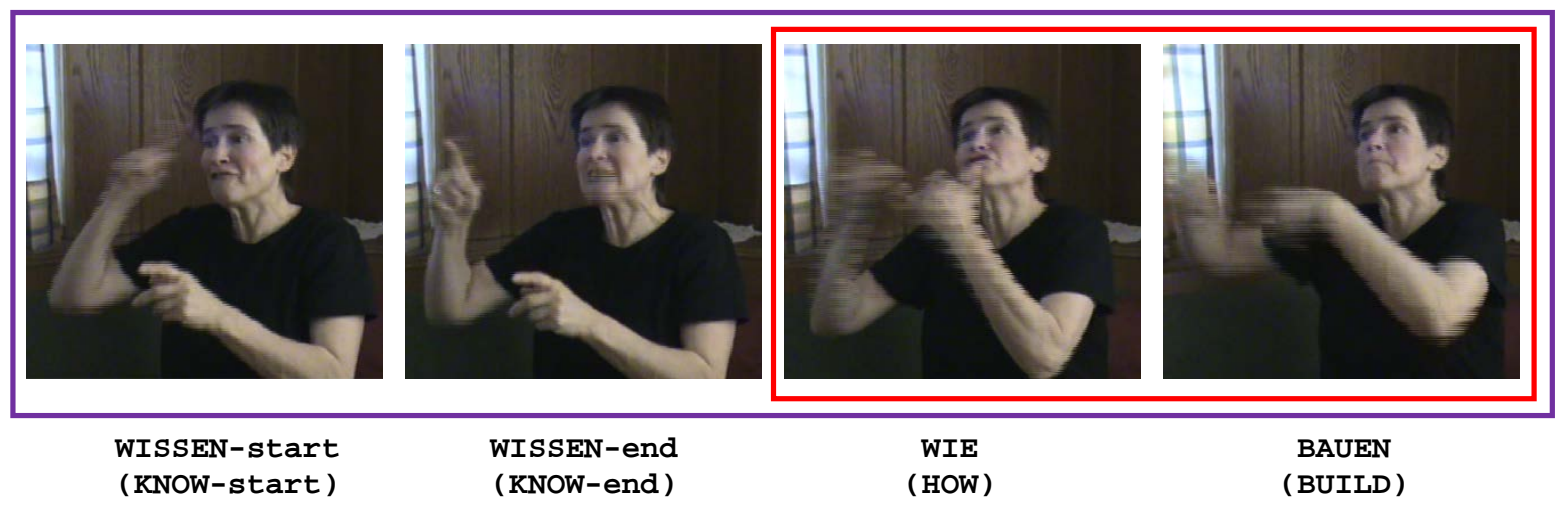

Figure 4.14 The embedded interrogative markers 'head forward' and 'chin up'

Figure 4.14 shows the occurrence of both markers. What is special in this illustration is that the marker head forward (edged violet) already starts with the expression of cognition while chin up (edged red) starts with the embedded clause (HOW BUILD). 
The data shows that in several instances both interrogative markers, performed on their own or together, only cover the embedded clause. But also in various instances the interrogative marker head forward also covers the preceding or following main clause, including the expressions of cognition, emotion or perception to which the interrogative clause is linked. Interestingly, in any example from the corpus, this spreading is done by the interrogative marker chin up. In other words, this marker always starts with the whquestion sign of the embedded clause.

Both Figure 4.14 (as well as the following example (71)) and 4.15 show the spreading of the head marker head forward along the expressions of cognition. The first illustrates only spreading to a preceding expression of cognition, the latter shows that the head marker can spread to both the preceding and following expression of cognition.

In the following example (71) (shown in Figure 4.14) the entire embedded content interrogative construction is covered by head forward, while the embedded clause is covered by chin up.

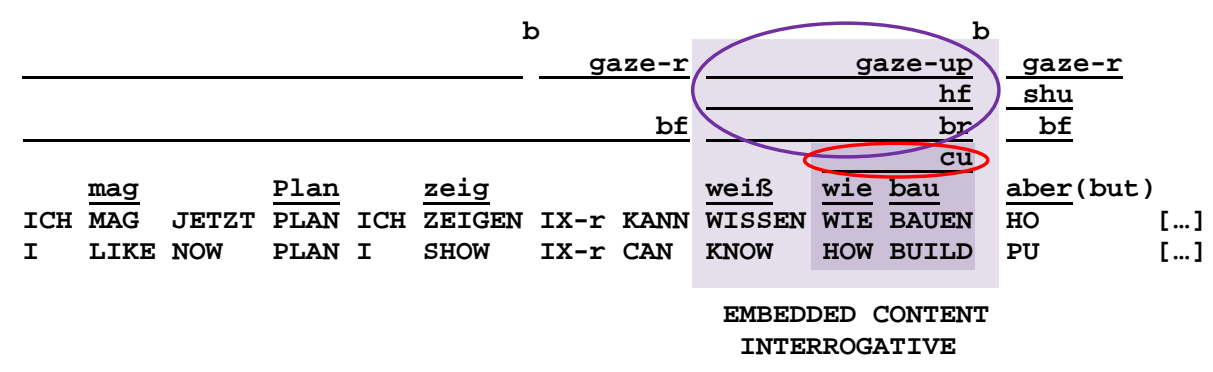

Ich möchte ihm heute den Plan zeigen, damit er weiß, wie zu bauen ist. Aber ... I want to show him the plan today so that he (can) know how to build (it). But ...

(F001_052_m_thoughts_00.16-00.21)

In (71), the relative clause, starting with the pointing sign IX-right which refers to the person to whom the narrator wants to show the plan, includes an embedded content interrogative clause (color-coded light violet). The expression of cognition (KNOW) takes an interrogative complement clause (color-coded dark violet) being composed of the signs HOW BUILD. Interestingly, the interrogative marker chin up (encircled red) covers only the embedded interrogative clause while the interrogative marker head forward co- 
occurring with the marker gaze up and brow raise (encircled violet) accompanies both the expression of cognition and the embedded interrogative clause.

So far it can be determined that the marker chin up exclusively starts with the question sign which is always found in clause-initial position of the embedded interrogative clause. The marker head forward can start in interrogative-initial position as well as in the initial position of the embedded clause.

In Figure 4.15, the annotators perceive that the signs I KNOW-NO which precede the embedded clause, as well as the expression of 'lack of knowledge', coded by a shoulder shrug, which follows the embedded clause, are covered by head forward (and also by head tilt sideward). An interrogative meaning is clearly ascribed to the marker by them.

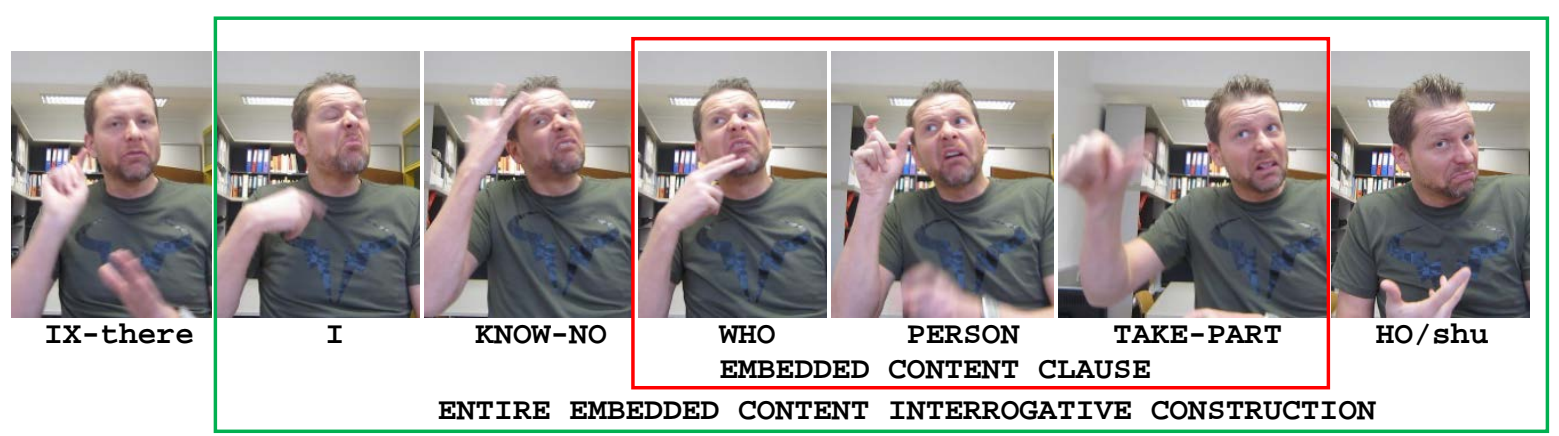

Ich weiß nicht, wer (an der Party) teilnimmt.

$I$ don't know who will be in (at the party).

Figure 4.15 Head forward covering the entire embedded content interrogative construction

The illustrated signer thinks about organizing a party and about the people who will go there (displaying the first picture in Figure 4.15 in which the head still is in neutral position). Then follows an embedded content interrogative of both the embedded content clause (encircled red) and the preceding and following expressions of lack of knowledge are covered by head forward (edged green). Also, during the entire interrogative the head is tilt sideward which is interpreted by the annotators as providing the construction with possibility (cf. 7.4.6). In addition, the signer looks upward referring to the 'hypothetical space' (cf. 6.4.3). Further, during the entire construction the forehead is frowned, the nose 
is wrinkled (cf. 7.3.2), and the brows are furrowed to a varying degree. Their particular functions require further investigations.

In sum, the interrogative marker head forward can spread to the signs of cognition, emotion, or perception which precede and/or follow the embedded interrogative clause. Consequently, similar to embedded polar interrogatives the phonological process of assimilation can be regressive and progressive.

So far, it cannot be determined in which cases the signers use one of the possible head markers or both together. There is one situation in which the signer uses the marker chin up as the marker head forward is required for another purpose. This is exemplified in (72).

\begin{tabular}{|c|c|c|c|c|c|}
\hline \multirow{4}{*}{$\frac{\text { vorher }}{\text { VORHER }}$} & \multirow[b]{3}{*}{ alle } & & \multicolumn{3}{|r|}{ gaze-r } \\
\hline & & hf & ht & & cu \\
\hline & & & wie wie & & \\
\hline & $\overline{\text { ALLE }}$ & alle-SCHAUEN-ich & WIE+ & $\mathbf{I C H}$ & GEBÄRDEN \\
\hline BEFORE & ALL & all-LOOK-I & HOW+ & I & SIGN \\
\hline
\end{tabular}

Zuerst schauen alle auf mich, wie ich gebärde.

First, all watch me how I sign. ${ }^{142}$

(M001_086_d_00.48-00.51)

Example (72) shows a situation in which a signer instructs the other participants how they have to sign. In doing so, they should look at him what he is signing. (He refers to the types of dialogues like producing a curriculum vitae, a joke, trains of thoughts, etc. which should be signed.) The head marker chin up co-occurs with the embedded content interrogative clause while two forward movements of the head accompanying the sign HOW and its repetition as well as the mouthing 'how', performed twice. Consequently, the first head marker indicates the content interrogative while the second one is used to emphasize the repeated question sign and mouthing. In (72) the gaze is directed to the audience and not to the hypothetical space.

\footnotetext{
${ }^{142}$ The embedded clause is interpreted as an interrogative/complementive clause. If the embedded clause would be interpreted as a relative clause, the translation would be 'Zuerst schaut auf mich, auf meine Art und Weise des Gebärdens. ('First, all watch how I sign.'). As analysed in the following section, an embedded clause which is covered by an interrogative marker is interpreted as an interrogative clause. However, the differentiation between embedded interrogative clauses and relative clauses needs further investigations.
} 
Concluding, both direct content questions and embedded interrogatives are indicated by chin up and/or head forward. The clear distinctive marking of the two interrogatives is that the first requires gaze contact with the dialogue partner while the latter is accompanied by gaze directed upward or somewhere to the front.

\subsubsection{The characteristics of the embedded clause}

Embedded content interrogatives, present in the data, are defined as constructions displaying a combination between a main clause, constituting a frame, and a further clause which displays the content to be filled into the frame. The embedded content interrogative clause is characterized by

- containing a wh-question-sign in clause-initial position,

- being covered by the interrogative marker chin up and/or head forward,

- frequently being accompanied by looking upward or somewhere to the front,

- being embedded in an expression of cognition, emotion or perception formulated within the main clause.

These characteristics are illustrated in example (73) and Figure 4.16.

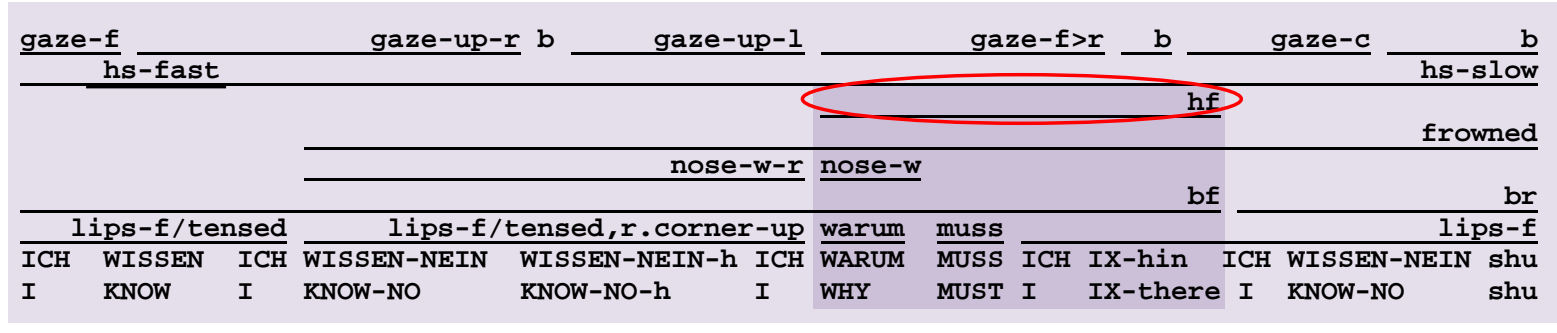

Ich weiß nicht warum ich dort hinmuss. Ich weiß es einfach nicht.

$I$ don't know why I have to go there. I really don't know.

(M007_2273_m_thoughts_00.07-00.10)

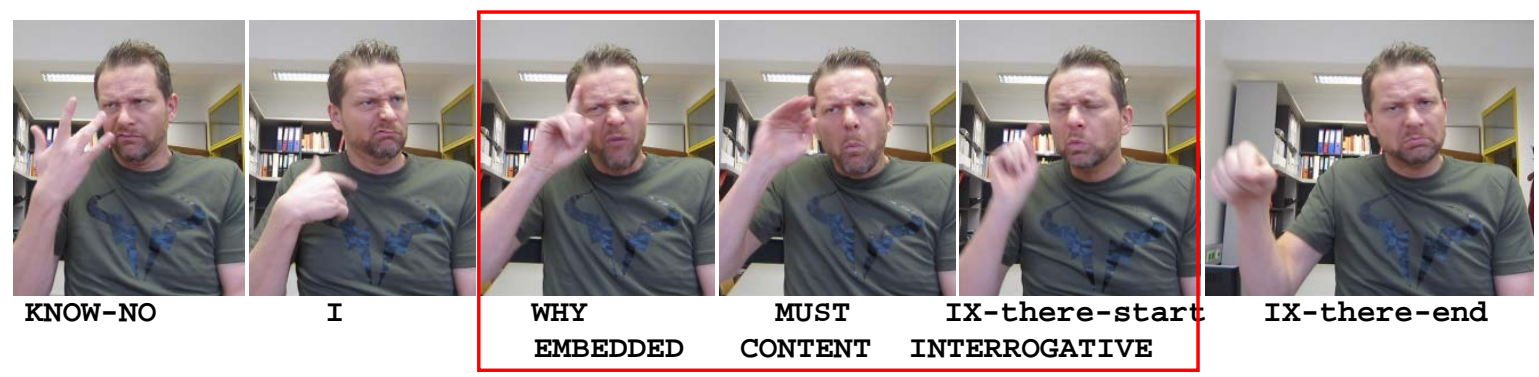

Figure 4.16 An embedded content interrogative marked by 'head forward' 
A signer imagines a situation in which he receives a letter indicating that he has to go to a special meeting, and he wonders why he has to go there. In doing so, the illustrated content interrogative construction (color-coded bright violet) including the embedded clause (color-coded dark violet) occurs which shows the characteristics listed above: First, the embedded clause is introduced by a wh-sign which is interpreted as interrogative-indefinite pronoun ${ }^{143}$. Second, the embedded clause is covered by the interrogative marker head forward (encircled/edged red in (73) and Figure 4.16). Third, the signer looks up or somewhere to the front during the signs of cognition as well as during the embedded clause, indicating the 'space of thoughts' (cf. 6.4.3) - similar to embedded polar interrogatives. Fourth, signs of cognition, expressing lack of knowledge (KNOW-NO), with which the embedded clause is associated/linked, precede and follow the embedded clause.

In example (73) other nonmanuals also precede, co-occur, or follow the interrogative clause. First, the entire construction is covered by slow non-assertive headshakes which are clearly overruled by fast negative headshakes co-occurring with the sign KNOW (cf. 7.4.3). Further, the forehead is frowned beginning with the sign KNOW-NO and lasting to the shoulder shrug. Also, the brows are furrowed from the beginning, when the signer starts expressing his wonder, and change to brow raise, when terminating the embedded content clause and adding the expressions of lack of knowledge. Moreover, the expression of lack of knowledge which precedes the embedded content clause is accompanied by wrinkled nose (one side up) and the mouth gesture 'lips forward-tensed, right corner up' (cf. 7.3.2). All the functions of these last-mentioned elements need further investigations.

\subsubsection{Distinguishing embedded content interrogatives from other constructions}

To begin with, the difference between embedded content interrogatives and wh-clefts (cf. 3.2.3.1) are clarified. Then, the difficulties in demarcating embedded content interrogatives

\footnotetext{
${ }^{143}$ Because I want to wait for results on further investigations on pronouns in ÖGS, I classify the pronouns occurring in this section as interrogative-indefinite pronouns. In doing so, I follow Bhat's (2004, 182-183) functional-based classification of pronouns of which one class displays interrogative-indefinite pronouns.
} 
from relative clauses which include a wh-sign are discussed. Finally, embedded interrogatives are distinguished from interrogatives occurring in 'constructed dialogues, ${ }^{144}$.

\section{Comparison with wh-clefts}

As shown in the sub-chapter on assertion (cf. 3.2.3.1) wh-clefts may give the impression that content interrogatives are present. In the following three clear differences between embedded content interrogatives and wh-clefts in ÖGS can be determined and are listed as follows:

- First, wh-clefts display focused structures and are characterized by a focused phrase which stands outside the wh-clause. Embedded content interrogatives fulfill the function described above (cf. 4.3.2.3). What is important is that embedded content interrogatives present in the ÖGS data are all associated with an expression of cognition, emotion, or perception. This is not a requirement of wh-clefts.

- Second, wh-clefts the wh-sign is in clause-final position while in embedded content interrogatives the wh-question-sign is in clause-initial position (and optionally also in clause-final position for reasons of emphasis, for example).

- Third, content interrogatives are covered by the interrogative marker(s) head forward and/or chin up, wh-clefts are not covered by an interrogative marker.

\section{Comparison with relative clauses}

The second aspect, I want to discuss in this section is distinguishing embedded content interrogatives from relative clauses which include a question sign. On the basis of example (74), illustrated by Figure 4.17, the difficulty of this demarcation is discussed.

\footnotetext{
${ }^{144}$ Following Herrmann \& Steinbach (2012) 'constructed dialogues in sign languages' is a phenomenon which is used for quotation and may be compared with direct quotation and reported speech in spoken languages.
} 
(74)

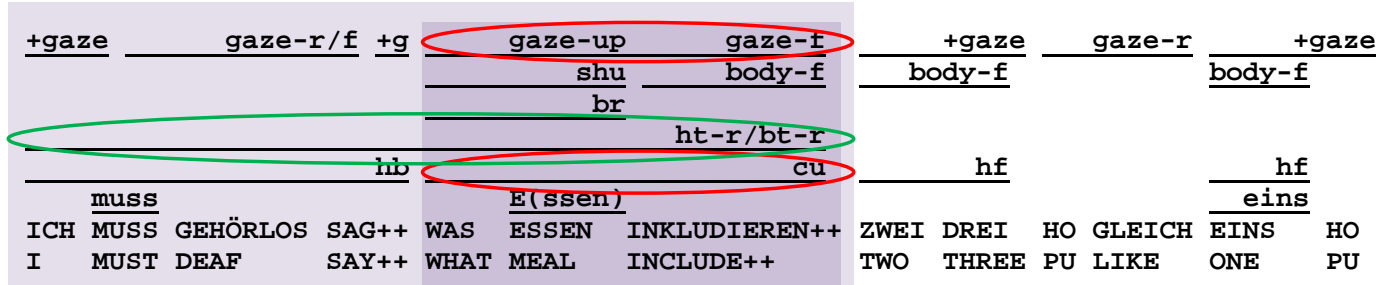

Ich muss den Gehörlosen sagen, was im Essen inkludiert ist. Zwei oder drei(Gänge) entsprechen einem (ausgiebigen Gang).

I have to tell all the Deaf what the meal includes. Two or three (courses) are like one (extensive course).

(M001_Film1_Szene3_d_02.35-02.42)
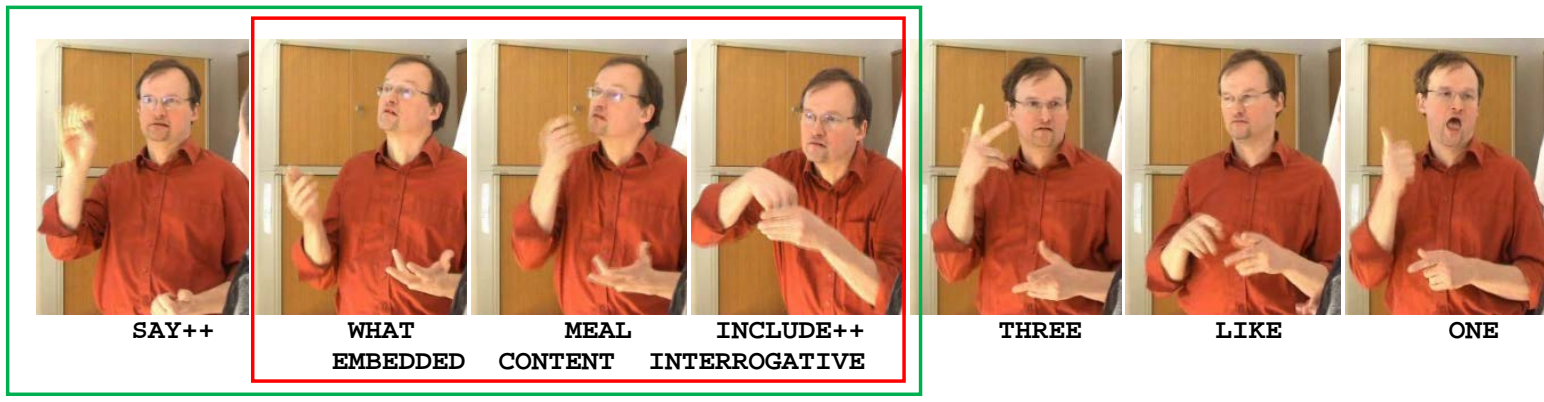

Figure 4.17 Embedded content interrogative characterized by chin up / head forward, gaze up or to the front, and being linked to an expression of perception ${ }^{145}$

As illustrated in example (74) and Figure 4.17, the clause WHAT MEAL INCLUDE+ $\left(2^{\text {nd }}\right.$ to $4^{\text {th }}$ picture) is covered by chin up and head forward which are clearly interpreted as interrogative markers by the annotators. In addition, the entire clause is accompanied by looking upward (during the signs WHAT MEAL) and somewhere to the front (during the repeated sign INCLUDE). Also, the content interrogative clause is associated with / linked to the predicate of perception (SAY++, meaning 'say to them') for which the interrogative clause serves as a complement. All these cues make it clear that the construction is an embedded content interrogative.

Moreover, the first two clauses (I MUST DEAF SAY++ WHAT MEAL INCLUDE+) are accompanied by head/body turn to the right which refers to the Deaf audience to whom the signer makes his statement. So again, the first clause is clearly brought into relation with the second clause as even the referential marker head/body turn to the right indicates that

\footnotetext{
${ }^{145}$ For reasons of space some signs are left out in the picture series. In example (74) they are present.
} 
the content interrogative refers to the Deaf audience to whom the interrogative is addressed. The following clause is not covered by turning the head to the right, only for a short while the signer moves the head to the right when signing LIKE. This movement to the right (including looking to the right) is not perceived as a language-relevant element but is rather interpreted as a means of holding the turn (cf. Lackner 2007, 113-116).

Some may suppose that the embedded cause of (74) as well as of the other illustrated examples in this sub-section serve as a relative clause ('I have to tell the Deaf (that) what the meal includes.'). This impression might be induced due to the fact that in various languages embedded wh-clauses and wh-relative clauses have homonymous or similar structures (cf. Givón 2009, 116-117). For example, Lehman (1984, 325-329), describing similarities of interrogative and wh-relative clauses with special focus on their particular pronouns, observes that in various spoken languages the wh-pronoun is found in clauseinitial position of both interrogative and wh-relative clauses, even though he provides different reasons for this phenomenon. The first is caused for reasons of focus and is part of an embedded clause while the latter is caused by 'empty place formation' and displays an 'endocentric nominal' which constitutes an empty clause gap (Lehmann 1984, 329 and 2010, 4).

However, what supports the interpretation of the present construction being an embedded interrogative clause is the observation that the embedded clause is covered by an interrogative marker. Bhat (2004, 182-183) argues that additional devices can support the interpretation of a pronoun as an interrogative-indefinite one which may occur in interrogative clauses. Two of her identified clues are the occurrence of interrogative elements within the clause and/or the occurrence of a different intonation pattern covering the clause. Consequently, the co-occurrence of the interrogative marker in example (74) as well as the co-occurrence of one or both interrogative markers in the examples of this sub-chapter - is a good device for interpreting the present construction as an embedded interrogative construction. 


\section{Comparison with constructed dialogues}

Also, the question may arise whether the present construction (74) more likely serve as a constructed dialogue. According to this interpretation the narrator takes the perspective of the protagonist (who is also the narrator) when formulating the question to the Deaf audience. However, the following reasons make it clear why the present constructions have to be interpreted as embedded interrogative clauses.

First, as previously mentioned (cf. 2.1.3.2), Schalber (2006, 140-141) observed that in polar or content interrogatives in ÖGS, occurring in constructed dialogues, the head is used to code the interrogative function while the height is only indicated by gaze direction or by both gaze direction and an intensification of the head marker, if the up or down movement of the head match the direction of the height. With example (74) the interrogative marker is chin up. Also the signer looks upward. What is clear is that looking upward does not coincide with the height of the Deaf audience (which would be looking somewhere to the right in the signing space). Rather looking upward / to the front indicates the hypothetical space.

A further reason why the constructions in this sub-chapter are interpreted as embedded interrogatives is the observation that the interrogative marker head forward can spread over the main clause containing the predicate (i.e. the expressions of cognition, perception, or emotion) which takes the complement. In constructed dialogues the interrogative marker cannot spread along the preceding clause which clarifies who is signing/acting/etc. with whom.

What is more, the data shows that when signing about unreal thoughts, wishes, and so forth which are self-addressed, the signer produces embedded interrogatives, if these lines of thoughts are formulated in an interrogative way. The resulting constructions are indicated by the markers of embedded interrogatives. However, if a signer produces a question when signing a course of action embedded in a narration and when taking the perspective of a protagonist or narrator, the signer produces the question within a constructed dialogue. In these cases the markers which occur in direct questions are used. Givón (2009, 118) supposes that wh-clauses embedded as a verb complement have been developed out of speech acts. Following his interpretation, it might be assumed that the present syntactic 
constructions (i.e. the embedded interrogatives) arose from paratactic constructions to which a constructed action can be allocated as it shows the character of a speech act.

Finally, one observation from the data is that with direct questions occurring in constructed actions it rarely occurs that the narrator quotes the predicate such as who is 'saying/signing' something to whom, who is 'asking' whom, and so forth. These signs of perception do not really precede the constructed dialogues, unless it is unclear who is addressing whom, then the signer quotes the person who takes the perspective in the constructed dialogue ${ }^{146}$ without labeling the sign of perception. Consequently, the signs of cognition, perception, or emotion do not really precede direct questions which are produced within a constructed dialogue.

\subsubsection{Conclusions on embedded interrogatives}

It is a new observation that embedded interrogatives do exist in sign languages, at least in ÖGS. Even the interrogative marker, used for embedded interrogatives, can spread over the main clause which comprises the sign of cognition, perception or emotion. To my knowledge, embedded interrogatives which are marked with a special non-manual configuration have been identified only in Brazilian Sign Language (LSB; Müller de Quadros 2006). Other sign language researchers even indicate that in sign languages indirect speech cannot be done by embedded interrogatives and therefore, sign languages use the means of constructed dialogues (cf. Herrmann \& Steinbach 2012, 211).

Thus, the difference between embedded interrogatives and direct questions is that

- the interrogative marker head forward of both the embedded polar interrogative and the embedded content interrogative can spread along the main clause, which includes a predicate of cognition, perception, or emotion which can take a complement.

\footnotetext{
${ }^{146}$ For instance, in example (11) the signs EDUCATOR, HEADMASTER and TEACHER precede the constructed dialogue in which the signer indicates that these persons thought that they were twins. With regard to the perspective of children (signing that at their young age, they didn't know what 'twins' were) no reference person is introduced. Also in (21), displaying a sequence of a constructed dialogue, the signer produces before the constructed dialogue the signs GRANDFATHER I+, making clear who is addressing whom. With (21) however, the latter sign (I+) is interpreted by some annotators as meaning he-SAY-I.
} 
- with polar interrogatives, different markers are used. For direct questions, the marker chin down is used, while for embedded interrogatives, the marker head forward is used.

- in direct questions, the signer always has eye contact with the counterpart, while in embedded interrogatives, the signer looks upward or somewhere to the front, indicating the hypothetical space.

- embedded interrogatives occur in different sorts of text than direct questions. The data shows that embedded interrogatives are primarily used when expressing self-addressed trains of thoughts, while direct questions are used in direct communication or in constructed dialogues, occurring in the data primarily in narrations in which a sequence of actions is given (but not a line of thoughts).

To be explicit, the characteristics of both embedded interrogative constructions are described as follows:

1. The interrogative markers are markers coded by the articulator 'head'. Embedded polar interrogatives are indicated by head forward, which is different than the polar question marker chin down. The embedded content interrogative marker is chin up and/or head forward which is similar to the direct content question marker.

All of the interrogative markers used for embedded interrogatives can only cover the part of the construction which is questioned. What is special is that the marker head forward of both types of embedded interrogatives can spread over the preceding or following predicate of cognition, perception or emotion, i.e. along the main clause. For this very reason, the present constructions are defined as 'embedded' from a syntactic point of view. What is special as well is that the marker head forward used in embedded polar interrogatives can be produced in a regular way when covering the entire construction or in an intensified way when covering that part of the construction which is actually put into question (see the following example (75)).

2. The data shows that embedded clauses can show additional interrogative markers, also indicating the interrogative character of the construction. In embedded polar questions, the marker brow raise can additionally be used to highlight the part of the 
construction which is put into question (similar to the intensification of interrogative marker head forward). Also, the embedded clause is frequently introduced by the element 'ob' ('whether') or immediately follows the element IX-up ${ }_{H Y P}$, which can occur in clause-initial position. 'Ob' only occurs in these embedded polar interrogatives. With embedded content interrogatives further investigations on this aspect are required.

3. Also, in embedded interrogatives, various indicators referring to the hypothetical space are frequently present. Primary is the marker gaze up or gaze somewhere to the front (but not to the dialogue partner) which frequently covers the entire construction. Also the pointing sign IX-upнур occurs which is frequently found in clause-initial position of the embedded clause. A displacement of the sign's place of articulation to a higher location is repeatedly observed, too.

4. What nearly all embedded interrogatives have in common is that these constructions are linked with a predicate of cognition, emotion, or perception for which the embedded clause constitutes its complement. These 'modality expressions' can precede, follow, or co-occur with the interrogative clause. They display modality signs or nonmanuals coding modality meaning (cf. 7.3).

5. What is more, the embedded interrogatives of the present data show the frequent occurrence of other modality markers, especially those coding epistemic modality. This co-occurrence may be due to the fact that the signers tend to express their attitude towards /evaluation of a proposition when thinking about a situation in an interrogative way.

To conclude, an embedded polar interrogative is presented in order to illustrate the characteristics listed-above. 
$(75)^{147}$

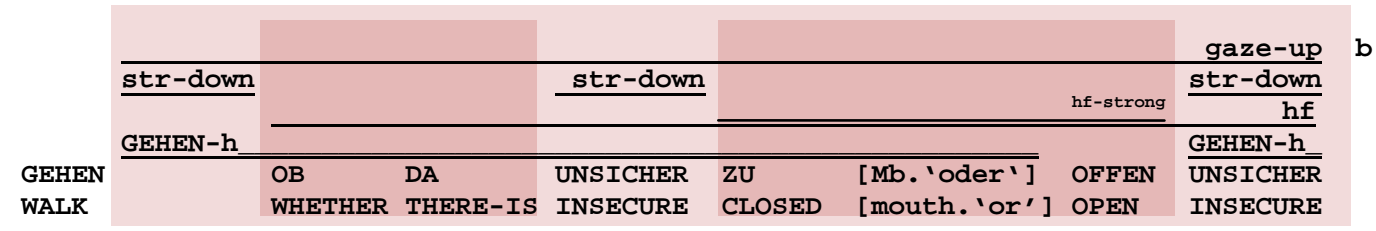

Ich bin mir unsicher, ob es etwas (eine Hütte oder ein Getränk in der Hütte) gibt, und ob es (die Hütte) geschlossen oder geöffnet ist.

I am unsure whether it (a hut or a drink in the hut) is available/there, and whether it (the hut) is closed or open.

(F002_109_m_thoughts_ex12_05.15-05.22)

Example (75) displays an embedded polar interrogative (color-coded bright rose), present in the data. First, the hf-marker covers both complement clauses (WHETHER THERE-IS and CLOSED 'or' OPEN; both color-coded dark rose) as well as the expressions of insecurity which intervene and follow the both embedded clauses. This is the sign INSECURE which is accompanied by the mouth action 'stretched down'. Also, the interrogative marker head forward is intensified when producing the second embedded clause. In doing so, the requested part is highlighted. Second, the construction comprises a further marker showing that an embedded polar interrogative is present. This is the sign WHETHER, which is found in clause-initial position of the first embedded clause. Third, the entire construction is accompanied by looking upward. The interpretation of this marker is indicating the hypothetical space, i.e. the space of thoughts. Of course, as the matter of subject - the hut - is also topographically found in an upper position, it cannot absolutely be excluded that looking upward is associated with the hut.

\footnotetext{
${ }^{147}$ What is also obvious in (75) is that the non-dominant hand is held while formulating the train of thoughts. Only when producing the two-handed sign OPEN, the non-dominant is used for this sign. Afterwards, the hand goes back to the holding position of the long action. The phenomenon of holding the non-dominant hand's configuration and position while formulating a line of thoughts with the dominant hand is frequently present in the data when signers produce trains of thoughts.
} 


\subsection{A special interrogative marked with 'head backward'}

In the data a special interrogative marker also occurs. This is the marker 'head backward' which covers a constituent and is identified as the relevant element which provides the construction with interrogativity.

\subsubsection{Marking with 'head backward'}

In the corpus containing the trains of thoughts, some of the interrogatives are indicated by the marker head backward as illustrated in Figure 4.18.
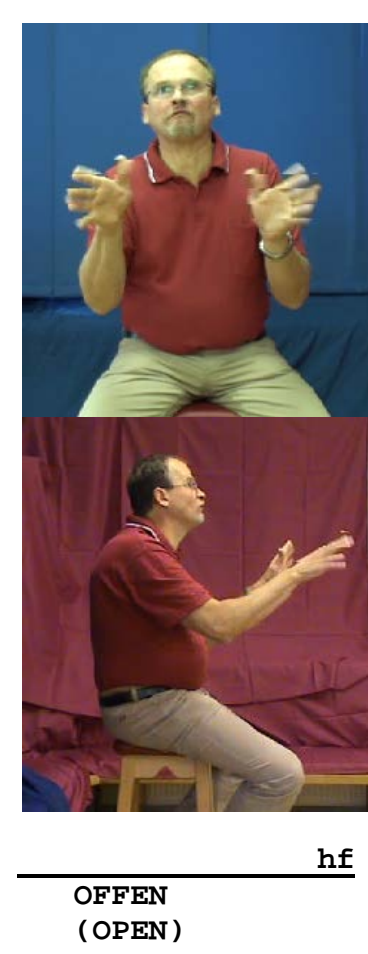

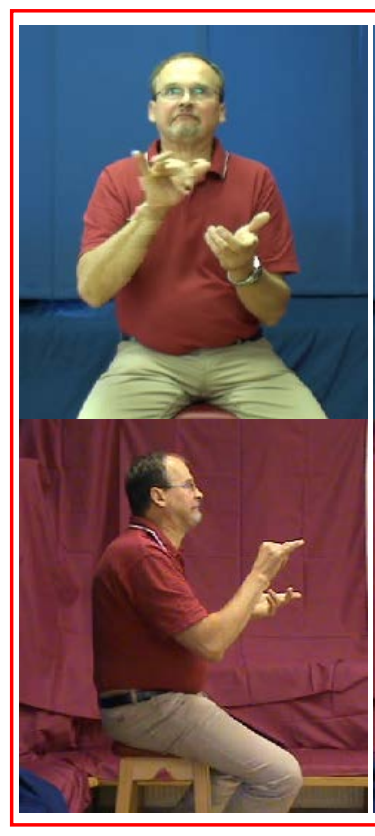

EINES-VON-BEIDEN (ONE-OF-BOTH)

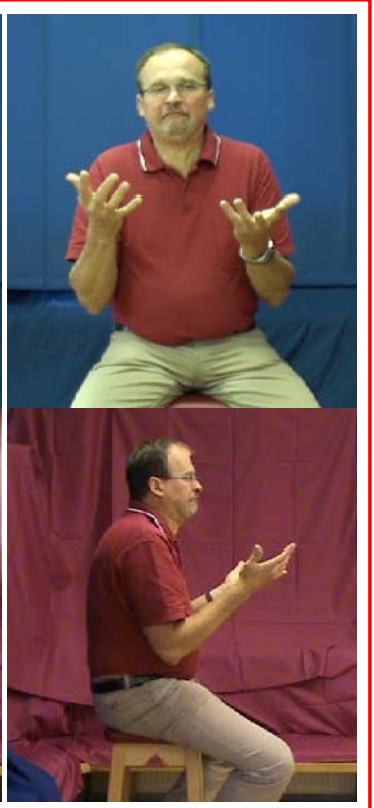

hb

PU

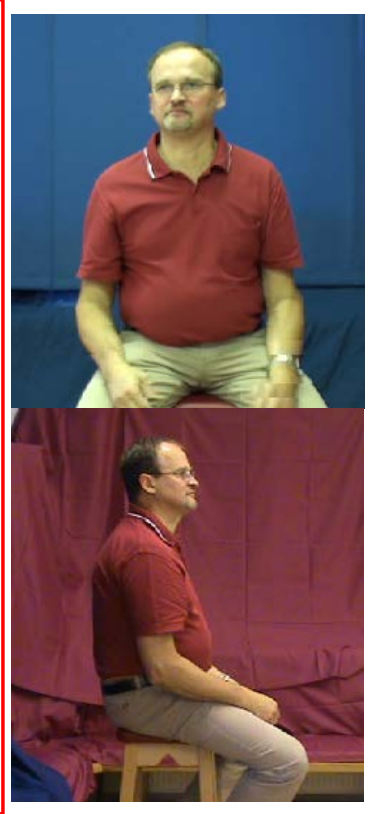

head neutral

$<$ rest position> $<$ rest position>

(Ich weiß nicht, ob die Hütte geschlossen oder) offen ist und bin mir unsicher, welches der beiden zutriff.

(I don't know whether the hut is closed or) open and I am unsure which is the case.

(M001_091,1183_m_thoughts_ex04_02.03-02.05)

Figure 4.18 The interrogative markers 'head backward'

In the following examples the occurrence of the marker head backward is illustrated; these will constitute the basis for the discussion on the construction's characteristics, discussed in 4.4.3. 


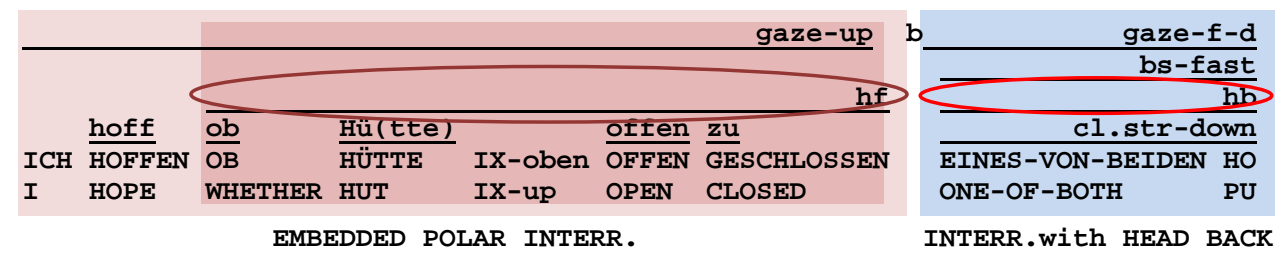

Ich hoffe, ob die Hütte offen oder geschlossen ist? (Aber) ich bin mir unsicher, welches der beiden zutrifft/zutreffen wird.

I hope whether the hut is open or closed? (But) I am unsure which is/will be the case.

(M001_091,1183_m_thoughts_ex07b_02.25-02.29)

In (76), the signer hopes/wonders that/whether a hut is open or closed (the entire embedded polar interrogative is color-coded bright rose, the embedded clause dark rose, the interrogative marker is encircled dark red). He keeps on thinking about the situation and asks himself insecurely which would be the case. While thinking which may be the case the signer positions the head backward (encircled red), sways the body sideward in a fast way, stretches downward his lips and looks somewhere unspecified into the front (colorcoded blue).

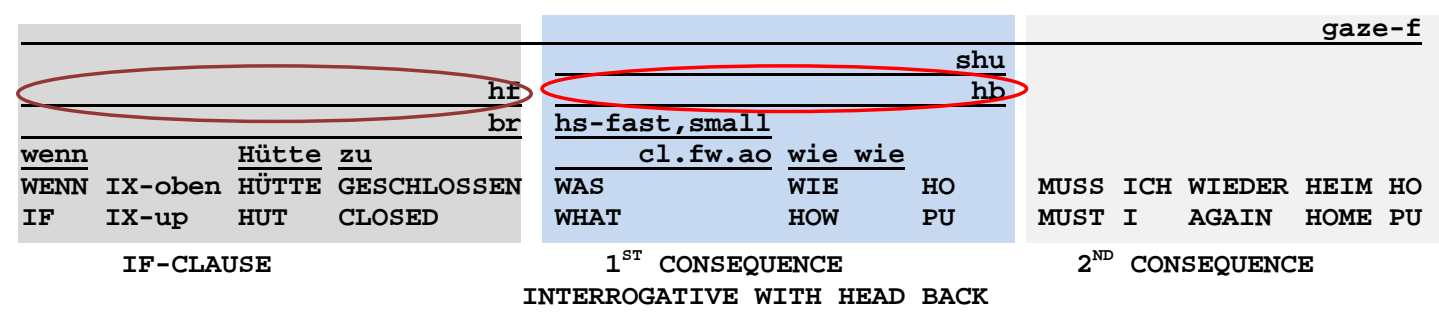

Wenn die Hütte geschlossen ist, was soll ich machen, wie geht es dann weiter? Dann muss ich wieder heim.

If the hut is closed, what should I do, how should I go on (then)? Then I have to go home.

(M001_091,1183_m_thoughts_ex09_03.18-03.22)

In (77), the first consequence of the conditional (the if-clause is color-coded grey, the conditional marker dark red) displays the discussed interrogative (color-coded blue) which is covered by positioning the head backward (encircled red), raising the shoulders and looking somewhere into the front. In addition, the signer performs fast small headshakes when signing WHAT which is implemented by fast shaking movements. In doing so, the signer expresses his insecurity and unawareness of what to do then. 
(78)

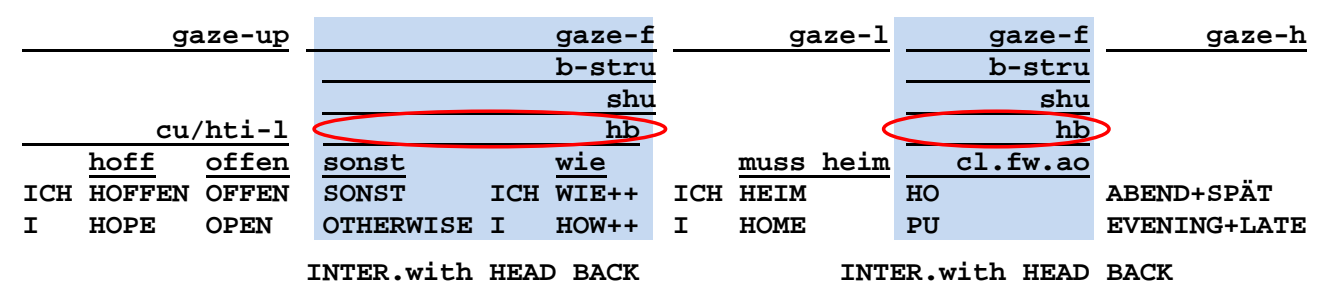

Ich hoffe, es ist offen, sonst weiß ich nicht wie (weiter). Dann muss ich heim, sonst wie (soll es weiter gehen)? Es ist ja schon spät abends.

I hope that it is open otherwise $I$ don't know whether to go on. Then $I$ must go home, otherwise what? It is already late in the evening.

(M001_091,1183_m_thoughts_ex07b_02.18-02.24)

In example (78), the signer is hoping that the hut has opened otherwise he does not know what to do. The second constituent (first block, color-coded blue) is covered by positioning the head backward (encircled red). It co-occurs with raising the shoulders, straightening the body and looking somewhere to the front. The entire non-manual configuration is repeated after signing I HOME (second block, color-coded blue). Once again this interrogative is interpreted that the signer does not know how things will come out.

All three examples show that an interrogative is present marked with head backward. According to the annotators' perception positioning the head backward is providing the construction with interrogativity, but also with a notion of negative/denial attitude towards the presented situation.

\subsubsection{The characteristics when marking an interrogative with 'head backward'}

The question arises 'what is common to all the interrogatives which are indicated by head backward'. In the following the characteristics of this interrogative construction are presented.

- First, all these interrogatives are indicated by the marker head backward. Also, in most instances a wh-sign occurs. To this, also the sign ONE-OF-BOTH, present in example (76), may be included in the group of wh-signs as it also can be interpreted as 'which'. Furthermore, occasionally the sign SONST (OTHERWISE) occurs in these interrogatives and is produced in clause-initial position of the interrogative. 
- Second, the marker head backward provides the construction with interrogativity, but also includes a negative/denial implication.

- Third, concerning the function of these interrogatives it becomes obvious that no content is to be asked for, even though a wh-sign is present. In all these instances the signer asks himself/herself in a reflective way, but no answer is offered. Somehow the present interrogative reminds one of a 'rhetorical question' in terms of increasing a self-made utterance, including one's opinion (here with a negative/denial notion) and receiving a denial or affirmation (here receiving a self-addressed denial, not an affirmation).

- Fourth, the data shows that the interrogative marked with head backward always follows a statement about which the interrogative is concerned. In (76), in the preceding embedded interrogative the state of being open or closed is asked for. This is questioned in the following interrogative. In (77), the state of being closed is the issue (formulated within a conditional clause) and again this is questioned in the following interrogative. In (78), the state of being open is hoped for in the preceding statement. Once more, the subsequent interrogative is related to the state of being open.

\subsubsection{Conclusions on interrogatives marker with 'head backward'}

Summing up, what is special in ÖGS is that some interrogatives are indicated with a head position (head backward) which is different from all other interrogative markers, regardless of whether used for direct or embedded interrogatives.

This special marked interrogative is furthermore characterized by

- being marking with the interrogative marker head backward which additionally provides the construction with a notion of negation/denial,

- the frequent occurrence of a wh-sign - sometimes also the sign SONST (OTHERWISE) is found in clause-initial position,

- the relation to a preceding statement,

- being not aimed at gaining some information, rather expressing one's denial attitude. 
The question that arises now is why the signers always position their heads backward instead of using one of the regularly used interrogative markers.

First, positioning the head and/or body backwards is very probably used for expressing pragmatic denial in ÖGS. Some lexical signs like ABLEHNEN (DENIAL) (cf. Skant et al. 2002, 226) or DAS-GEHT-MICH-NICHTS-AN (THAT-IS-NOT-MY-MATTER) imply a backward movement of the body and/or head. Without going into a detailed analysis this author can confirm that in the present ÖGS corpora many instances are present in which pragmatic denial is indicated by moving the head and/or body backward. Also, positioning the head or body backward for expressing negation and/or denial has been described for other sign languages too. For instance, Wilbur \& Patschke (1998) report that body lean backward can express the pragmatic function of denial as well as contrast in ASL. In NGT moving the body backward expresses the broader semantic meaning of negation and denial (Kooje et al. 2005, 1609-1610). In Turkish Sign Language (TID) tilting the head backward is one of the negation markers (Zeshan 2006c).

The second interesting aspect is the following. As in some of these interrogatives the element SONST (OTHERWISE) occurs, a comparison with German 'sonst-clauses' is not far to seek. In 'sonst-clauses' in German the most striking aspects are that two clauses, connected with 'sonst'('otherwise'), possess a conditional connection and imply a positivenegative contrast, obvious as frequently one of clauses is negated ${ }^{148}$. The present interrogatives have in common that they refer to a statement (the states of being open or closed are discussed) toward which the signer can have a positive or negative attitude. Also, a notion of conditionality is included as in all discussed examples the signer does not really know how things turn out or what to do next. Thus, both aspects of 'sonst constructions' - implying a positive/negative contrast and conditionality - are involved in the interrogatives marked with head backward too, even though both constructions are not the same.

\footnotetext{
${ }^{148}$ For example, the negative conditional 'If the weather is not nice, we can't go out' would be expressed in a 'sonst-construction' (otherwise-construction) in German as well as in English as 'The weather has to be nice, otherwise we can't go out'. So, the main clause is positive, the dependent clause is negated.
} 
To conclude, one aspect comes to the author's mind. If the signer can express interrogativity and denial by positioning the head backward, probably the signer can also express interrogativity and affirmation by positioning the head forward. And this seems to be the case as the regular interrogative markers, used for direct and embedded content interrogatives as well as for embedded polar interrogatives, is head forward. Consequently, it can be concluded that for these interrogatives (marked by head forward) a default affirmative attitude is implied. If a signer wants to emphasize the affirmative attitude, very likely the signer positions the head forward in an intensified way or produces an additional affirmative marker such as 'body lean forward'. 


\section{Conditionality coded by a head marker}

\subsection{Introduction}

Formulating thoughts or describing terminology (e.g. in academic lectures) are likely contexts for conditionals to occur. And indeed, in these contexts the informants produce conditionals, most of which are hypothetical ones.

Athanasiadou \& Dirven (1997, 61-64) list three main types of conditionals:

- course of event conditionals, which express two co-occurring events, one depending on the other. The relation between the two propositions is a factual one in the sense of 'whenever one event happens, the other event happens too'.

- hypothetical conditionals, where the prediction of the consequent follows the condition in the antecedent. Thus, there is a non-factual, hypothetical relation between the two propositions.

- pragmatic conditionals, which convey an indirect dependency of both propositions of the clauses and an interactional relation.

The hypothetical conditional is characterized by the authors as being (prototypically) a conditional that includes two different events or states which have a consecutive relation in the form of an antecedent-consequent relationship, and which convey a hypothetical character. This hypothetical conditional is the most frequent one in the ÖGS data and consequently, I will focus on this type. Counterfactual conditionals ${ }^{149}$ are also present in the data, but to a much lower extent. Their characteristics are briefly discussed in 5.3.

\footnotetext{
149 In German as well as in English 'counterfactuality' can be expressed by constructions such as 'counterfactual conditionals' or 'counterfactual wishes'. The latter construction implies that the speaker wishes that a situation is different from what it is now or it was in the past. In other words, the wish expresses the contrary of the fact (Iatridou 2000). This is illustrated by the following German example:

(79) 'Wäre er doch gekommen!' / 'Wenn er doch gekommen wäre!'

'If he had only come!')

In this example the fact 'he is not here' is given, while the speaker still wishes the person to come. As 'counterfactual wishes' could not been identified in my ÖGS data, I do not follow up that matter. More on 'counterfactuality' is discussed in Iatridou (2000).
} 
In the present subchapter, first, an overview of the literature on sign language research on conditionals $^{150}$ is given, focusing on which markers characterize such a construction. Second, the types of conditionals present in the data are discussed including a description of their hypotheticality and causality. Third, the particular indicators for conditionals in Austrian Sign Language are described, emphasizing the most significant one, the 'head forward marker'. Also, the different possible consequent clauses are presented. Finally, marking of other functions such as negation and assertion, alternativity, or modality, which also occur in conditionals, are discussed. To provide a clear illustration of the examples, the protasis of the conditionals is color-coded dark grey, the apodosis bright grey.

\subsection{Literature overview}

In some sign languages, conditional constructions have been described, but to varying degrees. In most cases the 'hypothetical conditionals' are focused on.

In all sign languages investigated up to now, one characteristic is significant of conditionals. When the antecedent conveying the condition is followed by a consequent constituting a statement, it is always the first clause that receives the 'conditional marker(s)'.

In American Sign Language (ASL) the identified non-manual markers are 'raised brows' and 'head tilt'. Both markers cover the antecedent which displays the condition. Additionally, the non-manual marker 'head thrust' occurs at the end of the if-clause, and an eyeblink follows the if-clause (cf. Baker \& Padden 1978, Liddell 1986, Reilly et al. 1990). However, thrusting the head is probably more likely a boundary marker that accompanies the last sign of a prosodic domain (cf. Wilbur 2000 or Sandler 1999). Pfau (2008, 6) analyzes the 'head thrust' as a 'mood marker' (in the sense that the non-manual marker is attached to the verb which moves to the functional head).

In Israeli Sign Language (ISL) the most prominent marker of hypothetical conditionals is 'raised brows' while those of counterfactual conditionals are 'raised brows' and

\footnotetext{
${ }^{150}$ A brief overview on conditional constructions in spoken languages is given by Podlesskaya (2001), an exhaustive description on conditionals by various linguists is found in Traugott et al. (1986) and Athanasiadou \& Dirven (1997). A description on conditionals within a framework is given, for instance, by Dancygier \& Sweeter (2005) who describe conditionals within the Mental Spaces Theory.
} 
additionally 'squinted eyes'. Additionally, in most conditionals there also is a head forward and downward movement marking the protasis. Also, at the end of the if-clause the forward and downward movement is intensified (cf. Dachkovsky 2005 and 2008, Dachkovsky \& Sandler 2009, Sandler 2011).

Sutton-Spence \& Woll (1999, 89-90) describe brow movements ('brow raised'), 'head tilt' and optionally the sign 'if' as indicators for conditionals in British Sign Language (BSL). In Auslan, conditional clauses can be marked by 'raised eyebrows' and a 'backwards head tilt'. These markers seem to be the same as those used for topicalization. In addition, fingerspelled 'i-f' or the sign 'IF' may occur (cf. Johnston \& Schembri 2007, 213-214).

In the Sign Language of the Netherlands (NGT) the conditional marker is 'raised eyebrows’' (cf. Pfau 2008).

In Croation Sign Language (HZJ) there is 'brow raised', 'head forward' and then 'head downward' (Ronnie B. Wilbur, personal communication, Jan. $7^{\text {th }}$, 2013). As described in the following, the first two markers of HZJ are also used to indicate conditionals in ÖGS. This observation shows, similar as for direct question markers, that the close relationship between these both languages in former times very likely caused the use of the same markers (cf. 4.2.1).

As Boyes Braem (1995, 108-109) describes for Swiss German Sign Language (DSGS), the clause including the condition is covered by non-manual markers. These are 'head tilt with a slightly forward thrust' and 'raised eyebrows'. Between the two clauses, there is a connective pause. In the consequent clause, the position of the head and upper part of the body changes depending on the non-manual markers which are used for a statement, a question, or an imperative. In her given examples the informant signs 'if the weather is fine'. In both examples the clause initial sign is an index sign which points to an upper (right) area in the signing space from the signer's perspective. The place of articulation of the following signs (REGEN (RAIN) or SCHÖN (FINE)) is performed closer to this upper location in the signing space. In addition, the gaze is directed to that upper location during the entire antecedent. These phenomena may be due to the perceived spatial location of weather as occurring in the sky, based on the absolute frame of reference. Probably, these 
examples also refer to the 'hypothetical space' (cf. 6.4.3) and hint at the hypothetical character of the utterance.

Summing up, the 'conditional marker' most frequently used in the sign languages investigated so far is 'raised eyebrows'. But in all of the sign languages mentioned, head markers may function as an alternative or additional conditional marker. Similar to Austrian Sign Language, Swiss German Sign Language and Israeli Sign Language also use 'head forward' as a conditional marker. As described in the following (5.4), in ÖGS the alternative or additional conditional marker, that is, 'raised eyebrows', is also a very frequent 'conditional marker'.

\subsection{The types of conditionals}

The most frequent conditional construction in the ÖGS data is the hypothetical conditional. This is first illustrated by Figure 5.1, and then, described in example (80).

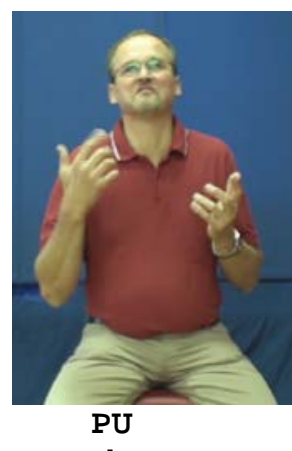

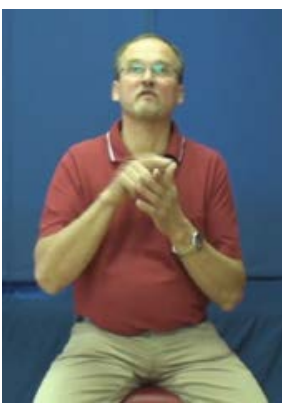

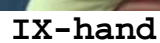
mouthing:wenn(if)

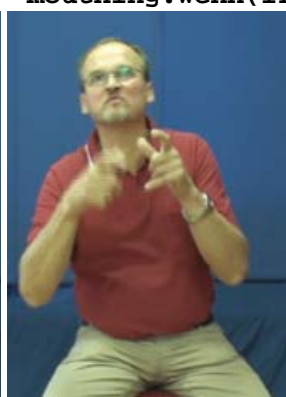

MILK

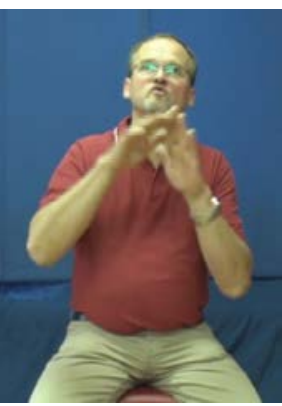

HUT

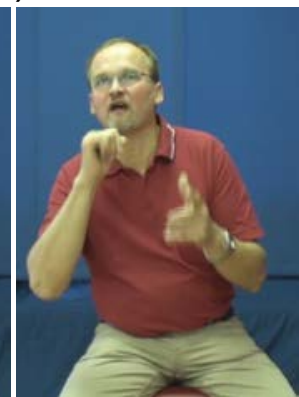

NOT

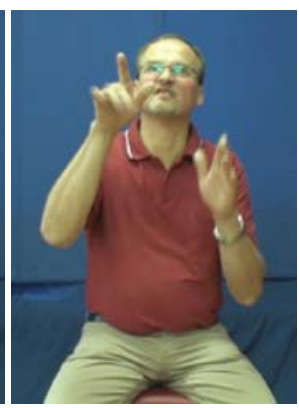

IX- up

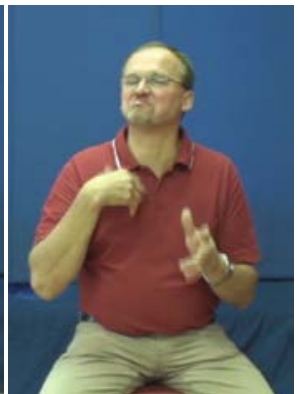

$\mathbf{I}$
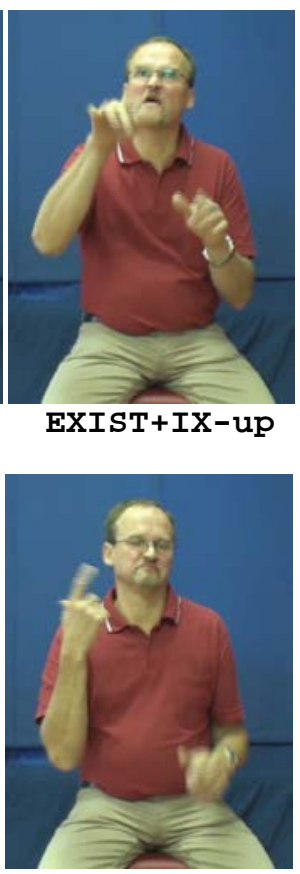

HOME

Figure 5.1 Marking of a hypothetical conditional（M001_091,1183_m_thoughts_ex04a_01.11-01.19) 
$(80)^{151}$

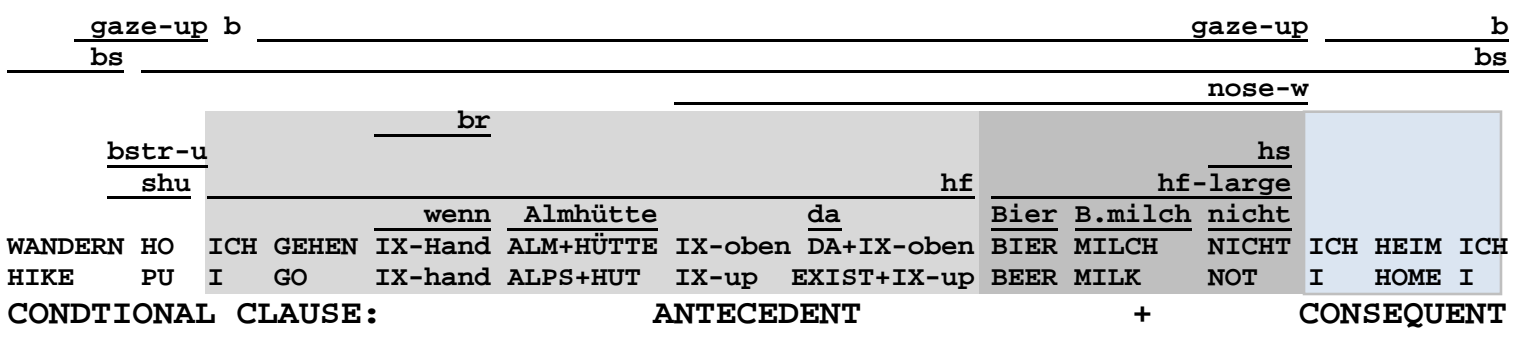

Während ich wandere bin ich mir unsicher. Wenn es in der Almhütte weder Bier noch Buttermilch gibt, gehe ich heim.

While I am hiking I am not sure. If there is neither beer nor buttermilk in the hut, $I^{\prime} l I$ go home.

(M001_091,1183_m_thoughts_ex04a_01.11-01.19)

In Figure 5.1 and example (80) the signer is hiking and thinking about a hypothetical situation. He considers that if in the hut there will be neither beer nor buttermilk, he will go back home. After having raised his shoulders and straightened his upper body (the hands are meanwhile held in a palm-up position) for expressing his lack of knowledge, the signer expresses a condition. The antecedent containing the condition is accompanied by positioning the head forward. This is identified as a language-relevant distinctive marker that characterizes the clause that conveys the condition by the annotators. They perceive the head forward marker as starting after the expressed lack of knowledge (color-coded light and dark grey). It is intensified during the last three signs (color-coded dark grey) conveying the condition. This antecedent is followed by the consequent (color-coded light blue) which is unmarked in this example. The head forward marker being produced in differing intensities is obvious in conditionals and embedded polar interrogatives in the ÖGS data, both using head forward as their most prominent marker. In both constructions the particular part of the construction which constitutes the 'exact condition' or the 'specifically asked part' are covered by the intensified marker.

The counterfactual conditional occurs in the ÖGS data too, but to a much lower extent. In counterfactual conditionals within the antecedent, a situation is described which did not

151 There are two kinds of body sways (abbreviated as 'bs'), the first co-occurring with the activity sign HIKE, the second covering the train of thoughts. Their different functions are discussed in 7.4.4. 
happen, but which might have happened. The consequent describes the results which would have happened.

The following example (81) illustrates the different parts of the conditional construction which are the same as in hypothetical conditionals. In the data, there is no obvious further marker that clearly distinguishes between a hypothetical and a counterfactual conditional. However, in the counterfactual conditional, extra information is provided which clarifies the interpretation.

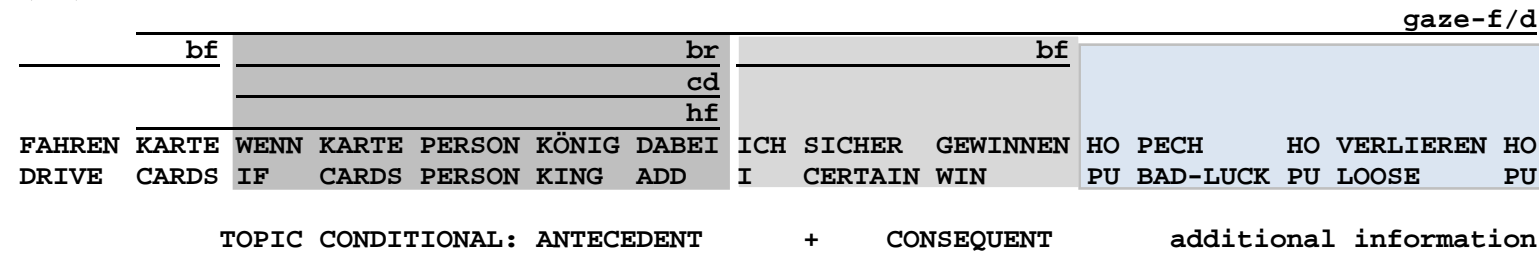

Während ich mit dem Auto fahre überlege ich, dass wenn ich beim Kartenspielen einen König gehabt hätte, ich sicherlich gewonnen hätte. Aber ich hatte Pech und verlor.

While driving I think that if I had had the king when playing cards, I would have won. But I had bad luck and lost.

(F001_039_m_thoughts_00.01-00.10)

The additional temporal information (color-coded blue) clarifies the chronological sequence of the events. As in the following information the actual fact (result) is given, the preceding conditional gets the counterfactual interpretation ${ }^{152}$.

In the educational corpus as well as in the corpus on trains of thoughts, and in the clarification corpus, the informants produce - apart from the (embedded) interrogatives conditionals. Both constructions convey a hypothetical character.

With regard to conditionals Comrie (1986) observes that the most important aspect of this construction is its degree of hypotheticality which goes together with its degree of potentiality of realization. Based on a typological comparison (of spoken languages) and following his interpretation, the resulting sets of conditionals are on the one hand conditionals which have a higher degree of hypotheticality. This means that the probability of realization is very low. On the other hand, there are conditionals with a low

\footnotetext{
152 Athanasiadou \& Dirven (1997) state that in 'course of event conditionals' the actual realization of the situation is clear while in 'hypothetical conditionals' this is not the case. Consequently, only the probability of the situation is expressed. This statement shows why the ÖGS-informants add this extra information.
} 
hypotheticality. Their probability of realization is very high. This differentiation comes close to the division of factual and counterfactual conditionals, although it is not the same.

Three characteristics which refer to the degree of hypotheticality are obvious in the conditionals (primarily hypothetical conditionals) present in the data.

- First, the hypothetical character results from the degree of possible realization of the two events which constitute unrealized events, states, situations, and so forth of which the outcome is unclear. So, in fact, it is the probability of realization that provides the hypothetical character and it is not really the 'causal relation' of the two considered propositions (compare to this the characteristics of hypothetical conditionals by Athanasiadou \& Dirven 1997, 64). But, in many of the produced conditionals two options are offered or implied, resulting in a reduction of the hypothetical character as a huge variety of outcomes is reduced to two possible options. Concerning the counterfactual conditionals, the hypothetical character as a whole is reduced. First, the antecedent already includes the unrealized event and so the answer is already on hand. Also, in the present counterfactual conditionals additional information is always given which follows the conditional clause. In doing so, not only a temporal anchor is offered, but also the outcome is presented.

- The second indication to the construction's hypotheticality can be provided by nonmanual cues. These are the markers gaze up or gaze to the front that may co-occur with the conditional construction. Looking upward to a 'hypothetical space' or sometimes staring (frontally) into space often occurs when the signer is thinking about an unrealized matter. Most of the conditionals which are produced in a monologue setting and some of the conditionals produced in a dialogue or lecture, all of which together express the signer's train of thoughts, have eye gaze directed upward or somewhere in front. Those conditionals which are directly addressed to the dialogue partner in the sense of 'If YOU come, I ...' or those which are in general addressed to the dialogue partner have gaze toward the dialogue partner or the audience (in lectures). A detailed description on the 'hypothetical space' is described in 6.4.3. 
- Third, the hypothetical character of the conditional construction may be expressed or even supported by modality markers providing the conditional construction with a higher degree of 'potentiality'. Which modality markers are obvious in conditional constructions is discussed briefly in 5.7.3.

With regard to causality, it can be summarized: It is true of all the present conditional constructions that they express an antecedent and a consequent. To this end, the protasis conveys the condition and the apodosis the consequence of that condition. In these cases a causal relation between two propositions is manifested. However, several of the conditional constructions have an interrogative as consequence. In these cases the signers express their indecision as to how to act when a special condition occurs. Moreover, counterfactual conditionals do convey a causality-relation between the two propositions, but the primary goal is not expressing this causal relation. For example, as shown in (97), an informant signs that 'if she had lost, she wouldn't have received 15 Euros; but this is not the case: she has won.' Indeed, the two propositions of the conditional clause convey a causal relation. However, the intention of the signer is to underline that she has won and what this entails.

Also, concerning causality, it has to be mentioned that causal relations between two propositions are not only expressed through conditional constructions. Interrogativeanswer-pairs can also be used to express a causal relation. This seems to be the most significant hint as to why the form of the non-manual markers for both constructions is so similar (more on that discussion cf. 8.3.3).

What stands out is that in all conditionals in the data the protasis (if-clause) precedes the apodosis and never the other way around. This phenomenon is also described by Pfau (2008) as one of the characteristics of conditionals (meaning the if-clause) as well as of topics in NGT and ASL ${ }^{153}$.

\footnotetext{
${ }^{153}$ Reilly et al. $(1990,375)$ reported that this arrangement is obligatory in ASL.

Auer (2000) observed that in spoken German there is a high preference on pre-positioning of 'wennclauses' ('if-clauses') conditionally used. To be exact, in German 'wenn' is used for both temporal
} 


\subsection{The conditional markers}

The conditionals present in the data are almost all indicated by positioning the 'head forward'. Occasionally, instead of head forward the marker 'chin down' occurs. The marker 'raised brows' also functions as conditional marker. It alternates with the head marker, can additionally occur with it, or is not produced. The marker 'shoulders forward' also provides the construction with conditionality. It occasionally co-occurs with the head or brow conditional markers. Finally, the element 'wenn' ('if') can occur in clause-initial position of the conditional clause.

\subsubsection{The marker 'head forward'}

\section{The hf-marker}

In the corpus in which the informants express their thoughts, the annotators identified 'positioning the head forward' as the most used marker for conditionals. It co-occurs with the antecedent of the conditional construction. Figure 5.2 shows three different head positions which occur in example (80) and Figure 5.1. The first and last picture display the neutral head position, the first occurring before the conditional clause, last when formulating the apodosis. The second and third picture shows the signer significantly moving his head forward ${ }^{154}$. Especially while formulating the condition, the head is moved forward in an intensified way. This is annotated as 'head forward-large'. The same pattern is obvious in embedded polar interrogatives (cf. 4.3.1.2.1).

('when') and conditional ('if') constructions. With 'wenn' conditionally used the protasis-apodosis sequence is with a ratio of 3:1 while in written German it is the other way around. Ford \& Thompson (1986) have comparable findings for spoken English. Following Auer's (2000) argumentation, one reason for the protasis-apodosis arrangement in sign languages is that in an online production the temporally sequential aspect of syntactic complex structures seems to be more important. Also, the advantage of a protasis-apodosis sequence is that first the background information (the condition) is introduced, and then the consequence can be clearly associated with this information. Other reasons for this restricted arrangement are also imaginable and need further analysis.

154 Especially the chin is moved forward, but all annotators describe this movement as 'head forward'. Investigations on phonetics go beyond the present thesis, but would provide important information on that matter. 

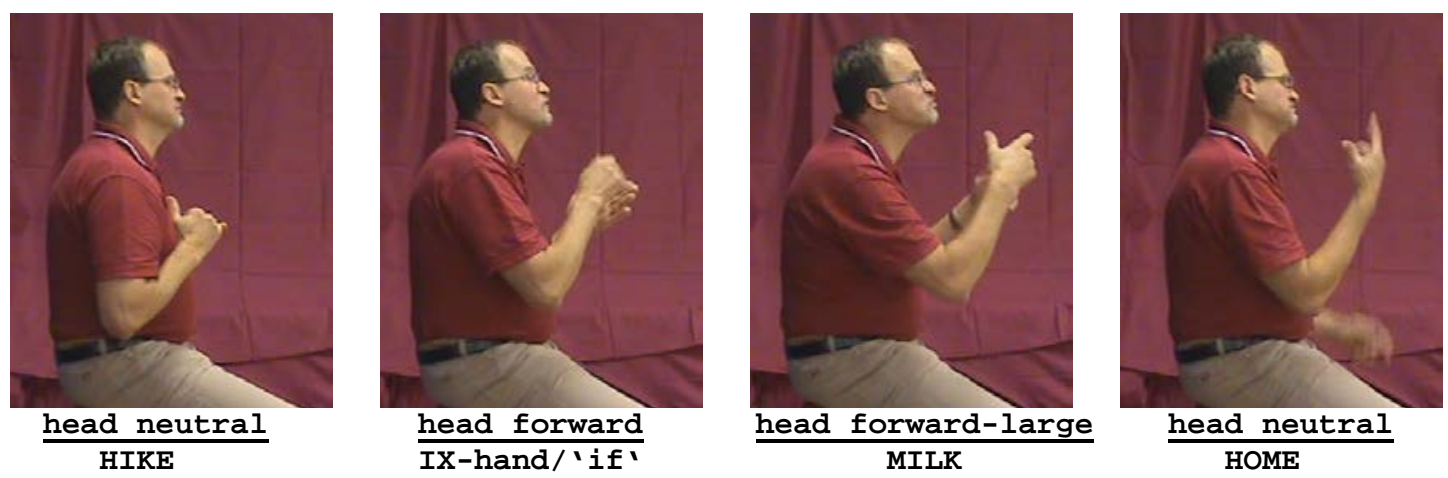

Figure 5.2 'Head forward' and 'head forward large'

The conditional marker head forward is used even if, for reasons of semantics, a forward and backward movement of the head would be expected. In (82), the oppositions of 'being open' versus 'being closed' are given. When this opposition occurs in discourse, it is frequently covered by a forward versus backward movement of the head and/or body. Example (82) clearly shows that both times when the head is positioned forward (encircled red), this constitutes pure conditional markers. First, the head movements cover the entire antecedent of the conditional construction, second, both options ('being open' and 'being closed') are covered by the same head marker, and finally, the annotators allocate a conditional function to the head marker. Moreover, further indicators are present that cooccur with a conditional construction like gaze up which starts with the sign THINK, covers the entire train of thought, and refers to the 'hypothetical space' (cf. 6.4.3).

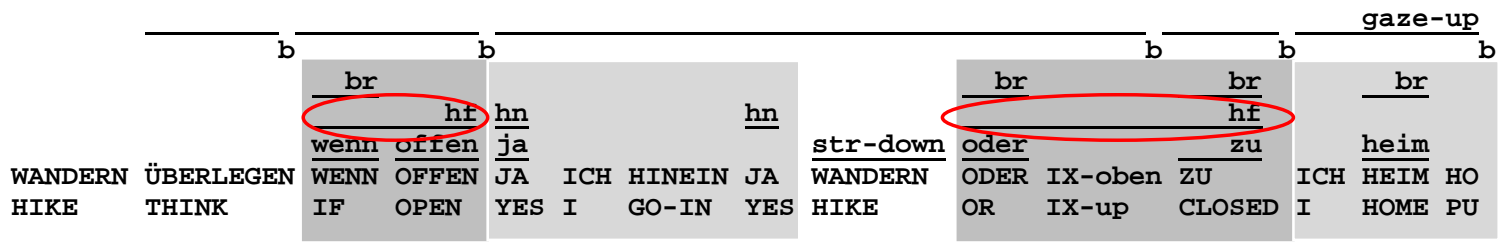

Während ich wandere überlege ich, dass wenn (die Hütte) offen hat, ich hineingehen werde und dass wenn sie zu hat, ich heimgehen werde.

While hiking I'm thinking that if (the hut) is open, I will go in; if it is closed, I will go home.

(M002_114,126_m_thoughts_ex06a_08.05-08.18) 


\section{The cd-marker}

In some conditional examples the annotators identify 'chin down' instead of or in addition to 'head forward' or 'brow raise' (see examples (82), (90) and (91)). The reasons for using chin down as alternative marker to head forward may be based on the similarity between question-answer-sequences and antecedent-consequent-sequences of conditionals (cf. 8.3.3). There may be other reasons which will be investigated in the future.

\section{The hf-marker and other head markers}

The 'head' as an articulator can code different functions. For example, the marker head forward can co-occur with headshakes (cf. 5.6.1) or with head tilt sideward (cf. 5.6.2). Also, a signer can mark a conditional clause with head forward and equally express listing items, which is coded by moving the head/body forward. This is illustrated in (83).

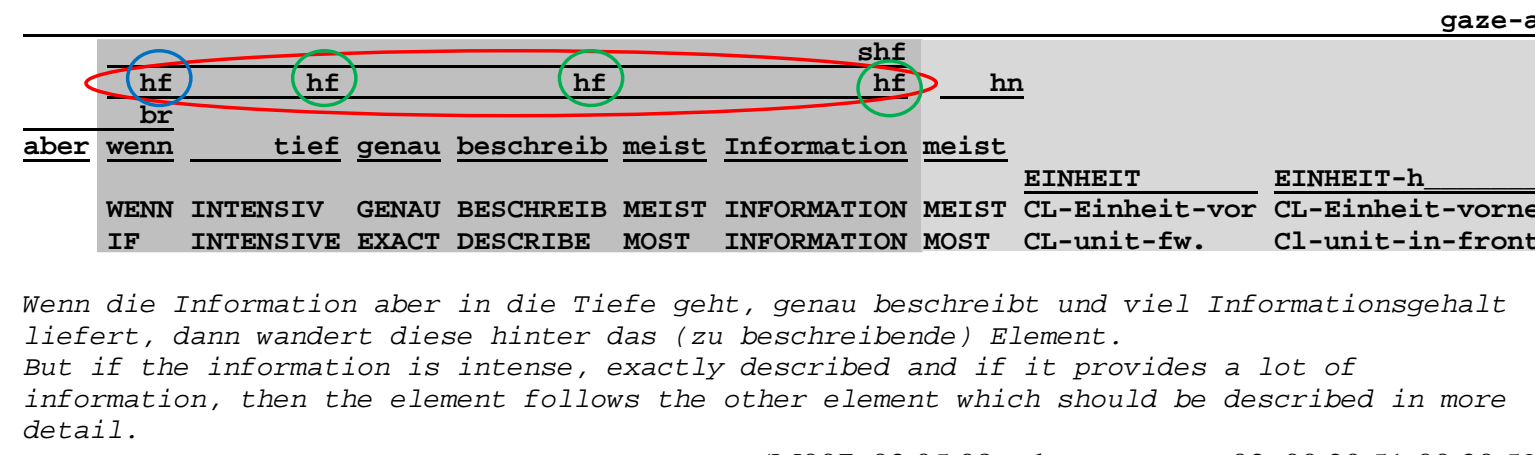

(M007_02.05.08_edu.course_part02_00.20.51-00.20.59)

In (3), the conditional marker head forward (encircled red) covers the protasis (color-coded dark grey) of the conditional. In formulating the conditional clause, the signer wants to list three conditions which cause a syntactic element to be produced after another syntactic element. As listing is also produced by moving the head and/or body forward along the sagittal axis, parallel to the frontal plane, the following pattern occurs. The signer starts positioning the head forward when signing 'wenn' ('if'), indicated by the first 'hf' (encircled blue). Afterwards, the head is held in a slightly forward position and moved further forward when listing the particular conditions. The particular end point of the forward movement is indicated by the abbreviations ' $\mathrm{hf}$ ' (each encircled green), occurring 
towards the end of the signs INTENSIVE, DESCRIBE, and INFORMATION. Consequently, only one marker is identified but this marker is intensified when indicated as 'hf'.

Summing up, it can be stated that the conditional marker head forward covers the entire protasis of the conditional construction. This is the marker which is used consistently by all informants, and many of them make use of only that conditional marker.

But some of the signers, in fact those having Deaf parents, differ in their use of conditional markers. These signers use raised brows as an alternative or additional marker to the described head forward, and sometimes also shoulder forward, as described in the following.

\subsubsection{The alternative/additional marker 'brow raise'}

In (84), the informant signs (more or less) the same situation as in example (84). However, it is significant that in (84) the antecedent of the conditional construction is covered by 'raised brows' (abbreviated as 'br-marker') instead of positioning the head forward.

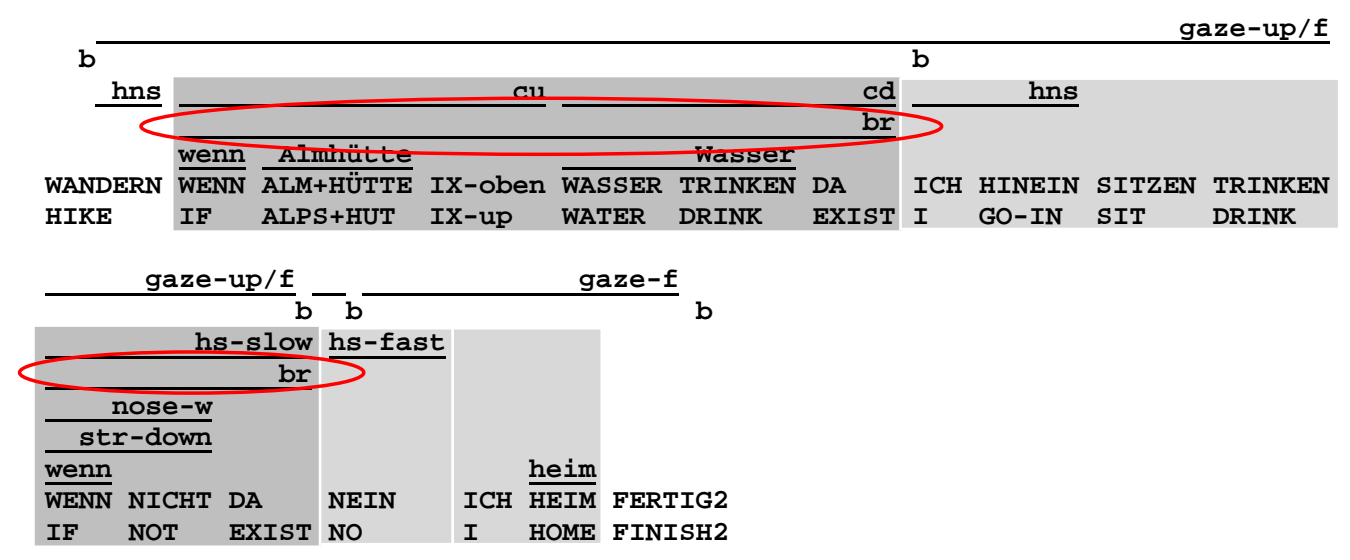

Während ich wandere überlege ich, dass wenn ich in der Almhütte Wasser zum Trinken bekomme, ich mich hineinsetzen und etwas trinken werde. Wenn es nichts gibt, dann bleibe ich nicht, sondern gehe ich heim.

While hiking I think that if I get water (to drink) in the hut, I will go in, sit down and drink water. If there is no water (to drink), I won't stay, but go home.

(M002_92,1184_m_thoughts_ex09a_02.12-02.24) 
In addition, the example (84) ${ }^{155}$ shows that the br-marker is used in positively formulated conditions as well as in conditions which are negated. The annotators identify it as having the same function as the above-mentioned head forward conditional marker. Using the alternative conditional marker raised brows is probably due to the fact that the head is used for other functions, and consequently, for disambiguation purposes, the brows take the function of indicating a conditional.

Independent of the context and thus of the particular lexical elements, the conditional marker raised brows is used instead of or in addition to the conditional marker head forward. In (85), the signer wonders whether the train will arrive on time (three o'clock) and whether the shops will still be open. The antecedent conveys the condition and is covered by raised brows (encircled red), the consequent constitutes an 'embedded polar interrogative' (more in 5.5.2) which is covered by head forward (encircled rose). The last sign of each syntactic clause is covered by moving the body forward. This probably is done to mark the boundary (compare to this the discussion on head thrust in ASL, cf. 5.2). $(85)^{156}$

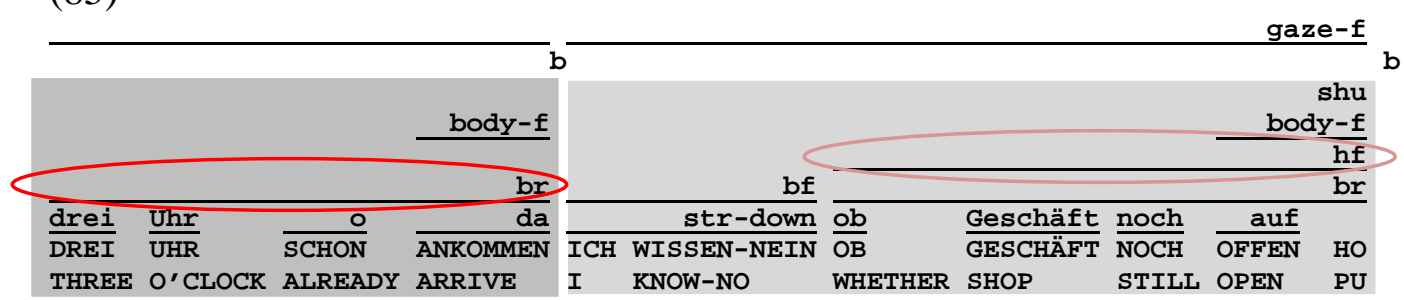

Wenn ich schon um drei Uhr ankomme, weiß ich nicht, ob die Geschäfte noch offen haben. If $I$ arrive at three o'clock, I don't know whether the shops will be still open.

(F001_024_m_thoughts_00.01-00.10)

\footnotetext{
${ }^{155}$ The informants always produced the short stories containing train of thoughts twice. In the present case, the change in the second production was significant in that the marker chin up only covers the sign WENN (IF); the remaining part of the antecedent is covered by chin down. The functions of these different head movements are unclear so far.

The informant starts looking upward when she starts her train of thought with a nodding movement expressing assertion/affirmation. Partly the gaze direction goes a bit downward to a non-specified location somewhere in the front (abbreviated as gaze-up/f). Afterwards, the signer clearly stares somewhere to the front.

In (84), the negator (here 'slow headshakes') covers the entire protasis of the conditional clause. Fast negative headshakes follow, expressing 'no, I won't stay' (more on that cf. 3.1.3 and 3.1.4).

156 The form of the sign ARRIVE is only in one feature different to the sign EXIST. It includes a forward movement which can be adapted to a specified location (which, for example, depends on a spatial location taken from an absolute frame of reference or a specified location for a referent).
} 
Summing up, the markers head forward as well as brow raised are clearly identified as conditional markers by the annotators. The data shows that both markers can be used together or separately. Moreover, it is obvious that when the head or the brows are used for indicating a conditional, the other marker will be used for other purposes, as shown in example (83), where the head constitutes the conditional marker and raised brows is used to make the first two lexical elements more prominent. This frequently occurs in the educational corpus when the Deaf lecturers want to add information on a topic. In example (84), the marker brow raise constitutes the conditional marker and the head is used for other purposes in both conditionals.

\subsubsection{The additional marker 'shoulders forward'}

In some conditionals, the signers thrust their shoulders forward when formulating the condition. This additional conditional marker optionally co-occurs with the markers head forward and raised brows and covers the entire antecedent. This is exemplified in Figure 5.3 and example (86).

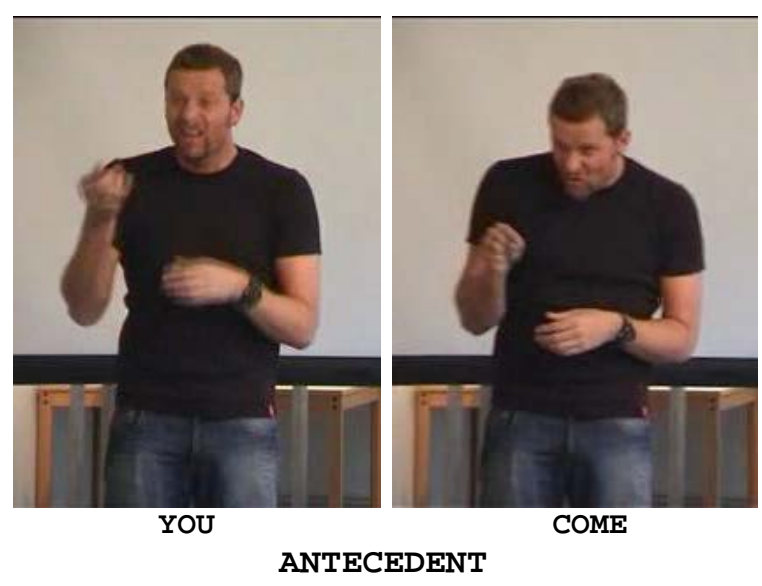

Figure 5.3 'Shoulders forward' in conditionals (M007_04.05.08_edu.course_part01_00.11.28-00.11.31)

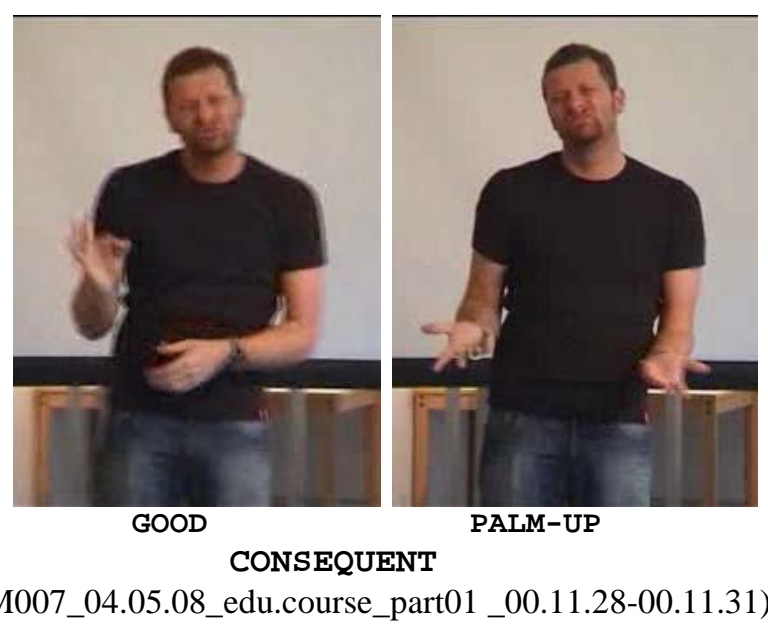


(86)

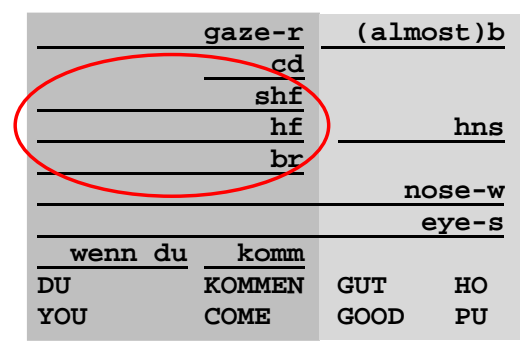

Wäre schön, wenn du kommen würdest. It would be nice if you came.

(M007_04.05.08_edu.course_part01_00.11.28-00.11.31)

The signer produces the antecedent (color-coded dark grey) with all listed non-manual markers, that is, head forward, brow raise, and shoulders forward (encircled red). The second lexical element additionally gets a downward movement of the chin which is identified as chin-down (or head nod). Furthermore, the signer produces the mouthing 'wenn' ('if') in clause-initial position of the apodosis. The consequent (color-coded bright grey) is covered by head nods expressing assertion in the sense of 'yes, it would be nice' (cf. 3.2.3.1 and 5.6.1). Two further non-manual markers co-occur with the entire conditional clause, which are squinted eyes and wrinkled nose (cf. 5.6.3 and 7.3.2).

\subsubsection{The marker 'wenn' ('if')}

In various conditionals the element 'wenn' ('if'), signed (see Figure 5.4 illustrating two variants ${ }^{157}$ of the sign WENN (IF) in the data) or only mouthed is found in clause-initial position of the protasis. When the mouthing 'wenn' ('if') occurs, it accompanies the sign IF or a pointing sign (cf. example (80)), or it goes together with the first sign of the ifclause.

${ }^{157}$ There are other variants of the sign IF in ÖGS too. 

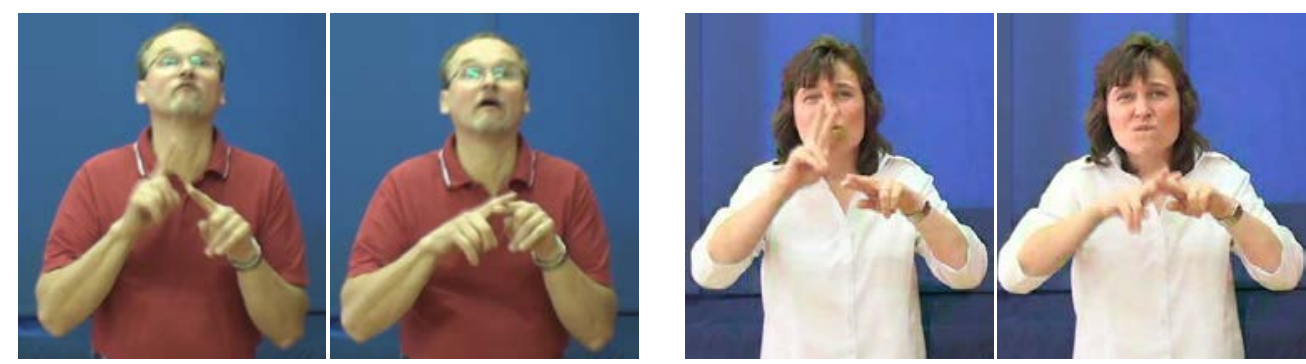

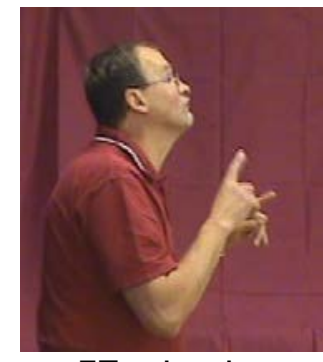

IF-start

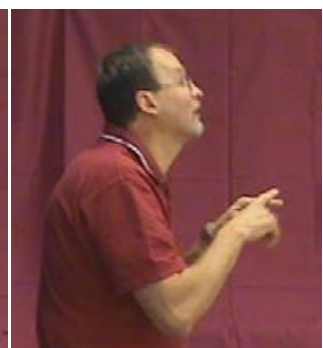

IF-end

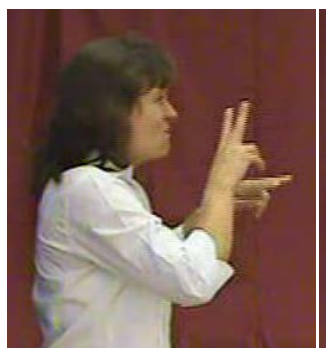

IF-start

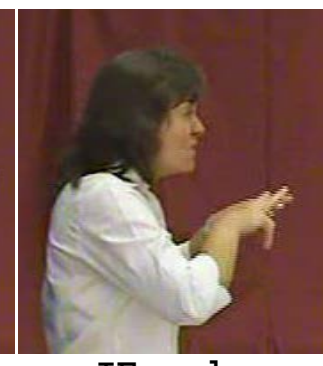

IF - end

Figure 5.4 Two variations of the sign WENN (IF) in frontal and side view

In Figure 5.4, in addition, all non-manual conditional markers which start with the if-sign are obvious, that is, head forward, raised brows and shoulders (slightly) forward.

\subsection{Consequent clauses}

The present conditionals all constitute two (in some cases even three) clauses which have a semantic as well as a syntactic relation. In all cases, the if-clause precedes and never occurs on its own. It always has at least one consequent. This can be a statement, an interrogative, or an exclamation. When an embedded polar interrogative construction follows, the consequent contains a verb of cognition and its interrogative complement.

The data shows too that it is possible that an antecedent has two consequents. In (11) the consequences constitute first, a content question and second, a polar question.

In all clauses, both protasis and apodosis, the predicate (usually a verb or a negation element) is in clause-final position. In all cases where the lexical elements 'if' (in the protasis) or 'head nod' (in the apodosis; cf. 5.6.1) occur, they are in clause-initial position. 
With regard to the non-manual markers which may co-occur with conditional constructions, it is significant that while some of them only cover one clause, others cover the entire conditional construction ${ }^{158}$.

\subsubsection{Statement as consequent}

The conditional construction cited most frequently in other sign languages is the one in which the antecedent receives a conditional marker, providing the if-clause, and the antecedent gets no conditional marker and serves as a statement which conveys the consequence.

This default case is illustrated in the following:

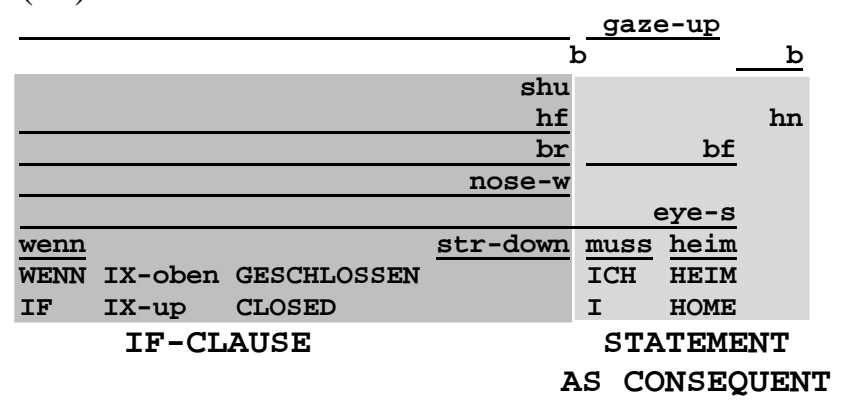

Wenn oben geschlossen ist, kann man nichts machen, dann muss ich heimgehen.

If it is closed up there, well then, ( $I$ guess that) I'll have to go home.

(F004_118,1210_m_thoughts_06.08-06.13)

In (87), the condition (IF IX-up CLOSED) together with the signer's comment on that situation (expressed by the mouth action 'stretched-down' and a shoulder shrug, both together conveying the meaning of 'well then, I guess that ...' ) are covered by the conditional markers head forward and brows raised. The consequent constitutes the statement 'then I'll have to go home'.

The consequent also can be a statement, even if the sign WAS (WHAT) precedes the formulated consequence. In this case the markers chin up and/or head forward, used for content questions, is not present. Following Wilbur (1996), in such a case a wh-cleft with a focusing function is present (cf. 3.2.3.1). Thus, I conclude that the consequent is not a

\footnotetext{
${ }^{158}$ For more on this, compare the following subchapter (5.6) on negation or modality in conditionals.
} 
content question. It is a clear consequence formulated as a statement of the preceding condition of the protasis. In (88), a signer explains the order of elements. This new information is displayed as the consequence under which conditions this arrangement occurs. The consequent is introduced by a head nod which follows the sign WHAT together with the mouthing 'das' ('that'). Subsequently, the new information is provided constituting the consequence of the preceding condition.

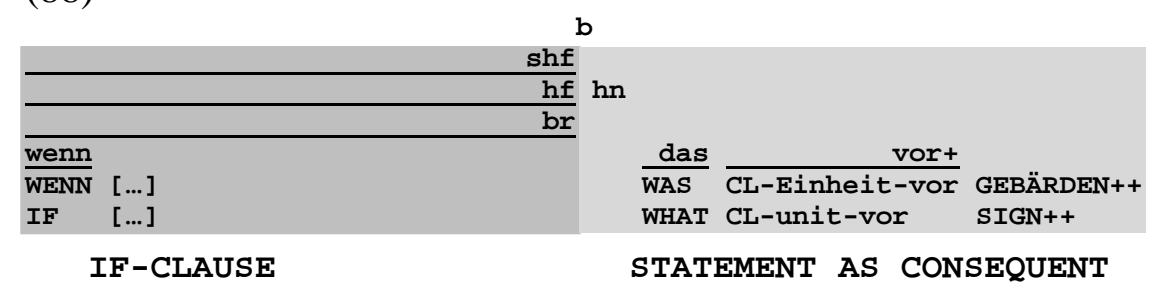

Wenn $[\ldots]$, dann rückt das Element nach vorne und es wird in dieser Reihenfolge gebärdet. If $[\ldots]$, then the element moves into a preceding position and it is signed in that order. (M007_02.05.08_edu.course_part02_00.20.42-00.20.51)

As the data shows, the consequent can also be a real interrogative, as will be discussed in the next section.

\subsubsection{Interrogative or other clauses as consequent}

Some sign language researchers have observed that the consequent may be an interrogative too. For instance, Johnston \& Schembri $(2007,214)$ quote the following conditional construction for Australian Sign Language (Auslan):

$(89)^{159}$

$\frac{\text { br-htb }}{\text { I-F WIN L-0-T-T-0 WHAT D-0 PRO-2 }}$
If you won Lotto, what would you do?
(Johnston \& Schembri 2007, 214)

In their example (89), the if-clause receives the conditional markers (brows raised and head tilt backward), and the consequent, constituting a content question, is covered by the whquestion marker brows furrowed. The same way of marking both clauses separately by the respective markers is present in ÖGS too. The protasis is covered by head forward and/or

$\overline{159}$ The abbreviations are: br - brows raised, htb - backward head tilt, bf - brows furrowed; 
brows raised, and optionally by shoulders forward. The apodosis is covered by the particular interrogative markers. If the consequent constitutes a content interrogative, the marker head forward is produced; If it is a polar question, the marker chin down is obvious; in the case of an embedded polar interrogative or a hope-that-construction, the marker used is head forward; If a special form of interrogatives, marked with head backward, constitutes the consequent of conditionals, this clause is also marked by head backward $^{160}$. The occurrence of a content interrogative as consequent of a conditional and the occurrence of two successively occurring interrogatives, each constituting a consequent of a conditional is exemplified in the following.

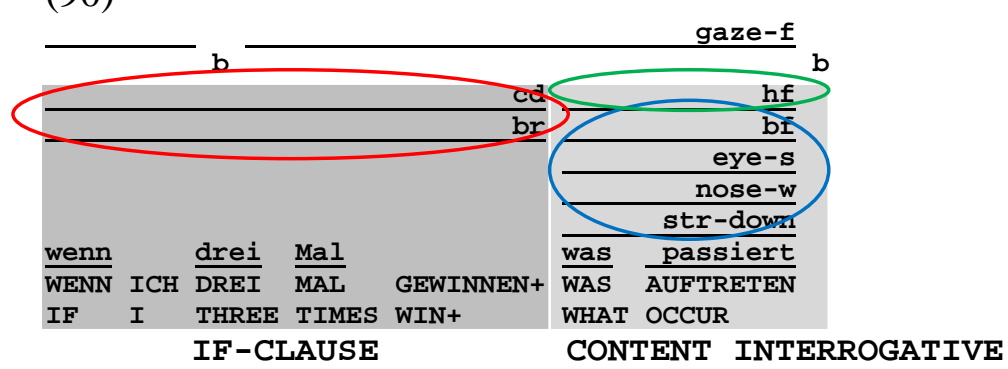

[Wärend ich Autofahre überlege ich, dass] Wenn ich drei Mal gewonnen hätte, was wäre dann passiert?

[While I drive I think that] If I had won three times, what would have happened?

(F001_037_m_thoughts_00.06-00.12)

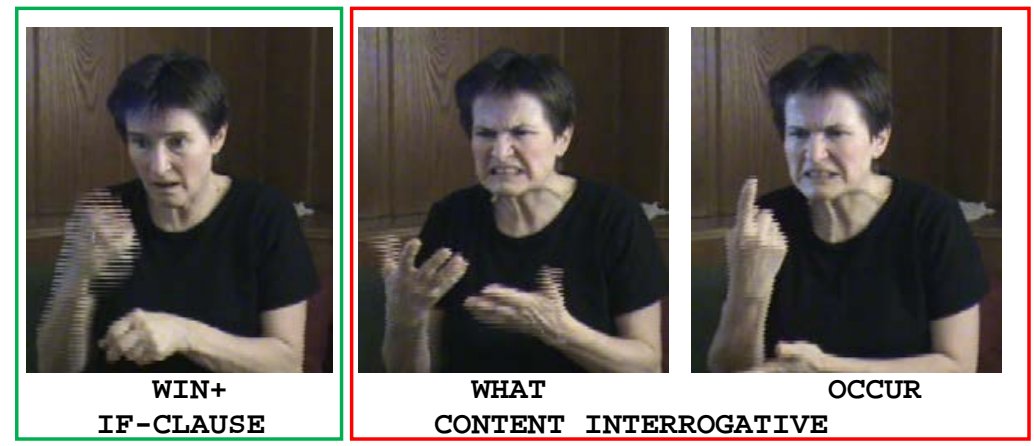

Figure 5.5 Marking the if-clause with brow raise \& chin down and the consequent with head forward

In example (90), illustrated in Figure 5.5, the signer wonders what she would get if she won three times. This statement was produced three times by the signer, each time with slight differences, but with the same head and brow markers covering the protasis and the

${ }^{160}$ A detailed description of the various markers is found in chapter 4. 
apodosis. So, the if-clause is covered by raised brows and chin down (encircled red). The content interrogative is covered by the marker head forward (encircled green). In addition, several other non-manual markers co-occur with the content question (encircled blue). One or more of these markers probably express modality in the sense of providing the construction with potentiality and (lack of) knowledge (cf. 5.6.3). During both clauses the signer looks somewhere into the front - clearly not to a present or imagined dialogue partner.

In (91), two consequents follow the antecedent, constituting a content and polar interrogative, the latter expressed in an exclamative way. The informant signs that while walking, she is feeling doubtful as to what would happen if she did not hand him the letter and whether he would be offended by her.

$(91)^{161}$

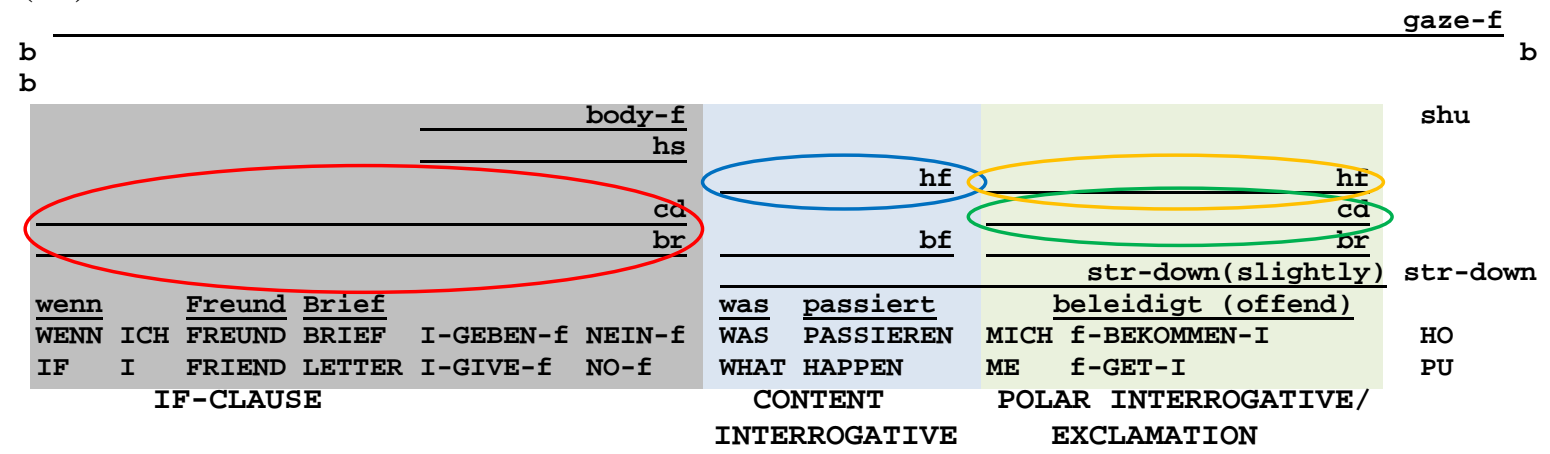

Ich weiß nicht, was passieren würde, wenn ich dem Freund nicht den Brief gebe und ob er auf mich beleidigt wäre?!

I don't know what would happen if I did not give the friend the letter and whether (in doing so) he would be offended (by me)?!

(F001_046_m_thoughts_00.01-00.10)

All clauses are covered by different non-manual markers that clarify their functions. The conditional markers (encircled red) are chin down and raised brows covering the if-clause (color-coded dark grey). The clearly identified interrogative marker (encircled blue) is head forward, covering the first consequent which displays a content interrogative (colorcoded blue). According to the annotators, the following clause is a polar interrogative

${ }^{161}$ I-GIBE-f means that the movement starts from the signer and goes somewhere to the front. 
(color-coded green) marked by chin down (encircled green) as well as an exclamation due to the co-occurring marker head forward (encircled orange).

Both interrogatives convey a meaningful linking to the condition in the way that they ask for the possible consequences. The first one - formulated in a content question - leaves open many possible consequences; the second one limits this huge variety of consequences and leaves open only two possible options. From a syntactic point of view, the if-clause requires at least one consequent, and two of them are offered.

An interrogative clause constituting the apodosis in conditional construction may also be an embedded polar interrogative marked by head forward. This is illustrated in (84). In this example, the verb phrase with the cognitive sign KNOW-NO on which the embedded interrogative clause depends on, precedes the part that is put into question. It could also be omitted so that the dependent interrogative clause directly follows the if-clause.

Although the conditional clauses, described in this sub-chapter, implies a hypothetical character, the conditional construction as whole also includes a speech act. Reilly et al. (1990, 376) investigated the child's acquisition of conditionals in ASL. For figuring out whether a child can understand the semantics of a conditional situation, they implemented a comprehension task. The given example for this task is a conditional, constituting an ifclause and an interrogative clause as consequent. To respond correctly to a conditional requires understanding more than just its hypothetical character or its causal relation. It also requires that the conditional has to imply a speech act to which the asked person can respond. For this reason it is supposed that the conditionals for the comprehension task were of this kind of conditionals. Dancygier \& Sweetser (2005, 16 and 113-117) label these conditionals as 'speech act conditionals'. According to them, a situation based on conditional information formulated in the if-clause is imagined in which the actor acts in the form of a speech act, given in the apodosis. In (90), the conditional information of if winning three times' is thought and then the speech act 'what would happen in that situation' is pictured. Consequently, the focus of 'speech act conditionals' is on the speech act rather than on the content. 
As has become obvious in previous examples, other functions are marked in conditionals too. In what follows, first, negation and assertion in conditionals are described. Second, the marking of alternativity in conditionals is presented. Finally, modality markers cooccurring with several conditional constructions of the ÖGS data are analyzed.

\subsection{Other functions marked within conditionals}

In the following, other functions which frequently occur in conditionals are described. These are negation and assertion coded by negative headshakes and assertive head nods, marking of alternativity coded by spatial indicators, and finally, the occurrence of possible modality markers in conditionals.

\subsubsection{Marking negation and assertion in conditionals}

Examples of negation are present in various conditionals in the data. In this subchapter, I first focus on headshakes which may co-occur with other head markers. Then, I discuss marking of assertion in conditionals.

\section{Marking of negation in conditionals}

Since the antecedent of conditionals is marked primarily by head forward in Austrian Sign Language, the interplay of head forward and headshakes is first demonstrated. Table 5.1 together with the examples shows the syntactic constituent that is covered by headshakes in conditionals. It is necessary to distinguish between headshakes that function for clausenegation and headshakes implying negative contrast (a detailed description on both functions is given in 3.1.3 and 3.1.4). In addition, the sign order is given in Table 5.1 with examples. 


\begin{tabular}{|c|c|c|c|c|}
\hline & $\begin{array}{l}\text { Syntactic constituent } \\
\text { covered by 'hs' }\end{array}$ & $\begin{array}{l}\text { Function in } \\
\text { protasis }\end{array}$ & $\begin{array}{l}\text { Function in } \\
\text { apodosis }\end{array}$ & Prototypical illustration \\
\hline a) & entire antecedent & $\begin{array}{l}\text { clause- } \\
\text { negation }\end{array}$ & & $\begin{array}{l}\frac{\mathbf{h s}}{\mathbf{h f}} \\
\text { HUT OPEN (N0) I HOME } \\
\text { If the hut is not open, I'll go home. }\end{array}$ \\
\hline b) & $\begin{array}{l}\text { negator only covers the } \\
\text { negated predicate of the } \\
\text { antecedent }\end{array}$ & $\begin{array}{l}\text { clause- } \\
\text { negation }\end{array}$ & & 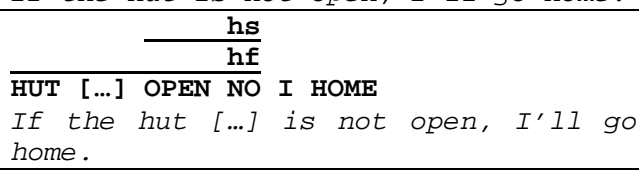 \\
\hline c) & $\begin{array}{l}\text { entire antecedent; first } \\
\text { part of the consequent }\end{array}$ & $\begin{array}{l}\text { clause- } \\
\text { negation }\end{array}$ & $\begin{array}{l}\text { implying } \\
\text { negative } \\
\text { contrast }\end{array}$ & $\begin{array}{l}\frac{\text { hs }}{\text { hf }} \frac{\text { hs }}{} \\
\text { HUT NOT OPEN No I HOME } \\
\text { If the hut is not open, no (then I } \\
\text { won't stay), then I'll go home. }\end{array}$ \\
\hline d) & $\begin{array}{l}\text { entire antecedent; } \\
\text { entire consequent }\end{array}$ & $\begin{array}{l}\text { clause- } \\
\text { negation }\end{array}$ & $\begin{array}{l}\text { implying } \\
\text { negative } \\
\text { contrast }\end{array}$ & $\begin{array}{l}\text { hs } \\
\text { HUT OPEN (N0) I HOME } \\
\text { If the hut is not open, I'll go home } \\
\text { and won't stay. }\end{array}$ \\
\hline e) & $\begin{array}{l}\text { entire antecedent; } \\
\text { entire consequent }\end{array}$ & $\begin{array}{l}\text { implying } \\
\text { negative } \\
\text { contrast }\end{array}$ & $\begin{array}{l}\text { implying } \\
\text { negative } \\
\text { contrast }\end{array}$ & $\begin{array}{l}\text { hs } \\
\text { hf } \\
\text { HUT CLOSED I HOME } \\
\text { If the hut is closed - so not open, } \\
\text { I'll go home and won't stay. }\end{array}$ \\
\hline f) & entire consequent & & $\begin{array}{l}\text { clause- } \\
\text { negation }\end{array}$ & \begin{tabular}{l} 
hf $_{$\cline { 2 - 2 } \text {$HUT OPEN I (NOT) HOME }}$ \\
If the hut is open, I won't go home.
\end{tabular} \\
\hline g) & $\begin{array}{l}\text { entire antecedent; } \\
\text { entire consequent }\end{array}$ & $\begin{array}{l}\text { clause- } \\
\text { negation }\end{array}$ & $\begin{array}{l}\text { clause- } \\
\text { negation }\end{array}$ & $\begin{array}{l}\text { hf } \\
\frac{\text { hs }}{\text { HUT OPEN (NO) }} \\
\text { If the hut is not open, I wo) }\end{array}$ \\
\hline h) & $\begin{array}{l}\text { second part of } \\
\text { antecedent }\end{array}$ & $\begin{array}{l}\text { clause- } \\
\text { negation } \\
\text { (covered part) }\end{array}$ & & $\begin{array}{l}\text { hf } \\
{ } \frac{\mathbf{h s}}{\text { NOT CLOSED I STAY }}\end{array}$ \\
If the hut is open and (if) not \\
closed, I'll stay.
\end{tabular}$} \\
$\hline$\end{array}$

Table $5.1^{162}$ Negation and implying negative constrast in conditionals

The possibility (a) in Table 5.1 is that the negative headshakes cover the antecedent and negate its content. The consequent remains non-negated. This is shown in example (92). In the corpus, this conditional clause follows another one which is formulated in a positive way. So, the informant signs that if the hut is open, she will sit down and drink something. This is the reason why in (92) the sign OPEN is not present. The negator is encircled orange, the conditional marker red and the presumed modality markers blue.

162 Both options are possible: NOT OPEN and OPEN NOT. For this, two different negation elements are used. The same is possible with the signs CLOSED or STAY. The sign HOME just occurs as NOT HOME. 
(92)

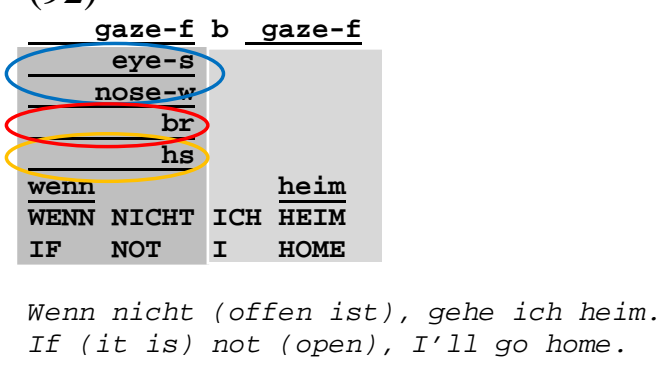

(M001_091,1183_m_thoughts_ex03a_01.08-01.10)

The same result is present when an ÖGS-signer only produces negative headshakes along the negated predicate (more on the spreading of negative headshakes and the influence of negative polarity context in 3.1.3.3 and 3.1.3.5), found in clause-final position of the protasis. Example (93) shows possibility (b) in Table 5.1.

$(93)^{163}$

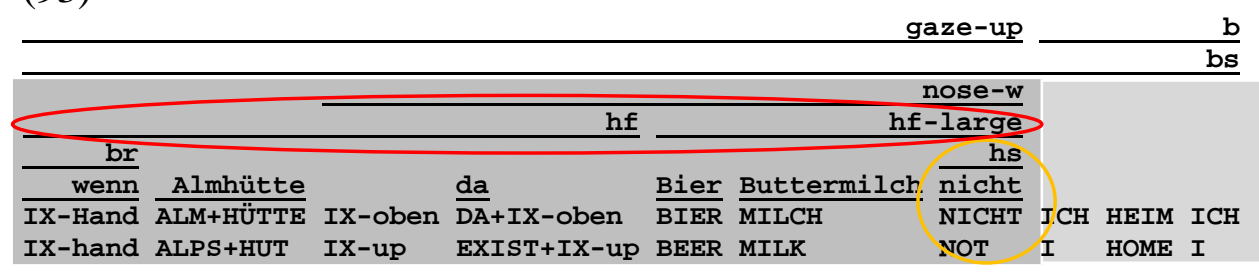

Wenn es in der Almhütte weder Bier noch Buttermilch gibt, gehe ich heim. If there is neither beer nor buttermilk in the hut, I'll go home.

(M001_091,1183_m_thoughts_ex04a_01.14-01.19)

As presented, only the sign NOT, being predicatively used and expressing non-existence, is covered by negative headshakes. So, in (93) the informant could also have signed GIBTNICHT (EXIST-NOT) or BEKOMMEN NEIN (GET NO) instead of NICHT (NOT) alone. In these cases, the negated verb or the verb together with the negation element are covered by headshakes ${ }^{164}$.

As a rule, the data shows that if the antecedent has few lexical elements like as (92), the entire clause is covered by the negator headshakes. If the antecedent contains more lexical elements as in (93), only the negated verb phrase is covered by headshakes.

\footnotetext{
${ }^{163}$ The example is the continuation of example (80), so only the conditional clause is given in (93). The marker head forward produced in different intensity is the conditional marker. Wrinkled nose and body sways very probably display modality markers (cf. 7.3.2 and 7.4.4).

${ }^{164}$ Both listed options are present in the data.
} 
A further variation, illustrating possibility (c) in Table 5.1, is present in the ÖGS data. In these conditionals, the signers negate the antecedent by headshakes. Afterwards, a negation element together with headshakes follows which is not covered by the conditional marker(s) and consequently, constitutes a part of the consequent. The remaining part of the consequent is not negated. This case is illustrated in (94):

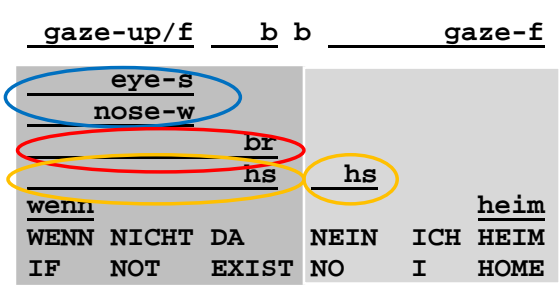

Wenn es nichts gibt, nein (dann bleibe ich nicht), dann gehe ich heim. If there isn't anything available, no (then I won't stay), then I'll go home.

(F001_092,1184_m_thoughts_ex11a2_02.55-02.58)

The next kind of occurrence of the negation marker headshake in conditionals is (95) (displaying possibility (d) in Table 5.1):

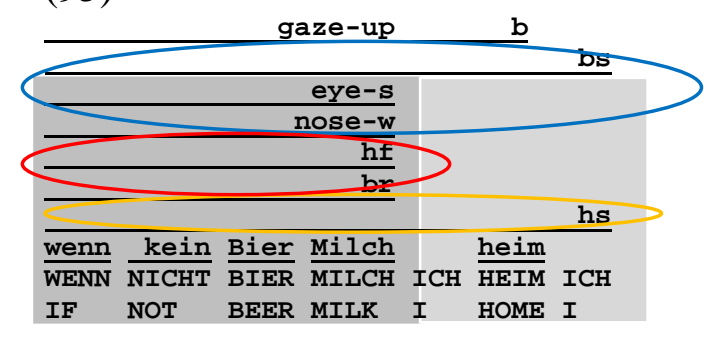

Wenn es in der Almhütte weder Bier noch Milch gibt, dann bleibe ich nicht, dann gehe ich heim.

If in the hut there is neither beer nor milk, then I won't stay, then $I^{\prime} l 1$ go home.

(M001_091,1183_m_thoughts_ex03a_01.08-01.10)

In example (95), the headshakes (hs - encircled orange) covers the entire conditional. The conditional markers head forward (hf) and raised brows (br) (encircled red) cover the antecedent. The markers squinted eyes (eye-s) and wrinkled nose (nose-w) co-occur with the antecedent, the marker body sways (bs) accompanies the entire construction (encircled blue). With regard to the negation marker, it turns out that only the antecedent should be negated, but not the consequent. The headshakes, co-occurring with the entire consequent, 
imply negative contrast. In this context the opposite activity of 'going home' is 'staying there' that is negated.

As example (96) demonstrates, headshakes can cover the entire conditional construction; however, neither of the clauses is directly negated. It is always the implied alternative option to which the headshakes are relevant.

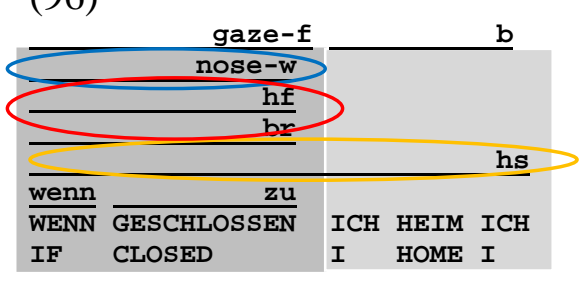

Wenn geschlossen (und nicht offen) ist, gehe ich heim (und bleibe nicht).

If it is closed (and not open), I'll go home (and won't stay).

(M001_091,1183_m_thoughts_ex10_03.33-03.35)

In (96) the opposite option, that is, 'being open' and 'staying', is negated. During the session the signer produces a positively formulated conditional clause which just precedes the one actually illustrated. In this conditional clause, the signer thinks that 'if the hut is open, he will be happy and stay'. So, when successively producing the second (illustrated) conditional, the headshakes associate to that content and imply a negative contrast. In the data the same pattern occurs when a signer formulates an embedded polar interrogative in which two opposed options are signed. When a conditional is produced successively, the same pattern as in (96) is seen.

Of course, it is also possible to only negate the consequent (illustrating possibility (f) in Table 5.1). In the counterfactual conditional (97), ${ }^{165}$ the consequent is covered by headshakes in order to express negation.

\footnotetext{
165 As described in 5.3, the counterfactual conditional provides additional information for identifying the construction as one. In (97) this is covered by head forward, raised brows and head nods. The latter expresses 'affirmation'. According to the annotators, positioning the head forward expresses pragmatic affirmation or head forward and/or raised brows should demonstrate that something is added to the already given information. For clarification this requires further investigation.
} 


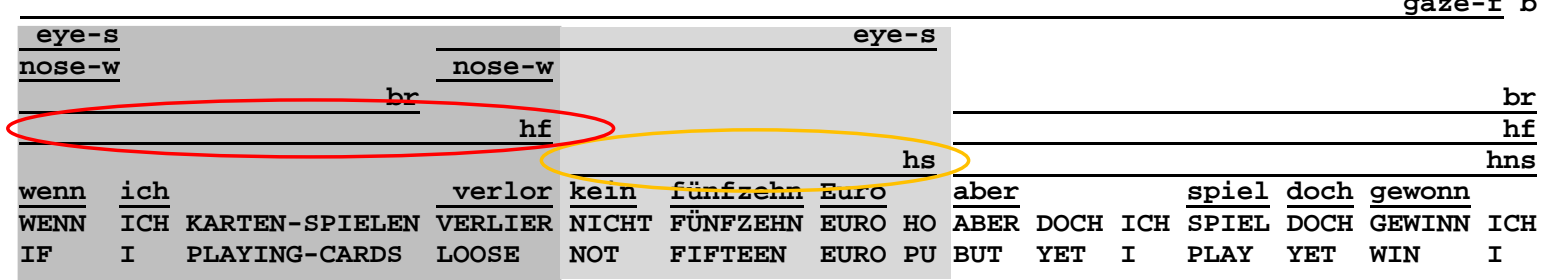

Wenn ich beim Kartenspielen verloren hätte, hätte ich leider keine 15 Euro (bekommen), aber ich habe doch gewonnen.

If I had lost when playing cards, I would not have (received) 15 Euros, but I won.

Furthermore, both antecedent and the consequent can be negated and are covered by negative headshakes. This displays the possibility g) in Table 5.1, exemplified in (98):

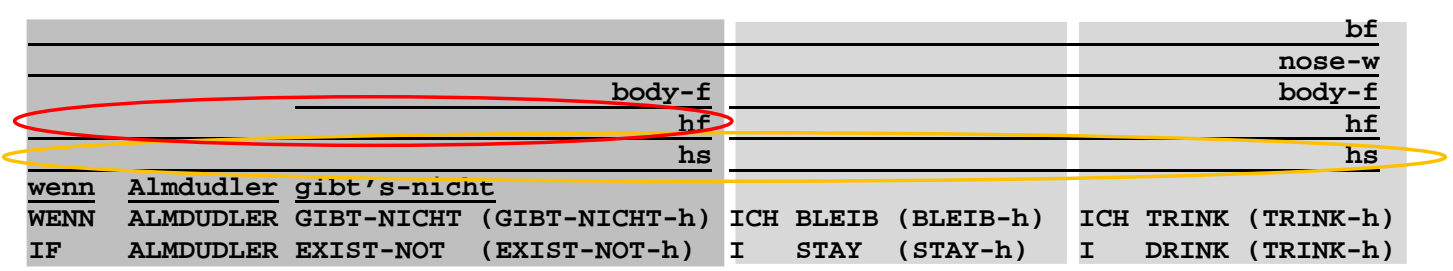

Wenn es keinen Almdudler gibt, dann bleibe ich nicht und trinke nichts.

If there is no almdudler, I won't stay and I won't drink anything.

(M007_1134_m_thoughts_ex.12a_00.00-00.06)

In (98), the entire conditional is covered by headshakes. The antecedent is covered by the marker head forward and in addition, the last sign, which is held for a while, is covered by a stronger forward movement of the head and the body. The consequent is covered by a forward position of the head (and also the body). The co-occurring non-manual markers (wrinkled nose and furrowed eye brows) seem to be modality markers (cf. 5.6.3).

Finally, two conditions can be formulated within a protasis of which one is negated. In such cases, only the negated one is covered by negative headshakes. This possibility (h) in Table 5.1 is illustrated in example (99). 
$(99)^{166}$

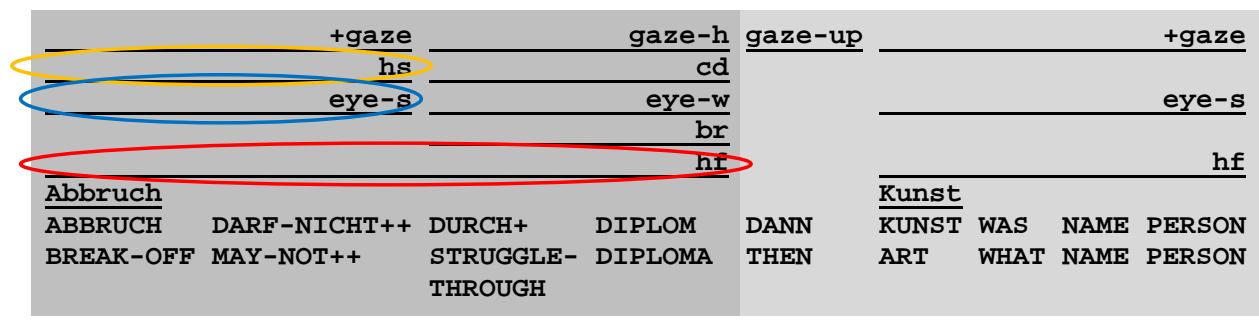

Wenn du nicht abgebrochen und (wenn du) bis zum Diplom durchgebissen hättest, dann wärst du was für ein Künstler?

If you had not stopped and (if you) struggled through to finish the diploma, then what kind of artist would you be?

(M001_Film2_Szene10_d_03.37-03.41)

In a dialogue situation, one of the partners asks the given conditional clause. The entire ifclause is covered by head forward. It is composed of two conditions of which one is negated. This negated condition is covered by negative headshakes and squinted eyes.

Summing up, the ÖGS data shows that in conditionals in which one or both clauses are negated or negative contrast is implied, the conditional marker head forward and the marker headshakes can co-occur. Furthermore, the data shows that in conditionals in which negation is present, there frequently are other non-manual markers, such as wrinkled nose or squinted eyes. This will be discussed in 5.7.3 and 7.3.2.

\section{Marking of assertion in conditionals}

If a consequent of a conditional is formulated in a positive way or even asserted, the following two marking patterns are present. The first pattern is that the positive consequent is introduced by a head nod. The head nod is produced alone or co-occurs with the sign YES or THEN. This head nod never is produced when the consequent is negated. The second pattern is that the positive consequent is completely covered by assertive head nods. Both phenomena are illustrated in the following examples.

\footnotetext{
${ }^{166}$ Gaze directed upward when signing THEN very likely is the reference to the 'hypothetical space' (cf. 6.4.3).
} 
$(100)^{167}$

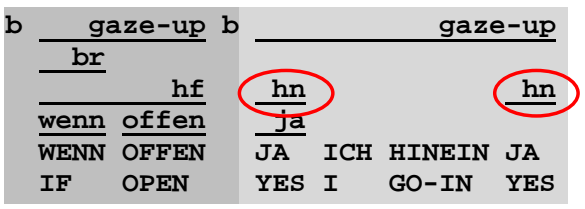

Wenn (die Hütte) offen hat, werde ich hineingehen. If (the hut) is open, I will go in.

(M002_114,126_m_thoughts_ex06a_08.05-08.18)

In example (100), the consequent is introduced by a head nod which covers the sign YES. The positive apodosis is terminated with an assertive head nod covering the sign YES.

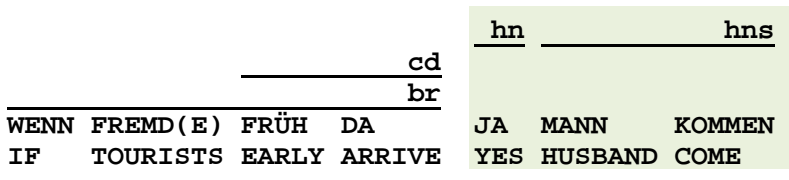

Wenn die Fremden (Touristen) früh da sind, dann kommt mein Mann. If the tourists arrive early, then my husband comes.

(F001_Film1_Szene7_d_informel_story_02.42-02.45)

Example (101) shows that the entire apodosis is covered by nodding movements. The first nodding movement, found in clause-initial position of the apodosis, introduces the consequence. At the same time it is the starting point of the assertion, that is, this intensified nodding movement is separate from the following nodding movements in (101), although it is already the start of the assertive nodding movements.

The subsequent conditional shows that the head movements clearly refer to the positive or negative consequence.

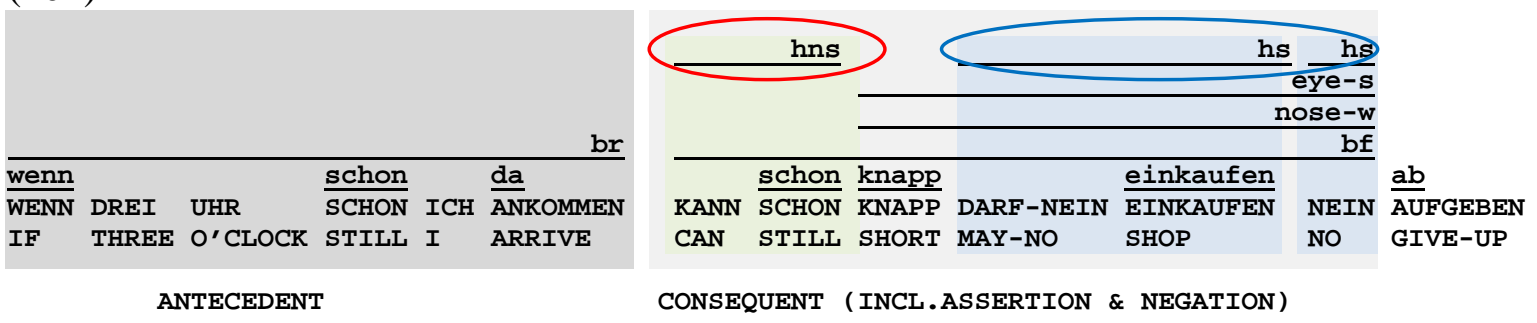

Wenn ich um drei ankomme, dann kann ich schon - ach nein, es wird zeitlich eng, dann schaffe ich es nicht mehr einzukaufen. Ich gebe das Vorhaben auf.

If I arrive at three o'clock, I still can - oh no, time will be too short for shopping. I give up my plans.

(F001_023_m_thoughts_00.02-00.10)

${ }^{167}$ Example (100) shows a part of the previously given example (82). 
In (102), the antecedent (color-coded dark grey), indicated by the conditional marker raised brows, is followed by the consequent. This is first formulated in a positive way. The signer thinks that if she arrives at three o'clock she will have time to go shopping. But then she becomes aware that time will be short and negates that she will go shopping. The positive consequence (color-coded green) is covered by assertive nodding movements (encircled red), the negative consequence (color-coded blue) by headshakes (encircled blue).

Summing up, the data shows that in a number of conditionals the apodosis is either covered by assertive nodding movements ${ }^{168}$ or negative headshakes. Consequently, there seems to be a high tendency that in ÖGS the positive or negative consequence of conditionals is marked. In other words, the ÖGS signers indicated whether - under the condition $X$ - the consequence is positive ('then $\mathrm{Y}$ ') or negative ('then not $\mathrm{Y}$ ').

\subsubsection{Marking alternativity in conditionals}

To begin with, the present data shows that in some instances an antecedent-consequentsequence of a conditional also displays two alternatives. If this is the case, the following implementation of the conditional clause is possible:

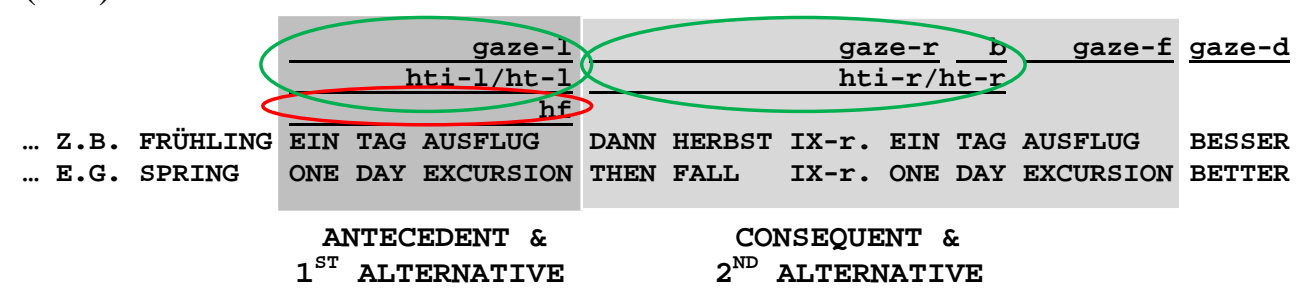

... zum Beispiel wenn man im Frühjahr einen Ausflugstag plant, dann kann man im Herbst noch einen Ausflugstag einplanen. Das wäre besser so.

... for example, if a day for an excursion is planned in spring, then a day for an excursion could be planned in fall too. This would be better.

(F001_Film1_Szene7_04.53-04.56)

In (103), in addition to the conditional marker head forward (encircled red) which covers the antecedent, the protasis is covered by tilting the head sideward, left from the signer's perspective, (which goes together with a head turn to the left and gaze directed leftward)

168 There are no instances in the data in which the protasis is covered by assertive head nods. 
(encircled green). The apodosis which constitutes the alternative option is covered by tilting the head to the opposite side; in this case it is the right side from the signer's perspective (which also goes together with a head turn to the right and gaze directed rightward) (encircled green) ${ }^{169}$.

The conditional clause as a whole can illustrate one alternative space too. In example (104), the entire conditional construction serves as first unmarked alternative space (colorcoded light grey). The conclusions which are drawn from the conditional constitute an alternative space of its own (color-coded dark grey). This is located sideward, on the right side of the signer, and characterized by leaning the body sideward and producing all signs allocated to this alternative space towards this location (all indicators are encircled red). $(104)^{170}$

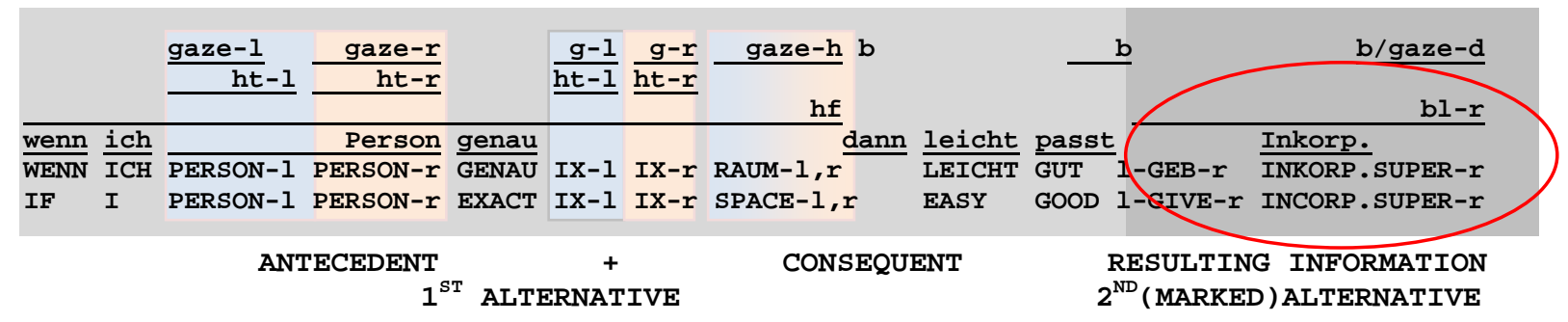

Wenn Personen im Gebärdenraum rechts und links positioniert werden, dann ist es $k l a r e r$. Dadurch ist der Raum inbegriffen. If persons are located in the right and left signing space, then it is easier (to understand). In doing so, space is incorporated.

(M009_13.01.08_part1_01.01.34-01.01.40)

In example (104), a lecturer explains that when the described condition is followed, it is easier to understand the utterance. Doing so (i.e. locating persons in the signing space) has the result that space is incorporated. The entire conditional clause, including the protasis and apodosis, constitute one alternative space. The result of doing so constitutes the second alternative space. In addition, in the present example (104), the 'referential space' is used (color-coded blue for a location to the left side of the signing space and color-coded red for a location to the right, both from the signer's perspective). The indicators of the 'referential

\footnotetext{
169 It remains unclear why the alternative marker does not cover the sign EXCURSION too. Further investigation is required.

${ }^{170}$ The marker 'ht-l' always includes a more downward positioning of the chin due to the specified referential location which is located left and slightly down.

The remaining gaze directions are always to the audience as the informant is holding a lecture.
} 
use of space' do not coincide with a clause of the conditional construction. But, as these indicators are also produced, among others, with the head, the example clearly shows that it is possible to produce both different head markers at the same time with different starting and end points. So, the annotators perceive that the entire antecedent is covered by positioning the head forward. While the particular right-left-locations are marked by head turn left or right, the forward positioning of the head is slightly reduced. But, despite this interference the annotators can perceive the conditional marker. Further studies on the phonetics of head movements would give more insights into that matter.

\subsubsection{Marking modality in conditionals}

One phenomenon which is discussed briefly in this chapter is the influence of modality in conditional constructions.

As described in 5.4, conditionals have different degrees of hypotheticality which go together with the degree of potentiality. It can thus be concluded that the present constructions must have some means of coding these aspects. Palmer ${ }^{171}(2001,124)$ mentions that in many spoken languages, conditionals are characterized by 'subjunctive' and/or 'modal tense'. In sign languages, some research on modal systems has been conducted, but little is known about modality markers. As will be described in chapter 7 on modality in ÖGS, two components are focused on when coding modality. First, 'knowledge' or 'lack of knowledge' - often coded by evidential markers in spoken languages - and second, the speaker's/signer's attitude and judgment on a proposition often coded by epistemic markers - are the two important components when studying 'propositional modality’ (cf. Palmer 2001,8).

Thus, when going through the conditional constructions of the various ÖGS corpora, some non-manual markers which frequently occur in conditionals become obvious. These seem to be good candidates for providing modality information - in the sense of providing

\footnotetext{
${ }^{171}$ Palmer (2001, 22, 207-209, 211-214 inter alia) describes the relation between modality and conditionals and adds that 'mood' is frequently marked in conditionals while 'irrealis' marking may occur, but is less frequent than 'mood' marking - an observation which is also based on spoken languages (more on modality in chapter 7).
} 
information on knowledge and on the degree of confidence about the possible realization of the implied options in the conditionals' propositions.

Two of these possible modality markers are illustrated in (105) and (106), which have been described previously (cf. examples (86) and (93)), and then the markers' occurrence in conditionals is analyzed.

$$
\text { (105) }
$$

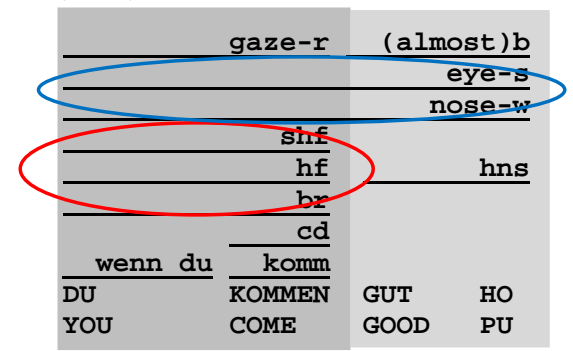

Wäre schön, wenn du kommen würdest. It would be nice if you came.
(106)

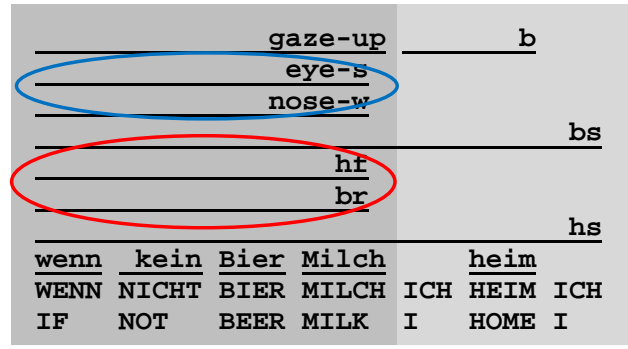

Wenn es in der Almhütte weder Bier noch Milch gibt, dann bleibe ich nicht, dann gehe ich heim. If in the hut there is neither beer nor milk, then I won't stay, then I'll go home.

(M007_04.05.08_edu.course_part01_11.28-11.31) (M001_091,1183_m_thoughts_ex03a_01.08-01.10)

Examples (105) and (106) have in common that in both constructions the markers wrinkled nose and squinted eyes (encircled blue) occur. The reason for their frequent occurrence in conditionals (the conditional markers are encircled red) is discussed below.

First, one non-manual marker which frequently accompanies conditionals is 'wrinkled nose'. For this, the nose is wrinkled and held in this position (without iteration movements) until the signer goes back to the original position. The marker wrinkled nose co-occurs with one or more lexical elements. This marker tends to occur in conditionals which are negated or which have a negative content. In all cited examples (12 through 18) which include negation, the marker wrinkled nose occurs. It is significant that the marker wrinkled nose goes with those contents of conditionals which express the negative option. For instance, it co-occurs with NOT OPEN (OPEN NO) or CLOSED but not with OPEN or - it co-occurs with NOT WIN (WIN NO) or LOSE but not with WIN. Modality markers, especially epistemic markers, express a person's degree of confidence of the fulfillment of a situation. Consequently, it may be supposed that wrinkled nose occurs in propositions which express potentiality, but the feeling of confidence is not very positively 
oriented. So, one possible interpretation is that this marker resembles the non-assertive marker 'fast, small headshakes' going together with 'head backward' (expressing pragmatic denial). It expresses a negative attitude on the outcome/fulfillment of a situation. But the marker does not negate the content in the way that the negation marker headshakes does. In sum, this may be one function of wrinkled nose. Based on the present ÖGS data it can be summed up that wrinkled nose tends to occur in the protasis of conditionals, but can cover the apodosis too. Those consequents which constitute a content question are covered by wrinkled nose.

The second non-manual marker which frequently occurs in conditionals and which probably is associated with possession or lack of knowledge is the marker 'squinted eyes'. Dachkovsky (2008) observes that in Israeli Sign Language (ISL) the protasis of counterfactual conditionals is marked by 'raised brows' and 'squinted eyes', while neutral conditionals are only marked by 'raised brows'. Dachkovsky \& Sandler (2009, 293, 302306) suggest that 'squint' is an indicator in ISL for "mutually retrievable or shared information" and that it occurs in several structures. 'Squint' is used when information is not directly presented in the discourse, but signed elements should be connected with background information shared by both dialogue partners. In their opinion, in counterfactual conditionals 'squint' is a hint for the addressee that the temporal reference of the clause is not actually accessible, but has to be 'retrieved'. As will be shown in 7.3.2, the association between squinted eyes and (lack of) knowledge is also evident in ÖGS. So, one possible interpretation is that squinted may be used to code the degree of knowledge on a condition or a conditional construction as a whole.

This brief description of two non-manual markers which are possible modality markers in conditional constructions shows that investigations on the form and function of these nonmanual markers, occurring in the various constructions, are required and may constitute the basis for further conclusions and interpretations. In 7.3.2 nonmanuals which very likely convey a modality meaning are discussed. 


\subsection{Conclusions on conditionals}

The present findings on conditionals confirm the findings of other sign language researchers insofar as only the if-clause of the conditional construction gets the conditional marker(s) in ÖGS as in other sign languages. Also, in ÖGS the protasis always precedes the apodosis, and never the other way around, as observed, for instance, for ASL or NGT (Reilly et al. 1990 and Pfau 2008). Moreover, the identified consequent clauses together with their marking are possible consequent clauses in other sign languages, too. More exactly, if a declarative clause follows, there is no clause marking, or if an interrogative follows, the appropriate interrogative marker covers the consequent (compare to this, among others, Reilly et al. 1990 for ASL or Johnston \& Schembri 2007 for Auslan).

What is new? For ÖGS, all present findings are new information as so far there have not been any investigations on conditionals in ÖGS. What the present findings contribute to sign language research is listed in the following:

- First, the present findings show that in a sign language one marker can be primarily used, but depending on language-internal and -external factors, alternative markers and/or additional markers which also code conditionality can occur. As for ÖGS, the most regularly used conditional marker is head forward. Chin down and brow raise function as alternative markers. Brow raise, shoulder forward and the sign/mouthing 'wenn' ('if') can additionally serve as conditional markers. Possible language-internal factors influencing the use of an alternative marker are, for example, the use of brow raise when the articulator 'head' is used for another function (cf. example (84)) or the semantic contiguity between a syntactic construction and a speech act. For instance, the contiguity between an antecedent-consequent sequence and question-answer sequence (cf. 8.3.3) may be one of the reasons for marking an if-clause with chin down (used for marking polar questions) instead of head forward. Language-external factors or influences such as distance from the audience can evoke the additional use conditional markers like shoulder forward, or the age of language acquisition (e.g. informants having Deaf parents vary more frequently between the conditional markers and more 
often use brow raise as alternative or additional marker) are further possible reasons for alternating or additionally using conditional markers.

In conclusion, the findings show that the signers neither use only one single conditional marker nor produce a single configuration of nonmanuals for indicating a conditional. Rather, they can alternate between more conditional markers (although one of these is primarily used) or use additional conditional markers, both depending on languageinternal and/or language-external reasons.

- It is striking that two constructions which show semantic similarities are indicated by the same non-manual marker. The most frequent conditional marker (head forward) is the same marker used for embedded interrogatives. As argued in chapter 8, I assume that this is due to the semantic contiguity of these constructions. Both constructions can comprise an unreal situation on which a person has his/her own state of knowledge and attitude concerning the probability of realization (cf. 8.3.3 for discussion).

- What is more, the present findings show that some other functions coded by other nonmanual markers frequently occur in conditionals.

First, marking of negation is frequently present. This is very likely due to the fact that conditionals express a positive or negative condition ('if $X$ ' or 'if not $X$ '). Only the negative condition is indicated by negative headshakes, in any case in the corpus a positive condition is covered by assertive head nods. Also, marking negative semantic contrast is frequently implied in conditionals, due to the fact that both the condition and the consequence can be formulated in a positive or negative way. In addition, in ÖGS there is a high tendency to underline a positive consequence with the result that the consequent can be introduced by a single assertive head nod or the entire apodosis is covered by assertive head nods.

Moreover, the two clauses of a conditional construction or the construction as a whole can also display alternatives, marked by the spatial alternative markers head/body tilt/lean sideward. 
Finally, various markers which very likely display modality markers occur in conditionals. First, the marker squinted eyes is presumed to indicate the state of knowledge. Second, wrinkled nose is presumed to indicate the negative epistemic presupposition on a situation. 


\section{Constructions based on space and coded by head and body markers}

\subsection{Introduction}

As some of the functions of head or body markers are based on the particular functional uses of the space, in this chapter first, an overview of the multifunctional use of the space in sign languages is presented focusing on differentiation of the 'topographic space' (spatial use of the signing space) and 'syntactic/referential' ${ }^{\text {, }}$ (72 space (symbolic use of the signing space). Subsequently, two special functional uses of the signing space present in the data are described, namely the 'alternative space' and the 'hypothetical space' which may be marked by the head or body.

\subsection{Multifunctional use of space}

\subsubsection{Spatial and linguistic use of space}

The signing space has a special characteristic. On the one hand, the space is used to provide information about location as well as about movement and shape. The given information may be either based on a coordinate system, that is, grounded on a physical reference frame or related to spatial information which is given without a physical reference frame. The latter may be 'prototypical deixis' in the sense of 'there', names of locations or a 'prototypical relation' which is implied in the absence of other local information. When a physical reference frame is included the following options for illustrating the spatial information (as well as the information of movement or even shape) are possible: First, spatial configuration of objects can be displayed. A means of coding this intrinsic reference frame is displaying the configuration with help of a [+ground] relation. Second, the spatial relations of the objects to each other constituting the relative reference frame may be coded. Third, the spatial relation of an object to a general coordinate system such as the four cardinal directions may be coded constituting the

\footnotetext{
${ }^{172}$ In this thesis I stick to the originally used terminology in order to distinguish between the 'topographical' and 'syntactic/referential'. With regard to this differentiation see, among others, Sutton-Spence \& Woll (1999, 129-135) in their introduction to British Sign Language (BSL).
} 
absolute reference frame. The above-described coding of spatial information of space has been described for spoken languages by Levinson (1996) and has been demonstrated to be evident in sign languages - especially in ÖGS - by Arik (2010).

On the other hand, the signing space can be used to code linguistic information. Using the space for various grammatical purposes can be found on different levels (cf. Emmorey 1996, 318-321). On the phonological (sublexical) level space is used to code phonological contrast. On the morphological level space can be used for various purposes like coding telic or atelic information (cf. Grose et al. 2007). On the syntactic/discourse level space may have referential function like co-reference ${ }^{173}$ or anaphora, coded by a pointing sign indexing to the locus, with gaze towards the locus, and/or with displacement of the signs at or toward that locus (cf. Emmorey 1996, 320-321), or space may code definiteness and specificity (cf. Barberà 2012). On the text/discourse level space may provide information of, for example, the narrative perspective that falls within the scope of constructed action/embodiment or constructed dialogue (cf. Cormier \& Smith 2011). This list of multifunctional use of space is non-exhaustive.

As the space in sign languages can be used to code spatial information as well as linguistic information, sign language researchers apply different terms for these two uses of space. Poizner et al. (1987) distinguish between a 'topographic space' and a 'syntactic space'. Barberà (2012, 38) adopts Quer et al.'s (2005) terminology, differentiating between 'descriptive' and 'non-descriptive localization' when talking about spatial and syntactic use of space, and states that the main difference between these two uses is that 'descriptive localizations' convey spatial meaning themselves while 'non-descriptive localizations' do not possess meaning on their own, but are used for syntactic and discursive purposes.

\subsubsection{Research on the spatial and linguistic use of space in ÖGS}

In Austrian Sign Language some research work on space has been conducted on the spatial (including motion and shape) function of space (cf. Arik 2010). Following Arik (2010), in

\footnotetext{
173 Emmorey \& Falgier (2004) define 'pronominal co-reference' which means that a nominal sign is associated with a special locus in the signing space, implemented by displacing the sign toward the location at which the referent is already located.
} 
ÖGS it is possible to display an 'intrinsic reference frame' by relating two classifiers. It is further possible to display the relative reference frame by the same means of coding. In my data it is evident that it is possible to use an absolute reference frame which means that the direction or directional movement follows cardinal points or spatial locations like 'up/down the mountain'. Hence, the coordinates for north are up, south are down, west are leftward and east are rightward. When a signer describes that $s /$ he is driving to a person located in one of the listed directions this special information is coded in the movement of the sign AUTO-FAHREN (DRIVE), resulting in AUTO-FAHREN in-den-Norden $_{\text {(DRIVE }}$ north). $_{\text {. }}$. Some linguistic functions of space have been analyzed in ÖGS but space has not been the primary research goal. Some of the findings of linguistic functions of space in ÖGS are: a contrastive function on the sublexical level (Skant et al. 2002, 17-31), coding telicity (Grose et al. 2007), providing locative information or linguistic information (multiplicity) through displacing signs ${ }^{174}$ in the signing space (cf. Chen Pichler et al. 2008, 450). Research on 'definitional structures in ÖGS'175 (Lackner 2009c, 2009d, Lackner et al. in prep.) shows that the signing space is frequently used for expressing cohesion by way of linking phrases/units, naming options that correlate or listing items which belong together. A study on turn-taking and dialogue-structures in ÖGS (Lackner 2007) describes some phenomena which are related with the signing space. Thus, the 'active signing space' is different from the 'feedback signing space'. Consequently, the different rest positions - in the signing space, in the feedback space or in the resting space - signal the dialogue partner whether turn-taking is wanted or not. Turn-taking is further structured within these spaces by how the dialogue partners hold their hands to show a competitive turn-taking structure, a collaborating turn-taking structure, a successively turn-taking structure, etc.

\footnotetext{
${ }^{174}$ In addition to locative information the displacement of classifiers may provide the general information of 'existence'.

${ }^{175}$ A 50-hour educational training course corpus of lectures was the basis for the study on 'definitional structures in ÖGS' (Lackner 2009c and 2009d). This corpus is composed of expository-based data of Deaf referents with different varieties of ÖGS from the university course for sign language teachers at the AlpenAdria-University in Klagenfurt, presenting selected topics (in the linguistic and pedagogical field).
} 


\subsubsection{Interferences of spatial and linguistic use of space}

Of course, following Clark (1973, 35-48) the 'perceptual space' which constitutes the basis for the 'spatial use of the space' (in sign language literature well-known as 'topographic space') while producing signs may influence the 'linguistic use of space' too. For example, in the 'perceptual space' the two sides of the frontal plane have two different values which are 'front' and 'back'. Thus, moving the head to a place in the front while signing results in a linguistic marker which is characterized by 'forward' and which conveys its own value. Moving the head to the 'back' displays another linguistic marker containing its own value. On the contrary, the human's right side and left side typically do not convey two different values. The human's body sides are symmetrical and consequently, the right-left distinction has no value. This fact is also displayed in the linguistic alternative space of ÖGS. Therefore, for using the right-left-alternative space it is not relevant whether the head or body lean goes to the right or to the left - except the signer wants to show a contrast (when the body or/and head have to go to both sides, again unimportant which sides these are $\left.^{176}\right)$. It is also possible that the 'spatial use of space' influences the 'linguistic use of space' in the sense that the contrasted elements are located in the real reference frame on the right and left side. In this case the relative spatial relation is coded taking into consideration the right and left values of perceptual space. Hence, the characteristic of the linguistic alternative space which is relevant for the head or body marker is only 'lateral'. Probably, the tendency to move the head or body more likely rightwards is due to the dexterity but other reasons are also possible. This requires further investigation.

A comparable comment on the influence of perceptual space on linguistic use of signing space is made by Malaia \& Wilbur (2010) in the course of the 'event visibility hypothesis'. The physics of motion and geometry of space become grammaticalized sign components, primarily for predicate signs.

\footnotetext{
${ }^{176}$ In the data - in some cases - the contrast is even done by covering the particular alternatives by the same body leans sideward. In these cases two body leans rightward cover the alternatives and these leans are produced successively.
} 
Figure 6.1 demonstrates the interaction of the spatial and linguistic uses of the signing space, present in the data.

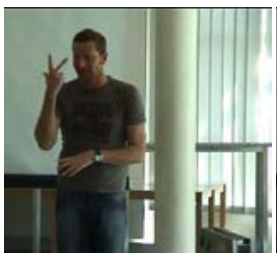

THREE

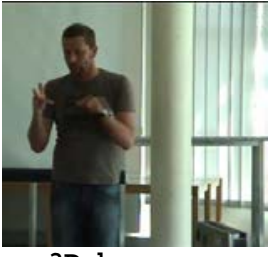

3D-h

IX-1.axis

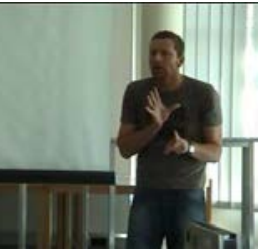

SpL-end

IX-1. space

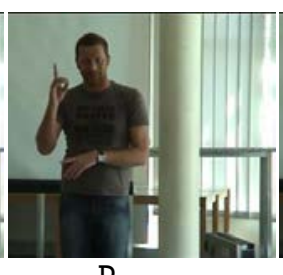

D

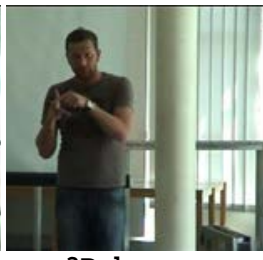

3D-h

IX-2.axis

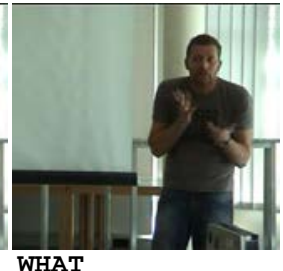

WHAT+IX-1. space

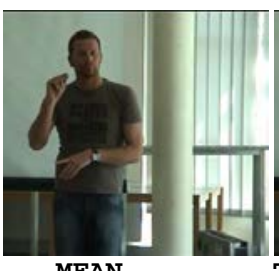

MEAN

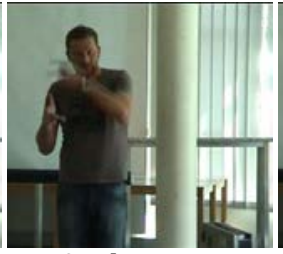

IX-3.axis

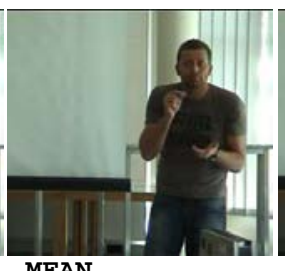

WHAT+IX-1. space

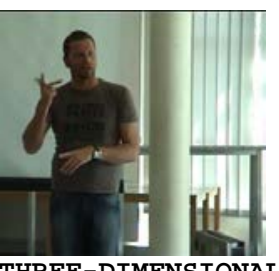

THREE-DIMENSIONAL
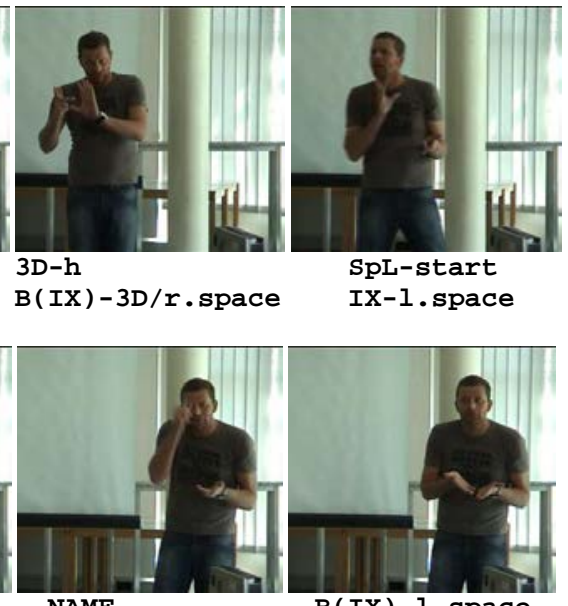

WHAT+IX-1. space B(IX)-1. space

... 3-D bedeutet Dreidimensionalität, d.h. alle drei Achsen im Raum werden genutzt. Dem gegenüber steht die gesprochene Sprache. Welche Dimenionaltität weist diese auf? ... ... 3-D means three-dimensional. This means that all three axis of the space are used. opposed to this is the spoken language. Which dimensionality does it show? ...

\section{Figure 6.1 Spatial and linguistic use of the signing space}

In the excerpt shown in Figure 6.1 the lecturer explains the semantics of 'threedimensional' by introducing a sign which shows the contrast of differentiation between providing additional spatial information in sign language and lack of spatial information in spoken language. The sign THREE-DIMENSIONAL (4 ${ }^{\text {th }}$ picture), which the lecturer redefines and introduces in his course, illustrates that intrinsic spatial information is present when using this sign. For illustrating the three axes the signer traces the three axes by holding the sign THREE-DIMENSIONAL and tracing successively with an index sign the three axis $\left(5^{\text {th }}-7^{\text {th }}\right.$ picture). In doing so, the signing space can be used to illustrate spatial information which can be expressed due to and based on our 'perceptual space' of reality. Finally, the lecturer compared the three-dimensional use of space in sign languages to the 
one-dimensional use in spoken languages. Demonstrating this, the lecturer first gives the three-dimensional expression its location in the right side along an imagined frontal axis in the signing space (by signing $\mathrm{B}(\mathrm{IX})$-right space) $\left(8^{\text {th }}\right.$ picture), then steps leftward $\left(9^{\text {th }}\right.$ picture) and finally, refers to the left space in the signing space. This is done by indexing first, with an index sign $\left(9^{\text {th }}-13^{\text {th }}\right.$ picture), and afterwards, with a pointing sign with Bhandform, performed two-handed ( $14^{\text {th }}$ picture). In doing so, two alternatives are expressed and hence, linguistic use of the signing space is obvious. The alternative information is only provided through opposing two differentiated locations in the signing space ${ }^{177}$; However, probably due to the above described perceptual properties of the real world an observer of the described phenomena gets the feeling that a kind of perceptual illustration is depicted (more on alternative space and particular indicators in 6.4.4).

\subsubsection{Multifunctional use of spatial indicators ${ }^{178}$}

In some instances spatial indicators are used to code spatial as well as linguistic functions. But having a closer look makes explicit that these markers are used differently that is, they are used in different syntactic positions, they are restricted to just special contexts, etc.; this aspect is demonstrated in the following:

One example from the corpus is the pointing sign IX-up which may be used for different purposes. It can be used in reference to a real space that is located 'somewhere up', i.e. at a higher place. For example, in all cases in the data in which the informants report about a 'hut in the Alps' all of them locate the item to the 'upper signing space' by signing ALM+HÜTTE (ALP+HUT) and postpositioning ${ }^{179}$ the pointing sign IX-up.

The second possible use of the pointing sign IX-up is syntactically found in clause-initial position of embedded self-addressed polar interrogatives, sometimes it is produced

\footnotetext{
${ }^{177}$ The two locations in the signing space are not identifiable by features which are purely defined due to the midsaggital plane of the signer (in the sense of ipsilateral and contralateral). As present, in Figure 6.1 the second specified signing space is not located 'contralateral' from the perspective of the signer but 'central' in front of his body in the signing space. More on this aspect is discussed in 6.4.4.

${ }^{178}$ In this thesis the term 'indicator' is used for all linguistic devices which characterize a special language structure. On the one hand, these are means of coding like chin up which are used to mark a special construction. On the other hand, these are language phenomena which co-occur with a special construction. The latter are, for example, the displacement of the sign's place of articulation.

${ }^{179}$ In some cases the pointing sign both precedes and follows.
} 
together with the question mouthing 'ob' ('whether'). In this case the pointing sign functions as a reference element which refers to a 'hypothetical space' - i.e. the space of thoughts (cf. 6.4.3). As described here (cf. 6.4.3) this space is referred to when a person formulates unrealized possibilities, uncertainty, obligations, and so on.

Comparing both uses of the pointing sign IX-up it can be noted that the first use has both referential uses, that is, it has a spatial function in the sense that an absolute reference frame $^{180}$ is involved and a linguistic function in the sense of establishing a reference location for the subject under discussion, the HUT. In most cases a pointing sign with these two functions follows the item which is allocated to the specified location in the signing space and it can be produced repeatedly if the signer refers to the item ${ }^{181}$ again. The second use of the pointing sign, i.e. referring to the hypothetical space, is clearly a linguisticsymbolic one. The element is only produced in the beginning of an embedded polar interrogative and consequently functions as a beginning phrase marker.

\subsection{The syntactic/textual/discourse use of space}

\subsubsection{The various linguistic uses of the signing space on syntactic / textual / discourse level}

A clear function of the sign language space is its possible syntactic, textual, or discourse use. This has been investigated by various sign language researchers. There are different points of view regarding how to interpret the use of the spatial arrangement. The two main perspectives are that on the one hand the 'spatial mapping view' considers a close interplay between the 'linguistic locus' and 'real space'. 'Locus' is interpreted as a location in the signing space to which a referent is allocated (cf., among others, Liddell 1990 or EngbergPedersen 1993). On the other hand the 'r-locus view' considers the formal relationship between the location and the referent as the relevant reason for using the signing space and

\footnotetext{
180 The present absolute reference frame may be due to a vertical coordinate system which conveys the points 'up on the mountain' and 'bottom/down the mountain' (comparable to languages which code 'toward the river' and 'from the river').

${ }^{181}$ Certainly, a pointing sign which has a syntactic function cannot be used indiscriminately and has some restrictions too.
} 
referential features are abstract features (cf., among others, Lillo-Martin \& Klima 1990 or Barberà 2012). For an overview on this topic see Barberà (2012, 46-79).

Special locations are used to express hypothetical propositions or alternatives. They are treated in the sign language literature in the following way: The first phenomenon 'hypothetical space' has not yet been identified as a prototypical location for thoughts. Barberà (2012, 121-127) describes the use of the upper and lower signing space for reasons of specificity. In her interpretation the upper part of the frontal plane may be used for a 'non-specific' nominal location in Catalan Sign Language (LSC). Probably this observation comes closest to the one which is described as hypothetical space in the present chapter. The second phenomenon, i.e. using the signing space for illustrating alternatives, has been included in describing discourse phenomena (cf., for example, Metzger \& Bahan 2001 on side comments in ASL marked by leaning or stepping sideward) or in listing contrasting meanings of body leans (cf. Wilbur \& Patschke 1998 on ASL; Kooij et al. 2006 on NGT).

\subsubsection{Common characteristics}

In the present ÖGS texts in all instances in which the signers use the signing space as a referential, alternative or hypothetical space, the common characteristics of the formatives (as listed in the following) become visible. The classifications made here are based on the production characteristics of the various indicators referring to a location in the space as well as to the kind of occurrence (independent occurrence or co-occurrence). In addition, the perspective, that is, the point of view toward or from the location as well as the signing space's own point of view is taken into consideration.

A) Production characteristics of the spatial indicators ${ }^{182}$
a) Orientation toward a location
b) Moving toward a location
c) Pointing toward a location

\footnotetext{
${ }^{182}$ Concerning this, only the 'point of view toward the location' and 'the signing space's own point of view' is implied.
} 
B) Kind of occurrence

a) Independent occurrence

b) Co-occurrence

C) Perspective

a) Signer's perspective is toward the location

b) Signer takes the perspective from the location

c) The perspective is determined from the signing space which functions as discrete/independent medium

With regard to the production characteristics the data shows that the gaze, the body orientation and the head orientation may be directed toward the locus in space to which the signer refers. In the matter of the articulators 'body' and 'head' the resulting markers are: 'body turn sideward', 'head turn sideward' and 'chin up ${ }^{183}$. Further indicators for making explicit that the signer refers to a location in the signing space are performed by moving parts of the body towards the targeted place in the signing space. This is possible by displacing the signs that refer to that location in the signing space toward that location or in case that the sign possesses a movement component which could be directed toward the specified location - signs which are allocated to the specified location direct their inherent movement toward the specified location. Further options are to move the body toward that direction resulting in the markers 'body lean sideward', 'body lean forward', or 'body lean backward'. The same option is possible for the head resulting in the language-relevant distinctive markers 'head tilt sideward' or positioning the 'head forward' or 'backward'. The body can be moved as a whole toward the particular location resulting in a step (or more steps) forward, backward or sideward which are also identified as language-relevant distinctive elements by the annotators. In addition, weight shifting sideward is possible too for referring to location in the signing space (also identified as a language-relevant marker by the annotators and present in the data). Finally, the signer may point to the particular

\footnotetext{
${ }^{183}$ At least in the present data, the head is never positioned downward resulting in a head nod which is used to indicate a location in the signing space to which the person refers.
} 
location in the signing space by directing and/or moving an articulator - the index finger, the head or rounded lips ${ }^{184}$ - toward the target location in the signing space.

Concerning the kind of occurrence the present data shows that some of the elements that refer to a location in the signing space occur independently, that is, they precede, intervene, or follow the lexical element(s) which are referred to by the specified location in the signing space. These elements occur alone or in a bundle. On the other hand, there are elements that co-occur with the lexical items to which they refer. These co-occurring elements are directed to the specified place in the signing space. Both kinds of occurrences are presented in Figure 6.2 and Figure 6.3.
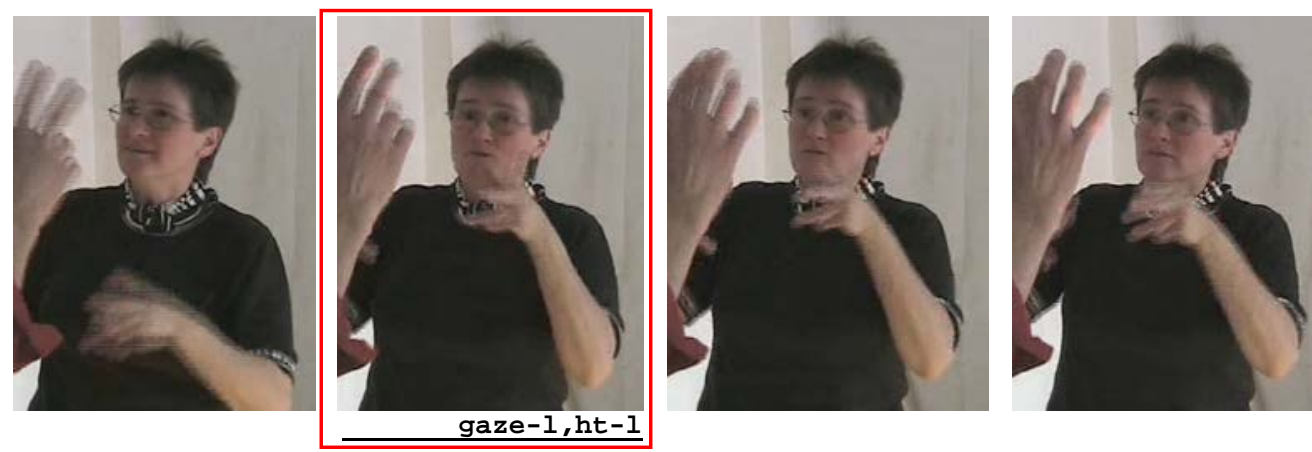

$<$ raising hands into start position

SCHNEIDEN (CUT) - start SCHNEIDEN (CUT) - end

Figure 6.2 Single occurrence of a spatial indicator

In Figure 6.2, the signer refers to a referential space by looking to that space, resulting in indicating the referential space by gaze left and head turn left (edged red) while the hands go into start position of the sign CUT which follows the indicators of the referential space.

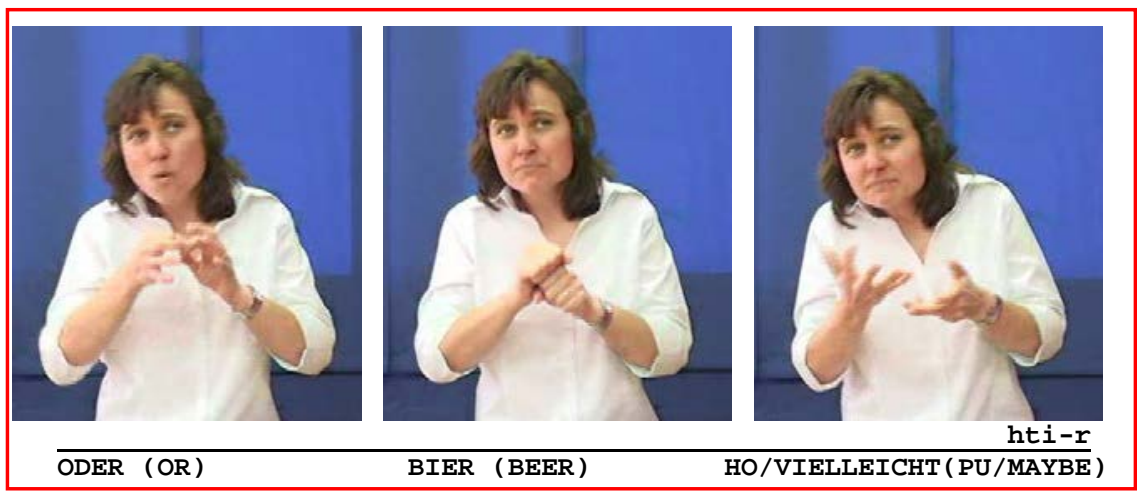

Figure 6.3 Co-occurrence of spatial indicators

184 'Mouth pointing' looks like a pout; the lips are closed, rounded and moved forward. 
In Figure 6.3, the signer tilts the head sideward when signing OR BEER PALMUP/MAYBE. This non-manual marker head tilt sideward (edged red) indicates the second alternative of two possibilities (cf. 6.4.4 on alternative space) and in this function it only can be produced simultaneously with the lexical elements which refer to that alternative space. In other words, in the data there is no instance in which the signer first tilts the head for indicating an alternative space and subsequently signs the lexical elements which refer to the particular alternative space. However, if the marker head tilt sideward is used for another function like expressing politeness and/or modality, the marker also can occur on its own and refers to the preceding lexical elements (cf. example (54) in 4.2.2.2).

With regard to perspective, the following three possibilities are present in the ÖGS data:

(1) The signer's perspective is toward the location which s/he establishes/specifies /allocates elements to. In the literature this is known the referential space.

(2) The second possibility is that the signer takes the perspective from the location. This occurs, for example, when in narratives the narrator takes the perspective of the actors or when in a constructed dialogue the signer takes the particular perspective of the dialogue partners.

(3) The perspective is determined from the signing space itself which functions as discrete/independent medium. This phenomenon occurs when using the signing space for naming alternatives or for allocating thoughts to a hypothetical space in the signing space (cf. 6.4.3 and 6.4.4).

\subsubsection{Resulting indicators ${ }^{185}$}

Based on the given characteristics of indicators for a location in the signing space, the following indicators are present in the data. Most of these indicators are identified as language-relevant distinctive elements by the annotators. Some of them such as displacing

\footnotetext{
185 The term 'indicator' is used to cover all phenomena which function as cues for a special linguistic structure. With regard to space, these are independent markers, co-occurring markers and phenomena like displacement of signs toward a location in the signing space.
} 
the signs toward the relevant locus in the signing space have been noticed by the annotators.

\subsubsection{Orientation-toward-indicators}

The first group of indicators have in common that they are all oriented toward a particular space in the signing space. The following indicators which all may function as elements that refer to a location in the signing space are 'body turn sideward', 'head turn sideward', 'head turn upward' ('chin up'), as well as 'gaze sideward', 'upward', 'downward', or 'straight forward'.

\section{a) Body turn sideward}

In the present data the signers use the marker body turn sideward to refer to one or more reference elements. The body turn can co-occur with head turn sideward and gazesideward. In most cases the sign/s for the referent element/s is/are also displaced to the particular location in the signing space or a pointing sign precedes or follows the reference sign/s to which it refers.

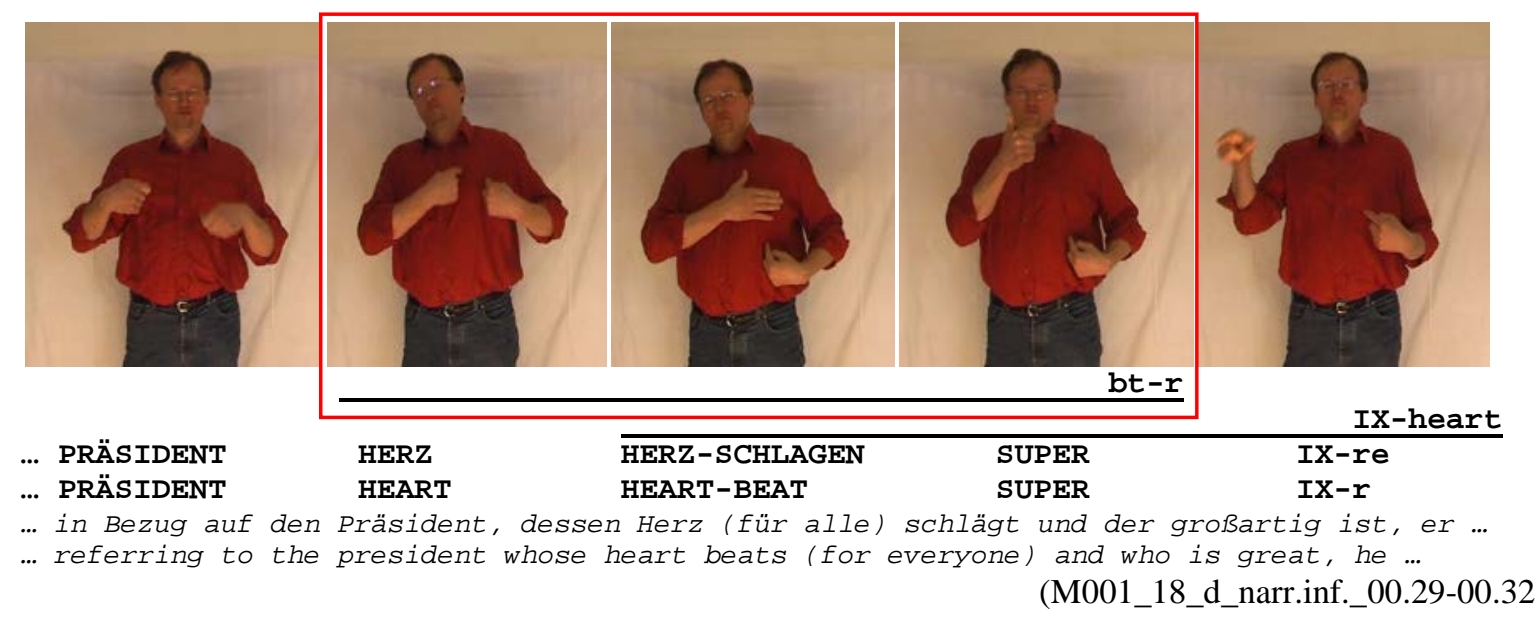

Figure 6.4 Body marker 'body turn sideward' used for indicating a referential space

In Figure 6.4 the signer narrates about the president of the association of disabled people and that this person's heart beats for everyone and that the person is great (in what he is doing). Turning the body toward the location in the signing space (edged red) to which the 
president has already been allocated to (see Figure 6.4), has been identified as a languagerelevant distinctive marker by the annotators. The non-manual marker refers to the person about whose qualities the signer is reporting. In addition, the signer is pointing to the heart (after having signed HEART while signing HEART-BEAT SUPER IX-r. This pointing refers to the heart and covers all signs which refer to the heart - in the sense of 'concerning that location which is the heart it has to be said that it beats for the others, it is great to the others and it refers to the president' (cf. 6.3.3.3).

In the ÖGS corpus the marker body turn sideward is also used to indicate 'side comments' which refer to a reference object. ${ }^{186}$ In most cases the reference object (mostly a person) is already allocated to a location in the signing space. In some cases the 'side comment' refers to a reference object that has not yet been allocated to the location to which the body turns to. However, out of the context and with the knowledge of prototypical locations in the signing space (which tend to be related with special reference objects) it is clear to whom/to what the side comment refers to or is brought in relation with, that is, the new referent is accommodated with help from the context and signer knowledge.

This is presented in example (107):

\begin{tabular}{|c|c|c|c|c|c|}
\hline \multicolumn{2}{|c|}{ gaze-c } & \multirow{2}{*}{\multicolumn{3}{|c|}{$\frac{\text { gaze-r/up }}{h t-r / b t-r}$}} & gaze $-f$ \\
\hline \multirow[b]{2}{*}{ hoffe } & \multirow[b]{2}{*}{ bleibt } & & & & bs/hthis \\
\hline & & so & weiter & & \\
\hline HOFFEN & $\overline{\text { BLEIBEN }}$ & $\overline{\text { IX-r/oben }}$ & WEITER & r-FINANZIEREN-Signer & HO \\
\hline HOPE & STAY & IX-r/up & KEEP - ON & $r$-FINANCE-signer & PU \\
\hline
\end{tabular}

Ich hoffe, dass es (wie gehabt) bleibt und so an uns weiterfinanziert wird. Aber ich weiß es nicht, wie und ob (es so weiter geht).

I hope that things stay the same and we will be financed in the same way. But I don't know how and whether (it will continue the same way).

(M001_18_d_narr.inf._01.03-01.06)

In (107) the signer expresses his hope that things will continue the same way, but that he is not certain if that will be the case. The side comment (color-coded grey) is composed of an index sign that refers to a location situated laterally (right sided) along the frontal axis and the signs KEEP-ON FINANCE. The latter sign conveys a movement from the specified location to the signer himself meaning that the money keeps coming to me/us. Also the place of articulation of the signs in the side comment is displaced toward the upper lateral

\footnotetext{
${ }^{186}$ In this thesis a reference object may be a person, an animate or inanimate object, or even an abstraction.
} 
location. The entire side comment is marked by body/head turn rightward. In addition, the gaze is directed rightward and upward. This is probably caused by the 'hypothetical space' (cf. 6.4.3) and/or the allocation of the side comment to the upper, lateral location referring to a higher authority that is financing the individuals.

Interestingly, 'side comments' can also be marked by head tilt sideward, body lean sideward, or step sideward. The use of one of these markers seems to be due to the spatial phenomena of 'alternative space' (cf. 6.4.4). Hence, when using one of these markers the side comment displays one of two facing options and has to be interpreted differently from a side comment that is marked by head and/or body turn and shows to whom the comment is made.

Furthermore, the element body turn (sideward) occurs in constructed actions and constructed dialogues which imply a perspective from the location. Occurrences and functions of this in ÖGS require further investigations.

\section{b) Head turn sideward}

Similar to the marker body turn sideward, the annotators identified head turns sideward to which they allocated the same function. It remains to be investigated what factors distinguish use of the head alone compared to use of the head and the body turned sideways (together with gaze direction sideward).

\section{c) Head turn upward (chin up)}

In the ÖGS corpus in which the informants express their thoughts, they turn the head upward resulting in the marker chin up. This upward orientation is done due to the 'space of thoughts'. An upward orientation can also be performed by eye gazes (see the following marker). This 'orientation-toward-location-marker' covers the entire train of thoughts, and in some instances the following or preceding cognitive predicate as well (more cf. 6.4.3).

\section{d) Gaze direction sideward/upward/downward/straight forward}

In the data the annotators identified various gaze movements which they labeled as markers on their own. Two possibilities of using gaze direction as a marker referring to a 
specified location in the signing space are frequently present in the data. These are gaze rightward and gaze upward. In using these markers, the informants specified a place in the signing space to which they allocated one single sign (mostly marked by gaze-r) or even more signs (mostly marked by gaze-up). When they allocated one or two signs to the location it was primarily a referent (person or an object). When they allocated more signs it was as 'trains of thoughts'.

Concerning the duration of production of the marker 'gaze-towards-specified-direction' the following two patterns can be distinguished:

First, in several instances the informants allocate a reference object to a specified location in the signing space. When the signers refer to the reference object at that specified location it is done by a pointing sign together with 'gaze-toward-the-specified-location'. Partly, this is done together with head and/or body orientation toward that locus too. In some cases the hand position of the last sign is held and only the gaze is directed toward the location (partly together with a head and/or body turn toward that location) and then the informant keeps on signing. The co-occurrence of all listed orientation indicators together with a pointing sign occurs most frequently in the data in those cases in which the reference object is set up in the specified location the first time. When the signers refer later on to the reference object allocated to the specified locus fewer orientation-towardmarkers are involved.
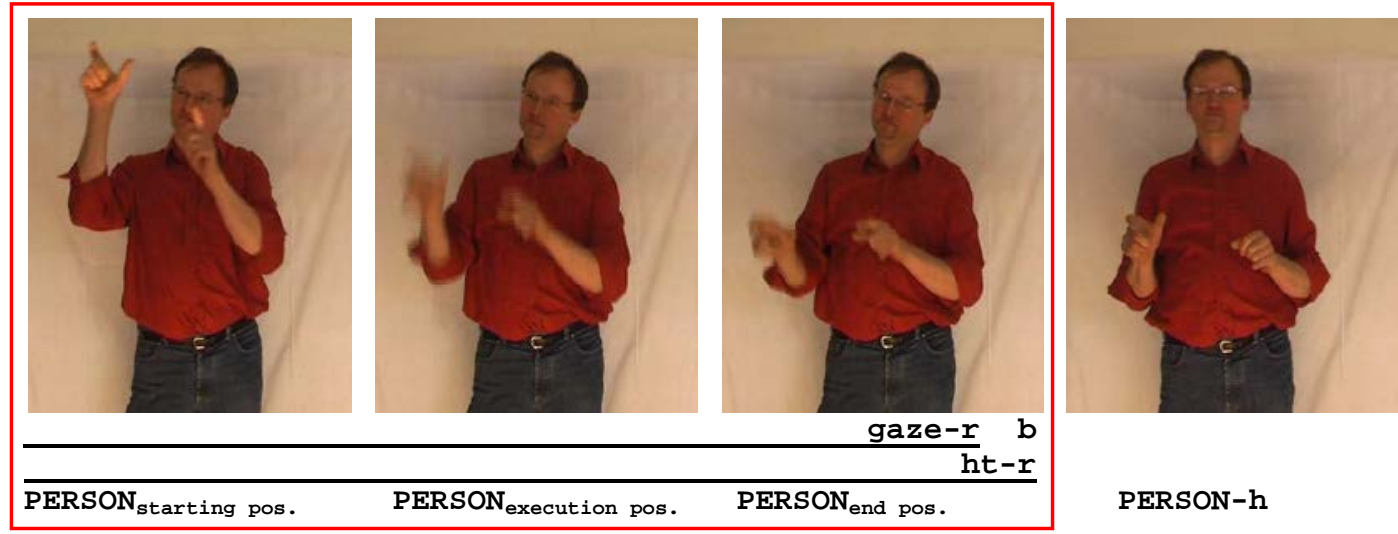

Figure 6.5 Head marker 'head turn sideward' used for indicating the referential space 
In Figure 6.5 the signer allocates a reference object - a person in this example - to a location on the right side of the signing space for the first time. This is done by the sign PERSON $^{187}$. Its place of articulation is displaced into a more rightward signing space (cf. 6.3.3.2). In addition, the markers gaze rightward (gaze-r) and head turn rightward (ht-r) ${ }^{188}$ (edged red) co-occur with the sign. Mirroring the sign with the non-dominant hand is probably due to emphasize the first allocation of the reference object. At the end of the sign movement a blink is performed. Subsequently, the hands are held in the end position until the head and gaze direction has gone back to the 'neutral position' and the informant keeps on signing.

Second, in those cases in which the signers formulate trains of thoughts, the gaze direction is directed upward to the 'space of thoughts' and frequently during the entire line of thoughts including the preceding or following cognitive predicate, the gaze direction upward (or somewhere to the front - definitely not to the dialogue partner or camera person) is maintained until the signer goes on narrating (more on the 'hypothetical space' in 6.4.3).

\subsubsection{Moving-toward-indicators}

The indicators which are grouped together in this subchapter all have in common that the particular articulators are moved toward the referred location in the signing space. In addition, all these indicators have in common that they co-occur together with lexical elements, or they are already bound to the signs which primarily are produced by the manuals (displacement of signs).

\section{a) Displacement of the sign's place of articulation}

One well-known phenomenon in SLs is displacing the signs towards a specified location (cf. Boyes Braem 1995). This is exemplified in Figure 6.5. In those cases in the data in

\footnotetext{
187 The sign PERSON does not require an indexing sign in addition and is comparable to the already investigated sign PERSON in DGS (cf. Pfau \& Steinbach, 2012). But it has to be investigated whether it has all the same features as the DGS sign PERSON.

188 Together with the marker head turn right the body orientation is slightly directed toward the right side.
} 
which the signers refer to a 'space of thoughts' one characteristic is the displacement of the sign’s place of articulation into a higher signing space (cf. 6.4.3.2).

\section{b) Body lean forward, backward, or sideward; step forward, backward, or sideward; weight shift sideward; head lean forward, backward, head tilt sideward}

The following head and body markers have been identified as language-relevant distinctive elements by the annotators. They always cover the signs with which they are associated. They have in common that a part of the body or the body as a whole is moved towards the specified location in the signing space. In doing so, the sign's place of articulation is automatically moved towards the specified location in the signing space. The 'movingtoward-location-markers' co-occur with the lexical element(s) which are referred to the specified location in the signing space. Consequently, the lexical items (displaying the reference object of that location or a proposition or modification made to this reference object themselves) are physically made in the displaced location or when the body as a whole moves to the specified location (e.g. by weight shift or step sideward), the result is that the signs are performed in the 'regular place of articulation'.

In the data 'moving-toward-location-markers' are: body lean forward, backward, or sideward; step forward, backward, or sideward; weight shift sideward; head lean forward, or backward; head tilt sideward. These markers only co-occur with signs which they cover. The listed 'moving-toward-location-markers' performed with the head and/or body refer to locations which convey the value 'front', 'back', and 'lateral'. The data shows that with regard to some functions the markers vary concerning their particular articulator. In addition, the sign's place of articulation is as a result closer to the specified location.

Finally, the data shows that these markers frequently function for contrasting elements, for naming alternatives, and for listing elements (cf. 6.4.4.6).

\subsubsection{Pointing elements to location}

The last group of elements that are used to refer to a special location in space has in common that orientation occurs together with moving towards the location. 


\section{a) Index sign}

A well-known and well-studied element which occurs in various sign languages is the index sign (which may have different forms in various sign languages) (for an overview see Barberà 2012, 91-95). The described functions in ÖGS are referential, spatial, and temporal (Skant et al. 2002, 82-83). This can be illustrated by the already described element IX-oben (IX-up) (cf. 6.2.4). It conveys the value 'up' and may have a spatial function in the sense of an absolute reference frame work ('up on the mountain'), or it may have a reference function ('the hut-up-there'), or may indicate the 'hypothetical space'. The latter function represents a new finding. On the discourse level the element INDEX touching-dialogue-partner has been observed and a discourse pragmatic function has been allocated to this element (Lackner 2008).

\section{b) Lips / tongue / head and/or body pointing}

Additional pointing elements toward a location in the signing space are 'lips pointing' which is performed by rounding and positioning the lips forward, orientated towards the specified location, 'tongue pointing' performed by sticking out the tongue and directing the head towards the specified location, and 'head and/or body pointing' performed by moving the head and/or upper part of the body toward the location. This occurs mostly together with orienting the front side of the body towards the specified location. These pointing elements occur rarely in the ÖGS data, but they are present. These pointing elements (except the index sign) are used in the data for referring to reference objects which already have been allocated to a place in the signing space. Primarily, these are reference objects displaying contrastive alternatives and the signer indicates one of the options by using one of these pointing signs. 


\subsubsection{Conclusions on the syntactic/textual/discourse use of space}

\section{Concerning characteristics:}

Summing up, the data shows that reference to a location in the signing space can be performed by

- orientation toward the location,

- moving a body part toward the location, and/or

- pointing toward the location.

The possible articulators for doing this are the more flexible ones. Consequently, apart from the manuals the articulators which come into consideration are the 'head' and the 'body'. Only the 'eye gaze' represents a further means of coding as the pupils may move toward different directions. This is probably one of the very reasons why various head and/or body markers are used.

\section{Concerning types:}

Two clear distinguishable types of functional uses of space become obvious. These are:

- Space used as discrete medium for displaying alternatives or hypothetical thoughts

The first type of use of space includes the values 'front', 'back', 'sideward', and 'upward' 189 . All indicators that refer to a location in the space and that constitute one of the listed values are therefore clearly identifiable and provide as additional information - apart from the information of the orientation or movement towards the location (this is, for example, the orientation information 'body turn' or the moving towards information 'body lean') - the particular value that makes the marker identifiable. This additional information is, for example, 'up’ (in case of, e.g., head turn up (chin up)) or forward (in case that the marker head lean forward is present). The two identified functional uses in ÖGS are the use of the 'hypothetical space' and the 'alternative space', described in the following subchapter.

\footnotetext{
${ }^{189}$ Probably the value 'down' exists too, but it is not evident in the present ÖGS data.
} 
To illustrate, the location in the signing space is first specified (this is done when referring to the 'hypothetical space' by directing the eye gaze upward, and/or turning the head upward, and/or indexing upward) and then, one or more lexical elements are allocated to that specified location. For instance, if the specified location conveys the value 'up', the information 'now we are at the space of thoughts' is provided. In this case the person just formulates a proposition - in the sense of train of thoughts - which refers to that specified location in the signing space.

\section{- Space used for referential purpose}

The second type of the functional use of space is the referential use of the signing space which has been described for various sign languages. Concerning this matter, the signing space does not constitute the above described values. In most instances in the corpus, the referential space is first specified together with first labeling the reference object. For instance, in Figure 6.5 the sign PERSON is locally specified by the markers 'gaze rightward' and 'head turn rightward'. In most cases the index sign follows sequentially as the next sign the spatial specified reference object toward which it is directed. Referring again to that specified location provides the information is done in order to refer again to the reference object or to refer/assert a proposition to/about the reference object.

\section{Concerning similarities}

Some behaviors both types of functional used of signing space have in common:

- First, the data shows that there is a high preference to locate the first reference object in the rightward location in the signing space and the second reference object in a leftward location in the signing space. This seems to display prototypical locations in the signing space, used for providing a specified space for the reference objects. Also, with the alternative space it is obvious that there is strong preference to use first an alternative space that is located to the right from the signer's perspective and afterwards an alternative space that is located to the left from the signer's perspective. 
Some research on sign language phenomena like 'dominance reversal', 'occurrence of buoys' (more on that see 6.4.3.3 and 6.4.4.4) or 'handedness' 190 in general (cf. Safár et al. 2010) was done which has a relation to the preference of using the particular locations in the signing space. But further investigations in ÖGS and other sign languages are required in order to get the bottom of this phenomenon.

- Second, the present data shows that if spatial information of the absolute frame of reference is available, the location in the signing space for allocating a reference object or for displaying an alternative (using sideward locations) follows this spatial information. Especially with referential use it is obvious that if an individual is sitting on the left side of somebody, the referential space is also located to the left from the signer's perspective. Also, if a referential subject has been allocated to a location to the right or left in the signing space, also indicators used for (sideward) alternative spaces which imply information on that referential subject, are directed to the location where the referential subject has been established ${ }^{191}$.

\section{Concerning differences}

With regard to the head and body markers an interesting observation is present in the data. For referential purposes the signers orient toward the specified/established location resulting in a 'body turn sideward' or 'head turn sideward'. For naming options and so for using the alternative space, the signers 'lean toward one of the alternative spaces'. Thus, the two language phenomena make use of the different body/head markers. In the first case an orientation-toward marker is used, in the second case a moving-toward marker is applied.

\footnotetext{
${ }^{190}$ Based on a questionnaire analysis Sáfár et al. (2010) found out that hand preference for activities of daily living and for signing have a close relation, but they do not always coincide.

${ }^{191}$ Geraci (2011) shows that with embedded structures in Italian Sign Language (LIS), the subject (main clause) is always located ipsilateral, the object (embedded clause) contra-lateral. In his illustrations, the particular signer produces a sign of cognition (constituting the main clause and displaying the subject) which is associated with an action performed by another person (constituting the embedded clause and displaying the object). With ÖGS the data shows that the alternative space follows the referential space which follows locations of the absolute reference frame. So, imagine a situation in which the subject is located in reality at a contra-lateral location of the signer's perspective, the question arises if in LIS in this case the fixed arrangement would be reversed.
} 


\subsection{Space as discrete medium}

In the present subchapter two linguistic uses of space which frequently occur in the ÖGS data, especially in the corpus containing trains of thoughts and the educational training corpus, are the 'hypothetical space' and the 'alternative space'. First, the characteristics of both are presented, and subsequently each of the two spaces are described in detail including a description of the location in the signing space, the functional use of the particular spaces, and the indicators of the particular spaces.

\subsubsection{The hypothetical space and the alternative space}

As described above, both spaces have in common that the location in the space conveys a meaningful value. These are 'up' for the hypothetical space and 'front' and/or/versus 'backward' or 'sideward-one-side' and/or/versus/without 'sideward-the-other-side' for the alternative space. The allocation of lexical elements to these spaces gives some information. Thus, referring to the 'upper space' provides the information that the informant is signing about his/her thoughts, referring to the 'alternative space' give some indication that the signer wants to oppose, add, or just list two or more facts, issues, activities and so forth.

Before describing both spaces in detail similarities of their indicators are analyzed.

\subsubsection{Characteristics of the indicators for the hypothetical and alternative space}

\subsubsection{Beginning or domain marking}

In the present ÖGS corpora the identified indicators which refer to the alternative or hypothetical space precede or cover the lexical elements that are allocated to the respective space. The data shows that the lexical elements that are allocated to the hypothetical or alternative space represent syntactic constituents. In other words, these indicators may function as beginning phrasal edge markers or phrasal domain markers. Some of the markers are primarily used as beginning phrasal edge markers, some tend to be used primarily as phrasal domain markers and some occur in both varieties. For instance, the pointing sign IX-up is used in most cases as beginning phrasal edge marker that refers to the space of thoughts or the space of alternatives and that precedes the phrase. The 
particular gaze direction, which is 'gaze up' for the hypothetical space and 'gaze right' versus 'gaze left' for the sideward alternative space, may occur in the beginning of the construction or may cover the entire construction (that is allocated to the particular space). Head and/or body markers are most frequently used as phrasal domain markers in that way that the entire lexical elements that are allocated toward a special space are covered by a particular head and/or body marker and constitute a phrase.

\subsubsection{Orientation toward location marking / moving toward location marking / pointing}

The elements used for indicating both types of space are the index sign(s), gaze direction toward the space, head/body lean toward or orientation toward the space, and displacement of the sign's place of articulation toward the space.

Depending on the particular space (hypothetic or alternative) these indicators have their own characteristics. The upper location in the signing space indicating the hypothetical space is marked by head turn upward (resulting in chin up) while indicating the sideward alternative space is marked by head tilt sideward and/or body leans sideward.

\subsubsection{The hypothetical space}

When first analyzing the embedded interrogative constructions it became obvious that the signers all pointed and referred to a special location in the signing space, situated in the upper field. It quickly turned out that all constructions that are concerned with thoughts are characterized by different indicators that refer to that upper location in the signing space. In this subchapter, first, the location of the 'space of thoughts' which is labeled 'hypothetical space' is described. Secondly, the indicators which refer to that space are listed and finally, the functional use of that space is analyzed.

\subsubsection{Location of the hypothetical space}

A signer describes the hypothetical space as illustrated in the following Figure 6.6. First, he signs that he is thinking - and looks up to a space in the upper signing space. In the 
second picture he specifies the 'space' in addition with a sign, a classifier that limits/establishes the space. In the third picture he points to that specified space.
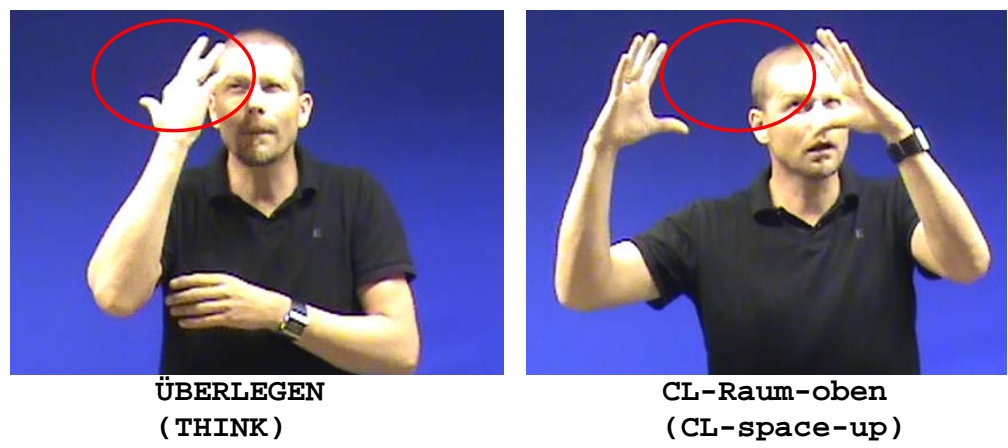

CL-Raum-oben

(CL-space-up)

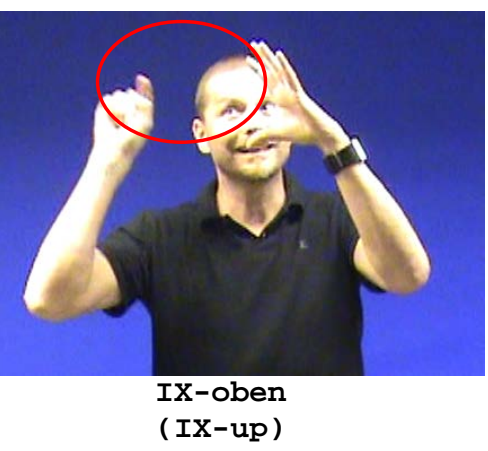

(M007_m_inf.story_00:37-00:37)

Figure 6.6 The location of the hypothetical space

The location of the hypothetical space is situated in the upper signing space with a minimal tendency to be located right-lateral. As all informants in the various corpora are right handed, it cannot be verified whether - in case that the location is slightly sideward apart from upward - this space of thoughts has to be located slightly at the right side in the signing space or whether the hypothetical space conveys as feature 'ipsilateral' which means that a left-hander would refer to an upper, slightly left-sided space. As research on gaze direction with hearing individuals shows that their gaze direction is rightward when formulating trains of thoughts ${ }^{192}$ it can be supposed that the hypothetical space is prototypically located at the upper, right-sided space in the signing space. However, the data shows that looking to a location in the upper, left-sided space is present too. Consequently, only the value 'up' is so far relevant for indicating this hypothetical space.

As already described, this space of thoughts is indicated by beginning phrasal edge markers or phrasal domain markers. If a signer refers to the hypothetical space and the train of thoughts imply a location or a located object that in the absolute reference

\footnotetext{
192 Kinsbourne (1973) conducted a study on gaze direction and thought processes. He found that hearing people look rightward when they talk about their thoughts. This fact occurs when all influence factors are neutralized. This study would confirm the fact that the 'hypothetical space' in ÖGS is located at the right side independent whether the signer is right-hander or left-hander.
} 
framework is situated at an upper place (like a hut in the Alps), the signers tend to use domain markers which refer to an upper location in the signing space. If the hypothetical space and the absolute reference space vary, the hypothetical space is indicated by a beginning phrasal edge marker (and in rare cases covered by a phrasal domain marker).

\subsubsection{Indicators and characteristics}

The present indicators for the space of thoughts in the various ÖGS corpora are the marker 'chin up' (or 'head forward' in case that interrogativity is included), the marker 'gaze up', 'IX-uрнур', and the 'displacement of the sign's place of articulation in the upper signing space'. In addition, the phenomenon 'buoying an ongoing action while thinking of a situation' is described.

\section{a) The marker chin up (or head forward)}

Bending the head toward the back or moving the head forward results in two markers, which the annotators perceive as ÖGS-relevant, distinctive elements. These two means of coding are the marker chin up and head forward. Both are used when the signers express lines of thoughts. The latter is used when these line of thoughts are formulated in an interrogative way. Both markers cover the entire train of thoughts, and optionally the cognitive element which precedes or follows such as the signs HOPE or KNOW-NO.

The marker chin up ${ }^{193}$ only indicates train of thoughts and consequently refers to the hypothetical space. It frequently co-occurs with the marker gaze up.

In (108), the entire line of thoughts and the cognitive expression of 'hope' (first time mouthing together with the sign, second time only the mouthing) are covered by the marker chin up ${ }^{194}$. This marker co-occurs with gaze up, both having the same starting and end point.

\footnotetext{
193 The marker chin up has other functions too like coding direct content questions (cf. 4.2.2.1).

${ }^{194}$ In (108), the annotators perceive - apart from the marker chin up - a slightly forward positioning of the head.
} 


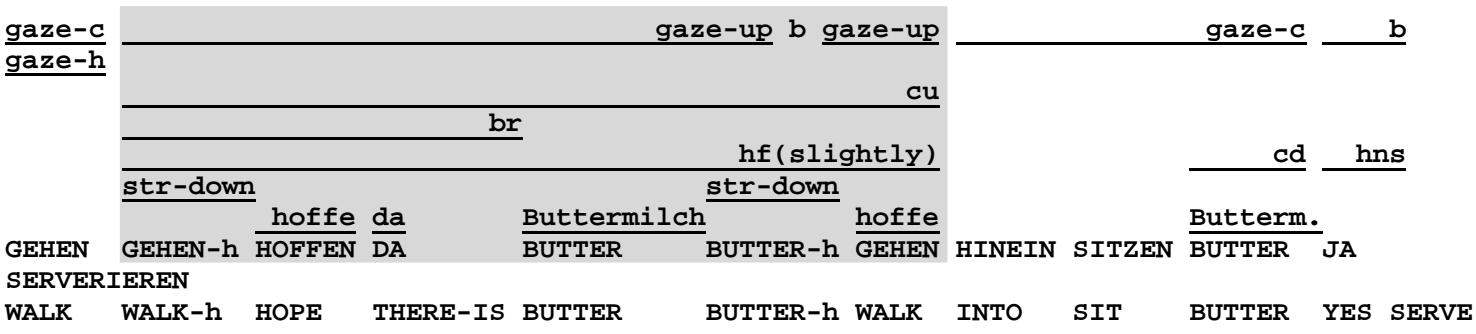

Unsicher hoffe ich, dass es Buttermilch gibt. Ich wandere weiter und setze mich (in die Hütte) hinein. Meine Frage nach Buttermilch wird bejaht und diese wird mir serviert. Doubtfully I hope that buttermilk is available. I keep on hiking and get to (the hut). My question for buttermilk is affirmed and it is served.

(F003_109_m_thoughts_ex3_02.31-02.38)

\section{b) The marker gaze up}

In the following Figure 6.7 the signer uses the indicator gaze up to refer to the hypothetical space. In this short story the signer first looks to the camera (gaze-c) when signing the long activity during which the line of thoughts takes place. When formulating the train of thoughts the gaze direction is upward (gaze-up). Afterwards the signer keeps on signing and produces a constructed dialogue. In this case the signer stares at a place located somewhere in the front in the signing space where the imagined dialogue partner would be (gaze-f).
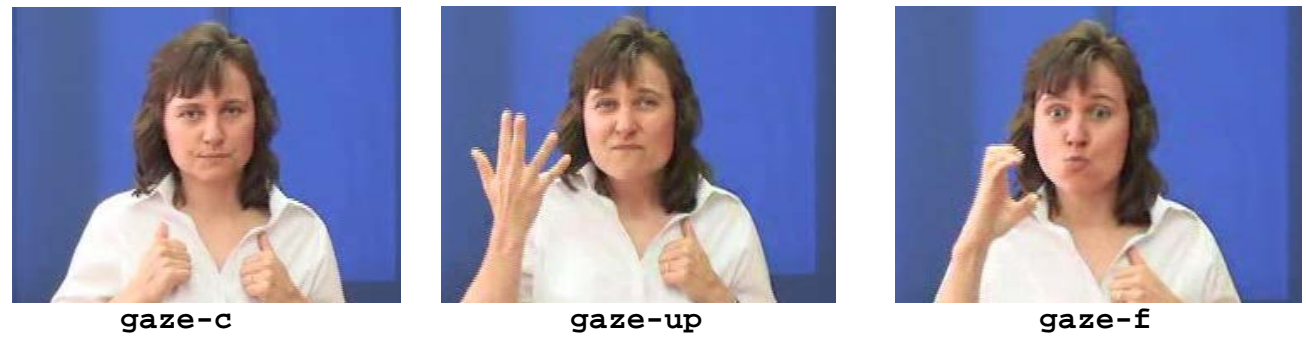

Figure 6.7 Gaze directions

Also in example (108) the gaze direction ${ }^{195}$ of the signer varies. In the beginning of the short story the gaze is directed toward the camera (gaze-c) while signing the long activity.

\footnotetext{
195 The various gaze directions together with their abbreviations in Figure 6.3 are gaze toward the camera (gaze-c), gaze directed upward (gaze-up) and gaze toward the hand(s) (gaze-h).
} 
During the train of thoughts the gaze is directed upward (gaze-up), only a short blinking movement intervenes. Subsequently, the informant keeps on signing the story and looks again toward the camera.

This marker gaze up is used in (nearly) all cases in which the signers express their thoughts. It frequently covers the entire line of thoughts. In embedded polar interrogative constructions it may start before the preceding expression of cognition or together with that expression (cf. example (108)). The last possibility when it starts is together with the pointing sign IX-up or the question element 'ob' ('whether'), both found in clause-initial position of the part of the construction put into question.

In (109), the signer first wonders whether the hut is open or closed and then expresses his hope that it is open. Both trains of thoughts are characterized by the marker gaze up (colorcoded grey). It starts with the first expression of lack of knowledge (a shoulder shrug) and is terminated by a blink at the end of the expression of indecision. The second time the marker gaze up starts with the expression of hope and co-occurs with the entire unit which is covered by tilting the head rightward. Thus, due to the marker gaze up the annotators identify two lines of thoughts.

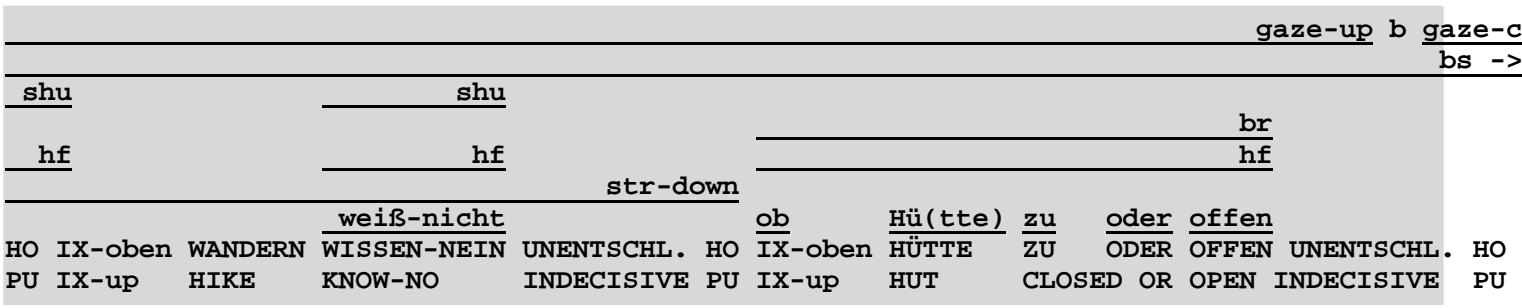

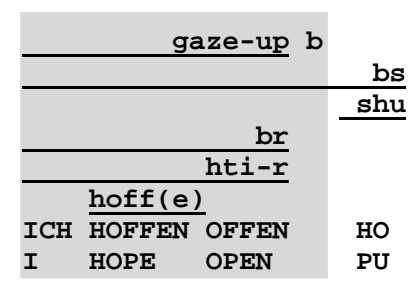

Während ich wandere denke ich darüber nach, dass ich unsicher und unwissend bin, ob die Almhütte geschlossen oder geöffnet hat. Ich hoffe, dass sie geöffnet ist. Aber ich weiß es nicht.

While I am hiking I am uncertain and wonder whether the hut is closed or open. I do hope that it is open. But I dont't know. 
The present indicator for the 'space of thoughts' is implemented in the data as a beginning phrasal edge marker too, even though there is a high tendency to use it as a phrasal domain marker. In case that it is a beginning marker, the marker gaze up is followed by staring in any direction, certainly not at the dialogue partner in dialogue settings, until the train of thoughts is terminated (cf. example (110)).

\section{c) Index sign}

A further indicator for referring to the hypothetical space is the index sign IX-up which may occur in the beginning of train of thoughts. Rarely IX-up co-occurs with the entire train of thoughts as the non-dominant hand is frequently held in the final position of the long action sign (which precedes) while formulating a chain of thoughts. However, in the corpus IX-up repeatedly intervenes in the train of thoughts. $(110)^{196}$

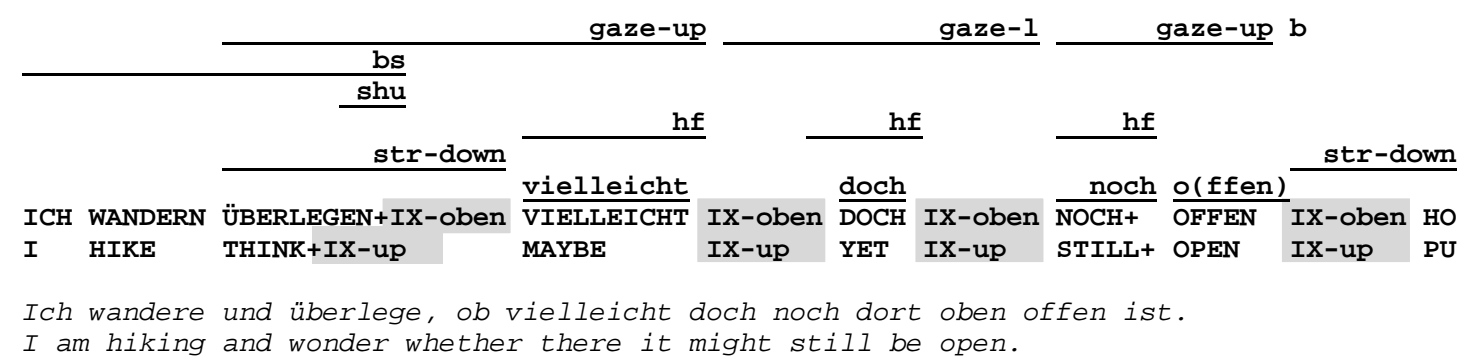

(M001_091,1183_m_thoughts_ex08a_02.44-02.53)

In (110), the four point signs intervene the chain of thoughts. They are all produced by the dominant hand. The first reference sign is produced together with the sign THINK ${ }^{197}$, which very likely functions as indicator for the 'hypothetical space'. The other reference signs (twice performed with an IX-hand form, once with a B-hand form) may refer to that space of thoughts or they may display a topographical hint, referring to a location situated upward.

\footnotetext{
${ }^{196}$ There are forward movements during the signs MAYBE, YET and STILL, functioning for emphasizing each of these expressions. The various index signs intervene between these signs. The illustration is misleading as the duration of each of the index signs is much shorter than the duration of the other signs.

${ }^{197}$ The pointing sign is identified due to the fact that the index finger is directed towards an upper location in the signing space while performing the sign THINK.
} 
Summing up, the pointing sign may function spatially or linguistically (in the sense of referring to the space of thoughts). Whether these functions can be distinguished or whether the pointing sign has multiple functions is still an open issue (and is shown in example (110)). However, in two special syntactic slots it is very likely that the pointing sign refers to the hypothetical space. First, when the pointing sign co-occurs with the expression of cognition (cf. example (110)) or second, when it occurs in initial position of the train of thoughts (cf. example (111)), it is very probably that IX-up refers to the space of thoughts. The very reason therefore is that on the one hand in these syntactic positions frequently other markers (like gaze up or chin up) which refer to the hypothetical space cooccur with IX-up or have their starting point of occurrence together with IX-up. Thus, the occurrence in a bundle shows that all indicators have the same function. On the other hand, if the spatial location of an object or an event has another spatial location than 'up(ward)', IX-up definitely functions for referring to the space of thoughts. Both arguments have been verified in an additional study ${ }^{198}$. In (111), a signer wonders whether the train will arrive in time. The part of the construction that is put into question starts with IX-up. While holding IX-up the signer is mouthing 'ob' ('whether'). Also, the gaze is directed upward (all markers are encircled red).

$(111)^{199}$

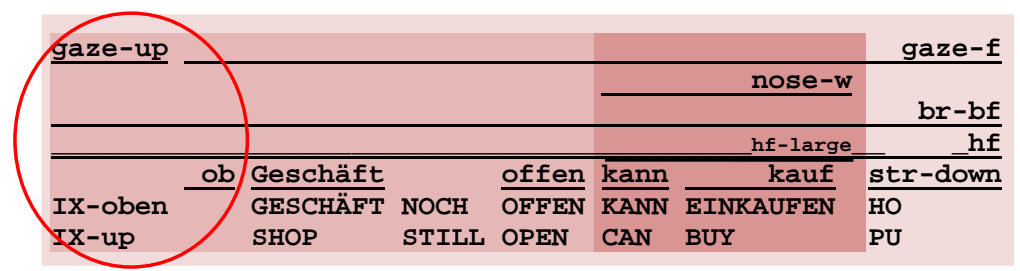

(Während ich mit dem Zug fahre überlege ich,) ob das Geschäft noch offen hat und ob ich noch einkaufen kann. Ich bin mir aber sehr unsicher.

(While I'm going by train I wonder) whether the shop will still be open and whether there will still be time to go shopping. But I am very uncertain.

(F001_24_m_thoughts_00:14-00:17)

\footnotetext{
198 In a clarification study the informants produced, among others, trains of thoughts in which they used various activity signs for a long action during which the line of thoughts was formulated. Both a common starting point of the indicators for the hypothetical space and the use of IX-up were frequently observed even though no reference object was allocated to a spatial area anywhere upward.

199 In (111), the entire embedded interrogative is color-coded bright rose, the first embedded interrogative clause mid rose and the second embedded interrogative clause dark rose.
} 
In (111), the index sign definitely refers to the hypothetical space and does not convey spatial information due to the fact that the shop is not located in an upper place in the absolute reference frame. This example further shows that the gaze direction is not influenced by spatial information. It shows that gaze up together with IX-up functions as beginning phrasal edge marker. Subsequently, the entire train of thoughts is covered by looking forward (gaze-f) to some undefined space.

\section{d) Displacement of the sign's place of articulation to a higher location}

Additionally, the signs of the trains of thoughts are produced in a higher position in the signing space. This phenomenon is probably due to the well-known phenomena of displacement of signs in space toward the particular loci. This phenomenon is illustrated in the Figure 6.8 in which the first time the sign is produced in the neutral signing space and the second time it is produced in higher position towards the imagined space of thoughts.

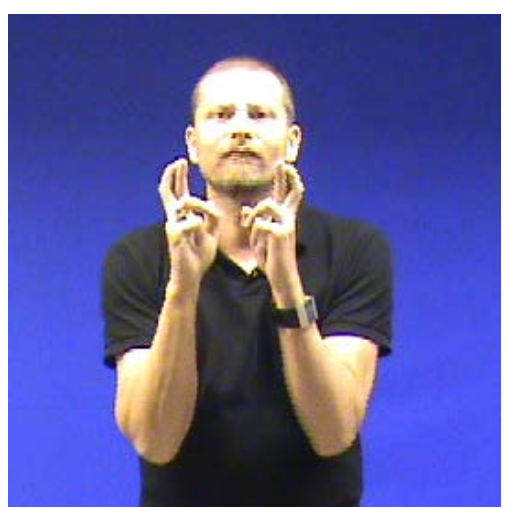

HOPE-neutral

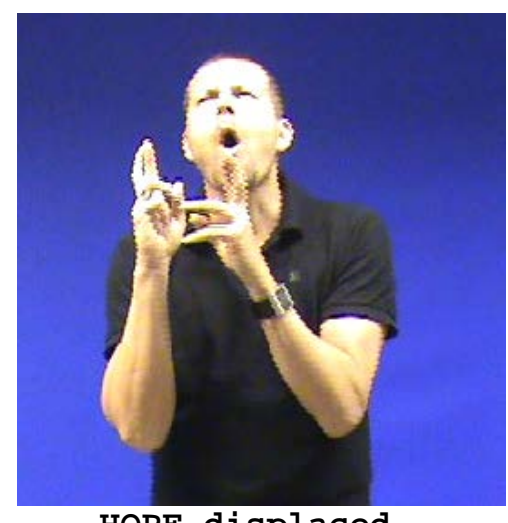

HOPE-displaced

Figure 6.8 Displacement of sign's place of articulation

In the ÖGS corpora there are many instances in which the signers think about a situation. In doing so, it happens that the sign's place of articulation is performed in a higher position. To this end, it could be supposed that the fact that the signer thinks about an event/location that is allocated spatially to an upper place in the signing space is the very reason for the phenomenon. However, in cases in which the signers express thoughts and no spatial information of a higher location is provided, the same phenomenon is perceived by the annotator. This makes it obvious that the displacement of the sign's place of 
articulation can be due to the hypothetical space. Investigating the exact phonetic place of articulation of the sign's during expressing train of thoughts however goes beyond the present thesis.

\section{e) Buoying the ongoing action}

When referring to the hypothetical space, this frequently is done during a long ongoing action. The data shows that in these cases the sign of action is frequently held by the nondominant hand, constituting a buoy of the long ongoing activity, while the dominant hand signs the line of thoughts. The term 'buoy' was described by Liddell (2003, 223-260) as maintaining the hand configuration of the 'weak hand' while the other hand continues providing information. In doing so, the weak hand displays a kind of anchor for the ongoing discourse structure. He lists various kinds of buoys for ASL like 'list buoys', where the weak hand functions as a numeration list to which the listed items, produced with the dominant hand, are allocated, or 'pointer buoys', where the weak hand points to an element about which the signer keeps on narrating (with the dominant hand).

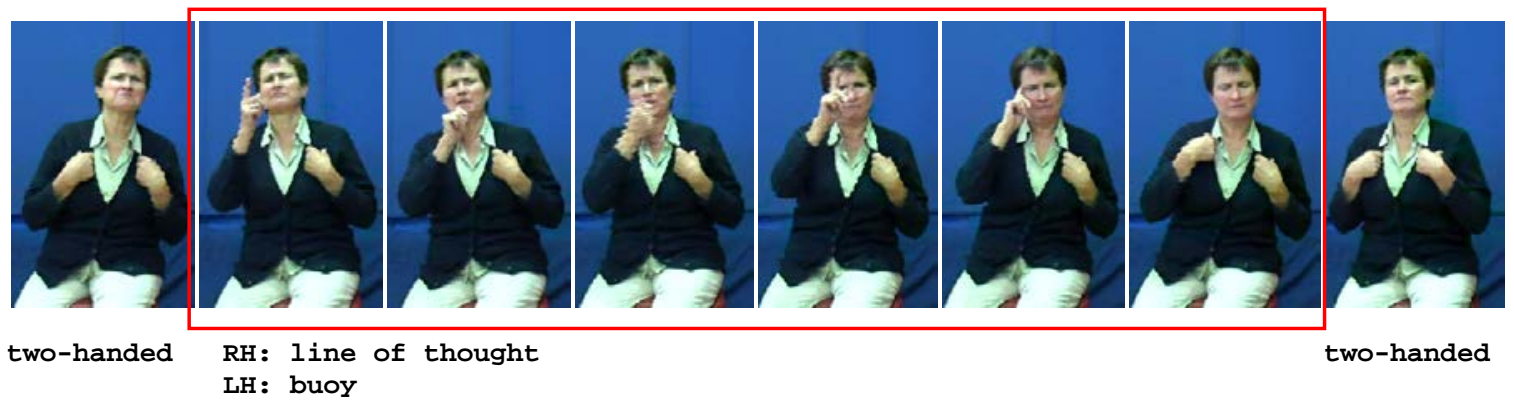

Figure 6.9 Buoying the ongoing action while formulating a line of thought

In Figure 6.9 the non-dominant hand is held in the position of the ongoing action while formulating a train of thought. Using the non-dominant as a buoy is also present when indication an alternative space (cf. 6.4.4.3).

\subsubsection{Functional use}

The main question is now in which circumstances an ÖGS-signer refers to the 'hypothetical space'. For clarifying under which conditions the reference to the 'space of 
thoughts' is required, I take a look at 'embedded polar interrogatives' (in which the signers always refer to the hypothetical space) which may provide some information about that. In embedded polar interrogative constructions the primary function is more likely to display the speaker's thoughts in an interrogative way than asking for an answer. The setting is a non-interactive one. The signers formulate within a monologue the thoughts which are put into question and which are floated into the signing space without being addressed towards a dialogue partner. Therefore, none of the signers is performing a 'constructed dialogue' in which the thoughts would be presented to an imagined counterpart. In addition, the annotators indicate that for them the thoughts formulated in an interrogative way are addressed to the signer himself/herself ${ }^{200}$. From this it follows that referring to the hypothetical space is a possibility to abstract away from the actual situation and consequently from the 'here and now'. It is an 'imagined window' that allows expression of thoughts about unrealized situations, possibilities, chance, and so on. In the case of embedded interrogatives in nearly all instances these considerations formulated in a proposition go back to an expression of cognition or uncertainty (including modality). So it might be supposed that the reference to the 'hypothetical space' is present in conditionals or when a person is using some expressions of modality which refer to unrealized situations, etc.

Following these aspects it can be concluded that when an ÖGS-signer is expressing unrealized situations, possibilities, etc. and these train of thoughts are addressed to the signer himself/herself the reference to a hypothetical space, that means the 'space of thoughts', is present.

The question arises as to what happens when a signer is formulating an unrealized situation, a hypothetical condition, and so forth which is directed a dialogue partner. The data shows that in some cases the signer indicates the 'hypothetical space' (in most instances with a phrasal beginning marker), in some cases not. This issue needs further investigations.

\footnotetext{
${ }^{200}$ This recognition follows Wilson \& Sperber (1988) who noted that there is a variety of questions, including 'rhetorical questions', 'expository questions', 'self-addressed questions', 'indirect questions', that have another or a further relevance/function than just asking for an answer. This aspect is formulated within the relevance theory framework, established by them.
} 


\subsubsection{Conclusions on the hypothetical space}

Summing up, in the ÖGS data the indicators chin up, gaze-up, IX-up and displacement of the signer's place of articulation at a higher space are cues that refer to a space of thoughts, located in the upper field of the signing space. This is labeled 'hypothetical space' in the present thesis. The signers refer to this space when formulating self-addressed trains of thoughts about unrealized situations, chances, possibilities, and so on. The listed indicators for the hypothetical space frequently occur in embedded, self-addressed interrogatives and partly in conditionals. Whether in other constructions the informants refer to the hypothetical space requires further investigations.

\subsubsection{The alternative space}

In the corpus in which the informants express their trains of thoughts as well as in the educational corpus of lectures it becomes apparent that the signers use the signing space for naming different objects, events or propositions. This is done in the way that mostly two options are listed in the sense of 'this and that', or two options are opposed in the sense of 'this or that'.

In the present subchapter first, the possible locations of the 'alternative space' in the signing space together with the possible amount of alternative locations are described. Secondly, the used indicators are listed. Subsequently, the various functional uses of the alternative space are analyzed as well as the 'underlying subject matters' of the particular alternative locations. Finally, the present phenomenon of functional use of space for distinguishing different options is distinguished from itemizing elements and so from listing.

\subsubsection{Locations of the alternative space}

In the ÖGS data two arrangements of alternative locations in the signing space for expressing two (sometimes three) options are present. These locations are defined relative to the signing space itself, that is, the signing space displays its own discrete and independent medium with its own division as illustrated in Figure 6.10. 


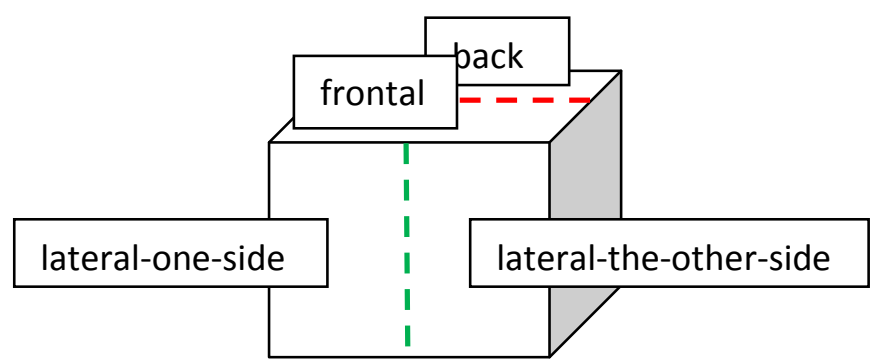

Figure 6.10 The signing space as independent medium with its divisions, used for the alternative space

As illustrated in Figure 6.10, the first two alternative locations (when two options are marked) are situated lateral of the midsaggital plane (indicated by the green dashed line), on the horizontal plane in the signing space. An occurrence in the data is illustrated in Figure 6.11 (the particular alternative space is encircled red, the signing space is circumscribed green). The other two possible alternative locations are situated in the front and back of an imagined frontal plane in the signing space (indicated by the red dashed green line), along the midsaggital plane. An example of this phenomenon is illustrated in Figure 6.12 (again the particular alternative space is encircled red, the signing space is circumscribed green).

\section{lateral alternative space}

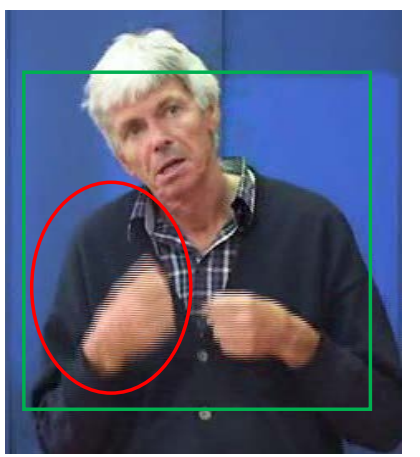

hti-r $\overline{\text { MILCH }}($ MILK)

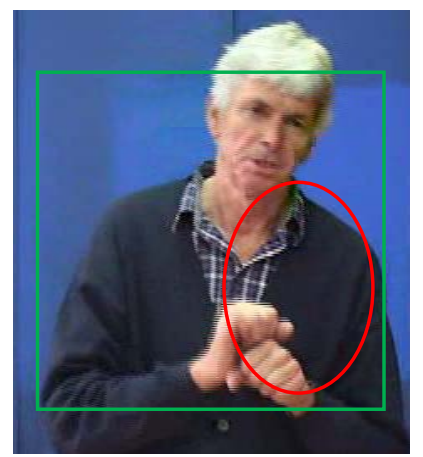

hti-1 BIER (BEER) lateral alternative space to the opposite side

Figure 6.11 Lateral alternative spaces 

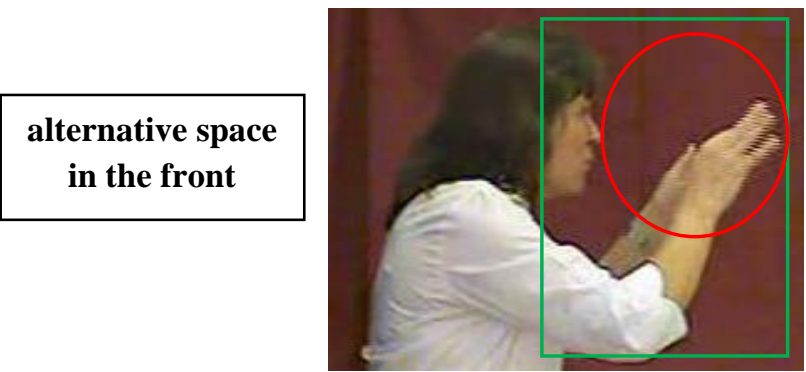

$\frac{h f}{\text { OFFEN }}$ (OPEN)

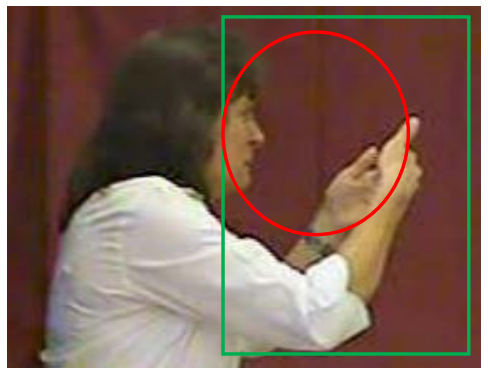

$\frac{\mathrm{hb}}{\mathrm{ZU}}$ (CLOSED) alternative space further back

Figure 6.12 Forward-backward alternative spaces

In regular cases the signing space is located in front of the signer's body, comprising the space in front of the head and upper part of body. In these cases the locations of the particular 'lateral alternative space' are situated 'ipsilateral' and 'contralateral' from the signer's perspective. The particular 'forward-backward alternative spaces' are located 'distal' and 'proximal' from the signer's perspective ${ }^{201}$. These are the locations where the ÖGS-signers frequently produce the described alternative spaces. These terms are also used for describing the actual locations of the respective alternative space. However, they do not display the underlying values of the respective alternative spaces. When using the terms 'ipsilateral', 'contralateral', 'proximal' and 'distal' the location is always brought into relation with a base point. For instance, 'ispilateral' implies that something is located on the same side of the reference point/object. With regard to the signing space two phenomena are possible. First, the signing space can be 'moved' to a place which is not located in front of the body. For instance, signers can hide their signs from a third individual by 'moving' the signing space to a location situated more lateral and lower to their body. When using in this circumstance the 'lateral alternative space', both alternatives are located 'ipsilateral' from the signer's body (or 'contralateral' when the signing space is moved to the left body side of the signer). Another possibility is that a signer formulates lines of thoughts. If the signs' place of articulation is performed closer to a hypothetical

\footnotetext{
${ }^{201}$ With regard to the sign structure's setting Brentari (1998, 4 and 130), among others, use the term 'distal' and 'proximal' for describing locations in the signing space that are situated further away from the body and closer to it. Locations that are located sideward from the signer in the signing space receive the features 'ipsilateral' and 'contralateral'.
} 
space that is situated upward and to the right, and if the signer makes use of the 'lateral alternative space', both actual locations of the particular alternatives are situated 'ipsilateral'. The second phenomenon with respect to the signing space is that it can be 'enhanced'. When using the 'lateral alternative space' in an educational setting, frequently the lecturers refer to an alternative by 'stepping sideward' and then indicate a location which actually is located just in front of them. Thus, the present alternative space is neither located 'ispilateral' from the reference point (the signer's body) nor 'contralateral'. There is no indication that this actual location is situated anywhere lateral/sideward at all with the reference to the signer's body ${ }^{202}$.

If one of these two phenomena with respect to the signing space occurs and if the signer uses the alternative spaces, the annotators notice a language-relevant difference which are the values described above. Thus, the relevant values are that one alternative space is situated 'lateral-to-one-side' with regard to the signing space as discrete medium, and the opposed alternative space is located 'lateral-to-the-other-side' with regard to the signing space as independent medium. In conclusion, the focus is on the absolute space as a discrete medium with its own division and therefore, the terms 'lateral-one-side' versus 'lateral-the-other-side' and 'front' versus 'back' are used.

Now, having the two different lateral alternative spaces in mind, the signer may name two options of which each of them is allocated to one location, that is, one option is allocated to one lateral location and the other option is allocated to the other lateral location. These lateral situated locations in the signing space are frequently indicated by body and/or head markers. This is due to the possible sideward movement distinctions of the upper part of the body and the head that is moving towards one side versus moving towards the other side. Furthermore, the data shows that when two options are listed or opposed, there is no apparent difference with respect to which lateral situated location in the signing space is referred to first. This is very likely based on the fact that the human body has two similar

\footnotetext{
${ }^{202}$ Of course, with regard to 'move' the signing space to another location as used for whispering, it can be interpreted that a 'tiny signer' is imagined to this moved signing space. In this case that features 'ipsilateral' and 'contralateral' are adequate too. With regard to use of 'enhanced' signing space, somebody would have to imagine that the signer still keeps standing in his/her original position for using the terms 'ipsilateral' and 'contralateral'. Anyway, this thesis focus on the absolute space used here as discrete/independent medium.
} 
body sides and consequently, only the value 'lateral' 203 ('sideward') is relevant (cf. 6.2.3). Moreover, the data shows that the signers have a preference to allocate one option to one of the specified locations and the other option to the other specified location. However, in the data there are also occurrences when the signers mention two alternatives which are allocated to the same location in the signing space, or they present only one alternative which they allocate to one of the lateral locations. With regard to mentioning two options which display events or activities that imply a time flow the first location for the first option is always located more 'leftwards' along the frontal axis than the second option.

All in all, it is obvious in the data that these two alternative locations along the frontal axis obtain the value 'lateral'. In the event that two or more options are present the signer has two possibilities to add the information that 'another' option is present. On the one hand, s/he can use indicators that move/orient/point towards two or more different locations, on the other hand, the indicators can move/orient/point toward the same location. Both possibilities provide the information that the value 'another' is relevant. When more than two options are named, two or more locations which are arranged next to each other along the frontal axis are specified.

In addition, spatial information, semantics of the entities or of the events of the options, hierarchical status of the different options, etc. may influence the actually implemented alternative location in the signing space of the allocated option(s). This aspect is not focused on in this thesis, but mentioned when describing the particular functional uses and examples, if required (more on this see Engberg-Pedersen 1993 or Barberà 2012, 115-117 on hierarchical relations).

Furthermore, as the alternative locations are situated in the lateral signing space it is mainly the head or body that indicates this space. The other indicators for the 'alternative space' such as pointing signs or gaze direction are more flexible and are not so restricted in movements as head and body markers. Consequently, the sideward movement of body

\footnotetext{
${ }^{203}$ In this thesis the term 'lateral' is favored to the term 'sideward', but both convey the same meaning. Pfau \& Steinbach (2006), for example, use the term 'sideward' when describing 'sideward reduplication' in German Sign Language (DGS). This comprises the phenomenon that a sign can be pluralized by producing the sign more times in laterally arranged locations.
} 
and/or head is significant and conveys a contrastive movement possibility (to one side and/or to the other side).

The second possibility is that the alternative locations for two or three (that is hardly ever the case in the data) options are situated along the midsaggital axis. When two options are named, the first one is situated 'in the front' of an imagined frontal plane in front of the signer; the second one is located 'in the back' of that imagined frontal plane. In the ÖGS corpora the use of the alternative spaces along the midsaggital axis has the following characteristics.

In all cases both alternatives are formulated and allocated to the particular location and both options are marked. The indicators that cover the two options are almost always head and/or body markers apart from the other indicators which may co-occur, precede or follow. One example in the corpus that is consistently marked by the use of alternative space is naming the two options 'open' or 'closed'. It is significant that the semantics of the two options already possess a contrasting meaning which is additionally marked by the contrastive alternative spaces. The sign for 'open’ is always produced in the distal location along the saggital axis while the sign 'closed' is produced in the proximal location along the saggital axis in the signing space. The values of the two alternative locations are 'front' and 'back'. The terms 'proximal' and 'distal' are only used for describing the actual location.

In the following subchapter the marked alternative locations are described. Hereunto, the 'lateral' alternative spaces are focused on, as these locations are much more frequently used than the 'frontal-back' alternative spaces.

\subsubsection{Indicating one or more alternative locations}

The number of locations used for the alternatives may vary. In most cases a binary-spatial arrangement of locations is offered which means that both options are named and marked. In quite a number of instances a one-place-spatial arrangement is indicated. This means that two options are present but only one is marked. Finally, in some cases three options 
are named and marked. This shows that expressing the relation of two or three alternatives $^{204}$ is done by marking one, two, or three locations in the signing space. If only one alternative location is marked, this is opposed to the unmarked one. The other possibility is to mark all options by indicating all alternative locations which are brought into a kind of relation to each other. These possible representations are described in the following:

\section{a) Indicating one alternative location}

In (112) and (113) only one option of two alternatives is indicated.

\begin{tabular}{|c|c|c|c|c|c|c|}
\hline & & & & bl-r & & \\
\hline OMA & AUFPASSEN & $\overline{\mathrm{ICH}}$ & ARBEIT(EN) & $I X-r$ & REDUZIERT & HO \\
\hline GRANNY & TAKE - CARE & I & WORK & $I X-r$ & REDUCED & PU \\
\hline UNN & IARKED & & MARKED & & & \\
\hline ALTEF & RNATIVE & AL & ERNATIVE & & & \\
\hline
\end{tabular}

Während ich arbeite passt Oma (auf die Kinder) auf. Das vermindert meine Zeit zuhause, in der ich mich meinen Kindern widmen kann.

While I am working granny takes care (of the children). This reduces my time being with the children at home.

(F003_d_Film1_Szene7_)

In (112), the signer informs her dialogue partner that she is working while her mother takes care of her children (at home) and that this fact reduces her time being at home and being with her kids. The 'being at work' is indicated by a body lean sideward. This option of 'being at work'(edged red) is consequently opposed to the state of 'being at home with the kids'. The second option (edged orange) is not explicitly marked. Hence, in this case a marked alternative is opposed to an unmarked alternative.

A further possibility of indicating only one alternative is illustrated in the example (113).

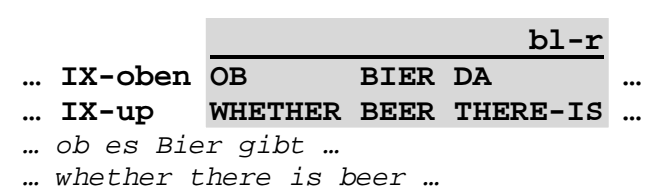

\footnotetext{
${ }^{204}$ In this thesis I use the term '(underlying) subject matter' or 'alternative' when referring to the content which is allocated to the respective alternative space (cf. 6.4.4.5).
} 
In (113), a signer wonders whether he will get some beer. While signing WHETHER BEER THERE-IS he leans the body sideward. According to the annotators the signer indicates one option out of further alternatives which are not itemized. Consequently, it can be concluded that indicating to one location of the two possible lateral locations along the frontal axis signals that the signer refers to alternatives. As illustrated, it is already sufficient to refer to one option for clarifying that probably further alternatives are possible too - that is, one marked option is indicated and opposed to further alternatives which, however, are not enumerated.

\section{b) Indicating two alternative locations}

As already mentioned, in most cases in the corpus the signers mark two alternative spaces. Therefore, it can be summed up that both alternatives are marked through indicating different (sometimes successively the same) alternative location. In doing so, both options are marked and opposed to each another.

\section{c) Indicating three alternative locations}

In some cases out of the ÖGS corpora the signers indicate three alternative locations.

Three options are named and allocated to a location along the frontal axis. The resulting alternative locations based on the signing space itself (which is situated in front of the signer and therefore, the signer's perspective refers to the space) are three locations of which two are located lateral at the opposite sides and one is located in between. These alternative spaces are in all cases marked by head and/or body markers. The body as a whole can be moved by stepping sideward ${ }^{205}$ and so indicating the particular alternative space. When stepping sideward to mark one alternative option, the specified alternative location is situated in front of the signer's body and all indicators refer to that space. In Figure 6.1 in which two options are distinguished by stepping sideward, the final picture of the sequence shows that the lecturer refers with a two-handed pointing sign to an alternative location in front of him (cf. 6.2.3). That is the very reason, why the signing

\footnotetext{
${ }^{205}$ Stepping forward and/or backward is only in some rare cases present in the ÖGS corpora for indicating three different alternative locations.
} 
space has its own values and these values do not relate to the signer's body. Therefore, the features 'contralateral' and 'ipsilateral' are not relevant.

Indicating three alternative spaces is used for illustrating three options like enumerating entities, for expressing two options and a further resulting option (out of the other two options), and for arranging a sequence of options such as a sequence of activities or events (more on these possible functions in 6.4.4.4).

In the following example (114), three listed alternatives (color-coded grey) are enumerated. The various indicators for the particular alternative locations are encircled (the non-manual indicators) and written in red, respectively.

$(114)^{206}$

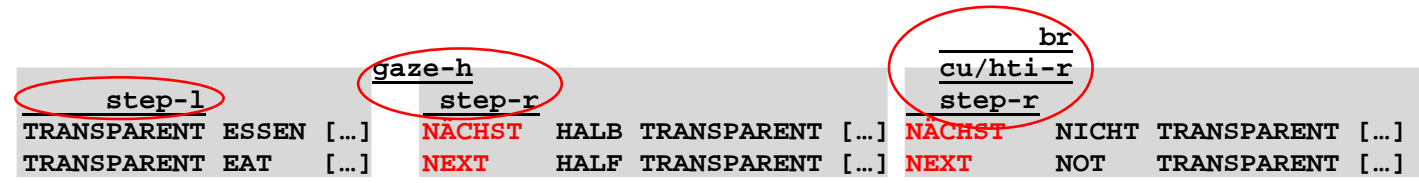

Transparent sind gestische Gebärden die die Handlungen Essen, (Sich-schlafen-legen oder Autofahren) ausdrücken. Halb transparent (sind teils gestisch ausgeführte Gebärden der Handlungen Schlafen, Autofahren oder Trinken). Nicht transparente Gebärden (sind Gebärden wie Geben. So ist das.)

Transparent are gestures which illustrate activities like eating, (going to bed, or driving) Half transparent (are gestural signs which are activities like sleeping, driving, or drinking). Not transparent signs (are signs like give. That's it.)

In (114), all subject matters which are 'transparent' versus 'half-transparent' versus 'not transparent' are allocated to three different alternative spaces. The first alternative location is indicated by stepping sideward (leftward) resulting in referring to a location in the 'enlarged' signing space in the lateral field along the frontal axis. This space refers to 'transparency'. The second alternative location is indicated by looking towards the signer's hand before starting the sign NEXT. Afterwards the informant performs this sign together with stepping sideward along the same frontal axis towards the opposite direction (resulting in a step rightward). In doing so, the signer refers to the second alternative location which is located between the first and the following alternative location. This alternative space refers to 'half-transparency'. Finally, the signer moves to the third alternative location by stepping once again in the same direction (resulting in a further step

\footnotetext{
${ }^{206}$ For reasons of simplification of the illustration, only the relevant non-manual indicators for the alternative space and the relevant signs are annotated. In the translation the whole content is given.
} 
rightward), signing NEXT and tilting the head sideward by an up and down nodding movement which is accompanied by raising the eye brows. In doing so, the signer indicates the third alternative space which refers to 'non-transparency'.

\subsubsection{Indicators and characteristics}

There are various indicators that refer to the 'alternative space'. First of all, the head and/or body markers are obvious. The body and head may be moved towards opposed directions which are sideward versus sideward or forward versus backward. As described, in most cases a dichotomy of two subject matters is intended. Consequently, the head and body are predesignated articulators for demonstrating this dichotomy. These articulators (head and upper part of the body or the body as a whole) may be moved towards the particular alternative location.

But there are other indicators as well, such as gaze direction marker, pointing sign(s), or displacing the place of sign's production - all toward the specified alternative space(s).

\section{a) Head and/or body markers}

The markers for indicating an alternative space along the frontal axis are 'head tilt sideward' (hti-r/l), 'body lean sideward' (bl-r/l) and 'step sideward' (step-r/l). The first two may occur together, or the three markers occur on their own. The co-occurrence of tilting the head sideward and leaning the body sideward is, among others, probably due to the possible movement abilities which depend on each other. The evident markers for indicating an alternative space along the saggital axis are positioning the head forward (hf) versus backward (hb), leaning the body forward (bl-f) versus backward (bl-b), and even stepping forward (step-f) versus backward (step-b). Similar to items already mentioned, the first two markers may occur together, or the particular markers occur on their own.

The data shows that the head and/or the upper body markers cover lexical elements that are allocated to the particular alternative space. The whole-body marker (stepping to an alternative space) is in most instances a beginning phrasal edge marker. In some instances head pointing is present which means that the head is pointing towards an already 
established alternative space. This pointing element only occurs when two contrastive alternatives are labeled along the frontal axis and the head refers to one of the options.

In the following, the implementation of the 'alternative space' along the frontal axis is illustrated. The examples are from the corpus containing train of thoughts. The same content is given in sitting and standing positions by all signers. The annotated results show that the signers have a tendency to use the marker head tilt sideward (hti-r/l) in case that they are sitting, and the marker body lean sideward (bl-r/l) in case that they are standing. But the differentiation of using one of the possible markers does not always depend on the sitting or standing position. However in standing position all signers move their body toward the particular alternative space, partly together with tilting their head while in sitting position the body is only optionally moved toward the particular alternative space.
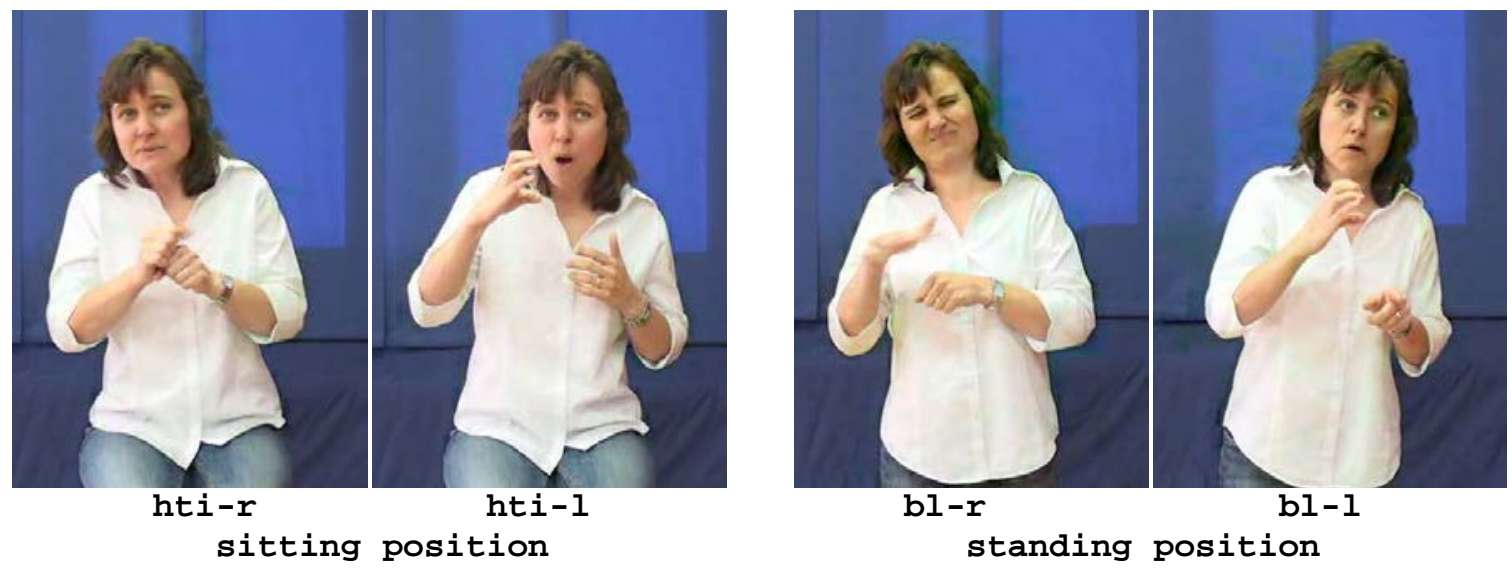

Figure 6.13 Use of head and body markers in sitting and standing position

The above-mentioned Figure 6.13 is taken from short stories in which the signer opposes entities of which she is wishing to get one. The head and body marker hti-l/bl-l and htir/bl-r cover further signs apart from the opposed entities. 
$(115)^{207}$

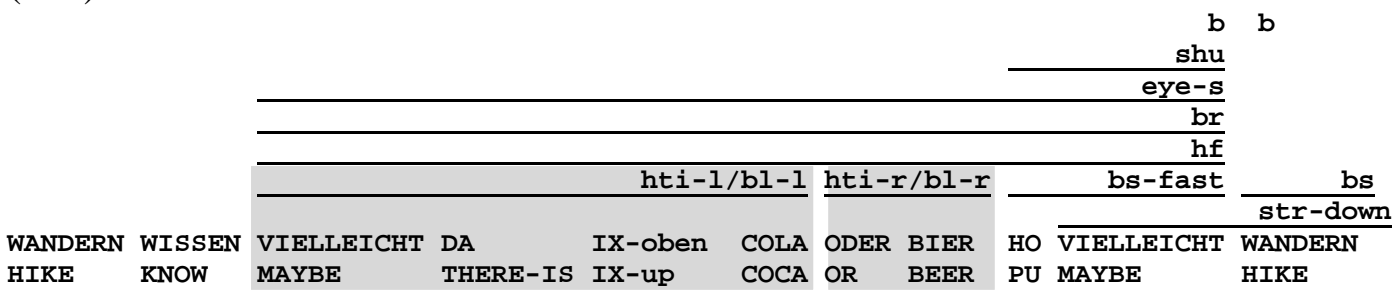

Während ich wandere denke ich, dass es dort in der Hütte vielleicht Cola oder Bier gibt. Jedenfalls bin ich mir unsicher.

While I am hiking I think that at the hut there might be cola or beer. Anyway, I am uncertain.

(F004_1117,1209_m_thoughts_ex06a_05.24-05.37)

The use of the alternative space may be established when two possibilities are available. The particular grey-colored block shows which lexical elements are covered by the particular head and body markers. This example (115) and the following example (116) clearly show that the head and body markers are phrasal domain markers, covering a syntactic constituent (and not just the particular entities). $(116)^{208}$

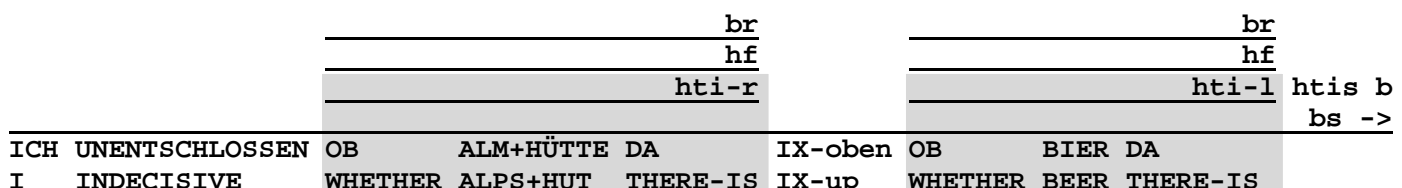

I INDECISIVE WHETHER ALPS+HUT THERE-IS IX-up WHETHER BEER THERE-IS

Ich bin unentschlossen, ob es etwas auf der Almhütte gibt, vor allem ob es Bier dort gibt. I am uncertain whether there will be anything available in the hut, especially whether there will be a beer.

(M002_114,126_m_thoughts_ex04a_05.05-05.18)

In (116), the signer wonders whether there is something to 'drink' and especially whether there is a specific drink - beer - available. The head is tilted rightward during the first train of thoughts concerning an option and leftward during the second train of thoughts concerning another option. The head tilt rightward and leftward are interpreted to function as a kind of contrast, but the annotators could not comment in which way the contrast is meant as not two equal subject matters are opposed. This means that 'existence' is not opposed to 'non-existence' and a 'kind of beverage' is not opposed to another 'kind of beverage'. In addition, the body sways sideways during the entire utterance. Following the

\footnotetext{
${ }^{207}$ The example has been cited in chapter 4 (cf. example (69)).

${ }^{208}$ The example has been cited in chapter 4. The ID-gloss INDECISIVE is maintained even though the actual meaning in example (116) is more likely 'uncertain'.
} 
annotators these body sways display the uncertain attitude toward the thought situation and what the signer is going to do now (more on the marker body sways cf. 7.4.4).

Head and body markers can have further functions too. For instance, moving the head and/or body forward is used to make an element more prominent. In doing so, the means of coding can be used to mark 'emphasis', for example. Moving the head and/or body forward versus moving it backward is expresses the pragmatic function of assertion versus denial (cf. Wilbur \& Patschke 1998 for ASL on this matter). The body can be moved forward or backward in order to allocate a turn or to avoid turn taking (Lackner 2007, 123126).

\section{b) Gaze direction}

Apart from the very obvious head and body markers other indicators are present in the data which may occur as the only indicators for the alternative space, but in nearly all instances these further indicators co-occur with the already described head and/or body markers. The following Figure 6.14 illustrates the use of the marker 'gaze-towards-the-particularalternative'. In addition, displacing the sign's place of articulation towards the particular location together with moving the sign towards that location is illustrated too. Finally, the second picture of the Figure 6.14 shows that the lecturer has changed his position resulting from stepping sideward (rightward from his perspective).

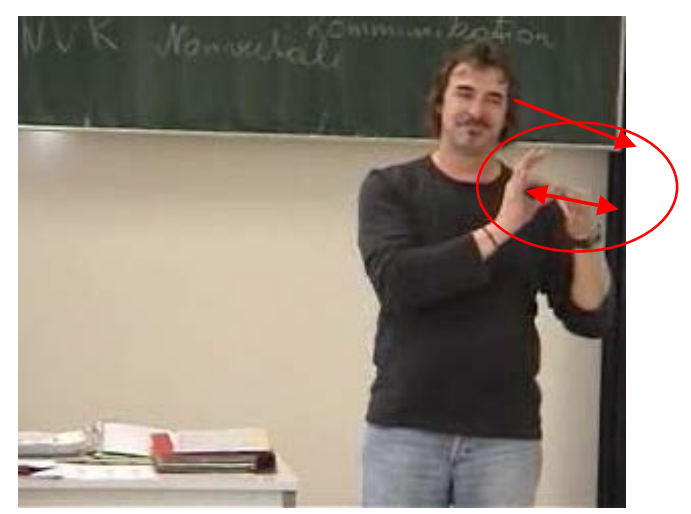

sign: left placed, performed towards the left space gaze: gaze-left 


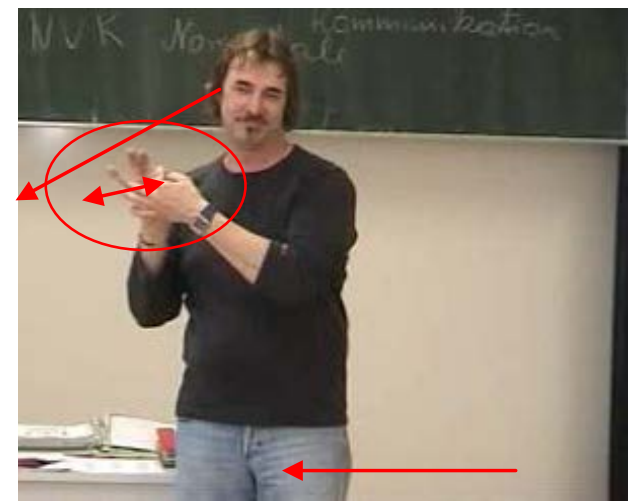

sign: right placed, performed towards the right space gaze: gaze-right step: step rightward (which has preceded)

Figure 6.14 Indicating two alternative spaces

\section{c) Signing at the respective alternative spaces or signing in a place that is closer to the particular alternative spaces}

As illustrated in Figure 6.14 above, the place of articulation of the signs may be moved toward the alternative space. So, when alternative spaces conveying the values 'lateral' versus 'lateral-the-other-side' or 'front' versus 'back' are present, the phenomenon 'displacement of the sign's place of articulation' results in signing more lateral (rightward or leftward from the signer's perspective) or more proximal or distal in front of signer's body. In Figures 6.16, 6.17 and 6.18, both possibilities are displayed. On the one hand, the signs referring the respective alternative space can be produced at these alternative locations (compare to this the three different places of articulation of the sign BEAUTIFUL in Figure 6.17 and also the three places of articulation of the signs FIRST, SECOND and THIRD in Figure 6.18) or on the other hand, the signs are produced in a place that is closer to the particular alternative locations (see the signs FIRST, SECOND and THIRD in Figure 6.17 which are produced with the non-dominant hand and constitute listing buoys which are performed closer at the respective alternative space).

\section{d) Signing production towards the alternative space}

The phenomenon that signs may move between different locations has been investigated, described, and interpreted in various ways by many sign language researchers (for an overview see Barberà 2012). In this thesis there is only brief mention that some of the 
signs that have the ability to be moved within the signing space are moved toward the particular alternative space in the event that these signs refer to an alternative location. This phenomenon is exemplified by the Figure 6.14 or example (119).

\section{e) Pointing signs}

One possibility to refer to an alternative space is pointing to that location. As present in the various ÖGS corpora this is done by different pointing signs.

The most prominent one is the index sign. Indexing is also possible when performing another sign which allows that one of the fingers (mostly the index finger, sometimes the middle finger or thumb) points towards the alternative location. This is shown in Figure 6.1. In the pictures nine and ten the lecturer points with the index sign, produced by the non-dominant hand, toward the second labeled alternative space. Subsequently (picture eleven to thirteen) the lecturer performs the hand form of the sign WHAT and simultaneously points with the index finger of the non-dominant hand towards the specified location. Then (picture fourteen) a further pointing $\operatorname{sign}^{209}$ refers to the alternative location which is performed with B-hand form, palm up, two-handed, and the tips of the fingers direct toward the specified location.

In the corpus of train of thoughts sometimes the sign DA (THERE-IS) occurs. When performing the sign the middle finger is directed downwards and the index finger is directed upwards. The downwards directed middle finger refers to 'here and now' and so expresses 'existentiality ${ }^{210}$. Directing the index finger upwards may refer the space of thoughts or/and a location situated in a higher absolute space in the reality.

Moreover, there is a further sign which is used to refer to alternative spaces. This is illustrated in Figure 6.15.

\footnotetext{
${ }^{209}$ In ÖGS the two pointing signs - index sign with stretched index finger and the index sign of which the middle finger directs toward the pointed location - have been described (Skant et al. 2002, 54 and 82-83).

${ }^{210}$ Schalber \& Hunger (2008) investigated expressing existentiality in ÖGS.
} 


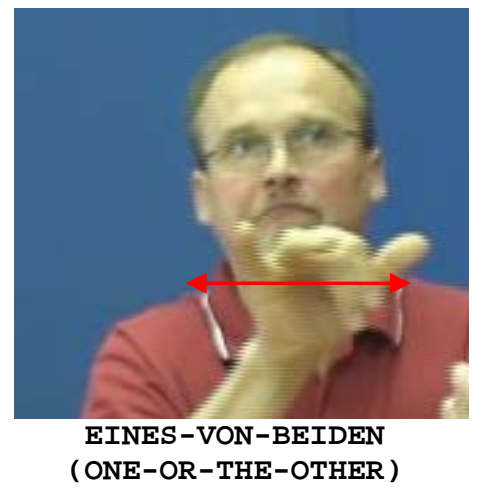

Figure 6.15 Pointing sign for alternative space

The sign ONE-OR-THE-OTHER ${ }^{211}$ occurs in the corpus when the signers establish two alternative spaces which refer to different propositions. Sometimes the sign is used to distinguish between two entities. The thumb and the little finger are pointing to the particular opposed space along the vertical axis. The pointing sign is used when the signer wonders ‘which' option will be true, occurring, chosen, etc.

\section{f) Dominance reversal, buoys}

In Figure 6.1, two phenomena are illustrated which characterize the alternative space several times. These are 'dominance reversal' and 'occurrence of buoys'. In Figure 6.1, the former dominant hand (right hand), signing THREE-DIMENSIONAL, is held at a place located more rightward in the signing space, constituting a 'buoy' and displaying one of the alternative spaces (pictures four to eight), while the up to now non-dominant hand (left hand) keeps on signing, resulting in a 'dominance reversal'. When the other alternative space is referred to, the up to now dominant hand (left hand) points to the left and is held in a location situated more leftward in the signing space (constituting a 'pointing buoy'), while the main information flow is continued by the original dominant hand (right hand).

The first phenomenon displays the occurrence of buoys when using the alternative space. As described in (f) under 6.4.3.2, Liddell (2003, 223-260) argues that buoys indicate

\footnotetext{
${ }^{211}$ In ASL this sign can mean 'same' and is also used to mean 'even'. In ÖGS the sign for GLEICH (SAME) is two-handed, has the hand form of the index sign, the index fingers direct from the body away, and the inside of the index fingers touch together.
} 
anchor points within a discourse structure. Three different kinds of buoys constantly occur when using the alternative space. These are 'list buoys', 'pointer buoys' (both as described in (f) under 6.4.3.2) and what I label 'placeholder buoys'. The latter phenomenon means that a sign is held while the other hand keeps on providing information. 'Holding a sign at the location of an alternative space' functions as a 'placeholder of an alternative' which is opposed to another alternative signed by the active hand. This phenomenon which occasionally occurs when using the alternative space is illustrated in Figure 6.16.
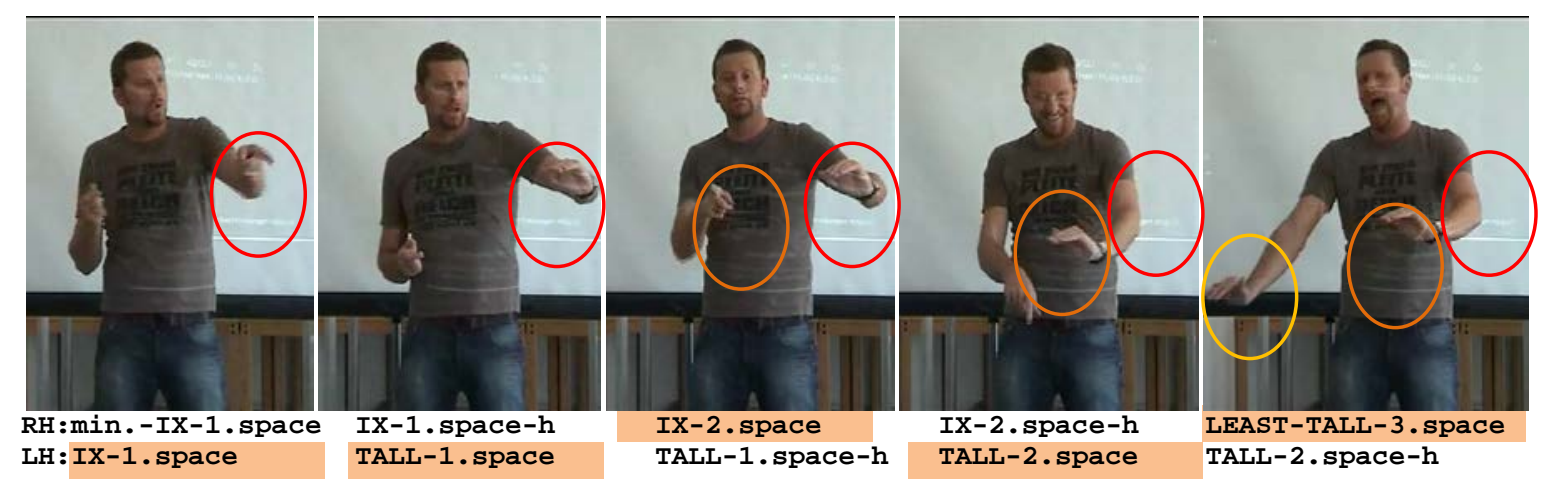

Er/sie/es ist groß. Im Vergleich dazu ist er/sie/es kleiner. Im Vergleich dazu ist er/sie/es am kleinsten.

s/he/it is tall. In comparison s/he/it is smaller. In comparison to that s/he/it is the smallest.

\section{Figure 6.16 Dominance reversal and buoys used for alternative spaces}

In Figure 6.16, the signer uses three alternative spaces, displaying the different degrees of comparison (in the fifth picture all three spaces are encircled). In addition to using the lateral alternative space, the signer also implies a downward gradient. The reason for this may be evoked by a prototypical comparison of height, but other reasons for implying a downward gradient are possible too. The three locations are indicated by gaze-directed-tothe-particular-space and indexing to the respective alternative space. With 'placeholder buoys' the sign TALL is always held in the respective alternative space (encircled red in picture three and orange in picture five) while the active hand keeps on signing. 
In addition, Figure 6.16 shows the phenomenon of 'dominance reversal' ${ }^{212}$. Frishberg (1985) described that based on the possibility that information can be coded separately by both hands in sign languages, active hand of a signer can alternate. This results in a 'dominance reversal'. The data shows that if the actual lateral alternative spaces are located 'ipsilateral' and 'contralateral' from the signer's perspective, in some cases the dominant hand of the signs which are allocated to the respective alternative space, is the right hand for the ipsilateral location and the left hand for the contralateral location. Thus, when opposing both spaces, that is, when producing first signs that refer to the ipsilateral space and subsequently producing signs that refer to the contralateral space, this results in a dominance reversal of the hands. In Figure 6.16, several times a dominance reversal occurs. For reasons of clear illustration, the particular sign which is performed with the dominant hand is marked orange in the glossing line. First, the left hand is used to indicate the first alternative space (encircled red) by signing IX-1.space and TALL. Both elements are produced with the hand which had been the non-dominant hand before (first dominance reversal). The last sign is held and the right hand points now to the second alternative space (encircled orange; second dominance reversal). The index sign is held and the left hand signs TALL-2.space (third dominance reversal). This sign is held and the right hand signs TALL-3.space (fourth dominance reversal). To sum up, signs and placeholder buoys that are allocated to the two alternative spaces which are situated at the left body side of the signer, i.e. contralateral (encircled red and orange), are produced by the left hand. The signs which are allocated to the actual ipsilateral alternative space are produced with the right hand. But if the left hand is already used for a placeholder buoy, the other hand has to produce the signs which are allocated to the other alternative spaces. So, in picture three the left hand displays the buoy while the right hand points to the second alternative space, located contralateral from the signer's perspective.

\footnotetext{
${ }^{212}$ Dominance reversal was discussed by various sign language linguists, among others, Vermeerbergen 1996 on Flemish Sign Language (VGT), Leeson \& Saeed 2004 on Irish Sign Language (ISL), Hendriks 2007b on Jordanian Sign Language (LIU), and Crasborn 2011 focusing on general aspects on dominance reversal in sign languages.
} 
With respect to 'list buoys' in the context of alternative space the following two phenomenon are of interest. To begin with, the data shows that frequently the nondominant hand is used for list buoys. When doing so, the listing hand always has the same place of articulation. In the context of alternative space the place of articulation can be different, as illustrated in Figure 6.17.

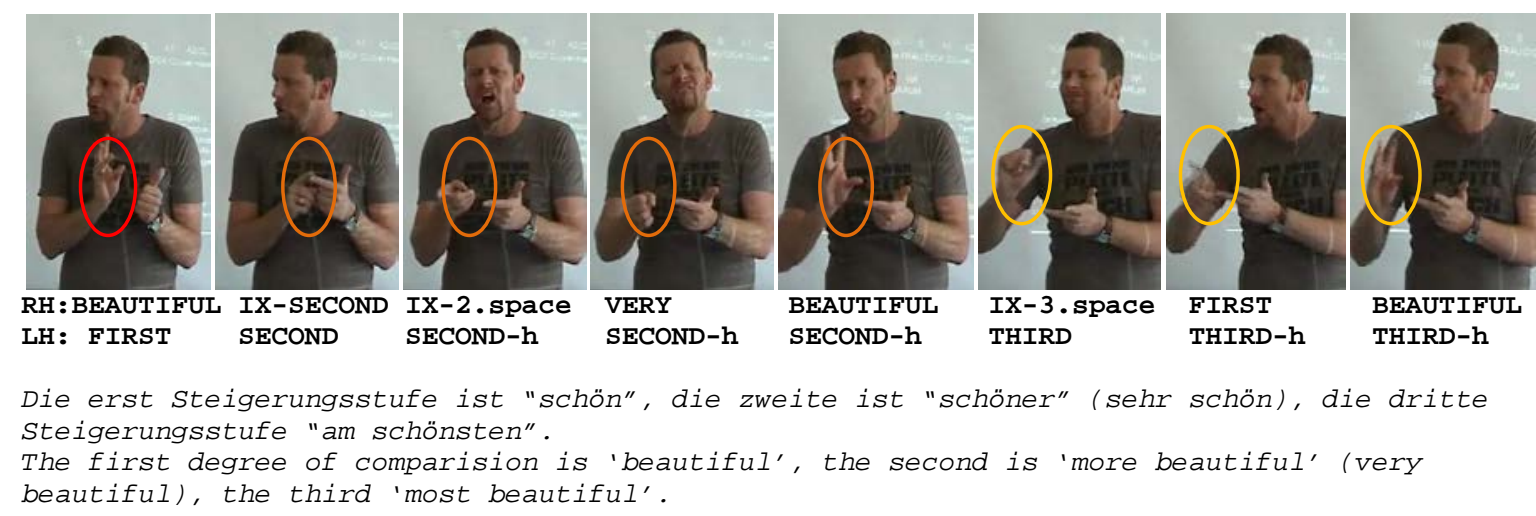

Figure 6.17 Moving of the listing signs towards the three alternative spaces

In Figure 6.17, the signer uses three lateral alternative spaces (encircled red, orange and yellow) for the different degrees of comparison. In addition, the non-dominant hand produces the list buoys FIRST, SECOND and THIRD. Interestingly, the place of articulation of each list sign is produced closer to the respective alternative space. The list buoy FIRST has its place of articulation below the shoulder in the height of the upper body, contralateral from the signer's perspective. The list buoy SECOND is produced in the same height of the upper body, but a bit closer to the vertical axis of the signer. The list buoy THIRD is signed even more to the right, having its place of articulation slightly ipsilateral from the vertical axis with respect to the signer's body.

In following Figure 6.18, in which listing occurs in the context of alternative space, the following phenomenon is present: 


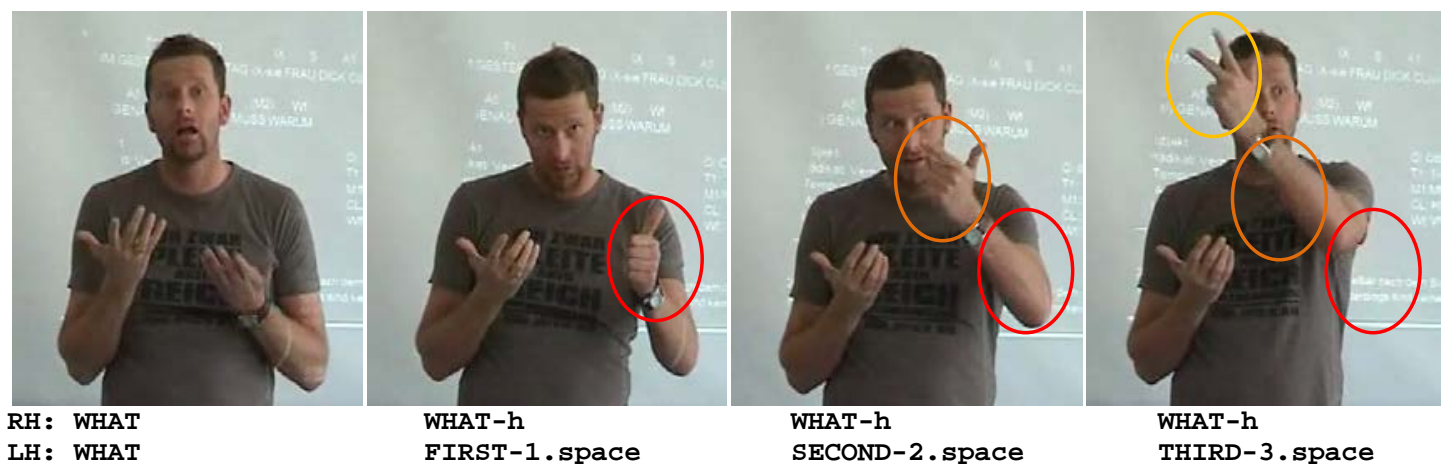

Welche ist es? Ist es die erste Steigerungsstufe? Ist es die zweite Steigerungsstufe? Oder ist es die dritte Steigerungsstufe?

Which one is it? (The signer refers to a preceding sign which is performed with a special degree of comparison.) Is it the first degree of comparison? Is it the second degree of comparison? or is it the third degree of comparison?

Figure 6.18 Listing signs constitute the the three lateral alternative spaces

In the present excerpt from the educational training corpus the lecturer asks which of the three degrees of comparison is expressed by the preceding sign. While holding the question $\operatorname{sign}^{213}$, the three possible alternative spaces are arranged laterally with an upward gradient (encircled red, orange and yellow). An ordinal sign is produced at each alternative space. In doing so, the three underlying subject matters 'first/second/third degree of comparison' are listed. In other words, in addition to list FIRST, SECOND and THIRD, a reference to the 'degree of comparison' is made by the use of the alternative space (more on 'underlying subject matters' see 6.4.4.5).

Figure 6.18 shows a further interesting aspect. The active hand for listing is the former non-dominant hand. In the data listing items is most frequently done be head and/or body forward movements (cf. 6.4.4.6) or by using 'list buoys'. Thus, the reason for a dominance reversal in the present case might be evoked by the usual performance of list buoys by the non-dominant hand.

Summing up, when using the alternative space the phenomena of 'dominance reversal' and 'occurrence of buoys' can characterize the alternative space. Looking at it the other way around, in the examples described above, both phenomena are clearly caused by using the

${ }^{213}$ The different head positions indicate the content and polar questions. Also the gaze is directed to the audience. 
alternative space. The various corpora show that other reasons which cause dominance reversal or the occurrence of buoys are possible too. A study on turn-taking in ÖGS (Lackner 2007) shows clearly that dominance reversal and the occurrence of buoys can be caused due to interactive reasons. The findings show that when a right-handed dialogue partner wants to maintain a turn, the last sign of the narration is held while interactive elements like WARTE (WAIT) or IX-touch-partner (in the sense of 'hey, you') are signed with the other hand, resulting in a dominance reversal. A study on definitional structures in ÖGS (Lackner 2009c and 2009d) includes that when introducing a new term, frequently the sign of the introduced term is held while the other hand defines the content of that term. Furthermore, the data shows that in the context of the referential use of space, both phenomena can occur too (see, for instance, example (147) in which the signer alternates the active hand when signing I-GIVE-he and continues with this hand signing what happens if a letter is not handed out to that person). All these listed possible contexts show that first the functional context in which the dominance reversal or the use of buoys occurs has to be identified and then, the reason for the dominance reversal can be investigated and clarified.

In conclusion, two facts stand out in the data which evoke a stronger tendency to make use of 'dominance reversal' when referring to lateral alternative spaces. First, those participants who have Deaf parents significantly more often reverse the dominance of the hands when referring to the respective alternative spaces. Secondly, the phenomenon 'dominance reversal' for demarcating the different lateral alternative spaces is significantly more frequent in the educational corpus than in the corpus in which the signers produce trains of thoughts including two opposed alternatives.

\subsubsection{Functional use}

The main function of the use of the alternative space is expressing two or three alternatives which are brought into relation. In the various ÖGS corpora the opposition of two alternatives is the most common case, so I will describe this in detail. A similar way of interpretation can subsequently be considered for three alternatives.

The representation of binarity is based on two parameters: 
First, an 'option A' is opposed by an 'option B' which means that using the alternative space requires at least two subject matters which are brought face to face. In most instances these subject matters already have a kind of relation or offer the opportunity to be contrasted or to be compared. They may represent alternatives of a collective area as in (117) in which two components of a description are listed. They may represent a decision which has been made between two or more options as in (118) in which two possible contrary executions of activities are labeled. They may illustrate two or more actions out of a collective action (Figure 6.22) or out of a sequence of activities (Figure 6.19 and 6.21) and so on.

The second parameter is the interplay of option A and option B. Toward this end, three aspects are possible: The relation between the two alternatives can be described, or two or more alternatives influence each other, or an external option (or even a person) influences the possible options.

Concerning the first aspect, there are the following kinds of relations: $\mathrm{A}$ and $\mathrm{B}$ (in the sense of listed elements illustrated as $\mathrm{A}+\mathrm{B})$; $\mathrm{A}$ is opposed to $\mathrm{B}(\mathrm{A}: \mathrm{B})$; $\mathrm{A}$ is different from $\mathrm{B}$ in $\mathrm{X}$ way (e.g. larger, bigger, faster, earlier, later, etc.); $\mathrm{A}$ and $\mathrm{B}$ are poles of a continuum, and so forth.

With regard to the second aspect unidirectional or mutual influence of the two options on each other may be: $A$ and $B$ result in $C(A+B=C)$; $A$ is deleted and $B$ is inserted; $A$ comes down to B, that is, B is crucial for A; A follows up with B, etc.

With respect to the third aspect an additional outstanding option or even 'person' affect the alternative options like somebody/something deletes A and inserts B, somebody/something likes $\mathrm{A}$ and hates $\mathrm{B}$, somebody/something prefers $\mathrm{A}$ to $\mathrm{B}$, and so forth.

In the following a selection of possible functional uses of the alternative space within the ÖGS corpora is presented and exemplified.

\section{a) A and B}

In many examples in the corpus the primary function of the spatial allocation of the subject matters is that two (or more) options are listed. In doing so, the importance is not only put 
on 'listing' alternatives, but on 'illustrating' two different elements which have a relation to each other.

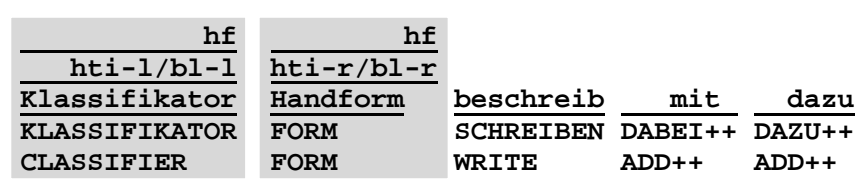

Klassifikator und Handform sind zu beschreiben, alles ist in Ausführlichkeit hinzuzufügen. The classifier and the hand form have to be described. Everything has to be done in a detailed way.

(M008-15.03.08-Teil4-01:00:01-01:00:06,5)

In (117), a lecturer names two aspects which have to be written down by the audience. When signing the two aspects each of them is covered by a head marker and a head/body marker. The sign CLASSIFIER is covered by moving the head forward and leaning the head together with the body sideward (leftward) while the sign FORM, accompanied by the mouthing 'Handform' ('hand form'), is covered by moving the head forward and leaning the head together with the body to the opposed side (rightward). The particular forward movement of the head is used for itemizing the elements (cf. 6.4.4.6) while the head/body marker express that two subject matters are facing each other in an 'additional' way.

\section{b) A versus (or) $B$}

A further function of allocating two alternatives to the opposed (in some cases in the corpus it is even the same location) alternative spaces is 'contrasting' them. Body leans have been described to have a contrasting function in various sign languages (cf. Wilbur \& Patschke 1998 for ASL, Kooij et al. 2006 for NGT).

In the ÖGS corpus frequently the alternative space is used in embedded polar interrogatives. In doing so, the two options of which the signer is thinking are named and additionally contrasted.

In the following Figure 6.19 the informant signs about the situation that her husband is not certain whether he should give up playing cards or whether he should keep on doing this 
activity. Both activities are located to the opposed places in the signing space, located laterally along the vertical axis. The contrary head tilts sideward covering the signs GIVEUP and KEEP-ON. The excluding-one-option sign OR intervenes. This opposition is followed by head tilts sideward which cover the manual element PALM-UP/MAYBE. Due to the head tilts' clear stops at the particular sideward end position, the annotators interpret that the first head tilting movement sideward refers to the first option and the following to the second option. All in all, this example demonstrates the possible contrastive function of using the alternative space.
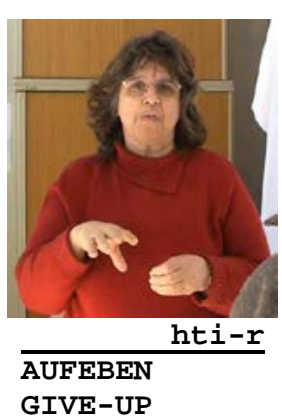

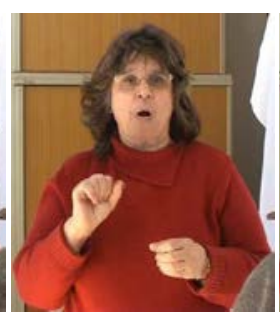

ODER OR
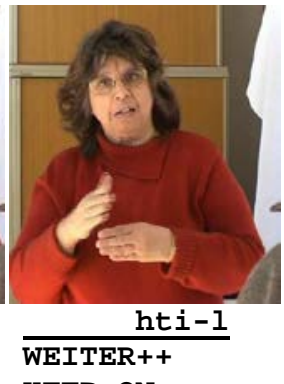

KEEP-ON++

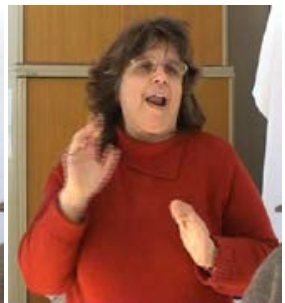

$\frac{\text { hti-r }}{\text { HO/VIELLEICHT }}$ PU/MAYBE

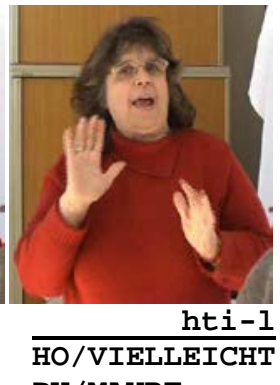

PU/MAYBE

Figure 6.19 Opposing activities by using the alternative space

(F002_d_Film1_Szene4_2.04-2.07)

\section{c) $B$ is highlighted}

A further function of using the alternative space is that two options are mentioned and one of them is highlighted. This can be implemented in the way that one alternative is made more prominent by marking it while the other one is unmarked. In the educational corpus this functional use of the alternative space is very often used when introducing a new terminology and demarcating this new term from an already known one.

In the following example (118) the lecturer wants to highlight that 'mouth gesture' is an important component of non-verbal communication while 'mouthing' is irrelevant. By stepping sideward and leaning the upper part of the body sideward the alternative 'mouth gesture' is made more prominent and, consequently, marked compared with the unmarked subject matter 'mouthing'. 


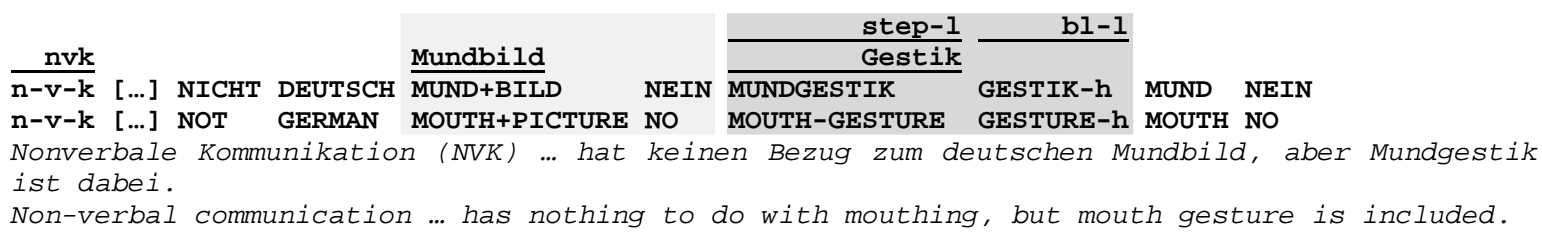

\section{d) A and B constitute C}

Illustrating the mutual influence of two different options may be a further functional use of the alternative space. One possibility is to express that two options together result in a further option which gets its own location in the signing space.

In (119), first two options are named by allocating each one to its own location in the signing space, representing two opposed locations lateral situated from the signer's perspective along the frontal axis. In doing so, the first two signs SAME MOVEMENT are covered by the marker body lean sideward, situated leftward from the signer's perspective. The following two signs SAME FORM are covered by the marker body lean sideward too, but to the opposite side resulting in a rightward movement from the signer's perspective. Hence, the first use of the alternative space is to express that option A is faced by option B. Now each named option gets its placeholder by signing UNIT with the right hand as well as with the left hand. This results in two placeholders for both options which are allocated to the ipsilateral and contralateral signing space. Subsequently, the lecturer brings the hands together (signing/indexing BOTH-TOGETHER) and in this way establishes a new location to which another option can be opposed. This is now done by stepping sideward (rightward) when introducing the new option, which is the terminology RULE DOMINANCE ${ }^{214}$.

First, this instance will be presented with a figure, second, the example is pictured (without an English translation of the glosses and a translation into German and English), and third, it is presented as a whole.

\footnotetext{
${ }^{214}$ The examples of the educational corpus are taken out of context and, consequently, in this case they do not represent a clear definition of the dominance rule of the hands.
} 

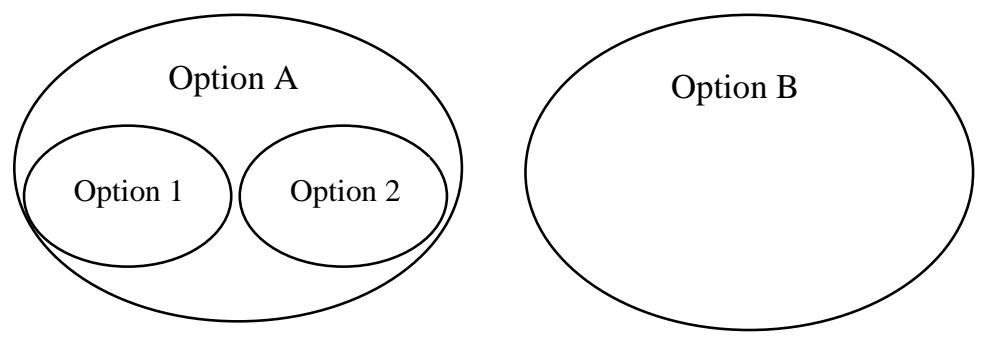

Figure 6.20 A and B constitute $C$

Figure 6.20 shows the two-time uses of the alternative space. The small circles constitute the first named options (option 1 and option 2), the bigger circles demonstrate the later on mentioned two alternative spaces (option A and option B).
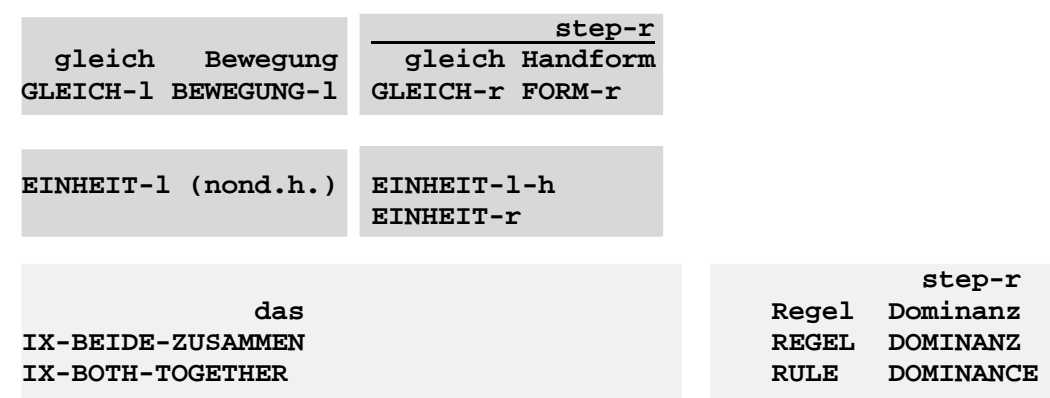

As illustrated in (119a), the signer is referring twice to the first mentioned alternative locations. For demonstrating this, first, the alternative locations are indicated by displacing the signs' place of articulation in the contralateral and ipsilateral signing space along the frontal axis. This displacement is performed when naming the particular options and when naming the placeholders for the options. Second, the alternative locations are marked by stepping sideward (rightward) when mentioning the second option.

The third block illustrates that the lecturer brings both options together and at the same time indexes to a place in front of him by signing IX-BOTH-TOGETHER. This new established alternative location is now opposed by the result, allocated to an alternative space that is placed more rightwards along the frontal axis referring to the other alternative space.

Finally, the example is presented as a whole. 


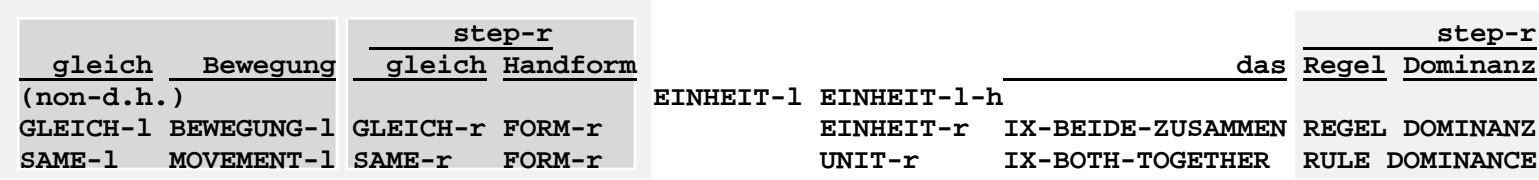

Gleiche Bewegung und gleiche Handform ergeben die Regel der Dominanz.

The same movement and the same hand shape result in the rule of dominance.

(M007-02.05.08-Teil3-1:07:18,5-1:07:24,5)

\section{e) A then $B$}

A further functional use of the alternative space is incorporating a temporal dimension in addition to naming two or more options. Hence, the temporal dimension of activities or events can be considered too, when numerating options. One possibility is shown by the temporal arrangement of activities in the sense of 'first A, then B'. This is exemplified in Figure 6.21. In a dialogue setting a signer narrates about the activities she has done in the Deaf club. On the one hand, she collaborated in doing the cooking and serving meals (summarized by signing WORK); on the other hand, she used the spare time for signing with the club colleagues.

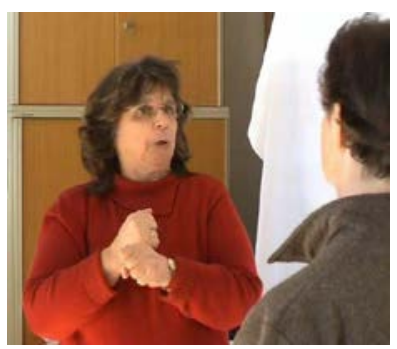

ARBEITEN

WORK
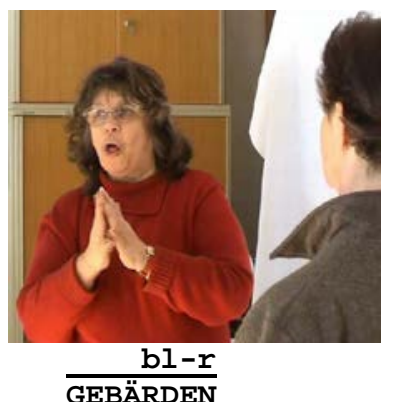

SIGN

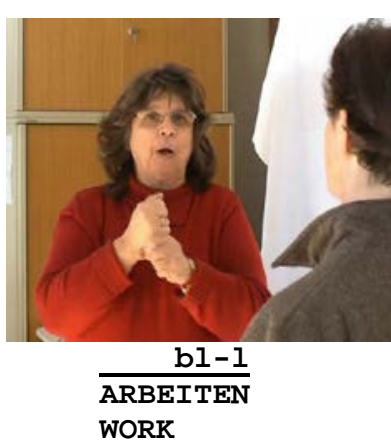

WORK

Figure 6.21 Use of space for displaying two temporal sequential activities

The temporal dimension of doing these activities is implemented by producing the first sign WORK in the neutral signing space in front of the upper body. The following sign SIGNING is performed in a lateral space along the frontal axis due to rightward movement of the upper part of the body. Afterwards she repeats that she is working. In this case the body leans toward the opposite lateral space along the frontal axis. 
In a further example (Figure 6.22) the same signer narrates about temporal sequential activities. The first sequence of activities including various eating preparations (cooking, preparing, serving the meal) are grouped together in a location that is allocated to lateral (exactly the rightward location from signer's perspective), indicated by the marker body lean lateral (rightward) and terminated by the sign FINISH $^{215}$. The second activity (writing) is allocated to the opposed lateral location (specifically the leftward location from the signer's perspective) along the frontal axis. This second activity is terminated by the sign FINISH too.

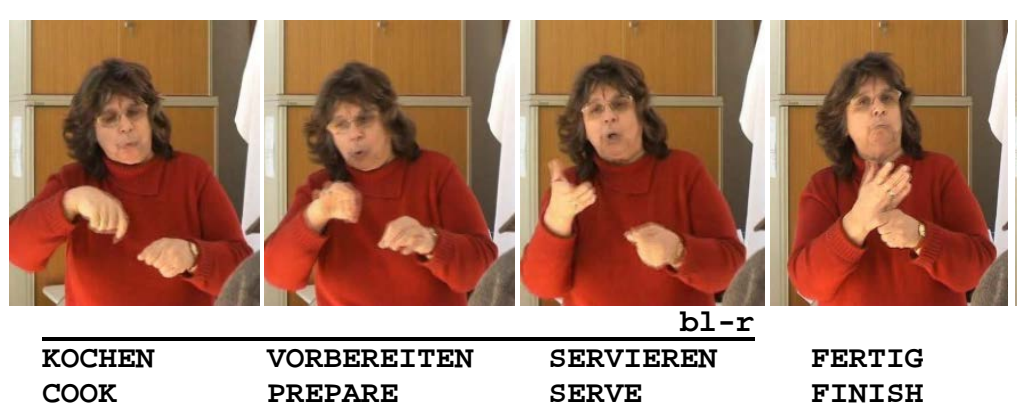

Figure 6.22 Use of space for displaying two complex temporal sequential activities

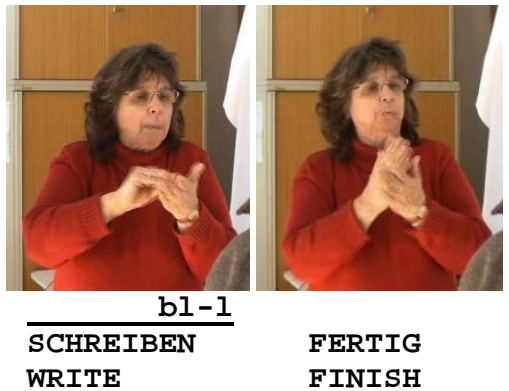

(F002_d_Film1_Szene4_0.56-0.59)

To sum up, the following conclusions may be drawn out of Figure 6.21 and Figure 6.22.

First, the two examples show that two single or complex activities are listed in the sense of A is faced to B (A : B). As the present options are activities the temporal sequential dimension of the single or complex activity is expressed too. This means that the spatial differentiation can be used to 'contrast' two listed (single or complex) activities and at the same time it can show that one single or complex activity precedes the other one.

Second, the underlying subject matters of the particular alternative spaces can be either expressed directly by the lexical elements, or it is expressed indirectly and forms the basis of the alternatives coded by the alternative space. For instance, in Figure 6.22 the first alternative space comprises all activities which are performed before starting the meal (that is, preparing the meal and serving it) while the second alternative space comprises the

\footnotetext{
${ }^{215}$ The sign FINISH has an aspectual function in ÖGS and is used to signal the completion of an activity (Lackner 2007, 92-94).
} 
activity which is done after having the meal (that is, writing the bill). Having these two comprehensive activities in mind, a temporal sequential dimension is included too.

\section{f) A beside B (result in a side comment)}

Metzger \& Bahan (2001) observe for ASL that with the help of spatial cues side comments $^{216}$ can be marked. In the ÖGS corpora these possible cues are primarily body markers like body lean sideward or step sideward. The latter one mostly occurs in the educational corpus. Further indicators are the markers gaze right/left, displacement of the sign's place of articulation toward a location situated sideward along the frontal axis as well as movements of the signs toward the location (if the sign allows this), and signs like DAZU (IN-ADDITION) or NEBEN (BESIDE) which introduce the side comment.

In (120), a lecturer describes Stokoe's work. His way to address the linguistic field is implemented as a side comment which is indicated by various cues (color-coded red and encircled red). With regard to the alternative location the side comment is allocated to a sideward (exactly rightward) location from the signer's perspective in the 'enhanced' signing space. As soon as the lecturer returns to telling about Stokoe's achievements in sign language research, he goes back to the original location which displays the location of the general story description.

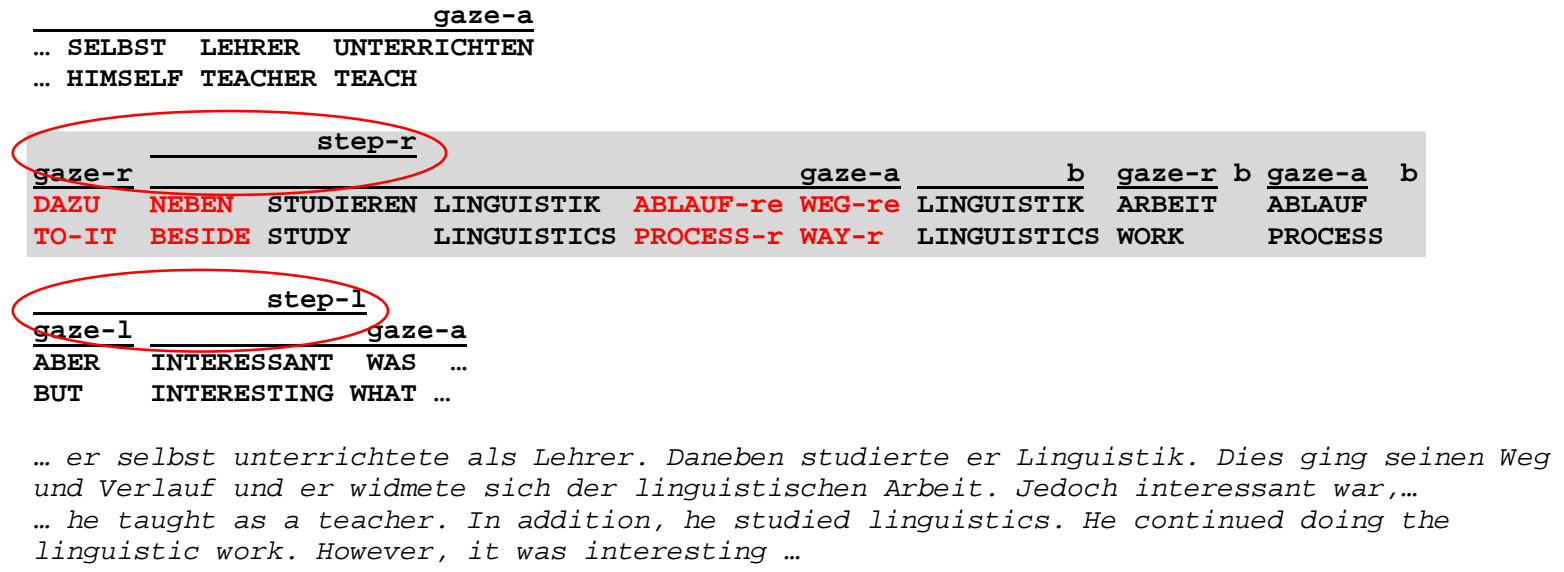

\footnotetext{
${ }^{216}$ In the present thesis a 'side comment' is defined as an additional remark of a fact, an observation, a person’s opinion, etc. which conveys additional information, attitudes, and so on.
} 
Finally, a further interesting use of the alternative space is present in the ÖGS corpora.

\section{g) If $\mathbf{A}$ then $B$}

In some conditionals the alternative space is used when illustrating that in addition two options are present. First, the antecedent of the conditional clause is allocated to an alternative space and second, the consequent of the conditional clause is allocated to the opposed alternative space. In the corpus the alternative spaces are the lateral locations along the frontal axis from the signer's perspective. Whether the two opposed locations along the saggital axis in front of the signer may be used for conditionals or not requires further investigations.

In (121), the antecedent of the conditional clause displays at the same time one alternative and the consequent displays the second alternative. The condition is marked by head forward, the particular alternative space is marked by head tilt sideward (first leftward, then rightward). In addition, alternativity is marked by gaze left/right which goes together with head turn left/right.

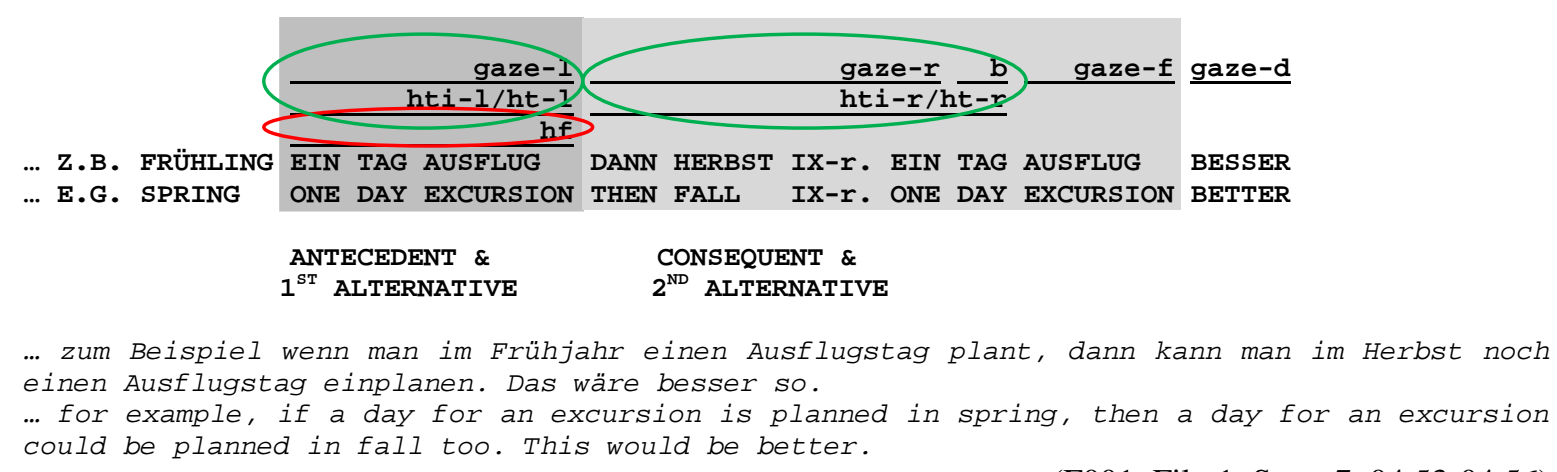

(F001_Film1_Szene7_04.53-04.56)

Summing up, it can be stated that the alternative space can be used for various purposes. The described functional uses are an incomplete list of possibilities and present the most frequent uses in the ÖGS corpora. Of paramount importance is that head and/or body

\footnotetext{
217 The gaze direction which covers the second alternative space does not cover the lexical item EXCURSION in the second option. This is probably due to the dialogue situation, as the signer looks at the dialogue partner (described as gaze-d). Furthermore, the example (121) is described in detail in chapter 5 (cf. example (102)).
} 
markers (including steps) are the most constantly and frequently used spatial cues for coding the alternative space.

\subsubsection{The underlying subject matters of the alternative space}

As described, beginning phrasal edge markers and/or phrasal domain markers indicate the alternative spaces. The first (i.e. the beginning phrasal edge markers) precede, the latter (i.e. the phrasal domain markers) cover the lexical elements which refer to underlying subject matters, that is generalized concepts. All these indicators for the alternative space are spatial cues that clarify which lexical items belong to which subject matter and are allocated to one of the alternative spaces. So, in fact it is 'an underlying subject matter' that is faced by 'another subject matter' and, in most cases, the relation between them or the influence of an external subject/factor is clarified. This observation is exemplified in (122). $(122)^{218}$

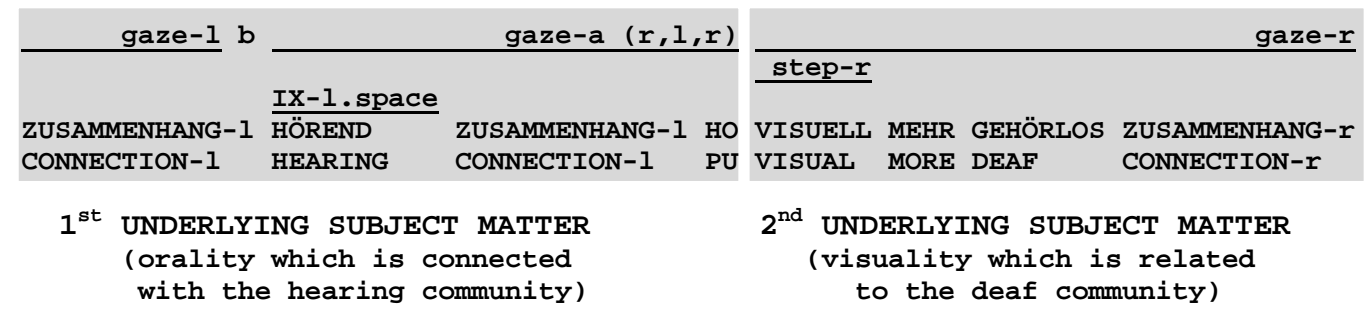

Verbalität wird mit Hörenden in Verbindung gebracht während Visualität mit Gehörlosen verbunden wird.

orality is related to hearing people while visuality is connected with Deaf people.

In the course of describing non-verbal communication a lecturer dwells on the meaning of visual communication. In doing so, he asserts what belongs to the hearing world, which is the spoken-auditory language modality, and opposes this to what belongs to the Deaf world, which is the visual language modality. Thus, neither the signs 'hearing' and 'deaf' nor the signs 'oral' (which is even not produced) and 'visual' are contrasted. The underlying subject matters are the concepts of 'visuality which is related to the Deaf community' and 'orality which is connected with the hearing community'. These two subject matters are opposed.

\footnotetext{
${ }^{218}$ The gaze directions in (122) are gaze directed to the left or right (gaze-l/r) and looking to the audience in the kind of a panorama view abbreviated as gaze-a $(\mathrm{r} / \mathrm{l} / \mathrm{r})$.
} 
Of course, in many instances the underlying subject matters are directly expressed by the lexical items on the surface of the language. But, as shown in (122), that does not have to be the case at all.

\subsubsection{Differentiation of listing}

The spatial cues - primarily the head and body markers - are directed by space. This is due to the spatial cues indicating the hypothetical space (marked by chin-up) and alternative space (marked by body lean / head tilt / step sideward versus body lean / head tilt / step sideward the other side or marked by body lean / head trust forward versus backward).

One of these markers - i.e. head thrust forward or body lean forward - can be used for other purposes too. A body lean forward / head trust forward covering one or more lexical items can either be used to put emphasis on the elements or, in case that the marker is performed more times always covering one element, it can be used as means of coding listing. In ÖGS both the marker head forward and the marker body forward can be used to give prominence to an lexical element. This prominence can function for 'emphasizing' or 'listing'.

However, these two listed markers conveying the functions emphasizing or listing are not directed by space. This means that no spatial location is established to which the head or body marker moves and to which a subject matter or a proposition is allocated. Especially with regard to the enumerating function of the head and body markers, it has to be clarified that the forward movement is not based on an established location in the signing space in front of the signer. Although when listing more lexical elements it is the fact that more alternatives are present, it is not the primary goal to illustrate that options oppose each other or that the relation between these options is described. Rather it is the aspect of 'listing' more options that is emphasized. In addition, in enumerations an opposed marker never occurs (e.g. head / body forward versus backward) and consequently, there is no opposed alternative space. Finally, as listing is based on giving prominence on the particular lexical items in the sense of 'itemizing', it is referred to the lexical elements on the language surface and not to an underlying subject matter.

The listed observations are illustrated in the following example (123) and Figure 6.23. 
(123)

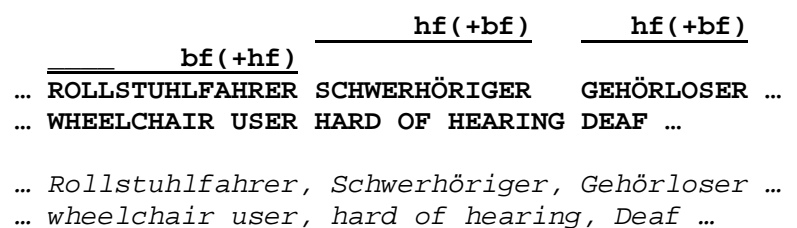

(M001_18_d_narr.inf._00.23-00.25)

In (123), an informant signs a joke. While listing various groups of people having different disabilities, the first lexical item WHEELCHAIR-USER is covered by a body lean forward that goes together with a slightly forward movement of the head, and each of the following signs, that is HARD-OF-HEARING and DEAF, are covered by a forward thrust of the head which goes together with a slightly forward movement of the head.

First, this example shows that performing a head or body marker for enumerating is - at least in this case - due to the place of articulation of the particular signs. So, the first sign is performed in the height of the stomach while the other two signs are produced in the height of the head. Second, the example illustrates that the direction of the movements are only forward and not contrasted to other elements. Consequently, it can be concluded that these markers have to be distinguished from spatial cues which allocate lexical elements to an established/specified location in the signing space.

In the second example (Figure 6.23) question signs are listed which are marked by head forward. In addition, the particular question sign is marked by chin up which is the obligatory head marker for constituent questions in direct communication in ÖGS (cf. 4.2.2.1). Thus, each listed sign is covered by the question markers (chin up as well as raised brows) and - in addition - the marker for itemizing (head forward). As illustrated and based on the annotators' feedback, in the beginning of the sign the marker chin-up is more significant while the marker head-forward becomes clearer at the end of the sign production because the head has reached its final position of the forward movement and additionally a short stop at this position is perceived. 

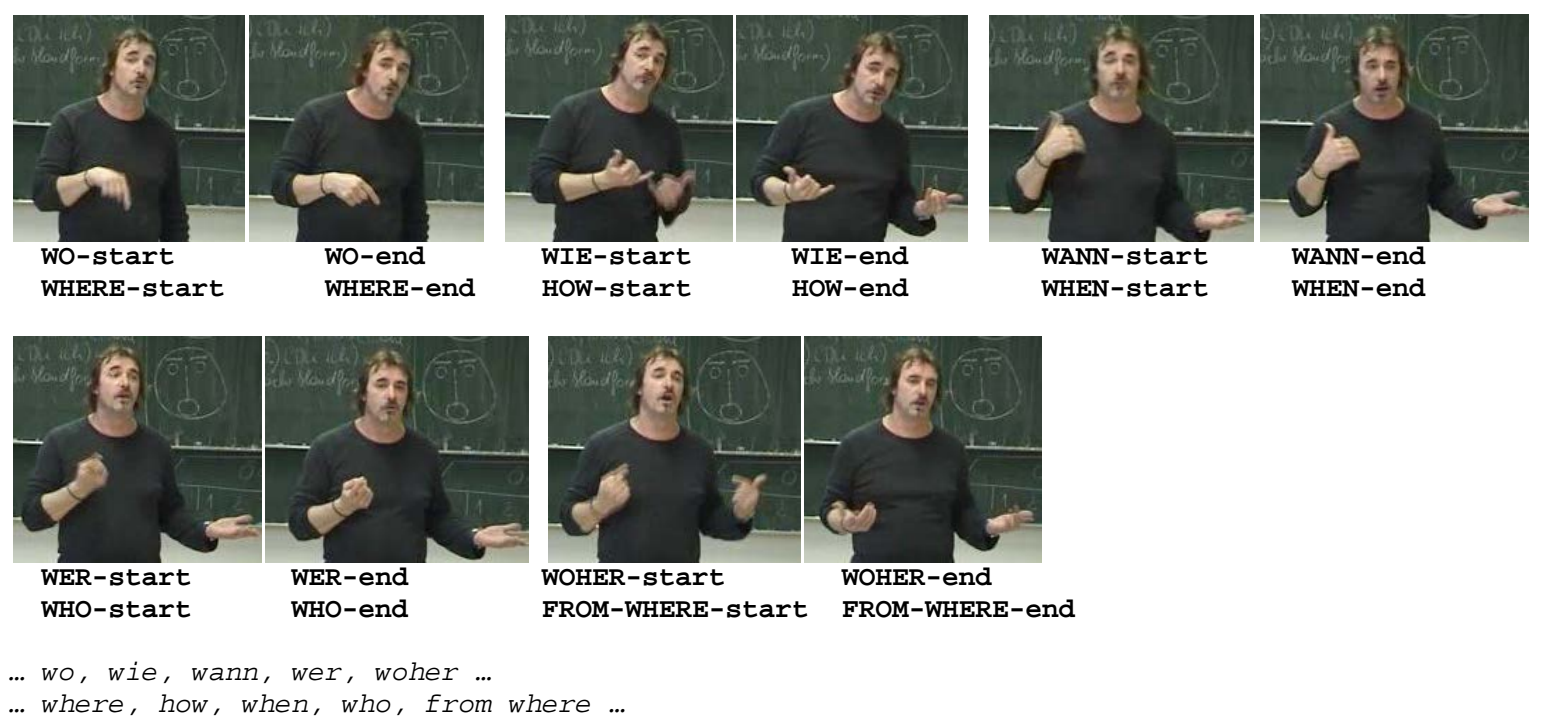

Figure 6.23 Listing question signs ${ }^{219}$

(M008-15.03.08-Teil4-0:23:41-0:23:45)

It can be concluded that one possibility for enumerating lexical elements is using the head and/or body marker ${ }^{220}$. Related to this matter the following obvious characteristics were found in the data: First, the particular listed lexical element is accompanied by a forward movement of the head and/or body which has a clear stop at the end position of the forward movement (which coincides with the endpoint of the sign movement). Second, the forward movements are performed in a regular way resulting in a constant, recurring rhythmical forward movement which is often perceived as regular forward nodding movements by the annotators. However, the present head and body markers are not spatial cues and, consequently, they are not directed by space. When naming options, one possibility is the use of the alternative space. This is done when the signer wants to put emphasis on expressing that two options face each other and stay in some kind of relation or are influenced by an external factor. The other possibility is focusing on the enumeration of elements. In doing so, it is demonstrated that more alternatives belong to a field and should be 'itemized' from the signer's point of view.

\footnotetext{
${ }^{219}$ Figure 6.23, taken out of the educational corpus, illustrates a picture sequence of pairs of pictures that show the beginning and end point of the sign as well as the head forward movement.

${ }^{220}$ Further possibilities for listing in ÖGS are listing by the non-dominant hand (by allocating each enumerated element to one finger), by using signs like ERSTENS (FIRST), ZWEITENS (SECOND) and so on, by regularly and successively performing downward movements of the particular listed element, etc.
} 


\subsubsection{Conclusions of the alternative space}

With regard to the present subchapter it can be stated that the alternative space is used to illustrate a subject matter A that sets it apart from subject matter B. In doing so, two alternatives are differentiated.

The markers for indicating an alternative space are spatial cues as they are directed by space. This is even the very reason why the locations of the alternative space are not described with features like 'ipsilateral' and 'contralateral'. Especially the educational ÖGS corpus demonstrates that for educational reasons the signing space can be 'enhanced'. So, for expressing alternatives, very frequently the marker step sideward is performed (instead of body lean sideward or head tilt sideward). In doing so, the lecturers refer to a location in the signing space that is found in the 'enhanced signing space'.

\subsection{Conclusions on spatial cues primarily coded by head and body markers}

In this section, two functional uses of the signing space, labeled 'hypothetical space' and 'alternative space' by the author, are described. The first has not yet been identified in any sign language; the latter only has been described with regard to contrast. In describing the characterization of the spatial phenomenon of both spaces, their particular spatial indicators and their respective functional uses, the present functional use of the signing space can be clearly distinguished from the linguistic-functional use of the referential space (summarized in Table 6.1), an intention which surely brings new insights into the discussion of the functional use of the signing space.

To begin with, the latest findings show that when ÖGS signers are thinking about unrealized or hypothetical situations which may occur or not or only under special conditions, they always allocate these lines of thoughts to a 'space of thoughts', labeled 'hypothetical space' in this thesis. To sum up, the hypothetical space is

- used when signing about unrealized or hypothetical situations, possibilities, etc.

- used when addressing signed thoughts to oneself. Thus, the lines of thoughts are self-addressed (not to a dialogue partner) or to a hypothetical audience (but not in 
the way as addressing a content to an 'imagined dialogue partner' in the course of a constructed dialogue).

- located in the upper field of the signing space.

- $\quad$ possessing the spatial value 'up'.

- indicated by gaze up (or gaze somewhere to an unspecified place in the front), chin up (or head forward if the thoughts are expressed in an interrogative way), IXup ${ }_{\text {HYP }}$, and/or displacement of the signs’ production to a higher signing place;

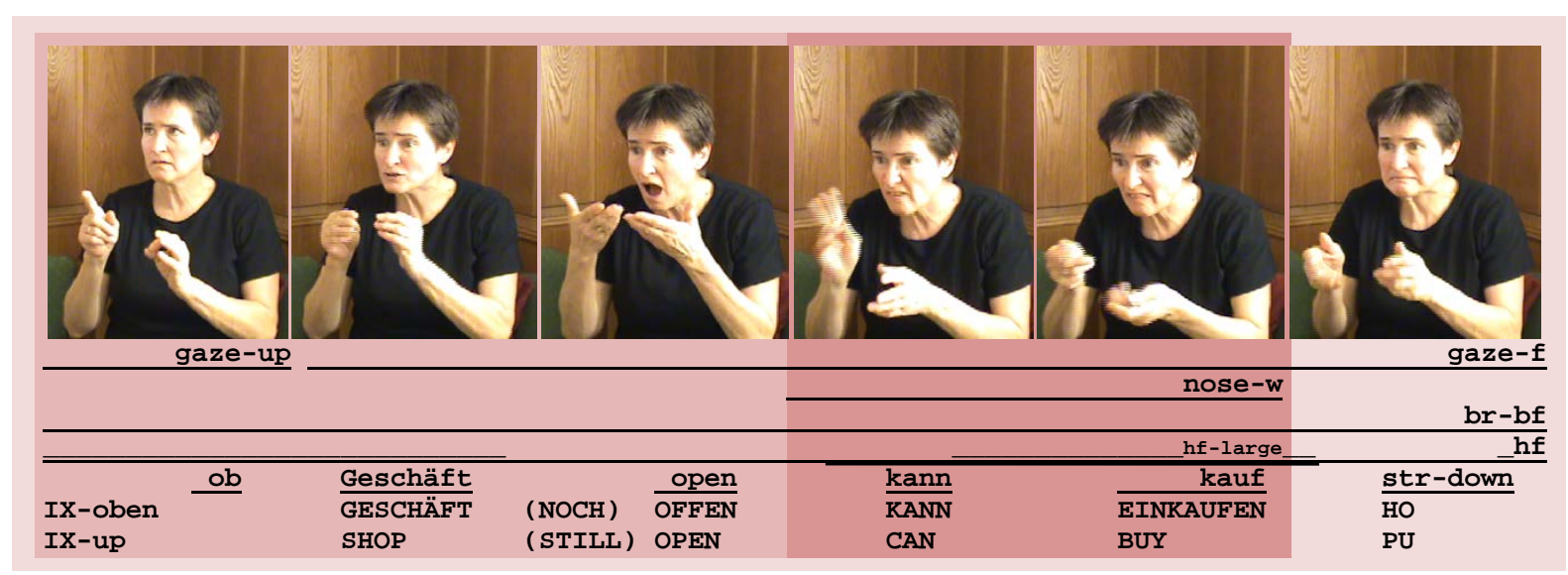

(Während ich mit dem Zug fahre überlege ich,) ob das Geschäft noch offen hat und ob ich noch einkaufen kann. Ich bin mir aber sehr unsicher.

(While I'm going by train I wonder) whether the shop will still be open and whether there will still be time to go shopping. But I am very uncertain.

Figure 6.24 Indicating to the 'hypothetical space'

As illustrated in Figure 6.24 (previously described by example (111)), the signer uses various indicators for referring to the hypothetical space. These are gaze up and gaze to the front, IX-upнур, and head forward instead of chin up as the present line of thoughts is displaying an embedded interrogative.

Using the hypothetical space is probably comparable to Barberà's (2012) observation on specificity. She describes that in Catalan Sign Language (LSC) the upper signing space can be used as a non-specific nominal location.

With regard to the 'alternative space', the present findings confirm Wilbur \& Patschke's (1998) as well as Kooij et al.’s (2006) observations that body indicators are used to imply 
contrast. However, what the present findings show in detail are the following characteristics of using, what I label the 'alternative space':

- First, the different alternative spaces possess the spatial values 'lateral-one-side' and 'lateral-the-other-side' or 'front' and 'back'.

- Second, it is used for displaying alternatives which possess different relations:

1. Opposing alternatives which do not influence each other (e.g. $A+B, A: B, A$ is different to B in X way, etc.);

2. Opposed alternatives which influence each other (e.g. $A+B=C, A$ is deleted and $\mathrm{B}$ is inserted, A comes down to B, etc.);

3. Outstanding factors influence the alternatives (e.g. somebody/something deletes $\mathrm{A}$ and inserts $\mathrm{B}$, somebody/something likes $\mathrm{A}$ and hates $\mathrm{B}$, somebody/something prefers A to B, etc.);

- Third, various possibilities of marked alternatives exist, i.e. marking one of two alternatives and marking two/three alternatives of two/three alternatives.

- Fourth, the lateral alternative spaces are indicated by head tilt and/or body lean sideward (to one side and the other side or twice to the same side), step sideward and/or gaze sideward (to the right and left). The front/back alternative spaces are indicated by head/body lean forward and backward and very rarely by step forward and backward.

- Fifth, the particular alternative spaces display an 'underlying subject matter' as illustrated in (124), previously described in example (122).

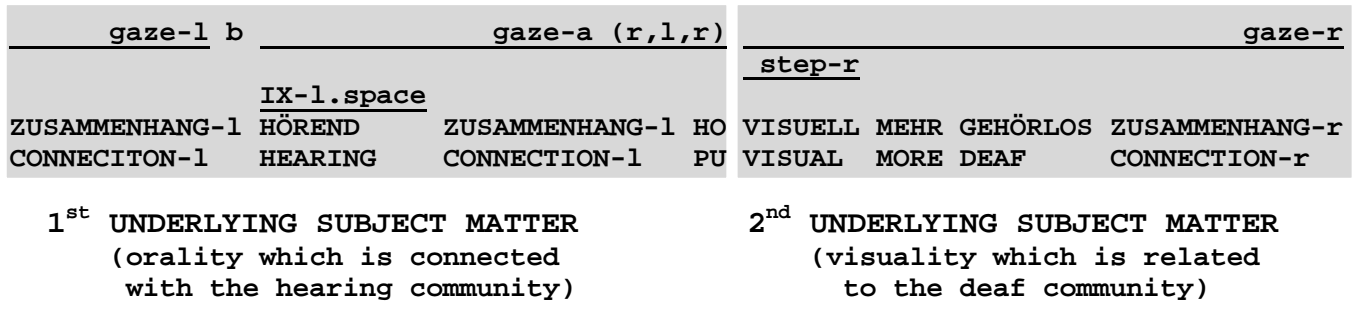

Verbalität wird mit Hörenden in Verbindung gebracht während Visualität mit Gehörlosen verbunden wird.

orality is related to hearing people while visuality is connected with Deaf people. 
In (124), neither the signs 'hearing' and 'deaf' nor the signs 'verbal' (which is not even produced) and 'visual' are contrasted. The underlying subject matters are the concepts of 'visuality which is connected with the Deaf community' and 'orality which is connected with the hearing community'. These two subject matters are opposed in (124). Of course, in the majority of cases the underlying subject matters are directly expressed by the lexical items (signs) on the surface of the language.

With respect to both spaces, the alternative and hypothetical space, the similarities are that first, the particular locations in the signing space are marked by a beginning and/or domain marker. Second, these spaces are indicated by 'orientation-toward markers' and/or 'moving-toward markers' and/or 'pointing elements', depending on the used articulator.

With regard to the differences and similarities between the alternative and hypothetical spaces and the referential space, the following points are summarized in Table 6.1, focusing on spatial markers coded by 'head' and/or 'body'.

\begin{tabular}{|c|c|c|}
\hline Concerning & $\begin{array}{l}\text { alternative and hypothetical } \\
\text { space }\end{array}$ & referential space \\
\hline $\begin{array}{l}\text { - the locations in the signing } \\
\text { space }\end{array}$ & $\begin{array}{l}\text { Locations in the signing space are } \\
\text { pre-established, that is, they have } \\
\text { their own values (up, sideward, } \\
\text { front, back) as a preliminary } \\
\text { determination. }\end{array}$ & $\begin{array}{l}\text { Locations in the signing space have } \\
\text { to be first established before } \\
\text { indicating later in the discourse to } \\
\text { them. }\end{array}$ \\
\hline - the functions & $\begin{array}{l}\text { displaying alternatives, } \\
\text { hypothetical thoughts }\end{array}$ & referential use \\
\hline $\begin{array}{l}\text { - the spatial indicators with } \\
\text { regard to 'head' and 'body' }\end{array}$ & $\begin{array}{l}\text { Alternativity is marked by } \\
\text { head/body tilt/lean sideward or } \\
\text { head/body lean forward/backward. }\end{array}$ & $\begin{array}{l}\text { Referentiality is marked by } \\
\text { head/body turn sideward. }\end{array}$ \\
\hline $\begin{array}{l}\text { - the occurrence of head/ } \\
\text { body indicators with regard } \\
\text { to the associated/referred } \\
\text { lexemes }\end{array}$ & $\begin{array}{l}\text { co-occur with the associated } \\
\text { lexemes }\end{array}$ & $\begin{array}{l}\text { both co-occurrence and single } \\
\text { occurrence with regard to the } \\
\text { reference object }\end{array}$ \\
\hline $\begin{array}{l}\text { - Varying between the } \\
\text { articulators 'head' \& 'body' }\end{array}$ & \multicolumn{2}{|c|}{$\begin{array}{l}\text { Markers indicating referentiality or alternativity can vary the articulator } \\
\text { (e.g. head tilt sideward or body lead sideward or step sideward; head turn } \\
\text { sideward or body turn sideward). }\end{array}$} \\
\hline
\end{tabular}

Table 6.1 Differences/similarities between the alternative/hypothetical space and the referential space 
Finally, based on, for example, Bühler’s (1934) 'two-field-theory’ ('Zweifeldtheorie’) that implies a 'deictic field' ('Zeigfeld') and a 'symbolic field' ('Symbolfeld'), it can be concluded that the linguistic use of space marked by spatial cues which are used for syntactic, textual, or discourse purposes fulfill a 'symbolic' function and are more than only 'deictic' cues. For instance, spatial cues for the alternative space provide more than only 'spatial' information like right and left in the absolute reference frame. They imply that two or more alternatives are opposed, stay in some kind of relation to each other, or are influenced by an external factor. Thus, making use of the alternative space may have the function of 'emphasizing an option', 'characterizing something as side comment', and so forth. 


\section{Modality coded by head and body markers}

\subsection{Introduction}

In this subchapter, I describe two coding systems for modality present in the ÖGS data.

The first modality system comprises the use of modal verbs and further cognitive, emotional, or perceptual signs which convey modality meaning. As shown in the literature overview, this possibility of coding modality was described in several sign languages. Also, some linguists included the non-manual components which cover these signs. In ÖGS only modal verbs have been previously investigated. The findings are briefly described in the literature overview on ÖGS research on modality.

The second modality system includes nonmanuals which code modality and co-occur with entire utterances. To this, I report for the first time of a set of non-manual markers which is primarily used to code 'epistemic modality'. These non-manual markers are primarily produced with the head or body (in addition to other nonmanuals). They serve to mark propositional modality, that is, the signer's knowledge and/or degree of confidence of the truth value of a proposition can be shown by these possibilities in ÖGS: convincedassertive head marker, non-assertive head maker, speculative body marker, and timitive ${ }^{221}$ head marker. In addition, I argue that the head (and body) marker which is used for marking alternatives (cf. 6.4.4) also functions as a deontic/dynamic marker, coding event modality.

Also, other nonmanuals which very likely code modality are mentioned and briefly described. These include 'wrinkled nose' and 'squinted eyes'.

It should be mentioned that the 'hypothetical space' discussed in the preceding chapter is used to express propositions which have not been realized and may convey the notion of possibility or irreality. Thus, referring to the 'hypothetical space' when formulating propositions can be related to modality (cf. 6.4.3).

\footnotetext{
${ }^{221}$ The term 'Timitive' is used in accordance with Palmer $(2001,22)$ when associating/coding the feeling 'concern/worry' with the outcome of a proposition.
} 


\subsection{Literature overview}

Modality $^{222}$ is a means to express unrealized possibilities, desires, wishes, intentions, obligations, and so forth. Following Bybee \& Fleischman (1995, 2), the factual or declarative semantic value of a proposition is supplemented by means of modality. In doing so, values like hypothetical, potential, desiderative, and so on are conveyed. Palmer (2001, 7-8, 24-85) distinguishes two categories of modality, namely 'propositional modality', composed of epistemic and evidential modality, and 'event modality', including deontic and dynamic modality. The first category refers to the speaker's/signer's attitude toward a proposition (epistemic modality) or provides information on the indication a speaker/signer has about a proposition (evidential modality) while the latter concerns events, situations, states, actions, etc. which did not happen in the past but could have happened. If the influencing resource on the 'event' is external, e.g. caused by permission or obligation, Palmer refers to deontic modality while if the influencing resource is internal, e.g. caused by ability or willingness, Palmer uses the term dynamic modality. To date, modality ${ }^{223}$ has been investigated in few sign languages. These sign languages make use of modal verbs and consequently, some part of the modal system has been described. Non-manual elements (face, head, body) that can co-occur with these modality verbs have also been described to some extent. But the emphasis has been put on the cooccurrence with modal verbs and signs that are used to express modality.

Ferreira Brito's article (1990) on modality in Brazilian Sign Language (LSB, Lingua Brasileira de Sinais, or LIBRAS) is one of the first papers focusing on that topic. She distinguishes the various modality signs of LSB in the three modality categories

\footnotetext{
${ }^{222}$ In accordance with Bybee and Dahl (1989), the term 'modality' is used, as it refers to the semantic domain. The term 'mood' is avoided as it is mostly associated with grammatical categories like indicative and subjunctive.

Kratzer (1991) introduces new terms (modal force, modal base, ordering source) for the interpretation of modals. As her theory grounds on modal systems (the data primarily come from English and German) and as the aim of the present thesis is to describe non-manual modality markers, each coding a special epistemic (or deontic) modality, her theory and description are not used here.

${ }^{223}$ The term 'modality' is used in sign language research more often for describing the channel of production and perception. So, the visual channel used in sign languages is opposed to the auditory-vocal channel of spoken languages.
} 
(epistemic, alethic, or deontic ${ }^{224}$ ) and describes their differences. She reports that signs used to express epistemic modality tend to have their place of articulation on the head (temple), while deontic signs are produced in front of the body and additionally can be performed in an energetic and non-energetic way (Ferreira Brito 1990, 255). The only nonmanual components which are described are headshakes or nods, which cover some of the modality signs.

With regard to American Sign Language (ASL), Long (1918) described the existence of modals and lists signs like CAN, CAN'T, MUST, and so forth. In the seventies and eighties, modal verbs are regularly mentioned for ASL (e.g. Padden 1988 among others). Wilcox \& Wilcox (1995; see also Wilcox 1996) first describe the modal system of ASL in detail and emphasize its function and use. They describe strong and weak forms of modals and discuss the path of grammaticalization for modals, determining that gestures become lexicalized elements and subsequently grammatical modals. They also list and describe in detail lexical elements conveying epistemic modality. They focus on the signs POSSIBLE, MAYBE, and IMPOSSIBLE ${ }^{225}$. In addition, they observe that epistemic modality may be expressed by signs of physical activity and perception like FEEL, SEEM, OBVIOUS. They note that epistemic modality may be exclusively expressed by nonmanuals, in the form of an added tag question which goes beyond the meaning of conveying a question and citing two examples in which the manuals are covered by 'head nods', 'squinted eyes' and 'furrowed brows'. For the latter, they describe an epistemic meaning, but notate the non-manual markers as ' $y$ ' and 'wh', referring to their occurrence in assertive constructions (' $y$ ' for nodding movements) and interrogative constructions ('wh' for whquestions which may be indicated by furrowed brows and squinted eyes). The authors

\footnotetext{
${ }^{224}$ Ferreira Brito’s (1990) classification of modality functions is based on Lyons (1981) who describes different perspectives on a propositional content following the notion of logical possibility and necessity. To this, the term 'alethic' modality conveys the speaker's estimation on the possibility or necessity of a proposition's content.

${ }^{225}$ It is of special interest for the present thesis that the illustrations of the signs POSSIBLE, MAYBE and IMPOSSIBLE (in Wilcox \& Wilcox 1995, 143-144) are covered by tilting the head sideward.
} 
conclude that similar non-manual patterns are present in imperatives ${ }^{226}$, obligations, requests for information, and epistemic probability. With regard to imperatives and obligation, both have to accompany the imperative verb; concerning deontic modals, the entire phrase is covered by the special nonmanuals.

In the last ten years, the focus has been put on detailed descriptions of different modality signs and their respective processes of grammaticalization. Shaffer (2000) continues the description of the modal system in ASL, treating the modals of necessity and possibility in her PhD thesis. Janzen \& Shaffer (2002) as well as Wilcox (2004) track the process of grammaticalization of modals. Wilcox \& Shaffer (2006) continue the description of modality in ASL. With regard to epistemic modality and the function of accompanying nonmanuals, they describe that 'furrowed bows' and 'head nod' go together with modal verbs, expressing certainty or conviction about a proposition.

Sutton-Spence \& Woll $(1999,126)$ briefly mention that modality ${ }^{227}$ can be expressed in British Sign Language (BSL) by modal auxiliaries like CAN or MUST, by changing the verb's tenseness, strength, or size, and by using nonmanuals expressing doubt or determination, which co-occur with the manual modality signs. A clear description of the non-manuals is lacking.

A comparison of modal verbs in Catalan Sign Language (LSC) and German Sign Language (DGS) with regard to syntax and negation shows that in both languages, modals tend to follow the lexical verb, may take agreeing verbs as their complement, and different modals cannot co-occur within one clause. Modals may cliticize negatives or even constitute suppletion, both covered by headshakes. These cliticized or suppletive forms always have to be used in negative contexts (Pfau \& Quer 2004).

Herrmann (2004) described in her MA thesis that in DGS as well as in Irish Sign Language (ISL) modal meaning is most frequently coded in the nonmanuals, even though the extent

\footnotetext{
${ }^{226}$ Wilcox \& Wilcox $(1995,147)$ quote an example in which the sign expressed in an imperative way is covered by a sharp head nod, leaning forward of the torso, squinted eyes, and furrowed brows.

${ }^{227}$ The authors use the term 'mood'. The description of the phenomenon refers to 'modality' following the definition of Palmer (1986/2001).
} 
of coding modality meaning in the nonmanuals varies. The author observed that especially in DGS modality meaning is coded to a high degree by nonmanuals such as 'head and body positions', 'eyebrow movements', and different 'facial expressions'.

Chien-hung \& Jung-hsing (2009) describe the syntactic position of modals in Taiwan Sign Language (TSL). They observe that deontic modals (like CAN) or epistemic modals (like DEFINITE) in pre-verbal position are covered by different non-manual markers than the same deontic or epistemic modals in clause-final position. They observed that in clausefinal position, these modals are all accompanied by an 'upward-backward head tilt' resulting in 'chin up'. In their interpretation, this is due to the "stronger subjectivity" (Chien-hung \& Jung-hsing 2009, 17-22). Further, their illustrations show that the sign DEFINITE is accompanied by a tensed face (Chien-hung \& Jung-hsing 2009, 20-21).

Herrero-Blanco \& Salazar-García (2010) describe modality in Spanish Sign Language (LSE). First, they list inherent modality coded by modal markers like CAN, WANT, or MUST; second, objective modality (expressing epistemic or deontic circumstances/conditions that stem from external sources and not the speaker) is described - also expressed by various modal signs; finally, epistemic modality is presented, exemplified with signs like SURE, THINK, OPINION-MINE, DOUBT, PERHAPS. As a rule, all these modal markers are found in a clause-final position or another position close to the end of the clause, except for the epistemic expressions of certainty (SURE) and probability/belief (THINK, OPINION-MINE), which precede the proposition which they refer to. With regard to non-manual markers accompanying modals, the authors state that these components seem to be "complementary elements of hand articulation" (HerreroBlanco \& Salazar-García 2010, 37). Concerning the exclusive occurrence of non-manual coding modality the authors state that these are non-existent in LSE and consequently that modality only can be expressed by manuals.

\subsection{Modal systems in ÖGS}

In this section, manual and non-manual elements which code modality in ÖGS are described. First, the modal signs constituting modal verbs and expressions which convey 
modality meaning are presented. This includes an overview on the known means of coding modality - which are modal verbs - in ÖGS. In addition, other signs implying modality meaning are presented.

The second possibility, on which I focus in this sub-chapter, is coding modality meaning by nonmanuals. To this, first all identified nonmanuals which can convey modality meanings are briefly described. Then, I go into detail on non-manual modality markers which are coded by the 'head' or 'body'. In particular, I present a set of head and body markers which primarily code epistemic modality.

\subsubsection{Modality signs}

One way of expressing modality meaning is coding by modal signs or by other signs which express cognition, emotion, or perception that imply such a modality meaning. As described in the literature overview, this possibility is used in several sign languages.

\section{Modal verbs}

In Austrian Sign Language, a study on modals was conducted by Schalber \& Hunger (2001), focusing on the syntactic position of modal signs. The results show that the position of modals is found in clause-medial position ${ }^{228}$. It was not explicitly formulated, but their examples demonstrate that the non-negated modal signs KÖNNEN (CAN), MÜSSEN (MUST), SOLLEN (SHOULD), DÜRFEN (MAY), WOLLEN (WANT), and MÖGEN (LIKE) ${ }^{229}$ precede their complements (which in most of the quoted examples is the performance of an activity), exemplified in (125):

\section{MÜSSEN STIEGENSTEIGEN \\ MUST GO-UP-STAIRS}

Ich/du/... müssen Stiegensteigen.

I/you/... have to go up the stairs.

\section{DU KÖNNEN TENNISSPIELEN \\ YOU CAN PLAY-TENNIS}

Du kannst Tennisspielen. You are able to / know how to play tennis.

(Schalber \& Hunger 2001, 43)

\footnotetext{
${ }^{228}$ It has to be mentioned that in the cited examples the clause-initial position is often filled by the agent which may be dropped.

229 The annotation conventions for ÖGS are that modal signs are glossed in the infinitive or first person form.

${ }^{230}$ The translation into English glosses as well as into German and English is added by the thesis author.
} 
In related investigations, Schalber \& Hunger (2000) show that modals may be copied and are reproduced in clause-final position. In this case, a pause has to occur before the doubled modal sign (cf. Wilbur 2005, 205).

In Skant et al. (2002, 204-208) negative modal verbs displaying a suppletive form are listed and described (cf. 3.1.3.4). Following the examples given by the authors, the particular negated modal sign either precedes its argument or follows the clause whose predicate it negates. In the latter case, a pause intervenes.

\section{Expressions implying modality meaning}

When formulating trains of thoughts, the signers produced different signs of cognition, emotion, or perception which imply modality meaning. In the majority of instances, these expressions are linked with a complement in which unrealized, hypothetical, or wished situations are expressed. The complement clauses ${ }^{231}$ in the data are primarily interrogative complement clauses, but there are also declarative complement clauses.

At the lexical level modality elements are manual signs that partly occur together with mouthings and further non-manual components. They convey concepts such as lack of knowledge, uncertainty, or indecisiveness. The modality signs in the present data which are frequently associated with trains of thoughts are DENKEN (THINK) ${ }^{232}$, WISSEN+NEIN (KNOW+NO), NEIN+SICHER / SICHER+NEIN (NO-SURE/SURENO), UNENTSCHLOSSEN (INDECISIVE) and UNSICHER (INSECURE) and HOFFEN (HOPE). These signs usually occur when a signer expresses his/her attitude towards or evaluation of a proposition. Further modality signs which convey the meaning of potentiality or eventuality are the signs VIELLEICHT (MAYBE) and MÖGLICH

\footnotetext{
${ }^{231}$ On a discussion and differentiation between complement clauses and complementation strategies see Dixon (2006).

${ }^{232}$ The signs WISSEN (KNOW) and DENKEN (THINK) are the same. To be sure that there is no misinterpretation of the meaning, the ID-gloss in the present thesis is DENKEN (THINK) as the sign conveys that meaning in the present construction. However, when the same sign is used together with the negation sign, the ID-gloss is WISSEN+NEIN (KNOW+NO) as it conveys the meaning of lack of knowledge.
} 
(POSSIBLE). These signs are used when a signer describes the potentiality of a situation or an event (cf. 7.4.6).

As these modality signs frequently occur with embedded interrogatives, in the following I illustrate their manner and syntactic places of occurrences in these constructions from the corpus containing the signers' trains of thoughts.

First, the sign which is used to express that somebody is thinking of a situation is DENKEN (THINK). In all cases the sign precedes the part that is put into question. More frequent are signs expressing lack of knowledge, insecurity, or indecisiveness towards a situation. The most frequently used modality sign expresses lack of knowledge. This information is conveyed by the compound signs WISSEN+NEIN (KNOW+NO), or just by the sign WISSEN that goes together with the mouthing 'nicht' ('not') and/or a headshake, or all components (WISSEN+NEIN together with the mouthing 'nicht' ('not') and a headshake) are performed together. In all examples from that corpus this sign precedes or follows the line of thoughts formulated in an interrogative way. Other signs that go together with embedded polar interrogatives and that occur in this position are SICHER+NEIN (SURE-NO) and UNENTSCHLOSSEN (INDECISIVE). The sign UNSICHER (INSECURE) precedes, follows, and even intervenes in the interrogative constructions. The signs VIELLEICHT (MAYBE) as well as MÖGLICH (POSSIBLE) are produced in initial or final position of the chain of thoughts and consequently are preposed and/or postpostioned in relation to the other lexemes that are put into question. So, they take a different syntactic position in the embedded interrogative constructions than the other modality signs.

Following Haspelmath \& König (1998, 578) who perceive an 'epistemic linking,233 between the protasis and the apodosis in embedded interrogatives, similar to concessive conditionals, the present constructions display in most cases an 'epistemic linking' between the means of coding modality (for lack of knowledge, insecurity, or hope) and the

\footnotetext{
${ }^{233}$ Epistemic (Greek) characterizes the status of knowledge (in German 'Erkenntnis'). Epistemic modality expresses the probability, doubt, possibility about the degree of knowledge and confidence of the unknown status/situation/thing (Glück 2000, 188).
} 
proposition which constitutes the train of thoughts. To conclude, from the semantic perspective a proposition is brought together with modality expressions. Consequently, the thought information, frequently put in question and displaying hypothetical alternatives in the present data, is associated with the expression(s) of not knowing, insecurity or hope ${ }^{234}$. As presented in chapter 4, in embedded interrogatives the non-manual marker head forward can also spread over a preceding, following or intervening modality sign. This clarifies that also from a syntactic perspective the described modality expressions are linked with the thought propositions (cf. 4.3), displaying syntactic embeddedness.

\subsubsection{Modality coded by nonmanuals}

The second possibility of expressing modality meanings is coding modality only by nonmanuals. That is, non-manual components which co-occur with modality signs (i.e. a modal verb of a modality expression, as described above) are excluded in this sub-chapter. In the data, on the one hand, there are nonmanuals coding modality meaning which can stand syntactically on their own. Frequently they co-occur with other nonmanuals having the same starting and end points and possessing alone or together a 'narrowed modality meaning', that is, a clear lexical meaning is ascribed to these elements by the annotators. On the other hand, there are nonmanuals coding modality meaning which co-occur with several lexical items and which do not stand syntactically on their own. Most of these nonmanuals express the sign's attitude on a proposition. Some code, for instance, the uncertainty of the proposition.

But, some of these nonmanuals can be both. So, they occur on their own possessing a clear modality meaning or cover several lexical items and provide them with modality meaning.

\footnotetext{
${ }^{234}$ Following this explanation, those instances in which no modality expression is produced, but in which the question element 'ob' ('whether') is present, the proposition is associated with the concepts of 'lack of knowledge' and 'implied insecurity' too. To be exact, in eight cases of the 37 embedded polar interrogatives none of the modality elements is present. However, in these cases the question element ' $\mathrm{ob}$ ' ('whether') always precedes the train of thoughts. Seven of these instances are produced by the same signer. To conclude, - from a semantic point of view - even in these cases 'epistemic linking' can be pointed to.
} 
In the following, the nonmanuals coding modality meaning are briefly described. They are present in my data, especially in the embedded interrogatives. The modality markers which are coded by 'head' and 'body' are described in detail in the following section.

\section{Shoulder shrug(s)}

An example of nonmanuals that frequently occurs in the context of embedded interrogative constructions but which may have a syntactic position on its own are one or more 'shoulder shrug(s)' (abbreviated as 'shu'). According to the annotators they convey the meaning of lack of knowledge and they are put on the same level as the lexical items like the sign KNOW+NO. In most cases the element precedes or follows the embedded interrogative clause, in some cases it intervenes.

\section{Mouth actions}

The data shows that some mouth actions are present which provide a modality meaning and which show both types of occurrence. On the one hand, these mouth actions can occur on their own (to be exact, they frequently co-occur with other non-manuals) and convey a clear lexical meaning. On the other hand, they cover an entire utterance and provide the construction with a 'broader meaning of insecurity and/or lack of knowledge'.

The most common mouth action, especially in the context of embedded interrogatives, is 'lips stretched-down (mostly with closed mouth)' (abbreviated as '(cl.)str-down'). From time to time the production of the mouth action is intensified resulting in a stronger stretching-down of the lips. This is identified by the annotators as 'mouth open and lips stretched-down-large' (abbreviated as 'open, str-down-large') conveying a very high degree of the broader meaning of insecurity. Finally, stretching-down only one side of the mouth corners is present too (annotated as 'cl., str-down-r/l') ${ }^{235}$. As illustrated in (126), both possibilities of occurrence of the mouth actions ‘stretched-down' are possible.

\footnotetext{
${ }^{235}$ Due to the status of research work on ÖGS it is unclear if the last described mouth action is a variation of the first one or a distinctive mouth action. That is, it is unclear, if - referring to the first one - the one-sided stretching-down of the lips conveys different semantics, if it is used in different positions in the various constructions, or if stylistic reasons are responsible for one-sided or two-sided mouth stretching-down movements.
} 


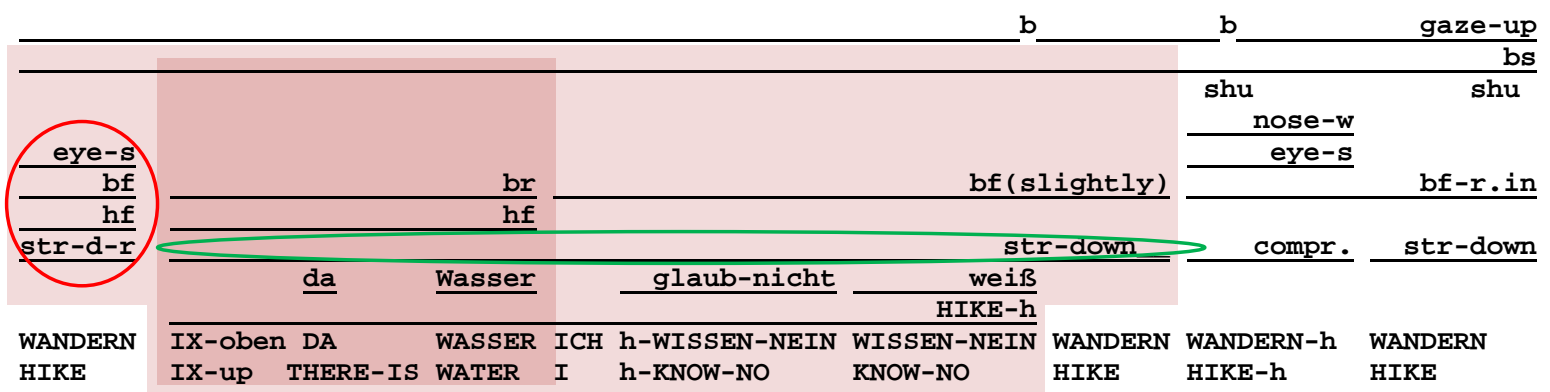

Während ich wandere überlege ich, ob es wasser (dort) gibt. Ich bin mir unsicher und weiß es auch nicht. Aber was soll's.

While I am hiking I wonder whether there is water. I am uncertain and $I$ dont't know. What the hey!

(F001_92,1184_m_thoughts_ex05_01.21-01.31)

In (126), the signer is hiking and thinking whether she will get some water. In the beginning of the short story she expresses her high degree of insecurity of the situation by producing the mouth action '(closed,) stretched-down, ${ }^{237}$ co-occurring with the nonmanual markers 'squinted eyes', 'brow furrowed' and 'head forward' (all encircled red). The latter's function is described by the annotators as putting an emphasis on the mouth action which they describe as expressing a high degree of 'insecurity about the situation ${ }^{\text {,238 }}$. This shows the first possibility in which the mouth action possesses its own meaning and, in this case, it is not associated with the sign (HIKE) with which the non-manual element(s) cooccur.

The signer keeps on thinking and stretches her lips downward (encircled green) during the entire line of thoughts and while she keeps on hiking, until she changes her mouth action to compressed lips ${ }^{239}$ what is interpreted as 'never mind'. This long co-occurring performance of the mouth action 'stretched-down', covering the embedded interrogative

\footnotetext{
${ }^{236}$ The entire embedded interrogative is color-coded bright rose, the embedded clause dark rose.

${ }^{237}$ The present mouth actions are the mouth actions 'stretched-down-right-side' (str-d-r), stretched-down (strdown) and compressed lips (compr.).

${ }^{238}$ Quite often in the corpus the mouth action 'stretched-down' co-occurs with 'squinted eyes' and 'furrowed brows' or 'wrinkled nose'. No sign is present, so the hands are held in rest position, in palm-up position, or the preceding sign is held. When these nonmanuals co-occur together, the annotators very likely give them the meaning of 'insecurity' and/or 'lack of knowledge'.

${ }^{239}$ The mouth action looks like as if the signer is compressing her lips strongly together. Its semantic is translated by the annotators in casual German as 'Hm, was soll's' ('What the hey!'). Also, the two shoulder shrugs (shu) in example (126) are not interpreted as lack of knowledge, rather in the way of 'What the hey!’.
} 
clause, is even maintained for a moment while signing HIKE. It is interpreted as lack of knowledge and being insecure about the situation the signer is thinking about ${ }^{240}$.

\section{Squinted eyes and wrinkled nose}

Further movements or positions of various nonmanuals are identified by the annotators that especially occur in the context of embedded interrogatives and conditionals. These are described as distinctive language-relevant elements, however in many circumstances their functions are assumed by annotators but have not yet been analyzed in terms of linguistic investigations on ÖGS. Two of these identified markers which frequently co-occur are 'squinted eyes' (abbreviated as ‘eye-s') and ‘wrinkled nose’ (abbreviated as ‘nose-w’).

With embedded interrogatives, these markers frequently precede or follow the requested part and go together with the modality sign or as already mentioned with the mouth action 'stretched-down'. The annotators describe their meaning as expressing security or insecurity and possessing knowledge or lacking knowledge, respectively ${ }^{241}$. This kind or occurrence is illustrated in (127), described in detail in example (127), in which the markers wrinkled nose and squinted eyes co-occur with the mouth action 'stretched down'.

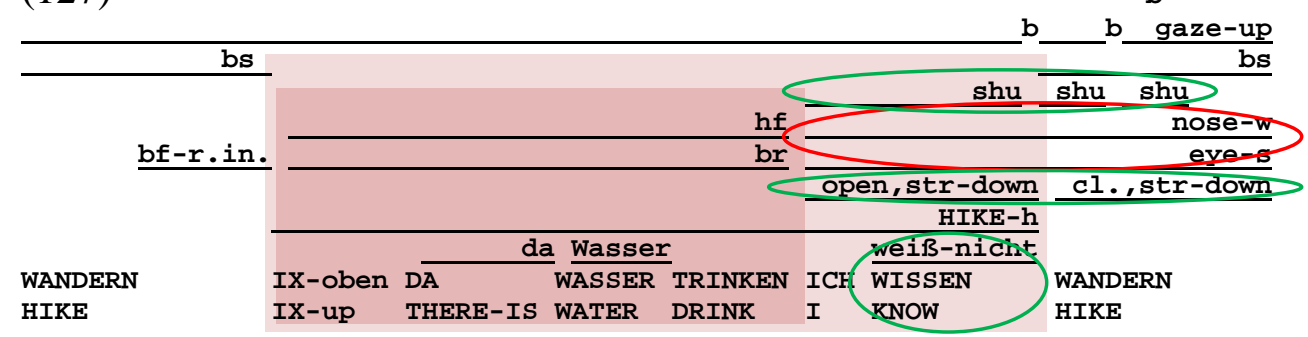

Ich bin am Wandern und weiß nicht, ob ich Wasser zum Trinken bekomme. While I am hiking $I$ don't know whether $I^{\prime} l l$ get water to drink.

(F001_92,1184_m_thoughts_ex06_01.34-01.43)

\footnotetext{
${ }^{240}$ The phenomenon that more lexical elements are covered by a mouth action has been described for various sign languages (see, among others, Boyes Braem 2001a and 2001b on the extension of mouthing in Swiss German Sign Language (DSGS) or Crasborn et al. (2008) on spreading behavior of different mouth actions in British Sign Language (BSL), Sign Language of the Netherlands (NGT), and Swedish Sign Language (SSL)). Moreover, Brentari \& Crossly (2002) describe the behavior 'lower face tension' (LFT), a tension of the muscles at the mouth's corner, which is analyzed as a primary cue to the prosodic structure of ASL. This behavior is probably comparable to mouth action 'stretched-down' in ÖGS. But, further investigations on mouth actions in ÖGS are required in order to make clear statements.

${ }^{241}$ cf. among others, (F004_1117,1209_m_thoughts_ex04_), (F001_92,1184_m_thoughts_ex05_01.2101.31), (F001_92,1184_m_thoughts_ex06_01.34-01.43)
} 
In (127), wrinkled nose and squinted eyes (encircled red) co-occur with the mouth action 'stretched down' and in the context of other elements expressing lack of knowledge. These are the sign KNOW, produced with the mouthing 'weiß-nicht' ('know-not'), and shoulder shrugs (shu) and (each of them encircled green).

With conditionals, these both markers frequently co-occur in the protasis, if this is negated or signs conveying a negative meaning are involved. Also, in various conditionals both markers cover the entire conditional construction. This is re-illustrated in the following examples (128) and (129). Both examples have been previously described in detail (cf. examples (105) and (106)).

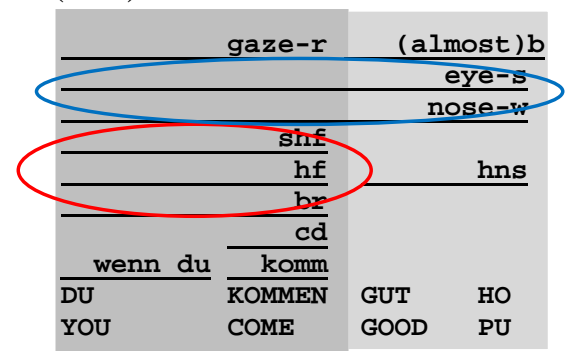

Wäre schön, wenn du kommen würdest. It would be nice if you came.

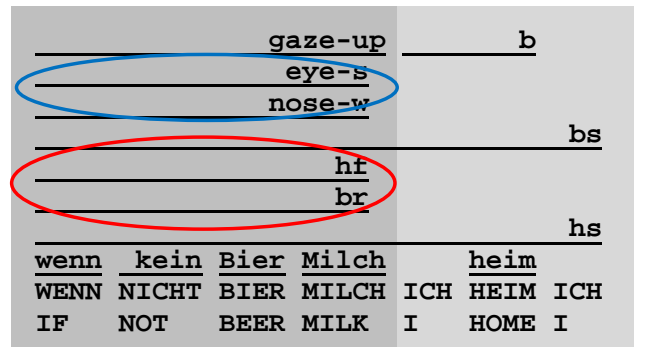

Wenn es in der Almhütte weder Bier noch Milch gibt, dann bleibe ich nicht, dann gehe ich heim. If in the hut there is neither beer nor milk, then I won't stay, then I'll go home.

(M007_04.05.08_edu.course_part01_11.28-11.31) (M001_091,1183_m_thoughts_ex03a_01.08-01.10)

In (128), the two described markers (encircled blue) cover the entire conditional construction, in (129) the negated protasis is covered by wrinkled nose and squinted eyes (encircled blue). The red encircled markers display the conditional markers (cf. 5.4). A deeper discussion on the occurrence of both markers in conditionals is discussed in 5.7.3.

To sum up, the present data shows that when wrinkled nose occurs in conditionals, it frequently occurs in the context of negation or lexical elements conveying negative meanings. With embedded interrogatives, wrinkled nose frequently occurs when the thought proposition possesses a very high degree of uncertainty. So, based on the current knowledge it is supposed that when wrinkled nose occurs in these hypothetical constructions, the non-manual element is used for coding negative presupposition. 
Probably its function is comparable with non-assertive marker 'fast, small headshakes', described in 7.4.3.

In other sign languages the various forms and functions of this marker have either not been investigated at all, or only to a minimal extent. Coulter described the occurrence of wrinkled nose in relative clauses in ASL as early as 1978. Wood (1996) states that repeated wrinkling of the nose in ASL provides referential or pragmatic information. Pfau \& Steinbach $(2004,2)$ describe an NGT example in which they allocate to wrinkled nose both the function to signal 'shared knowledge', and also to provide pragmatic information which can be compared to the function of modal particles in spoken languages as in German. The function of 'signaling shared knowledge' leads to the second non-manual marker - ‘squinted eyes' - which probably also has a modality function.

In the ÖGS data, the sign WISSEN-NEIN (KNOW-NO), which frequently precedes embedded polar constructions, is covered by squinted eyes together with furrowed brows and the mouth action 'stretched-down'. The marker 'squint' also co-occurs when an ÖGSsigner narrates a story and keeps inserting the question WISSEN-DU (KNOW-YOU), describing some details (persons, locations, etc.) both dialogue partners know. When doing this, the eyes are frequently squinted. To conclude, a relation between shared knowledge and squinted eyes is apparent. For those cases where squint occurs in conditionals the following has to be clarified in advance: Most of the conditionals are produced in monologue settings, when the informants formulate trains of thoughts which are addressed to a 'general audience'. As the signers did not know the camera man it may be supposed that there was no common knowledge on diverse subject matters available. Moreover, the filmed people did not know to whom the signed content would be addressed. So, a 'generalized addressee' is the target of the utterance. In Israel Sign Language (ISL) and other sign languages 'squinted' has been stated to function for signaling that the signer thinks the addressee can retrieve knowledge (cf. Dachkovsky 2008 and Dachkovsky \& Sandler 2009, 293 and 302-306). Because in the ÖGS data a clear dialogue partner, to whom the information is addressed, is missing, it may be supposed that the marker squinted eyes does not have that strong interactive character in the sense of 'retrieving 
common knowledge'. Possession or lack of knowledge is known to be coded in many spoken languages as a means of coding modality (often coded in the form of existentials). So, one possible interpretation is that squinted may be used to code the 'degree of knowledge' on a possible proposition.

Finally, another hint for the interpretation of both described markers (especially when occurring together) is that they provide the construction with 'potentiality'. For instance, example (128) shows a conditional which is as a whole covered by wrinkled nose and squinted eyes. According to the feedback of annotators, these markers are used to provide 'potentiality' (in the sense that all possible alternatives are open) for reasons of politeness. The use of modality (as polite directive) for implying more alternatives for reasons of politeness has been described for spoken languages (cf. Ford 1997, 387-389). The interpretation that these markers are used to 'imply the potentiality of various alternatives' would also clarify why in example (88) the consequent, constituting a content question ('If ..., what would happen?'), is covered by these markers.

To conclude, the nonmanuals described very likely possess modality meanings. However, further investigations are required to make clear statements and interpretations on their places of occurrence, their co-occurrence and their functions.

\section{A set of head and body markers coding modality}

The corpus in which informants produced trains of thoughts while performing an ongoing activity has turned out to be a good source of contexts in which the signers express epistemic modality. When formulating lines of thoughts, the signers expressed propositions towards which they had different attitudes. They expressed their conviction about the positive outcome of a situation, they wondered whether something would turn out in a positive way, they expressed their feelings that something was not likely to end up well when formulating a proposition, and so forth. In formulating all these different attitudes or evaluations towards a proposition, different head and body movements were produced. Going through the corpus with the annotators, it became apparent that a set of non-manual markers is coding epistemic modality. In addition, there is a head/body marker which 
expresses possibility on an unrealized event. These non-manual markers are focused on and described in detail as next.

\subsection{Types of modality coded by head and body movements in ÖGS}

\subsubsection{Introduction}

Propositional modality is defined as the speaker's/signer's attitude towards a 'proposition' which is based on the person's judgment/evaluation or it is formulated due to some given evidence (Palmer 2001, 24). In the ÖGS corpus which is composed of lines of thoughts many propositions are present, expressed with different attitudes towards them. A detailed description of the co-occurring modality head/body markers follows within this subchapter. Evidential markers coded by non-manual markers have not yet been identified in ÖGS. For this reason, evidential modality is not included in the present chapter on modality.

Based on a typological comparison, Palmer (2001, 24-35) distinguishes three main types of human judgment on propositions: First, the speaker/signer expresses his/her general uncertainty about a formulated proposition; second, the speaker's/signer's judgment is based on conclusions drawn from observable and evidential indicators; third, the speaker/signer judges a proposition due to generally known facts. These types of judgment are defined as Speculative (Dubitative) ${ }^{242}$, Deductive, and Assumptive, respectively. These modality meanings are expressed and illustrated by the following three German and English sentences. In these spoken languages, the particular modality information is expressed by modal verbs, and no separate marker for coding these three epistemic types exists.

$$
\begin{array}{lll}
\text { Speculative meaning: } & \text { Es dürfte etwas geben. } & \text { There may be something. } \\
\text { Deductive meaning: } & \text { Es muss etwas geben. } & \text { There must be something. } \\
\text { Assumptive meaning: } & \text { Es wird etwas geben. } & \text { There will be something. }
\end{array}
$$

\footnotetext{
${ }^{242}$ A further term for the Speculative is 'Dubitative'. However, this term only includes the negative aspect, thus 'being in doubt about something', and excludes a positive, deliberative way of thinking (cf. Bybee 1985, 179).
} 
In Austrian Sign Language it is also possible to express these types of judgment by means of modal verbs. As follows, these types of judgment can also be expressed exclusively by head and body movements. These non-manual modality markers co-occur with the particular modality signs, they occur in the context of modality signs and cover further non-modality signs too, or they occur on their own, covering signs which do not include the appropriate modality signs.

In the following, first, two head movements are focused on which fall within the scope of deductive-assumptive markers (the convinced-assertive marker and the non-assertive marker). Second, the speculative marker is described. Third, a timitive marker is presented which possesses epistemic as well as dynamic modality meaning.

The second option is that the speaker/signer expresses conditional factors with regard to an unrealized event which conveys a high degree of potentiality to be realized. This means that there is a lot of potential, i.e. uncertainty, associated with the event and its realization. These conditional factors may be caused by external or internal reasons. If external factors are expressed with reference to unrealized events, this can be done by expressing permission or obligation. If internal factors are expressed with reference to unrealized events, this can be done by expressing willingness or ability. The first is labeled 'deontic modality', the second 'dynamic modality' (Palmer 2001, 8-10).

The last described head/body marker expresses event modality and refers to the possibility of the implementation of an unrealized event.

There may be further modality markers coded by the head or body. In the present thesis I focus on those which have been realized by individual signers and which have been clearly identified by the annotators.

Each sub-chapter on a modality marker is structured in the way that first, its form, its meaning and its scope ${ }^{243}$ are described. Then, the frequently co-occurring elements are

\footnotetext{
${ }^{243}$ In this section on coding modality by nonmanuals, the term '(semantic) scope' is used in accordance with Lehmann (2012). Here, 'scope' indicates that part of an utterance which is covered by the non-manual modality marker.
} 
discussed. If required, the marker is distinguished from other markers which convey similarities with respect to its form or meaning.

\subsubsection{Convinced-assertive head marker}

Wilcox \& Shaffer (2006, 226-229) describe for ASL non-manual components accompanying epistemic signs. One of the described sets of co-occurring nonmanuals are 'furrowed brows' and 'head nod(s)' covering modal verbs. They provide the construction with the meaning of 'being certain'. This co-occurring set of non-manuals probably is comparable in form and meaning with the convinced-assertive head marker and furrowed brows expressing the signer's uncertainty on something in ÖGS.

\section{Form}

In the data two formatives are present whose form and meaning are clearly identified by the annotators. The first form is fast, small head nodding movements which may differ in the phonetic implementation of the nodding movement. So, the annotators identified 'fast, small head nodding movements' which were performed with a slightly forward rotating movement, or a slightly forward movement, or only with an up and down nodding movement (see Figure 7.1). The second form is slow, large head nodding movements, each co-occurring with one sign (see Figure 7.2).

Ferreira Brito (1990, 255) observes that in LSB deontic signs may be performed in an energetic and non-energetic way. Wilcox \& Wilcox (1995, 155-156) distinguish strong and weak forms of modal signs. The two distinct formatives of head nodding movements used for epistemic modality in ÖGS show that it is also possible to produce non-manual markers more or less energetically, and consequently, they are distinguished as a weak and a strong form by the annotators. These non-manual nodding movements in ÖGS are mostly performed in a regular nodding way. However, the larger, slow nodding movements tend to be performed in a less regular way than head nods which are used to express assertion. Nevertheless, a clear beginning and endpoint of the nodding movements is perceived by the annotators. This means that the annotators clearly allocate the nodding movements which are used for modality purpose to the relevant lexical items. 

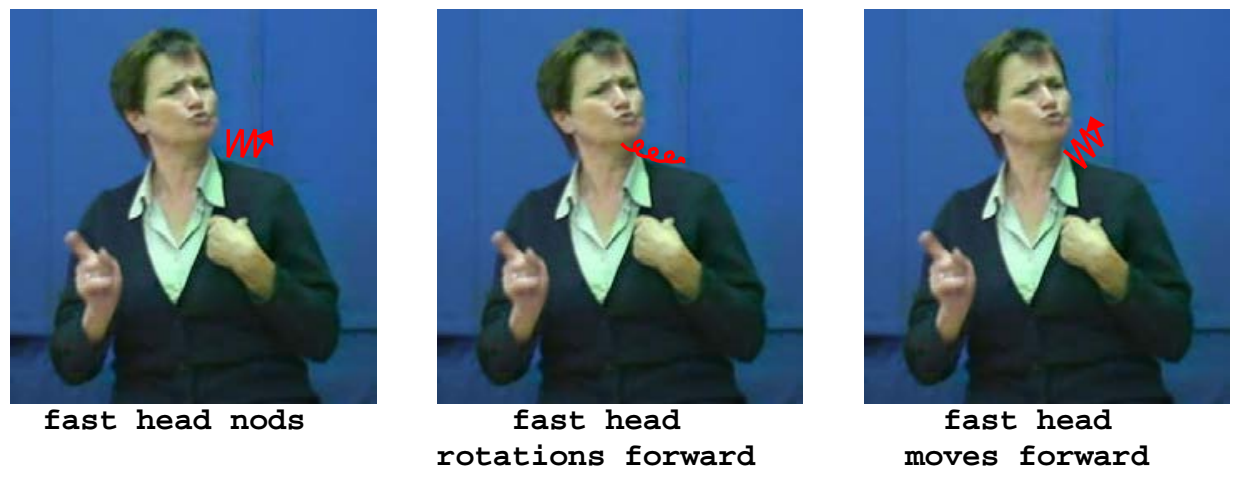

Figure 7.1 Fast, small head nodding movements

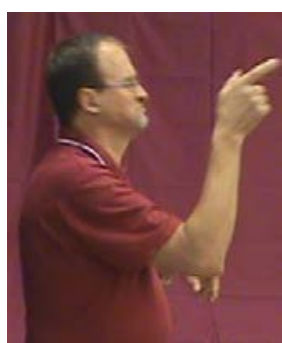

IX-up

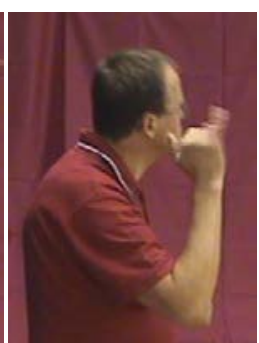

YES large nod

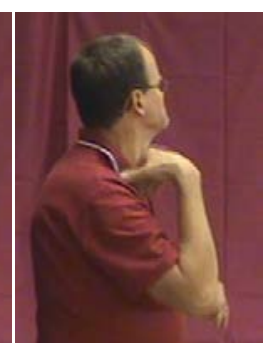

$\mathbf{I}$

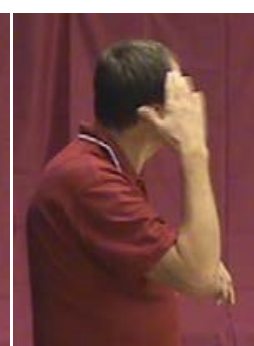

KNOW large nod

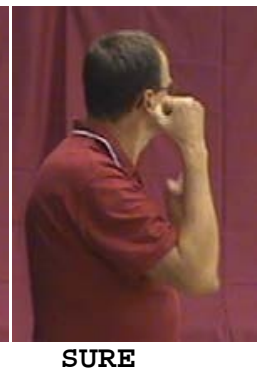

SURE large nod

Figure 7.2 Slow, large head nodding movements ${ }^{244}$

\section{Meaning}

The 'convinced-assertive marker' as well as the 'non-assertive marker' described in the following subchapter (cf. 7.4.3) are used for expressing the signer's judgment/evaluation on a self-addressed proposition. These judgments/evaluations are based on a reasonable inference or on situations/facts which in regular circumstances are the case. In other words, with help of the convinced-assertive marker (or the non-assertive marker, respectively) the signer's confidence in the truth value of what $s /$ he is thinking about is expressed. This is based on deductive reasoning from facts which are known to the person (deductive interpretation) or the signer's experience or common sense (assumptive interpretation). Thus, the degree of confidence is combined with the knowledge a person has. However, this knowledge is not explicitly formulated and consequently where it comes from is not specified. So, no evidential connotation is coded.

\footnotetext{
${ }^{244}$ The picture series corresponds to example (131).
} 
With regard to the convinced-assertive marker, the indicator shows that it is the signer's judgment on a proposition that s/he is convinced that the outcome of the unrealized thoughts will be fulfilled. In indicating this, the signer commits the truth value of the thought proposition, that is, the signer asserts the truth value of the proposition. The conviction and the assertion of the truth value of the proposition are based on deductive reasoning from facts, or on established common sense, or on previous experience. As there is no instance in the data where the reason for the conviction about a proposition as well as the assertion on the truth value of the proposition is given, all the listed reasons are possible. For instance, in (131) the signer is convinced that he will get something in a hut. This certainty can be based on the knowledge that generally during summer time, the hut is open and that drinks are offered, or he had the experience the last time the hut was open, or somebody informed him of that fact. The source of his certainty about his knowledge is not coded in the utterance.

With regard to the two distinguished forms of convinced-assertive markers, the annotators report that the epistemic meaning is forced when the nodding movements are performed in a larger and slower way ${ }^{245}$ (cf. Figure 7.2) compared to small, fast nodding movements (cf. Figure 7.1).

\section{Co-occurring nonmanuals and signs}

One non-manual marker constantly co-occurs with the nodding movements of the head, namely 'furrowed brows'. In most instances where the signer implements the convincedassertive marker, the eyes are also squinted.

Both markers together with the sign SICHER (SURE) performed by different signers are illustrated in the following:

\footnotetext{
245 The description of the particular nodding movement is made in comparison to the other formative. Of course, the speed and size of nodding movements with regard to one of the described modality marker may differ. For instance, in example (131) the signer performs the large, slow nodding movements in the beginning of his train of thoughts in a slower and more irregular way than in the end of the utterance (illustrated in the second line). These nodding movements (in the second line of the illustration) are moreover perceived as conveying reinforced epistemic modality compared to the nodding movements performed in the beginning of the utterance.
} 

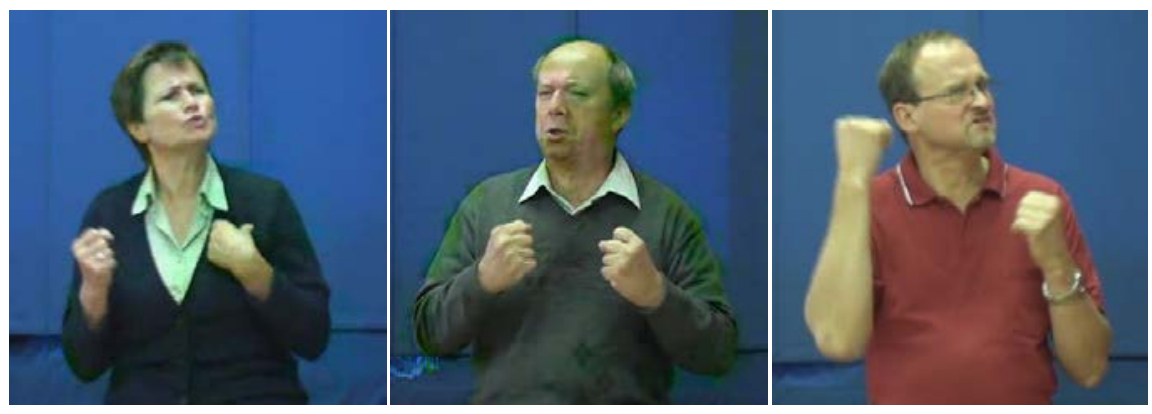

Figure 7.3 Co-occurring nonmanuals with the convinced-assertive marker

In the following, an utterance in which the signer expresses his conviction about the positive outcome of a situation is displayed. It illustrates the co-occurrence of additional non-manual markers apart from the modality marker performed by head.

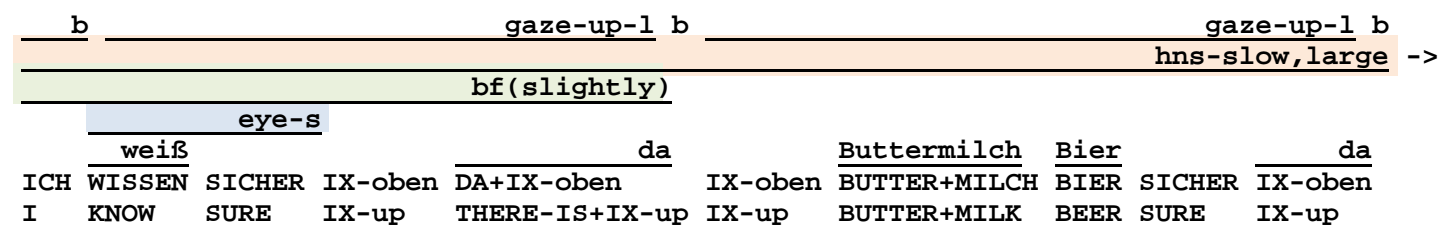

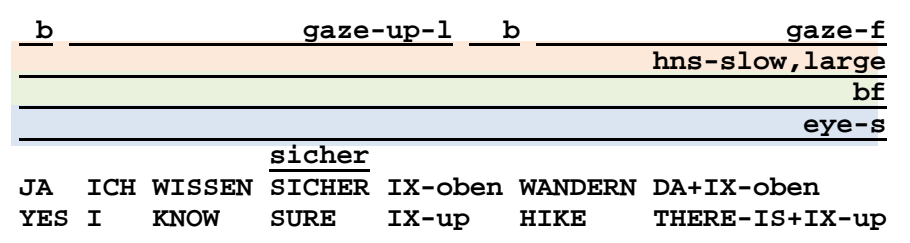

[...] Ich weiß, dass es oben (auf der Hütte) sicherlich Buttermilch und Bier gibt. Ja, ich bin der Überzeugung, dass es oben etwas gibt. Und wahrlich es gibt etwas.

[...] I know that there (at the hut) must be buttermilk and beer. Yes, I am convinced that there will be something up there. Truly, there is something.

(M001_091,1183_m_thoughts_ex01_00.18-00.25)

In example (131), the entire train of thought is covered by slow, large head nods (performed in a way slightly rotating forward; color-coded rose). These are performed in a more or less uniform way. During the last part of the line of thought (constituting the second line of the example), the head nods are performed more uniformly and tend to cooccur on each sign. The annotators state that the head marker indicates the person's conviction about the positive outcome of the proposition. This means that the signer is convinced that he will get something to drink up there. 
As displayed in example (131), two additional non-manual markers are annotated. First, the brows are slightly furrowed during the first part of the line of thought. The last part of the line of thought, in which the signer expresses his absolute conviction about the situation, is covered by (strongly) furrowed brows. The second non-manual marker which constantly co-occurs in the context of the modality marker head nods is squinted eyes. As obvious in (131), this marker tends to occur in the context of the cognitive sign of knowledge in the present ÖGS data. In this example squinted eyes first covers the constituent KNOW SURE; the second time the entire clause which refers to the sign of knowledge is covered by squinted eyes.

Further investigations are required concerning the interplay of the listed non-manual markers and whether they are required together for providing the construction with the described epistemic modality or whether the epistemic marker head nods may occur on its own conveying the same meaning. What can be determined at the present stage is that when one of the modality indicators (a modality sign or a non-manual modality marker) is intensified, the other co-occurring modality indicators are also performed in an intensified way (see second line of the example (131)).

Apart from the co-occurring non-manual markers, three signs tend to occur in the context of the convinced-assertive marker. These are the signs SICHER (SURE), MUSS (MUST) and WISSEN (KNOW). SICHER expresses sureness, certainty or determination. It occurs in all examples from the ÖGS corpora where the convinced-assertive marker is used. MUSS is one of the modal verbs in ÖGS. As a rule, it precedes its complement or it includes existence and is used in the way of MUSS-GEBEN/DA (MUST-THERE-IS) (see example (132) in which the sign MUST is first used as 'must-exist' and the second time it precedes the sign of existence). WISSEN refers to the fact that the present epistemic marker expresses the judgment/evaluation of a proposition which is based on some kind of knowledge (wherever this stems from). The data shows that the listed signs can change the way of implementation depending on which formative of the head marker is used or the intensity with which the particular head marker is performed. Thus, the epistemic marker 
small, fast head nods means that signs which usually are made with a single downward movement tend to be performed with two or more downward movements. This results in two or more movement iterations of the particular sign as illustrated in (132).

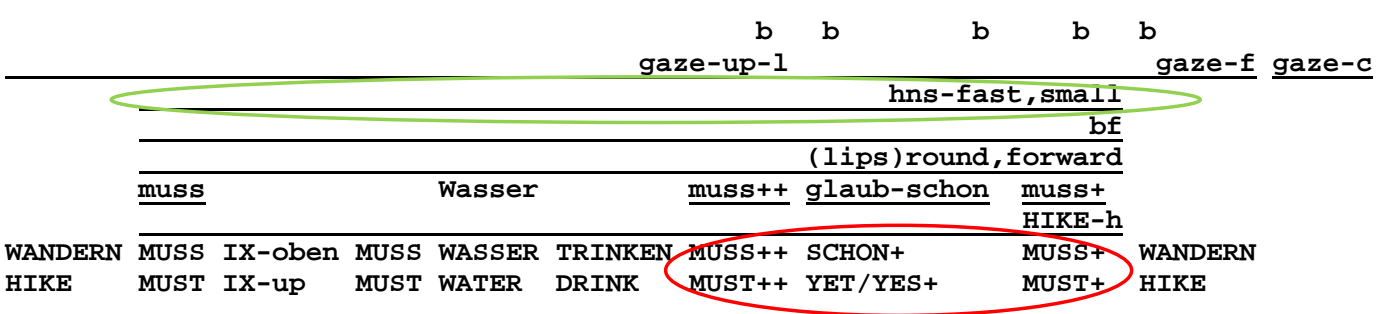

[...] Während ich wandere komme ich zur Überzeugung, dass es oben Wasser geben wird.

[...] While I am hiking I am convinced that there must be water up there.

(F001_092,1184_m_thoughts_ex08_01.58-02.05)

In (132), the particular downward movement of the signs MUST and YET/YES (encircled red) is performed two or three times.

If the head marker is intensified, the sign is performed in a more energetic way, too. This is the case in example (132), in which the signs of the last part of the train of thought (second line of the example) are performed in a more energetic way.

\section{Scope}

All instances in which the convinced-assertive marker occurs in the ÖGS data show the marker covers exactly the content to which it refers. Consequently, the covered part is the (semantic) scope of the convinced-assertive marker.

$$
(133)^{246}
$$

b b $\quad$ b $\quad$ b $\quad$ b $\quad$ b

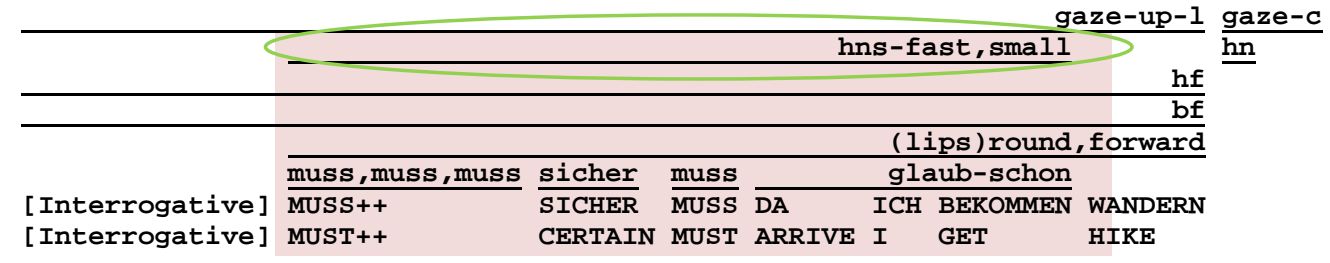

[Interrogative] Ich bin überzeugt, es muss so sein und ist sicher so, dass ich dort etwas bekommen werde.

[Interrogative] I am convinced and it must be and certainly is the case that I will get something there.

(F001_092,1184_m_thoughts_ex07_01.51-01.56)

\footnotetext{
${ }^{246}$ Example (133) has previously been quoted in chapter 3 (example (40)).
} 
In example (133), a signer wonders whether she will get something at an inn. This is expressed in a preceding embedded interrogative which is not illustrated in the example. She is convinced that she will get something. Exactly that part of the train of thought in which the signer expresses her conviction (color-coded rose) is covered by the epistemic marker fast, small head nods (encircled green). The marker furrowed brows already covers the interrogative and is maintained during expressing the conviction of the positive outcome.

Summing up, it can be determined that the described head nodding movements are used and identified as an epistemic modality marker coding a person's conviction of the positive outcome of a proposition. There are two formatives which are distinguished by the annotators.

The head marker frequently co-occurs with additional non-manual markers which are furrowed brows and squinted eyes. It also tends to occur together with the signs SICHER (SURE), MUSS (MUST), and WISSEN (KNOW). The implementation of the particular form of convinced-assertive marker as well as the intensification of production interplays with the production of co-occurring elements (further nonmanuals or signs).

Finally, the modality marker always covers the part to which it refers.

Example (134) shows how a signer first wonders whether there will be the wished-for thing. He keeps on thinking and expresses his hope that there will be something. Finally, he becomes convinced that there must be something. The various attitudes on the proposition -wondering, hoping, and being convinced - are clearly covered by different head movements as follows: 


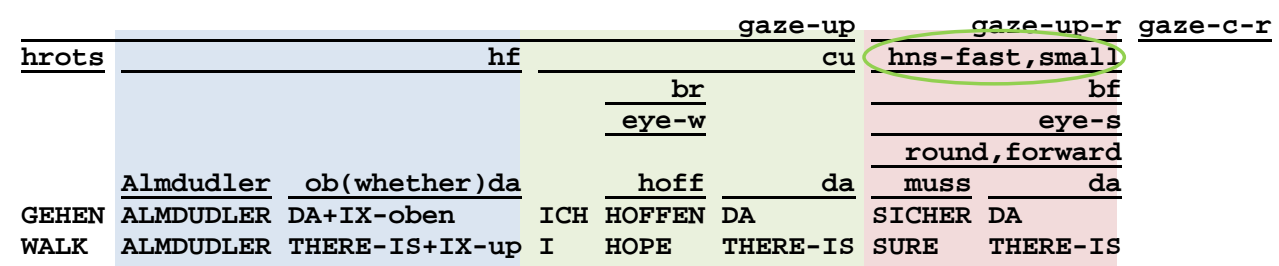

Während ich stetig dahingehe überlege ich, ob es Almdudler geben wird. Ich hoffe, dass es diesen gibt. Ich komme zur überzeugung, dass dieser vorhanden ist.

While I am steadily walking I wonder whether there will be Almdudler [Austrian brand of soda]. I do hope that there will be some. I become convinced that there surely must be some.

(M003_106,1198_m_thoughts_ex06_01.53-02.01)

In the beginning the sign for expressing the motion of walking is accompanied by small, fast head rotations forward. The first part of the line of thought is formulated in an interrogative way, marked by head forward (cf. 4.3.1.2.1). The second part of the line of thought is covered by moving the chin up and down. The final part of the thoughts is covered by fast, small head nods. These are performed together with furrowed brows and squinted eyes. Also, the sign SURE together with the mouthing 'muss' ('must') occurs.

The second head marker which is categorized as one of the deductive-assumptive markers is discussed in the next section:

\subsubsection{Non-assertive head marker}

The annotators quickly noticed that not all headshakes are used solely for clause negation. With regard to one of these headshakes, which tends to occur in the context of trains of thoughts, they could identify headshakes which clearly code the signer's attitude towards a proposition. Both in form and meaning these headshakes are different from those which are used for clause negation. This modality marker is described in the present subchapter.

\section{Form}

The identified means of coding are slow headshakes which tend to be small in size. These headshakes seem to be performed in a rather tentative way compared to headshakes which are produced more definitively and which negate a lexical sign / syntactic constituent. An utterance which is accompanied by the described modality headshakes is the following: 


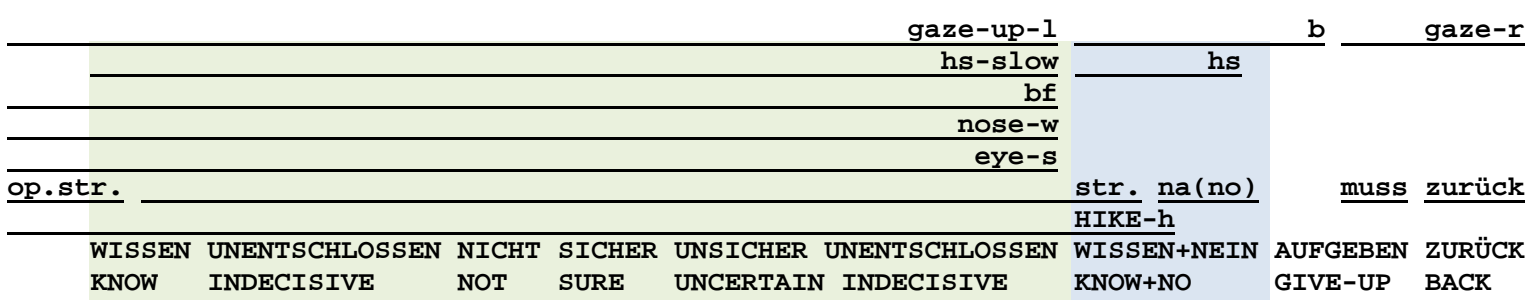

Während ich wandere bin ich mir unsicher (ob es etwas gibt) und denke, dass es eher nichts geben wird. Ich komme zur überzeugung, dass es nichts gibt, gebe (meine Pläne) auf und kehre zurück.

While hiking I am uncertain (whether there will be anything available). I tend to think more likely not. I become convinced that there won't be anything and give up (my plans) and go back.

(F004_118,1210_m_thoughts_ex02_04.18-04.21)

A signer expresses her negative attitude to the outcome of a situation while hiking which precedes the present annotations of example (135) in the data. The negative line of thought is covered by slow, non-uniform and tentative headshakes (color-coded green). These headshakes are followed by uniform and definite headshakes (color-coded blue) covering the signs KNOW+NO.

\section{Meaning}

Similar to the convinced-assertive marker, the present head marker is used to express the signer's judgment/evaluation on a proposition which is based on a reasonable inference or on facts which under regular circumstances are the case. With regard to the knowledge on which the degree of confidence is based on, the signer for some reasons feels that a result or outcome more likely will not occur. In other words, the signer thinks that the conclusion/result of a proposition will have a negative outcome, even though the reason for this negative attitude towards such an outcome is not explicitly formulated.

In example (135) above, the first headshake pattern illustrates the non-assertive marker used for coding epistemic modality. The second headshake pattern represents a clear negative marker which is used for clause-negation. 


\section{Co-occurring headshakes used for negation}

In the context of the non-assertive head marker, negative signs which evoke headshakes for clause-negation tend to occur. Following the annotators perception the following patterns can be determined:

The first pattern (see example (135)) is that first non-uniform tentative headshakes are produced, and then distinctive, uniform and definite headshakes occur. This pattern is present when a signer first is indecisive and more likely not convinced that a situation will occur. Then, the signer becomes convinced that the situations will not arise and rejects the plans.

The second pattern is that the annotators identify a non-assertive head marker which is interrupted by headshakes which possess a clear distinctive form and meaning. These headshakes are performed in a regular and definite fashion. Their function is a clear negation. This second phenomenon is illustrated in the following two examples.

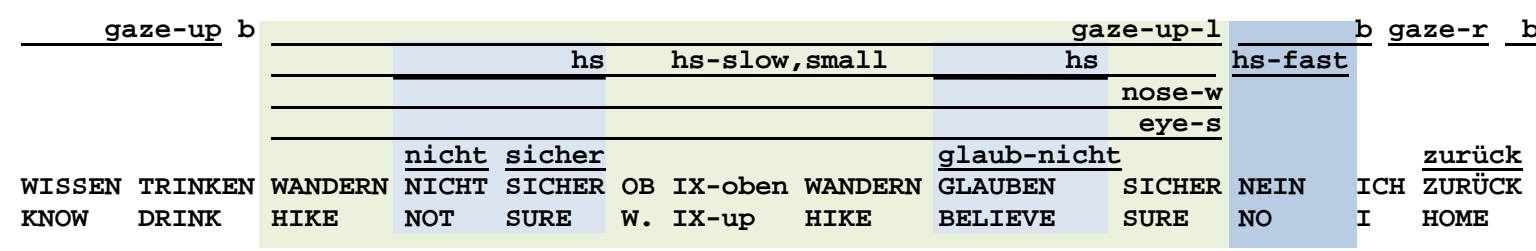

Wenn ich an das Trinken denke, bin ich mir nicht sicher, ob (es etwas gibt). Ich bin eher der überzeugung, dass es nichts gibt. Nein, ich denke nicht (,dass es etwas gibt). Ich kehr zurück.

When thinking about a drink, I am not sure (if there will be something). I tend to suppose that there won't be anything. No, I don't think (that there will be anything). I go back.

(F004_117,1209_m_thoughts_ex04_04.30-04.37)

In example (136), the signer expresses her negative attitude towards the positive outcome of 'getting a drink'. The entire line of thought (which is twice interrupted by the long activity (HIKE) during which she let her mind wander) is accompanied by slow, small headshakes (color-coded green). They are terminated when the signer becomes aware that she will not get anything. This is expressed by the sign NO which is covered by fast, rhythmic headshakes (color-coded dark blue). This interplay displays the first pattern described above. Interestingly, the non-assertive head marker is interrupted by two clear headshakes too (color-coded blue). They are produced when the informant signs NOT 
SURE and BELIEVE together with the mouthing 'glaub-nicht' ('believe-not'). This phenomenon displays the second pattern described above which is only present in (137).

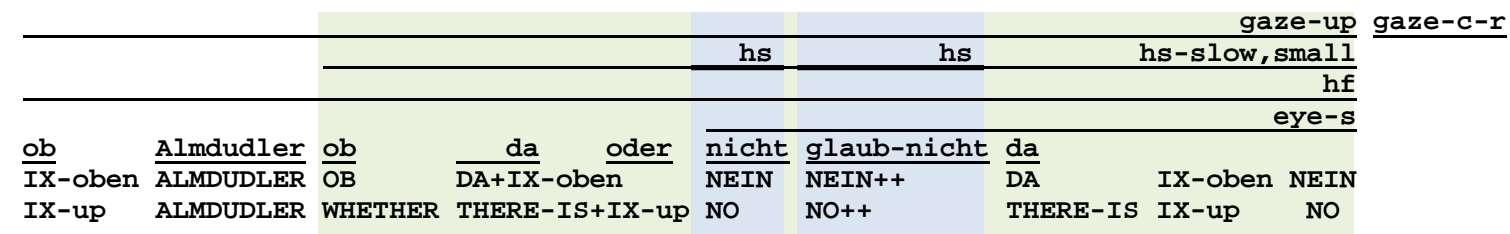

Ich frage mich, ob es Almdudler gibt oder nicht. Ich glaube eher nicht.

$I$ wonder whether there is Almdudler or not. I believe more likely not.

(M003_106,1298_m_thoughts_ex02_00.32-00.40)

In example (137), the signer restarts an embedded interrogative which is accompanied by slow, small headshakes (color-coded green). The annotators clearly perceive that the last sign (NO) of the interrogative as well as the first lexical elements (NO++ together with the mouthing 'believe-not') of the following statement are covered by more regular and more definite headshakes (color-coded blue).

Examples (136) and (137) show that the annotators could clearly determine the scope of the non-assertive marker. In addition, they identified headshakes used for negation which interrupt the non-assertive head marker. These headshakes accompany only a negative sign (cf. the first identified negative headshakes in example (137)) or a negated predicate (cf. the other co-occurring negative headshakes that interrupt the non-assertive marker in examples (136) and (137)). An interpretation of this phenomenon is that the headshakes used for clause negation are grammatically required and therefore overlay the nonassertive head marker used for coding epistemic modality.

\section{Co-occurring nonmanuals and signs}

Two non-manual markers tend to occur in the context of a non-assertive marker, namely wrinkled nose and squinted eyes (cf. 7.3.2). Wrinkled nose consistently occurs in negated clauses. Also, the occurrence in negated conditionals or conditionals including signs with negative meaning (like CLOSED) strongly suggests that wrinkled nose occurs in propositions which express 'potentiality', but also conveys the attitude that the outcome of a proposition is negative oriented. The second non-manual marker is squinted eyes. As 
already described in the preceding subchapter in 7.3.2, this non-manual is supposed to have a connection with knowledge or lack of knowledge. The occurrence of both non-manual markers together with the non-assertive head marker is exemplified in the following: $(138)^{247}$

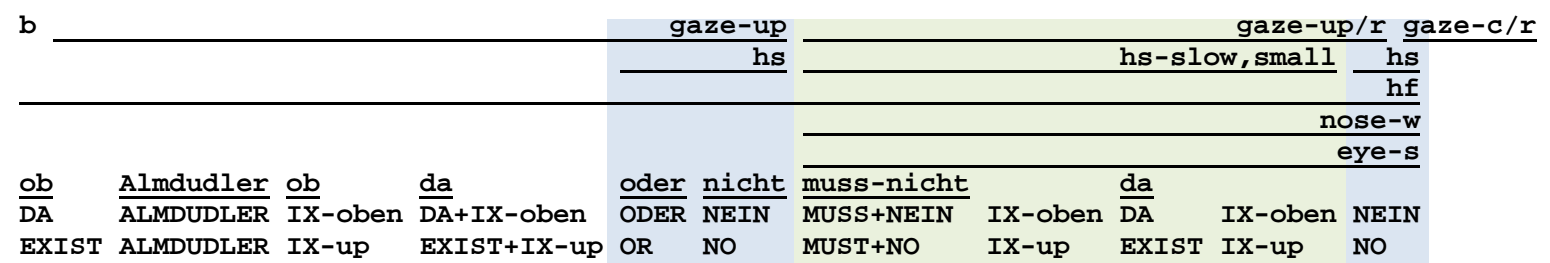

Ich frage mich, ob es Almdudler gibt oder nicht. Ich vermute eher nicht und komme zum Schluss, dass es nichts geben wird.

I wonder whether there is Almdudler or not. I suppose that is more likely not the case and I conclude that there won't be anything.

(M003_106,1298_m_thoughts_ex03_00.43-00.50)

In example (138), the second part of the chain of thought which follows the embedded interrogative is covered by slow, small headshakes. The annotators allocate a 'more-likelynot' meaning to that part of the chain of thought which concludes with clear headshakes covering the chain of thought and concluding that the desired thing will not be there.

The non-manual markers which co-occur with the non-assertive head marker and the following negative headshakes are squinted eyes and wrinkled nose. With regard to the latter, the present finding confirms that wrinkled nose tends to occur in negative contexts which possess a kind of potentiality. Concerning squinted eyes, the connection with knowledge may be assumed in (138), but further investigations on this have to be conducted in order to make clear statements on the context of occurrence as well as the exact meaning of squinted eyes. This is the same with the non-manual marker wrinkled nose.

Lack of confidence about the outcome of a proposition can be conveyed by lexical elements as well as by the non-assertive head marker. Consequently, signs of cognition or feelings expressing uncertainty, indecisiveness, or doubtfulness occur together with this epistemic marker. Furthermore, negation signs are present in these contexts.

\footnotetext{
${ }^{247}$ The utterance, illustrated in example (138), follows the utterance in example (137), and repeats the same content.
} 
Summing up, the non-assertive marker and the convinced-assertive marker are deductiveassumptive markers which express the judgment/evaluation on a proposition.

The form of the non-assertive marker is very similar to the negation marker - both indicators are coded by headshakes. Nevertheless, the epistemic marker is different in form and meaning than the clause negator headshakes. As demonstrated by the example above, the non-assertive marker covers the content to which it refers. The ÖGS data show that the epistemic marker in the majority of cases occurs in the context of trains of thoughts. Further, the data demonstrate that the non-assertive marker is in most cases used in declarative utterances. In some instances, the epistemic marker occurs in embedded interrogatives too.

\subsubsection{Speculative body marker}

The next non-manual marker codes epistemic possibility, which, following Palmer (2001, 8-9, 24-28), is labeled 'speculative marker'. The term 'dubitative marker' has been avoided. This is in accordance with Bybee (1985, 179), who argues that dubitative modality requires at least one dubitative element. As the present marker expresses the signer's deliberation/consideration on the truth value of a proposition and - depending on the signs and other non-manuals conveying a more deliberative, indecisive, or insecure meaning -conveys different degrees of uncertainty. The term 'dubitative' for the present epistemic marker as including only the negative notion of a consideration is too narrow.

\section{Form}

The means of coding this epistemic marker is a slow and regular swaying movement of the upper body. The movement can be described as a purely side-to-side movement of the torso, like a pendulum swaying from side to side. The annotators could determine a clear starting and ending point of these movements, as well as a clear description of the meaning.

When body sways co-occur with an utterance, a single sway to one side can accompany one sign (see Figure 7.5) or several signs, i.e. a complete unit (see Figure 7.4). The body sways may be performed in a more or less regular way. As illustrated in example (140), in 
which the body sways accompany the entire utterance, in the rose color-coded part of the utterance the side-to-side movements tend to be timed to coincide with each sign and are large in size. In those parts of the utterance in which the signer expresses his train of thought, the side-to-side movements are implemented less regularly, are smaller in size and tend to cover more lexical elements. Hence, there is a close correlation between the lexical elements and the body's movement direction and its change of direction. In other words, there is no instance in which a signer changes the movement direction of the body in the middle of a sign. This is illustrated in Figure 7.4 and Figure 7.5.
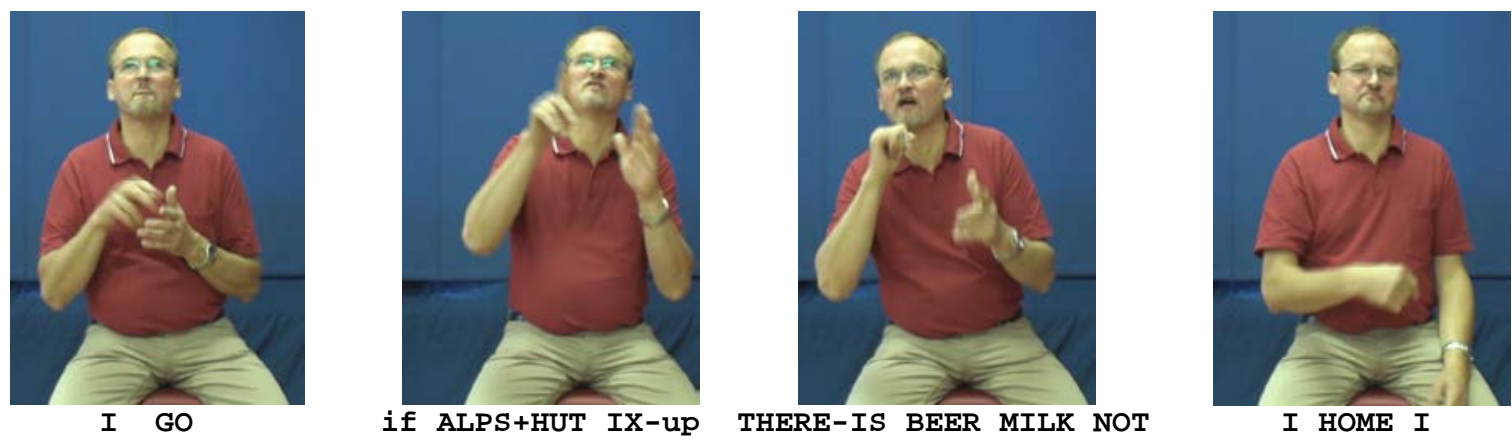

Figure 7.4 The speculative marker with deliberative meaning

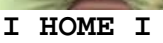

As depicted in the picture series of Figure 7.4 and described subsequently by example (139), the body sways cover more lexical signs which are aligned with syntactic constituents.

The following Figure 7.5 (illustrated in example (140)) shows that on each sign (twice including the sign for I), the torso sways to one direction:

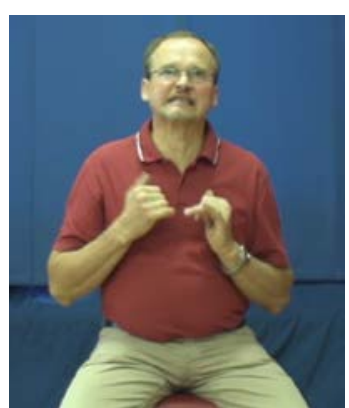

I HIKE

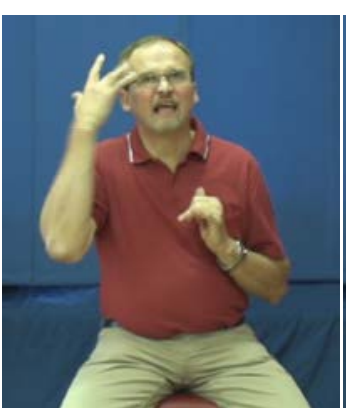

KNOW-NO

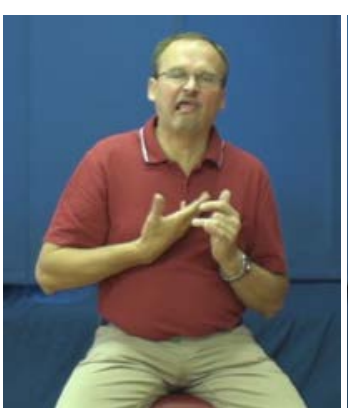

I INDECISIVE

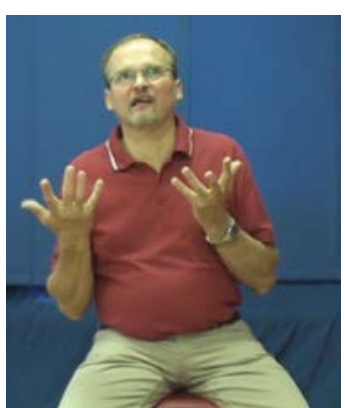

PU

Figure 7.5 The speculative marker with indecisive/doubtful meaning 
In Figure 7.5, the body sways first slightly to the left when signing I HIKE, then slightly to the right when signing KNOW-NO. Subsequently, the body sways left when signing I INDECISIVE and to the right when using the element PALM-UP.

\section{Meaning}

The speculative marker, primarily present in the trains of thoughts, is used when the particular signers judge or evaluate in a deliberative, insecure or doubtful way about the truth value of a proposition. This judgment goes together with a 'potentiality of implementation' of the event/situation/etc. formulated in the proposition. This combination results in a speculative judgment on a proposition.

When doing the annotations, the annotators quickly noticed that the marker body sways used for expressing epistemic modality conveys different degrees of uncertainty paired with a certain amount of potentiality.

The two poles of the continuum of uncertainty are first, the concept of uncertainty that conveys a more deliberative or reasoning meaning, while second, the concept of uncertainty conveys a more indecisive, insecure, or even doubtful meaning. This is exemplified in the following two examples:

$(139)^{248}$

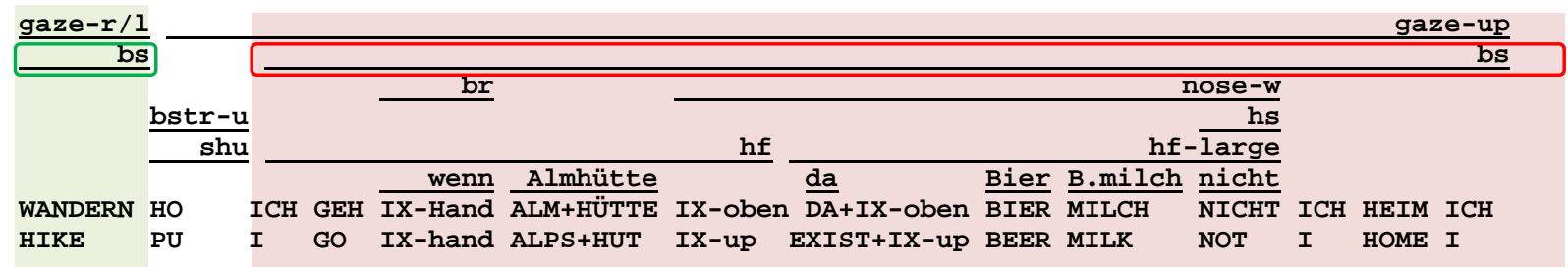

Während ich wandere bin ich mir unsicher / überlege ich. Wenn es in der Almhütte weder Bier noch Buttermilch gibt, gehe ich heim.

While I am hiking I am insecure / I deliberate. If in the hut there is neither beer nor buttermilk, I will go home.

(M001_091,1183_m_thoughts_ex04a_01.11-01.19)

In (139), the entire chain of thought (color-coded rose) is accompanied by slow body sways (edged red) whose movement direction (illustrated in Figure 7.4) coincides with the syntactic constituents. Thus, the body sways to the right while signing I GO, to the left while signing/mouthing 'wenn' ('if') ALPS+HUT IX-up, to the right when signing

\footnotetext{
${ }^{248}$ The example (139) has previously been cited in chapter 5 (see example (80)).
} 
EXIST+IX-up BEER MILK NOT, and back to neutral position when signing I HOME I. With regard to the meaning of the body sways, the annotators allocate a deliberative or reasoning meaning - always additionally mentioning that a 'general uncertainty' is present which is also due to the potential meaning of the body marker. As a result of the conditional, the entire construction is also provided with potentiality. The scope of the bsmarker (color-coded rose) is that part which is covered by the bs-marker. The preceding body sways (edged green) belong to the sign HIKE and do not provide a modality meaning.

In example (140), the annotators perceive and describe a higher degree of uncertainty:

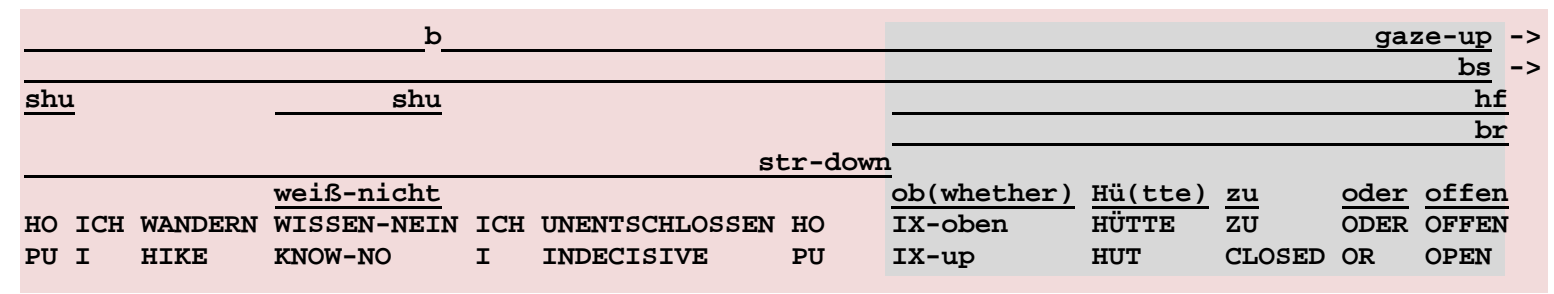

\begin{tabular}{|c|c|c|c|c|c|c|}
\hline & gaze-up b & gaze-d b & & ga: & ze-up & gaze-d \\
\hline & & & & & $h t i-r$ & $\overline{\text { shu }}$ \\
\hline & & & & & $\frac{b r}{b r}$ & \\
\hline & & str-down & & & & \\
\hline UNENTSCHLOSSEN1 & UNENTSCHLOSSEN2 & HО & ICH & HOFFEN & OFFEN & WISSEN - NEIN \\
\hline INDECISIVE1 & INDECISIVE2 & PU & I & HOPE & OPEN & KNOW-NO \\
\hline
\end{tabular}

Während ich wandere überlege ich unentschlossen (abwägend/unsicher), ob die Hütte geschlossen oder offen ist. Ich hoffe, sie ist offen, weiß es (aber) nicht.

While I am hiking I wonder indecisively (deliberatively/insecurely), whether the hut is open or not. I hope that it is open, (but) I don't know.

(M001_091,1183_m_thoughts_ex05_01.47-01.57)

In example (140), the body sways are described as conveying an indecisive and insecure meaning by the annotators. Some even have the impression the signer is in doubt whether the hut is open or closed. All of them perceive that the body sways accompany the entire utterance and consequently, the entire utterance is embedded in the context of this uncertainty displaying the scope of the bs-marker (color-coded rose).

When comparing these examples ((139) and (140) as well as the following example (141)) it becomes obvious that differences in the perception of the degree of uncertainty about the 
truth value of the proposition are evident, although in all examples the same epistemic body marker is present. Furthermore, it becomes obvious that the annotators' description of the meaning of the body sways depends on which lexical elements are used in the particular utterance. Thus, if expressions of indecisiveness or insecurity are used in the utterance, the annotators tend to allocate these meanings to the epistemic markers in addition to the more neutral, deliberative meaning.

These perceptions of the annotators lead to the conclusion that the present epistemic body marker possesses a kind of 'broader meaning of uncertainty' which implies some degree of 'potentiality'. The narrowing of the epistemic meaning of the modality marker 'body sways' is effected by use of lexical elements conveying a special modality meaning. That is, if the signer uses the epistemic body marker together with emotional or cognitive signs like UNENTSCHLOSSEN (INDECISIVE) or WISSEN-NEIN (KNOW-NO), or if the signer produces nonmanuals like the mouth gesture 'stretched-down' conveying, among others, the meaning of 'uncertainty', then the modality meaning is narrowed. This probably leads to the consequence that - like in example (140) - the annotators describe that the body sways possess - apart from the deliberative meaning - a more indecisive and insecure meaning.

\section{Co-occurring nonmanuals and signs}

Lexical elements which occur in constructions that are accompanied by epistemic body sways are emotional signs expressing insecurity or indecisiveness and signs of cognition expressing knowledge or lacking of knowledge, respectively.

The non-manuals which tend to occur in connection with the speculative marker are squinted eyes, wrinkled nose and the mouth action 'stretched' or 'stretched-down'.

The co-occurrence of the listed elements is exemplified in the following: 
$(141)$

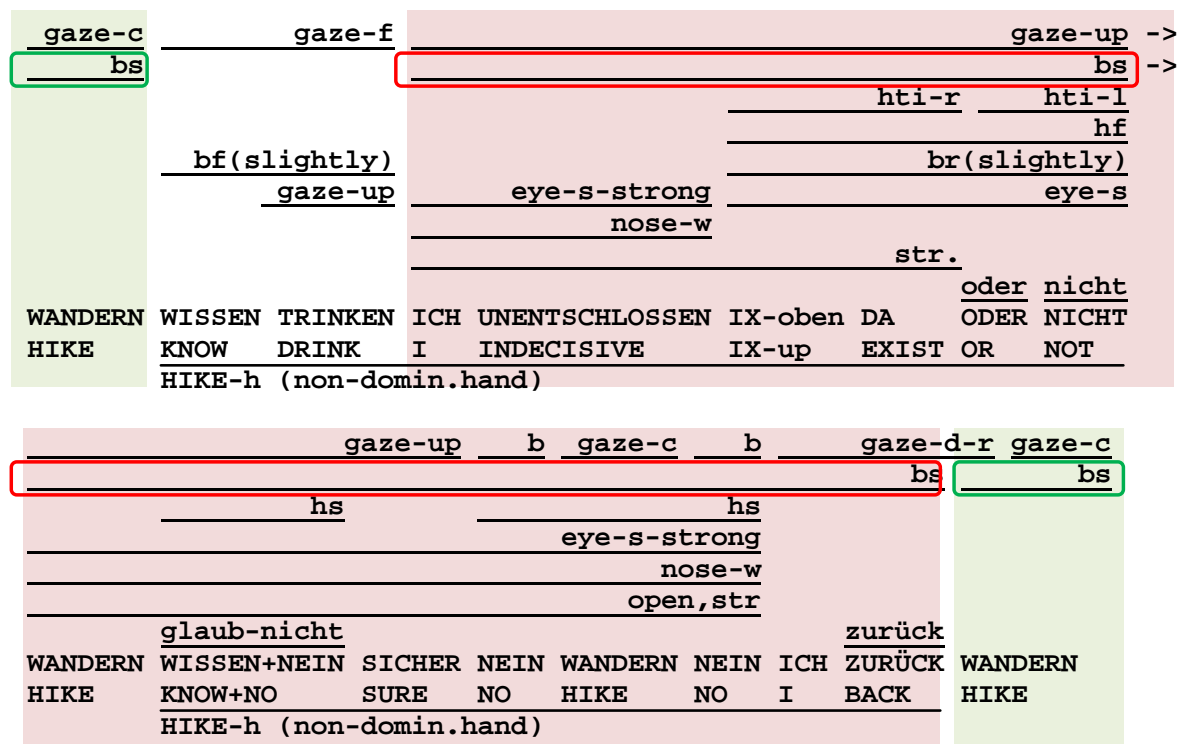

Während ich wandere überlege ich unentschlossen (abwägend/unsicher), ob die Hütte geschlossen oder offen ist. Ich hoffe, sie ist offen, weiß es (aber) nicht.

While I am hiking I wonder indecisively (deliberatively/insecurely), whether the hut is open or not. I hope that it is open, (but) I don't know.

(F004_1117,1209_m_thoughts_ex03_04.11-4.25)

In (141), the epistemic body marker (edged red; both bs-markers illustrate one ongoing swaying movement) accompanies the entire line of thought which possesses the modality meaning of uncertainty and potentiality and displays the scope of the bs-marker (colorcoded rose). The signs which occur in this context are INDECISIVE, KNOW-NO, and SURE NO. All nonmanuals listed above, i.e. squinted eyes, wrinkled nose and the mouth action 'stretched', occur in this example (color-coded rose). They clearly cover those parts of the utterance which include the emotional or cognitive signs of insecurity and lack of knowledge. During the proposition (IX-up EXIST OR NOT), these markers are not performed or if they are, then in a less intense way. In contrast to them, the epistemic marker body accompanies the entire train of thought which conveys the epistemic meaning. As described below, the preceding and following body sways (edged green), covering HIKE, differ in form and function. 


\section{Distinguishing features from other kinds of body sways}

The body sways coding epistemic modality differ in form and/or meaning from other kinds of body sways which are present in ÖGS.

Boyes Braem (1999, 187-200) investigated body sways accompanying narratives in Swiss German Sign Language (DSGS). Comparable body sways serving a discourse-structuring function are present in ÖGS too. Apart from the different function (epistemic versus discourse-structuring), these discourse-structuring body sways may vary the articulator in ÖGS. The right and left movements can be implemented by weight shift as well as by moving the torso from side to side. What is more, at the points where the side-to-side movement changes direction, the movement can be held for a while. In contrast, it is also possible for the epistemic modality marker to be performed unevenly, but longer holds never occur. Moreover, the epistemic body sways only can be performed by side-to-sidemovements of the torso as opposed to the whole body.

Other body sways are present in the data which are used for signing a 'steady and uniform forward motion' (edged and color-coded green in the examples (139) and (141)). These body sways are not pure side-to-side movements. They may differ in form (e.g. imply an alpha-movement or a forward movement alternating to the right and left) and are articulated differently by the various ÖGS signers. In most cases, they are described as constructed actions. This means that the signer slips into the role of a person who is walking, hiking, etc.

Summing up, the epistemic marker 'body sways' is used to express the signer's deliberative, insecure, or even doubtful judgment/evaluation of the truth value of a proposition as well as the potentiality in implementation of the proposition's outcome. Similar to the other epistemic markers, the covered part is exactly the part the modality marker refers to.

The marker tends to occur when the signers express their insecurity in trains of thoughts, especially when these thoughts are expressed in an embedded, self-addressed interrogative (cf. 4.3). 
Boyes Braem (1999) notes that early and late learners of DSGS use body sways for discourse-structuring purposes differently. Late learners use body sways less frequently and their movements are less displaced from the center lines. This difference is observed with the signers' use of the epistemic body marker in ÖGS. Those who have Deaf parents use the marker more often and perform the side-to-side movements more clearly and evenly. Especially when the informants formulate a proposition, the swaying movements tend to be less regular or reduced by those Deaf individuals who do not have Deaf parents but who started signing at a very early age.

\subsubsection{Timitive head marker}

Expressing one's thoughts may imply that the person has wishes, hopes, or concerns/worries concerning the truth value of a formulated proposition or an unrealized event. Following Palmer (2001, 13, 22, 131-135), coding these emotions implies that both epistemic and deontic modality can be coded. He distinguishes 'Desiderative' for coding wishes and 'Timitive' for coding fears. Coding these emotional judgments on a proposition is made possible either by lexical means or by moods like the Latin subjunctive. Rare, but still evident, is the coding of one or all of these emotional attitudes by one or more markers conveying only this modality function. For instance, Lehmann (2012) cites the timitive morpheme '-ege' in Hocank ${ }^{249}$ expressing 'I am afraid that ...'.

\section{Form}

In the data on trains of thoughts, some of the signers use trembling movements in which the head is the primary articulator. These tend to be very small nodding or head forward movements, performed in a trembling way, while the body is completely tensed.

The following Figure 7.6 shows a signer uttering the timitive marker. It should demonstrate the tensed body; the trembling movement is so minimal that they cannot be depicted by a picture series.

\footnotetext{
${ }^{249}$ Hocank is a Siouan Language which is spoken by the Wisconsin Hocanks and the Nebraska Winnebagos. The language is known for its rich verb morphology (cf. Department of Linguistics of the University of Erfurt 2007).
} 


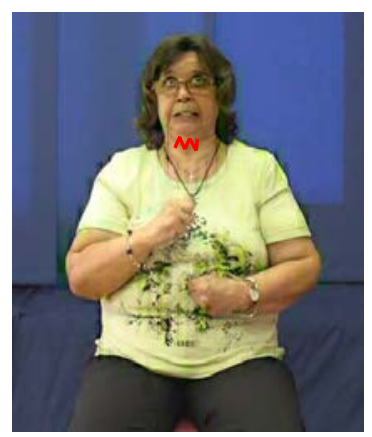

Figure 7.6 The timitive marker

\section{Meaning, co-occurring elements}

In the ÖGS corpus, the timitive marker occurs only a couple of times. In all these cases, the marker is used to express the signer's doubt and even concern/worry on the outcome of a proposition. The annotators all conclude that it is the concern about the outcome which is expressed by the modality marker, while in most cases the hands express hope. This combination is illustrated in Figure 7.6.

In the following example (142), the production of the timitive marker is exemplified. $(142)^{250}$

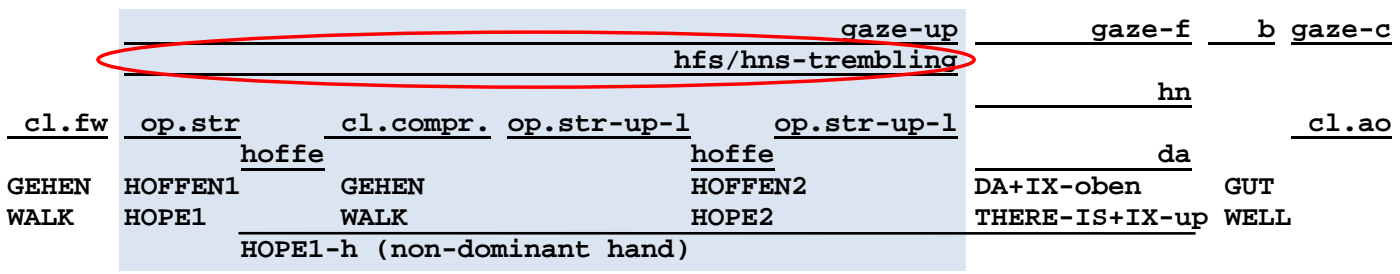

Während ich spaziere hoffe ich inständig, aber auch mit großer Sorge (Angst), dass es dort etwas gibt. (Als ich ankomme sehe ich, dass) es etwas gibt. Ich bin wirklich erleichtert. While I am walking I am afraid - but I desperately hope - that I can get something there. (As $I$ arrive, I see that) there is something. I am really relieved.

(F002_109,1201_m_thoughts_ex07_03.56-04.06)

In example (142), the signer expresses her concern/worry as well as her hope with the timitive marker (encircled red). Its scope is exactly the covered part (color-coded blue). These emotions refer to a proposition in the sense of 'I am afraid that ... / I hope that ...'.

${ }^{250}$ The abbreviations for the mouth actions are: lips closed, forward (cl.fw.); lips open, stretched (op.str.); lips closed, compressed together (cl.compr.); lips open, stretched, left corner up (op.str.up-l); lips (nearly) closed, air comes out (cl.ao.);

The sign for HOPE2 differs from the sign HOPE1 in that it includes a trembling movement and it is covered by the mouthing 'hoffe' and subsequently by the mouth action 'open mouth, lips stretched and left corner up'. 
But at the same time, these emotions can refer to an unrealized event. So, both interpretations are possible. In this example the proposition/event is not explicitly formulated, but the signer's line of gaze directed upward reveals that she refers to the hypothetical space, which is the space of thoughts to which unrealized events, activities, situations, etc. are allocated. This implies that the signer is thinking about something (a proposition or an event) and not only expressing her emotions. Finally, (142) shows that in the context of this trembling marker various mouth actions are possible. They all have in common that the lips are tensed by stretching them or pressing the lips together.

In conclusion, a head marker for expressing concern concerning a proposition/event has been identified by the annotators. It would be very interesting to obtain and compare ÖGS texts in which different emotions and attitudes, such as wishes or desire with regard to propositions/events are expressed. Probably other non-manual modality markers exist that have not been described before.

All in all, the present timitive marker (exemplified in (142)) shows that whenever a person wants to express more emotions at the same time (here hope together with concern), it is very helpful to code these using different articulators. While concern is coded by the head, the hands express hope. Of course, concern/worry can be coded in the hands too, performing a trembling movement. However, it would be very difficult maintaining this trembling movement while performing several signs, when a signer wants to express concern/worry along a larger text unit. So, in this aspect the timitive marker is a very helpful means of coding modality.

\subsubsection{Possibility head/body marker}

The epistemic modality marker described above is the marker 'body sways' which is used to express the signer's insecurity on a proposition. When using this epistemic marker, in addition, the potentiality of the implementation of the described event in the proposition is implied. The marker described in this subchapter only refers to the possibility (of realization) of an unrealized event, formulated within a thought proposition. Based on Palmer’s (2001, 22) categories, the present marker displays a ‘dynamic modality marker'. 
However, someone may suggest that the possibility of realization of an unrealized event is evaluated by an external source, namely the signer himself/herself. Following this interpretation, the present marker can be defined as 'deontic modality marker' at the same time.

In various occurrences in the corpus, the signers only describe the possibility of the implementation of an unrealized event. This information can be provided by lexical elements which refer to the potentiality of an unrealized event. In ÖGS, these are the signs VIELLEICHT (MAYBE) and MÖGLICH (POSSIBLE) as well as 'fast head tilts' and/or 'fast body sways' conveying a meaning of possibility. Another indicator expressing modality meaning is 'tilting the head sideward' and/or 'leaning the body sideward', described in the next section.

\section{Form, meaning, and co-occurring elements}

In the preceding chapter (6.4.4), the 'alternative space' together with its indicators head tilting sideward (hti-r/l), body lean sideward (bl-r/l), and step sideward (step-r/l) have been discussed. These indicators are used to mark different alternatives. A characteristic of expressing the potentiality of an event is to list different alternatives. Expressing possibility by listing a positive and a negative outcome of the unrealized event is a very common way in ÖGS to imply the modality interpretation of an utterance. For expressing potentiality on an unrealized event, the alternative markers body lean sideward and/or head tilt sideward are used. Consequently, these two alternative markers function additionally as potentiality markers coding the information 'maybe this or maybe that'.

Apart from using these two head/body markers for listing alternatives, the data shows that in most instances in which the signs VIELLEICHT (MAYBE) or MÖGLICH (POSSIBLE/POSSIBLY) occur, these signs are accompanied by tilting the head sideward and frequently forward too. As described in the literature overview, nonmanuals which accompany modality signs have been mentioned (cf. 7.2). Interestingly, tilting the head sideward when using modality signs (which express possibility) has also been described for ASL (cf. Wilcox \& Wilcox 1995, 142-144). In the following, the implementation of the signs MÖGLICH (POSSIBLE) and VIELLEICHT (MAYBE) is illustrated. First, two 
occurrences from the ÖGS corpora are cited. For comparison then, the ASL signs POSSIBLE and MAYBE are given. The great similarity - also with regard to the nonmanual component - is highly significant since the languages are not known to be related.

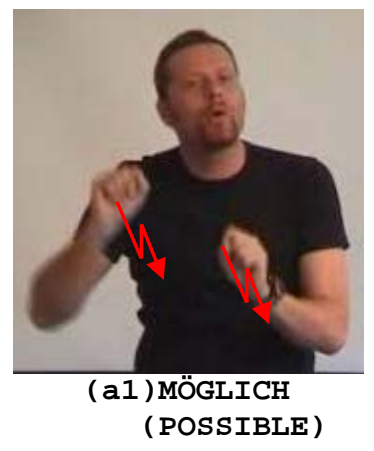

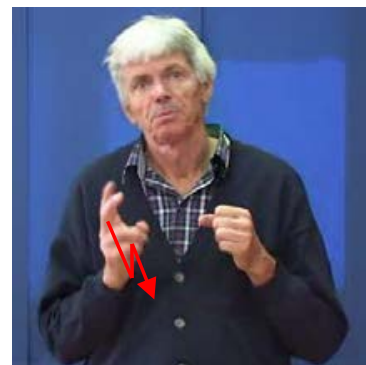

(a2) KANN+/MÖGLICH (COULD/POSSIBLE) ${ }^{251}$

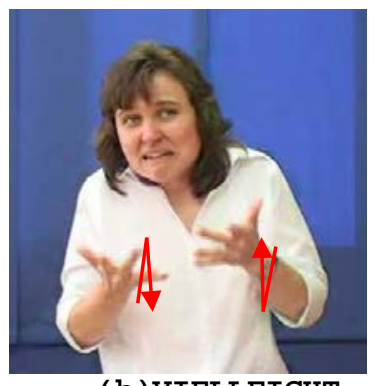

(b) VIELLEICHT (MAYBE)

Figure 7.7 POSSIBLE and MAYBE in ÖGS

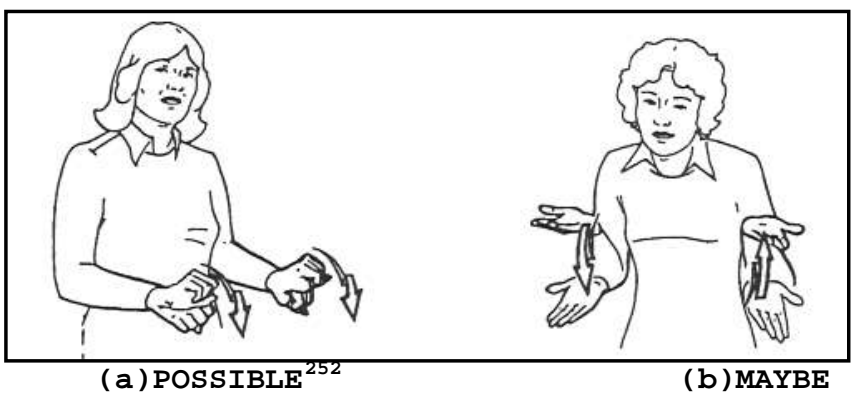

(cf. Wilcox \& Wilcox 1995, 143)

Figure 7.8 POSSIBLE and MAYBE in ASL

As obvious in all pictures, the signers tilt their head. In addition, in ÖGS the head is pushed forward in many instances.

\section{Co-occurrence, Occurrence of the modality elements}

In the following, the co-occurrence of the various modality elements is illustrated by two examples which show typical characteristics of the deontic/dynamic modality construction.

251 In the data the meaning of 'possible/might' is implemented by producing the sign MÖGLICH (POSSIBLE) which looks identical to the illustration of POSSIBLE in ASL. The second way of producing potentiality in the sense of 'could be possible' is the use of the sign KANN (CAN) which is conducted with at least two iterations, performed in a non-intensified way, and optionally covered by the mouthing 'möglich' ('possible') or 'kann' ('can').

252 The illustrated sign can also mean CAN in ASL (cf. Wilcox \& Wilcox 1995, 141) which usually has one downward movement which can be repeated. 
Thus, the examples illustrate the co-occurrence and place of occurrence of the manual and non-manual modality signs and markers (encircled red).

$(143)^{253}$

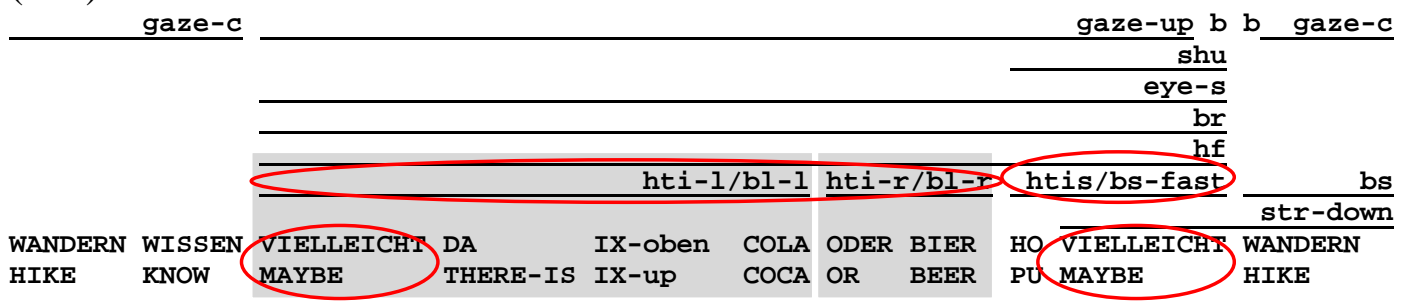

Während ich wandere denke ich, dass es dort in der Hütte vielleicht Cola oder Bier gibt, bin mir aber unsicher.

While I am hiking I think that at the hut there might be cola or beer available. But I am unsure.

(F004_1117,1209_m_thoughts_ex06a_05.24-05.37)

In (143), the signer uses the sign MAYBE, found in clause-initial position of the proposition, and fast head tilts / body sways (htis/bs-fast), found in clause-final position of the proposition, both conveying a modality interpretation. Also, the signer uses the marker head tilt sideward / body lean sideward (hti-l/bl-l; hti-r/bl-r) to code the alternatives as well as the deontic/dynamic modality. Thus, the head/body marker is used to illustrate the two alternatives and, in addition, the potentiality of the unrealized event. $(144)^{254}$

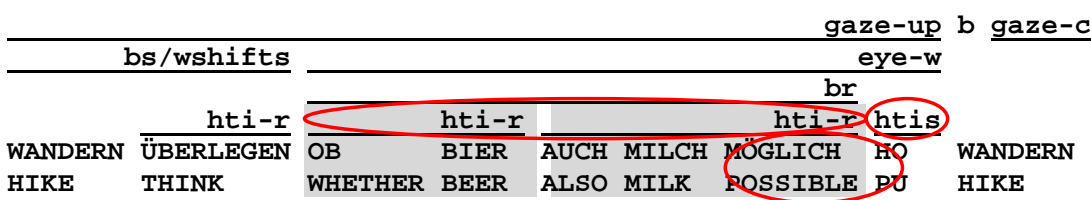

Ich bin am Wandern und überlege, ob es Bier bzw. auch Milch gibt. Es ist möglich. While I am hiking I wonder whether there will be beer or even milk. It is possible.

(M002_114,126_m_thoughts_ex03a_03.47-03.57)

In (144), the deontic/dynamic modality is expressed by three means of coding (encircled red). First, the construction that attributes the deontic/dynamic interpretation is terminated by the sign POSSIBLE. Second, this modality part is followed by head tilts (htis) conveying the meaning of 'maybe'. Third, the head marker used for alternative and modality purpose covers that construction which possesses the modal interpretation.

\footnotetext{
253 The example has been previously cited in chapter 4 (cf. example (69)).

${ }^{254}$ The example has been previously cited in chapter 4 (cf. example (65)).
} 
Both examples show a prototypical occurrence of the modality signs/markers. Thus, the modality indicators may be produced while formulating a line of thought that is selfaddressed and implies an interrogative/irrealis way of interpretation. Concluding, both examples show that the concepts of modality and interrogativity are closely related, which probably is due to their semantic contiguity ${ }^{255}$.

Summing up, ÖGS possesses a head/body marker which can function to display alternatives and which may additionally function as deontic/dynamic modality marker. Also, tilting the head is a component which frequently co-occurs with the modality signs VIELLEICHT (MAYBE) and MÖGLICH (POSSIBLE) in ÖGS. Whether there are further markers coding event modality requires further and deeper investigations on that topic.

\subsection{Conclusions on modality markers in ÖGS}

This description of how to code modality in a sign language (in this case, ÖGS) brings brand-new insights into sign language research insofar as first, two modality systems within a sign language are distinguished, and second, a set of head and body markers is presented which codes modality meaning.

To begin with, the first modal system in ÖGS is composed of modality signs. This includes:

- modal verbs

- $\quad$ signs of cognition, emotion, or perception which convey a modality meaning In the majority of instances from the data, the latter take a complement, implying a hypothetical situation which the signers evaluate or have an opinion on. Both modal verbs and modality signs can include a non-manual component. As the literature overview shows, sign language research is focused on this modal system, when discussing 'modality/mood' in sign languages.

\footnotetext{
${ }^{255}$ Palmer (2001, 11-13, 120-121) describes the close relation between coding interrogativity (as well as negation) and modality. He cites as example Latin, among other languages, which uses the subjunctive in reported (indirect) questions.
} 
The second modal system in ÖGS includes nonmanuals which code modality meaning and show different characteristics:

- First, there are nonmanuals which code a 'narrowed modality meaning', that is, a clear lexical meaning is ascribed to them by the annotators. They can stand syntactically on their own; frequently they co-occur with other nonmanuals constituting together this 'narrowed (i.e. lexical) modality meaning'.

- Second, there are nonmanuals which code modality meaning by co-occurring with an utterance with which they are associated. Several of the identified markers, especially head and body markers, express the signer's attitude on a proposition.

But some of these nonmanuals can be both. So, they occur on their own possessing a clear modality meaning or cover an utterance and provide this with modality meaning. Both possibilities are illustrated in Figure 7.9.
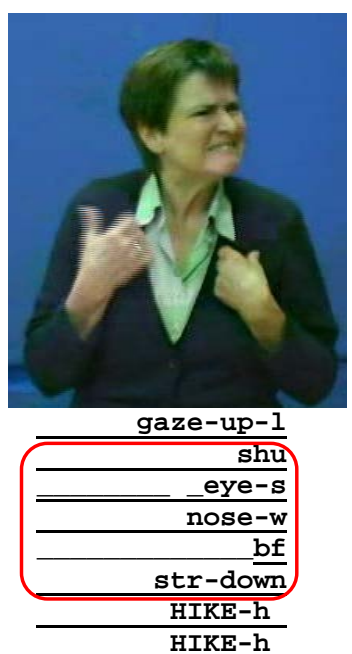
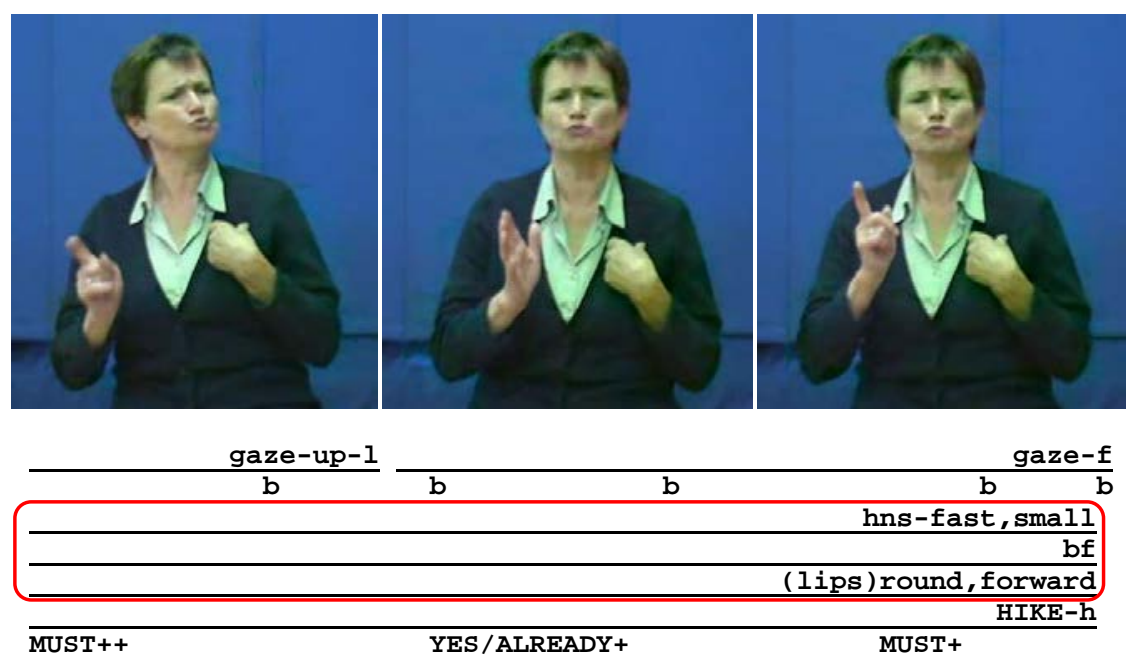

Figure 7.9 Nonmanuals coding modality meaning

In Figure 7.9, first a co-occurrence of various nonmanuals, some of which very likely code modality meaning. In particular, the mouth action 'stretched-down' is used to express being unsure; the shoulder shrug implies 'lack of knowledge'; the squinted eyes quite likely also express lack of knowledge and/or the degree of possessing/lacking of knowledge; wrinkled nose may express negative presupposition. All these nonmanuals, co- 
occurring with gaze up and somewhere to the left, occur while the preceding sign HIKE is held. Only the dominant hand is slightly raised when producing all these nonmanuals.

The following picture sequence, taken from example (132), illustrates that nonmanuals which code modality meaning co-occur with lexical items with which they are associated. Here, the assertive head marker, displaying small fast forward/upward rotating head movements and co-occurring with furrowed brows shows the signer's convinction of the positive outcome of a situation.

The second new aspect with coding modality in sign languages is a set of head and body markers which primarily code epistemic modality. They serve to mark propositional modality, that is, the signer's knowledge and/or degree of confidence of the truth value of a proposition can be shown by the following possibilities: convinced-assertive head marker, non-assertive head marker, speculative body marker and timitive head marker. One identified head/body marker, used for marking alternatives, also functions as a possibility marker, coding event modality. The identified markers, their form and functions are summarized in Table 7.1. With regard to the markers' scope, the present findings show that the scope displays the covered part of an utterance.

\begin{tabular}{|c|c|c|c|}
\hline $\begin{array}{l}\text { Type of } \\
\text { modality }\end{array}$ & Marker & Form & Meaning \\
\hline \multirow[t]{4}{*}{$\begin{array}{l}\text { propositional } \\
\text { modality }\end{array}$} & $\begin{array}{l}\text { Convinced- } \\
\text { assertive } \\
\text { head marker }\end{array}$ & $\begin{array}{l}\text { fast, small or slow, large } \\
\text { head movements/rotations } \\
\text { forward ( } \& \text { upward) }\end{array}$ & $\begin{array}{l}\text { signer is convinced of the positive outcome; } \\
\text { comments the truth value of the thought } \\
\text { proposition }\end{array}$ \\
\hline & $\begin{array}{l}\text { Non-assertive } \\
\text { head marker }\end{array}$ & $\begin{array}{l}\text { slow headshakes, small in } \\
\text { size, performed tentatively }\end{array}$ & $\begin{array}{l}\text { signer thinks that the conclusion/result of a } \\
\text { proposition will have a negative outcome }\end{array}$ \\
\hline & $\begin{array}{l}\text { Speculative } \\
\text { body marker }\end{array}$ & $\begin{array}{l}\text { slow, regular body sways } \\
\text { (only with torso) }\end{array}$ & $\begin{array}{l}\text { signer judges/evaluates in a deliberative, } \\
\text { insecure or doubtful way about the truth } \\
\text { value of a proposition }\end{array}$ \\
\hline & $\begin{array}{l}\text { Timitive } \\
\text { head marker }\end{array}$ & $\begin{array}{l}\text { small nods or forward } \\
\text { movements of the head, } \\
\text { performed in a trembling } \\
\text { way, body is tensed }\end{array}$ & $\begin{array}{l}\text { signer expresses his/her concern/worry on } \\
\text { the outcome of a proposition; }\end{array}$ \\
\hline $\begin{array}{l}\text { event } \\
\text { modality }\end{array}$ & $\begin{array}{l}\text { Possibility } \\
\text { head/body } \\
\text { marker }\end{array}$ & $\begin{array}{l}\text { head tilt sideward }(\mathrm{r} / \mathrm{l}) \\
\text { and/or body lean sideward } \\
(\mathrm{r} / \mathrm{l})\end{array}$ & $\begin{array}{l}\text { expresses the potentiality/possibility of an } \\
\text { unrealized event, displaying alternatives } \\
\text { (maybe this or maybe that) }\end{array}$ \\
\hline
\end{tabular}

Table 7.1 A set of head and body markers coding modality

\footnotetext{
256 The presented types of modality follow Palmer’s (2001, 7-8, 24-85) classification.
} 
What is more, the presented examples show that when an ÖGS signer is thinking about unrealized possibilities, alternatives, conditions and so on and when expressing his/her certainty, insecurity, deliberation, worry, and so forth on these self-addressed thoughts, the signer is always referring to the hypothetical space (cf. 6.4.3). It is striking that in all examples presented in this chapter, the marker gaze up or somewhere to the front is present, which is used to indicate the space of thought (although in all examples in which the reference object is located somewhere in the upper field, this may also be a reason for looking upward). To conclude, referring to the hypothetical space is also closely connected with modality.

With regard to methodology, it has to be mentioned that the present findings are based on 'signed texts' and not 'elicited sentences'. Primarily, the findings stem from the corpus in which the signers produced trains of thoughts, expressing them with different attitudes. The corpus of the educational training as well as various previously annotated dialogues (on diverse topics) also offered constructions in which modality has been coded. Elicited sentences were not helpful for finding the non-manual marker as the signers tended to code the modality meaning almost exclusively by modality signs. 


\section{New insights, summary and generalizations on head and body markers}

In the final chapter of my thesis, all new insights for sign language research and for Austrian Sign Language in particular are presented. Also, each chapter of the thesis is summarized. This includes the new insights as well as findings which support previous analyses for ÖGS, but also in general for sign languages. Then, I focus on generalizations of the head and body markers based on the ÖGS data. First, I report that many functions are coded by the 'head'. Second, I show that the identified head and body markers can be distinguished in three subclasses based on their production, the units which they cover, and the dependency on other factors like space. Third, I analyze the semantic contiguity of head markers as it is significant that various related functions are coded by the same markers. Fourth, the co-occurrence and sequential occurrence of head/body marker are discussed. Finally, I conclude with the requirements for future research.

\subsection{New insights}

What is new for Austrian Sign Language, but for sign languages in general too, are the observations that

- several functions can be coded by means of head and/or body movements, namely negation, assertion, interrogativity (even in embedded interrogatives, in which it may be interpreted as irreality), conditionality, alternativity, hypotheticality and modality (especially epistemic modality) in ÖGS (cf. chapter 3 to 7).

- markers which additionally co-occur with the respective non-manual marker can have the same function or a different function. If the latter is the case, these markers tend to occur in the context of the investigated function. In other words, on the one hand the thesis shows that various functions are primarily coded by a head marker, but there are other markers which function as alternative markers or additional markers. Their occurrence depends on language-internal and/or -external factors. On the other hand the thesis shows that other head markers which frequently co-occur in a special 
construction are not 'secondary markers' (coding the same function), but are markers which fulfill a different function. A detailed analysis of different head markers cooccurring in interrogatives is found in chapter 4.

- in sign languages - at least in ÖGS - the possibility exists that interrogativity/ irreality is marked in embedded interrogative clauses. What is special in ÖGS is that first, the interrogative marker can spread along the main clause, and second, there are different markers for indicating direct polar questions and embedded polar interrogatives (cf. chapter 4).

- in some special constructions such as interrogatives, adversative constructions or conditionals headshakes used for clause negation or for implying contrast have a high tendency to cover the entire clause. Reasons for this, such as 'influence of negative epistemic presupposition', are discussed in chapter 3.

- in a sign language, the signing space can function as 'discrete/independent medium' possessing values such as 'up', 'front', 'back', 'lateral-one-side', 'lateral-the-otherside'. For this reason, no location in the signing space has to be established (as it has to be done for referential space) as these locations in the signing space have their own values (cf. chapter 6).

- two phenomena concerning the signing space exist which are the 'hypothetical space' and the 'alternative space', both possessing values as listed above. The first refers to the 'space of thoughts'. It is referred to when a signer is addressing hypothetical lines of thoughts to himself/herself. The latter is used for opposing alternatives (which implies contrast, also described for other sign languages), showing the mutual influence of alternatives, or displaying the influence of external factors on alternatives. With the alternative space, the thesis analyzes for the first time that 'underlying subject matters' are opposed/contrasted and not the lexemes on the surface, even though in the majority of instances the underlying subject matters are directly expressed by the lexical items on the surface of the language (cf. chapter 6).

- epistemic modality can be coded by head and/or body markers (and other nonmanuals too), covering that part of an utterance which is associated with the modality meaning. 
Various types of epistemic modality markers have been identified in ÖGS, including the convinced-assertive head marker, the non-assertive head marker, the speculative body marker, and the timitive head marker. In addition, a possibility head/body marker, coding event modality, has been identified. Consequently, this thesis shows that in sign languages two modal systems can exist. The first codes modality with modality signs, implying modal verbs and signs conveying a modality meaning. Both can imply a nonmanual component. The second modal system codes modality by nonmanuals. These can occur (frequently with other nonmanuals) on their own, implying a clear lexical modality meaning, or they cover part of an utterance which they provide with the respective modality meaning (cf. chapter 7).

Concluding, the present data together with the Deaf individuals' annotations show that several head and body movements/positions are present in ÖGS which vary in form and meaning. More precisely, movements along a body plane like headshakes can have many different formatives and functions. As described in 2.2.4.4, head and body movements/positions show the following characteristics:

\section{- clear form-function allocation}

First, they are clearly identified by the annotators with regard to their form, description of 'what the element means for the annotator', and reference to which lexical elements the marker refers.

\section{- clear allocation along one of the three axes}

Second, the annotators clearly allocated the various head and body movements to one of the movement options along the three axes, that is, these movements can be upward or downward, forward or backward, and to both sides.

\section{- head/body markers possess additional features to movement direction}

Third, the movement direction is not the only criterion for identifying a head and/or body movement/position. Further relevant features are an intensified performance or different sizes of performances (e.g. positioning the head forward versus positioning the head forward in an intensified way, or producing headshakes with a small radius versus headshakes with a large radius), the speed of performance (e.g. producing fast 
headshakes versus producing slow headshakes), the degree of body tension (e.g. producing head nods with an non-tensed articulator versus performing head nods in an trembling way with a tensed articulator), and an additional movement component (e.g. head nods with trembling movement or headshakes with alpha-shaped movement).

- some markers can vary the articulator, others cannot

Fourth, some of the head and body movements/positions can vary which articulator they are made with, others cannot. For instance, indicating one or more alternative spaces can be done by head tilt sideward, body lean sideward or step to the side. The choice of the respective articulator depends on several conditions, such as whether a signer is sitting or standing when signing or whether the addressee/audience is close or far away from the signer. In contrast, the various interrogative markers can only be performed by the articulator 'head'.

\section{- co-occurrence of several head (and body) markers}

Fifth, more head (and body) movements indicating a special function can co-occur. This means that the annotations show that, for instance, two or three head markers can be produced at the same time.

\subsection{Summary}

In the following, first I summarize chapter 2 in which the research object and the methodological approach are elaborated on. Then, I sum up the findings of chapters 3 to 7 in which I describe functions and forms of head and/or body movements/positions. Through this, I address the main research questions of my thesis, asking which head and body movements/positions exist in ÖGS, what their formatives look like, which meanings/functions they possess, and whether there are different forms with different meanings/functions of head/body movement along a body plane, addressed in chapter 1 .

In Chapter 2 I first describe the possible head and body movements along the various body axes, focusing on those which are also relevant for ÖGS. Second, I review the research done on these articulators, or implying findings with relevance for head and body 
movements. Then, I present all the head and body movements/positions which have been identified by the various Deaf annotators up to now.

- With regard to the 'head', these include the head movements/positions chin up, chin down, chin up-large, head nod(s) upward, head nod(s) downward, head nods performed in a trembling way, head turn to one side, head turn to the other side, various headshakes (fast or slow headshakes, regular in size, used for clause negation or implying negative contrast; small, fast headshakes co-occurring with signs like RATHER; a fast single headshake used, for instance, to underline the realized speed of an action; fast, non-tensed headshakes occurring in content questions; small, slow and tentative headshakes displaying a non-assertive modality marker), head tilt to one side, head tilt to the other side, ongoing side-to-side head tilting movements, head forward, head forward-large, head backward, slow or fast head forward movements, fast ongoing head forward and upward rotation movements, side-to-side movements performed in an alpha-shaped movement of the head, and head forward movements which alternate between the right and left side forward.

- With regard to the 'body', the following movements/positions have been identified by the annotators: Body lean forward, body lean backward, body leans forward, body turn to one side, body turn to the other side, body lean to one side, body lean to the other side, shifting of the weight to one side, shifting of the weight to the other side, step to one side, step to the other side, step forward, (rarely) step backward, hip shift to the side (performed only by one signer), body sways, and body weight shifts;

- With regard to language-relevant 'shoulder' movements/positions, the following language-relevant elements have been identified for ÖGS: Moving one or both shoulders upward (one time, or several times in an equal or alternating way), moving both shoulders forward, and straightening the body up.

The second part of chapter 2 deals with the methodological approach. To begin with, I show that the intention of this thesis is first, only to analyze signed texts, and second, to include all additional language-relevant nonmanuals when illustrating the particular 
function of a head/body marker. The result is that not only that the data shows that a lot of information is coded by non-manual means in signed texts, but also that co-occurring nonmanual markers which occur in a special functional construction posses the same or different functions. If the latter is the case, the analyses show that several of these additionally co-occurring non-manual markers (possessing different functions as the described markers) have a high tendency to occur in these functional construction which are indicated by a particular marker.

To sum up my empirical and inductive approach, first, the language phenomena are identified and described, second, they are classified, and third, generalizations on the results are made. Finally, explanations of some generalizations are included too, such as the fact that a special marker is used for different constructions which possess contiguity. Following these steps, my implemented approach concurs with what Croft $(2003,2)$ calls ‘empirical scientific approach’.

The resulting data comprises formal and informal stories formulated in monologues and dialogues, communicative contents produced in dialogues, trains of thoughts addressed to the signers themselves and lecture recordings. The annotations of the material were primarily done by four or five Deaf annotators who identified the particular head and body markers and described which meaning/function the particular element had for them. With regard to the Deaf informants and Deaf annotators, the thesis includes a description of their former and current living situation in the valley 'Großarl' - as this is unique in Europe. Finally, in chapter 2, the characteristics of the head and body markers are described (as mentioned above).

In Chapter 3 I describe negative and assertive constructions primarily coded by head markers. First, the two formatives of headshakes are described, i.e. headshakes performed with a regular speed and headshakes performed in a very fast way. Both are used for clause negation. The first more often tends to cover the clause or part of it which it negates. The latter more often tends to follow the clause which it negates. In a negated clause which is covered by headshakes, at least the predicate has to be marked by it. The remaining part of the clause optionally can be covered by headshakes. But, the findings show some 
conditions which cause the headshakes to cover the entire clause. So, negative headshakes have the tendency to cover the entire antecedent of conditionals or an entire polar question. For the present, the primary reason for this is ascribed to 'negative epistemic presupposition'. Also, pragmatic-communicative reasons or turn-construction may be further reasons for the spreading of negative headshakes. Furthermore, the data shows that the negative sign which most frequently co-occurs with negative headshakes is the sign NEIN (NO) in ÖGS. What is more, negative headshakes which follow a clause more likely function as 'speech act negation' than clause negation.

A further important function of headshakes is to 'imply negative contrast'. The data shows that headshakes covering the apodosis of adversative constructions are used to imply negative semantic contrast. Most headshakes which cover the consequent of conditionals possess the same function. Interestingly, in 'rather/anyway-constructions' negative contrast is implied, however only the signs RATHER and ANYWAY are covered by small, fast headshakes.

Finally, headshakes can also possess related or other functions than clause negation. For instance, signs conveying negativity like CLOSED or CANCEL can be covered by headshakes. In these cases, the shaking movement starts and ends somewhere close to that sign but it does not depend on syntactic constituents. Furthermore, one or more headshakes are used for other purposes too. For instance, a fast single headshake is used to illustrate the speed of an action, or small, fast headshakes covering a lexeme are used to add the meaning 'incredible’.

In the same chapter head nods functioning as means of assertion are described. Like negative headshakes, two different formatives are identified. The first form is slow, intensively performed nodding movements. Each movement is performed together with a sign or with a sign and an unstressed element. The second form is fast, small, and regularly performed nodding movements co-occurring with a lexical element, or a syntactic constituent (phrase, clause, and sentence). Both forms are used to assert content. The data shows that in all cases in which the signers wants to make clear that the content has to be understood in an assertive way, the head nods cover that syntactic part. 
Furthermore, the data shows that in ÖGS there is a high tendency to show that the consequence of a condition is positive or negative. Thus, similar to the phenomenon of 'implying negative contrast', assertive head nods are used to 'imply positive contrast' in the consequence of conditionals. Also the apodosis of adversative constructions can be covered by assertive head nods for reasons of implying positive contrast.

What is more, one or more assertive head nods which follow a clause more likely function as 'speech act assertion', a phenomenon comparable with headshakes functioning as speech act negation.

Finally, assertive head nods are distinguished from confirmative head nods. The latter occur especially in dialogue situations and show the characteristic that they can be performed in different speeds and sizes, and that they are not restricted to syntactic constituents.

Concluding, negative headshakes and assertive head nods both associate with a clause which they negate or assert. The latter only asserts the covered content while headshakes can cover a part of a clause and refer to the entire clause. Both non-manual markers tend to cover the consequent of conditionals and the apodosis of adversative constructions for reasons of implying contrast.

In Chapter 4 I show different interrogative constructions (polar question, content question, embedded polar interrogative, embedded content interrogative, and a special interrogative marked with a different head marker) which are all indicated by head markers. With regard to the direct questions this thesis demonstrates (and confirms Schalber's 2002 results) that chin down is present in polar questions and chin up and/or head forward in content questions. It is shown too, that in special functional contexts an alternative marker or an additional marker co-occurs, focusing on head markers. Thus, in polar question the marker head forward is used to imply an exclamative emotion. Tilting the head is not an additional question marker, but it tends to occur in polar questions. Two already clearly identified functions for tilting the head in polar questions are expressing possibility (so functioning as modality marker) and expressing politeness. With regard to the marker chin down it was highlighted that this marker is used in two further 
constructions which are not targets of asking a counterpart something. The first, labeled 'confirmation question', is used to receive a confirmation from the dialogue partner to a content which is known by both dialogue partners. The second, defined as 'attention-getter question', occurs in narratives in which the sign of 'knowledge' together with the pointing sign for 'you' are covered by the marker for reasons of getting the addressee's attention.

With regard to content questions the analysis shows that the marker chin up or head forward are clearly identified by the annotators, but in many instances the annotators comment that both markers seem to occur together. Especially, annotating head forward in addition to chin up is argued by the annotators to be the result of perceiving a tensed neck (see Figure 4.3 and 4.4) 257 $^{25}$. Co-occurring head markers in content questions are negative headshakes, head tilting expressing politeness or a notion of modality (possibility), and special for content interrogatives, headshakes which do not function as negator in the sense of structural negation. The latter probably can be interpreted as being unaware of something ${ }^{258}$. Both the polar question and the content question have in common that they are directed toward a counterpart.

The data, especially the corpus containing trains of thoughts, shows that the signer can address himself/herself or an imagined addressee in the space of thoughts. When these lines of thoughts are expressed in an interrogative way referring to two options of which one or both are expressed, the signers use another marker rather than the polar question marker. In these cases the signers position their head forward. The marker's function can be interpreted in two ways. On the one hand the marker functions to express 'interrogativity' in the sense of requesting something which is 'self-addressed' or directed to an 'imagined addressee'. On the other hand the function can be interpreted to express the 'questionability' of a proposition. Consequently, head forward can be defined not only

\footnotetext{
${ }^{257}$ This fact shows that the focus on moving the chin to a position or rotating the head as whole to a special position is not only a difference in the way of annotation by various sign language researchers, it is also a discussion of 'what is perceived as being more relevant'. To solve this problem, investigations on the perception of the different head features are required.

${ }^{258}$ In this thesis 'structural negation' is distinguished from 'inherent negative context'. The first means the function of negating content by means of structure (cf. chapter 4), the latter may included different forms of semantic negation. In this sense someone may interpret 'being unaware' as a form of semantic negation.
} 
as an 'interrogative marker', but also as an 'irrealis marker'. Supporting the second function is the circumstance that the marker also occurs in 'hope-constructions' in which a questionable proposition is hoped. In these 'hope-constructions' the annotators indicate that the marker head forward primarily indicates the 'questionability' of the construction. The interrogative character of asking oneself a proposition is not perceived to be that relevant for them. The marker head forward is perceived to be performed in different intensities. One very common pattern is that the entire questionable proposition is covered by the marker; however, the part which is questioned is covered by an intensified forward movement of the head or additional by raised brows. If an embedded polar interrogative is present the element 'ob' ('whether') optionally precedes the questionable proposition. Also a reference to the 'hypothetical space' is present which means that the proposition precedes, starts with, or is covered by indicators that refer to the space of thoughts such as gaze directed upward or pointing upward. Further, epistemological concepts like knowledge or insecurity (expressed by signs and non-manual elements) are linked with the questionable proposition. So, in most cases such an expression of cognition, perception, or (doubtful) feeling precedes, follows or co-occurs with the questionable proposition. With regard to 'embeddedness' of the embedded polar interrogatives, the head marker also functions as a complementizer which (optionally) can cover the preceding or following expressions of cognition/feeling/perception such as being aware of something (KNOW), being unaware of something (KNOW-NO; mouth action 'stretched-down'; shoulder shrug), being insecure about something (INSECURE, INDECISIVE), and so forth.

With regard to embedded content interrogatives, the present data show that they are characterized by being covered by the interrogative markers chin up and/or head forward, including a wh-question-sign in clause-initial position, frequently being accompanied by looking upward or somewhere to the front, and being embedded in an expression of cognition, emotion or perception formulated within the main clause. What is special is that first, the head markers are the same markers as in direct content question, and second, the marker head forward can spread along the main clause. 
Finally, chapter 4 shows an interrogative head marker (head backward) which is different than all other interrogatives markers. When using this marker, the construction is provided with interrogativity and also with a notion of negation/denial. These constructions include in most instances a wh-question-sign, sometimes the sign SONST (OTHERWISE), both found in clause-initial position. In all instances in the data these interrogative constructions, covered by head backward, are related to a previously made statement. They are not aimed at gaining some information, rather expressing one’s denial attitude.

In chapter 5 I focus on conditional constructions which are also primarily coded by a head marker. The protasis (antecedent) is covered by positioning the head forward while the apodosis (consequent) does not receive a conditional marker. In addition, the data shows that other markers can co-occur when formulating the condition. The first is raised brows which constitutes an alternative marker or an additional marker to head forward. The second indicator is positioning the shoulder forward which is an additional marker to head forward. Moreover, the antecedent optionally can start with the sign IF; an assertive consequent optionally can begin with a head nod. To make clear that only the if-clause is covered by the conditional marker, in chapter 5 examples are presented in which the consequent constitutes an interrogative which is covered by the required interrogative marker.

The chapter also shows the interplay with other functions which are coded by 'head'. Two clauses of a conditional can be indicated by head tilt and/or body lean to the side of which each movement to one or the other side covers one clause. In doing so, each clause displays an alternative. Also an entire conditional clause can constitute one alternative (i.e. the entire conditional clause is covered by head tilt and/or body lean to the side) which is distinguished from another alternative. The second function which regularly occurs in conditional is expression of negation. In Table 5.1 all options which are present in the data are listed (cf. 5.6.1). On the one hand, this shows the co-occurrence possibilities of the conditional marker head forward and the negation marker headshakes. On the other hand, this demonstrates that both markers operate independently. For instance, the entire protasis of a conditional clause always has to be marked by the conditional marker independently 
of whether a part or the entire antecedent is covered by headshakes. Finally, the cooccurrence of other non-manual markers in conditionals are given and described with regard to their form and their possible function. As a result, I demonstrate that these cooccurring markers tend to occur in conditionals but they do not display 'additional conditional markers'. Their function is more likely located in the field of modality or negation. It is very likely that there is a connection between squinted eyes and possessing or lacking knowledge which refers to a questionable proposition. The second frequently co-occurring non-manual marker is wrinkled nose. This marker tends to co-occur in the context of negation, indicated by headshakes, or it occurs when content conveys a negative notion but is not indicated by headshakes. For example, this is caused by signs like CLOSED. The current interpretation is that this marker expresses on the one hand 'potentiality' of a proposition; on the other hand the signer's negative feeling concerning the outcome of the questionable proposition is expressed.

In Chapter 6 I described and argue that there is a set of head and body markers which are directed by the linguistic use of the signing space. They have in common that they are either oriented toward a specified/established location in the signing space, or they move toward that place, or they point - that is, they orient and move toward that place. Further, the use of the particular head and/or body indicator depends on the perspective. First, the perspective can be toward the specified/established location in the signing space. This is the case for the referential use of space which is indicated by head turn sideward and/or body turn sideward in ÖGS. Further indicators are gaze sideward and pointing to that space. Second, the perspective can be from a specified/established location in the signing space. This is the case in constructed dialogues. For instance, chin up and/or gaze up is used for representing the perspective of a child or chin down and/or gaze down is used to refer to the perspective of an adult toward a child (cf. Figure 2.11). Indicating height by both gaze direction and head position was first observed by Schalber (2006, 140 141) for ÖGS. I do not go into detail of this aspect in chapter 6 . Third, the perspective is governed by the signing space which functions as an independent, discrete medium. This means that a location in the signing space itself has a meaningful value. The described 
values are 'up', ‘front', ‘back', 'lateral-one-side’ and 'lateral-the-other-side’. In ÖGS the space conveying the value 'up' is used for the 'hypothetical space'. Indicating this location in the signing space provides the information that an informant is signing about his/her thoughts. The other values ('front' and 'back' as well as 'lateral-one-side' and 'lateral-theother-side') are used for indicating the alternative space. This is used in ÖGS when a signer wants to oppose/add/etc. two or more subject matters which can be facts, issues, circumstances, or activities.

All these 'spatial indicators' have in common that they refer to a location in the signing space and consequently, they can vary with regard to their articulators. For instance, the alternative space can be indicated by head and body movements sideward which are head tilt sideward, body lean sideward and step sideward. But other indicators can also refer to that alternative space like gaze directed to that location, pointing towards that location and even signing in a place that is closer to the particular alternative space. Which spatial cues or bundles of cues are used for indicating the particular alternative space, depends on various reasons. One discussed reason is that in lectures those spatial cues tend to be used which are more perceptible to the audience (like body lean sideward or step sideward). With regard to the 'space of thoughts' the indicators chin up, gaze up, pointing upward and the displacement of the signer's place of articulation to a higher location are the spatial cues used in ÖGS. All of the above indicators for the alternative and hypothetical space can occur as phrasal domain markers and the first three indicators of the hypothetical space can even occur as phrasal beginning markers.

In the final part of chapter 6 I describe head and/or body markers which are used for 'listing items'. In describing these markers I show the difference between 'spatial indicators' and 'non-spatial indicators'. When listing items each of them is marked by a forward movement of the head and/or body. This has nothing to do with a specified/established place in the signing space or with a discrete space in the signing space which governs the forward movement. To provide the forward movement of listing with the value 'front' is useless. This value 'front' only becomes of use if it is used for displaying alternatives such as for opposing the signs OPEN versus CLOSED (covered by 
a forward movement versus a backward movement), or if a special place in the signing space has been specified/established to which the forward movement refers.

In chapter 7 I describe a set of head and body markers, reported for the first time for sign language, which are used to code modality. They serve to mark propositional modality, that is, the signer's knowledge and/or degree of confidence of the truth value of a proposition can be shown by these possibilities: convinced-assertive marker, non-assertive maker, speculative marker, and timitive marker. In addition, a possibility marker has been identified, coding deontic modality. All modality markers co-occur with the entire utterance on which this modality meaning is intended.

With help of the 'convinced-assertive head marker' and the 'non-assertive head marker' the signers express their convinced/assertive or their negative judgment on the positive or negative outcome of a situation formulated within a thought proposition. The first marker has two formatives. First, these are fast, small head nodding movements which are preformed with a slightly forward rotating movement, or a slightly forward movement, or only with an up and down nodding movement (see Figure 7.1). The second implementation is slow, large head nodding movements which tend to be produced in a less regular way than the faster movement implementation. Elements which are frequently co-occurring with this convinced-assertive marker are furrowed brows and the sign SURE. The second marker is implemented by slow headshakes which tend to be performed in a smaller size. Compared to headshakes functioning as negator these headshakes are performed in a tentative way. If an utterance is covered by the non-assertive marker and a part in between is negated, exactly this negated part is covered by clearly distinctive negative headshakes and the parts before and afterwards are covered by the non-assertive marker. Other nonmanuals which tend to co-occur in the context of the non-assertive marker are squinted eyes and wrinkled nose.

The signers use the 'speculative body marker' for judging in a deliberative, insecure or doubtful way the truth value of a proposition. This marker is slow, more or less regular performed body sways. Co-occurring elements are expressions of insecurity or indecisiveness as well as expressions of knowledge or lack of knowledge. Body sways 
conveying the modality function are demarcated from body sways used to structure discourse and body sways used to indicate a steadily and uniformly performed forward motion. The side-to-side movement for structuring a discourse can vary the articulator which produces it. So, it can also be implemented by weight shifting sideward. The body sways indicating a steadily and uniformly performed forward motion are not pure side-toside movements; mostly they have an additional movement feature. For instance, these swaying movements tend to implement an alpha-shaped movement or an additional forward movement with each side movement.

For expressing the signer's concern/worry on the outcome of a situation formulated within a proposition (epistemic modality) or for expressing the fear on the fulfillment of an uncertain situation (event modality) a special marker has been identified in the data. The 'timitive head marker' is implemented by very small nodding or head forward movements, which are performed in a trembling way. In addition the body is completely tensed.

The last identified modality marker is a 'probability head marker'. Expressing potentiality on an unrealized event is implemented by tilting the head sideward and/or leaning the body sideward. The marker which also constitutes an alternative marker consequently offers the information 'maybe this or maybe that'. Tilting the head sideward also accompanies the signs VIELLEICHT (MAYBE) and MÖGLICH (POSSIBLE) and an ongoing head tilting sideward is interpreted as MAYBE.

\subsection{Generalizations on head and body markers}

After having summarized the identified functions and forms of head and body movements/positions, I address the hypotheses in the introduction (cf. 1.1). First, I show that the annotations of the data show that head markers are one of the most structured nonmanual markers in ÖGS. Second, I demonstrate that the various head and body movements/positions can be classified in groups based on their characteristics. Third, I show that some of the linguistic structures marked with the same non-manual marker feature a functional common ground which has semantic/pragmatic contiguity. Finally, I show the co-occurrence and sequential occurrence of head/body marker. 


\subsubsection{The 'head' as one of the most structured non-manual markers in ÖGS}

Having the various described constructions in mind, first of all it becomes obvious that a lot of information is coded by the articulator 'head'. Second, various linguistic functions are coded by several head movements/positions. Thus, functions like negation, assertion, interrogativity, irreality, conditionality, and modality (especially epistemic modality) are primarily coded by movements or positions of the head. Further, among other spatial cues coded by other articulators, the head is one of the articulators which is used for referring to the hypothetical space, displaying alternatives, or referring to a specified/established place in the signing space. Third, in the present thesis the described formatives of movements/positions of the head together with their functions could be clearly identified by the various annotators ${ }^{259}$. This includes the informant who signed the text as well as three/four other individuals. Fourth, the present ÖGS texts show that in functional contexts which require the discussed head movements/positions, these elements very regularly and consistently occur. For example, with regard to the interrogative/irrealis marker head forward (cf. 4.3.1.2.1) I show that in 35 of the 37 embedded polar interrogative constructions present in the data, the marker head forward could be clearly identified.

All these reasons suggest that the 'head' is one of the most systematically structured nonmanual articulators in Austrian Sign Language (ÖGS).

\subsubsection{Classification of head and body movements/positions}

In the present thesis various head and body movements/positions are described which all have in common that they are clearly identified by the annotators. However, when having a closer look at all these head and body movements/positions it turns out that subgroups of them convey the similar characteristics that differ from the other subgroups. Three classes could clearly be distinguished. Other classes of head and body movements/positions are also present (cf. 8.4) but since their forms, functions, co-occurrence and occurrence are not investigated in detail, no generalizations can be made here.

The classification of the three subclasses is based on

\footnotetext{
259 Other movements and positions have been perceived by the annotators too, but up to now their function and systematic occurrence could not be clearly determined (cf. 8.4).
} 
- the difference in the production with regard to its conformity/regularity of movement execution and/or

- the different units which are covered by the particular head/body movement/position and/or

- the dependency on other factors like space.

1. The first classified group constitutes several head and body movements/positions which form a clear form-function pair and cover syntactic constituents. These are head movements/positions which convey functions like negation, assertion, interrogativity, irreality, conditionality, or deontic modality ${ }^{260}$ (described in chapter 3, 4, 5 and 7). A distinct start and endpoint of the head movements/positions is perceived by the annotators. As will be seen in discussion of the third group, the first group differs in the 'uniformity' of the movement. The head movements of this group are very regularly performed. For instance, the two forms of negative headshakes are regularly performed shaking movements. They are unambiguously allocated to the particular constituent associated to them. Their clause negation function is clearly identified. Another example is the marker chin down in polar questions. This downward positioning is constantly held during the entire interrogative constituent. The interrogative function is clearly identified. A third example is the marker head tilt sideward. This clearly identifiable tilting position of the head is maintained during the entire constituent associated to it. In using this marker the probability of the realization of an event is expressed. One very significant observation is that in the data the signers always perform these movements with the articulator 'head'. This differs from the movements/positions of the second class which can vary the articulator under special conditions. Another aspect of movements/positions of the first class is that these head movements/positions are more 'resistant' compared to those of the third class. For instance, an informant signs that a situation (formulated within a proposition) will more likely not be the case. This feeling of unease can be expressed by the non-assertive

\footnotetext{
${ }^{260}$ The thus far identified modality marker which falls within this class is the marker head tilt sideward which codes the possibility of the realization of an event.
} 
head marker (an epistemic modality marker). If within this proposition which is covered by slow, small, tentative headshakes a part is negated, the negative headshakes clearly 'override' the modality headshakes during this negated part. This observation leads to the conclusion that this first class of head movements are language elements that possess functions which are more relevant for the language structure and first have to be expressed compared to elements of the third class.

2. The second classified group includes those head and body elements which depend on the signing space. A subgroup of this classified group are head and body movements which are directed by specified/established spaces in the signing space. Another subgroup are head and body movements that are governed by the space itself which function as 'discrete medium' conveying values like 'up' or 'lateral'. The head and body movements of the latter subclass - on which I have focused in the present thesis tend to occur as phrasal domain markers, sometimes as phrasal beginning markers. As described above, the most significant aspect is that these head and body movements can vary the articulator as a result of various reasons. For instance, in a sitting position the signers tend to mark alternatives by tilting the head to one side for the first option and tilting the head to the other side for marking the second option. In standing position the sideward movement is performed by the body. So the signer leans to the side successively to both sides - for indicating the particular alternatives.

3. The third classified group comprises head and body movements which are also clearly identified through their form(s) and function by the annotators. The data of the various informants shows that the head and body movements are less uniformly performed (than those of the first group) but the particular movement execution is perceived as relevant for a specific utterance. This means that the annotators could make out a distinct beginning and endpoint of these head and body movements. This classified group is a set of non-manual markers used to code 'epistemic modality' (cf. chapter 7). They serve to mark propositional modality, that is, the signer's knowledge and/or degree of confidence of the truth value of a proposition is expressed. This 
judgment can be expressed in a convinced-assertive, a non-assertive, a doubtful or deliberative, or in a trembling way.

\subsubsection{Semantic contiguity}

When analyzing the several functions of the identified head and body indicators, three interesting observations on patterns arise.

- First, some of the constructions are marked with the same non-manual marker. The interesting aspect concerning this matter is that these constructions and the functions of the indicating markers seem to have a semantic/pragmatic contiguity as will be illustrated below.

- The second interesting aspect is that some of the head / body indicators are movements/positions which are performed along a body axis but to the opposite side. It is obvious that the resulting two indicators mostly mark functions which are 'related' to each other.

- Third, the data shows that some language structures convey similar 'constructions', that is, the sequential and simultaneous composition of the manual and non-manual elements of different constructions (e.g. interrogatives and conditionals) convey similarities for both the semantic and the formative (primarily syntactic) aspect.

With regard to the first pattern the Deaf individuals' annotations of the data show that they have identified some head and body positions/movements which have the same form but which differ in their functional use. Two of these indicators I want to focus on in the following are the marker head forward and the marker head tilt sideward. Both markers are described by the annotators as 'position markers', that is, the annotators perceive that the signer is positioning the head forward or tilting sideward. They distinguish the 'position markers' from the 'movement indicators' head forward which is perceived as moving the head forward and backward or tilting the head sideward and back to the neutral position ${ }^{261}$.

\footnotetext{
${ }^{261}$ To find out the correlation between what is perceived as 'movement' and what is perceived as 'position' further investigations are required.
} 
In order to avoid a wrong interpretation due to a possible movement-position-difference, I only take for discussion here those head forward / head tilt sideward markers which are described as positioning the head forward or tilting the head sideward (for a while) by the annotators. So, the forward movement of the head used for 'emphasizing' or for 'itemizing' is excluded in my discussion here. Further, the marker head forward used as alternative marker in a semantic pair OPEN versus CLOSED is excluded too as the forward movement is governed by the signing space. With regard to head tilting movements, ongoing head tilting interpreted as maybe as well as head tilting due to spatial dependency (e.g. for displaying an alternative space) are not taken into consideration in my discussion here.

The first marker head forward is used by the various ÖGS-signers for indicating interrogativity (in direct and embedded content questions), irreality (in embedded polar interrogative constructions but also in 'hope-constructions'), conditionality (in conditionals), and exclamation (in pure exclamation constructions and in interrogatives which are expressed in an exclamative way). Moreover, the possibility head marker head tilt sideward tends to include a forward positioning of the head too.

The second marker head tilt sideward is used in the data for indicating possibility. This can refer to the realization of an event in the sense of 'probably' or 'estimated' something is possible. The data further shows that head tilt sideward can express politeness (occurring in polar and content questions).

However, one finding of the preliminary study conducted by Lackner \& Stalzer (2010) (cf. 2.2.1) shows that there probably is a difference. The presented video material contains examples in which in the first round the first two signs are covered by positioning the head forward; afterward, the same signs are covered by moving the head forward. Half of the participants who watched the examples figured out that the first head marker is a conditional marker; the second indicates that the two signs are 'emphasized'. The participants even described that the forward movement of the first marker goes quickly forward (during the first sign) for indicating the conditional marker while the forward movement goes forward later (and has its peak on the second sign) when head forward is used for 'emphasizing the two signs'. But half of the participants could not figure out a difference with regard to the form. They only could make out the functional difference due to the context. To clarify this, an investigation on the perception of movement/position correlation with domain of one sign versus more than one sign is needed. 
In the following the functions of these two head markers are illustrated by a semantic map $^{262}$. To provide an overview, all described head markers which fall within the first classified group are included in the illustration. Thereby, I aim to show the various functions which are coded by the same and different head markers from a synchronic perspective.

In advance, I have to mention that - as described in the different chapters - the listed functions coded by the illustrated head markers do not illustrate the entire possibilities of coding the particular function. So, it has to be kept in mind that alternative or additional markers are present in ÖGS which can express this function too or add some information to the expressed function. For instance, the conditional marker head forward can be replaced by the alternative marker raised brows and sometimes by the alternative marker chin down. It can be complemented by additional means such as moving the shoulders forward. Furthermore, the illustrated head markers do not show all functions which can be coded by the articulator 'head'.

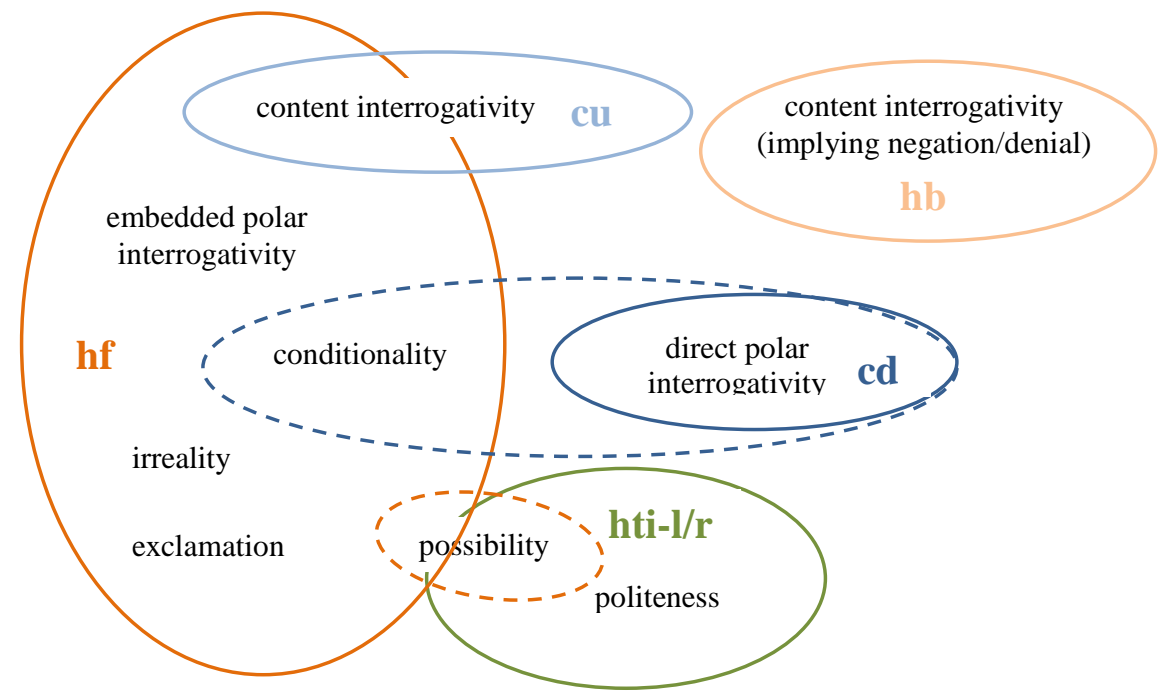

Figure 8.1 Semantic mapping of head markers in ÖGS ${ }^{263}$

\footnotetext{
${ }^{262}$ An introduction on the use of semantic maps is given by Haspelmath (2000).

263 Abbreviations: hf - head forward, hb - head backward, cu - chin up, cd - chin down, hti-l/r - head tilt to the right or to the left;
} 
At a first glance it is obvious that the various functions of the above-mentioned head markers head forward and head tilt sideward have a semantic/pragmatic contiguity. With regard to the marker head forward four (eventually five) listed functions can be expressed. Summed up, the properties of these functions can be described as follows: Content interrogativity includes that a signer/speaker demands information of a counterpart in order to complete a proposition (cf. Siemund 2001, 1018). Following Michaelis (2001, 10391041) the concept of exclamation/exclamative conveys the speaker's perspective and his/her judgment on a situation that is not canonical; especially exclamatives express propositional content which is thought to be true. They function as speech acts. As described in chapter 5 (5.4), conditionality ${ }^{264}$ comprises hypotheticality and a degree of potentiality of the realization of a proposition as well as causality. Irreality as it is expressed in the present data with the marker head forward and described in chapter 4 (cf. 4.3.1) includes that alternatives are offered within propositions, which have not yet been realized, but which could be realized. Consequently, a notion of hypotheticality and potentiality is included. This is based on the signer's lack of knowledge or insecurity of judgment on that unrealized proposition. Going through the summarized semantic properties of these functions makes clear that all these functions have common aspects which they - all or a part of them - share such as referring to a proposition or expressing a judgment.

A similar observation can be applied to the functions which are coded by tilting the head to the left or to the right. As described in chapter 7 (cf. 7.4.6), possibility coded by head tilt to the side expresses an event modality, that is, the potentiality/possibility of an unrealized event is coded. Following Blum-Kulka (1987) using modality has the side effect that a speaker (here signer) is less definite. This effect of losing directness is also achieved by formulating questions. Both means are used in several languages to express politeness (for an overview on literature on politeness see Fraser 2001). Thus, tilting the head to the side in the context of a question displays an honorific element in ÖGS texts. Concluding, tilting the head sideward can be described as a head marker which possesses these two functions,

\footnotetext{
${ }^{264}$ I focus on conditionality which is expressed by the marker 'head forward' in 'hypothetical conditionals' in the ÖGS data.
} 
or it only conveys the function 'potentiality' which, embedded in questions, evokes less directness and thus, implies a notion of politeness. Interestingly, optionally the head moves forward too, when using head tilt as possibility markers. And indeed, it is obvious that there is a semantic contiguity (for instance with regard to hypotheticality) with at least some of the functions indicated by head forward.

The second interesting aspect is that some pairs of the markers which constitute movements to the opposite sides along a body plane have in common that they display 'related' functions. As illustrated in Figure 8.1 the marker chin down is used for polar questions while the marker chin up is used for content questions. Content questions can be marked by positioning the head forward too, while interrogatives which include a wh-sign or the sign SONST (OTHERWISE) and which imply negation/denial are marked by head backward. Further, head nods displaying assertion and headshakes functioning as negator also have to be kept in mind since both are shaking/nodding movements which differ in that they are performed along different body planes $^{265}$.

The third identified aspect is that some linguistic structures convey similar 'constructions', that is, the sequential and simultaneous composition of the manual and non-manual elements of different constructions show/imply similarities with regard to semantics and formatives. This phenomenon is clearly present in the comparison of the implementation of question-answer-sequences and antecedent-consequent-sequences of conditionals as exemplified in the following. A lecturer wants to show the audience how modality can be expressed in ÖGS. In doing so, the signer first formulates an antecedent-consequentsequence displaying a conditional (Figure 8.2 and example (145)) and subsequently a question-answer-sequence (Figure 8.3 and example (146)) which is also interpreted as conditional by the annotators.

\footnotetext{
${ }^{265}$ Of course, these two markers can have further differences like the size or speed of shaking/nodding movement.
} 

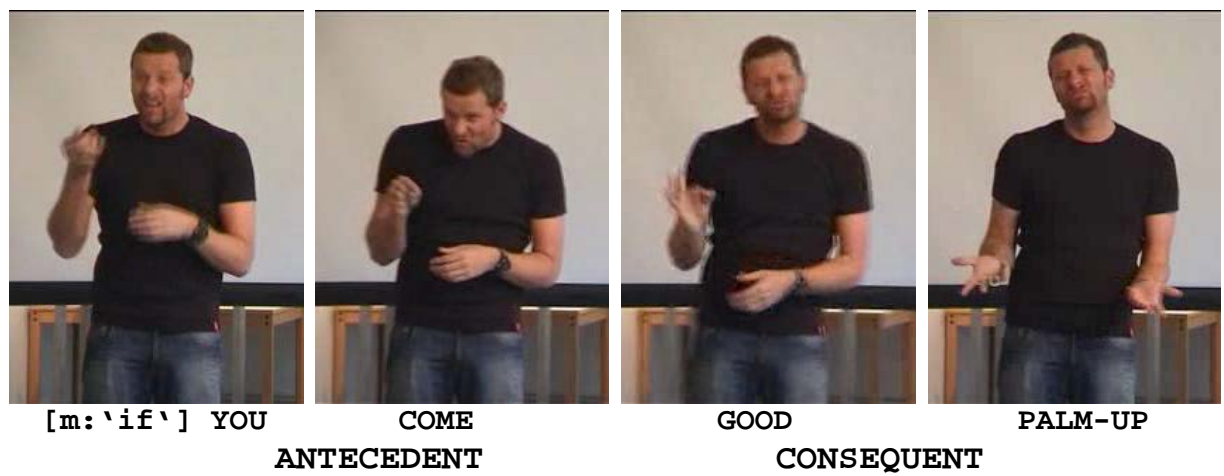

Figure 8.2 Antecedent-consequent-sequence (illustrating example (145))
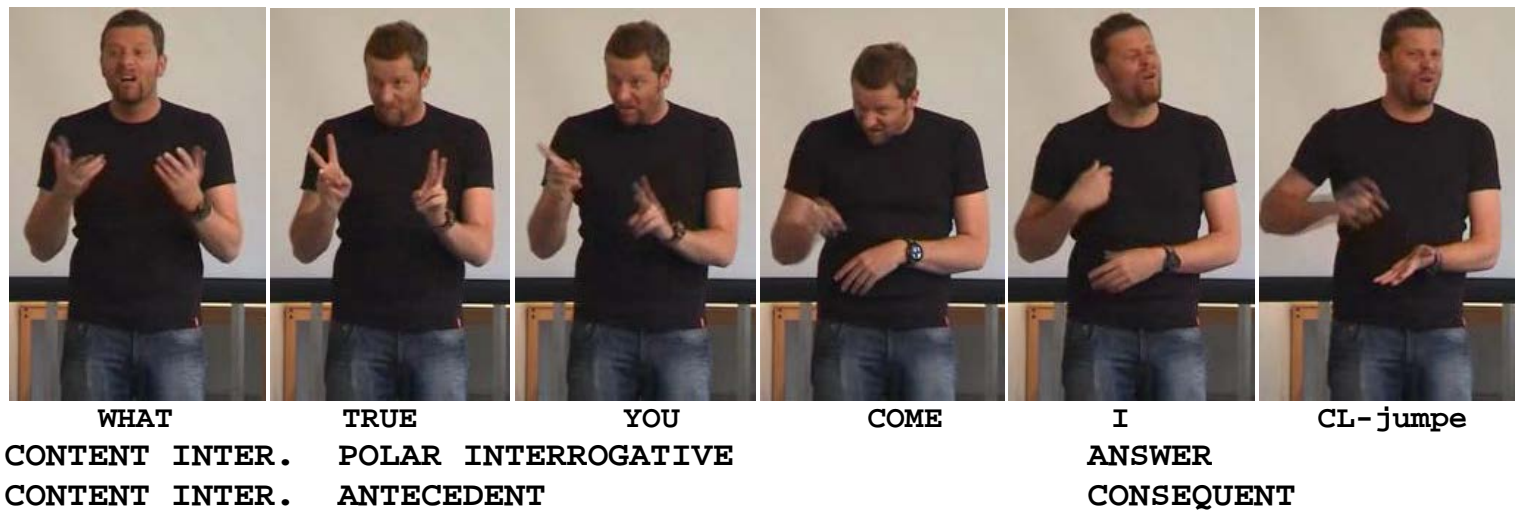

Figure 8.3 Question-answer-sequence or antecedent-consequent-sequence (illustrating example (146))

(145)

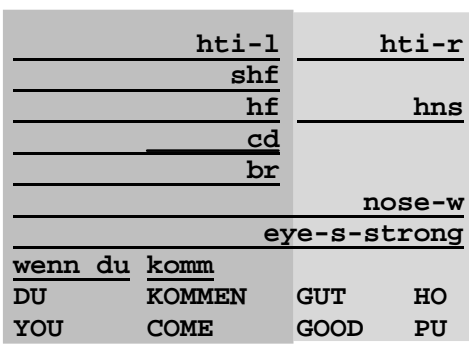

Wäre schön, wenn du kommen würdest. It would be nice, if you came.

(146)

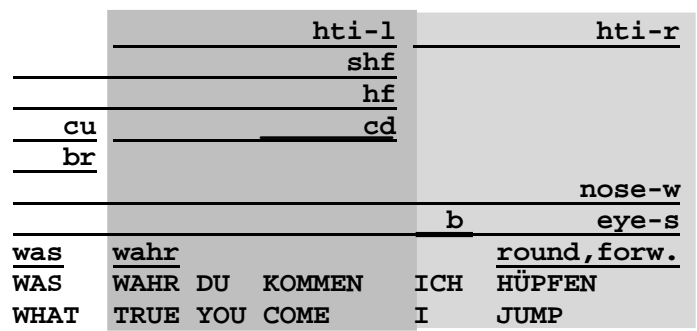

Also, du kommst wirklich? Da würde ich mich freuen. So, you really come? I'd be very happy. OR Also, wenn du kommst, würde ich mich sehr freuen. So, if you come, I would be very happy.

(M007_04.05.08_edu.course_part01_11.28-11.31)(M007_04.05.08_edu.course_part01_11.40-11.43)

Comparing both figures, it becomes obvious that similar non-manual markers are present in the if-clause and the polar question. Both are covered by positioning the chin down. This downward positioning is intensified during the last sign of the antecedent and the polar 
question (illustrated by the bold underline) ${ }^{266}$. Further, both constructions are accompanied by head forward and shoulder forward. Only in the if-clause are further non-manuals perceived or are perceived to be performed stronger. This refers to the markers raised brows, squinted eyes, and wrinkled nosed. In addition, in both constructions the sequential constituents are accompanied by tilting the head subsequently to the different sides. With regard to the semantic component of both constructions it becomes obvious that a strong semantic contiguity is present with regard to the properties potentiality (modality) and interrogativity (so, asking for the possibility).

\subsubsection{Co-occurrence and sequential occurrence of head/body marker}

Different head markers (and body markers) can co-occur as shown in the following: $(147)^{267}$

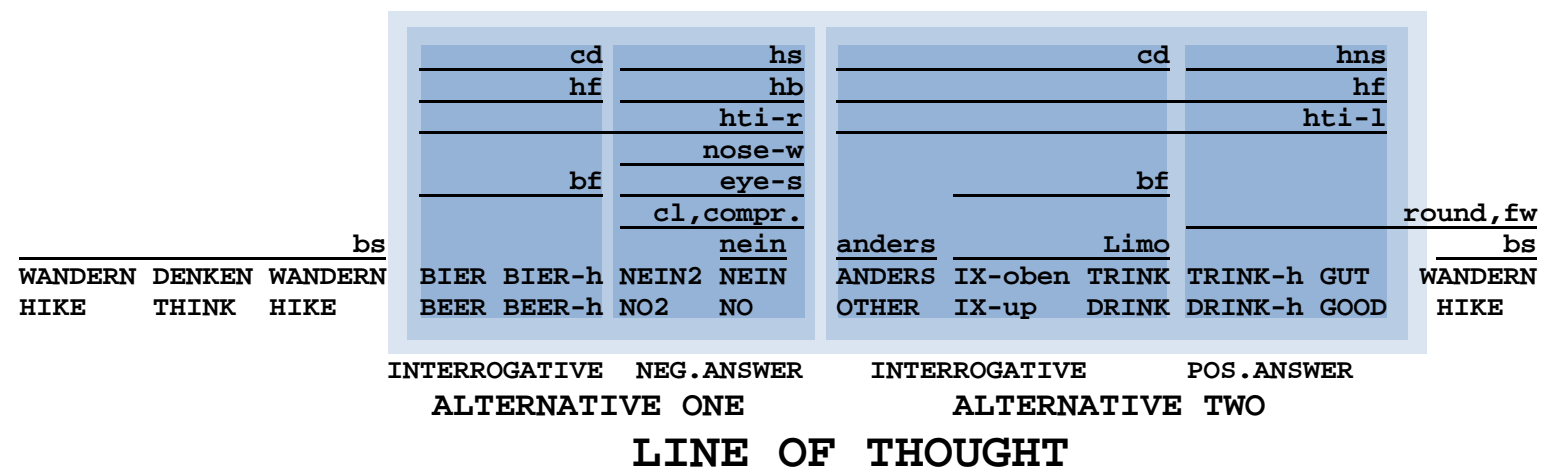

Während ich wandere überlege ich, ob ich ein Bier trinken soll. Ich denke nein, lieber etwas anderes - ja eher eine Limonade.

While I am hiking I think whether I should drink a beer. I think no, better something else - yes, better a lemonade.

(F004_117,1209_m_thoughts_03.26-03.37)

This example shows that as many as three head markers can co-occur such as chin down together with head forward and head tilt sideward, or headshakes together with head backward and head tilt sideward, or head nods together with head forward and head tilt sideward'. Also, (147) makes it clear that if head (and body) markers co-occur, some of them tend to co-occur based on their meaning/function. So, the data shows that headshakes

\footnotetext{
266 The ÖGS data shows that signs of existence and arrival are constantly accompanied by a downward movement of the head. This is very likely one of the main reasons for the intensification of the downward movement of the marker 'chin down' in both examples.

${ }^{267}$ Example (147) has been previously described by example (5) in chapter 2.
} 
and head backward tend to co-occur with each other while head nods and head forward more frequently co-occur. To go into detail on this, further investigations on the cooccurrence of different non-manuals in ÖGS are required.

The sequential occurrence of head (and body) markers has been demonstrated in various examples in the present thesis. The sequential occurrence of the same head marker is implemented by the various signers in two different ways. To begin with, the marker is produced once and maintained during the subsequent construction which requires the same indicator. A pause optionally intervenes between the two constructions. The second option is that the first construction is indicated by a regular performance of the marker, the subsequently following construction is covered by an intensified performance of the same marker. Both options are exemplified in (148) and (149) by the marker head forward.

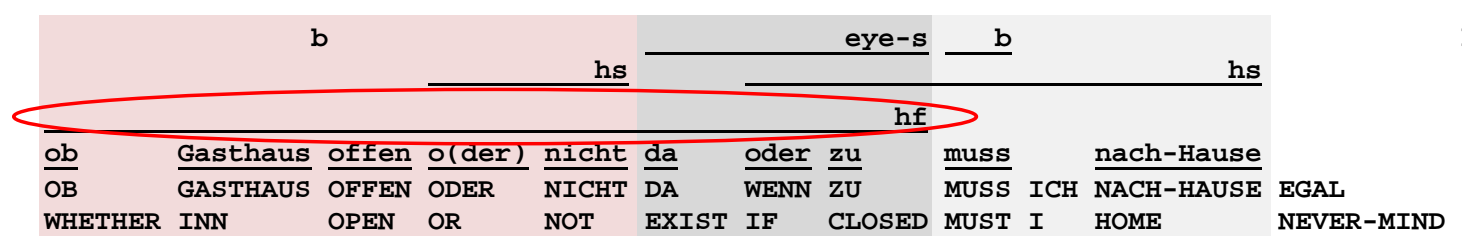

Ich überlege, ob das Gasthaus geöffnet hat oder nicht. Wenn es dort geschlossen ist, muss ich nach Hause. Macht nichts.

I wonder whether the inn is open or not. If it is closed, I'll have to go home. Never mind.

(M003_116,1198_m_thoughts_ex09_03.06-03.17)

$(149)^{268}$

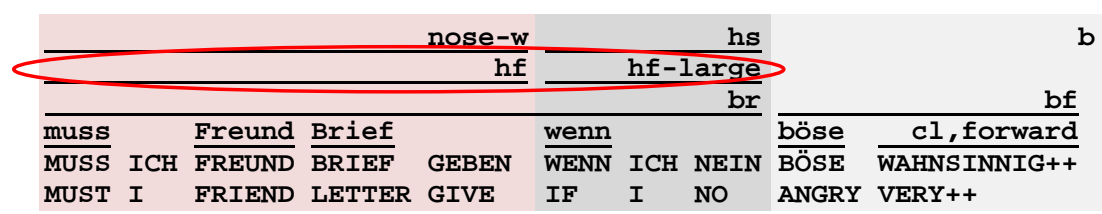

Ich überlege, dass ich meinem Freund den Brief geben muss. Falls ich das vergesse, wird er wahnsinnig böse auf mich sein.

I think that I have to give the letter to my friend. If I forget that, he will be very angry with me.

(F001_044_m_thoughts_00.01-00.07)

In the examples (148) and (149), two successively occurring constructions are indicated by positioning the head forward. In (148), the marker functions first as interrogative/irrealis

268 The signer alternates the active hand when signing I-GIVE-he and continues with this hand signing what happens if a letter is not handed to that person. 
marker, then as conditional marker. In (149), it is vice versa. In example (148), both the embedded polar construction and the protasis of the conditional clause are covered by the marker head forward. The marker is only produced once, that is, the marker is maintained during the second construction as well. In example (149), the interrogative/irrealis construction is covered by head forward. The successive conditional construction is covered by an intensified forward positioning of the head. Probably the intensified forward positioning provides some extra information, for instance, increasing the imperative character of the request. In example (149), this would mean that the signer herself desperately has to keep in mind that she has to hand the letter over to him. Both options (maintaining the marker or intensifying the marker during the subsequently following construction) are present in the data. The clear differences with regard to a regular and intensified execution of one of the two subsequently produced markers need further investigation.

The detailed description of several functions in ÖGS which are indicated by various head and body movements/positions as well as the presented classification and generalizations on head and body movements/positions in ÖGS show that further research on this topic is still required. In the following, I list some aspects which have been noticed by myself and to which future investigations will be addressed.

\subsection{Future research}

To begin with, further head and body movements/positions have been identified by the annotators which have not yet been investigated in detail. For instance, one of these head positions is head tilt sideward. This frequently occurs with signs of cognition (like KNOW or HOPE) which have their place of articulation on the side of the temple. This tilting movement not only accompanies the sign of cognition, it co-occurs with the entire constituent which is governed by that verb of cognition. Further, in some instances the tilting position is maintained during the proposition which is embedded by the sign of cognition. More detailed investigation of these head and body movements are required to make generalizations. 
Second, the annotators identified several head and body movements to which they could not ascribe a clear meaning/function. Within an ongoing project on segmentation and structuring of ÖGS-texts (cf. 2.1.2) several of these head and body movements have been ascribed a segmentation function. These results date from a pilot study (cf. Mallinger 2013, Lackner et al. in prep., Wiener et al. 2012) in which informants had to segment a signed text. Subsequently, they had to identify the cues of the particular segmenting boundaries. Thus, on this topic further results will be determined in the near future.

Other head and body movements have also been identified as language-relevant elements by the various annotators. Their meaning/functions have not been clearly identified nor investigated, but some of them seem to possess a 'rhythmical' or 'discourse-structuring' function. One of these movement elements is the following:

As illustrated in Figure 8.4, a signer constantly nods by moving the head downward and upward (partly the body too). These head movements are implemented in a slightly downward to upward rotating movement. The first nodding movements have an assertive meaning, but the signer keeps on performing these head movements. The viewer gets the impression that the narrated text gets a kind of 'rhythmical beat'. This 'beat' changes with the sign HIKE (which follows the last illustrated sign). The signer positions the head forward (especially the chin) and performs very small head forward and upward rotations. These small movements are interpreted to display the 'regularity and constancy' of the ongoing action (here HIKE). These small movements perfectly connect to the preceding head movements, only the size differs. In the following the constantly head nods are illustrated: 


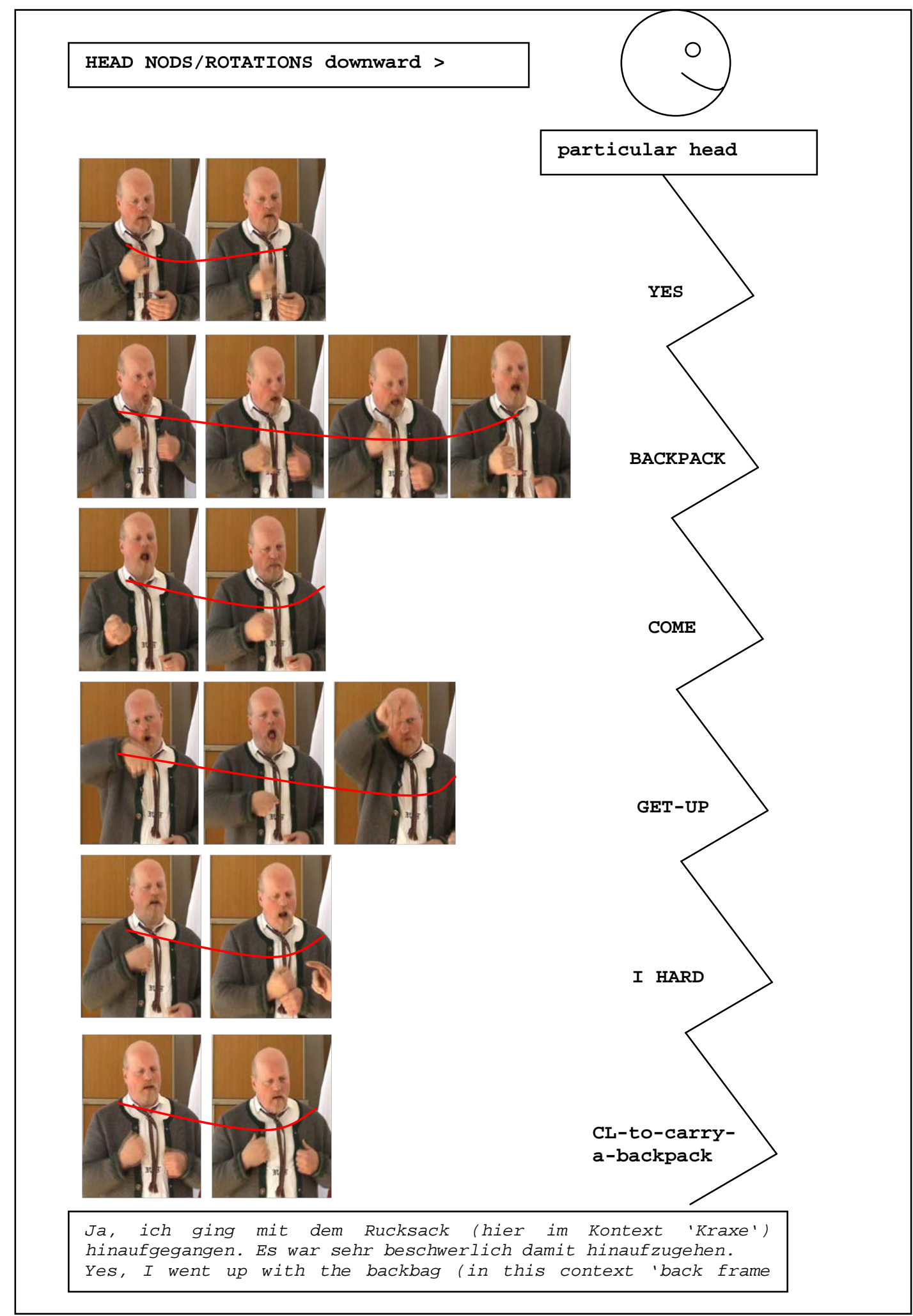

Figure 8.4 Ongoing head nods/rotations downward to upward 
Third, the thus far conducted investigations on head and body movements show that it is a must to keep movements of different articulators in mind in order to make clear statements on a phenomenon and on its functions, respectively. This correlation is demonstrated by the following Figure 8.5.

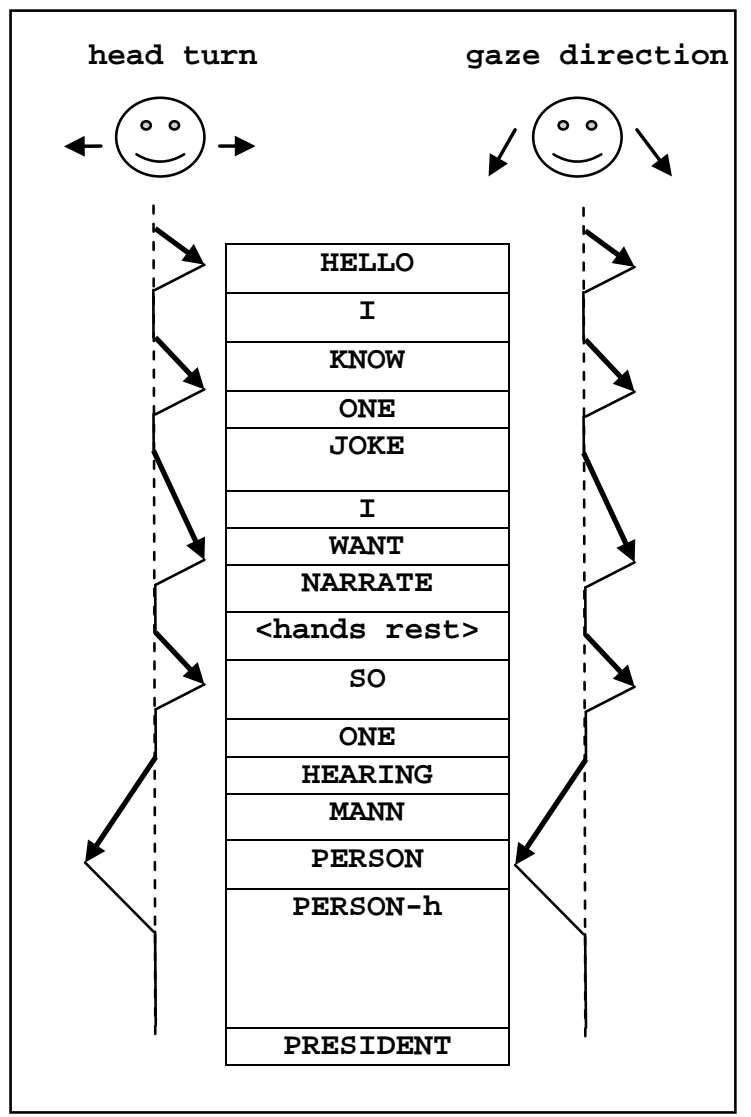

CORRELATION OF HEAD TURN

AND GAZE DIRECTION
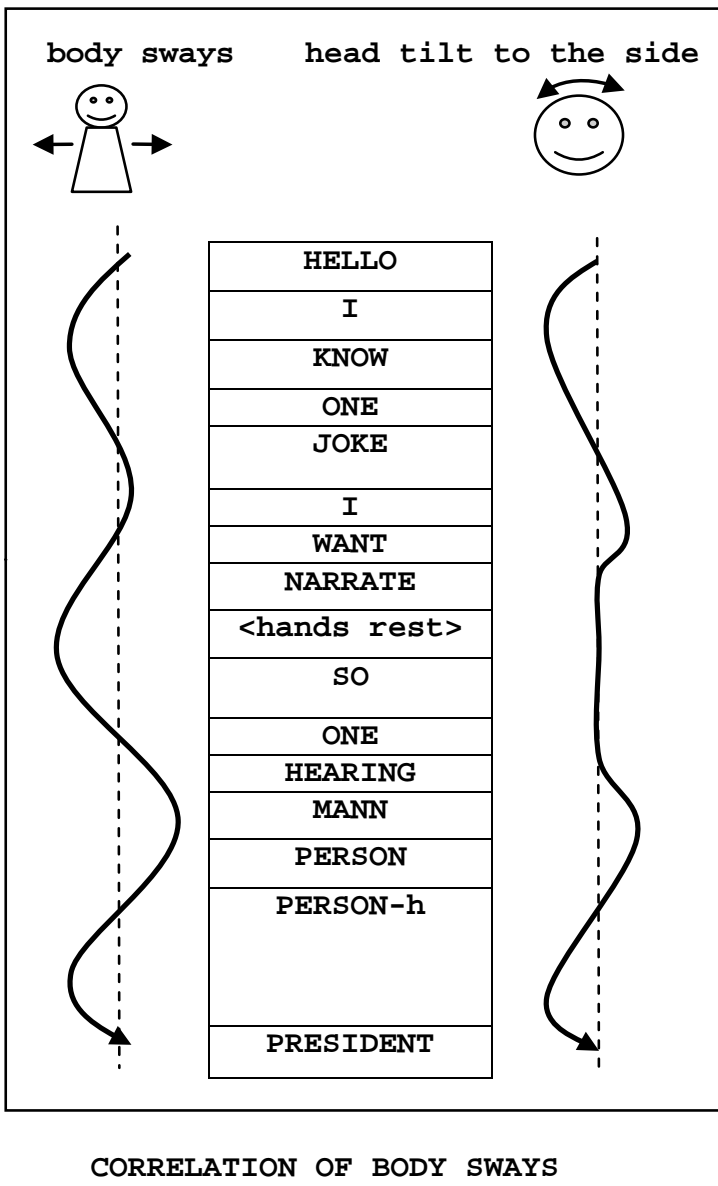

AND HEAD TILTS TO THE SIDE

Figure 8.5 Correlation of movements of different articulators

Both illustrations show that movements of different articulators correlate. In the first illustration the head turn direction obviously correlates with the gaze direction; in the second illustration the body sways correlate with the head tilts. Whether each of these cooccurring elements has its own function, an additional function, an alternative function, or 
the bundled elements together have a function, whether they possess a language-relevant or an interactive function requires further investigations.

In conclusion, there are various other head and body movements in Austrian Sign Language which 'are only waiting to be discovered'. 


\section{APPENDIX A: Corpora}

Corpus 1

\begin{tabular}{|l|l|}
\hline defined as & basilectal, dialogue-based corpus \\
\hline $\begin{array}{l}\text { organization } \\
\text { of text }\end{array}$ & dialogues \\
\hline type of text & informal stories, interactive exchange \\
\hline $\begin{array}{l}\text { content of } \\
\text { text }\end{array}$ & $\begin{array}{l}\text { various, free-chosen topics of former or daily experiences (frequently } \\
\text { very personal experiences) }\end{array}$ \\
\hline variety of SL & Pongau-Salzburg variety of ÖGS \\
\hline recorded & 2005 \\
\hline annotated & $\begin{array}{l}2006 \text { by the present thesis writer and various Deaf annotators from } \\
\text { Pongau (a region of the federal state of Salzburg) }\end{array}$ \\
\hline resource & electronic resource \\
\hline access rights & Lackner Andrea \\
\hline
\end{tabular}

Corpus 2

\begin{tabular}{|l|l|}
\hline defined as & educational training course corpus \\
\hline $\begin{array}{l}\text { organization } \\
\text { of text }\end{array}$ & $\begin{array}{l}\text { lectures show: teacher-centered teaching (monologues) and teaching } \\
\text { in an interactive mode (dialogues or exchange within a group) }\end{array}$ \\
\hline type of text & conducted analyzed so far: definitional structures \\
\hline $\begin{array}{l}\text { content of } \\
\text { text }\end{array}$ & $\begin{array}{l}\text { conducted analyzed so far: introductions of linguistic terminology } \\
\text { relevant for spoken and signed languages }\end{array}$ \\
\hline variety of SL & Styrian and Viennese variety of ÖGS \\
\hline recorded & 2008 \\
\hline annotated & $\begin{array}{l}2008 \text { and 2009 by the present thesis writer and Deaf annotators from } \\
\text { Styria and Vienna }\end{array}$ \\
\hline resource & electronic resource \\
\hline access rights & Lackner Andrea (of annotations) \\
\hline
\end{tabular}

Corpus 3

\begin{tabular}{|l|l|}
\hline defined as & corpus containing trains of thoughts \\
\hline $\begin{array}{l}\text { organization } \\
\text { of text }\end{array}$ & monologues \\
\hline type of text & trains of thoughts formulated within a short story \\
\hline $\begin{array}{l}\text { content of } \\
\text { text }\end{array}$ & hypothetical thoughts embedded in a short story \\
\hline variety of SL & Pongau-Salzburg variety of ÖGS \\
\hline recorded & 2010 \\
\hline annotated & $\begin{array}{l}2011 \text { by various Deaf annotators from Pongau (a region of the federal } \\
\text { state of Salzburg) }\end{array}$ \\
\hline resource & electronic resource \\
\hline access rights & Lackner Andrea \\
\hline
\end{tabular}


Corpus 4

\begin{tabular}{|l|l|}
\hline defined as & basilectal, monologue-based corpus \\
\hline $\begin{array}{l}\text { organization } \\
\text { of text }\end{array}$ & primarily monologues \\
\hline type of text & informal and formal stories \\
\hline $\begin{array}{l}\text { content of } \\
\text { text }\end{array}$ & $\begin{array}{l}\text { narrations of former or daily experiences (informal stories); jokes and } \\
\text { curricula vitae (formal stories) }\end{array}$ \\
\hline variety of SL & Pongau-Salzburg variety of ÖGS \\
\hline recorded & 2010 \\
\hline annotated & $\begin{array}{l}\text { since } 2012 \text { the corpus has been annotated by sign language } \\
\text { interpreter students and in addition by two Deaf individuals via video }\end{array}$ \\
\hline resource & electronic resource \\
\hline access rights & Lackner Andrea (responsible for the access rights) \\
\hline
\end{tabular}

Corpus 5

\begin{tabular}{|l|l|}
\hline defined as & clarification corpus containing trains of thoughts \\
\hline $\begin{array}{l}\text { organization } \\
\text { of text }\end{array}$ & monologues \\
\hline type of text & short stories containing trains of thoughts \\
\hline $\begin{array}{l}\text { content of } \\
\text { text }\end{array}$ & hypothetical thoughts embedded in a short story \\
\hline variety of SL & Pongau-Salzburg variety of ÖGS \\
\hline recorded & 2010 \\
\hline annotated & $\begin{array}{l}2011 \text { by various Deaf annotators from Pongau (region situated in the } \\
\text { federal state of Salzburg) as well as by sign language interpreter } \\
\text { students and in addition by two Deaf individuals via video since 2012 }\end{array}$ \\
\hline resource & electronic resource \\
\hline access rights & Lackner Andrea \\
\hline
\end{tabular}




\section{APPENDIX B: Metadata of the participants from Großarl ${ }^{269}$}

Demographic data regarding the valley 'Großarl', deafness, and education

\begin{tabular}{|c|c|c|c|c|c|}
\hline $\begin{array}{l}\text { Infor- } \\
\text { mant }\end{array}$ & $\begin{array}{l}\text { Decade } \\
\text { of year } \\
\text { of birth }\end{array}$ & $\begin{array}{l}\text { Relation to the valley } \\
\text { 'Großarl' }\end{array}$ & $\begin{array}{l}\text { Deaf } \\
\text { since }\end{array}$ & $\begin{array}{l}\text { Deafness in } \\
\text { family }\end{array}$ & $\begin{array}{l}\text { Elementary } \\
\text { education }\end{array}$ \\
\hline A & $50 \mathrm{~s}$ & $\begin{array}{l}\text { grew up and lives in } \\
\text { Hüttschlag (smaller village } \\
\text { of the valley Großarl) }\end{array}$ & birth & $\begin{array}{l}\text { Deaf parents, } \\
\text { Deaf sibling }\end{array}$ & $\begin{array}{l}\text { boarding school for } \\
\text { the Deaf }\left(1^{\text {st }}-9^{\text {th }}\right. \\
\text { grade })\end{array}$ \\
\hline B & $40 \mathrm{~s}$ & $\begin{array}{l}\text { married to a person who grew } \\
\text { up in the valley Großarl }\end{array}$ & age 2 & $\begin{array}{l}\text { married to a } \\
\text { Deaf person }\end{array}$ & $\begin{array}{l}\text { boarding school for } \\
\text { the Deaf }\left(1^{\text {st }}-8^{\text {th }}\right. \\
\text { grade })\end{array}$ \\
\hline C & $50 \mathrm{~s}$ & $\begin{array}{l}\text { grew up and lives in Großarl } \\
\text { (bigger village in the valley } \\
\text { Großarl) }\end{array}$ & age 3 & $\begin{array}{l}\text { married to Deaf } \\
\text { person }\end{array}$ & $\begin{array}{l}\text { boarding school for } \\
\text { the Deaf }\left(1^{\text {st }}-9^{\text {th }}\right. \\
\text { grade })\end{array}$ \\
\hline D & $30 \mathrm{~s}$ & $\begin{array}{l}\text { grew up and still lives in } \\
\text { Hüttschlag (smaller village in } \\
\text { the valley Großarl) }\end{array}$ & birth & $\begin{array}{l}\text { married to a } \\
\text { Deaf person }\end{array}$ & $\begin{array}{l}\text { boarding school for } \\
\text { the Deaf }\left(5^{\text {th }}-9^{\text {th }}\right. \\
\text { grade })\end{array}$ \\
\hline $\mathbf{E}$ & $60 \mathrm{~s}$ & $\begin{array}{l}\text { grew up in Großarl (larger } \\
\text { village in the valley Großarl), } \\
\text { lives in Hüttschlag (smaller } \\
\text { village in the valley Großarl) }\end{array}$ & birth & $\begin{array}{l}\text { Deaf sibling; } \\
\text { married to a } \\
\text { Deaf person }\end{array}$ & $\begin{array}{l}\text { boarding school for } \\
\text { the Deaf }\left(1^{\text {st }}-9^{\text {th }}\right. \\
\text { grade })\end{array}$ \\
\hline $\mathbf{F}$ & $40 \mathrm{~s}$ & $\begin{array}{l}\text { grew up and lives in Großarl } \\
\text { (bigger village in the valley } \\
\text { Großarl) }\end{array}$ & birth & $\begin{array}{l}\text { three Deaf } \\
\text { siblings; married } \\
\text { to a Deaf person }\end{array}$ & $\begin{array}{l}\text { boarding school for } \\
\text { the Deaf }\left(1^{\text {st }}-8^{\text {th }}\right. \\
\text { grade })\end{array}$ \\
\hline G & $50 \mathrm{~s}$ & $\begin{array}{l}\text { grew up in Hüttschlag } \\
\text { (smaller village in the valley } \\
\text { Großarl) }\end{array}$ & birth & $\begin{array}{l}\text { Deaf parents, } \\
\text { Deaf sibling; } \\
\text { married to a } \\
\text { Deaf person }\end{array}$ & $\begin{array}{l}\text { boarding school for } \\
\text { the Deaf }\left(1^{\text {st }}-9^{\text {th }}\right. \\
\text { grade })\end{array}$ \\
\hline $\mathbf{H}$ & $60 \mathrm{~s}$ & $\begin{array}{l}\text { close contact to Deaf } \\
\text { inhabitants of the valley } \\
\text { Großarl (lives in Pongau) }\end{array}$ & birth & $\begin{array}{l}\text { married to a } \\
\text { Deaf person }\end{array}$ & $\begin{array}{l}\text { boarding school for } \\
\text { the Deaf }\left(1^{\text {st }}-9^{\text {th }}\right. \\
\text { grade })\end{array}$ \\
\hline I & $40 \mathrm{~s}$ & $\begin{array}{l}\text { lives in Großarl (bigger } \\
\text { village in the valley Großarl) }\end{array}$ & birth & $\begin{array}{l}\text { Deaf sibling; } \\
\text { married to a } \\
\text { Deaf person }\end{array}$ & $\begin{array}{l}\text { boarding school for } \\
\text { the Deaf }\left(1^{\text {st }}-9^{\text {th }}\right. \\
\text { grade })\end{array}$ \\
\hline $\mathbf{J}$ & $70 \mathrm{~s}$ & $\begin{array}{l}\text { lives (in the city) next to the } \\
\text { valley Großarl }\end{array}$ & birth & not specified & $\begin{array}{l}\text { boarding school for } \\
\text { the Deaf }\left(1^{\text {st }}-9^{\text {th }}\right. \\
\text { grade })\end{array}$ \\
\hline
\end{tabular}

${ }^{269}$ For keeping the data anonymous each informant is indicated by a letter. 


\section{Information on language use}

\begin{tabular}{|c|c|c|c|c|}
\hline $\begin{array}{l}\text { Infor- } \\
\text { mant }\end{array}$ & $\begin{array}{l}\text { Language in school / } \\
\text { boarding house }\end{array}$ & $\begin{array}{l}\text { Main means of } \\
\text { communicatio } \\
\mathbf{n}\end{array}$ & $\begin{array}{l}\text { Means of } \\
\text { communication at } \\
\text { work }\end{array}$ & $\begin{array}{l}\text { Preferred } \\
\text { language }\end{array}$ \\
\hline A & $\begin{array}{l}\text { school - oral orientated } \\
\text { / boarding house - } \\
\text { signing }\end{array}$ & sign language & $\begin{array}{l}\text { spoken language } \\
\text { (German) }\end{array}$ & sign language \\
\hline B & $\begin{array}{l}\text { school - oral orientated } \\
\text { / boarding house - } \\
\text { signing }\end{array}$ & sign language & $\begin{array}{l}\text { spoken language } \\
\text { (German) }\end{array}$ & sign language \\
\hline C & $\begin{array}{l}\text { school - oral orientated } \\
\text { / boarding house - } \\
\text { signing }\end{array}$ & sign language & $\begin{array}{l}\text { spoken language } \\
\text { (German) }\end{array}$ & sign language \\
\hline D & $\begin{array}{l}\text { school - oral orientated } \\
\text { / boarding house - } \\
\text { signing }\end{array}$ & sign language & $\begin{array}{l}\text { (home) signs known by } \\
\text { the colleagues }\end{array}$ & sign language \\
\hline $\mathbf{E}$ & $\begin{array}{l}\text { school - oral orientated } \\
\text { / boarding house - } \\
\text { signing }\end{array}$ & sign language & $\begin{array}{l}\text { sign language, written } \\
\text { German }\end{array}$ & sign language \\
\hline F & $\begin{array}{l}\text { school - oral orientated } \\
\text { / boarding house - } \\
\text { signing }\end{array}$ & sign language & $\begin{array}{l}\text { sign language, spoken } \\
\text { language (German), } \\
\text { (home) signs known by } \\
\text { the colleagues }\end{array}$ & sign language \\
\hline G & $\begin{array}{l}\text { school - oral orientated } \\
\text { / boarding house - } \\
\text { signing }\end{array}$ & sign language & $\begin{array}{l}\text { spoken language } \\
\text { (German), written } \\
\text { German }\end{array}$ & sign language \\
\hline $\mathbf{H}$ & $\begin{array}{l}\text { school - oral orientated } \\
\text { / boarding house - } \\
\text { signing }\end{array}$ & sign language & $\begin{array}{l}\text { spoken language } \\
\text { (German) }\end{array}$ & sign language \\
\hline I & $\begin{array}{l}\text { school - oral orientated } \\
\text { / boarding house - } \\
\text { signing }\end{array}$ & sign language & $\begin{array}{l}\text { spoken language } \\
\text { (German), written } \\
\text { German }\end{array}$ & sign language \\
\hline $\mathbf{J}$ & $\begin{array}{l}\text { school - oral orientated } \\
\text { / boarding house - } \\
\text { signing }\end{array}$ & sign language & $\begin{array}{l}\text { Signed German referred } \\
\text { to as LBG (Lautsprach- } \\
\text { begleitendes Gebärden) }\end{array}$ & sign language \\
\hline
\end{tabular}




\section{APPENDIX C: Questionnaire}

\section{Questionnaire - original version in German}

Die Videoaufnahmen werden nur für die Sprachforschung verwendet und sind nicht als Lehrmaterial gedacht. Die Aufnahmen werden vertraulich behandelt.

Der Fragebogen ist ein Zusatz zu den Videoaufnahmen. Er dient als zusätzliche Information und wird ebenfalls vertraulich behandelt, in anonymer Form gespeichert und aus statistischen Gründen angeführt, d.h. in einer Form, dass kein Rückschluss auf einzelne Personen gezogen werden kann!

\section{ALLGEMEIN}

Name:

Geburtsjahr:

Wo sind Sie aufgewachsen?

Wo haben Sie in den letzten Jahren gelebt?

Seit wann sind Sie gehörlos?

Sind Ihre Eltern: $\quad 0$ beide gehörlos

0 beide hörend

0 Mutter gehörlos / Vater hörend

0 Mutter hörend / Vater gehörlos

Haben Sie gehörlose oder hörende Geschwister?

0 hörend (Wie viele?

0 gehörlos (Wie viele?

Wie sieht Ihre Familie jetzt aus? 0 ledig

0 gehörlose Frau

0 geschieden / getrennt

0 hörende Frau

0 gehörloser Mann

0 gehörlose Kinder

0 hörende Kinder

0 hörender Mann

(Wie viele?

(Wie viele?

Wie sieht Ihr Freundeskreis aus?

0 viele Gehörlose - wenig Hörende

0 viele Hörende - wenig Gehörlose

0 halb Gehörlose - halb Hörende

0 nur Gehörlose

0 nur Hörende 


\section{KOMMUNIKATION}

Was ist Ihr Hauptkommunikationsmittel?

0 Gebärdensprache (GS)

0 Lautsprache (LS)

Wie kommunizieren Sie mit:

1. Frau / Mann 0 Gebärdensprache 0 Lautsprache 0 LBG 0 Schrift 0 Sonstiges

2. Kindern 0 Gebärdensprache 0 Lautsprache 0 LBG 0 Schrift 0 Sonstiges

3. Eltern 0 Gebärdensprache 0 Lautsprache 0 LBG 0 Schrift 0 Sonstiges

4. Arbeitskollegen 0 Gebärdensprache 0 Lautsprache 0 LBG 0 Schrift 0 Sonstiges

5. hörende Freunde 0 Gebärdensprache 0 Lautsprache 0 LBG 0 Schrift 0 Sonstiges

6. gehörlose Freunde0 Gebärdensprache 0 Lautsprache 0 LBG 0 Schrift 0 Sonstiges

Welches Kommunikationsmittel benützen Sie am liebsten?

0 Gebärdensprache 0 Lautsprache 0 LBG 0 Schrift 0 Sonstiges

Ab wann hatten Sie regelmäßig Kontakt mit Gebärdensprache?

0 Geburt 0 Kindergarten 0 Schulalter 0 Erwachsenenalter (Wie viel Jahre?

\section{SCHULE / AUSBILDUNG}

Welchen Kindergarten haben Sie besucht?

0 keinen 0 Regelkindergarten 0 Hörgeschädigten Kindergarten 0 Sonstiges

Welche Schule haben Sie besucht?

1. Hörgeschädigten Schule

0 Gehörlosenklasse

0 Schwerhörigenklasse

\begin{tabular}{|c|c|}
\hline $\begin{array}{c}\text { Klasse } \\
\text { (von - bis) }\end{array}$ & $\begin{array}{c}\text { Kommunikation } \\
\text { in GS oder LS }\end{array}$ \\
\hline & \\
\hline & \\
\hline & \\
\hline & \\
\hline
\end{tabular}

0 Bilinguale Klasse

0 Sonstiges

2. Internatsbesuch 0 ja $\quad 0$ nein $\quad$ Kommunikation in: $\quad 0$ GS $\quad 0$ LS

3. Regelschule - Volksschule: 0 ja 0 nein

Kommunikation in: 0 GS 0 LS

- Hauptschule: 0 ja 0 nein

Kommunikation in: 0 GS 0 LS 
4. Andere Schule

Welchen Beruf haben Sie erlernt?

Was machen Sie jetzt: $\quad 0$ in Ausbildung 0 Hausfrau/-mann bzw.

Kinderbetreuung
0 Arbeit
0 arbeitslos / Krankenstand
0 Pension
0 Sonstiges

\section{Danke für die Mitarbeit!}




\section{Questionnaire - translated version in English}

The recordings are only used for linguistic purposes. They are not intended to be used for teaching. The recordings are treated confidentially.

The questionnaire is used additionally to the recordings to gain more information and is also treated confidentially, saved in an anonymous form, and used for statistic purposes. In other words, the questionnaire is saved in such a way that none of the informants can be identified.

\section{GENERAL INFORMATION}

Name:

Year of birth:

Where did you grow up?

Where have you been living the last years?

Since when are you Deaf?

Are your parents: $\quad 0$ both Deaf

0 both hearing

0 mother Deaf / father hearing

0 mother hearing / father Deaf

Have you Deaf and/or hearing siblings?

$\begin{array}{ll}0 \text { hearing } & \text { (How many? } \\ 0 \text { Deaf } & \text { (How many? }\end{array}$

What is your family status? /

Who is part of your family?

$\begin{array}{ll}\text { 0 single } & \text { 0 separated / divorced } \\ \text { 0 Deaf wife } & \text { 0 Deaf husband } \\ \text { 0 hearing wife } & \text { 0 hearing husband } \\ \text { 0 Deaf children } & \text { (How many? } \\ \text { 0 hearing children } & \text { (How many? }\end{array}$

Who is part of your circle of friends?

0 many Deaf persons - few hearing persons

0 many hearing person - few Deaf persons

0 half are hearing persons - half are Deaf persons

0 only Deaf persons

0 only hearing persons 


\section{COMMUNICATION}

What is your main means of communication? 0 sign language (SL)

0 spoken language (spL)

How do you communicate with your

1. wife / husband $0 \mathrm{SL} 0 \mathrm{spL} 0$ Signed German (LBG) 0 written language 0 other

2. children $\quad 0 \mathrm{SL} 0 \mathrm{spL} 0$ Signed German (LBG) 0 written language 0 other

3. parents $\quad 0 \mathrm{SL} 0 \mathrm{spL} 0$ Signed German (LBG) 0 written language 0 other

4. colleagues at work $0 \mathrm{SL} \quad 0 \mathrm{spL} 0$ Signed German (LBG) 0 written language 0 other

5. hearing friends $0 \mathrm{SL} \quad 0 \mathrm{spL} 0$ Signed German (LBG) 0 written language 0 other

6. Deaf friends $\quad 0 \mathrm{SL} \quad 0 \mathrm{spL} 0$ Signed German (LBG) 0 written language 0 other

Which means of communication do you prefer?

$0 \mathrm{SL} \quad 0 \mathrm{spL} \quad 0$ Signed German (LBG) 0 written language 0 other

Since when do you have regular contact with sign language?

0 birth 0 kindergarten 0 school 0 adult age (For how many years have you been signing?_)

\section{SCHOOL / EDUCATION}

Which type of kindergarten did you attend? other

0 non 0 regular kindergarten $\quad 0$ kindergarten for 'hearing-impaired children' 0

Which type of school did you attend?

1. school for 'hearing-impaired children'

0 Deaf class

0 Hard of hearing class

0 Bilingual class

0 Other

\begin{tabular}{|c|l|}
\hline $\begin{array}{c}\text { grade } \\
\text { (from - to) }\end{array}$ & $\begin{array}{l}\text { communication } \\
\text { in SL or spL }\end{array}$ \\
\hline & \\
\hline & \\
\hline & \\
\hline & \\
\hline
\end{tabular}

2. school boarding house 0 yes 0 no means of communication: 0 SL $0 \mathrm{spL}$

3. regular school - primary school: 0 yes 0 no means of communication: 0 SL 0 spL - secondary school: 0 yes 0 no means of communication: 0 SL 0 spL 
4. other school

Which profession did you learn?

Which profession do you carry out at the present?

$\begin{array}{ll}0 \text { profession-in-training } & 0 \text { homemaker / at home due to child care } \\ 0 \text { work } & 0 \text { out-of-work / sick leave } \\ 0 \text { retired } & 0 \text { other }\end{array}$

Thank you for your participation! 


\section{APPENDIX D: Preliminary study on the identification of head and body movements in ÖGS}

Department of Linguistics \& Department of Translation Studies

Karl-Franzens-University Graz, Austria

\section{The interplay of different head movements and their functions in Austrian Sign Language}

Andrea Lackner MA andrea.lackner@edu.uni-graz.at Christian Stalzer christian.stalzer@uni-graz.at

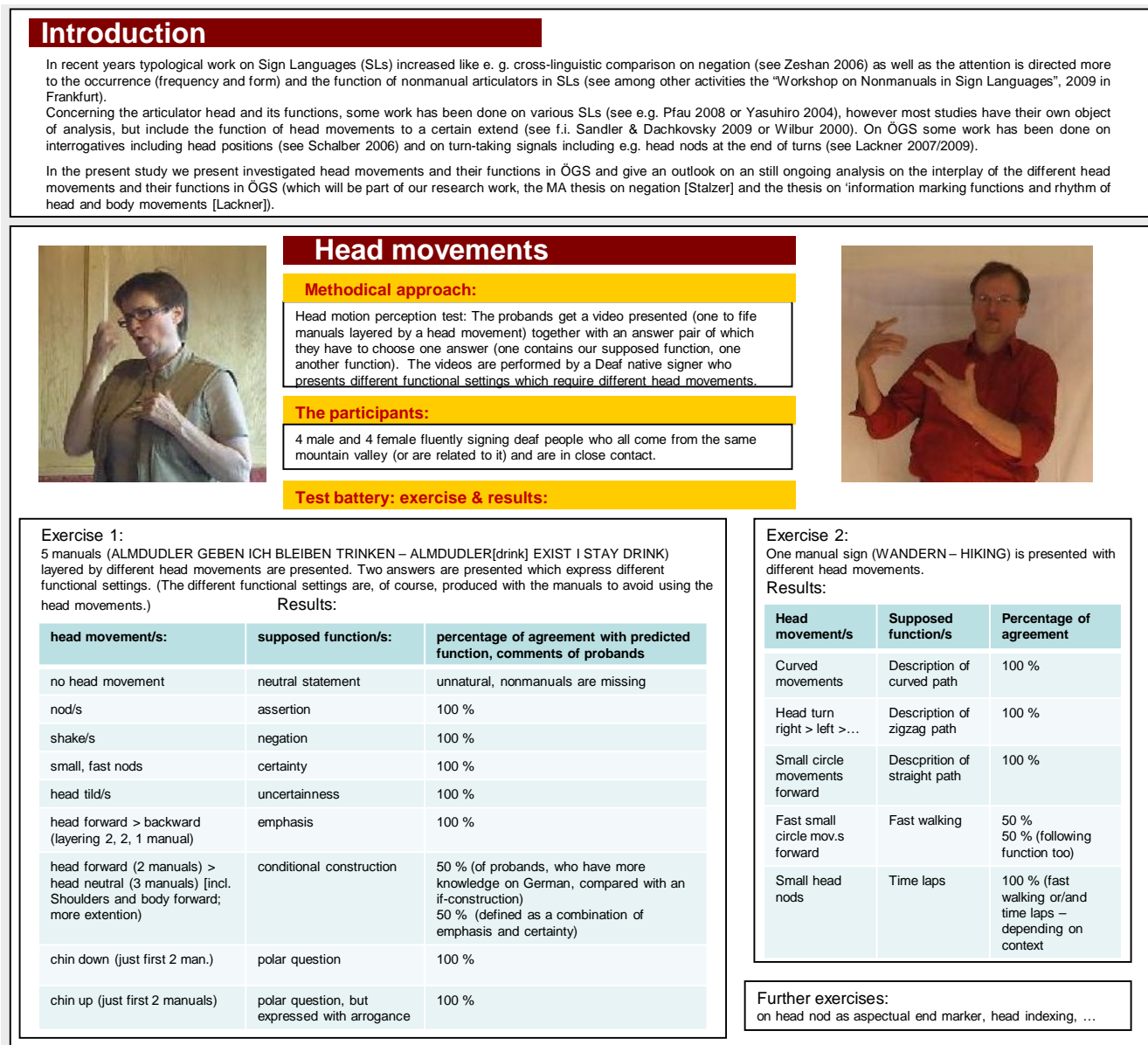

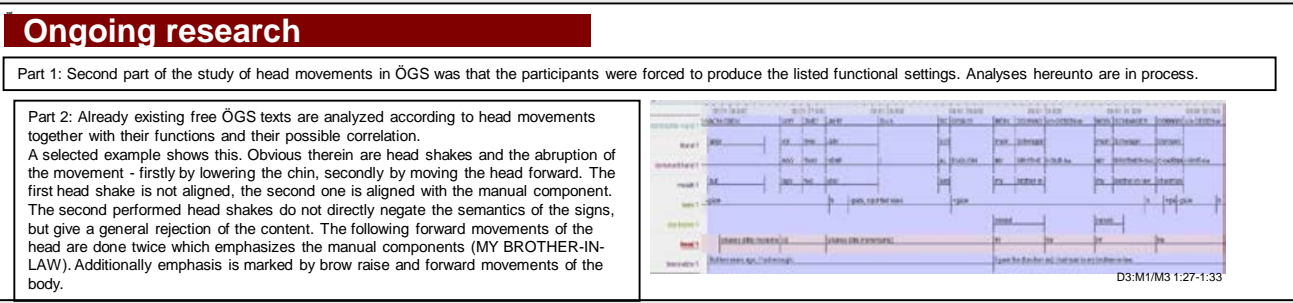

(Lackner \& Stalzer 2010) 


\section{References}

Antzakas, Klimis (2006) The use of negative head movements in Greek Sign Language. In: Zeshan, Ulrike (2006a) Interrogative and negative constructions in sign languages (Sign language typology series no. 1). Nijmegen: Ishara Press, 258-269.

Arik, Engin (2010) A crosslinguistic study of the language of space. Newcastle upon Tyne: Cambridge Scholars Publishing.

Athanasiadou, Angeliki \& Dirven, René (1997) Conditionality, hypotheticality, counterfactuality. In: Athanasiadou, Angeliki \& Dirven, René (eds.) On conditionals again. Amsterdam: John Benjamins, 61-96.

Auer, Peter (2000) Pre- and post-positioning of wenn-clauses in spoken and written German. In: Couper-Kuhlen, Elisabeth \& Kortmann, Bernd (eds.) Cause, condition, concession, contrast: cognitive and discourse perspectives. Berlin / New York: Walter de Gruyter, 173-204.

Baker, Carl L. (1970) Double negatives. In: Linguistic Inquiry 1, 169-186.

Baker, Charlotte \& Padden, Carol (1978) Focusing on the nonmanual components of American Sign Language. In: Siple, Patricia (ed.) Understanding language through sign language research. New York: Academic Press, 27-57.

Barberà, Gemma (2012) The meaning of space in Catalan Sign Language (LSC). Reference, specificity and structure in signed discourse. PhD thesis, Universitat Pompeu Fabra.

Bhat, Shankara (2004) Pronouns. Oxford: Oxford University Press.

Biezma, María \& Rawlins, Kyle (2012) Responding to alternative and polar questions. In: Linguistics and Philosophy, 35(5), 361-406.

Blum-Kulka, Shoshana (1987) Indirectness and politeness in requests: same or different? In: Journal of Pragmatics 11, 131-146.

Bowerman, Melissa (2011) Linguistic typology and first language acquisition. In: Song, Jae Jung (ed.) The Oxford handbook of linguistic typology. Oxford: Oxford University Press, 591-617.

Boyes Braem, Penny (1995) Einführung in die Gebärdensprache und ihre Forschung. $3^{\text {rd }}$ Edition, Hamburg: Signum.

Boyes Braem, Penny (1999) Rhythmic temporal patterns in the signing of deaf early and late learners of Swiss German Sign Language. In: Language and Speech, 42(2-3), 177-208. 
Boyes Braem, Penny (2001a) Functions of the mouthings in the signing of Deaf early and late learners of Swiss German Sign Language (DSGS). In: Boyes Braem, Penny \& SuttonSpence, Rachel (eds.) The hands are the head of the mouth. The mouth as articulator in sign languages. Hamburg: Signum, 99-131.

Boyes Braem, Penny (2001b) Functions of the mouthing component in Swiss German Sign Language. In: Brentari, Diane (ed.) Foreign vocabulary in sign languages. Mahwah, NJ: Lawrence Erlbaum Associates, 1-45.

Braffort, Annelies (2008) Traitement automatique de la Langue des Signes Française. Mémoire préparé en vue de l'obtention de l'habilitation à diriger des recherches d'informatique. Paper of the presentation at Atelier TALS (Traitement Automatique des Langues des Signes) 2008, Avignon, June 9-13, 2008.

Brentari, Diane (1998) A prosodic model of sign language phonology. Cambridge: MIT Press.

Brentari, Diane \& Crossley, Laurinda (2002) Prosody on the hands and face. Evidence from American Sign Language. In: Sign Language \& Linguistics 5(2), 105-130.

Buyssens, Eric (1959) Negative contexts. In: English Studies 40, 163-169.

Bühler, Karl (1934) Sprachtheorie. Die Darstellungsfunktion der Sprache. Jena: Gustav Fischer.

Bybee, Joan L. (1985) Morphology: A study of the relation between meaning and form (Typological studies in language 9). Amsterdam / Philadelphia: John Benjamins.

Bybee, Joan L. \& Dahl, Östen (1989) The creation of tense and aspect systems in the languages of the world. In: Studies in Language 13, 51-103.

Bybee, Joan L. \& Fleischman, Suzanne (1995) Modality in grammar and discourse: An introductory essay. In: Bybee, Joan L. \& Fleischman, Suzanne (eds.) Modality in grammar and discourse. Amsterdam: John Benjamins, 1-14.

Campbell, William W. (2005) DeJong's the neurologic examination. $6^{\text {th }}$ Edition, Balitmore: Lippincott Williams \& Wilkins.

Chen Pichler, Deborah \& Schalber, Katharina \& Hochgesang, Julie \& Milković, Marina \& Wilbur, Ronnie B. \& Vulje, Martina \& Pribanić, Ljubica (2008) Possession and existence in three sign languages. In: de Quadros, Ronice Müller de (ed.) Sign languages: Spinning and unraveling the past, present, and future. TISLR9, forty five papers and three posters from the $9^{\circ}$ Theoretical Issues in Sign Language Research Conference, Florianopolis, Brazil, December 2006. Petrópolis, Brazil: Editora Arara Azul, 440-458. 
Chien-hung, Lin \& Jung-hsing, Chang (2009) Modality in Taiwan Sign Language. Presentation at the International Conference on Role and Reference Grammar, University of California, Berkeley, California, August 7-9, 2009.

Clark, Herbert H. (1973) Space, time, semantics, and the child. In: Moore, Timothy E. (ed.) Cognitive development and the acquisition of language. New York: Academic Press, 27-63.

Comrie, Bernard (1986) Conditionals: A typology. In: Traugott, Elizabeth C. \& Meulen, Alice ter \& Reilly, Judy S. \& Ferguson, Charles A. (eds.) On conditionals. Cambridge: Cambridge University Press, 77-99.

Cormier, Kearsy \& Smith, Sandra (2011) Defining and annotating constructed action, constructed dialogue and role shift. In: Proceedings of the $33^{\text {rd }}$ Annual Meeting of the German Linguistic Society, Workshop on Sign Language Discourse. University of Gottingen, Germany, February 23-25, 2011.

Coulter, Geoffrey R. (1978) Raised eyebrows and wrinkled noses: The grammatical function of facial expression in relative clauses and related constructions. In: Caccamise, Frank \& Hicks, Doin (eds.) American Sign Language in a bilingual, bicultural context. Proceedings of the Second National Symposium on Sign Language Research and Teaching. Silver Spring: NAD, 65-74.

Crasborn, Onno (2011) Are left and right like high and low? Presentation at the Workshop on Complex Sentences and Beyond in Sign and Spoken Languages, Lichtenberg-Kolleg, Georg-August-Universität Göttingen, Germany, October 13-14, 2011.

Crasborn, Onno \& Kooij, Els van der \& Waters, Dafydd \& Woll, Bencie \& Mesch, Johanna (2008) Frequency distribution and spreading behavior of different types of mouth actions in three sign languages. In: Sign Language \& Linguistics, 11(1), 45-67.

Croft, William (2003) Typology and universals. $2^{\text {nd }}$ Edition, Cambridge: Cambridge University Press.

Dachkovsky, Svetlana (2005) Facial expression as intonation in ISL: The case of conditionals. MA thesis, University of Haifa.

Dachkovsky, Svetlana (2008) Facial expression as intonation in Israeli Sign Language: The case of neutral and counterfactual conditionals. In: Quer, Josep (ed.) Signs of the time. Selected papers from TISLR 2004. Hamburg: Signum, 61-82.

Dachkovsky, Svetlana \& Sandler, Wendy (2009) Visual intonation in the prosody of a sign language. In: Language and Speech 52(2-3), 287-314. 
Dancygier, Barbara \& Sweetser, Eve E. (2005) Mental spaces in grammar: Conditional constructions. Cambridge: Cambridge University Press.

Department of Linguistics of the University of Erfurt (2007) Homepage for the documentation of the Hočąk Language (last update: 12/2012). Retrieved June 15, 2012, from www2.uni-erfurt.de/sprachwissenschaft/Vgl_SW/Hocank/index_frames.html

Dimon, Theodore (2008) Anatomy of the moving body: a basic course in bones, muscles, and joints. $2^{\text {nd }}$ Edition, Berkeley, California: North Atlantic Books.

Dixon, Robert M. W. (2006) Complement clauses and complementation strategies in typological perspective. In: Dixen, Robert M. W. \& Aikhenvald, Alexandra (eds.) Complementation. Oxford: Oxford University Press, 1-48.

Dubuisson, Colette \& Boulanger, Johanne \& Desrosiers, Jules \& Lelièvre, Linda (1991) Les mouvements de tête dans les interrogatives en langue des signes québécoise. In: Revue québécoise de linguistique 20(2), 93-121.

Dubuisson, Colette \& Miller, Christopher (1992) Nonmanual behaviors in interrogatives in Quebec Sign Language. Paper presented at TISLR 4 (Theoretical Issues in Sign Language Research Conference), San Diego, California, June 12-14, 1992.

Edmondson, Jerry A. (1981) Affectivity and gradient scope. In: Chicago Linguistics Society $17,38-44$.

Emmorey, Karen (1996) The confluence of space and language in signed languages. In: Valli, Clayton \& Lucas, Ceil (eds.) Linguistics of American Sign Language: An introduction. Washington, DC: Gallaudet University Press, 318-346.

Emmorey, Karen \& Falgier, Brenda (2004) Conceptual locations and pronominal reference in American Sign Language. In: Journal of Psycholinguistic Research 33(4), 321-331.

Engberg-Pedersen, Elisabeth (1993) Space in Danish Sign Language. The semantics and morphosyntax of the use of space in a visual language. Hamburg: Signum.

Fauconnier, Gilles (1975) Pragmatic scales and logical structure. In: Linguistic Inquiry 6, 353-375.

Fenlon, Jordan (2010) Seeing sentence boundaries: The production and perception of visual markers signalling boundaries in sign languages. $\mathrm{PhD}$ thesis, University College London. 
Ferreira Brito, Lucinda (1990) Epistemic, alethic, and deontic modalities in a Brazilian Sign Language. In: Fischer, Susan D. \& Siple, Patricia (eds.) Theoretical Issues in Sign Language Research. Vol. 1: Linguistics. Chicago / London: University of Chicago Press, 229-260.

Fischer, Susan D. (2006) Questions and negation in American Sign Language. In: Zeshan, Ulrike (2006a) Interrogative and negative constructions in sign languages (Sign language typology series no. 1). Nijmegen: Ishara Press, 165-197.

Ford, Cecilia E. (1997) Speaking conditionally: Some contexts for if-clauses in conversation. In: Athanasiadou, Angeliki \& Dirven, René (eds.) On conditionals again. Amsterdam: John Benjamins, 387-413.

Ford, Cecilia E. \& Thompson, Sandra A. (1986) Conditionals in discourse: A text-based study from English. In: Traugott, Elizabeth C. \& Meulen, Alice ter \& Reilly, Judy S. \& Ferguson, Charles A. (eds.) On conditionals. Cambridge: Cambridge University Press, 353-372.

Frishberg, Nancy (1985) Dominance relations and discourse structures. In: Stokoe, William C. \& Volterra, Virginia (eds.) SLR'83. Proceedings of the Third International Symposium on Sign Language Research. Rome, June 22-26, 1983. Silver Spring, MD: Linstok Press / Rome: Consiglio Nazionale delle Ricerche (CNR), 79-90.

Fraser, Bruce (2001) The form and function of politeness in conversation (Form und Funktion von Höflichkeit im Gespräch). In: Brinker, Klaus (ed.) Text- und Gesprächslinguistik (Linguistics of Text and Conversation) HSK 16.2. Berlin / New York: Walter de Gruyter, 1405-1425.

Geraci, Carlo (2011) Sentential complementation in SL: The case of Italian Sign Language and more ... Presentation at the Workshop on Complex Sentences and Beyond in Sign and Spoken Languages, Lichtenberg-Kolleg, Georg-August-Universität Göttingen, Germany, October 13-14, 2011.

Givón, Talmy (2009) The genesis of syntactic complexity: Diachrony, ontogeny, neurocognition, evolution. Amsterdam: John Benjamins.

Glück, Helmut (2000) Metzler Lexikon Sprache. $2^{\text {nd }}$ Edition, Stuttgart / Weimar: J. B. Metzler.

Gökgöz, Kadir (2011) Negation in Turkish Sign Language. In: Herrmann, Annika \& Steinbach, Markus (eds.) Nonmanuals in sign language: Special issue of Sign Language \& Linguistics 14(1), 49-75. 
Grose, Donovan \& Wilbur, Ronnie B. \& Schalber, Katharina (2007) Events and telicity in classifier predicates: A reanalysis of body part classifier predicates in ASL. In: Lingua 117(7), 1258-1284.

Haspelmath, Martin (1997) Indefinite pronouns. Oxford: Clarendon Press.

Haspelmath, Martin (2000) The geometry of grammatical meaning: Semantic maps and crosslinguistic comparison. In: Tomasello, Michael (ed.) The new psychology of language. Vol. 2. Mahwah, NJ: Lawrence Erlbaum, 211-242.

Haspelmath, Martin (2004) Coordinating constructions: An overview. In: Haspelmath, Martin (ed.) Coordinating constructions. Amsterdam: John Benjamins, 3-39.

Haspelmath, Martin (2007) Coordination. In: Shopen, Timothy (ed.) Language typology and syntactic description. Vol. 2. Cambridge: Cambridge University Press, 1-51.

Haspelmath, Martin (2010) Framework-free grammatical theory. In: Heine, Bernd \& Narrog, Heiko (eds.) The Oxford handbook of linguistic analysis. Oxford: Oxford University Press, 341-365.

Haspelmath, Martin \& König, Ekkehard (1998) Concessive conditionals in the languages of Europe. In: Auwera, Johan van der (ed.) Adverbial construction in the languages of Europe. (Empirical Approaches to Language Typology - EUROTYP 20-3). Berlin: Mouton de Gruyter, 563-640.

Heine, Bernd \& Narrog, Heiko (2010a) The Oxford handbook of linguistic analysis. Oxford: Oxford University Press.

Heine, Bernd \& Narrog, Heiko (2010b) Introduction. In: Heine, Bernd \& Narrog, Heiko (eds.) The Oxford handbook of linguistic analysis. Oxford: Oxford University Press, 1-25.

Hendriks, Bernadet (2007a) Negation in Jordanian Sign Language: A cross-linguistic perspective. In: Perniss, Pamela M. \& Pfau, Roland \& Steinbach, Markus (eds.) Visible variation: Comparative studies on sign language structure. Berlin: Mouton de Gruyter, 103-128.

Hendriks, Bernadet (2007b) Simultaneous use of the two hands in Jordanian Sign Language. In: Vermeerbergen, Myriam \& Leeson, Lorraine \& Crasborn, Onno (eds.) Simultaneity in signed languages: Form and function. Amsterdam: John Benjamins, 237-256.

Herrmann, Annika (2004) Modal particles and modal meaning in sign language. A crosslinguistic comparison of spoken German with German Sign Language (inter-modal variation) and further investigation into Irish Sign Language (intra-modal variation) and English. MA thesis, Johannes Gutenberg-Universität Mainz. 
Herrmann, Annika \& Steinbach, Markus (2012) Quotation in sign languages - A visible context shift. In: Buchstaller, Isabelle \& Alphen, Ingrid van (eds.) Quotatives. Crosslinguistic and cross-disciplinary perspectives. Amsterdam: John Benjamins, 203-228.

Herrero-Blanco, Ángel \& Salazar-García, Ventura (2010) The expression of modality in Spanish Sign Language. In: Wanders, Gerry \& Keizer, Evelien (eds.) Webpapers in Functional Discourse. Special Issue: The London papers II. Amsterdam, 19-42.

Hoeksema, Jack (2012) On the natural history of negative polarity items. In: Linguistic Analysis 38(1-2), 3-33.

Hofstätter, Karin \& Stalzer, Christian (2001) Sag niemals nie - Zur Semantik der Negation in der ÖGS. In: Grazer Linguistische Studien 56, 1-14.

Horn, Laurence R. (1996) Flaubert triggers, squatitive negation, and other quirks of grammar. In: Taalkundig Bulletin 26, 183-205. Reprinted (2001) in: Hoeksema, Jack \& Rullmann, Hotze \& Sánchez-Valencia, Víctor \& Wouden, Ton van der (eds.) Perspectives on negation and polarity items. Amsterdam / Philadelphia: John Benjamins, 173-200.

Hrastinski, Iva (2010) Negative structures in Croatian Sign Language (HZJ). MA thesis, Purdue University.

Hunger, Barbara (2003) Nomen/Verb-Paare in der Österreichischen Gebärdensprache. MA thesis, University of Graz.

Hunger, Barbara \& Schalber, Katharina \& Wilbur, Ronnie B. (2000) 'Bub wollen lernen, wollen?' Further investigations into the modals in the Styrian dialect of Austrian Sign Language with a particular focus on repetition and pauses. Poster presented at TISLR 7 (Theoretical Issues in Sign Language Research Conference), Amsterdam, July 23-27, 2000 .

Iatridou, Sabine (2000) The grammatical ingredients of counterfactuality. In: Linguistic Inquiry 31(2), 231-270.

Ichida, Yasuhiro (2004) Head movement and head position in Japanese Sign Language. Poster presented at TISLR 8 (Theoretical Issues in Sign Language Research Conference), Barcelona, September 30 - October 2, 2004. In: TISLR 8 Barcelona, September 30 - October 2. Program and Abstracts, 71-72.

Israel, Michael (2011) The grammar of polarity: Pragmatics, sensitivity, and the logic of scales. Cambridge: Cambridge University Press. 
Janzen, Terry \& Shaffer, Barbara (2002) Gesture as the substrate in process of ASL grammaticalization. In: Meier, Richard P. \& Cormier, Kearsy \& Quinto-Pozos, David (eds.) Modality and structure in signed and spoken languages. Cambridge: Cambridge University Press, 199-223.

Jarmer, Helene (2004/5) Einführung in die Gehörlosenpädagogik. Wien. Script.

Johnston, Trevor (2010) Auslan corpus annotation guidelines. Paper presented at the $3^{\text {rd }}$ Sign Linguistics Corpora Network, Stockholm University, Stockholm, June 14-16, 2010.

Johnston, Trevor \& Schembri, Adam (2007) Australian Sign Language. An introduction to sign language linguistics. Cambridge: Cambridge University Press.

Kimura, Harumi \& Ichida, Yasuhiro (1995) Hajimete no Shuwa [Shuwa for Beginners]. Tokyo: Nihon-Bunkei-Sha.

Kinsbourne, Marcel (1973) Direction of gaze and distribution of cerebral thought processes. In: Neuropsychologia 12(2), 279-281.

Klima, Edward S. (1964) Negation in English. In: Fodor, Jerry A. \& Katz Jerrold J. (eds.) The structure of language: Readings in the philosophy of language. Englewood Cliffs, NJ: Prentice-Hall, 246-323.

Kooij, Els van der, Crasborn, Onno \& Emmerik, Wim (2006) Explaining prosodic body leans in Sign Language of the Netherlands: Pragmatics required. In: Journal of Pragmatics 38, 1598-1614.

Kooij, Els van der \& Crasborn, Onno (2008) Syllables and the word-prosodic system in Sign Language of the Netherlands. In: Lingua 118, 1307-1327.

Kortschak, Irfan (2010) Where everyone speaks Deaf talk. In: Kortschak, Irfan (ed.) Invisible people: Poverty and empowerment in Indonesia. Jakarta, Indonesia: The Lontar Foundation, 76-89.

Kratzer, Angelika (1991) Modality. In: Strechow, Arnim von \& Wunderlich, Dieter (eds.) Semantik (Semantics). Ein internationales Handbuch zeitgenössischer Forschung (An international handbook of contemporary research) HSK 6. Berlin / New York: Walter de Gruyter, 639-650.

Krausneker, Verena (2006) taubstumm bis gebärdensprachig. Die österreichische Gebärdensprachgemeinschaft aus soziolinguistischer Perspektive. Klagenfurt: Drava Verlag. 
Krausneker, Verena (2008) Language use and awareness of Deaf and hearing children in a bilingual setting. In: Plaza-Pust, Carolina \& Morales-López, Esperanza (eds.) Sign bilingualism. Language development, interaction, and maintenance in sign language contact situations (Studies in Bilingualism 38). Amsterdam: John Benjamins.

Krausneker, Verena \& Schalber, Katharina (2007) Sprache Macht Wissen. Zur Situation gehörloser SchülerInnen, Studierender \& ihrer LehrerInnen, sowie zur Österreichischen Gebärdensprache in Schule und Universität Wien. Script. Retrieved September 10, 2012, from http://www.univie.ac.at/sprachemachtwissen/files/SpracheMachtWissen_Nov.pdf

Krifka, Manfred (1994) The semantics and pragmatics of weak and strong polarity items in assertions. In: Harvey, Mandy \& Santelmann, Lynn (eds.) Proceedings from Semantics and Linguistic Theory IV, Ithaca, Cornell University, 195-219.

Krifka, Manfred (1995) The semantics and pragmatics of polarity items. In: Linguistic Analysis 25, 1-49.

Krifka, Manfred (2011) Questions. In: Heusinger, Klaus von \& Maienborn, Claudia \& Portner, Paul (eds.) Semantics. An international handbook of natural language meaning. Vol. 2. Berlin: Walter de Gruyter, 1742-1785.

Krifka, Manfred (to appear) Negated polarity questions as denegations of assertions. In: Kiefer, Ferenc \& Lee, Chungmin (eds.) Contrastiveness and scalar implicatures. (proposed by editors to be published in Springer).

Lackner, Andrea (2007) Turn-Taking in der Österreichischen Gebärdensprache. Eine Gesprächsanalyse der Salzburger Variante. MA thesis, University of Graz.

Lackner, Andrea (2008) 'Palm-up' and 'Touching' in Austrian Sign Language - Two interactive elements at the transition relevance place in the turn-taking process. In: Proceedings of the First Sign Typ Conference, University of Connecticut, Storrs, USA, June 26-28, 2008.

Lackner, Andrea (2009a) Turn-Taking in der Österreichischen Gebärdensprache. Eine Gesprächsanalyse der Salzburger Variante. In: Klagenfurter Beiträge zur Sprachwissenschaft. Klagenfurt: Veröffentlichungsreihe des ZGH.

Lackner, Andrea (2009b) Turn-Taking und Dialogstruktur in der Österreichischen Gebärdensprache: Eine Gesprächsanalyse der Salzburger Variante. In: Das Zeichen 23: 81, 90-104. 
Lackner, Andrea (2009c) Linguistische Terminologie zur Österreichischen Gebärdensprache. Eine Beschreibung der Bildung(sprozesse) und der definitorischen Strukturen von sprachwissenschaftlichen Begriffen im ULG GebärdensprachlehrerIn (Linguistic terminology in Austrian Sign Language. A description on the formation of linguistic terminology and its definitional structure in an educational training course). Manuscript.

Lackner, Andrea (2009d) Linguistische Terminologie zur Österreichischen Gebärdensprache. Eine Beschreibung der Bildungs(prozesse) und der definitorischen Strukturen von sprachwissenschaftlichen Begriffen im ULG GebärdensprachlehrerIn (Linguistic terminology in Austrian Sign Language. A description on the formation of linguistic terminology and its definitional structure in an educational training course). Presentation at the $37^{\text {th }}$ Austrian Conference on Linguistics, Verbal-Workshop on Sign Language Research, University of Salzburg, December 5-7, 2009.

Lackner, Andrea \& Stalzer, Christian (2010) The interplay of different head movements and their functions in Austrian Sign Language. Poster presented at TISLR 10 (Theoretical Issues in Sign Language Research Conference), Purdue University, West Lafayette, USA, September 30 - October 2, 2010.

Lackner, Andrea \& Mallinger, Lena \& Dotter, Franz \& Riemer, Nikolaus \& Auersperg, Bernadette \& Dürr, Xenia \& \& Stalzer, Christian \& Wiener, Anna \& Graf, Isabel \& Koppendorfer, Marlen \& Cornelia Harratzmüller (in prep.) Segmentation units and segmentation cues in Austrian Sign Language (ÖGS).

Ladd, D. Robert (1981) A first look at the semantics and pragmatics of negative questions and tag questions. In: Proceedings of the Chicago Linguistic Society. Chicago, 164-171.

Ladusaw, William (1980) Polarity sensitivity as inherent scope relation. New York: Garland.

Lakoff, Robin (1971) If's, and's, and but's about conjunction. In: Fillmore, Charles J. \& Langendoen, D. Terence (eds.) Studies in linguistic semantics. New York: Holt, Rinehard and Winston, 114-149.

Lang, Ewald (1991) Koordinierende Konjunktionen. In: Stechow, Arnim von \& Wunderlich, Dieter (eds.) Semantik (Semantics). Ein internationales Handbuch zeitgenössischer Forschung (An international handbook of contemporary research) HSK 6. Berlin / New York: Walter de Gruyter, 597-623.

Langacker, Ronald W. (2010) Cognitive grammar. In: Heine, Bernd \& Narrog, Heiko (eds.) The Oxford handbook of linguistic analysis. Oxford: Oxford University Press, 87-109. 
Leeson, Lorraine \& Saeed, John I. (2004) Windowing of attention in simultaneous constructions in Irish Sign Language. In: Cameron, Terry \& Shank, Christopher \& Holley, Keri (eds.) Proceedings of the Fifth High Desert Linguistics Conference, University of New Mexico, Albuquerque, November 1-2, 2002, 1-18.

Lehmann, Christian (1984) Der Relativsatz. Typologie seiner Strukturen - Theorie seiner Funktionen - Kompendium seiner Grammatik. Tübingen: Gunter Narr.

Lehmann, Christian (1986) On the typology of relative clauses. In: Linguistics 24, 663-680. Modified version (2010) retrieved April 7, 2013, from http://www.christianlehmann.eu/publ/typology_relative_clauses.pdf

Lehmann, Christian (2012) Semantik. Theoretische und empirische Bereiche. (last update: 05/14/2012) Script. Retrieved December 1, 2012, from http://www.christianlehmann.eu/ling/lg_system/sem/index.html

Levinson, Stephen C. (1996) Language and space. In: Annual Review of Anthropology 25, 353-382.

Liddell, Scott (1977) An investigation into the syntactic structure of American Sign Language. PhD thesis, University of California, San Diego.

Liddell, Scott (1978) Non-manual signals and relative clauses in American Sign Language. In: Siple, Patricia (ed.) Understanding language through sign language research. New York: Academic Press, 59-90.

Liddell, Scott (1980) American Sign Language syntax. The Hague: Mouton.

Liddell, Scott K. (1984) THINK and BELIEVE: Sequentiality in American Sign Language. In: Language 69(2), 372-399.

Liddell, Scott K. (1986) Head thrust in ASL conditional marking. In: Sign Language Studies 52, 243-262.

Liddell, Scott K. (1990) Four functions of a locus: Reexamining the structure of space in ASL. In: Lucas, Ceil (ed.) Sign language research: Theoretical issues. Washington, DC: Gallaudet University Press, 176-198.

Liddell, Scott K. (2003) Grammar, gesture, and meaning in American Sign Language. Cambridge: Cambridge University Press.

Lillo-Martin, Diane \& Klima, Edward (1990) Pointing out differences: ASL pronouns in syntactic theory. In: Fischer, Susan D. \& Siple, Patricia (eds.) Theoretical issues in sign language research. Vol. 1: Linguistics. Chicago: University Chicago Press, 191-210. 
Long, John S. (1918) The sign language: A manual of signs. Washington, DC: Gallaudet College Press.

Lyons, John (1981) Language, meaning and context. London: Fontana.

Maas, Utz (2009) Franz Boas und Edward Sapir. Zur Grundlegung der deskriptiven Sprachwissenschaft. In: Schmuhl, Hans-Walter (ed.) Kulturrelativismus und Antirassismus: Der Anthropologe Franz Boas (1858-1942). Bielefeld, 251-275.

Malaia, Evie \& Wilbur, Ronnie B. (2010) Representation of verbal event structure in sign languages. In: Bertinetto, Pier M. \& Korhonen, Anna \& Lenci, Alessandro \& Melinger, Alissa \& Schulte im Walde, Sabine \& Villavicencio, Aline (eds.) Proceedings of the Interdisciplinary Workshop on Verbs. The Identification and Representation of Verb Features, Pisa, Italy, November 4-5, 2010, 165-170.

Mallinger, Lena (2012) Grenzsignale in der Österreichischen Gebärdensprache. MA thesis, University of Graz.

McGinnis, Peter M. (2005) Biomechanics of sport and exercise. Champaign, IL: Human Kinetics.

Metzger, Melanie (1995) Constructed dialogue and constructed action in American Sign Language. In: Lucas, Ceil (ed.) Sociolinguistics in Deaf communities. Washington, DC: Gallaudet University Press, 255-271.

Metzger, Melanie \& Bahan, Ben (2001) Discourse analysis. In: Lucas, Ceil (ed.) The sociolinguistics of sign language. Cambridge: Cambridge University Press, 112-144.

Michaelis, Laura A. (2001) Exclamative constructions. In: Haspelmath, Martin \& König, Ekkehard \& Oesterreicher, Wulf \& Raible, Wolfgang (eds.) Language typology and language universals: An international handbook. Vol. 2. Berlin / New York: Walter de Gruyter, 1038-1050.

Miller, Christopher (2004) The bases of LSQ syntax: Constituency, order, and information structure, Papers of the presentation at the Sixth High Desert Linguistics Conference, University of New Mexico, Albuquerque, November 4-6, 2004.

Morgan, Michael W. (2006) Interrogatives and negatives in Japanese Sign Language (JSL). In: Zeshan, Ulrike (ed.) Interrogative and negative constructions in sign languages (Sign language typology series no. 1). Nijmegen: Ishara Press, 91-127.

Nassira, Nicola (2008) Dire N'IMPORTE-Q: Identifying a polarity item in Quebec Sign Language. In: Lima, Suzi (ed.) Proceedings of the SULA 5: Semantics of underrepresented language of the Americas. Amherst, MA: GLSA Publications, 157-172. 
Nespor, Marina \& Sandler, Wendy (1999) Prosody in Israeli Sign Language. In: Language and Speech 42(2-3), 143-176.

Nonhebel, Annika, Crasborn, Onno \& Kooij, Els van der (2004) Sign language transcription conventions for the ECHO project. Retrieved May 5, 2010, from http://www.let.kun.nl/sign-lang/echo/docs/transcr_conv.pdf

Padden, Carol A. (1988) Interaction of morphology and syntax in American Sign Language. New York: Garland Publishing.

Palastanga, Nigel \& Field, Derek \& Soames, Roger (2006) Anatomy and human movement: Structure and function. $5^{\text {th }}$ Edition. Edinburgh / New York: Butterworth.

Palmer, Frank R. (1986/2001) Mood and Modality. Cambridge: Cambridge University Press.

Perlmutter, David M. (1989) A moraic theory of American Sign Language syllable structure. University of California, San Diego, unpublished paper.

Petkova-Schick, Ivanka (1998) On the contrast relation in the Balkan languages in comparison to Russian and German. In: Dimitrova-Vulchanova, Mila \& Lellan, Lars (eds.) Topics in South Slavic syntax and semantics, 41-58.

Petronio, Karen (1993) Clause structure in American Sign Language. PhD thesis, University of Washington.

Pfau, Roland (2008) Topic and conditionals in sign languages. Presentation at the $30^{\text {th }}$ Annual Meeting of the German Linguistic Society (DGfS), Workshop on 'Topicality', Bamberg, February 28, 2008.

Pfau, Roland \& Steinbach, Markus (2004) On grammaticalization: Do sign languages follow the well-trodden paths? Paper of the poster presented at TISLR 8 (Theoretical Issues in Sign Language Research Conference), Barcelona, September 30 - October 2, 2004.

Pfau, Roland \& Quer, Josep (2004) On the syntax of negation and modals in Catalan Sign Language and German Sign Language. Presentation at the $26^{\text {th }}$ Annual Meeting of the German Linguistic Society (DGfS), Workshop on sign languages: A cross-linguistic perspective, Mainz, February 27, 2004.

Pfau, Roland \& Steinbach Markus (2006) Pluralization in sign and in speech: A cross-modal typological study. In: Linguistic Typology 10(2), 135-182. 
Pfau, Roland \& Steinbach, Markus (2012) PERSON climbing up the tree (and other adventures in sign language grammaticalizcation). Presentation at FEAST (Formal and Experimental Advances in Sign Language Theory) 2012, Warsaw, Poland, June 1-2, 2012.

Podlesskaya, Vera (2001) Conditional constructions. In: Haspelmath, Martin \& König, Ekkehard \& Österreicher, Wulf \& Raible, Wofgang (eds.) Language typology and language universals: An international handbook. Vol. 2. Berlin / New York, 998-1009.

Poizner, Howard \& Klima, Edward \& Bellugi, Ursula (1987) What the hands reveal about the brain. Cambridge: MIT Press / Bradford Books.

Quadros, Ronice Müller de (2006) Questions in Brazilian Sign Language (LSB). In: Zeshan, Ulrike (ed.) Interrogative and negative constructions in sign languages (Sign language typology series no. 1). Nijmegen: Ishara Press, 270-283.

Quer, Josep et al. (2005) Gramàtica bàsica LSC. CD-ROM. Barcelona: Federació de persones sordes de Catalunya.

Quinto-Pozos, David \& Cormier, Kearsy \& Ramsey, Claire (2009) Constructed action of highly animate referents: Evidence form American, British and Mexican Sign Languages. In: Proceedings of the $35^{\text {th }}$ Annual Meeting of the Berkeley Linguistics Society (Special Session on Non-Speech Modalities): Berkeley, CA.

Reilly, Judy S. \& McIntire, Marina \& Bellugi, Ursula (1990) The acquisition of conditionals in American Sign Language: Grammaticalized facial expressions. In: Applied Psycholinguistics 11(4), 369-392.

Richmond, Frances J. \& Vidal, Pierre P. (1988) The motor system: Joints and muscles of the neck (1988) In: Peterson, Barry W. \& Richmond, Frances J. (eds.) Control of head movement. Oxford: Oxford University Press.

Sáfár, Anna \& Crasborn, Onno \& Ormel, Ellen (2010) Handedness in the corpus NGT. Poster presented at TISLR 10 (Theoretical Issues in Sign Language Research Conference), Purdue University, West Lafayette, USA, September 30 - October 2, 2010.

Saladin, Kenneth (2007) Human anatomy. New York: McGraw-Hill.

Sandler, Wendy (1986) The spreading hand autosegment of American Sign Language. In: Sign Language Studies 50, 1-28.

Sandler, Wendy (1999) Prosody in two natural language modalities. In: Language and Speech, 42(2-3), 127-142. 
Sandler, Wendy (2011) Prosody and syntax in sign language. In: Transactions of the Philological Society 108(3), 298-328.

Savolainen, Leena (2006) Interrogatives and negatives in Finnish Sign Language: An overview. In: Zeshan, Ulrike (ed.) Interrogative and negative constructions in sign languages (Sign language typology series no. 1). Nijmegen: Ishara Press, 284-302.

Schalber, Katharina (2002) The nonmanual marking of yes/no and wh-questions in Austrian Sign Language. MA thesis, University of Graz.

Schalber, Katharina (2006) What is the chin doing? An analysis of interrogatives in Austrian Sign Language. In: Sign Language and Linguistics 9(1/2), 133-150.

Schalber, Katharina \& Grose, Donovan (2008) The semantics, syntax and phonology of adverbial nonmanuals in Austrian and American Sign Language. In: Quadros, Ronice Müller de (ed.) Sign languages: Spinning and unraveling the past, present and future. TISLR9, forty five papers and three posters from the $9^{\circ}$ Theoretical Issues in Sign Language Research Conference, Florianopolis, Brazil, December 2006. Petrópolis, Brazil: Editora Arara Azul, 552-565.

Schalber, Katharina \& Hunger, Barbara (2000). wollen bub fussball spielen, wollen: Further investigation into the modals in the Styrian dialect of Austrian Sign Language, with a particular focus on repetition and pauses. Poster presented at the TISLR 7 (Theoretical Issues in Sign Language Research Conference), Amsterdam, The Netherlands, July 2327, 2000.

Schalber, Katharina \& Hunger, Barbara (2001) BUB FUSSBALLSPIELEN KÖNNEN Untersuchungen zur Stellung von Modalverben in der Steirischen Variante der Österreichischen Gebärdensprache. In: Grazer Linguistische Studien 56, 37-46.

Schalber, Katharina \& Hunger, Barbara (2008) Possession in Austrian Sign Language (ÖGS) - with existentials on the side. In: Zeshan, Ulrike \& Perniss, Pamela M. (eds.) Possessive and existential constructions in sign languages (Sign language typology series no. 2). Nijmegen: Ishara Press, 151-180.

Schünke, Michael \& Schulte, Erik \& Ross, Lawrence M. \& Schumacher, Udo \& Lamperti, Edward D. (2006) Thieme atlas of anatomy: General anatomy and musculoskeletal system. Stuttgart: Georg Thieme.

Shaffer, Barbara (2000) A syntactic, pragmatic analysis of the expression of necessity and possibility in American Sign Language. PhD thesis, University of New Mexico. 
Siemund, Peter (2001) Interrogative constructions. In: Haspelmath, Martin \& König, Ekkehard \& Oesterreicher, Wulf \& Raible, Wolfgang (eds.) Language typology and language universals: An international handbook. Vol. 2. Berlin / New York: Walter de Gruyter, 1010-1028.

Simons, David G. \& Travell Janet G. \& Simons Lois S. (1999) Travell \& Simons' myofascial pain and dysfunction: The trigger point manual. Upper half of body. Vol. 1. $2^{\text {nd }}$ Edition. Baltimore, USA: Williams \& Wilkins.

Skant, Andrea \& Dotter, Franz \& Bergmeister, Elisabeth \& Hilzensauer, Marlene \& Hobel, Manuela \& Krammer, Klaudia \& Okorn, Ingeborg \& Orasche, Christian \& Orter, Reinhold \& Unterberger, Natalie (2002) Grammatik der Österreichischen Gebärdensprache. Klagenfurt (Veröffentlichungen des Zentrums für Gebärdensprache und Hörbehindertenkommunikation 4).

Stalzer, Christian (in prep.) Negation in der Österreichischen Gebärdensprache (ÖGS). MA thesis, University of Graz.

Statistics Austria: Ein Blick auf die Gemeinde Hüttschlag <50413> retrieved June 6, 2012, from http://www.statistik.at/blickgem/blick1/g50413.pdf

Statistics Austria: Ein Blick auf die Gemeinde Großarl <50411> retrieved June 6, 2012, from http://www.statistik.at/blickgem/blick1/g50411.pdf

Stokoe, William C. (1974) Motor signs as the first form of language. In: Wescott, Roger W. \& Hewes, Gordon W. \& Stokoe, William C. (eds.) Language origins. Silver Spring, MD: Linstok Press, 35-50.

Sutton-Spence, Rachel \& Woll, Bencie (1999) The linguistics of British Sign Language. An introduction. Cambridge: Cambridge University Press.

Sze, Felix (2004) Blinks and intonational phrases in Hong Kong Sign Language. Paper presented at TISLR 8 (Theoretical Issues in Sign Language Research Conference), Barcelona, September 30 - October 2, 2004.

Šarac, Ninoslava \& Schalber, Katharina \& Alibašić, Tamara \& Wilbur, Ronnie B. (2007) Cross-linguistic comparison of interrogatives in Croatian, Austrian, and American Sign Languages. In: Perniss, Pamela M. \& Pfau, Roland \& Steinbach, Markus (eds.) Visible variation: Comparative studies on sign language structure. Berlin: Mouton de Gruyter, 207-244.

Šarac Kuhn, Ninoslava \& Wilbur, Ronnie B. (2006) Interrogative structures in Croatian Sign Language. Polar and content questions. In: Sign Language \& Linguistics 9(1/2), 151167. 
Traugott, Elizabeth C. \& Meulen, Alice ter \& Reilly, Judy S. \& Ferguson, Charles A. (1986, eds.) On conditionals. Cambridge: Cambridge University Press.

Veinberg, Silvana C. \& Wilbur, Ronnie B. (1990) A linguistic analysis of the negative headshakes in American Sign Language. In: Sign Language Studies 68, 217-244.

Vermeerbergen, Myriam (1996) ROOD KOOL TIEN PERSOON IN: Morfo-syntactische Aspecten van Gebarentaal. PhD thesis, Vrije Universiteit Brussel.

Vermeerbergen, Myriam \& Leeson, Lorraine \& Crasborn, Onno (2007) Simultaneity in signed languages: Form and function. Amsterdam: John Benjamin.

Vos, Connie De (2011) A signers' village in Bali, Indonesia. In: Minpaku Anthropology Newsletter (Where sign language studies can take us) 33, 4-5.

Watson, Katherine L. (2010) WH-questions in American Sign Language: Contributions of non-manual marking to structure and meaning. MA thesis, Purdue University.

Weast, Traci (2008) Questions in American Sign Language: A quantitative analysis of raised and lowered eyebrows. PhD thesis, University of Texas.

Wiener, Anna \& Lackner, Andrea \& Dotter, Franz \& Auersperg, Bernadette \& Dürr, Xenia \& Graf, Isabel \& Koppendorfer, Marlen \& Mallinger, Lena \& Ni, Dawei \& Riemer, Nikolaus \& Stalzer, Christian (2012) The power of the source text and the responsibilities of team members - advantages and challenges of working in a bilingual team. Presentation at the EFSLI (European Forum of Sign Language Interpreters) Conference, Vienna, September 15-16, 2012.

Wilbur, Ronnie B. (1991) Intonation and focus in American Sign Language. In: No, Yongkyoon \& Libucha, Mark (eds.) ESCOL '90: Proceedings of the Seventh Eastern States Conference on Linguistics. Columbus, OH: Ohio State University Press, 320-331.

Wilbur, Ronnie B. (1996) Evidence for the function and structure of wh-clefts in American Sign Language. In: Edmondson, William \& Wilbur, Ronnie B. (eds.) International review of sign linguistics. Hillsdale, NJ: Lawrence Erlbaum, 209-256.

Wilbur, Ronnie B. (1999) Stress in ASL: Empirical evidence and linguistic issues. In: Language and Speech 42, 229-250.

Wilbur, Ronnie B. (2000) Phonological and prosodic layering of non-manuals in American Sign Language. In: Emmorey, Karen \& Lane, Harlan (eds.) The signs of language revisited: An anthology to honor Ursula Bellugi and Edward Klima. Hillsdale, NJ: Lawrence Erlbaum, 190-214. 
Wilbur, Ronnie B. (2002) Phrase structure in ASL and Austrian Sign Language (ÖGS). In: Schulmeister, Rolf \& Reinitzer, Heimo (eds.) Progress in sign language research: In honor of Siegmund Prillwitz (Fortschritte in der Gebärdensprachforschung: Festschrift für Siegmund Prillwitz). Hamburg: Signum, 235-248.

Wilbur, Ronnie B. (2005) Evidence from American Sign Language and Austrian Sign Language (ÖGS) for asymmetries in universal grammar. In: Di Sciullo, Anna Maria (ed.) Universal grammar and external systems. Language, brain and computation. Amsterdam: John Benjamins, 193-212.

Wilbur, Ronnie B. (2006) Discourse and pragmatics in sign language. In: The Encyclopedia of Language and Linguistics - $2^{\text {nd }}$ Edition (EEL2) 11. Oxford, England: Elsevier, 303307.

Wilbur, Ronnie B. (2009) Effects of varying rate of signing on ASL manual signs and nonmanual markers. In: Language \& Speech 52(2-3), 245-285.

Wilbur, Ronnie B. \& Patschke, Cynthia G. (1998) Body leans and the marking of contrast in American Sign Language. In: Journal of Pragmatics 30, 275-303.

Wilcox, Phyllis P. (1996) Deontic and epistemic modals in ASL: A discourse analysis. In: Adele E. Goldberg (ed.) Conceptual structure, discourse and language. Cambridge: Cambridge University Press, 481-492.

Wilcox Sherman (2004) Gesture and language: Cross-linguistic and historical data from signed languages. In: Gesture 4(1), 43-73.

Wilcox, Sherman \& Shaffer, Barbara (2006) Modality in ASL. In: Frawley, William (ed.) The expression of modality. Berlin: Mouton de Gruyter, 207-238.

Wilcox, Sherman \& Wilcox, Phyllis (1995) The gestural expression of modality in ASL. In: Bybee, Joan L. \& Fleischman, Suzanne (eds.) Modality in grammar and discourse. Amsterdam: John Benjamins, 135-162.

Wilcox, Sherman \& Wilcox, Phyllis P. (2010) The analysis of signed languages. In: Heine, Bernd \& Narrog, Heiko (2010a) The Oxford handbook of linguistic analysis. Oxford: Oxford University Press, 739-760.

Wilson, Deirdre \& Sperber, Dan (1988) Mood and the analysis of non-declarative sentences. In: Dancy, Jonathan \& Moravcsik, Judith M. E. \& Taylor, Christopher C. (eds.) Human agency: Language, duty, and value. Philosophical essays in honor of J. O. Urmson. Standford: Standford University Press, 77-101. 
Wood Sandra K. (1996) Nose wrinkles in ASL: A discourse particle for co-construction. Paper presented at the Annual Meeting of the American Association for Applied Linguistics (AAAL), Chicago, Illinois, March 23-26, 1996.

Wouden, Ton van der (1994) Negative contexts. PhD Thesis, University of Groningen.

Zeshan, Ulrike (2004) Interrogative constructions in sign languages: Cross-linguistic perspectives. In: Language 80(1): 7-39.

Zeshan, Ulrike (2006a) Interrogative and negative constructions in sign languages (Sign language typology series no. 1). Nijmegen: Ishara Press.

Zeshan, Ulrike (2006b) Negative and interrogative constructions in sign languages: A case study in sign language typology. In: Zeshan, Ulrike (ed.) Interrogative and negative constructions in sign languages (Sign language typology series no. 1). Nijmegen: Ishara Press, 28-68.

Zeshan, Ulrike (2006c) Negative and interrogative structures in Turkish Sign Language (TID). In: Zeshan, Ulrike (ed.) Interrogative and negative constructions in sign languages (Sign language typology series no. 1). Nijmegen: Ishara Press, 128-164. 